\title{
ENERGY EFFICIENCY OF DISTRIBUTED ENVIRONMENTAL CONTROL SYSTEMS
}

\section{Final Report}

Prepared by Syracuse University

Under US DOE Contract Number:

DE-FG02-03ER63694

June 30,2006 


\section{PREFACE}

This report is the result of a research effort sponsored by the US Department of Energy under Grant No. DE-FG02-03ER63694 to Syracuse University. Professor H. Ezzat Khalifa is the Principal Investigator; Professors Can Isik and John Dannenhoffer are co-principal investigators, all of the College of Engineering and Computer Science at Syracuse University. The research team also included Professor Peter Wilcoxen of the Syracuse University Maxwell School, and three engineering graduate students who performed the analytical and computational work under the supervision of Professors Khalifa, Isik, Dannenhoffer and Wilcoxen, namely: Mr. Ian Cosden who developed and implemented the thermal model of the building and its environmental control system; Mr. Seckin Ari who developed the comfort model and performed the optimization and control analyses; and Mr. Xuanhang (Simon) Zhang, who developed the CFD model of intra- and inter-cubicle air and energy transport and refined the thermal and comfort models accordingly. 


\section{A. ABSTRACT}

Recent research has indicated that allowing building occupants to customize their own local environment increases satisfaction and workplace performance. However, concern about the possible increase of energy consumption associated with the implementation of distributed localized environmental control has limited the widespread adoption of such systems. In this report, we present an analytical evaluation of the potential of occupant-regulated distributed environmental control systems (DECS) to enhance individual occupant thermal comfort in an office building with no increase, and possibly even a decrease in annual energy consumption. To this end we developed and applied several analytical models that allowed us to optimize comfort and energy consumption in partitioned office buildings equipped with either conventional central HVAC systems or occupant-regulated DECS. Our approach involved the following interrelated components:

1. Development of a simplified lumped-parameter thermal circuit model to compute the annual energy consumption. This was necessitated by the need to perform tens of thousands of optimization calculations involving different US climatic regions, and different occupant thermal preferences of a population of $\sim 50$ office occupants. Yearly transient simulations using TRNSYS, a time-dependent building energy modeling program, were run to determine the robustness of the simplified approach against time-dependent simulations. The simplified model predicts yearly energy consumption within approximately $0.6 \%$ of an equivalent transient simulation. Simulations of building energy usage were run for a wide variety of climatic regions and control scenarios, including traditional "one-size-fits-all" (OSFA) control; providing a uniform temperature to the entire building, and occupantselected "have-it-your-way" (HIYW) control with a thermostat at each workstation. The thermal model shows that, un-optimized, DECS would lead to an increase in building energy consumption between 3-16\% compared to the conventional approach depending on the climate regional and personal preferences of building occupants. Variations in building shape had little impact in the relative energy usage. The model also allowed us to study the effect of the thermal resistance of the partitions between adjacent workstations, which can have a significant influence on the energy cost (3\% to $78 \%$ increase), absent full system optimization.

2. Development of a gradient-based optimization method to minimize energy consumption of DECS while keeping each occupant's thermal dissatisfaction below a given threshold. The DECS energy usage was calculated using the aforementioned simplified thermal model. Traditional OSFA control; providing a uniform temperature to the entire building, and occupant-selected HIYW control with a thermostat at each workstation were implemented for 3 different cities representing 3 different climatic regions and control scenarios. It is shown that optimization allows DECS to deliver a higher level of individual and population thermal comfort while achieving annual energy savings between 14 and 26\% compared to OSFA, depending on the climate region and personal preferences of building occupants. The coupled comfort-energy consumption optimization model also allowed us to study the influence of the partitions thermal resistance and the variability of internal heat loads at each office. These influences didn't make significant changes in optimized DECS energy consumption relative to conventional OSFA systems. The results have shown that it is possible to provide thermal comfort for each occupant of a building while saving energy compared to a traditional system. Furthermore, in order to simplify the implementation of 


\section{Report No. DOE $\backslash$ ER63694-1}

this distributed control approach, a fuzzy logic system has been developed to generalize the overall optimization strategy of the gradient-based system. Its performance was almost as good as the gradient system, while avoiding its implementation difficulties. That is, the fuzzy logic system provided thermal comfort to each occupant, and saved energy compared to a traditional OSFA system. The energy savings of the fuzzy logic system were not as high as that of the gradient-optimized system, but in return, the fuzzy logic system avoided the complete connectivity requirement of the optimum system, and the optimization did not have to be repeated for each population.

3. In the third component of our research, we employed a detailed CFD model of adjacent occupied cubicles to extend the abovementioned thermal-circuit model in three significant ways: (a) relax the "office wall" requirement by allowing energy to flow between zones via advection as well as conduction, (b) improve the comfort model to account both for radiation as well as convection heat transfer, and (c) support ventilation systems in which the temperature is stratified, such as in underfloor air distribution systems. Initially, threedimensional CFD simulations of several cubicle configurations, with an adjoining corridor, were performed both to understand the advection between cubicles and the resulting temperature stratification. These simulations showed that the advective flow between cubicles is very significant and severely limits the occupants' ability to control the personal micro-environments by simply controlling the temperature of the incoming air. Subsequently, the existing thermal-circuit model (see item 1 above) was extended to include the phenomena described above. The modifications to the thermal-circuit model, which were incorporated such that the simulation time was only slightly impacted, showed that accounting for room stratification resulting from the use of floor swirl diffusers could lead to $10 \%-26 \%$ reduction in the annual energy consumed for HVAC in non-temperate climates. This trend was evident in both OSFA and HIYW scenarios. However, the ratio of energy usage in the two scenarios was little affected by the enhancements in the thermal model.

In a separate project, work is currently underway to verify experimentally the findings of the CFD model and to incorporate the improved thermal model of item 3 in the optimization model of item 2 above. 


\section{B. REPORT SUMMARY}

\section{BACKGROUND}

Current central HVAC systems are designed to satisfy the thermal comfort needs of $80 \%$ of building occupants ${ }^{1}$. These "one-size-fits-all" (OSFA) designs contribute to thermal discomfort and suboptimum indoor environmental quality (IEQ) [1]. Annual productivity loss resulting from poor IEQ in large office buildings has been estimated as high as $\$ 250 \mathrm{~B}$ [3]. Occupant satisfaction and performance can be markedly improved by providing local environmental control [4-7]. However, if not specifically addressed within the overall system optimization, such decentralized control could result in higher energy consumption. The strong link between IEQ and building energy consumption is highlighted in "A priority agenda for energy related indoor environmental quality research" [8].

The complexity of HVAC systems in large buildings presents formidable challenges in providing local environmental control. Acceptable IAQ within these buildings is traditionally maintained through dilution of recirculated air with outside air in accordance with the ventilation procedure of ASHRAE Standard 62 [9]. However the use of large amounts of outside air for contaminant dilution results in higher energy consumption, and would be ineffective when outdoor air quality is poor. Ventilation systems in most of these buildings are of the mixing type, contributing to rapid dispersion of contaminants from localized sources, and higher energy consumption (they condition both occupied and unoccupied spaces within the same zone). Mixing type ventilation systems also make it impracticable to provide local environmental control within connected spaces. Displacement and localized ventilation systems have been proposed to overcome the shortcomings of mixing-type ventilation systems $[10,11]$. For example, underfloor air distribution systems promise improved comfort and lower energy consumption and facilitate individual control [12, 13]. Localized (Task) ventilation systems include Johnson Controls' "Personal Environments" system, which provides individual workers with controllable environmental conditions, resulting in a 3-15\% performance improvement after installation [4, 5], and Centercore developed the Airflow system, a user-controllable fan-filter that improves IAQ as well as user satisfaction, health, and performance $[6,7]$.

Task ventilation systems that regulate only air flow and direction and rely on central temperature and humidity control systems to condition air supply, are not likely to achieve full benefit in human performance possible by local environmental control. Enhanced human comfort, satisfaction and performance could be achieved if local control also extends to temperature and humidity, e.g., [14]. However, if not optimized within the framework of an integrated building system, such decentralized local environmental control may result in increased energy consumption, particularly in the case of high occupant density within contiguous or partially connected spaces, e.g., an open classroom or a cluster of office cubicles. In an office building partitioned into offices, cubicles, or other semi-open work spaces, individual control would result in significant cross-flows between adjacent spaces as a result of the varying adjustments of the conditioned air supply or temperature to suit the preferences of the individual occupants. With individual control of ventilation and the thermal environment in connected, partitioned or semi-open work spaces (e.g., cubicles),

\footnotetext{
${ }^{1}$ Percentage of dissatisfied occupants may be as high as 30\% [1].
} 
imbalances in supply air flow and temperature may cause significant fluxes of air, contaminants and energy between adjacent cubicles. These airflows would negate some of the benefits of individual control. The presence of these energy fluxes in occupant controlled scenarios has lead some to suspect that that the wide use of DECS would increase building energy consumption for HVAC.

The essential enablers of this "have it your way" (HIYW) vision is the development of distributed environmental control systems (DECS), by which many interacting (coupled) personal microenvironments $(\mathrm{P} \mu \mathrm{Es})$ within a building comprising a very large number of such $\mathrm{P} \mu \mathrm{Es}$ can be optimized for increased thermal comfort without increasing the building's overall energy consumption. Prior research has focused largely on a "one-size-fits-all" (OSFA) central approach that left a large fraction of the building occupant either dissatisfied or at a higher risk of exposure to harmful pollutants. Nowadays, increasing attention is being paid to detailed studies of the $\mathrm{P} \mu \mathrm{E}$, e.g., $[15,16]$, but little work is done on the energy and comfort implications of their interaction with the context of a complex, integrated built environmental system - BES remains largely unexplored $[17,18]$.

\section{Project Scope}

The underlying hypothesis of the research described in this report is that considerable gains in human performance and satisfaction can be obtained through "mass-customization" of the individuals' personal micro-environment $(\mathrm{P} \mu \mathrm{E})$. The challenge in providing individuals with a wider range of control of their personal thermal microenvironment while optimizing the entire system for higher occupant thermal satisfaction and lower energy consumption is a formidable one. An assessment of the energy efficiency implications of distributed environmental control systems (DECS) within typical light commercial and institutional buildings is the aim of the study documented in this report.

This project is focused on analytical and computational evaluations of individual thermal comfort and energy consumption in typical partitioned office suites (e.g., Fig. 1), under two environmental control scenarios:

1) conventional central HVAC control in which the entire office suite is controlled by a small number of thermostats adjusted to achieve a uniform average thermal environment for all occupants. We designate this scenario "one size fits all” (OSFA);

2) distributed environmental control (DECS) in which each individual is allowed to regulate his/her thermal environment based on personal preferences. We designate this scenario as "have it your way" (HIYW).

In both scenarios, cooling is provided by an electric vapor-compression system and heating by a gas-fired furnace. It is also assumed that each individual has a characteristic thermal preference defined, a priori by a preferred neutral temperature and a temperature tolerance range. Effects of humidity, draft, asymmetrical radiation, temperature gradients, actual or perceived air quality, and a host of other environmental factors that affect comfort and energy consumption were not considered. These could be included in future studies of DECS.

By adopting a HIYW rather than a OSFA environmental control strategy, system complexity, as measured by the number of degrees of freedom, increases considerably (Fig. 2), necessitating the use of intelligent optimization and control methodologies to achieve 
improved thermal comfort without increasing energy consumption. This is primary motivation for the present investigation.

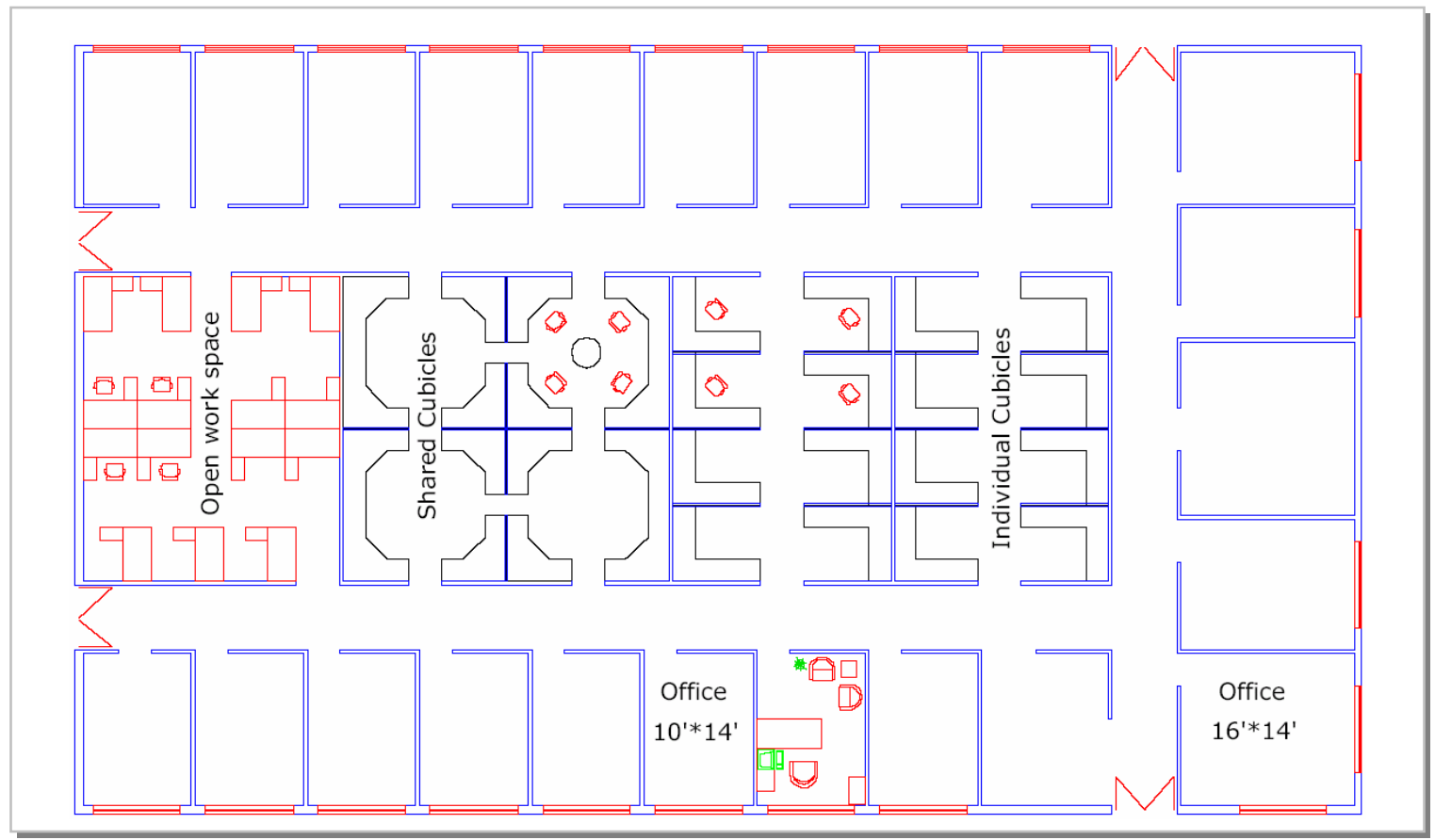

Figure 1. Typical Office Floor with Private Offices, Cubicles and Open Workspace

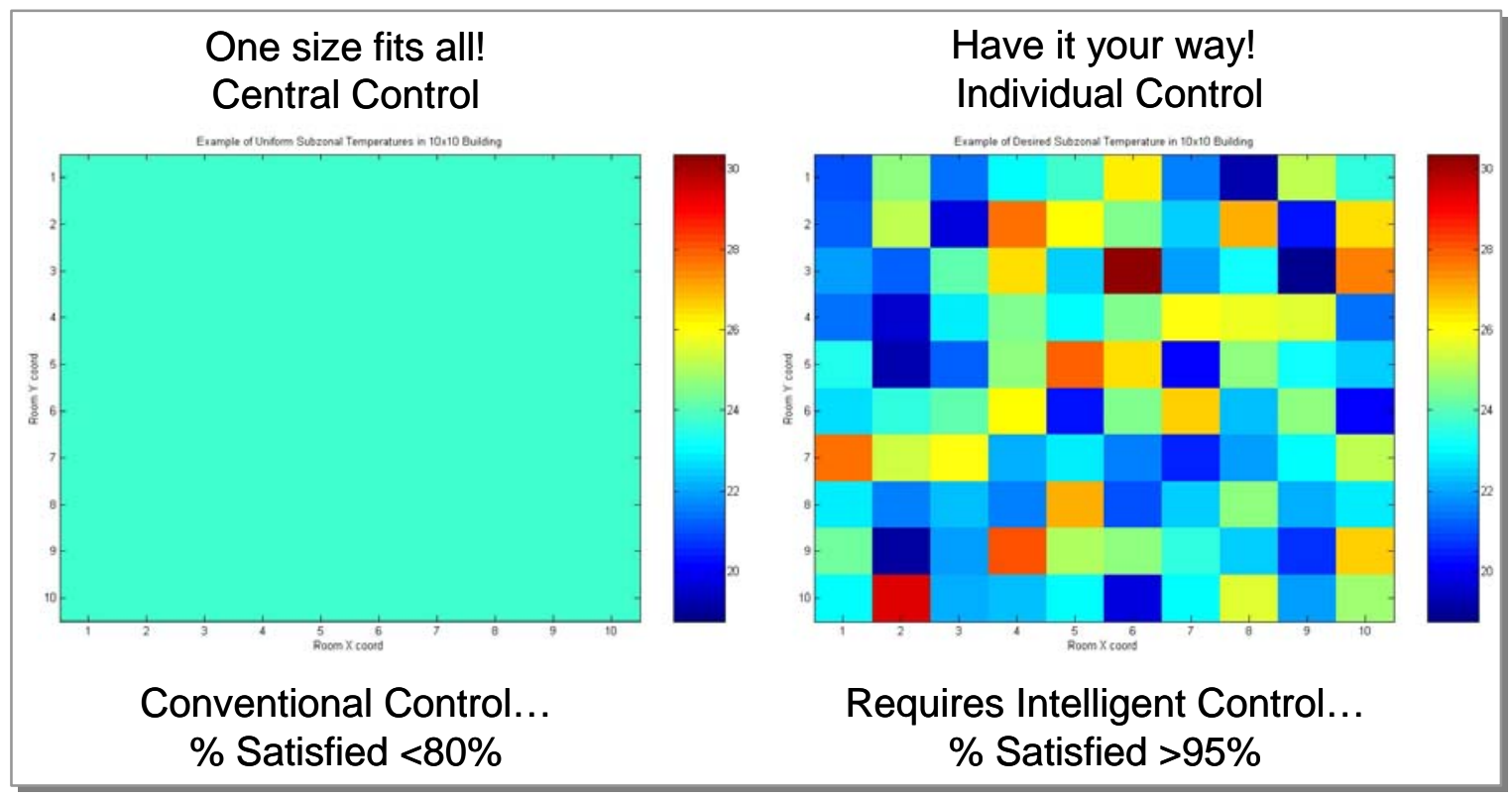

Figure 2: OSFA vs HIYW Scenarios

\section{ReleVANCE to DOE}

DOE has long recognized that building IEQ and energy consumption are intimately related [19]. In the US, buildings account for over one-third of the total energy consumption and building HVAC is close to $45 \%$ of that [20]. Given the strong influence of IEQ on human 
health and performance [3], and the importance of energy conservation to the national economy and environmental sustainability, it is important to develop building environmental control systems that enhance IAQ and comfort while reducing energy consumption. However, these two imperatives do not always go hand in hand; IEQ enhancement practices may increase or decrease energy consumption and energy conservation measures may result in improved, worsened or neutral IEQ [8].

This project addresses a class of building environmental control systems that has shown a significant promise in improving IEQ through distributed local control, yet received little attention insofar as their impact on overall energy consumption, and their optimal integration into densely populated commercial buildings. Advancing the technology of energy efficient DECS would support the dual DOE objectives of improved IEQ and lower energy consumption for building environmental control.

\section{Project Objective}

The objective of this project is twofold:

a) to quantify the HVAC energy efficiency for buildings equipped with occupantregulated distributed environmental control systems (DECS) relative to the same buildings when equipped with conventional central HVAC systems,

b) to develop optimization and control strategies that optimize overall energy consumption and improve individual thermal comfort in DECS-equipped typical office buildings.

A further goal of this work is to identify technical barriers and future research needs for DECS-equipped buildings.

\section{TECHNICAL APPROACH}

In order to fulfill the project objectives, we conducted a research effort organized into three interrelated technical thrusts, namely:

\section{Development of a Simplified Building Thermal Model}

We developed a simplified lumped parameter thermal circuit model for an office building that can be controlled by any number of thermostats, from one to as many as there are rooms. The model was developed so that it can be executed very rapidly in order to allow its use in gradient and fuzzy optimization techniques that require tens of thousands of repeated calls to the energy calculation routine for several US climatic regions and occupant thermal preferences (thermostat settings). Yearly transient simulations using TRNSYS ${ }^{2}$, a timedependent building energy modeling program were found to be very slow for repeated use in the comfort/energy optimization calculations. Consequently, TRNSYS was used only as a check on the robustness of the simplified model against time-dependent simulations. The annual energy consumption in the simplified model was based on a modified temperaturebin method, whereas the TRNSYS simulations were based on hourly typical meteorological

\footnotetext{
${ }^{2}$ DOE2 or Energy + could have been used for the same purpose but we opted for TRNSYS because of its well developed user interface.
} 
year weather data. Both were run for a range of climatic conditions, spanning the spectrum from cold (heating-dominated), to moderate (temperate), to hot (cooling-dominated).

In order to compute the annual energy consumption of the building and optimize its operation for IAQ, comfort and energy efficiency, it is necessary to incorporate models of the performance characteristics of the HVAC systems ${ }^{3}$ under varying indoor (zone) and outdoor conditions, and under various control settings. Therefore, the building thermal model included a representation of commercially available HVAC system performance as a function of indoor and outdoor conditions, as well as the effect of HVAC system cyclic degradation for part-load (off-design) operation. The HVAC system model also included the option to use an economizer when the ambient conditions permit the use of this energy saving feature.

The simplified thermal model accounts for differences in external envelope and internal partition thermal resistance, building configuration (e.g., floor aspect ratio), supply air flow rate, internal loads, and thermostat settings. This model, and the results obtained from it are described in detail in Section $\mathbf{C}$ of this report.

\section{Development of DECS Optimization and Control Strategies}

Control of advanced HVAC systems can be viewed as a multi-criteria optimization problem. Some of these criteria are objective, such as energy consumption and indoor air quality. Such variables are measurable in a physical setting, and computable in numeric implementations. Some are subjective, and difficult to quantify, such as individual comfort.

It has been established that as a result of a traditional central control, about $80 \%$ of the occupants are satisfied on average. If a hypothetical distributed control system provides to each occupant the exact environment they desire, then close to $100 \%$ of the occupants would be satisfied. One class of control strategies views the individual selections of environmental variables such as the thermostat setting as inputs to an optimization problem, rather than absolute targets to be met. Then, the distributed control of the HVAC can be formulated as finding a solution (a distribution of target HVAC variables) that satisfies a predetermined percentage of the occupants (between $80 \%$ and $100 \%$ ) at the smallest possible energy cost. In this preliminary study we evaluated both gradient-based algorithms and genetic algorithms [21] and selected the former for its computational efficiency.

The starting point of the system optimization effort was the development of a computational statistical model of the response (comfort) of an individual to the thermal environment (characterized by the dry-bulb temperature only). The thermal comfort model was calibrated such that it produces the same population statistics as the thermal comfort model specified in ASHRAE Standard 55 (based on Fanger's work) [22]. This thermal comfort model, along with the abovementioned simplified thermal model have been integrated into optimization routines (gradient method and fuzzy rules) and used to study the energy consumption and predicted percent dissatisfied (PPD) of a population of 49 office workers in a variety of climatic conditions and building thermal properties (wall and partition thermal resistances, ventilation rate, etc). Initial results indicate that it is possible to optimize the operation of a building equipped with DECS for both reduced energy

\footnotetext{
${ }^{3}$ We use the term "system" here to include both the air-side and the "refrigerant" or heat transfer fluid side of the HVAC system; each side will be treated as a coupled subsystem of the overall HVAC system.
} 
consumption and decreased PPD. The energy and comfort models, coupled with the control strategies yield predictions of the overall HVAC system (DECS) annual energy consumption for a given occupancy profile (distribution of occupants with differing thermal preferences) and use schedule.

To assess the sensitivity of the predicted performance statistics (that is, objectives and constraints) to the arrangement of building occupants (each with his/her own optimal comfort setting), we ran several $(\sim 50)$ full simulations, each with a different random arrangement of building occupants. The performance statistics from each of the runs are then combined to determine the sensitivity of these statistics, including worst-case, best-case, and average behaviors. For each case metrics of energy efficiency and comfort were computed, e.g. relative annual energy consumption and predicted percent dissatisfied (PPD) [22]. Because DECS control algorithms need to run in real time, on platforms with modest computational power, neural network and fuzzy logic approximations have been investigated and applied as reduced order modeling tools in the development of the DECS control algorithm [23].

Detailed description of this thrust are given in Section $\mathbf{D}$ of this report.

\section{Refinement of the Thermal Model Through CFD Simulations}

In the third thrust of our research, we employed a detailed CFD model of adjacent occupied cubicles to extend the abovementioned thermal-circuit model in three significant ways: (a) relax the "office wall" requirement by allowing air and energy to flow between zones via advection as well as conduction, (b) improve the comfort model to account both for nonuniform radiation as well as convection heat transfer from the human body (rather than assume that the body is exposed to the uniform temperature of well mixed room air, and (c) model ventilation systems in which the temperature is stratified, such as in underfloor air distribution systems. Initially, three-dimensional CFD simulations of several cubicle configurations, with an adjoining corridor, were performed both to understand the advection between cubicles and the resulting temperature stratification. This effort was motivated by indications that significant advective flows between adjacent cubicles would defeat the purpose of HIYW control, i.e., limit the occupants' ability to control the personal microenvironments by regulating the temperature of the incoming air. Subsequently, the existing thermal-circuit model (see item 1 above) was modified and extended to include the phenomena described in items (a), (b) and (c) above.

The simulations were performed using the commercially-available CFD solver FLUENT. The simulations were performed for a pair of adjacent cubicles sharing a corridor using the $\mathrm{k}-\varepsilon$ turbulence model. Each cubicle was equipped with an L-shaped desk, heated blocks representing a seated person, a computer and a task lamp, a floor-mounted swirl diffuser, and a ceiling exhaust vent.

Detailed description of this thrust are given in Section $\mathbf{E}$ of this report.

\section{Results AND ConClusions}

The following paragraphs are organized into the same three thrusts described above in the Technical Approach: 


\section{Development of a Simplified Building Thermal Model}

The lumped parameter, TCTB model, presented in this study provided us with an accurate and quick method to simulate yearly building energy usage. From the comparison with TRNSYS we were able to determine that, despite neglecting transient effects, for a yearly simulation the TCTB model predicted energy usage within approximately $0.6 \%$ of the transient TRNSYS solution (without solar radiation), while executing in one six-hundredth of the time. The effect of solar radiation was neglected, and proved to be a minor assumption (approximately 3\% energy difference over a yearly simulation) when the direct beam radiation through the windows was removed. The TCTB model's major advantage over the transient TRNSYS simulations is its significantly faster execution time.

The TCTB model was able to run a yearly simulation for the two control strategies (HIYW and OSFA) for all 15 cities in approximately thirty seconds. Running the equivalent TRNSYS simulations would require 30 independent simulations each requiring between ten and fifteen minutes of computation time. This would make running large-scale optimization or parametric studies using a TRNSYS nearly impossible in a reasonable time frame. The lumped parameter approach was ideal for the exploration of energy costs in buildings equipped with distributed environmental control systems.

The cost associated with the adoption of a HIYW approach to environmental control is dependent on a number of factors including: climate region, interior partition thermal resistance, building inhabitants preferences, and to a lesser extent building shape (excluding solar heat gain, which depends strong on building shape and orientation). The remainder of the results and conclusions in this thrust are based on analyses without comfort and energy optimization.

At extreme temperatures, both hot and cold, the heat transfer associated with the small internal temperature deviations between offices in a building becomes insignificant compared to the heat transfer with the outside ambient air. The opposite is true for mild outdoor temperatures. At these temperatures the building energy usage is lowest. Internal heat transfer between offices at different temperatures becomes more significant and has an appreciable effect on building energy use. This is further impacted by the presence of an economizer. In the HIYW case, the economizer is unable to meet the cooling load of the building at outdoor temperatures at which it can in the OSFA case.

The climate in which a building is located is a major factor in the cost associated with HIYW compared to OSFA. Cities, and their corresponding climate zones, with a significant portion of the year having extreme temperatures (Miami, Phoenix, Fairbanks, etc.) show the lowest increase in yearly energy usage when switching to HIYW control (between 2-6\%). It is in these cities that adoption of personal environmental control systems appears to be the best choice. Cities with a moderate climate, such as San Francisco, show the largest increase in yearly energy usage, ranging from $10-20 \%$ and would be the least practical to implement personal control. The majority of the US population lives in climates that fall between these two extremes and would likely see a $5-10 \%$ increase with the adoption of personal control.

The thermal resistance of the interior partition of a building also greatly affects the energy cost associated with HIYW. Thermal resistances ranging from partial height cubicles to full office walls, appear to provide sufficient resistance to internal heat transfer to keep building energy usage within reasonable limits. However, open workspaces, where there are no physical boundaries between personal zones, may create significant internal heat transfer that 


\section{Report No. DOE $\backslash$ ER63694-1}

translates to increased energy costs. However, the well-mixed model assumption is a poor representation of the physical problem and more research must be done to determine true energy transfer characteristics of such open office spaces.

The impact of the randomness of the personal preferences of building occupants varies depending on the climatic location of the building. In most cities the random populations fell within approximately $3-10 \%$ increase for common interior walls and a $4-15 \%$ increase for partial height partitions. For a building in San Francisco, or a comparable climate, the impact of the building occupants' desired temperatures was significantly larger. The HIYW energy usage premium ranged from $10-35 \%$ for common interior walls and $23-78 \%$ for partial height partitions depending on the specific occupants within the building. Regardless of building occupants, in every case, except two populations, HIYW used more energy over the course of a year than OSFA. The only two instances where HIYW saved energy were in extreme climates (Miami and Fairbanks) for an unusually fortunate arrangement of building inhabitants. In both these cases the yearly savings was less than 1\%. For all other arrangements and in every other city HIYW resulted in an increase in energy usage, in some cases as high as $78 \%$ more than OSFA. A summary of the unoptimized results for several cities and a 100 office populations is presented in Fig. 3.

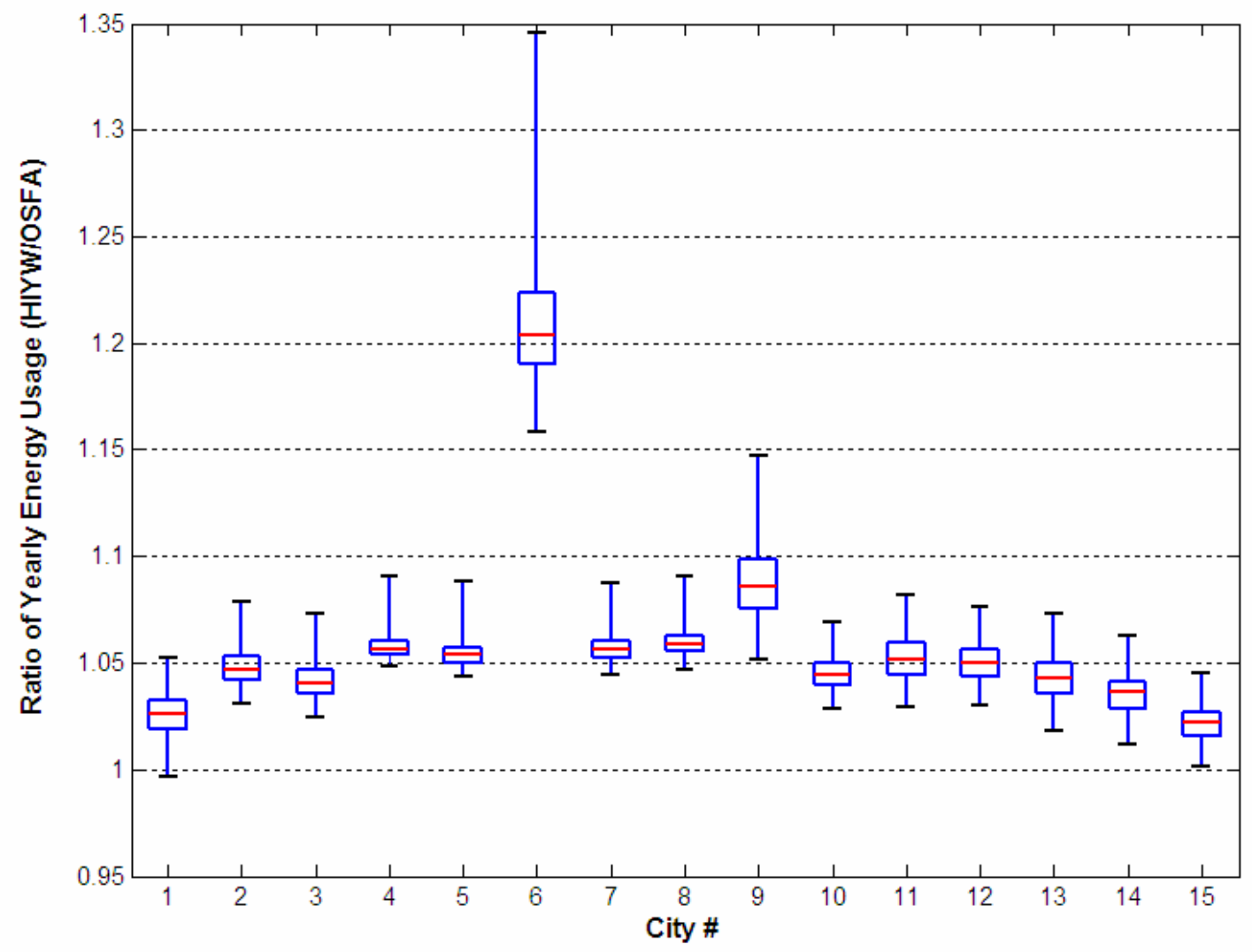

Figure 3: Comparison of non-optimized OSFA and HIYW Scenarios (100 populations)

Building shape appears to have a very small influence on the relative cost between HIYW and OSFA. Most office buildings tend to be rectangular with at least some core offices or 
cubicles. Small changes in the aspect ratio of the building have a negligible effect on HIYW costs. Even drastic changes, such as a corridor of only exterior offices, actually slightly lowered the energy penalty related to the adoption HIYW.

By providing the building occupants' with slightly different temperatures than their desired perfect temperature, energy savings can be realized. No longer will a personalized approach to environmental control result in increased yearly energy costs. A small $1{ }^{\circ} \mathrm{C}$ deviation from the occupants' neutral temperature can help create an energy savings from OSFA in nearly every city, while still catering to the occupants' desires. This promising result has been explored in other studies by the author and others. It was shown that using advanced optimization techniques to intelligently adjust the occupants' temperature set points, optimized HIYW can result in an energy savings and a better overall thermal satisfaction than OSFA.

Without optimization, the increase in energy associated with HIYW compared to OSFA in the worst case scenario was approximately $78 \%$. This corresponded to the worst of 100 populations in a partial height partitioned building in San Francisco.

The TCTB model can be improved in the future to include even more physics, especially solar radiation and humidity effects. The temperature bin approach could be expanded to some form of three dimensional weather bins to include humidity as well as dry bulb temperature. The effect of solar radiation on building energy use is large when windows are involved and would be beneficial to include in the TCTB model, either directly or through some correlation with the outdoor temperature. If it is possible to correlate the solar gain on a vertical surface with different azimuths, a correction factor could be added to the dry bulb temperature. A new effective temperature could then be used for the outer surfaces and windows. Shading factors could then be calculated and a better approximation for windows exposed to solar gains could be made. More work must to be done in an effort to understand and quantify the energy flux between partial height partitions. Field testing and experimental office settings may provide some of the answers and help to further validate the computed results.

\section{Development of DECS Optimization and Control Strategies}

This study has shown that, through optimization and intelligent control, it is possible to provide improved thermal comfort to all occupants of a building with no increase in energy consumption. In fact, our analysis indicates that it would be possible to increase thermal comfort while achieving energy savings. More specifically, in this study, a methodology called Optimized HIYW has been developed that takes advantage of an individual's range of insensitivity to small deviations from his/her preferred temperature setting, and minimizes the annual energy consumption for environmental control in a building subject to a maximum dissatisfaction level constraint for all individual occupants.

A straightforward gradient based optimization, as well as a fuzzy logic generalization of the underlying principles of the optimum solutions have been used as alternative implementations. Both results have been compared with a traditional OSFA solution to demonstrate their improvements in both thermal comfort and energy consumption (Fig. 4).

The numeric results about individual dissatisfactions showed that both HIYW and fuzzy logic approximation provide a Degree of Individual Dissatisfaction (DID) level of no more than $20 \%$ for each occupant. The OSFA method, on the other hand, does not guarantee any 
level of individual thermal comfort. For instance, for Phoenix, AZ, OSFA is likely to cause the DID level to exceed the $20 \%$ limit for $\sim 15 \%$ of the occupants, and to exceed $50 \%$ for $\sim 5 \%$ of the occupants. Similar violations of individual comfort levels by the OSFA method were observed for other cities as well (Fig. 5).

Occupants' comfort has been improved while reducing energy consumption by optimized HIYW system. While HIYW approach requires the use of all sensor network connectivity in the building, it is not required in fuzzy logic approximation. Reduction of sensor connectivity would not only reduce system complexity, but also cause modest decrease in energy savings relative to fully connected HIYW system.

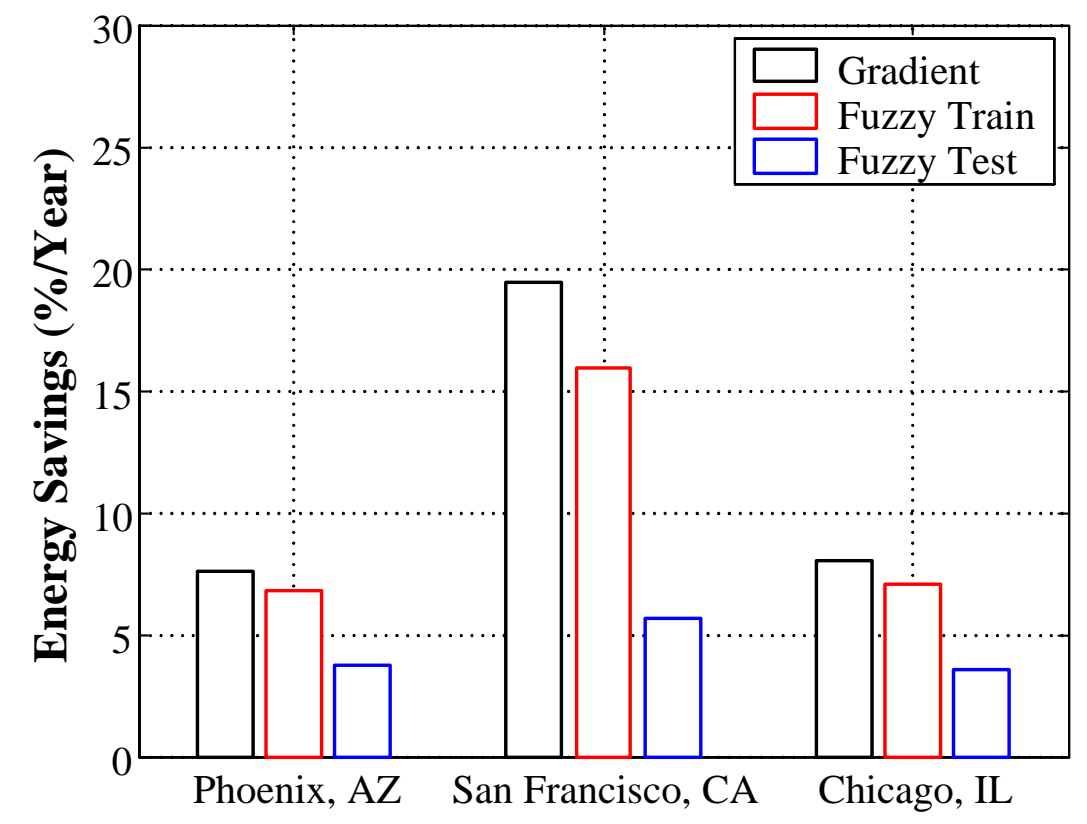

Figure 4: HIYW Annual Energy Savings Relative to OSFA.

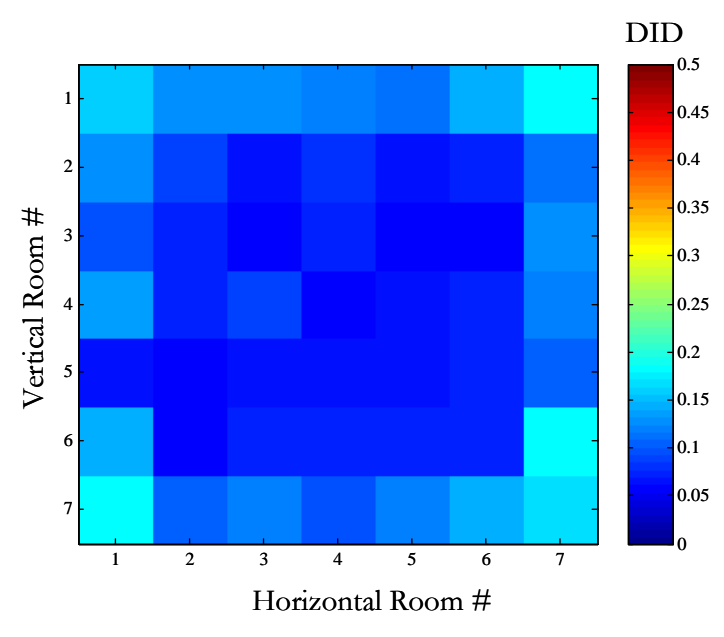

HIYW

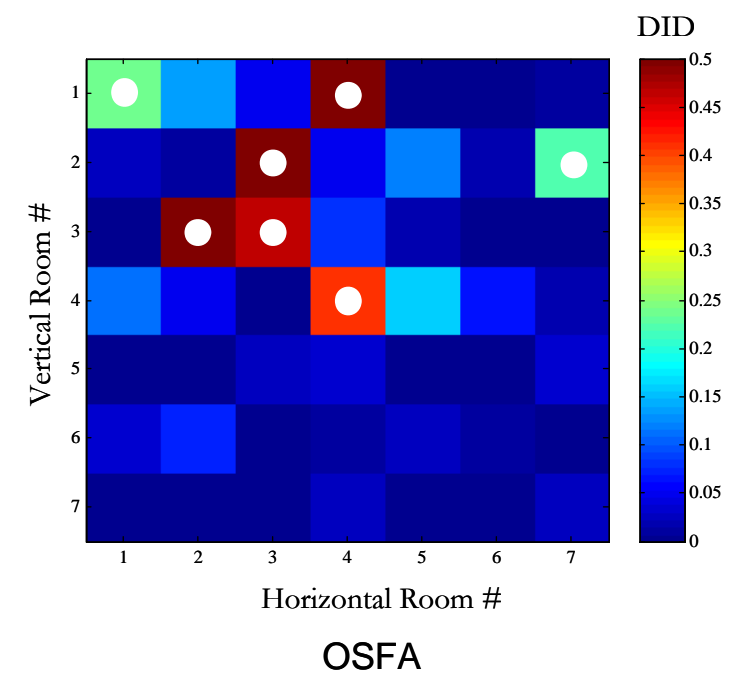

OSFA

Figure 5: Comparison of Degree of Individual Dissatisfaction 
Optimal results for lower internal resistances, and non-uniform heat loads are also encouraging to apply HIYW system in real world applications. There is always between trade-off between energy consumption and thermal comfort. However, while optimized HIYW make occupant reasonably satisfied within an acceptable temperature range, it also improves the cost of the system under the different thermal conditions such as outside temperature, desired temperatures of occupants, heat loads in offices, and resistance values of internal walls.

\section{Refinement of the Thermal Model Through CFD Simulations}

For two cubicles and an adjoining corridor, the advective flow between zones is very significant and severely limits the occupants' ability to control the personal microenvironments via inlet temperature control. CFD calculations were performed for pairs of cubicles with an adjoining corridor in which the supply temperatures in the two cubicles differed by up to $8{ }^{\circ} \mathrm{C}$.

In cases where the air was allowed to freely traverse through the corridor, the temperatures "felt" by the occupants in the two cubicles differed by less than $25 \%$ of the supply temperature difference.

Flow visualization of the CFD results indicated that this loss of personal temperature control was caused by the cold supply flow sinking to the floor and traversing through the corridor into the other cubicle; at the same time, the warm supply in the other cubicle ascended into the space above the cubicles and had little effect on the temperatures "felt" by the occupant (Fig. 6). This cross flow counteracts the purpose of a "HIYW" and must be controlled to achieve a higher degree of individual control of one's own environment.

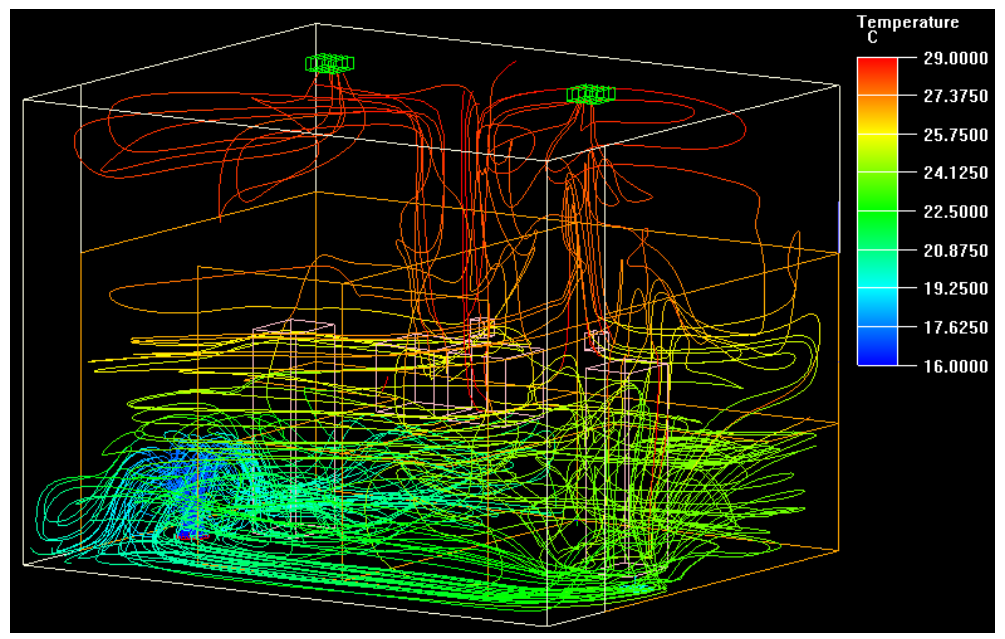

Figure 6: Corridor Cross-flow between Adjacent Cubicles

Through a series of parametric studies, it was found that the effectiveness of personal environmental control could best be improved by installing doors at the openings between the cubicles and the corridor; other strategies, such as changing the height and insulation of the cubicle walls, changing the configuration of the cubicles, and altering the swirl direction and location of the inlet diffusers had a less significant effect on the control of the person temperature in the two cubicles (Fig. 7). 
A thermal comfort model that accounts for stratified temperature profiles and radiation is required for configurations that employ an underfloor air distribution (UFAD) system. While Section C's original thermal circuit (TC) model is suitable for predicting the annual energy consumption of buildings with mixing ventilation, its well-mixed assumption is not valid for the stratified flows associated with UFAD. In the current work, detailed CFD simulations were performed to predict the vertical air temperature profiles associated with UFAD, and a simple model was developed to approximate these profiles.

The thermal circuit model was subsequently modified to account for the thermal stratification, both for the temperature experienced by the occupant and also for the energy transfer between zones via convection and conduction. Additionally, Section C's model was modified to include a thermal comfort model that accounts both for convection/conduction and radiation to/from the occupant. Care was taken to include these additional physical phenomena into Section C's model via linear relationships, and thus had a very small impact on the overall execution time of the model.

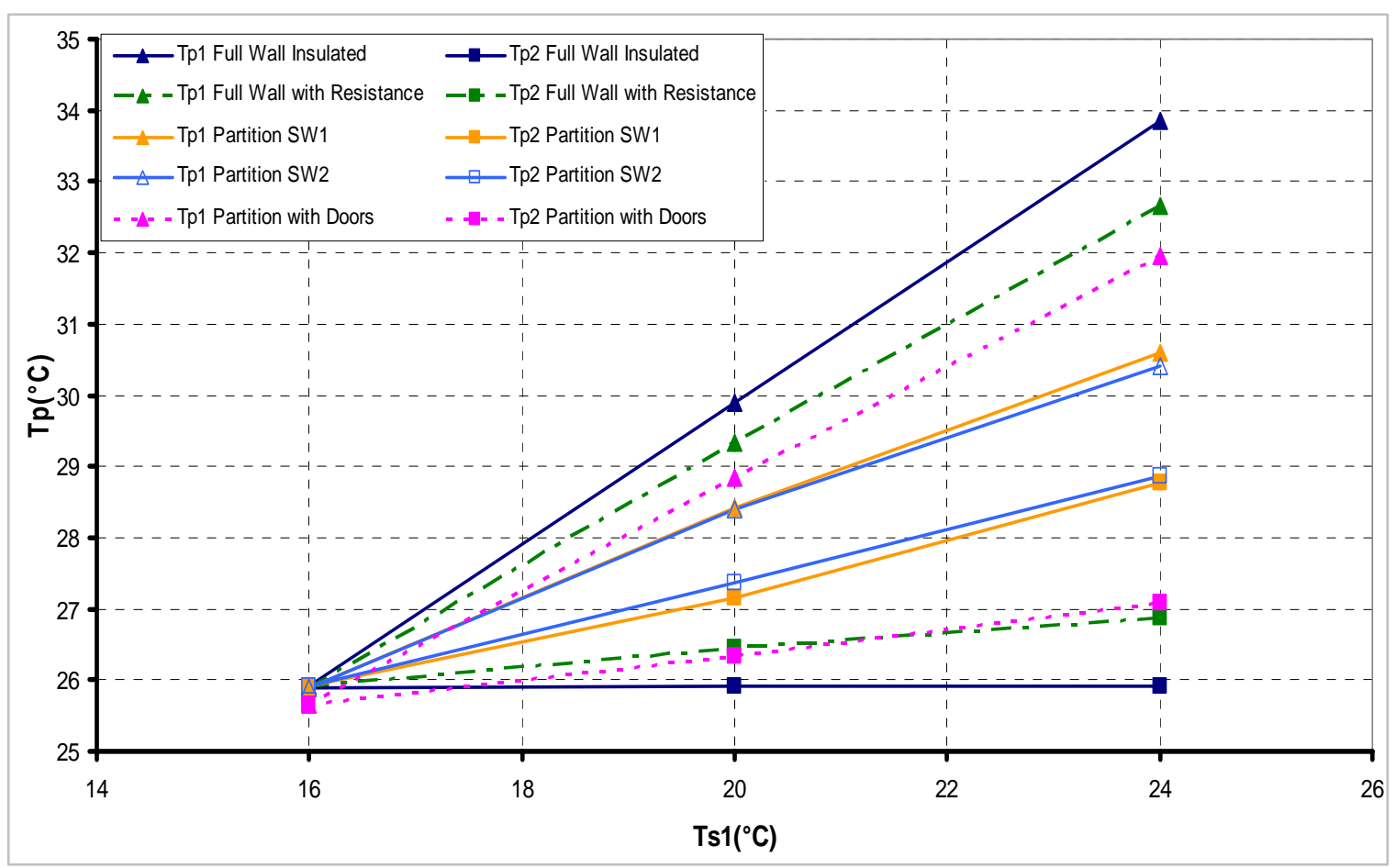

Figure 7: Effect of Cubicle Configuration and Supply Temperature $\left(\mathrm{T}_{\mathrm{s}}\right)$ on Personal Temperature $\left(\mathrm{T}_{\mathrm{p}}\right)$

Including room stratification in the thermal model results in a reduction in the predicted energy usage for UFAD in non-temperate cities by between 10 and 26\% annually, as compared with Section C's simplified (well mixed room) model. This highlights one of the advantages of UFAD systems. These results apply both to Section C's three-thermostats-forall (TTFA) and have-it-your-way (HIYW) control strategies. For cities with moderate climates such as San Francisco, the modified model, absent optimization, predicts about $17 \%$ more energy is required, due largely to increased frequency of use of the economizer.

Only slight changes were observed between the simplified and modified thermal models in the ratio of HIYW yearly energy usage to TTFA yearly energy usage. 


\section{SUgGESTIONS FOR FUTURE WORK}

Given the promising results of this initial effort, it is suggested that the following efforts be undertaken: (1) expand the optimization and control analysis to include advective and radiative coupling between adjacent offices, cubicles and workstations; (2) include a more realistic model of DECS performance; (3) account for transient weather and occupancy effects on comfort and energy use; (4) account for humidity, ventilation and radiation asymmetry in the assessment of comfort; (5) develop algorithms for sensor information fusion in DECS-equipped buildings; (6) demonstrate the effectiveness of DECS optimization and control strategies in a building testbed.

\section{REFERENCES}

1. Fanger, O. P., "IAQ in the $21^{\text {st }}$ Century," presentation at the Second Environmental Quality Symposium at Syracuse, October 29-30 (2002).

2. Spengler, J. D., J.M. Samet, and J.F. McCarthy, Editors, Indoor Air Quality Handbook, McGraw-Hill (2001).

3. Fisk, W. J., "Estimates of Potential Nationwide Productivity and Health Benefits from Better Indoor Environments," in Indoor Air Quality Handbook, J.D. Spengler, J.M. Samet, and J.F. McCarthy, Editors, McGraw Hill. (2001).

4. Kroner, W., J.A. Stark-Martin, and T. Willemain, "Using Advanced Office Technology to Increase Productivity," Center for Architectural Research and Center for Services Research and Education: Rensselaer, Troy, New York, U.S.A. (1992).

5. Arens, E. and F. Bauman, "A Field Study of PEM Performance in Bank of America's San Francisco Office Buildings," Department of Architecture, Center for Environmental Design Research, the University of California, Berkeley (2000). See also (http://arch.ced.berkeley.edu/resources/bldgsci/research/pem.htm).

6. Hedge, A., M.G. Martin, and J. McCarthy, "Breathing-Zone Filtration Effects on Indoor Air Quality and Sick Building Syndrome Complaints," in Healtby Buildings - LAQ '91, ASHRAE, p. 351-357. (1991).

7. Hedge, A., M.G. Mitchell, and J. McCarthy, "Effects of a Furniture-Integrated BreathingZone Filtration System on Indoor Air Quality, Sick Building Syndrome, Productivity, and Absenteeism," Indoor Air, 3(4): p. 328-336. (1993).

8. Fisk, W. J., G. Brager, H. Burge, J. Cummings, H. Levin, V. Loftness, M. J. Mendell, A. Persily, S. Taylor, and J.S. Zhang, "Energy-related Indoor Environmental Quality Research: A Priority Agenda”, LBNL Report No. 51328. (2002).

9. ASHRAE, Ventilation for Acceptable IAQ - Standard 62 (2001).

10. Yuan, X. and Q. Chen, "A critical review on displacement ventilation," ASHRAE Transactions. Pt.2. (1998).

11. Tsuzuki, K. et al., "Individual thermal comfort control with desk-mounted and floormounted task/ambient conditioning (TAC) systems," Proceedings of Indoor Air 99 (2): 368373. (1999).

12. McCarry, B.T., "Underfloor Air Distribution Systems: Benefits and When to use the System in Building Design," ASHRAE Transactions, Vol. 101(2). (1995). 


\section{Report No. DOE $\backslash E R 63694-1$}

13. Bauman, F. and T. Webster, "Outlook for underfloor air distribution," ASHRAE Journal, June (2001).

14. Tsuzuki, K., E.A. Arens, F.S. Bauman, and D.P. Wyon, "Individual Thermal Comfort Control with Desk-Mounted and Floor-Mounted Task/Ambient Conditioning (TAC) Systems," Proceedings of Indoor Air 1999. Edinburgh, Scotland, p. 8-12. August (1999).

15. Murakami, S., S. Kato, J. Zeng. "Flow and temperature fields around human body with various room air distribution, CFD study on computational thermal manikin---Part I", ASHRAE Transactions, 103: 3-15 (1997).

16. Murakami, S., "Analysis and Design of Micro-climate around the human body with respiration by CFD”. Indoor Air, 14, pp.144-156 (2004).

17. Melikov, A. K., "Personalized ventilation", Indoor Air, 14, pp.157-167 (2004).

18. Cermak, R., J. Holsol, K.E. Meyer, A.K. Melikov, "PIV Measurements at the breathing

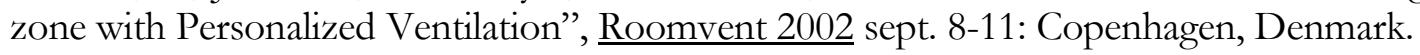

19. http://www.eere.energy.gov/buildings/research/roadmaps.cfm

20. http://www.eia.doe.gov/neic/historic/hconsumption.htm

21. Emerging Optimization Techniques in Production Planning and Control, G.C. Onwubolu, Imperial College Press, (2002).

22. ASHRAE Standard 55-2004, "Thermal Environmental Conditions of Human Occupancy", (2004).

23. Application Of Fuzzy Optimization In Energy Saving, L. Da Silva, G.L. Torres, L. Dos Reis, Rev. Ciênc. Exatas, Taubaté, v. 5-8, p. 21- 35 1999-2002. Also : http://www.unitau.br/prppg/publica/exatas/artigo2-99-02.pdf 


\title{
C. MODELING THE ENERGY EFFICIENCY OF DISTRIBUTED ENVIRONMENTAL CONTROL SYSTEMS
}

\author{
By \\ Ian A. Cosden \\ B.M.E., University of Delaware, Newark, DE
}

\begin{abstract}
THESIS
Submitted in partial fulfillment of the requirements for the

degree of Master of Science in Mechanical Engineering in the Graduate School of Syracuse University
\end{abstract}

July 2005

Approved:

Dr. H. Ezzat Khalifa 
Report No. DOE $\backslash E R 63694-1$

Date:

Copyright (C) 2005 Ian A. Cosden

All Rights Reserved 


\section{Table of Contents}

List of Figures viii

List of Tables $\quad$ xi

Nomenclature $\quad$ xii

1 Introduction 1

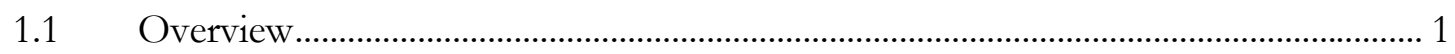

1.2 Implementing Personal Environmental Control .......................................................... 3

1.3 Personal Environmental Control Increases Productivity ............................................... 4

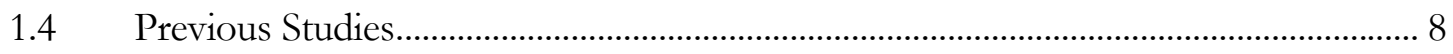

1.5 Building Energy Simulation Programs ........................................................................... 10

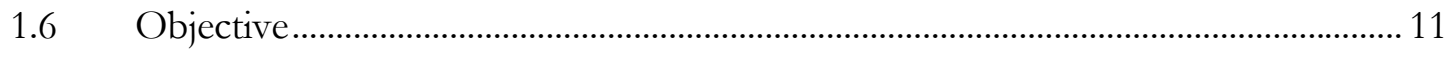

2 Method and Model Development 13

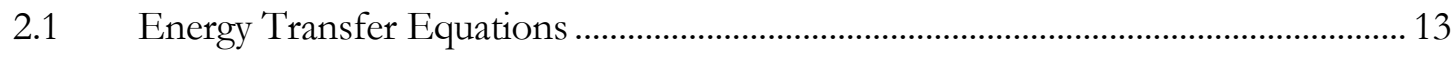

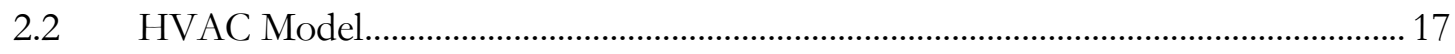

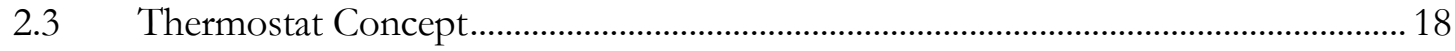

2.4 Building Model as a Thermal Circuit Network ................................................................ 19

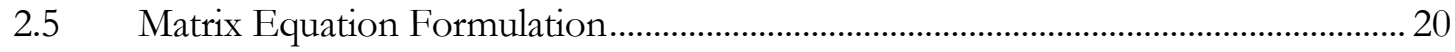

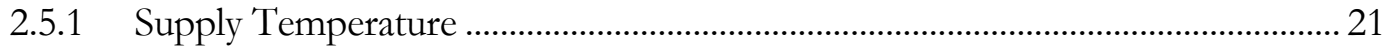

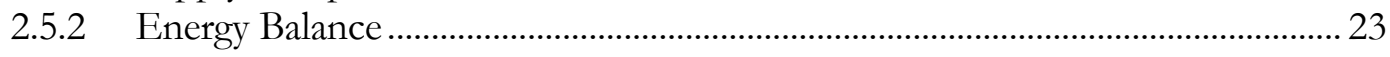

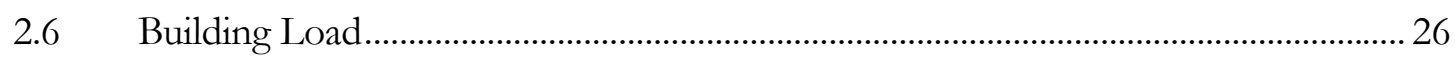

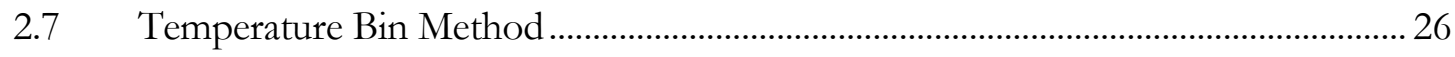

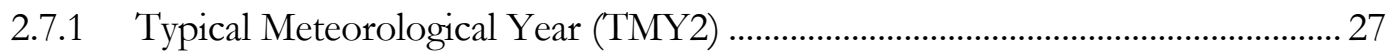

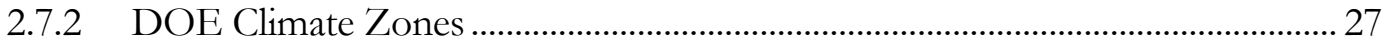

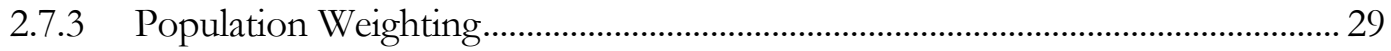

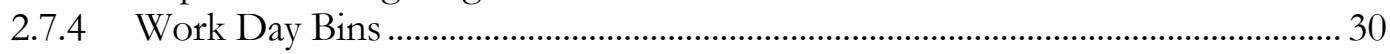

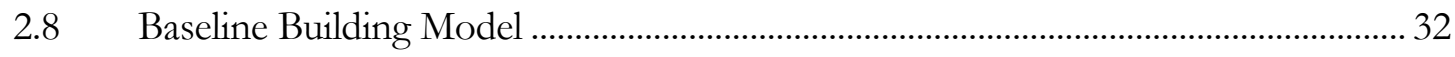

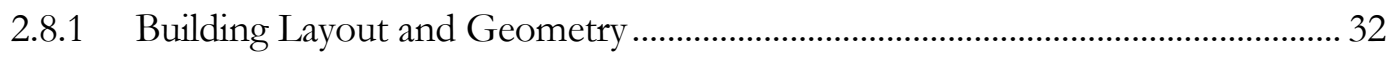

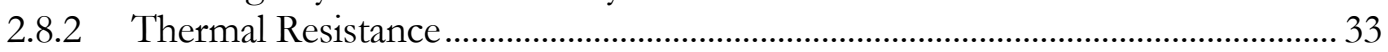

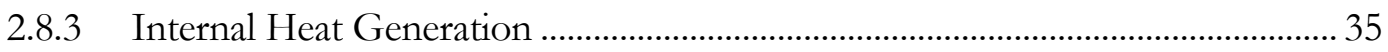

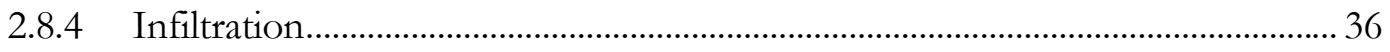

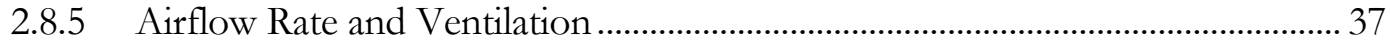

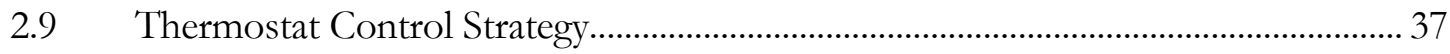




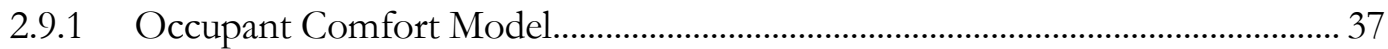

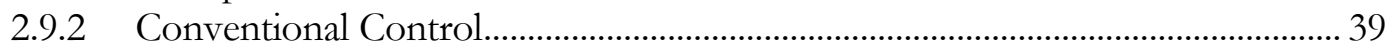

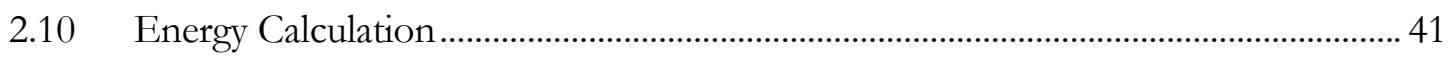

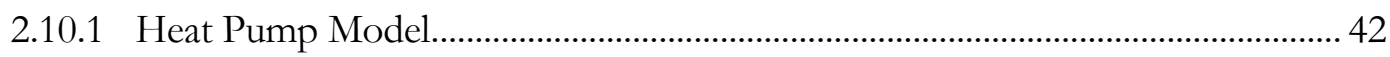

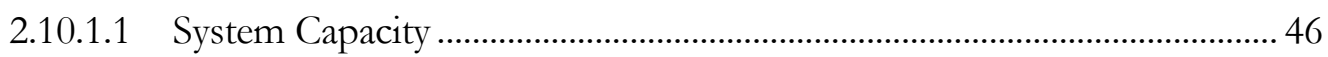

2.10.1.2 Economizer .................................................................................................. 49

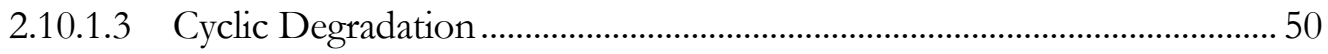

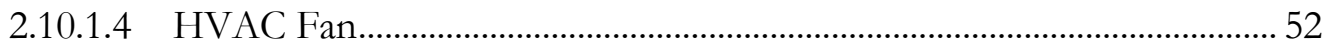

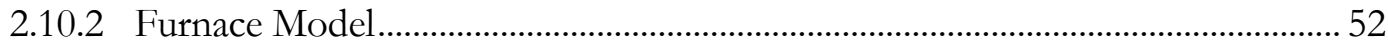

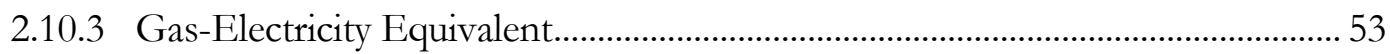

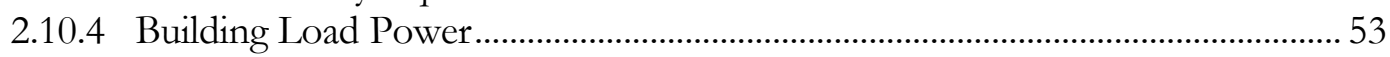

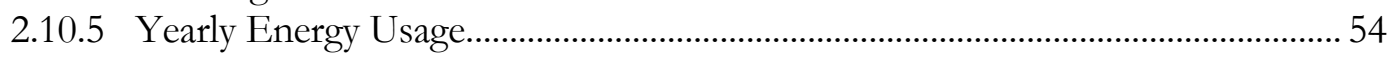

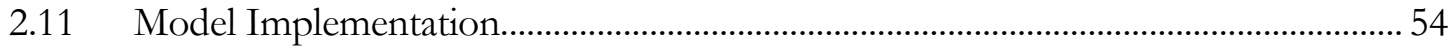

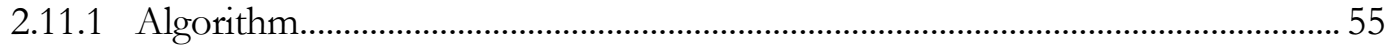

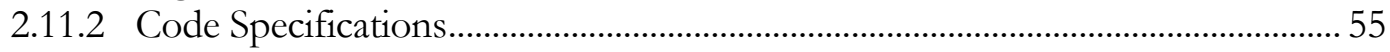

$\underline{3}$ Validation $\quad 58$

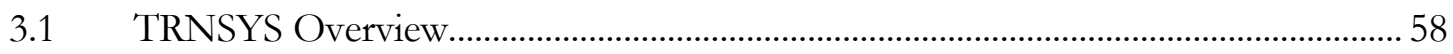

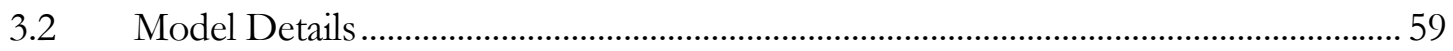

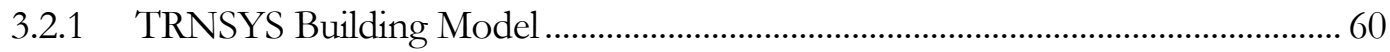

3.2.2 Thermostat Control in TRNSYS ........................ Error! Bookmark not defined.

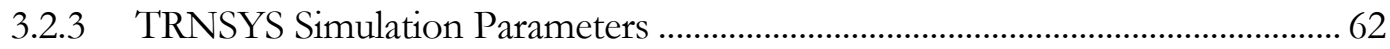

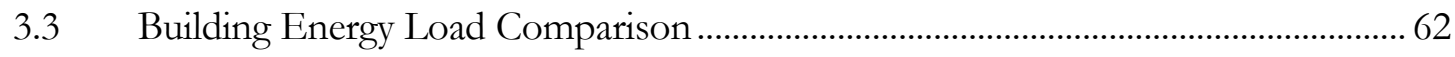

4 Computed Results $\quad 65$

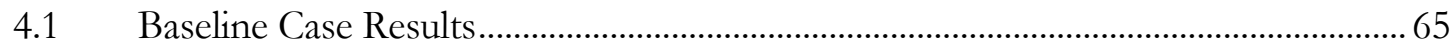

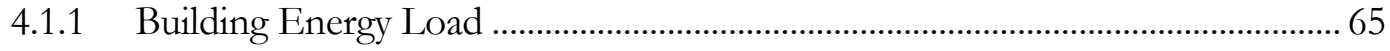

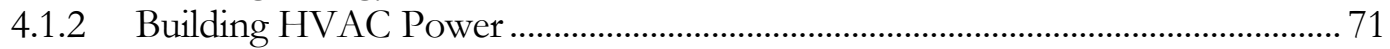

4.1.3 Energy Cost Associated with HIYW for Various Climates................................... 74

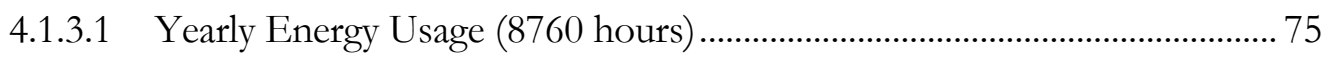

4.1.3.2 Work Day Energy Usage (4750 hours) …………........................................ 78

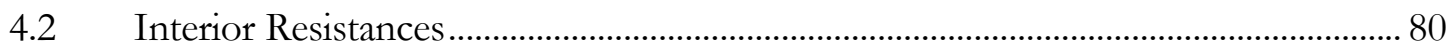

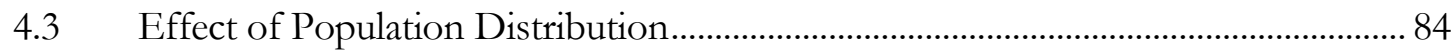

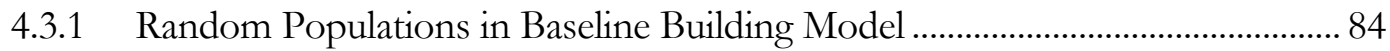

4.3.2 Random Populations in Partial Height Partition Building .................................. 87

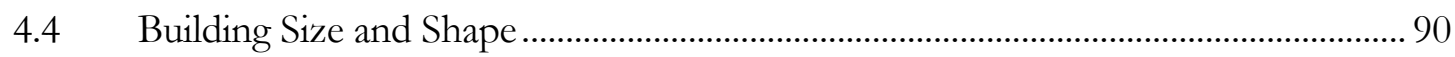




\section{Report No. DOE $\backslash E R 63694-1$}

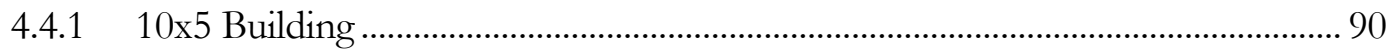

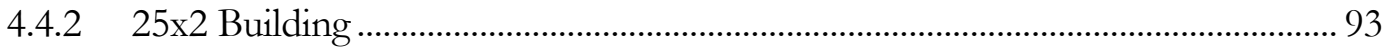

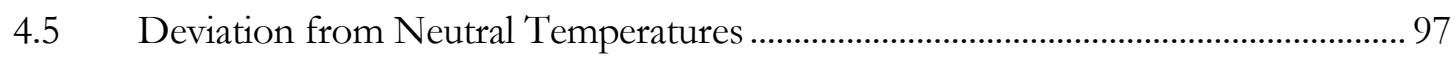

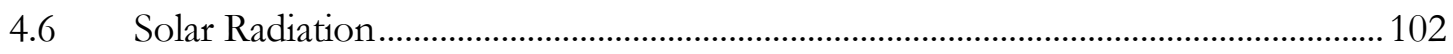

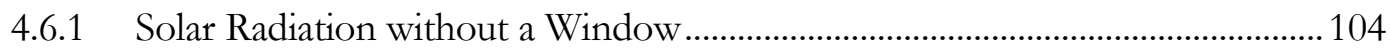

$\underline{5}$ Conclusions 107

$\underline{6}$ Appendix 112

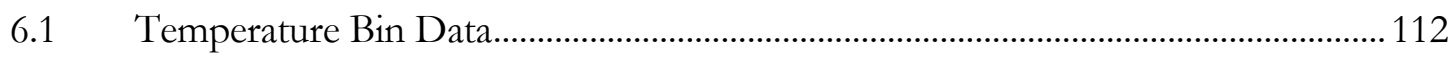

6.2 Thermodynamic Properties..................................................................................... 115

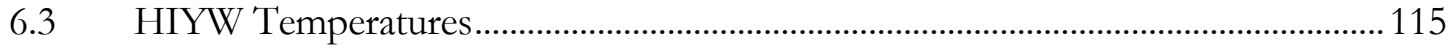

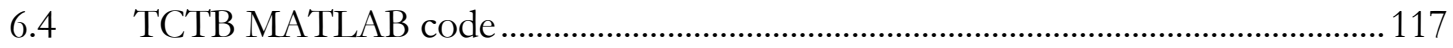

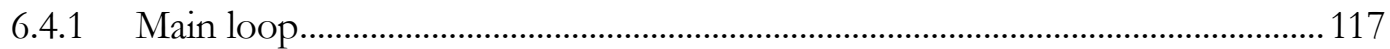

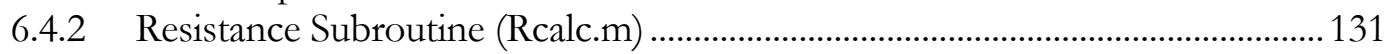

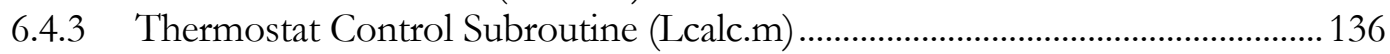

6.4.4 COP Calculation Subroutine (COPcalcCity.m) .................................................... 139

6.4.5 Cyclic Degradation COP calculation (CYCDEGcalc.m) .................................... 141 


\section{Report No. DOE $\backslash E R 63694-1$}

\section{List of Figures}

Figure 1.1 - Average annual commercial building expenses (in 1995 US dollars) [19]................ 7

Figure 2.1 - Example 4-zone domain with thermostats in zones $b, c$, and $d$................................. 19

Figure 2.2 - Cut-out example of a thermal circuit network around zone $i$.................................. 20

Figure 2.3 - Example 4-zone domain with thermostats in zones 2, 3, and 4.............................. 22

Figure 2.4 - Map of DOE US climate zones [30] ......................................................................... 28

Figure 2.5 - Total population in each climate zone (US Census Bureau 2000) ........................... 30

Figure 2.6 - Histogram of the number of work-day hours per temperature bin for Chicago, IL

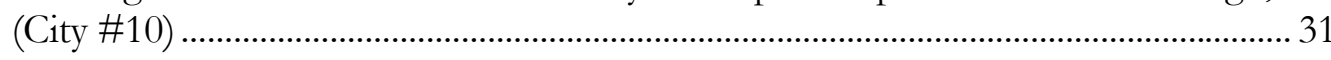

Figure 2.7 - Baseline building model floor plan ................................................................................. 33

Figure 2.8 - Histogram of 49 random individuals' neutral temperature setpoints taken from

Ari, Cosden et al. [32] Gaussian distribution.................................................................. 38

Figure 2.9 - Example individuals' neutral temperatures distributed randomly in building........ 39

Figure 2.10 - Thermostat placement and controlled zones for OSFA......................................... 40

Figure 2.11 - OSFA temperature distribution in baseline building ............................................... 41

Figure 2.12 - Calculated COP for three indoor dry bulb (db) temperatures for 10 ton, constant speed, standard efficiency, Carrier rooftop heat pump ................................................. 45

Figure 2.13 - Cooling capacity for three indoor entering dry bulb (Edb) temperatures for 10 ton, constant speed, standard efficiency Carrier rooftop heat pump ......................... 47

Figure 2.14 - Flow chart of the main structure of the TCTB code ................................................. 56

Figure 2.15 - Flow chart of bin loop calculations .............................................................................. 57

Figure 3.1 - Yearly building energy demand comparison between TCTB and TRNSYS models for the baseline building

Figure 4.1 - OSFA building load power usage for bin temperatures for baseline case (no HVAC equipment)

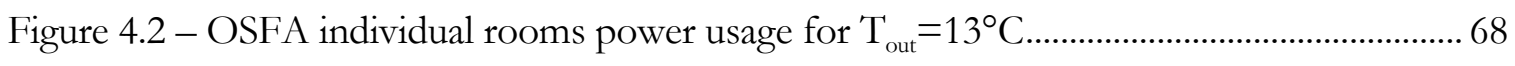

Figure 4.3 - HIYW building load power usage for bin temperatures for baseline case (no HVAC equipment)

Figure 4.4 - Building load power demand comparison between OSFA and HIYW for baseline

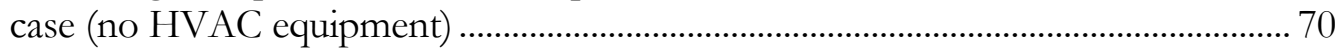

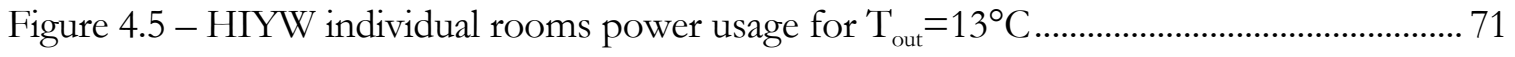

Figure 4.6 - HVAC power usage for OSFA and HIYW baseline building model case in Baltimore, MD (city \#7) 
Figure 4.7 - HVAC power usage for OSFA and HIYW baseline building model case in San Francisco, CA (city \#6)

Figure 4.8 - Yearly energy usage for baseline case for OSFA and HIYW .................................... 75

Figure 4.9 - Ratio of HIYW to OSFA energy usage (8760h) ........................................................ 77

Figure 4.10 - Work day energy usage for baseline case OSFA and HIYW ................................. 78

Figure 4.11 - Ratio of HIYW to OSFA energy usage (4750h) ....................................................... 79

Figure 4.12 - Relative energy cost of HIYW to OSFA for various internal resistances.............. 83

Figure 4.13 - 100 population energy usage comparison for baseline case ..................................... 85

Figure 4.14 - Ratio of HIYW to OSFA energy usage of 100 random populations for baseline

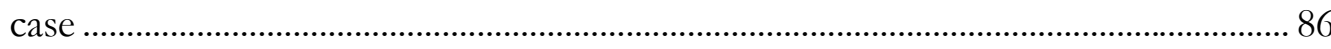

Figure 4.15 - Energy usage by city for 100 random populations for $R_{\text {int }}=0.44 \mathrm{~m}^{2} \mathrm{~K} / \mathrm{W}$ (partial

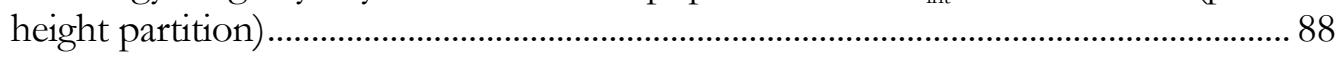

Figure 4.16 - Range of HIYW to OSFA yearly energy usage by city for 100 random

populations for $\mathrm{R}_{\mathrm{int}}=0.44 \mathrm{~m}^{2} \mathrm{~K} / \mathrm{W}$ (partial height partition) …................................... 89

Figure 4.17 - Plan view of an example HIYW temperature distribution for 10x5 building...... 90

Figure 4.18 - Yearly energy usage for 100 random populations for 10x5 building.....

Figure 4.19 - Range of HIYW to OSFA yearly energy usage by city for 100 random

populations for $10 \times 5$ building 92

Figure 4.20 - Plan view of an example HIYW temperature distribution for 25x2 building...... 94

Figure 4.21 - Energy usage for 100 random populations for 25x2 building ................................ 95

Figure 4.22 - Range of HIYW to OSFA yearly energy usage by city for 100 random

populations for $25 \times 2$ building. 96

Figure 4.23 - Influence of deviation from neutral temperature on HIYW to OSFA energy usage ratio for Miami, Baltimore, and Fairbanks.............................................................. 98

Figure $4.24-$ HIYW to OSFA energy ratio with heating $(-\Delta \mathrm{T})$ and cooling $(+\Delta \mathrm{T})$ temperature

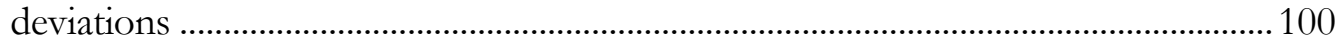

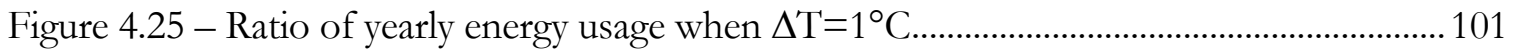

Figure 4.26 - Percent increase in TRNSYS-calculated OSFA yearly energy usage associated with addition of solar radiation

Figure 4.27 - Increase in yearly energy consumption for OSFA and HIYW associated with the addition of solar radiation.

Figure 4.28 - Percent increase in yearly energy usage with the inclusion of solar radiation for baseline building without windows.

Figure 6.1 - HIYW neutral temperature (thermostat set point) random room assignment distribution within building.. 
Report No. DOE $\backslash E R 63694-1$ 


\section{Report No. DOE $\backslash E R 63694-1$}

\section{List of Tables}

Table 2.1 - DOE climate zone definitions and representative US cities [29] .............................. 29

Table 2.2 - Baseline building model zonal physical dimensions .................................................. 33

Table 2.3 - Thermal resistance values in baseline building model.................................................. 34

Table 2.4 - Internal heat gains in each zone [27] ........................................................................... 36

Table 2.5 - Manufacturer's published cooling data for Carrier WEATHERMAKER 50TFQ012 [33] standard efficiency 10-ton heat pump operating at $3750 \mathrm{cfm}$, and

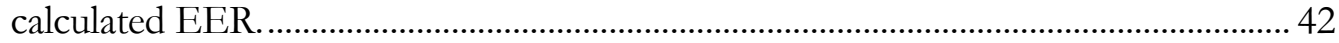

Table 2.6 - Ratio of 10-ton capacity heat pump with required building capacity for each city. 48

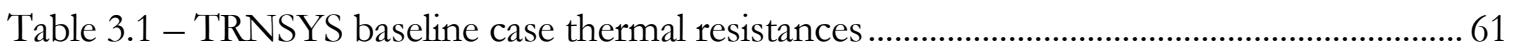

Table 3.2 - Effect of TRNSYS simulation time step for baseline building in Chicago, IL ....... 62

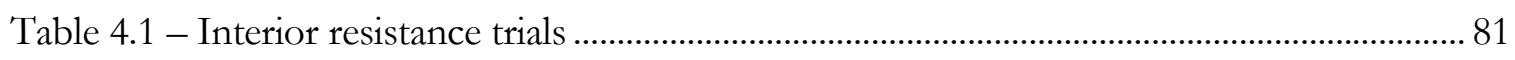

Table 4.2 - Increase in outside wall surface area from baseline case............................................... 93

Table 6.1 - Yearly temperature bin data for 15 representative U.S. cities (number of hours per

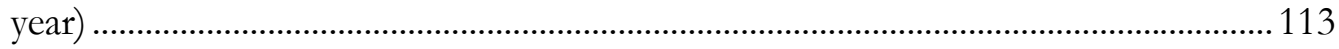

Table 6.2 - Thermodynamic properties of air used in simulations................................................ 115

Table 6.3 - HIYW neutral temperatures (thermostat set points) by assigned room (in ${ }^{\circ} \mathrm{C}$ ).... 116 


\section{Nomenclature}

\begin{tabular}{|c|c|c|}
\hline A & Area & $\mathrm{m}^{2}$ \\
\hline $\mathrm{C}$ & Combined mass flow rate and specific heat & $\mathrm{J} / \mathrm{sK}$ \\
\hline $\mathrm{c}_{\mathrm{d}}$ & Cyclic degradation coefficient & - \\
\hline$c_{\mathrm{p}}$ & Specific heat of air & $\mathrm{J} / \mathrm{kgK}$ \\
\hline COP & Coefficient of performance of heat pump & - \\
\hline$\eta$ & Efficiency & - \\
\hline h & Convective heat transfer coefficient & $\mathrm{W} / \mathrm{m}^{2} \mathrm{~K}$ \\
\hline $\mathrm{L}$ & Length & $\mathrm{m}$ \\
\hline$\dot{m}$ & Mass flow rate of conditioning air & $\mathrm{kg} / \mathrm{s}$ \\
\hline $\mathrm{k}$ & Thermal conductivity & $\mathrm{W} / \mathrm{mK}$ \\
\hline $\mathrm{P}$ & Electrical power & W \\
\hline$\dot{q}$ & Heat flux & W \\
\hline$\dot{Q}$ & Energy flux & $\mathrm{W}$ \\
\hline $\mathrm{r}$ & Thermal resistance of building material & $\mathrm{m}^{2} \mathrm{~K} / \mathrm{W}$ \\
\hline $\mathrm{R}$ & Thermal resistance of zonal interface & $\mathrm{K} / \mathrm{W}$ \\
\hline TC & Total capacity of heat pump & W \\
\hline $\mathrm{T}$ & Temperature & ${ }^{\circ} \mathrm{C}$ \\
\hline $\mathrm{T}^{*}$ & Desired room temperature & ${ }^{\circ} \mathrm{C}$ \\
\hline$\Delta \mathrm{T}$ & Deviation from neutral temperature & ${ }^{\circ} \mathrm{C}$ \\
\hline$T_{k}^{s}$ & Supply air temperature to room $\mathrm{k}$ & ${ }^{\circ} \mathrm{C}$ \\
\hline $\mathrm{U}$ & Overall heat transfer coefficient & $\mathrm{W} / \mathrm{K}$ \\
\hline
\end{tabular}




\begin{tabular}{|c|c|}
\hline \multicolumn{2}{|c|}{ Subscripts and Superscripts } \\
\hline $\mathrm{adv}$ & Advection \\
\hline con & Convection \\
\hline cond & Conduction \\
\hline comp & Compressor \\
\hline cool & Cooling \\
\hline cyclic & Cyclic degratation \\
\hline ewall & Exterior wall \\
\hline ewindow & Exterior window \\
\hline fan & HVAC fan \\
\hline furnace & Furnace \\
\hline heat & Heating \\
\hline hp & Heat pump \\
\hline HVAC & HVAC system \\
\hline in & Inside \\
\hline iwall & Interior wall \\
\hline out & Outside \\
\hline $\mathrm{rad}$ & Radiation \\
\hline tot & Total \\
\hline
\end{tabular}




\section{Introduction}

\subsection{Overview}

Today, typical heating, ventilation, and air-conditioning (HVAC) systems account for 40-60\% of the overall energy consumption of a building [1]. Before the 1970s energy crisis, HVAC systems were designed to provide quality occupant comfort with little regard for energy conservation. Systems often had simultaneous heating and cooling, and building envelopes were minimally insulated. Following the energy crisis, however, HVAC system design has shifted to reduce energy usage by lowering outdoor ventilation rates while keeping building temperatures on the threshold of acceptable levels. These energy conservation measures resulted in reduced comfort and unhealthy indoor air quality (IAQ). The quality of the indoor environment is critically important to human health and performance. It is estimated that in technologically developed countries people spend more than $90 \%$ of their time in a man-made environment (buildings, vehicles, etc.) [2].

The American Society of Heating Refrigeration and Air-conditioning Engineers (ASHRAE) and the International Organization for Standardization (ISO) have published standards that outline necessary conditions for human thermal comfort. ASHRAE Standard 55-2004 [3] titled "Thermal Environmental Conditions for Human Occupancy," in close agreement with ISO Standard 7730 [4], specifies the criteria for providing acceptable thermal conditions in an indoor environment based on a combination of personal factors (clothing and activity level) and environmental factors (air temperature, humidity, radiant temperature, air velocity). The standard defines acceptable conditions as those under which $80 \%$ of a building's occupants consider the thermal conditions satisfactory. It is therefore not only possible, but also acceptable to provide conditions deemed unsatisfactory by $20 \%$ of a building's occupants. The 
$20 \%$ dissatisfied is broken into two parts: $10 \%$ for temperature discomfort and $10 \%$ for local non-uniformities (i.e. draft). The standards, by nature, are based on a population average comfort. They aim to provide a more or less uniform temperature and humidity to the entire group of building occupants and concede that some of the occupants will be dissatisfied. Given the diversity of human preferences, this has long been agreed on as the best method for design.

Unfortunately, in practice achieving $80 \%$ satisfaction may be optimistic. Occupant satisfaction with overall office air quality and comfort has been found to be as low as $40 \%$ in some studies [5]. In a large field study of building thermal comfort, Schiller et al. [6] found less than $25 \%$ of the subjects surveyed were either moderately or very satisfied with the air temperature. The study consisted of 10 office buildings in the San Francisco Bay area with 304 participants. Of the workstation temperature measurements, $78.2 \%$ (winter) and $52.8 \%$ (summer) fell within the ASHRAE standard 55-81 comfort zones. In the buildings surveyed, 38\% (winter) and 41\% (summer) of the occupants felt the desire to be either warmer or cooler.

In a large survey of office tenants by the Building Owners and Managers Association (BOMA) and the Urban Land Institute (ULI) tenants were asked to rate the importance of 53 different office amenities and features and rate their satisfaction of their current office in the same 53 categories [7]. The tenants' ability to control their own temperature, or the lack thereof, is the only feature that appears on both the most important list ( $97 \%$ of surveyed) and least satisfied list (65\% of surveyed). The study suggests that upgrading the HVAC system to allow personal control would create an immediate, positive impact on tenants' positive perception of the building environment. 
Report No. DE-FG02-03ER63694-F1

\subsection{Implementing Personal Environmental Control}

To achieve personal environmental control, the traditional HVAC approach of mixing ventilation needs to be modified. Mixing ventilation supplies conditioned air through a ceiling diffuser and relies on the inlet jet momentum and buoyancy to mix the supply air and room air [2]. The goal is to create as uniform a temperature as possible in the space (and therefore for the occupants) and supply enough clean air to dilute contaminants to a safe level. This type of ventilation system does not lend itself well to personal control in any type of setting other than a fully enclosed single-occupant room.

Underfloor air distribution (UFAD) supplies conditioned air at floor level through numerous supply locations, while the returns are on the ceiling above the occupied space. Supply temperatures are slightly higher than traditional mixing ventilation since the supply vents are significantly closer to the occupants. Underfloor air distribution with low supply velocity conditions is similar to displacement ventilation [8], which relies solely on thermal buoyancy to provide occupants with conditioned air. The thermal plume generated by an occupant pulls cool, conditioned air from the floor level into the breathing zone. As a result underfloor air distribution, despite slightly higher supply air velocity than displacement ventilation, provides a greater ventilation efficiency at the breathing level than mixing ventilation since it is not mixed with a significant amount of the existing room air [8]. UFAD systems have the ability to provide occupants with local control by adjusting damper levels on the local floor diffusers [9]. Underfloor air distribution systems lend themselves easily to open plan office settings where each individual cubicle/workstation has its own floor diffuser and can therefore allow a person to adjust the damper according to his/her personal preference. In a field study of a building equipped with occupant controlled underfloor air distribution in an open plan office, Bauman 
et al. [8] observed temperature differences in the range of $1^{\circ} \mathrm{C}$ to $2.5^{\circ} \mathrm{C}$ between adjacent workstations.

Desktop (task) conditioning systems also provide occupants with a significantly higher amount of personal control than mixing ventilation systems [10]. While individual designs vary and are the subject of current research, in general desktop conditioning systems provide conditioned air through some type of diffuser (nozzle, grille, etc) at the desk level very close to the occupant's breathing zone [10]. The advantage is clear; the conditioned air is supplied directly to the breathing zone nearly eliminating mixing with the room air.

Manufacturers, such as Johnson Controls, have begun developing commercial desktop conditioning systems. The Johnson Controls Personal Environments ${ }^{\circledR}$ unit consists of a user control unit, task lighting, radiant heat panel, diffusers, fan, and particle filter to provide the occupant significant control over temperature, airflow, lighting, and acoustic levels [11].

\subsection{Personal Environmental Control Increases Productivity}

There is a growing list of researchers and practitioners who have found that allowing occupants control over their personal environment leads to increased comfort and productivity. Considering a traditional approach to thermal comfort, acceptable to $80 \%$, overall workplace productivity is likely more affected by large numbers of slightly dissatisfied employees rather than a very few hypersensitive employees [5]. Therefore, if productivity is to be increased effectively the majority of employees, not just those that complain, must be given some level of personal control. In addition to comfort, physical illnesses have been attributed to poor ventilation and HVAC control. Sick Building Syndrome (SBS) symptoms are characterized by acute building-related health symptoms (e.g. irritation of eyes, headache, 
fatigue). Despite being affected by psychosocial factors many building factors have been shown to influence SBS such as: outside air ventilation rate, temperature, humidity, and the level of chemical and microbiological pollutants [12]. Fisk [13] estimates the potential U.S. annual productivity gain from improved worker performance from changes in thermal environment and lighting to be between $\$ 20$ billion and $\$ 160$ billion (1996 U.S. \$). Reduced respiratory illness and sick building syndrome could contribute an estimated additional annual savings of \$16-34 billion from improved indoor environmental condition. Both displacement ventilation and especially task ventilation help provide more fresh air to the occupant's breathing zone and can help limit exposure to pollutants and provide control to the individual.

Lorsch and Abdou [14] found the lowest rate of industrial accidents to occur in the $67-69^{\circ} \mathrm{F}$ (19.4-20.6 $6^{\circ}$ ) range. Accidents increased significantly at temperatures above and only slightly below the temperature range. Tham [15] studied the effects of temperature and outdoor air supply rate on the performance of call center operators. He observed that the operators were (a) distinctly able to distinguish a difference between $22.5^{\circ} \mathrm{C}$ and $24.5^{\circ} \mathrm{C}$, (b) more productive at the lower temperature $\left(22.5^{\circ} \mathrm{C}\right)$, despite the common building practice of maintaining $24.5^{\circ} \mathrm{C}$, and (c) able to tolerate, without a decrease in productivity, the higher temperature $\left(24.5^{\circ} \mathrm{C}\right)$ if the outdoor air supply rate was doubled from $5 \mathrm{l} / \mathrm{s} /$ person to $10 \mathrm{l} / \mathrm{s} /$ person.

Wyon [16] predicted that by providing individual control equivalent to $\pm 3^{\circ} \mathrm{C}$, productivity would increase $2.7 \%$ for logical thinking, $7.0 \%$ for general office work, $3.4 \%$ for very skilled manual work, and $8.6 \%$ for very rapid manual work. In a later study with field observations, Wyon [17] showed that poor air quality, resulting from low outdoor air supply rate and uncomfortable temperatures, can have a negative effect on office productivity in the range of 6-9\%. 
Johnson Controls performed a case study on the West Bend Mutual Insurance Company in West Bend, WI to determine the effectiveness of personal ventilation devices [18]. The building in question was a 15,000 sq. ft. company headquarters with over 500 employees in open-plan offices. Johnson Controls Personal Environments ${ }^{\circledR}$ systems were installed in each workstation allowing individual control of temperature, airflow, lighting, as well as background noise masking. As a result, employee productivity increased by an impressive $16 \%, 2.8 \%$ of which was attributed to the individual control of the workstation environment. The $2.8 \%$ increase was estimated to be a savings of $\$ 364,000$ in annual payroll. Also, nearly all thermal condition complaints were eliminated; HVAC service calls decreased from more than 40 per day (at an estimate of $\$ 25$ per call plus $\$ 250$ in maintenance) to only two per week.

Figure 1.1 shows average annual commercial building costs in dollars per square foot. Employee salaries are nearly ten times more costly than the next highest expense, rent. Utilities which we can consider to include all HVAC operation costs, is less than $1 \%$ of employee salaries. This means that if it were possible to increase the productivity of the employees by $1 \%$, the equivalent monetary gain is equal to the entire building utility cost. Surely this is motivation enough to explore the ability to increase worker productivity through increased comfort. 


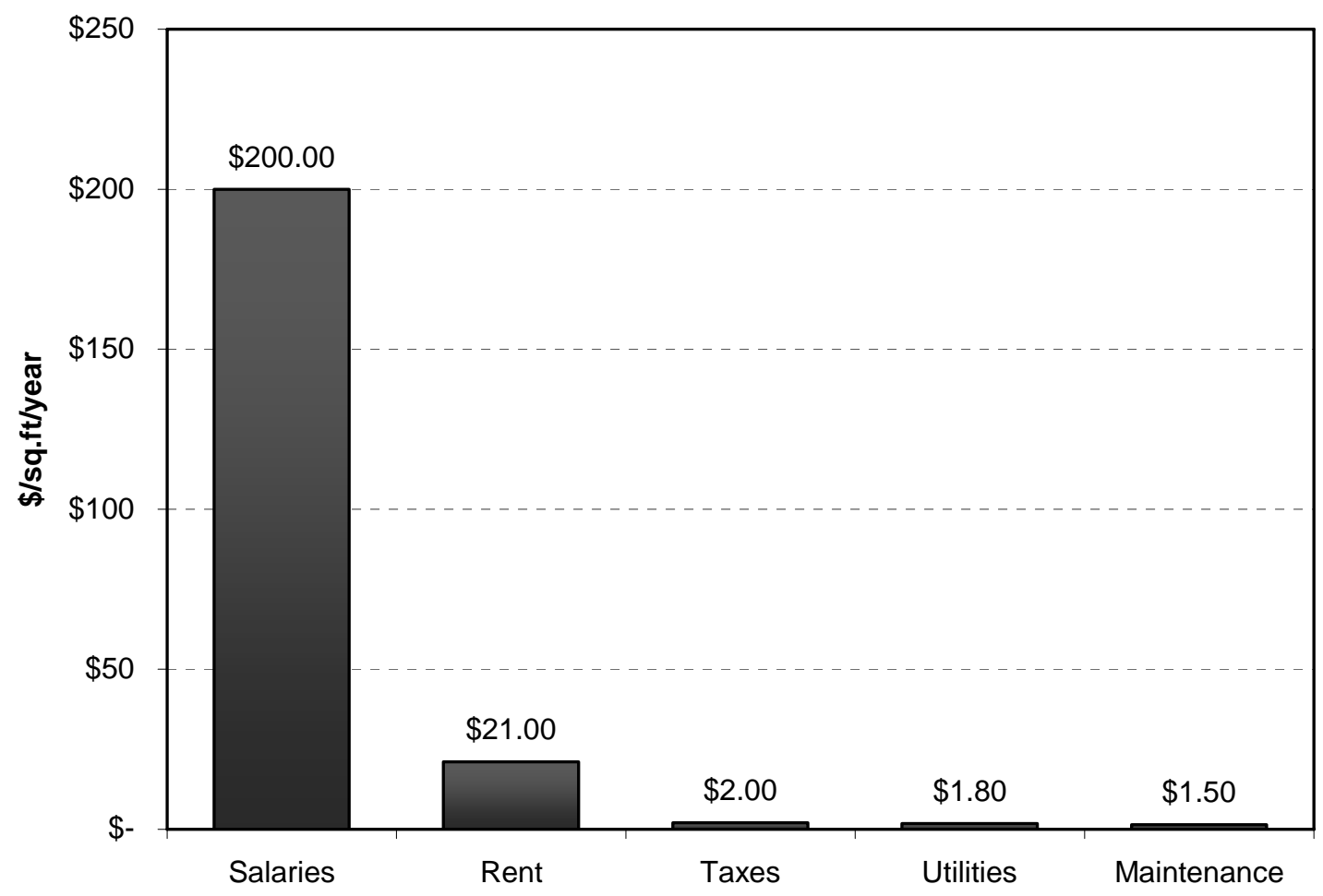

Figure 1.1 - Average annual commercial building expenses (in 1995 US dollars) [19].

With the benefits of personal environmental control (PEC) so overwhelmingly clear, there must be a reason for the industry's reluctance for widespread adoption of individual control. The explanation is two fold: (1) the high uncertainty of measuring an increase in productivity, and (2) increased energy costs. As mentioned before, typical HVAC systems account for between $40-60 \%$ of a commercial building's overall energy consumption. Building owners are reluctant to adopt personal environmental control systems that will likely increase the energy usage of a building for the promise of more productive employees, given the high uncertainty of the latter. Energy costs, while small compared to employee salaries, are still a major commercial expense and are very easily measured, whereas productivity gains due to improved indoor environmental quality are much more difficult to measure. In addition, there is an 


\section{Report No. DE-FG02-03ER63694-F1}

associated increase in initial construction costs. Lorsch and Abdou [20] note one instance of a federal building manager who spent $\$ 1$ million on personal control and never saw concrete evidence of an increase in productivity. With experiences like this it is easily understand why building owners are hesitant to risk the increased costs associated with personal environmental control. Unfortunately the true energy cost associated with the adoption of personal environmental control is relatively unknown. Often PEC systems are installed in conjunction with other green building, energy saving enhancements that make it difficult to isolate the PEC contribution to building energy costs observed in field studies [18].

\subsection{Previous Studies}

There have only been a handful of researchers who have modeled the energy usage of buildings equipped with occupant controlled distributed environmental control systems. Seem and Braun [21] compared HVAC and lighting costs of personal environmental control (PEC) with those of conventional variable air volume (VAV) systems through computer simulations. Each occupant had a desktop PEC equipped with an occupancy sensor. The sensor also controlled local task lighting. The PEC allowed occupants to control the temperature and flow rate of the airflow through a radiant heating panel and local fan. In addition to each PEC, underfloor diffusers were also included to provide overall conditioning to the space. Cooling was provided through a chilled water system while heat was provided through localized heating coils. Seem and Braun modeled one floor (45,000 sq. ft) of a multi-story building in Madison, WI with 360 total occupants. The building was modeled using TRNSYS [22] by dividing the building into three well mixed zones. Each PEC had different occupied and non-occupied heating and cooling set points, so when an occupant leaves his/her workstation the set points 


\section{Report No. DE-FG02-03ER63694-F1}

are relaxed. Electricity was used to provide cooling power while natural gas was used to provide heating power at an assumed one-third the cost of electricity. Radiant heating panels, however, used electricity. In their analysis Seem and Braun neglected the heat transfer between the individual workstations/offices. In each of the three zones, there were at least 80 occupants, all of whom were modeled as having the same temperature. The energy costs associated with maintaining different personal temperatures within these zones was not considered. That is, each workstation/office was assumed to be maintained at the same temperature when occupied. Overall the operating costs of PEC systems costs were found to be between $2-15 \%$ greater than the VAV systems. However, if occupancy rates of workstations fell below 60\% then PEC systems resulted in a net savings of energy because of the occupancy sensor adjusting the lighting and workstation temperature.

Glicksman and Taub [23] modeled occupant controlled HVAC systems using computer simulations. Unlike the previous study by Seem and Braun, Glicksman and Taub accounted for the heat transfer between individual zones. A model was created where individual nodes represented conditioned cubicles equipped with occupant controlled HVAC devices. The area between the cubicles and the ceiling was treated as a single well-mixed zone. Glicksman and Taub modeled an interior region on a floor of a multiple story office building. This interior region was an open-plan office space consisting of only cubicles with no interaction with outside weather conditions. A conventional mixing ventilation system was compared with a new occupant controlled underfloor air distribution system. Random processes were used to model the inhabitance of the occupants at their workstations as well as their desired temperature set points. The local occupant fans as well as the lighting and equipment (computer) is controlled by an occupant sensor. The preferred temperature for each occupant was taken from a Gaussian distribution with a mean of $23^{\circ} \mathrm{C}$ and a standard deviation of 


\section{Report No. DE-FG02-03ER63694-F1}

$1.5^{\circ} \mathrm{C}$. From this distribution, temperatures were taken and assigned at random within the building model. This study estimated a substantial savings in the adoption of a floor based system. They predicted an annual energy savings of $13 \%$ for a non-uniform temperature distribution and a $21 \%$ savings for a uniform temperature distribution over a conventional mixing ventilation system using the same HVAC equipment. This is largely a result of the stratification within the room, reduced conditioning of the corridor, and occupancy sensors which reduce the heat load in unoccupied rooms. The energy penalty $(8 \%)$ associated with the non-uniform (i.e. individual control) temperature distribution over a uniform distribution was driven by the energy penalty of the cooler than average rooms.

\subsection{Building Energy Simulation Programs}

There are a number of software programs dedicated to the simulation of building energy usage. TRNSYS (TRaNsient SYstem Simulation program) is a commercially available program designed for the transient simulation of thermal systems [22]. Main applications include: solar systems (solar thermal and photovoltaic systems), building energy simulation and HVAC systems, and renewable energy systems. TRNSYS has a polished front-end user interface and has become reference software for researchers and engineers doing building energy simulations. EnergyPlus, often referred to as $\mathrm{e}^{+}$, is another building energy simulation program for modeling building heating, cooling, lighting, ventilating, and other energy flows [24]. First introduced in 1999, EnergyPlus is based on the most popular features and capabilities of past building energy simulation programs BLAST and DOE-2. However, due to its recent inception it lacks a graphical user interface and has yet to gain significant use amongst researchers. 
Report No. DE-FG02-03ER63694-F1

While some of the details of these programs are slightly different, the building energy simulation programs are all time-dependant codes and are designed to account for the transient nature of building environments (weather, occupant schedules, HVAC equipment cycling etc). One of the drawbacks of such programs is the execution time of yearly building energy simulations. Depending on a number of factors such as the total number of zones in a building and the HVAC equipment, the total computation time of a yearly simulation using a transient simulation program can take anywhere from a few minutes to a few hours.

\subsection{Objective}

This study is part of a larger project intended to explore building energy consumption minimization and occupant comfort maximization of distributed environmental control systems using optimization techniques. In order to accomplish this task, a quickly executing building energy model must be created to use as an objective function for the optimization routine. Using a gradient based optimization routines the building energy model will need to be executed thousands of times before an optimum is reached. For this reason we require a building energy simulation model unlike the aforementioned transient programs. If a simulation using a transient program executed in ten minutes, five thousand iterations of the program would take nearly thirty-five days to execute. This would make running large-scale optimization trials and parametric studies completely infeasible. The building model must execute orders of magnitude quicker than the transient building energy simulation programs to serve as a realistic objective function.

Therefore, the objective of this study is to develop an accurate, fast-executing lumped parameter building model to quantify the energy costs associated with allowing occupants to 


\section{Report No. DE-FG02-03ER63694-F1}

control their own micro environment through the use of distributed environmental control systems. For the purposes of this study we will consider the micro environment to be affected only by the dry bulb temperature since humans are far more sensitive to changes in temperature in normal conditions than humidity [1], and temperature control is the most desired office feature not currently available [7]. Also we will determine how factors, such as building shape and climate, affect the energy cost of distributed environmental control.

In this report, we will start by outlining the necessary physical principles and assumptions used to develop a novel, temperature bin based, lumped parameter building model. We will then illustrate the mathematical formulation of the building model and the ensuing computer program. A discussion of the computed results and comparison of the new building model with a transient building energy simulation using TRNSYS will follow. Lastly, we will end with some concluding remarks and suggestions for future studies. 
Report No. DE-FG02-03ER63694-F1

\section{Method and Model Development}

Here we will discuss the formulation of the simplified lumped-parameter model. First we will present the physical situation and the assumptions to develop the mathematical problem. Then we will discuss how to apply the physics to create a realistic, yet simple, model of a building. We will treat the building as steady state using a quasi-steady temperature bin approach. The influence of the neglected transient effects will be addressed later through a comparison with TRNSYS in Chapter 3.

\subsection{Energy Transfer Equations}

Before we can define the interaction between office zones, we must first discuss the applicable heat transfer mechanisms. Heat transfer between building zones can be by one or more of the following mechanisms: radiation, conduction, and/or advection.

Radiative heat transfer can occur between any two surfaces at different temperatures. Assuming steady state, the net radiative heat transfer from a surface $a$ at one temperature $\left(T_{a}\right)$ and a surface $b$ at another temperature $\left(T_{b}\right)$ is given by [25],

$$
\dot{q}_{a b}^{r a d}=\varepsilon \sigma f A_{b}\left(T_{a}^{4}-T_{b}^{4}\right)
$$

where $\varepsilon$ is the emissivity of the surface, $\sigma$ is the Stephan-Boltzmann constant $\left[\mathrm{W} / \mathrm{m}^{2} \mathrm{~K}^{4}\right], f$ is the view factor between surface $a$ and $b, A_{s}$ is the area of the surface $b\left[\mathrm{~m}^{2}\right], T_{a}$ is the temperature of surface $a[\mathrm{~K}]$, and $T_{b}$ is the temperature of surface $b[\mathrm{~K}]$.

Since the purpose of this study is to predict heat transfer between adjacent office workstations it is unlikely that surfaces will have significantly different temperatures. Furthermore the view 


\section{Report No. DE-FG02-03ER63694-F1}

factors are small and we therefore do not expect to have appreciable radiative heat transfer. For this reason we will neglect the heat transfer between zones by radiation.

Conductive heat transfer refers to the transport of energy as a result of a temperature gradient in a stationary medium. From Fourier's Law [25] we can write the expression for onedimensional steady state conduction for a plane wall as,

$$
\dot{q}_{a b}^{\text {cond }}=\frac{k A\left(T_{a}-T_{b}\right)}{L},
$$

where $k$ is the thermal conductivity of medium $[\mathrm{W} / \mathrm{mK}], \mathrm{A}$ is the surface area of medium $\left[\mathrm{m}^{2}\right]$, $\mathrm{L}$ is the thickness of medium $[\mathrm{m}], \quad T_{a}$ is the temperature of surface $a\left[{ }^{\circ} \mathrm{C}\right]$, and $T_{b}$ is the temperature of surface $b\left[{ }^{\circ} \mathrm{C}\right]$.

In our analysis we will examine the heat transfer in office buildings, so it is expected that conduction will occur between adjacent workstations across solid boundaries. It is also necessary to include the thermal resistance associated with convective heat transfer. Convection is the heat transfer between a moving fluid (in our case air) and a surface (in our case the wall/cubicle interface). From Newton's law of cooling [25],

$$
\dot{q}^{\text {conv }}=h A_{s}\left(T_{f}-T_{s}\right)
$$

where $h$ is the convective heat transfer coefficient $\left[\mathrm{W} / \mathrm{m}^{2} \mathrm{~K}\right], A_{s}$ is the area of surface $\left[\mathrm{m}^{2}\right], T_{f}$ is the temperature of fluid $\left[{ }^{\circ} \mathrm{C}\right]$, and $T_{s}$ is the temperature of surface $\left[{ }^{\circ} \mathrm{C}\right]$. The convective heat transfer coefficient $(b)$ is dependent on boundary layer conditions that are influenced by a number of factors, such as surface geometry and fluid motion (e.g. Reynolds and Grashoff numbers). Determining appropriate convective heat transfer coefficients is challenging and will be addressed in Section 4.2 . 


\section{Report No. DE-FG02-03ER63694-F1}

The heat transfer through walls by the conduction and convection can be analyzed using the concept of thermal resistance. Named for its analogy to electrical resistance (Ohm's Law), thermal resistance is defined as the ratio of the driving potential (temperature difference) to the rate of heat transfer. From equation (2.2) and (2.3) we get the thermal resistance for conduction and convection, respectively as,

$$
\begin{gathered}
R_{a b}^{\text {cond }}=\frac{\left(T_{a}-T_{b}\right)}{\dot{q}_{a b}^{\text {cond }}}=\frac{L}{k A}, \\
R_{f s}^{\text {conv }}=\frac{\left(T_{f}-T_{s}\right)}{\dot{q}_{f s}^{\text {conv }}}=\frac{1}{h A_{s}} .
\end{gathered}
$$

The total thermal resistance is the sum of the individual thermal resistances in series. If we take the driving temperature difference between two fluid zones $a$ and $b$ separated by a plane wall we can write the total heat transfer $\dot{q}_{a b}^{\text {tot }}$ as,

$$
\dot{q}_{a b}^{\text {tot }}=\frac{T_{a}-T_{b}}{R_{a b}^{\text {tot }}}
$$

where, $R_{t o t}$ is the total thermal resistance between zone $a$ and zone $b$ and is given by the sum of the individual resistances,

$$
R_{a b}^{\text {tot }}=R_{a b}^{c o n d}+R_{a b}^{c o n v}=\frac{1}{A h_{a}}+\frac{L}{k A}+\frac{1}{A h_{b}}
$$

It is often more convenient to write (2.6) in terms the conductance $\left(U^{\text {tot }}\right)$ which is simply defined as $U^{\text {tot }}=\frac{1}{R^{\text {tot }}}$. As a result (2.6) becomes,

$$
\dot{q}_{a b}^{\text {tot }}=U_{a b}^{\text {tot }}\left(T_{a}-T_{b}\right) .
$$




\section{Report No. DE-FG02-03ER63694-F1}

Advective heat transfer is the heat flux associated with a fluid flow [26]. In the case of an office setting, heat transfer associated with air flow through the door of an office or air flow over one workstation into another is considered to be advection, which is represented as,

$$
\dot{q}_{a b}^{a d v}=\dot{m}\left(h_{a}-h_{b}\right),
$$

where, $\dot{m}$ is the mass flow rate $[\mathrm{kg} / \mathrm{s}]$ and $h$ is the enthalpy $[\mathrm{kJ} / \mathrm{kg}]$. If we neglect the latent terms and look only at the sensible load, we can assume that the enthalpy is a function of the dry bulb temperature only and Eqn. (2.9) reduces to,

$$
\dot{q}_{a b}^{a d v}=\dot{m} c_{p}\left(T_{a}-T_{b}\right),
$$

where $c_{p}$ is the specific heat of air at constant pressure.

Both $\dot{q}_{c o n d}$ and $\dot{q}_{a d v}$ are linear functions of the temperature difference $\left(\mathrm{T}_{1}-\mathrm{T}_{2}\right)$. If we add these equations together, we get the total heat flux between zones $a$ and $b$,

$$
\begin{aligned}
& \dot{q}_{a b}^{\text {tot }}=\dot{m} c_{p}\left(T_{a}-T_{b}\right)+U_{a b}^{\text {tot }}\left(T_{a}-T_{b}\right), \\
& \dot{q}_{a b}^{\text {tot }}=U_{a b}^{\text {tot }}\left(T_{a}-T_{b}\right)
\end{aligned}
$$

where $U_{a b}^{e q v}=\left(\dot{m} c_{p}+U_{a b}^{\text {tot }}\right)$ is the equivalent conductance. For simplicity we drop the superscript eqv and use just $U_{a b}$ 


\section{Report No. DE-FG02-03ER63694-F1}

\subsection{HVAC Model}

We will assume the HVAC system is an air delivery system and therefore the energy supplied to zone $i$ from the HVAC system is given by the corresponding advective flux,

$$
\dot{q}_{i}^{H V A C}=\dot{m}_{i} c_{p}\left(T^{s}-T_{i}\right)
$$

where $T^{s}$ is the supply temperature from the HVAC system. For the time being, the specific system which delivers the air is not important; however later in section 2.10 we will discuss the introduction of a specific air handling unit.

In many buildings, ventilation air from the HVAC system is supplied at a single temperature. In order to maintain the desired temperature, the airflow rate is modulated either through a damper in the diffuser or by cycling air on and off. This is what is known as Variable Air Volume (VAV). For VAV, $\dot{m}_{i}$ in (2.13) could be different for each zone while $T^{s}$ would be constant. Just as easily we could treat use a fixed volume, variable temperature system, where $T_{i}^{s}$ is different for each room and $\dot{m}$ is fixed. This is equivalent to having a controllable heater or chilled water coil in each zone. In both approaches the total energy supplied by the

HVAC system $\left(\dot{q}^{H V A C}\right)$ remains the same. The difference lies solely in the free parameter we choose to control. The advantage in choosing a variable temperature system is that when following the method described in section 2.5 , the resulting system equations remain linear. Using the VAV approach will result in a non-linear system of equations. Since the timely execution of this system is desired, we will choose the approach to ensure the equations remain linear and quickly solvable. We will treat the airflow rate to each zone as constant and equal, and modulate the supply temperature for each zone. As a result equation (2.13) becomes, 
Report No. DE-FG02-03ER63694-F1

$$
\dot{q}_{i}^{H V A C}=\dot{m} c_{p}\left(T_{i}^{s}-T_{i}\right)
$$

\subsection{Thermostat Concept}

A thermostat-controlled zone will be defined as a zone which contains a thermostat that adjusts HVAC energy flow into the zone. The desired set point temperature, $T^{*}$, will be considered to be perfectly achieved through the HVAC system. The HVAC system will deliver the correct amount of heating/cooling energy necessary to maintain the temperature in the zone to the desired set point for the zone.

Each zone in the building must be controlled by one thermostat only; however, the location of this thermostat could vary and may not be in the controlled zone. The number of thermostats can range from a single thermostat in one zone that controls all other zones, to one thermostat in each zone, which control only the individual zone.

For example, consider 4 zones of equal dimensions as shown in Figure 2.1. Zones $b$, $c$, and $d$ contain thermostats with set points $T_{b}^{*}, T_{c}^{*}$, and $T_{d}^{*}$, respectively. Since zone $a$ does not have a thermostat it must be controlled by a thermostat located in another zone, say zone $b$. Zone $b$, will be supplied with the necessary energy from the HVAC system to ensure that the temperature in the zone remains perfectly at the set point temperature, $T_{b}^{*}$. This same temperature is also supplied to zone $a$ since it is controlled by the zone $b$ thermostat. Zones $c$ and $d$ receive energy from the HVAC system independently of zones $a$ and $b$ since they have a local thermostat. Therefore, the temperature in zones $b, c$, and $d$ can be independently set, whereas that in zone $a$ cannot. 
Report No. DE-FG02-03ER63694-F1

\begin{tabular}{|l|l|}
\hline$a$ & $b$ \\
& $T_{b}^{*}$ \\
\hline$c$ & $d$ \\
$T_{c}^{*}$ & $T_{d}^{*}$ \\
\hline
\end{tabular}

Figure 2.1 - Example 4-zone domain with thermostats in zones $b$, c, and $d$

The location of the thermostats within the building will depend on the nature of the individual control and will be discussed as it applies to the particular building in the section on thermostat control strategy (Section 2.9).

\subsection{Building Model as a Thermal Circuit Network}

With much of the necessary theory reviewed we can now discuss the development of the numerical model. The basic model consists of a single story office building divided into $n \times m$ zones. Each zone will be treated as a well-mixed zone of constant temperature. A single zone will correspond to an occupant's workstation, whether it is a private office or cubicle. Each zone will correspond to a node in a thermal resistance network. Continuing with the electrical circuit analogy, each node in the network is connected to the other nodes via a thermal resistance. Figure 2.2 shows a visual example of a portion of a thermal circuit network. Zone $i$ (at temperature $T_{i}$ ) is connected to three neighboring zones (at temperatures $T_{i-1}, T_{i+1}$, and $T_{i+1}$ ) by a resistance appropriate to the dividing interface $\left(R_{\text {in }}\right)$. It is also connected to the outside (at temperature $\left.T_{0}\right)$ by a resistance appropriate to the exterior wall $\left(R_{o u t}\right)$. It is in this way that we will connect each of the various zones in the building to each other and the outside temperature conditions. 


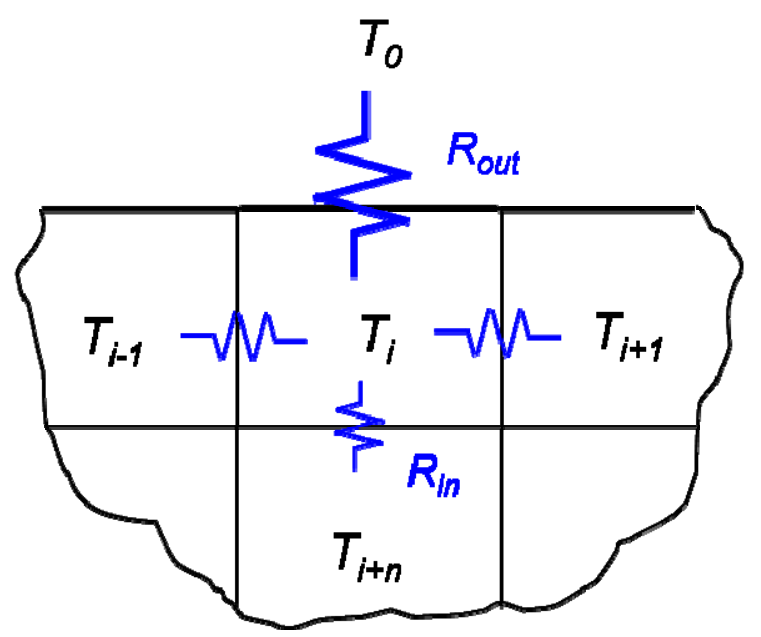

Figure 2.2 - Cut-out example of a thermal circuit network around zone $i$

As previously detailed, latent effects will be neglected and all heat transfer between zones will be treated as sensible. We will also neglect heat transfer by radiation; however, in Section 4.6 we will quantify the effect of this assumption. The model described in the following sections will be referred to as the Thermal Circuit Temperature Bin (TCTB) model.

\subsection{Matrix Equation Formulation}

We will treat the specific heat of air as constant regardless of the air temperature and as a result it can be easily combined with the mass flow rate, in an effort to reduce the cumbersome equations, as

$$
C_{i j}=\dot{m}_{i j} C_{p}
$$

Here, $\dot{m}_{i j}$ represents the mass flow rate of air to zone $i$ from zone $j$, and $c_{p}$ is the specific heat of air. $C_{i j}$ is now an effective flow rate with units $\mathrm{J} / \mathrm{sK}$. We will treat $C_{i i}$ as the effective flow 


\section{Report No. DE-FG02-03ER63694-F1}

rate coming into zone $i$ from the HVAC system. Take note that we will not be using summation notation.

As described in Eqn. (2.11), since both advection and conduction are a function of the same temperature difference it will be convenient to start with an overall conductance, $U_{i j}$ as given by,

$$
U_{i j}=C_{i j}+\frac{1}{R_{i j}},
$$

where $R_{i j}$ is the conductive and convective resistance of the interface (treated as a plane wall) between zone $i$ and zone $j$. We can now define the heat flux between any zones $i$ and $j$ as,

$$
\dot{Q}_{i j}=U_{i j}\left(T_{j}-T_{i}\right)
$$

which is Eqn. (2.11) applied to a system of zones. Eqn. (2.17) can apply to heat transfer with the outside environment which we will call zone 0 , hence the heat transfer with outside and zone $i$ can be written as, $\dot{Q}_{i 0}=U_{i 0}\left(T_{0}-T_{i}\right)$.

\subsubsection{Supply Temperature}

The supply temperature for each zone is dependent on the thermostat controlling that zone. We need a linkage operator, $\mathbf{L}$, which given the location of thermostats within the building, defines which zones are controlled by which thermostat. Since, by rule, a zone may be controlled by only one thermostat we will define the linkage between each room with the

linkage operator matrix $\mathbf{L}$, defined such that $L_{i k}=1$ when zone $i$ is controlled by a thermostat in 


\section{Report No. DE-FG02-03ER63694-F1}

zone $k$, otherwise $L_{i j}=0$. To illustrate the application of $\mathbf{L}$, let us revisit the earlier example of a $2 \times 2$ building consisting of four zones numbered 1, 2, 3, and 4 .

\begin{tabular}{|c|c|}
\hline 1 & $\begin{array}{l}2 \\
T_{2}^{*}\end{array}$ \\
\hline 3 & 4 \\
\hline$T_{3}^{*}$ & $T_{4}^{*}$ \\
\hline
\end{tabular}

Figure 2.3 - Example 4-zone domain with thermostats in zones 2, 3, and 4.

Zones 2, 3, and 4 have their own thermostats that will control each room respectively. Zone 2's thermostat will also control zone 1 . As a result, $L_{12}=1, L_{22}=1, L_{33}=1, L_{44}=1$, and all other elements of the $\mathbf{L}$ matrix are zero. For this 4-room example the entire $\mathbf{L}$ matrix would then look like,

$$
\mathbf{L}=\left[\begin{array}{llll}
0 & 1 & 0 & 0 \\
0 & 1 & 0 & 0 \\
0 & 0 & 1 & 0 \\
0 & 0 & 0 & 1
\end{array}\right]
$$

Notice the sum of every row is identically equal to one. This is a check that a zone is not being controlled by more than one thermostat. In more general terms, for any zone $i$,

$$
\sum_{k=1}^{N} L_{i k}=1
$$


Using the $\mathbf{L}$ matrix, and Eqn. (2.14), we can define the heat flux supplied by the HVAC system for zone $i$ as,

$$
\dot{Q}_{i}^{H V A C}=C_{i i} \sum_{k=1}^{N} L_{i k}\left(T_{k}^{s}-T_{i}\right)
$$

where $T_{k}^{s}$ is the supply temperature from the HVAC system to zone $k, T_{k}^{*}$ is the thermostat setpoint for zone $k$, and $\mathrm{C}_{\mathrm{i}}$ is defined between zone $i$ and the HVAC system as described above by Eqn. (2.15).

For example, if zone $i$ contains a thermostat, $L_{i i}=1$ and therefore $\dot{Q}_{i, H V A C}=C_{i i}\left(T_{i}^{s}-T_{i}^{*}\right)$. This is simply Eqn. (2.14) with the assumption that the zone temperature achieved is equal to the thermostat set point temperature. $\left(T_{i}=T_{i}^{*}\right)$.

\subsubsection{Energy Balance}

Now we will consider the entire building as a system of zones. Since the model is steady state, from the first law of thermodynamics [26], for any zone $i$ the sum of the heat fluxes into and out of the zone must sum to zero,

$$
\begin{gathered}
\sum \dot{Q}=0 \\
\sum \dot{Q}_{i}^{\text {cond }+a d v}+\sum \dot{Q}_{i}^{\text {cond }}+\dot{Q}_{i}^{H V A C}=0 .
\end{gathered}
$$

Here we break the heat flux into three unique types: conduction and advection (cond + adv), internal heat generation (gen), and HVAC supplied energy (HVAC). Substituting the appropriate terms, as detailed in the previous sections above, for any zone $i$ in subscript notation we get, 


$$
\sum_{j=0}^{N} U_{i j}\left(T_{j}-T_{i}\right)+G_{i}+C_{i i} \sum_{k=1}^{N} L_{i k}\left(T_{k}^{s}-T_{i}\right)=0 .
$$

Here we introduce $G_{i}$ as the sum of the internal heat generation sources (computer, person, lighting, etc.) within zone i (see section 2.8.3 for more detail). Notice that we will include the outside as zone 0 in the first sum. We can write (2.19) for zone $k$, which we will define as a local thermostat-controlled zone (i.e. zone $k$ has a thermostat set to temperature $T_{k}^{*}$ ):

$$
U_{k 0}\left(T_{0}-T_{k}^{*}\right)+\sum_{j=1}^{N} U_{k j}\left(T_{j}-T_{k}^{*}\right)+G_{k}+C_{k k}\left(T_{k}^{s}-T_{k}^{*}\right)=0
$$

Since zone $k$ is a locally controlled zone we know $T_{k}=T_{k}^{*}$. Rearranging and solving for the only unknown, $T_{k}^{s}$,

$$
T_{k}^{s}=\sum_{j=0}^{N} \frac{U_{k j}}{C_{k k}}\left(T_{k}^{*}-T_{j}\right)-\frac{G_{k}}{C_{k k}}+T_{k}^{*}
$$

Combining and simplifying equations (2.19) and (2.21) we get,

$$
\begin{aligned}
\sum_{j=1}^{N} U_{i j} T_{j} & -\sum_{j=0}^{N} U_{i j} T_{i}-C_{i i} T_{i}-C_{i i} \sum_{j=1}^{N} \sum_{k=1}^{N} \frac{U_{k j} L_{i k} T_{j}}{C_{k k}}=-U_{i 0} T_{0}-G_{i} \\
& -C_{i i} \sum_{k=1}^{N} L_{i k}\left[\frac{U_{k 0}}{C_{k k}}\left(T_{k}^{*}-T_{0}\right)+\sum_{j=1}^{N} \frac{T_{k}^{*}}{C_{k k}} U_{k j}-\frac{G_{i}}{C_{k k}}+T_{k}^{*}\right]
\end{aligned}
$$

We can re-write equation (2.22) in matrix notation, with the unknown temperature vector $\mathbf{T}$, as in equation (2.23).

$$
\mathbf{A T}=\mathbf{B}
$$

where, 


$$
\begin{aligned}
& A_{i j}=U_{i j}-C_{i i} \sum_{k=1}^{N} \frac{U_{k j} L_{i k}}{C_{k k}}, \quad i \neq j, \\
& A_{i i}=-\sum_{j=0}^{N} U_{i j}-C_{i i} \sum_{k=1}^{N} \frac{U_{k i} L_{i k}}{C_{k k}}-C_{i i}, \\
& B_{i}=-U_{i 0} T_{0}-G_{i}-C_{i i} \sum_{k=1}^{N} L_{i k}\left(\frac{U_{k 0}}{C_{k k}}\left(T_{k}^{*}-T_{0}\right)+\sum_{j=1}^{N} \frac{T_{k}^{*}}{C_{k k}} U_{k j}-\frac{G_{i}}{C_{k k}}+T_{k}^{*}\right) .
\end{aligned}
$$

Eqn. (2.23) now gives us a linear set of equations which we can use to solve for the unknown zonal temperatures for any given thermostat control strategy (i.e., any $L_{i k}$ ), building conductances $\left(U_{i j}\right)$, and outdoor temperature $\left(T_{0}\right)$. The unknown temperatures need to be found only in the zones without local thermostats because of the assumption that the thermostat set point temperature is the zone temperature. In these thermostat-controlled zones we must find the local supply temperature. The local HVAC supply temperatures can be found by substituting the zone temperatures into (2.21). For a given $T_{0}, \mathbf{L}, \mathbf{U}$, and $\mathbf{T}^{*}$ set points we now can find the corresponding zonal temperatures and local HVAC supply temperatures.

Had we chosen to use a VAV system we would have been unable to successfully create a linear system. This would have lead to the product of unknown temperature $\left(T_{i} T_{j}\right)$ terms in equation (2.22) and we would have been unable to solve the system as neatly. While solving a non-linear system is possible, it would require an iterative process and slow the computation time considerably, thus making it unsuitable for optimization. 
Report No. DE-FG02-03ER63694-F1

\subsection{Building Load}

The building load can be determined using the zone and supply temperatures found in Section 2.5. The building load will be defined as the rate of sensible cooling and/or heating energy supplied by the HVAC system. This number is independent of any HVAC equipment (see Section 2.10 for equipment calculations). Eqn (2.18) gives an expression for the power (rate of energy) supplied to each zone by the system. We can sum heating and cooling power separately as,

$$
\begin{gathered}
\dot{Q}_{\text {cool }}=\sum_{i=1}^{N}\left\{\begin{array}{ccc}
\dot{Q}_{i}^{H V A C} & \text { if } & \dot{Q}_{i}^{H V A C}<0, \\
0 & & \text { otherwise }
\end{array}\right\}, \\
\dot{Q}_{\text {heat }}=\sum_{i=1}^{N}\left\{\begin{array}{ccc}
\dot{Q}_{i}^{H V A C} & \text { if } & \dot{Q}_{i}^{H V A C}>0 \\
0 & & \text { otherwise }
\end{array}\right\} .
\end{gathered}
$$

\subsection{Temperature Bin Method}

The model, as described thus far, is inherently steady state. As mentioned, the outdoor temperature $\left(T_{0}\right)$ is assumed to be constant or quasi-steady. Since outdoor weather is transient by nature, an approximation must be made for computing the annual energy consumption. Here we will use a common method used in building energy simulation known as the temperature bin method [27]. The yearly temperature profile of a city is grouped by the number of hours per year in various temperature intervals (bins). The temperature bin method involves computing the building energy consumption as a steady state calculation for each constant outdoor temperature bin and then multiplying by the number of hours in each bin. Common practice in the U.S. is to use $5^{\circ} \mathrm{F}$ bins, but since we will be using Celsius as the unit for temperature, $3^{\circ} \mathrm{C}$ bins will be used. Bins will be referred to as the average bin temperature, 
Report No. DE-FG02-03ER63694-F1

as this is the value that will be used in the energy calculations. For example, the $19^{\circ} \mathrm{C}$ bin consists of temperatures between $17.5^{\circ} \mathrm{C}$ and $20.5^{\circ} \mathrm{C}$. See Table 6.1 for a complete list of temperature bin data.

\subsubsection{Typical Meteorological Year (TMY2)}

The National Renewable Energy Laboratory (NREL) has produced hourly values of metrological data for a 1-year period for hundreds of US locations [28]. Temperature, solar radiation, wind speed, and so on were recorded for a 30 year period from 1961-1990. The average data from this 30 year period is stored in what is commonly called TMY2 (Typical Meteorological Year) files. The TMY2 data is a collection of typical values for a given location, not extremes, as they were intended to be used for computer simulations of building and solar systems. Since the TMY2 data contains dozens of meteorological phenomena that are beyond the scope of this model, only the hourly temperature data, for all 8760 hours per year, was extracted for the cities described below.

\subsubsection{DOE Climate Zones}

The US Department of Energy (DOE) has developed a new classification for US climates that will be adopted by the new energy codes and standards [29]. The classification system divides the country into 8 zones based on dry bulb temperature with 3 further subdivisions $(A, B$, and C) based on moisture (Figure 2.4) [29]. For each zone, a representative US city was chosen based on the following three criteria:

1. The city should have average weather conditions of the climate zone; not favoring mild or harsh conditions. 
2. The city should preferably be located centrally within the zone.

3. The city should be close to where buildings are predominately located (population centers).

Given the above criteria, 15 representative cities were chosen, by DOE, to represent the climatic diversity of the US as shown in Table 2.1. Note that zones $1 \mathrm{~B}$ and $5 \mathrm{C}$ do not occur in the US. It will be the temperature data, extracted from TMY2 data files, from these 15 cities that will be used in the TCTB (Thermal Circuit Temperature Bin) model.

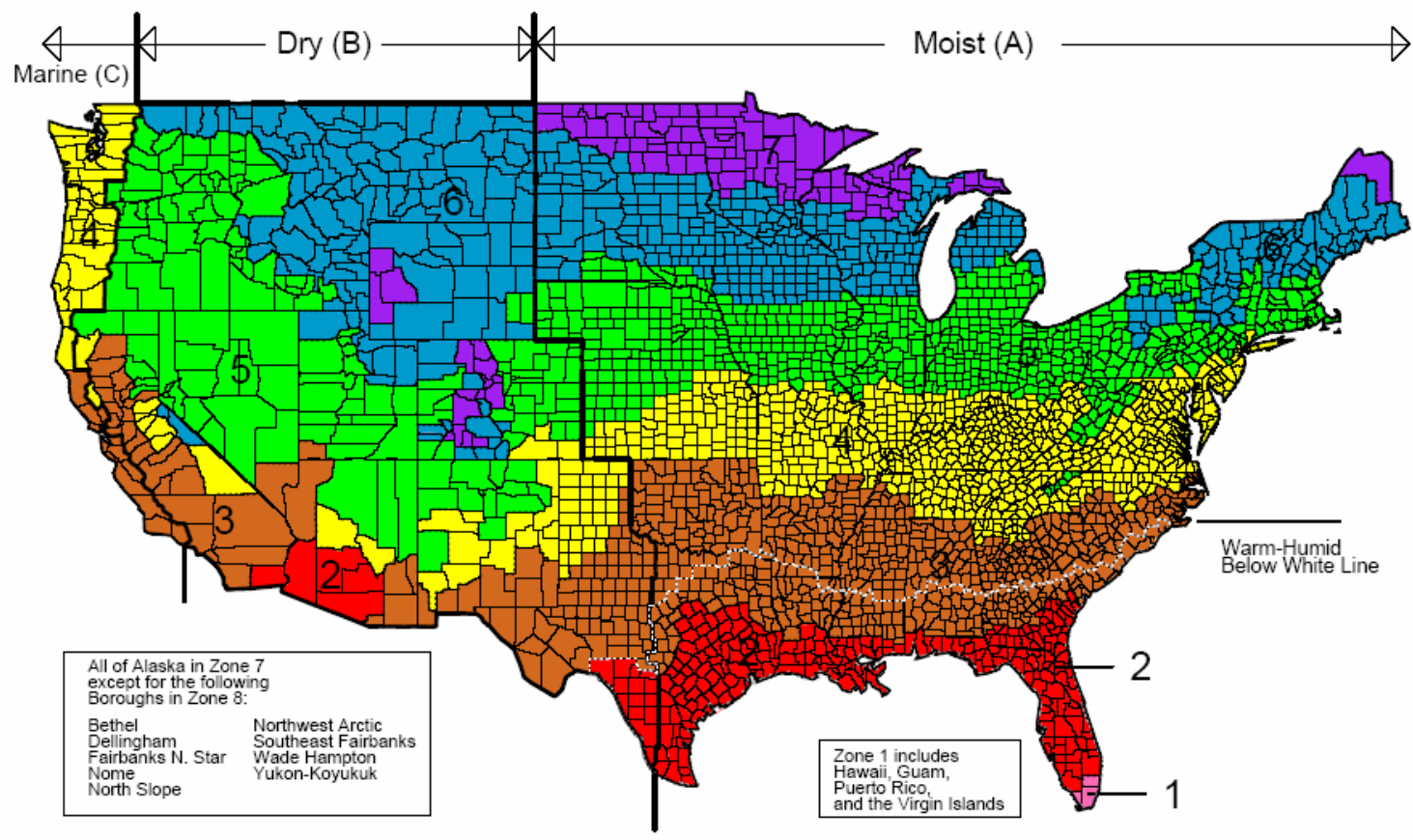

Figure 2.4 - Map of DOE US climate zones [30] 
Report No. DE-FG02-03ER63694-F1

Table 2.1 - DOE climate zone definitions and representative US cities [29]

\begin{tabular}{|c|c|c|c|}
\hline Zone No. & Climate Zone Name and Type & Representative US City & TCTB City No. \\
\hline $1 \mathrm{~A}$ & Very Hot - Humid & Miami, FL & 1 \\
\hline $1 \mathrm{~B}$ & Very Hot - Dry & --- & --- \\
\hline $2 \mathrm{~A}$ & Hot - Humid & Houston, TX & 2 \\
\hline $2 \mathrm{~B}$ & Hot - Dry & Phoenix, AZ & 3 \\
\hline $3 \mathrm{~A}$ & Warm - Humid & Memphis, TN & 4 \\
\hline $3 \mathrm{~B}$ & Warm - Dry & El Paso, TX & 5 \\
\hline $3 \mathrm{C}$ & Warm - Marine & San Francisco, CA & 6 \\
\hline $4 \mathrm{~A}$ & Mixed - Humid & Baltimore, MD & 7 \\
\hline $4 \mathrm{~B}$ & Mixed - Dry & Albuquerque, NM & 8 \\
\hline $4 \mathrm{C}$ & Mixed - Marine & Salem, OR & 9 \\
\hline $5 \mathrm{~A}$ & Cool - Humid & Chicago, IL & 10 \\
\hline $5 \mathrm{~B}$ & Cool - Dry & Boise, ID & 11 \\
\hline $5 \mathrm{C}$ & Cool-Marine & --- & --- \\
\hline $6 \mathrm{~A}$ & Cold - Humid & Burlington, VT & 12 \\
\hline $6 \mathrm{~B}$ & Cold - Dry & Helena, MT & 13 \\
\hline 7 & Very Cold & Duluth, MN & 14 \\
\hline 8 & Subarctic & Fairbanks, AK & 15 \\
\hline
\end{tabular}

\subsubsection{Population Weighting}

To capture the relative importance of each climate zone we can weight their importance based on total population within each zone. Along with a description of the climate zone every US county and the climate zones to which they belonged were included in the new climate definitions. Using the 2000 United States Census Bureau [31] county population data, an approximate total of the number of people living in each climate zone was calculated. Figure 


\section{Report No. DE-FG02-03ER63694-F1}

2.5 shows the approximate zonal population per zone. It can be quickly observed that zones 4A (Baltimore) and 5A (Chicago) are by far the most populated of the climate zones.

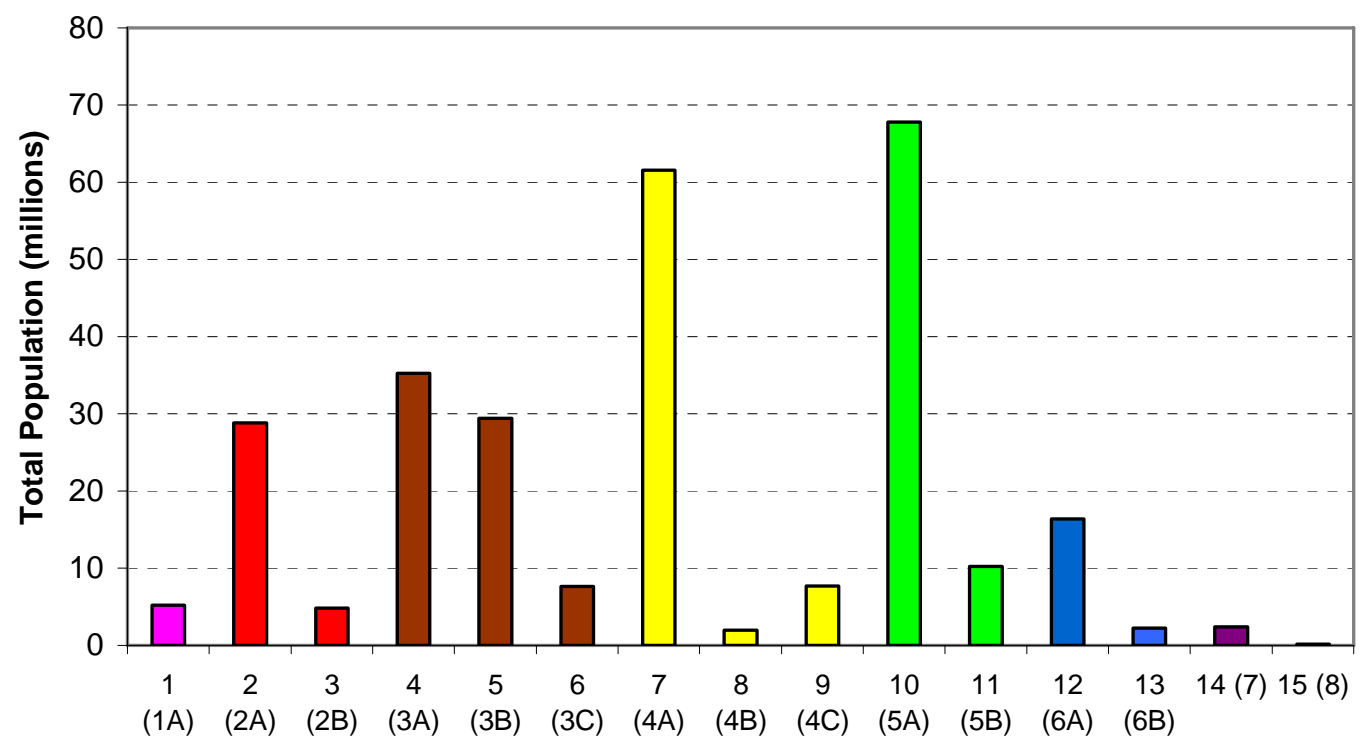

City \# (DOE Zone)

Figure 2.5 - Total population in each climate zone (US Census Bureau 2000)

\subsubsection{Work Day Bins}

Since this study was focused on office buildings, it was desired to monitor the energy usage of the building during occupied hours only, since it is common to have an office building that is largely unoccupied during nighttime hours. During these unoccupied hours, the HVAC system is set back to a point of minimal energy usage since occupant comfort is no longer a priority, and for this reason will be neglected. Also, during unoccupied hours, it is unlikely to observe a difference between conventional and personal control, since unique temperatures are no longer necessary and both will be operating at similar set back temperatures. Therefore, it was decided that only the hours between 6am and 7pm, should be observed. The hourly data 
provided by the TMY2 was truncated between $7 \mathrm{pm}$ and 6am, 7 days a week, so only the 4750 hours corresponding to the intervals within this time frame were preserved. The temperature bin intervals as described above will remain the same, however the hours per bin will be adjusted to include only the 4750 work day hours. Figure 2.6 shows an example histogram of hours per bin for the work day time frame.

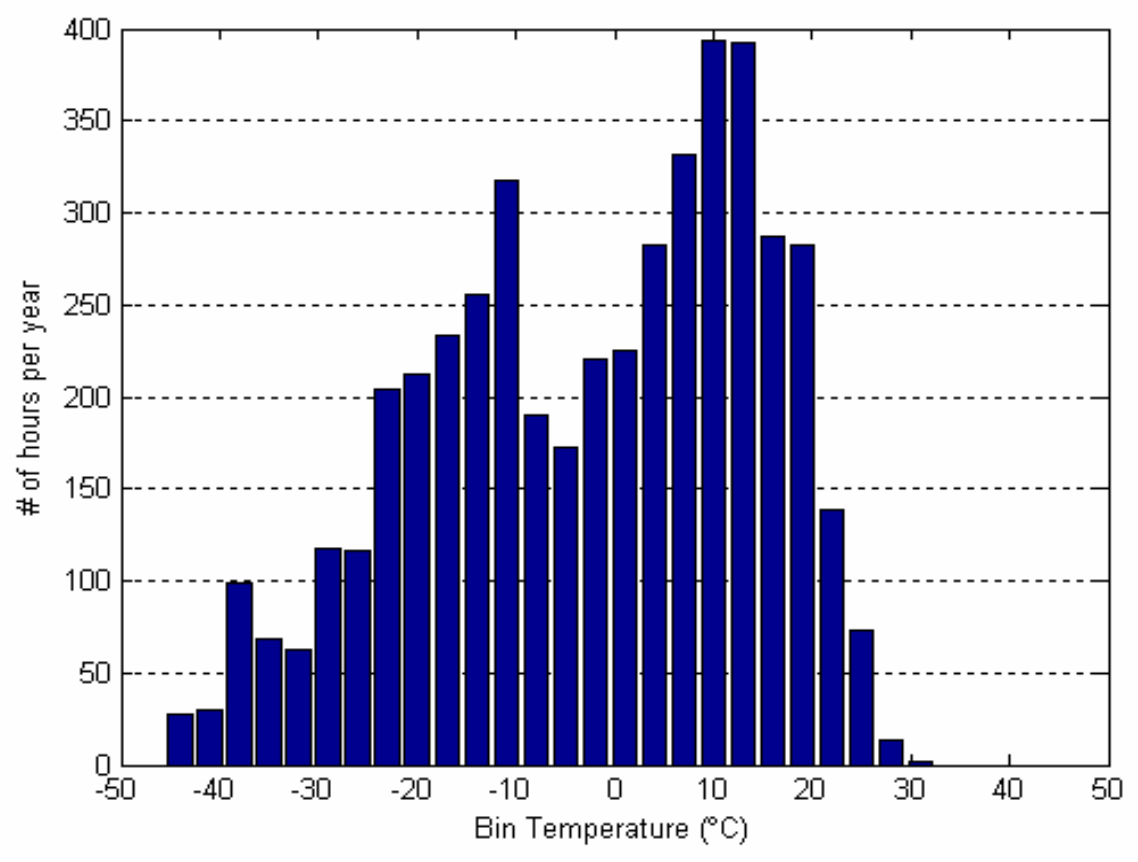

Figure 2.6 - Histogram of the number of work-day hours per temperature bin for Chicago, IL (City \#10)

The work day hours could be further truncated to exclude weekend hours, however this was not done for two reasons. First, weekends do not fall on the same days every year and therefore there is no reason to assume that weekend weather will be any different that nearby weekday weather. Second, it is not uncommon for some offices operate six or seven days a week (e.g. call centers). Therefore, for the purposes of this study we will include all seven days 
a week. If it were desired to calculate the energy usage for only the weekdays we could use a 5/7 multiplier to scale accordingly.

\subsection{Baseline Building Model}

In this section, the specifics of the baseline building model will be discussed. Details of the building layout, building envelope materials (e.g. walls and windows) and the approximations regarding human occupants and other heat gains will also be outlined.

\subsubsection{Building Layout and Geometry}

As formulated, the matrix equations are capable of modeling any rectangular building of $n \times m$ zones. Typical office buildings are quite varied is size, shape, and interior layout. To get an understanding of an average office building we will need to make some simplifying assumptions about the layout of a building. We will neglect corridors hallways etc, as they tend not to be a primary place of occupancy for office workers. Also, we would like a significant number of offices/workstations inside the building to better account for the variation in personal preference.

For the baseline building model, a square building consisting of 49 square zones $(7 \times 7)$ was chosen. Figure 2.7 shows a schematic of the floor plan of the baseline building. Each room is identical in dimensions (Table 2.2) with a total zonal volume of $36.75 \mathrm{~m}^{2}$. The only difference between zones is that each exterior wall of a perimeter zone will have a $5 \mathrm{~m}^{2}$ window, the details of which are explained in Section 2.8.2. This configuration creates a large core of 25 
zones that do not have an exterior wall. This is quite common in large office buildings with a significant concentration of workstations in the central core.

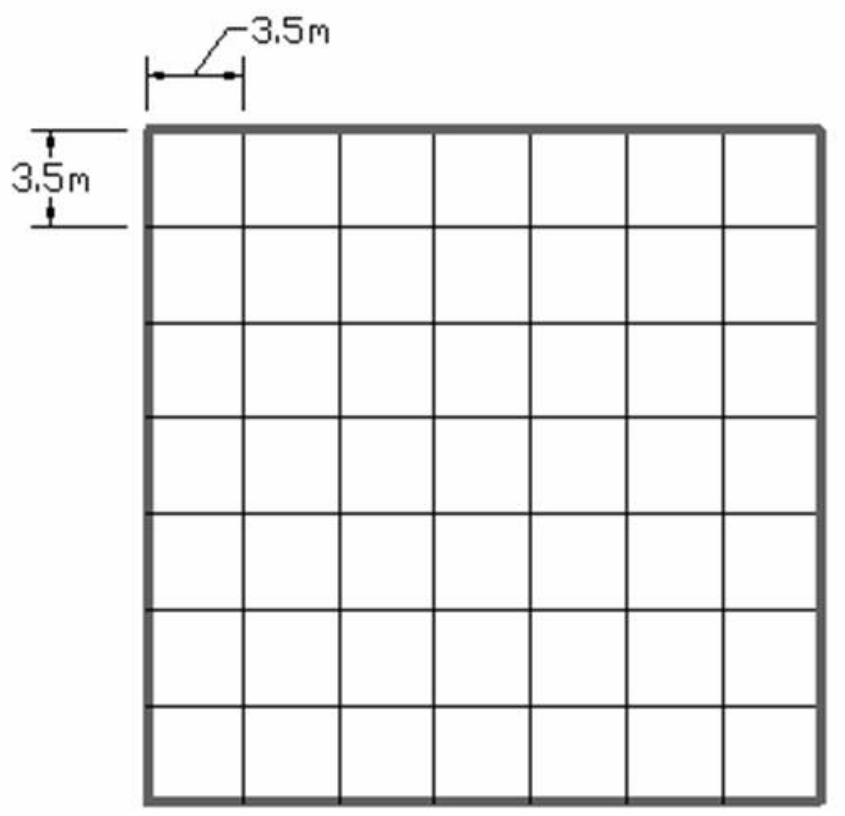

Figure 2.7 - Baseline building model floor plan

Table 2.2 - Baseline building model zonal physical dimensions

\begin{tabular}{lc}
\hline Dimension & Area $\left(\mathrm{m}^{2}\right)$ \\
\hline Interior wall & 10.5 \\
Exterior wall (including window) & 10.5 \\
Exterior window & 5.0 \\
Ceiling/Floor & 12.25 \\
\hline
\end{tabular}

\subsubsection{Thermal Resistance}

Integral to the nature of the building model is the numerical values for the thermal resistances between adjacent zones and the outside. Table 2.3 lists the values of the thermal resistances 


\section{Report No. DE-FG02-03ER63694-F1}

used in the baseline building model. The partitions between adjacent workstations, for the baseline case, will be treated as interior walls, i.e. private offices. Later, in Section 4.2, the interior resistance will be modified to a value more appropriate to model cubicle workstations with partial height partitions.

Table 2.3 - Thermal resistance values in baseline building model.

\begin{tabular}{|c|c|c|c|}
\hline Type & Notation & Value & Notes \\
\hline Interior wall & $r_{\text {iwall }}$ & $0.392 \mathrm{~m}^{2} \mathrm{~K} / \mathrm{W}$ & Gypsum wall board wood frame [27] \\
\hline $\begin{array}{l}\text { Exterior wall } \\
\text { (and roof) }\end{array}$ & $\mathrm{r}_{\text {ewall }}$ & $2.47 \mathrm{~m}^{2} \mathrm{~K} / \mathrm{W}$ & Insulated wood frame [27] \\
\hline Exterior window & $\mathrm{r}_{\text {window }}$ & $0.353 \mathrm{~m}^{2} \mathrm{~K} / \mathrm{W}$ & Double paned air-filled window [22] \\
\hline $\begin{array}{l}\text { Inside convective heat } \\
\text { transfer coefficient }\end{array}$ & $\mathrm{h}_{\text {in }}$ & $3.06 \mathrm{~W} / \mathrm{m}^{2} \mathrm{~K}$ & $\begin{array}{l}\text { Common building energy simulation } \\
\text { indoor value [22] }\end{array}$ \\
\hline $\begin{array}{l}\text { Outside convective } \\
\text { heat transfer } \\
\text { coefficient }\end{array}$ & $\mathrm{h}_{\text {out }}$ & $17.78 \mathrm{~W} / \mathrm{m}^{2} \mathrm{~K}$ & $\begin{array}{l}\text { Common building energy simulation } \\
\text { outdoor value [22] }\end{array}$ \\
\hline
\end{tabular}

It is important to note the difference between the lower case $\mathrm{r}$ and upper case $\mathrm{R}$. A lower case $r$ has the units $\mathrm{m}^{2} \mathrm{~K} / \mathrm{W}$ while the upper case includes the area of the interface and has units $\mathrm{K} / \mathrm{W}$. Simply, $R=r / A$. The thermal resistance published for building materials is the lower caser $r$, however for building energy calculation purposes we need the overall interface resistance $(\mathrm{R})$.

The values listed in Table 2.1 provide a means to calculate to resistance matrix $\left(\mathrm{R}_{\mathrm{ij}}\right)$ used in Eqn. (2.16). Before the matrix can be constructed, overall interface resistances $\left(R_{\text {tot }}\right)$ must be calculated as shown in Eqn (2.7). For example, the resistance of an interface between a zone 


\section{Report No. DE-FG02-03ER63694-F1}

and the exterior consists of an inside convective heat transfer coefficient in series with the combination of the exterior wall and window in parallel, and then an outside convective heat transfer coefficient given by,

$$
R_{\text {ext }}=\frac{1}{A h_{\text {in }}}+\left(\frac{1}{\frac{A_{\text {ewall }}}{r_{\text {ewall }}}+\frac{A_{\text {window }}}{r_{\text {window }}}}\right)+\frac{1}{A h_{\text {out }}}
$$

where, $A$ is the total wall area including windows $\left[\mathrm{m}^{2}\right], A_{\text {evall }}$ is the area of exterior wall $\left[\mathrm{m}^{2}\right]$, $A_{\text {window }}$ is the area of exterior window $\left[\mathrm{m}^{2}\right], b_{i n}$ is the inside convective heat transfer coefficient

$\left[\mathrm{W} / \mathrm{m}^{2} \mathrm{~K}\right], h_{\text {out }}$ is the outside convective heat transfer coefficient $\left[\mathrm{W} / \mathrm{m}^{2} \mathrm{~K}\right], r_{\text {ewall }}$ is the exterior wall resistance $\left[\mathrm{m}^{2} \mathrm{~K} / \mathrm{W}\right]$, and $r_{\text {window }}$ is the exterior window resistance $\left[\mathrm{m}^{2} \mathrm{~K} / \mathrm{W}\right]$.

Determination of convective heat transfer coefficients is difficult to approximate with a single number since they are inherently dependent on many variables such as surface geometry, fluid motion, etc. The values chosen represent common building energy simulation estimates. As mentioned, the interior resistance values will be modulated later and it will be possible to determine the importance and influence of the convective heat transfer coefficients.

\subsubsection{Internal Heat Generation}

Inside each zone there was an average person doing moderate office work, a desktop computer with flat panel monitor, and lighting. The specific heat gain values used, obtained from the ASHRAE handbook of Fundamentals [27], are show in Table 2.4. Note that since the model does not account for humidity, the latent gain of the occupants will not be considered. Therefore, the total internal heat gain in each zone is $260 \mathrm{~W}$. This is the value that will be used for $G_{i}$ in equation (2.23). 
Report No. DE-FG02-03ER63694-F1

Table 2.4 - Internal heat gains in each zone [27].

\begin{tabular}{lll}
\hline Source & Gain & Notes \\
\hline Person & $75 \mathrm{~W}$ & $\begin{array}{l}\text { Moderate seated office work } \\
\text { Only sensible gain (note: latent }=55 \mathrm{~W})\end{array}$ \\
Computer CPU & $55 \mathrm{~W}$ & Average computer \\
Computer Monitor & $55 \mathrm{~W}$ & Average small monitor \\
Printer & $10 \mathrm{~W}$ & Small personal desktop printer, idle \\
Lighting & $65 \mathrm{~W}$ & Typical dual lamp fluorescent fixture \\
\hline
\end{tabular}

\subsubsection{Infiltration}

Infiltration is the unintentional air leakage through small cracks in the building envelope due to a pressure difference (from wind, mechanical ventilation, stack pressure, etc.) across the opening. Infiltration will not be considered explicitly (see section 2.8.5) in this study for a few reasons. First, commercial building envelope leakage tends to be much less than that of residential buildings. Commercial buildings tend to have a more airtight construction and their ventilation systems are typically designed to minimize infiltration by providing a slight pressurization [27]. In buildings with large cores, as commercial buildings often have, the effect of the perimeter air leakage becomes less significant. Also, infiltration through the building envelope tends to be much less than that of internal passages (elevator shafts etc.) [27]. 
Report No. DE-FG02-03ER63694-F1

\subsubsection{Airflow Rate and Ventilation}

The airflow rate for each zone was chosen to be a standard $5 \mathrm{ACH}$ [2], of which a standard 20 cfm was fresh, outdoor air, while the remaining was re-circulated air. The fresh air was introduced to each zone at outdoor conditions, before the heat balance. This $20 \mathrm{cfm}$ will be assumed to be much larger than any possible infiltration to the zones. Converting $20 \mathrm{cfm}$ to SI units, we get an outdoor air flow rate of $0.00944 \mathrm{~m}^{3} / \mathrm{s}$. For our baseline building, with an office volume of $36.75 \mathrm{~m}^{3}$, we get a total airflow rate of $0.051 \mathrm{~m}^{3} / \mathrm{s}(5 \mathrm{ACH})$. Applicable air properties used in the calculation of the associated advective heat flux are listed in Appendix 6.2 .

\subsection{Thermostat Control Strategy}

With the physical building described, we are now faced with the task of modeling the use and energy consumption of the distributed environmental control systems, and the comparable traditional systems. We suggest the phrase "Have It Your Way" or "HIYW" to refer to the personal control case, where each occupant has a thermostat in his/her office or workstation with the ability to control its environment. With a conventional system, the system seeks to achieve the same temperature in every zone in the building. For this case, we suggest the phrase "One Size Fits All” or “OSFA,” meaning everyone gets the same.

\subsubsection{Occupant Comfort Model}

In Chapter 1 we discussed the inherent difference in personal preference of temperature. Unfortunately, standards like ASHRAE 55-2004 are concerned mainly with the average comfort of a population. The individual preference is not of addressed by the standards, only 
the aggregate of the group. Ari et al. [32] proposed an individual comfort model derived from the published ASHRAE standard. The comfort model consists of a Gaussian distribution for neutral temperatures (the temperature at which an individual occupant votes 0 on Fanger's thermal comfort scale [3]), and a Gaussian distribution for individual temperature tolerance range, $\Delta \mathrm{T}$ (the sensitivity to deviations from the neutral temperature). For this study we will only employ the neutral temperature distribution and study the effect of the temperature tolerance only parametrically. A detailed optimization study accounting for the variation of both neutral temperature and $\Delta \mathrm{T}$ from one individual to another is described in Ari, Cosden, et al. [32]. The mean of this neutral temperature distribution is $24^{\circ} \mathrm{C}$ with a standard deviation of $1.2^{\circ} \mathrm{C}$. We can sample randomly from this distribution to obtain a population for our building. Figure 2.8 is an example histogram of 49 individuals' neutral temperatures.

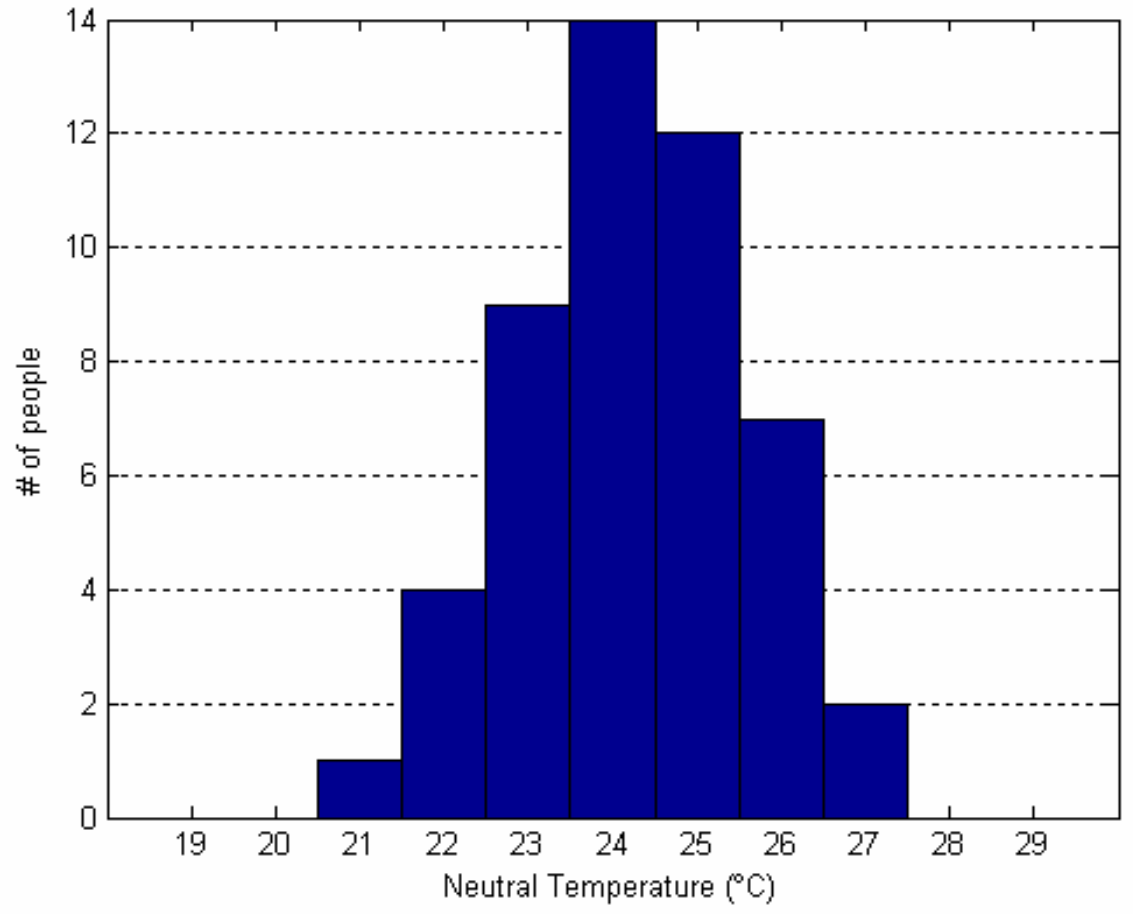

Figure 2.8 - Histogram of 49 random individuals' neutral temperature setpoints taken from Ari, Cosden et al. [32] Gaussian distribution 
These individuals could then be assigned randomly to zones, or "offices," in the building model as their prescribed workstations (Figure 2.9). Note that a different random selection of 49 individuals from the population distribution could produce a different histogram.

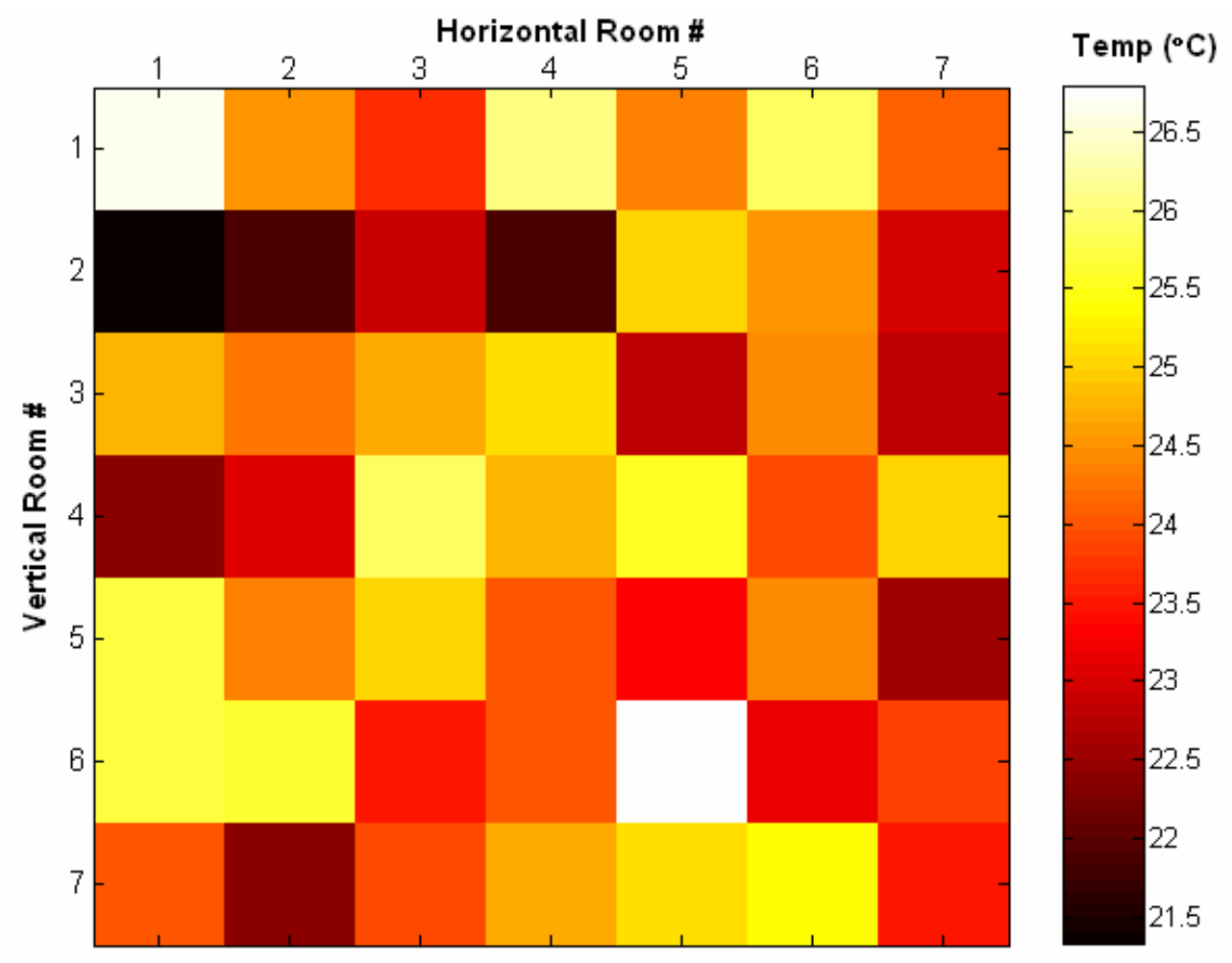

Figure 2.9 - Example individuals' neutral temperatures distributed randomly in building

\subsubsection{Conventional Control}

We would like to mimic conventional practice as closely as possible, which usually means attempting to provide a building with a uniform temperature through a small number of thermostats. In deciding where to place thermostats we will take advantage of the nature of the $7 \times 7$ model assumptions. As described before the baseline building model has three types of 


\section{Report No. DE-FG02-03ER63694-F1}

zones with unique interfaces with the exterior: corner, perimeter, and center zones. Since we do not consider any potential asymmetries with the outside temperature (i.e., solar radiation, directional wind) $U_{i 0}$ has three unique values corresponding to these 3 types of zones. Therefore, if we place a single thermostat, each set to the identical temperature, in one zone of each type (corner, perimeter, and center) and use that thermostat's signal to control the other zones of like type, we can achieve a uniform temperature distribution in the building (OSFA). Figure 2.10 shows the placement of three thermostats $x, y$, and $z$ : one in each type of zone. The zones controlled by these thermostats are correspondingly shaded.

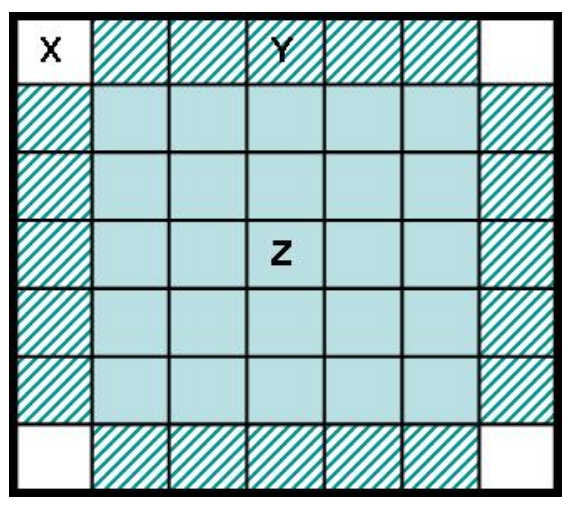

Figure 2.10 - Thermostat placement and controlled zones for OSFA

As a result, if we eliminate heat transfer between interior zones by having the same temperature in every zone, the only heat transfer term that is different between zones is with the outside. Hence, the building can be held at a constant temperature through three strategically placed thermostats, each set to the same temperature. While this may not be entirely realistic, it gives the best possible outcome of a conventional approach. Deviations from this (two thermostats, etc.) would cause temperatures to differ in various zones in the building and would not follow the ASHRAE guidelines as closely. 
Also, to get as close as possible to the ASHRAE guideline ideal, OSFA thermostats will each be set to $24.0^{\circ} \mathrm{C}$, the mean neutral temperature for a population distribution. Figure 2.11 shows the OSFA temperature distribution in the 49-zone building. As expected the temperature in each zone is exactly $24.0^{\circ} \mathrm{C}$.

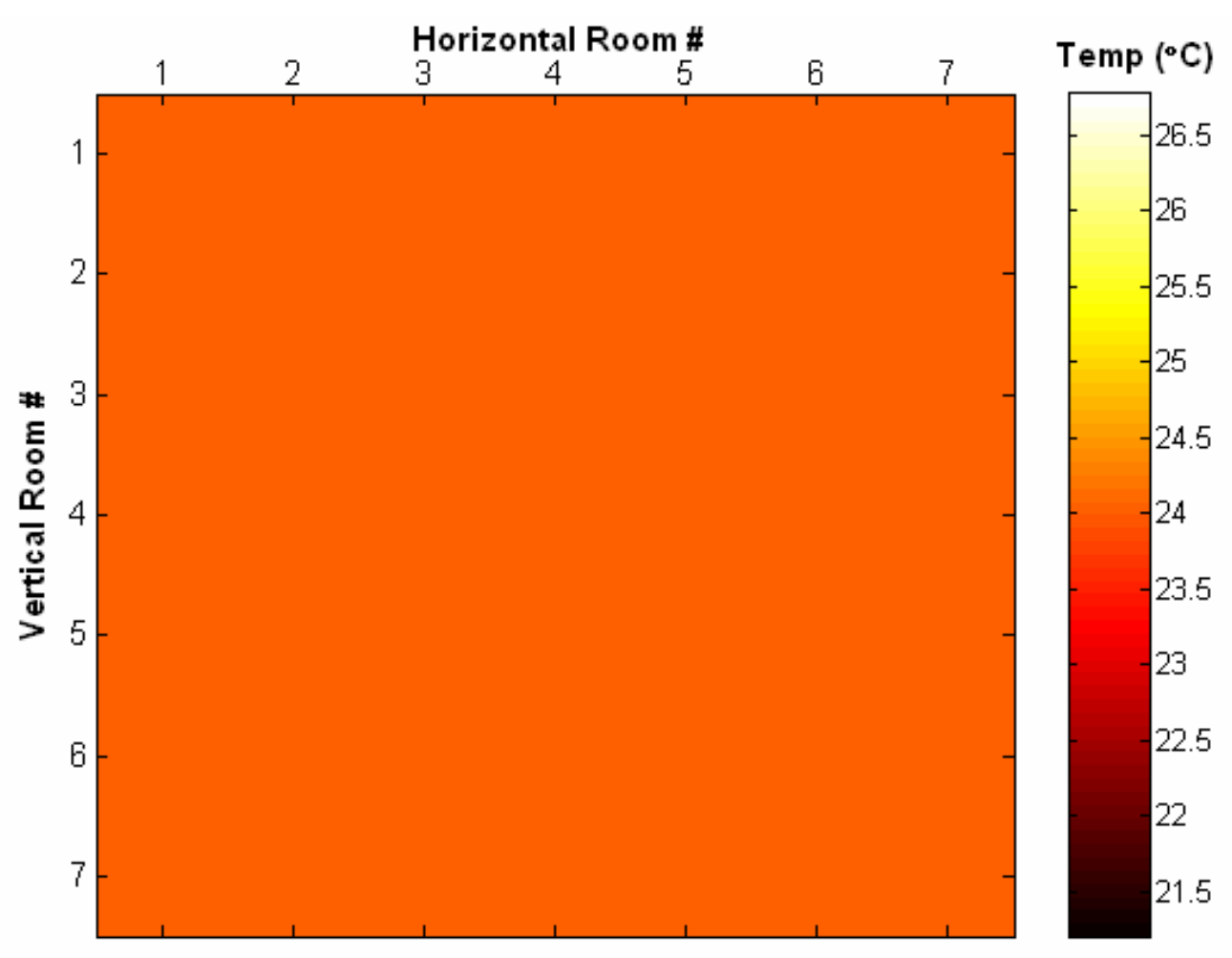

Figure 2.11 - OSFA temperature distribution in baseline building

\subsection{Energy Calculation}

For the purpose of this study, we assumed that the HVAC system of the building consists of a roof top heat pump, furnace, and an indoor fan. The heat pump is solely used to provide cooling while the furnace solely provides heating. The indoor fan, used to carry conditioned air to the offices, is the same for both the furnace and the heat pump 


\section{Report No. DE-FG02-03ER63694-F1}

\subsubsection{Heat Pump Model}

The model for the roof top heat pump is based on the Carrier 10-ton Weathermaker® 50TFQ012 [33]. This is a standard efficiency, constant speed, heat pump equipped with an economizer. The 10-ton model was chosen to provide realistic coefficient of performance (COP) and capacity data for a heat pump of approximate size for the 49 zone, $600 \mathrm{~m}^{2}$ building. Capacity scaling of the heat pump will be discussed in the following section. Performance data, published by the manufacturer is available for the heat pump for a variety of indoor temperatures, outdoor temperatures, and airflow rates. The data was published for four discrete flow rates. Since we want to provide the 49-zone $1800 \mathrm{~m}^{3}$ building with approximately $5 \mathrm{ACH}$, of which between 3.5 and $4 \mathrm{ACH}(3700 \mathrm{cfm}$ and $4200 \mathrm{cfm})$ is recycled indoor air, we will choose the closest published indoor return-air fan setting, $3750 \mathrm{cfm}$. Table 2.5 shows the performance data for a return-air flow rate of $3750 \mathrm{cfm}$. Also shown in Table 2.5 is the calculated energy efficiency ratio (EER), which is defined as the ratio of the total cooling capacity (TC) and the compressor power input $\left(\mathrm{P}_{\text {comp }}\right)$.

Table 2.5 - Manufacturer's published cooling data for Carrier WEATHERMAKER 50TFQ012 [33] standard efficiency 10-ton heat pump operating at $3750 \mathrm{cfm}$, and calculated EER.

Ewb is the Entering Wet-bulb temperature, $\mathrm{P}_{\text {comp }}$ is the compressor motor power input, TC is the Total Capacity (1000Btuh) gross, and EER is the calculated energy efficiency ratio. 
Report No. DE-FG02-03ER63694-F1

\begin{tabular}{|c|c|c|c|c|}
\hline \multirow{2}{*}{\multicolumn{2}{|c|}{$\begin{array}{c}\text { Temp }\left({ }^{\circ} \mathrm{F}\right) \text { Outdoor } \\
\text { Entering Air } \\
(\mathrm{Edb})\end{array}$}} & \multicolumn{3}{|c|}{ Indoor Entering Air - Ewb $\left({ }^{\circ} \mathrm{F}\right)$} \\
\hline & & \multirow{2}{*}{$\begin{array}{c}87 \\
134.2\end{array}$} & \multirow{2}{*}{$\begin{array}{c}81 \\
126.2\end{array}$} & \multirow{2}{*}{$\begin{array}{c}75 \\
116.2\end{array}$} \\
\hline \multirow{3}{*}{75} & $\mathrm{TC}$ & & & \\
\hline & $\mathrm{P}_{\text {comp }}$ & 8.54 & 8.34 & 8.04 \\
\hline & EER & 15.7 & 15.1 & 14.5 \\
\hline \multirow{3}{*}{85} & $\mathrm{TC}$ & 131 & 122 & 112.4 \\
\hline & $\mathrm{P}_{\text {comp }}$ & 9.5 & 9.24 & 8.98 \\
\hline & EER & 13.8 & 13.2 & 12.5 \\
\hline \multirow{3}{*}{95} & TC & 126.2 & 117.4 & 107.4 \\
\hline & $\mathrm{P}_{\text {comp }}$ & 10.46 & 10.18 & 9.9 \\
\hline & EER & 12.1 & 11.5 & 10.8 \\
\hline \multirow{3}{*}{105} & TC & 122.4 & 112.4 & 102.8 \\
\hline & $\mathrm{P}_{\text {comp }}$ & 11.64 & 11.28 & 10.9 \\
\hline & EER & 10.5 & 10.0 & 9.4 \\
\hline \multirow{3}{*}{115} & $\mathrm{TC}$ & 116.6 & 107 & 98 \\
\hline & $\mathrm{P}_{\text {comp }}$ & 12.78 & 12.42 & 12 \\
\hline & EER & 9.1 & 8.6 & 8.2 \\
\hline \multirow{3}{*}{125} & $\mathrm{TC}$ & 110.4 & 101.2 & 93.6 \\
\hline & $\mathrm{P}_{\text {comp }}$ & 13.96 & 13.62 & 13.22 \\
\hline & EER & 7.9 & 7.4 & 7.1 \\
\hline
\end{tabular}

The published data needs to be converted to appropriate SI units $\left({ }^{\circ} \mathrm{F}\right.$ to ${ }^{\circ} \mathrm{C}$ and BTUh to $\left.\mathrm{kW}\right)$ and since the TCTB model does not account for humidity the web bulb temperatures need to 


\section{Report No. DE-FG02-03ER63694-F1}

be approximated by equivalent dry bulb temperatures. All entering wet bulb temperatures were converted to equivalent dry bulb temperatures at 50\% relative humidity. Then, the COP (like the EER in the other units) can be calculated from,

$$
C O P=\frac{T C}{P_{\text {comp }}}
$$

where, TC is the total heat pump capacity in the cooling mode $[\mathrm{kW}]$, and $P_{\text {comp }}$ is the compressor motor power input $[\mathrm{kW}]$.

Figure 2.12 shows calculated COPs for various outdoor and indoor dry bulb temperatures. Also shown are the best-fit linear trend lines used to interpolate and extrapolate the COP data when necessary. The calculated COP data, along with the extrapolated values, will be used to create a table look-up for the model heat pump. 
Report No. DE-FG02-03ER63694-F1

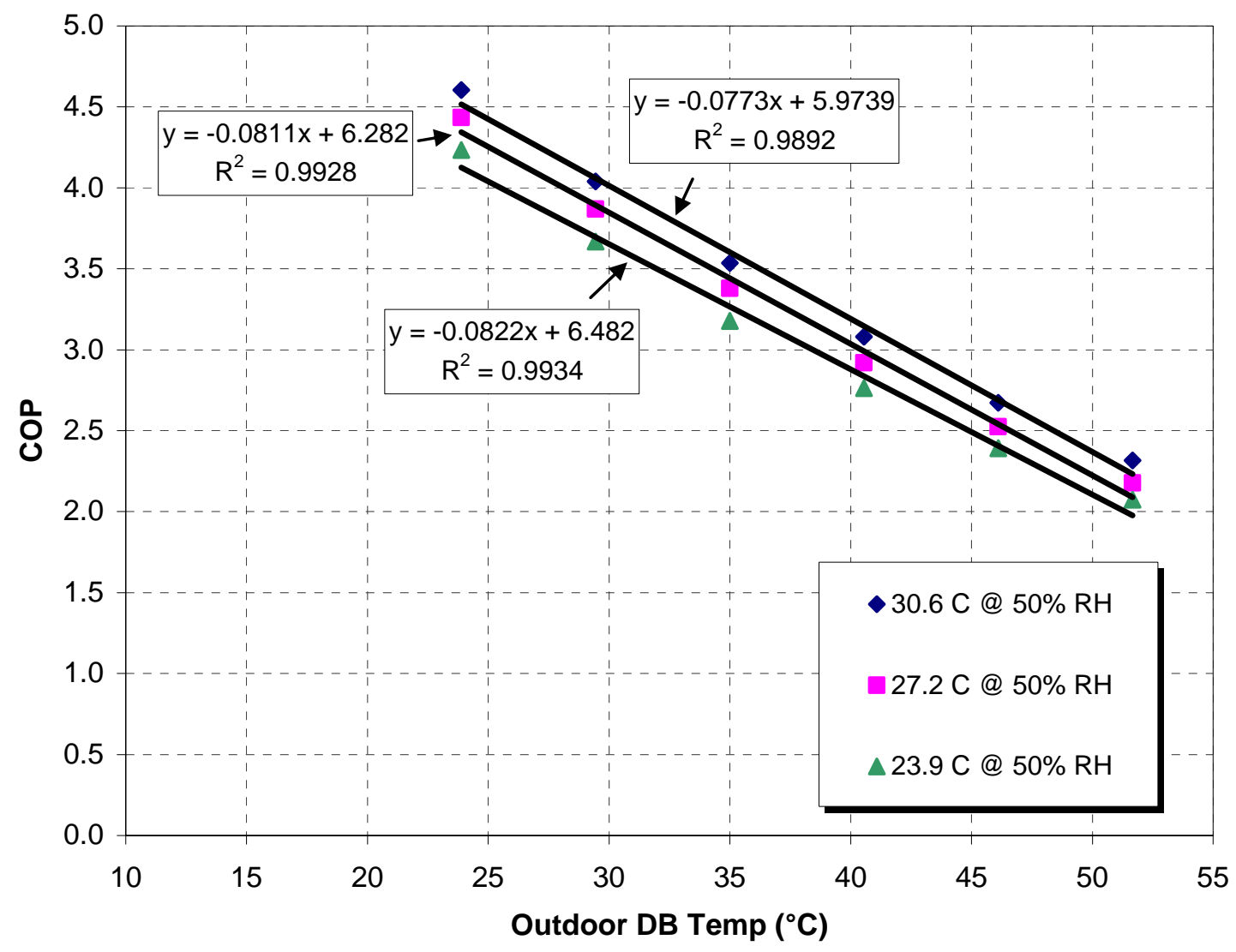

Figure 2.12 - Calculated COP for three indoor dry bulb (db) temperatures for 10 ton, constant speed, standard efficiency, Carrier rooftop heat pump

From our previous calculations, $\dot{Q}_{\text {cool }}$ in equation (2.24) is the total amount of cooling energy required by the building that must be provided by the heat pump. To convert this to electrical energy, we must determine the power required to run the compressor $\left(P_{\text {comp }}\right)$. To do this, we simply calculate the average building return temperature (mean of the zonal temperatures) along with the outdoor dry bulb temperature $\left(T_{0}\right)$ and find the corresponding COP. Then the power used by the heat pump, which we will now call $P_{h p}$ is given by,

$$
P_{h p}=\frac{\dot{Q}_{\text {cool }}}{C O P} .
$$


This equation is only valid if $\dot{Q}_{\text {cool }}$ is equal to the total capacity (TC) of the heat pump and does not account for the cyclic degradation at part loads. We must account for the fact that if the required load is less than the total capacity, then the heat pump will not operate at full efficiency (i.e. lower COP).

\subsubsection{System Capacity}

As seen in Table 2.5 the manufacturer publishes the total heat pump capacity (cooling) depending on the indoor and outdoor air conditions. Following the same procedure as the $\mathrm{COP}$, the capacity data was converted to $\mathrm{kW}$ and the wet bulb temperatures were converted to corresponding dry bulb temperatures at 50\% relative humidity. The capacity was then plotted as a function of indoor and outdoor dry bulb temperature along with the best-fit linear trend line as shown in Figure 2.13. These capacities will be used as a table look up in the heat pump model and cyclic degradation calculations. 
Report No. DE-FG02-03ER63694-F1

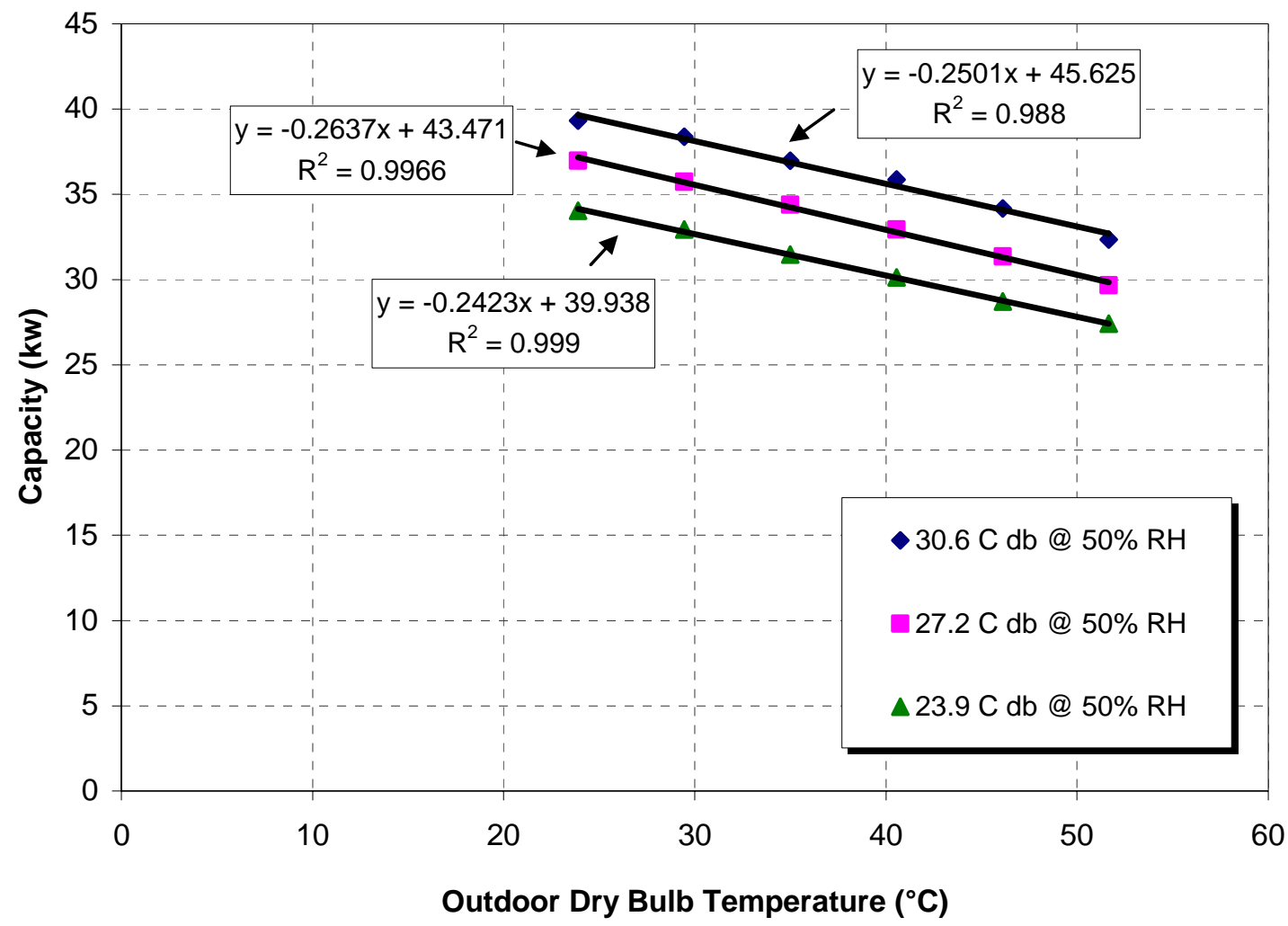

Figure 2.13 - Cooling capacity for three indoor entering dry bulb (Edb) temperatures for 10 ton, constant speed, standard efficiency Carrier rooftop heat pump

The capacity of the heat pump required for the selected building is expected to change significantly in each climate location. It would be unwise to design a building with the same size heat pump in Fairbanks, AK as in Phoenix, AZ. Until now all calculations have been assuming the standard 10-ton heat pump data as published by Carrier. To approximate the varying sizes of heat pumps a scaling factor will be used from the baseline 10-ton unit. The scaling factor will be based on the proper sized heat pump for each city as outlined in the section on climate zones. 


\section{Report No. DE-FG02-03ER63694-F1}

To properly choose the size of a heat pump, we will use the maximum design temperature method [27]. The design temperature is that which is not exceeded more than a prescribed maximum percentage of time in each city. Common practical design temperatures are $0.4 \%$, $1 \%$, and $2 \%$. The $0.4 \%$ design temperature is one that is not exceeded more than 35 hours per year. Because 35 hours is very small in comparison with a yearly simulation, we will use a $0.0 \%$ design temperature to simplify calculations. The $0.0 \%$ design temperature is the maximum temperature for each representative city, as listed in the TMY2 file. To determine the total cooling capacity (TC) the last non-zero-hours bin temperature was used as the outdoor temperature in the TCTB model.

The baseline building model (Section 2.8) set up was used in the computer model (Section 2.11). The total cooling load $\left(\dot{Q}_{\text {cool }}\right)$ calculated is the required cooling power to be supplied by the heat pump. This cooling load was set as the total capacity for heat pump for each city. The ratio of these calculated capacities and the total capacity of the 10-ton heat pump were found to determine the necessary scaling of the manufacturers data (Table 2.6). These ratios will be used as the scaling factor for the heat pump size, i.e. each city will have a heat pump with a capacity matching the design temperature load. In reality, an installer will choose a manufacture's size just larger than this capacity, which may be quite different from our scaling factor given the discrete number of capacities within a heat pump product line.

Table 2.6 - Ratio of 10-ton capacity heat pump with required building capacity for each city. 


\section{Report No. DE-FG02-03ER63694-F1}

\begin{tabular}{|c|c|c|}
\hline $\begin{array}{l}\text { TCTB City } \\
\text { No. }\end{array}$ & Representative US City & $\begin{array}{l}\text { Ratio of } 10 \text {-ton Capacity to } 0.0 \% \\
\text { design temperature required capacity }\end{array}$ \\
\hline 1 & Miami, FL & 0.721 \\
\hline 2 & Houston, TX & 0.841 \\
\hline 3 & Phoenix, AZ & 1.196 \\
\hline 4 & Memphis, TN & 0.841 \\
\hline 5 & El Paso, TX & 0.967 \\
\hline 6 & San Francisco, CA & 0.746 \\
\hline 7 & Baltimore, MD & 0.841 \\
\hline 8 & Albuquerque, NM & 0.841 \\
\hline 9 & Salem, OR & 0.760 \\
\hline 10 & Chicago, IL & 0.760 \\
\hline 11 & Boise, ID & 0.967 \\
\hline 12 & Burlington, VT & 0.841 \\
\hline 13 & Helena, MT & 0.841 \\
\hline 14 & Duluth, $\mathrm{MN}$ & 0.626 \\
\hline 15 & Fairbanks, AK & 0.606 \\
\hline
\end{tabular}

\subsubsection{Economizer}

An economizer is an energy savings feature available on many commercial heat pumps including the Carrier Weathermaker ${ }^{\circledR}$. The economizer's function is to turn off the compressor when the ambient conditions are sufficient to meet the building cooling load. The heat pump fan operates as normal, supplying some fraction (up to 100\%) of outside air directly to the occupied zones. 


\section{Report No. DE-FG02-03ER63694-F1}

Since humidity is not directly considered in the present model, only the ambient dry bulb temperature will be used to decide economizer use. The maximum cooling available by the heat pump when the economizer is operating, assuming 100\% outside air supplied, is given by (2.28).

$$
\dot{Q}_{e c o n, \max }=\dot{m} c_{p}\left(T_{a m b}-T_{i}\right)
$$

Given the specifications of the model, in order to sufficiently meet the entire building load and

not require the compressor, $\left|\dot{Q}_{i}^{\text {econ }}\right| \geq\left|\dot{Q}_{i}^{\text {cool }}\right|$ for all zones $i$, where $\dot{Q}_{i}^{\text {cool }}$ is the calculated required cooling (Section 2.6). That is, the economizer must be able to supply the necessary cooling load, using ambient air, for every zone in the building. If this condition is met, we can assume the economizer can completely meet the building cooling load and therefore turn off the compressor power requirements. Following the same assumption as before, it will be assumed that the economizer will provide the necessary cooling by some type of damper controlling the fraction of outside air and return air. In other words, the supply temperature to each zone can be modulated to provide the necessary cooling, instead of the flow rate (section 2.2). When ambient and interior conditions make the use of the economizer possible, since $P_{h p}=0$, the power usage of the HVAC system reduces to $P_{f a n}$. Scenarios in which the economizer provides on a fraction of the cooling load while the compressor provides the balance were not considered.

\subsubsection{Cyclic Degradation}

The calculated COP data from Section 2.10.1 lists COPs for continuous operation of the heat pump at the full capacity possible at eh ambient and indoor conditions. However, only when 
the cooling load of the building matches the heat pump capacity will it operate continuously. Variable speed heat pumps can operate nearly continuously matching the load. A constant speed heat pump, as in our study, must cycle on and off at some given rate to match the cooling supplied to the building load. This cycling has a negative effect on the efficiency (i.e. COP) of the heat pump since the compressor, fans, etc, must overcome inertia upon start up. Therefore an approximation of the lowered efficiency must be used. An approximation of part load efficiencies for heat pumps, modified to apply to the heat pump COP, is given by [34],

$$
C O P_{\text {cyclic }}=C O P_{\text {cont }}\left(1-C_{d}(1-P L R)\right)
$$

where $C O P_{\text {grlic }}$ is the new COP due to cycling, $C O P_{\text {cont }}$ is the published COP for continuous operation, PLR is the partial load ratio defined as the hourly load divided by the available capacity, and $C_{d}$ is the degradation coefficient.

If the partial load ratio is one $(\mathrm{PLR}=1)$, i.e. the building load exactly matches the capacity and there is no cycling, then the equation reduces to $C O P_{\text {gclic }}=C O P_{\text {cont }}$ as expected. Here, the available capacity is the total capacity (TC) of the heat pump for a given city as calculated from the scaling factor shown in Section 2.10.1.1. The instantaneous load is the building load calculated by the TCTB model. The degradation coefficient $\left(C_{d}\right)$, depends on the start up time constant and maximum cycling rate of the heat pump. Measured $C_{d}$ values for typical heat pump systems range from 0.10 to 0.20 [34]. For the purposes of this study, $C_{d}=0.20$ will be used. 


\section{Report No. DE-FG02-03ER63694-F1}

\subsubsection{HVAC Fan}

The fan used to deliver the conditioned air from the heat pump will be assumed to run at a constant indoor return air rate of $3750 \mathrm{cfm}$. We will assume that the fan included in the heat pump will operate for the furnace as well. From the Carrier published data the fan uses 1438 W of power at this flow rate [33]. We will use this value whenever the HVAC system (heating or cooling) is active.

\subsubsection{Furnace Model}

To supply the necessary heating to the building a model of a standard efficiency, natural gas fire furnace was used. Commercial furnaces are required by federal law to operate at a minimum of $78 \%$ annual fuel utilization efficiency (AFUE) [35]. The AFUE is a measure of the amount of fuel converted to space heat in proportion to the total fuel entering the furnace. High efficiency furnaces, as defined by Energy Star, operate at a minimum of 90\% AFUE [35]. For our building model an $85 \%\left(\eta_{\text {furnace }}=0.85\right)$ efficient unit was chosen. This efficiency was constant regardless of the temperature of the indoor recirculating air and the outdoor temperature. As a result, to find the power required to run the furnace for the building we use the following equation,

$$
P_{\text {furnace }}=\frac{\dot{Q}_{\text {heat }}}{\eta_{\text {furnace }}},
$$

where $\dot{Q}_{\text {heat }}$ is as defined in equation (2.24). 


\section{Report No. DE-FG02-03ER63694-F1}

\subsubsection{Gas-Electricity Equivalent}

Since the heat pump operates on electricity and the furnace uses natural gas, a direct comparison of the energy usage in kilowatt-hours would be misleading. To bring gas and electricity to a common, comparable quantity, cost per unit energy supplied will be used. The Energy Information Administration (EIA) [36] publishes average energy costs for the United States. In 2003, the average cost per kWh in the United States was $\$ 0.0765$ and the average cost per $1000 \mathrm{ft}^{3}$ of natural gas was $\$ 8.32$. Converting the price of natural gas to $\mathrm{kWh}\left(1000 \mathrm{ft}^{3}=\right.$ $30.2 \mathrm{kWh}$ ) we get an average cost for natural gas of $\$ 0.0275$ per $\mathrm{kWh}$. To convert natural gas $\mathrm{kW}$ to equivalent electricity $\mathrm{kW}$ we use the following equation,

$$
P_{f_{\text {eqv }}}=\frac{P_{\text {furnace }}}{C}=\frac{C_{\text {gas }}}{C_{\text {elect }}} P_{\text {furnace }} .
$$

Here $P_{f_{e q v}}$ now expresses the power used by the furnace as an electric equivalent power, still in $\mathrm{kW}, \mathrm{c}$ is the equivalent gas to electricity conversion factor. Substituting $\mathrm{c}_{\text {elect }}=0.0765$ and $\mathrm{c}_{\text {gas }}=0.0275$ we get $P_{\text {feqv }}=P_{\text {furnace }} / 2.78$, which indicates that natural gas is nearly three times less expensive than electricity.

We now have the ability to calculate the power (and equivalently the yearly energy) used by the building in terms of equivalent electric $\mathrm{kW}$,

$$
P_{\text {tot }}=P_{h p}+P_{f_{\text {eqv }}}+P_{\text {fan }}
$$

\subsubsection{Building Load Power}

It is often useful to observe the building power consumption before the HVAC equipment calculation. To do this we will assume that the COP for cooling supplied energy is equal to the gas to electricity unit price ratio. This is reasonable assuming considering a COP of 
approximately three and the gas to electricity ratio of approximately three. We can therefore express the total load is given by,

$$
P_{\text {load }}=\left|\dot{Q}_{\text {cool }}\right|+\left|\dot{Q}_{\text {heat }}\right| \text {, }
$$

where $\dot{Q}_{\text {cool }}$ and $\dot{Q}_{\text {heat }}$ are the cooling and heating power, as given by equation (2.24).

\subsubsection{Yearly Energy Usage}

We now have a method by which to determine the total power usage for the building at a given outdoor temperature $\left(\mathrm{T}_{0}\right)$. As mentioned in section 2.7 the weather bin method uses the constant outdoor bin temperature, and calculates the total building power (Eqn. (2.32)) at that temperature. The total building power for each bin must then be integrated over the number of hours in each bin for a total yearly energy usage.

\subsection{Model Implementation}

Until this point, the method by which we solve the building energy problem has been outlined, but the implementation has been overlooked. As mentioned earlier, the execution time of the code is an important feature for the temperature bin method. In the future it is desired to use this model to run optimization exercises which require the model be run thousands of times. MATLAB, a commercially available software which stands for matrix laboratory, lends itself well to calculations involving matrices and vectors [37]. MATLAB code was written following the mathematical description described in this chapter. The complete code is included in Appendix 6.4. Here, we will show an outline of the format and structure of the code. 


\subsubsection{Algorithm}

Figure 2.14 shows a flow chart depicting the structure of the algorithm used within the code to solve for the yearly building energy usage. Figure 2.15 shows in more detail a flow chart of the calculations occurring in the bin loop.

\subsubsection{Code Specifications}

The total execution time of the code, for the baseline case, for 15 cities and two control schemes (OSFA and HIYW), is approximately 30 seconds on a PC with a Pentium $42.4 \mathrm{GHz}$ processor with $2 \mathrm{~GB}$ of RAM. As we will see later, this is close to two orders of magnitude faster than a transient simulation of the same building using TRNSYS. 


\section{Report No. DE-FG02-03ER63694-F1}

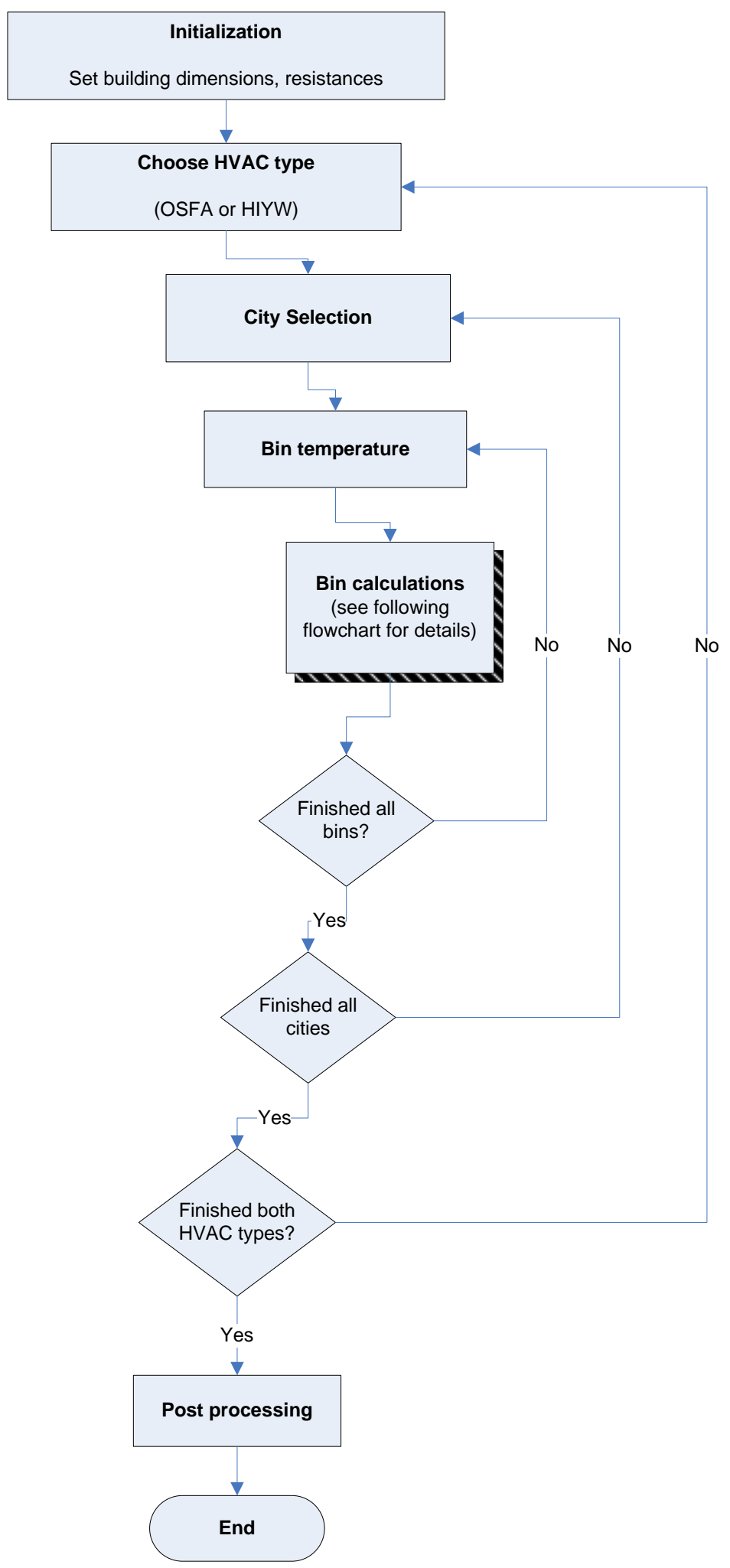

Figure 2.14 - Flow chart of the main structure of the TCTB code 
Report No. DE-FG02-03ER63694-F1

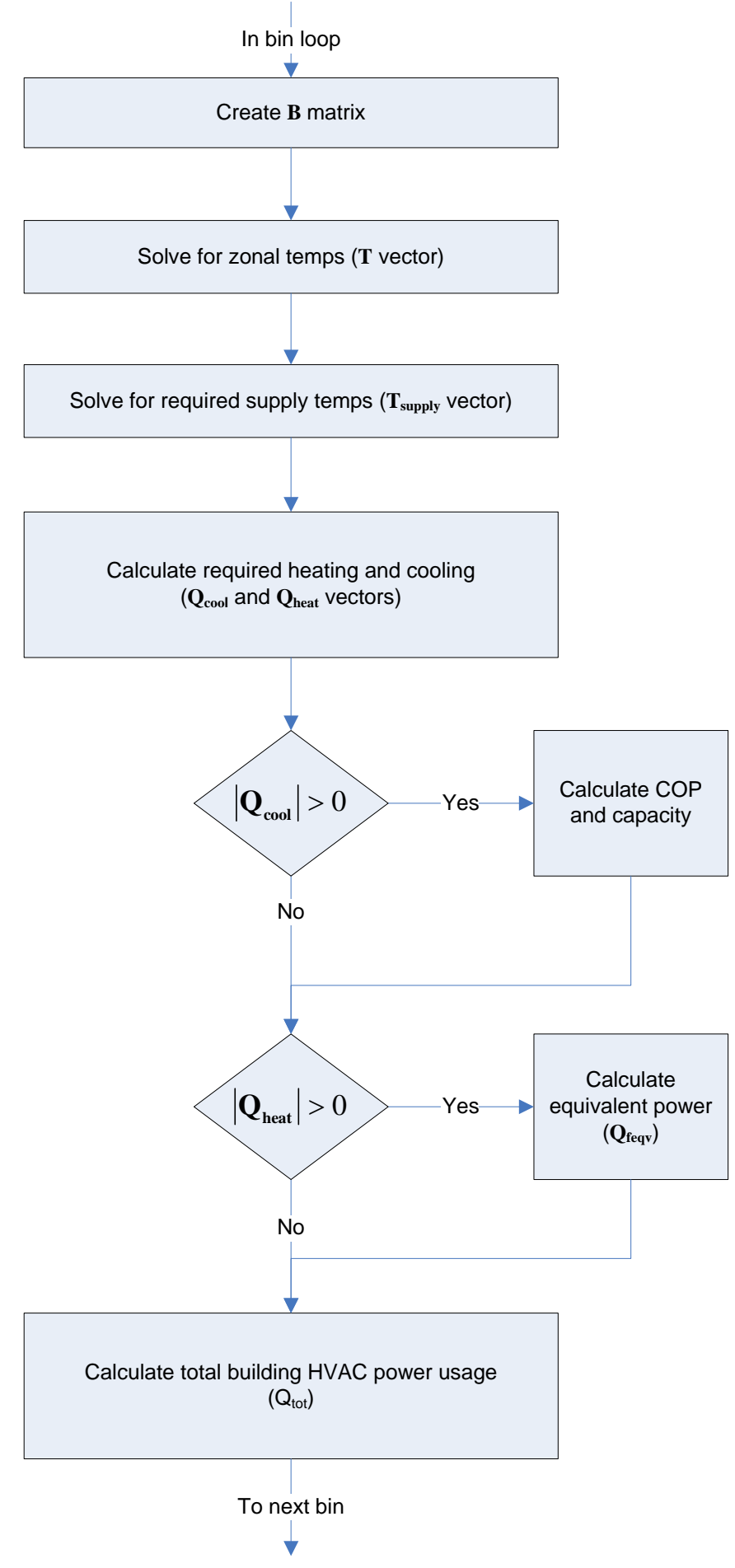

Figure 2.15 - Flow chart of bin loop calculations 
Report No. DE-FG02-03ER63694-F1

\section{Validation}

We would like to have confidence that the results we will obtain are realistic and valid. To accomplish this we will compare the computed results from the TCTB model with those generated by the industry accepted building energy simulation program TRNSYS. TRNSYS provides us the ability to perform a higher fidelity building energy simulation than the TCTB model. Most importantly TRNSYS offers a fully transient simulation, which unlike the TCTB model can account for the inherent nature of a building and weather time-dependency. Walls, especially exterior, tend to be thick with significant thermal inertia necessitating the use of transfer functions to capture the transient energy transfer. Since weather is also inherently transient, and the TCTB model cannot account for this, it will be useful to compare a TRNSYS simulation with a TCTB simulation to determine the importance of the transient nature of weather and the thermal inertia of the building envelope.

\subsection{TRNSYS Overview}

TRNSYS [22] (TRaNsient SYstem Simulation program), originally developed at the University of Wisconsin-Madison in 1975, is now a commercially available program designed for the transient simulation of thermal systems, including multi-zone buildings. Users create a text file input and TRNSYS, an open-source FORTRAN code, uses a modular approach to solve a group of components describing the system. TRNSYS includes a graphical user interface through which the user can specify and connect components of the system and set necessary simulation parameters (time step, convergence tolerance, etc.). 


\section{Report No. DE-FG02-03ER63694-F1}

For building simulations TRNSYS has two important software programs: the TRNSYS simulation studio (TRNSYS studio) and the building input interface (TRNBuild). First the user must specify the details of the building in question in TRNBuild by defining: geometric specifications, building construction materials, infiltration, internal heat gains, etc. Then TRNBuild creates the Type 56 input files to be used by the TRNSYS studio. Type 56 is the name of TRNSYS component corresponding to a detailed multi-zone model. In the TRNSYS studio other necessary simulation components are specified including: weather, solar radiation, HVAC equipment, etc. Simulation parameters can be modified (simulation start and end, time step, etc.) and then the transient simulation can be executed.

The International Energy Agency, in conjunction with the U.S. National Renewable Energy Lab, created a benchmark for building energy simulation programs entitled the IEA Building Energy Simulation Test (BESTEST) and Diagnostic Method [39]. The qualification tests start with the basic structure of a building and then manipulate it by moving the windows, adding exterior shading, changing the wall constructions, modifying the coupling with the ground and adding a sunspace. In doing so, any possible bugs in the program should surface and the program's accuracy can be determined. TRNSYS v14.2 was subjected to the simulation guidelines of BESTEST and fell with the range of acceptable values for all output parameters, and no bugs were found [38].

\subsection{Model Details}

In order to compare the TCTB model to TRNSYS a comparable TRNSYS simulation needed to be created. Using TRNBuild the baseline building model was created following the same guidelines as given in Section 2.8. Since TRNSYS is capable of extremely detailed simulations a 
certain amount of care needed to be taken to ensure that the two models would be compared on equal footing. We want to isolate the temperature bin approximation of the TCTB model by making the TRNSYS simulation as close as possible to the TCTB in all respects except the transient nature.

\subsubsection{TRNSYS Building Model}

The baseline building geometry was created in TRNBuild as shown in earlier in Section 2.8.1.

Internal heat generation was transferred purely by convection (no latent or radiative components). Outside ventilation was assigned to each room. Humidity ratios were set to zero and all humidity calculations were turned off in TRNSYS. Solar radiation processors were not included.

The interior and exterior wall materials were chosen, as close as possible to the physical make of the walls given in Table 2.3, from the TRNSYS library of common building materials. The resulting TRNSYS resistances and convective heat transfer coefficients are given below in

Table 3.1. Since there are some slight discrepancies between the TRNSYS resistance values and the previously used TCTB values (Table 2.3) for the purpose of these comparisons only, the TCTB values will be modified to be identical to the resulting TRNSYS resistances. 
Report No. DE-FG02-03ER63694-F1

Table 3.1 - TRNSYS baseline case thermal resistances

\begin{tabular}{|c|c|c|c|}
\hline Type & Notation & Value & Notes \\
\hline Interior wall & $\mathrm{r}_{\text {iwall }}$ & $0.397 \mathrm{~m}^{2} \mathrm{~K} / \mathrm{W}$ & $\begin{array}{l}\text { Gypsum wall board with airspace } \\
\text { between }\end{array}$ \\
\hline Exterior wall & $\mathrm{r}_{\text {ewall }}$ & $2.34 \mathrm{~m}^{2} \mathrm{~K} / \mathrm{W}$ & $\begin{array}{l}\text { Inside surface: gypsum wall board. } \\
\text { Insulation: rigid foam insulation. } \\
\text { Outside surface: stucco }\end{array}$ \\
\hline Exterior window & $r_{\text {window }}$ & $0.353 \mathrm{~m}^{2} \mathrm{~K} / \mathrm{W}$ & Double paned air-filled window \\
\hline $\begin{array}{l}\text { Inside convective heat } \\
\text { transfer coefficient }\end{array}$ & $\mathrm{h}_{\text {in }}$ & $3.06 \mathrm{~W} / \mathrm{m}^{2} \mathrm{~K}$ & Recommended value $\left(11 \mathrm{~kJ} / \mathrm{hm}^{2} \mathrm{~K}\right)$ \\
\hline $\begin{array}{l}\text { Outside convective } \\
\text { heat transfer coefficient }\end{array}$ & $\mathrm{h}_{\text {out }}$ & $17.78 \mathrm{~W} / \mathrm{m}^{2} \mathrm{~K}$ & Recommended value $\left(64 \mathrm{~kJ} / \mathrm{hm}^{2} \mathrm{~K}\right)$ \\
\hline
\end{tabular}

ERC was used with the heating and cooling set points for each room coincident and equal to the thermostat set points discussed earlier for OSFA and HIYW. For example, for OSFA each zones heating set point and cooling set point was set to $24^{\circ} \mathrm{C}$. Then, TRNSYS calculates the necessary heating energy required to maintain the zonal temperature at $24^{\circ} \mathrm{C}$ or if necessary the amount of cooling energy required to maintain $24^{\circ} \mathrm{C}$. Since the two set points (heating/cooling) were coincident there were no floating temperatures. The absolute value of the resulting energy demands of each zone could be summed together for a yearly simulation to find the total building energy demand ${ }^{1}$. The TRNSYS yearly building energy demand can be compared to the TCTB building load power demand integrated over the year.

\footnotetext{
${ }^{1}$ This is equivalent to assuming that the COP is equal to the electricity to gas unit price ratio. See Section 2.10.4.
} 
Report No. DE-FG02-03ER63694-F1

\subsubsection{TRNSYS Simulation Parameters}

TMY2 weather files were used to provide hourly temperature data for each city. A simulation time step of one hour was chosen. When a time step of less than one hour is chosen the temperature data from the TMY2 file is interpolated, however, as shown in Table 3.2 there is no benefit in using smaller time steps. A single yearly simulation, for one city, executed in approximately 10 minutes using a one hour time step. The execution time increased linearly with the time step.

Table 3.2 - Effect of TRNSYS simulation time step for baseline building in Chicago, IL

\begin{tabular}{cc}
\hline Time step $(\mathrm{h})$ & OSFA Yearly Energy Demand (MWh) \\
\hline 1 & 80.548 \\
0.5 & 80.564 \\
0.25 & 80.613 \\
\hline
\end{tabular}

\subsection{Building Energy Load Comparison}

To compare the TCTB model with the TRNSYS model both were used to run simulations for all 15 cities with both OSFA and HIYW strategies. To ensure that the HIYW population did not skew the results, 10 random populations were chosen. Therefore, for each city a total of 11 simulations were run. The yearly energy demand from TRNSYS was then compared with the building load power demand integrated over the year (8760h) from the TCTB model. Since the TRNSYS calculation uses the ERC method, which is before HVAC equipment, we cannot use the HVAC power. The calculation of yearly energy demand is identical however. The building 


\section{Report No. DE-FG02-03ER63694-F1}

power demand per bin is multiplied by the appropriate number of hours per bin for each city (Table 6.1).

The resulting TRNSYS computed energies were then plotted against the corresponding TCTB yearly energy for the same city, population, and control strategy. If there was perfect agreement between the two models the points would fall precisely along the $45^{\circ}$ line.

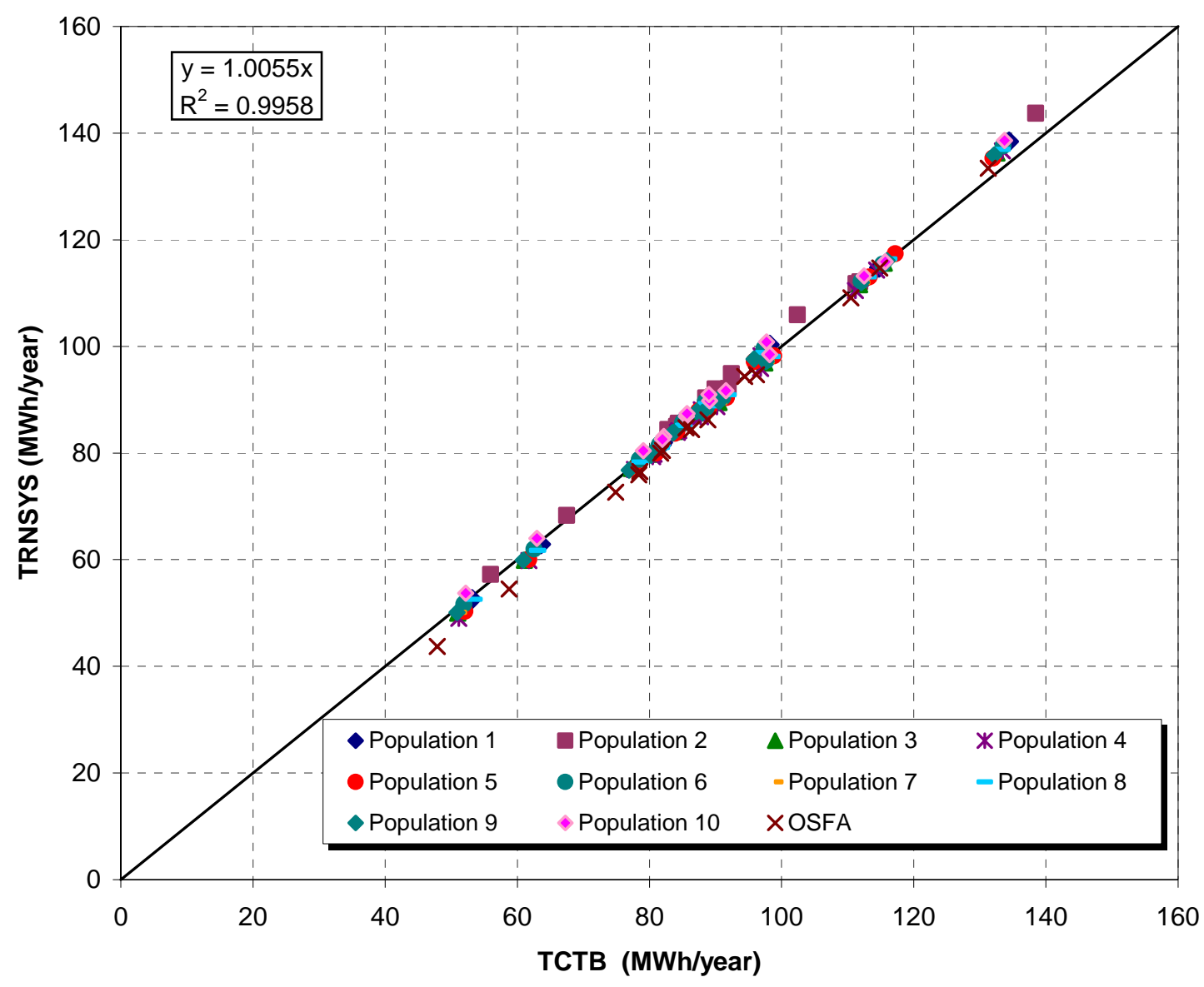

Figure 3.1 - Yearly building energy demand comparison between TCTB and TRNSYS models for the baseline building 


\section{Report No. DE-FG02-03ER63694-F1}

While the two models predict nearly the same energy demand, there are some small deviations. Clusters of points on the graph are indicative of a city. For example, the cluster of points at the very top right of the plot correspond to the 11 simulations of Fairbanks, AK, while the lower most cluster consists of San Francisco, CA simulations. The equation and $\mathrm{R}^{2}$ value on the plot are those given if we were to fit the data to a linear trend line forced through zero. Not only is the trend line a very good fit $\left(\mathrm{R}^{2}=0.9958\right)$ but it also gives us an idea about the relative error associated with the TCTB model. It reveals that the TCTB model under predicts the energy demand as calculated by TRNSYS by only about $0.55 \%$. The agreement with the higher fidelity TRNSYS model gives us confidence that the assumptions used in the TCTB model are reasonable for yearly energy calculations. Despite the considerable simplicity of the TCTB, it appears as though the transient factors, most importantly the thermal inertial of the building envelope, do not have a strong influence on the yearly energy consumption. 
Report No. DE-FG02-03ER63694-F1

\section{Computed Results}

In this chapter we will test a variety of conditions to explore the effect different parameters have on the building energy consumption for both HIYW and OSFA. We seek to quantify the costs associated with the implementation of HIYW compared to the traditional OSFA. With an understanding of the relative influence of the climate and interior partition resistances, we can begin to understand the situations in which HIYW could prove to be a feasible solution to occupant thermal comfort. Conversely, we will also be able to determine those scenarios which would make HIYW a less attractive feature from an energy perspective.

\subsection{Baseline Case Results}

The first scenario we will explore is the baseline case as described earlier. Section 2.8 provided details of the building geometry, heat loads, and thermal resistances. First we will explore the behavior of a single population consisting of 49 individuals chosen randomly from the Gaussian distribution described in Section 2.9.1. Later, we will explore the effect of the population distribution.

\subsubsection{Building Energy Load}

The first item of interest is the building energy load as calculated in Equation (2.33). This only looks at the energy demand of the building, without regard for equipment or location. Figure 4.1 shows a plot of the OSFA $\left(\mathrm{T}_{\mathrm{in}}=24^{\circ} \mathrm{C}\right)$ power demand of the building as a function of the outdoor temperature. The behavior is as expected. The $\mathrm{V}$ shape of the graph reaches a minimum at a value lower than $24^{\circ} \mathrm{C}$ due to the large internal heat gain of the building. The 


\section{Report No. DE-FG02-03ER63694-F1}

linear portion of the curve to the right of the minimum represents cooling only, while the linear portion to the left of the minimum represents heating only. The rounding of the curve near the minimum consists of some combination of heating and cooling. The curve never reaches zero because nature of the three unique exterior interfaces within the building. For example the exterior offices may need to be heated when the interior zones are still receiving cooling. Figure 4.2 illustrates this phenomenon when the outside temperature is $13^{\circ} \mathrm{C}$. The figure shows the power consumption of each individual room in the building. A positive value indicates heating, while a negative value indicates cooling. The corner offices require significant heating (positive), the perimeter offices require a small amount of heat, while all the center offices require cooling (negative). 


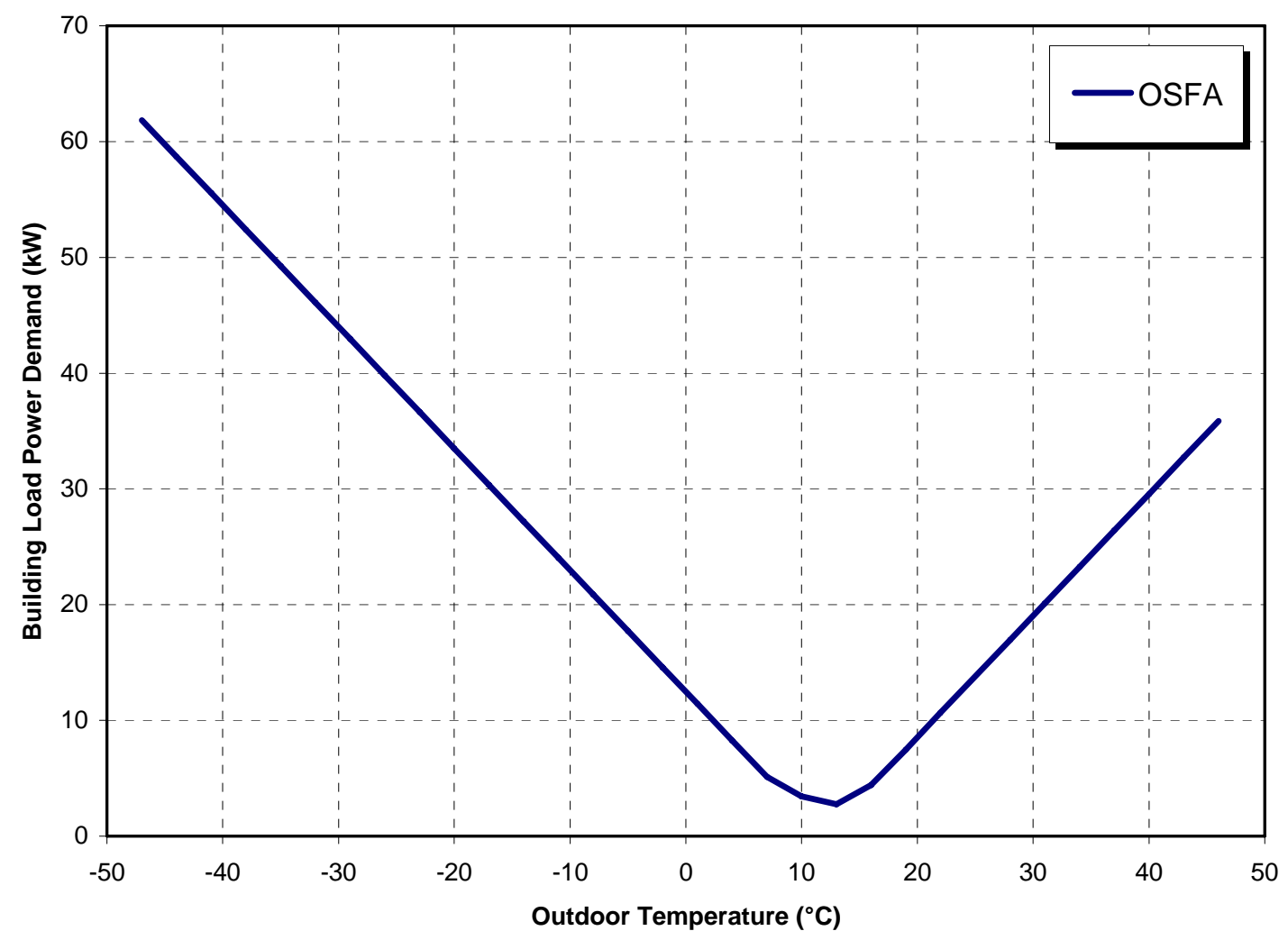

Figure 4.1 - OSFA building load power usage for bin temperatures for baseline case (no HVAC equipment) 


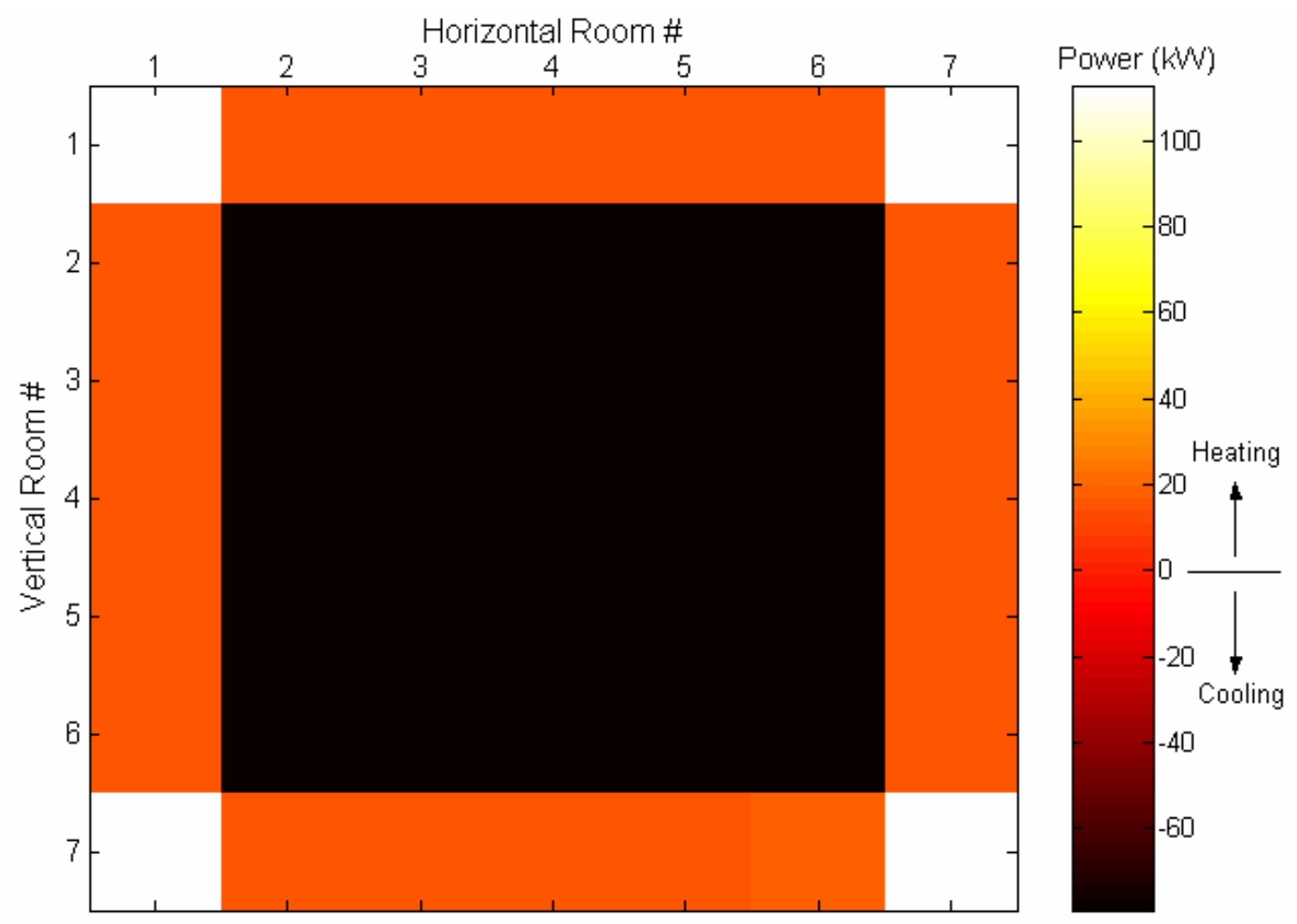

Figure 4.2 - OSFA individual rooms power usage for $\mathrm{T}_{\text {out }}=13^{\circ} \mathrm{C}$

When we allow occupants to choose their own temperatures qualitatively the building power consumption behaves very similarly. Figure 4.3 shows the power demand of the building as a function of the outdoor temperature for HIYW. The thermostat set points were chosen at random from the population distribution, and assigned randomly to an office. The exact neutral temperatures (set point temperatures) and their locations are shown in Appendix 6.3. Like the preceding OSFA case, the HIYW building has a V shape, with a more pronounced curve at the minimum. The difference between the OSFA and HIYW can be seen more clearly in Figure 4.4. Here, the points denote the bin values, and we can see that as the bin temperature deviates from that at which the building load is a minimum, the difference between OSFA and HIYW becomes nearly indistinguishable. Only between bins $4^{\circ} \mathrm{C}$ and $19^{\circ} \mathrm{C}$ is there a noticeable power demand increase associated with HIYW. This fact will be 


\section{Report No. DE-FG02-03ER63694-F1}

important when we explore the energy consumption of the HVAC equipment as well as the overall energy consumption in various cities.

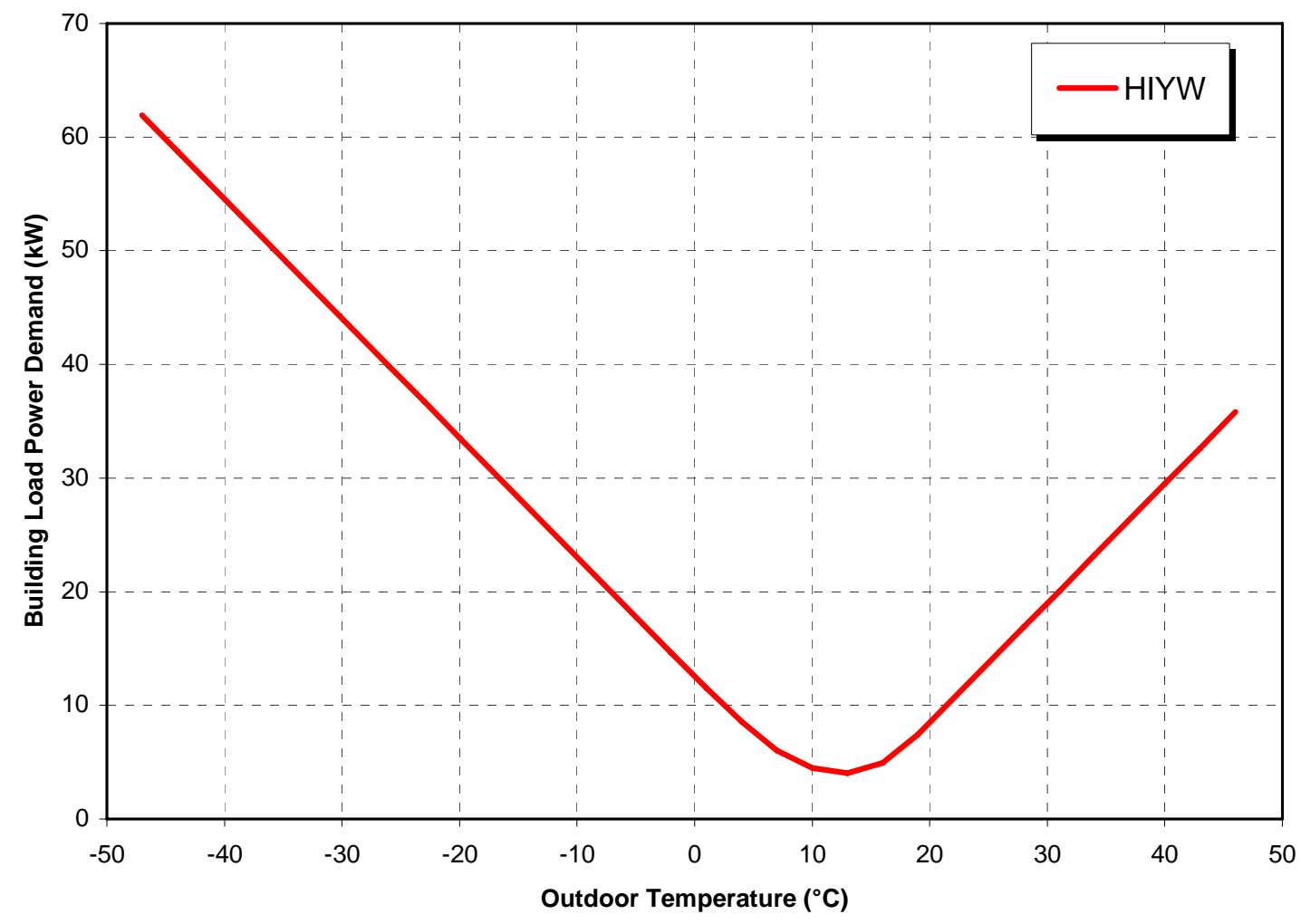

Figure 4.3 - HIYW building load power usage for bin temperatures for baseline case (no HVAC equipment) 


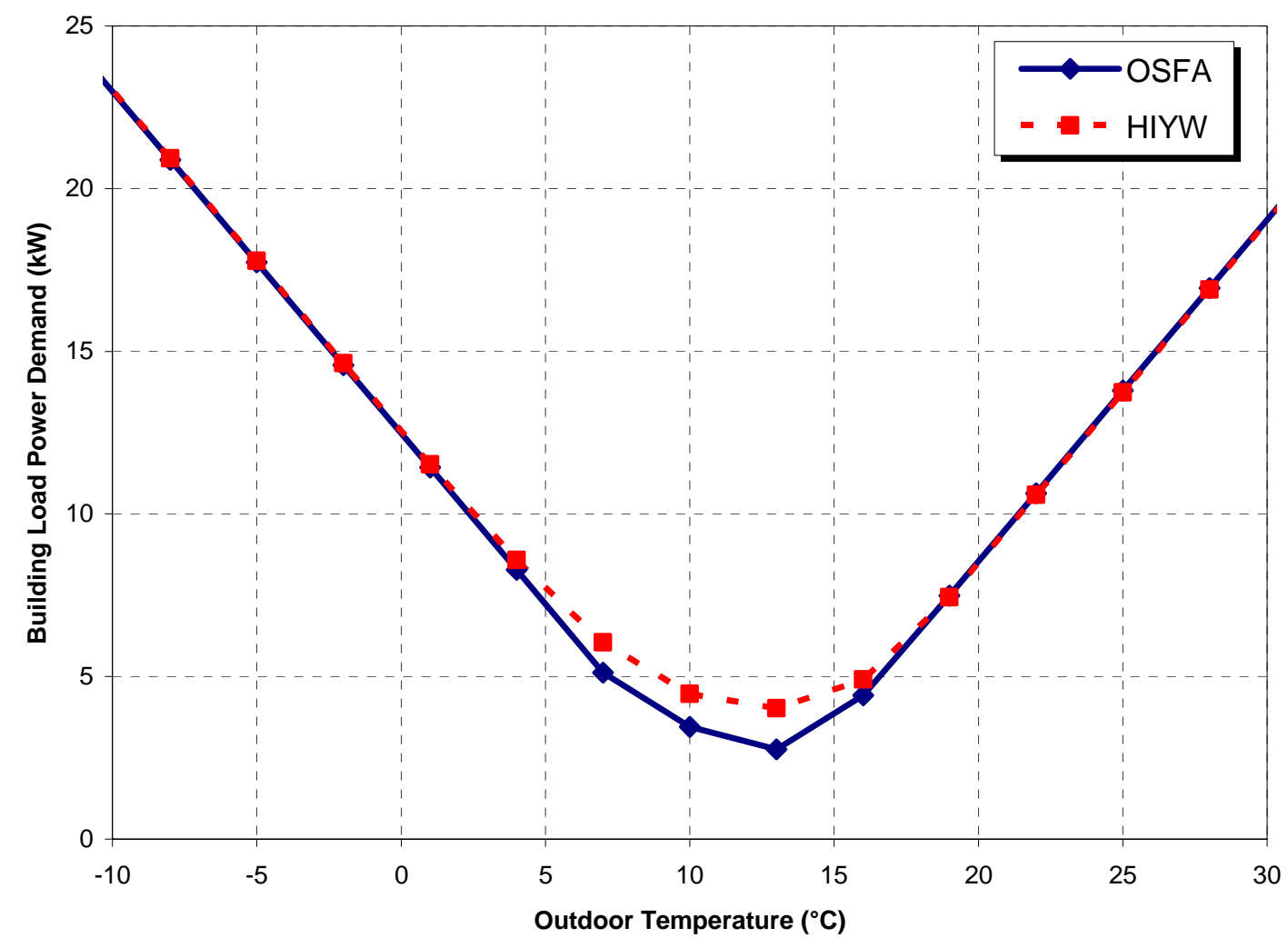

Figure 4.4 - Building load power demand comparison between OSFA and HIYW for baseline case (no HVAC equipment)

Similar to the OSFA case we can also examine the energy demand of the individual rooms. Figure 4.5 shows an illustration of the difference in the power supplied to each room for the same population distribution (Appendix 6.3) at an outside temperature of $13^{\circ} \mathrm{C}$. Unlike the OSFA case, the uniformity in power usage is far less defined. Most of the perimeter zones require heating, and most of the interior zones require cooling, however, there are many exceptions. For example, zone $(1,4)$ has one of the coldest set point temperatures $\left(22.66^{\circ} \mathrm{C}\right)$ and therefore requires a significant amount of cooling despite being on the perimeter, while both its perimeter neighbors (with set points of $25.74^{\circ} \mathrm{C}$ and $24.66^{\circ} \mathrm{C}$ ) require heating. 


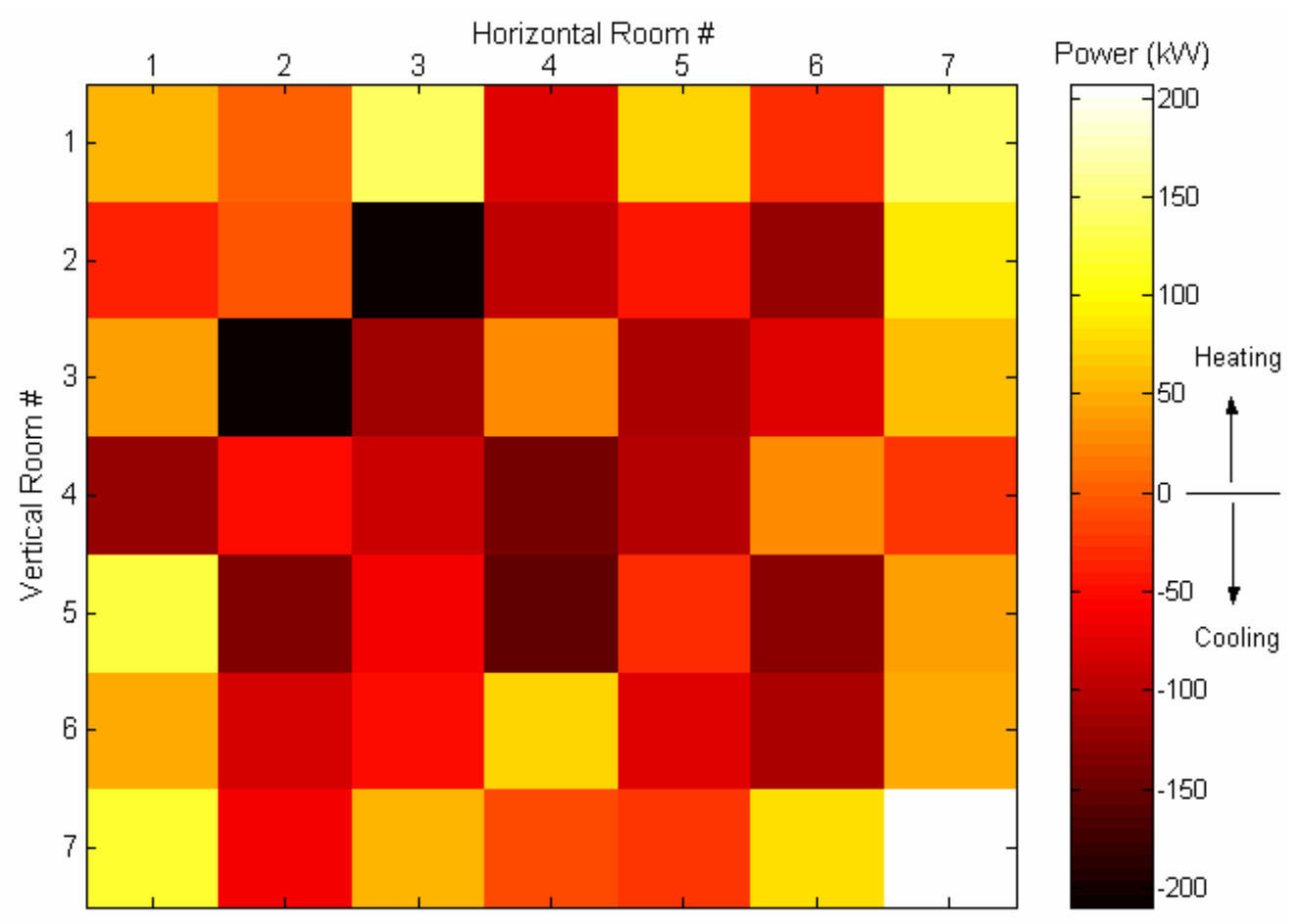

Figure $4.5-\mathrm{HIYW}$ individual rooms power usage for $\mathrm{T}_{\text {out }}=13^{\circ} \mathrm{C}$

\subsubsection{Building HVAC Power}

Now that we have computed the building power demand as a function of the outdoor temperature we can examine the introduction of HVAC equipment. The power specifications of the heat pump, fan, and furnace are outlined in Section 2.10. Unlike the building load power demand, the HVAC power usage is now dependant on the location (city) of the building. The sizing of the heat pump varies based on the maximum design temperature, and therefore the COP (after accounting for cyclic degradation) will not be identical in every city. We will explore the difference between cities later, for now let us examine an example city: city \#7, Baltimore, MD. Baltimore provides us with a good mix of warm temperatures and cold temperatures, and is a good example case. Figure 4.6 shows the HVAC power usage for both OSFA and HIYW for all applicable bin temperatures. That is, only the non-zero-hours bin 


\section{Report No. DE-FG02-03ER63694-F1}

temperatures (for the entire year) were used since they are the only realistic possibilities. Qualitatively the HVAC power usage curves appear very similar to the building load power demand curves shown in Figure 4.4.

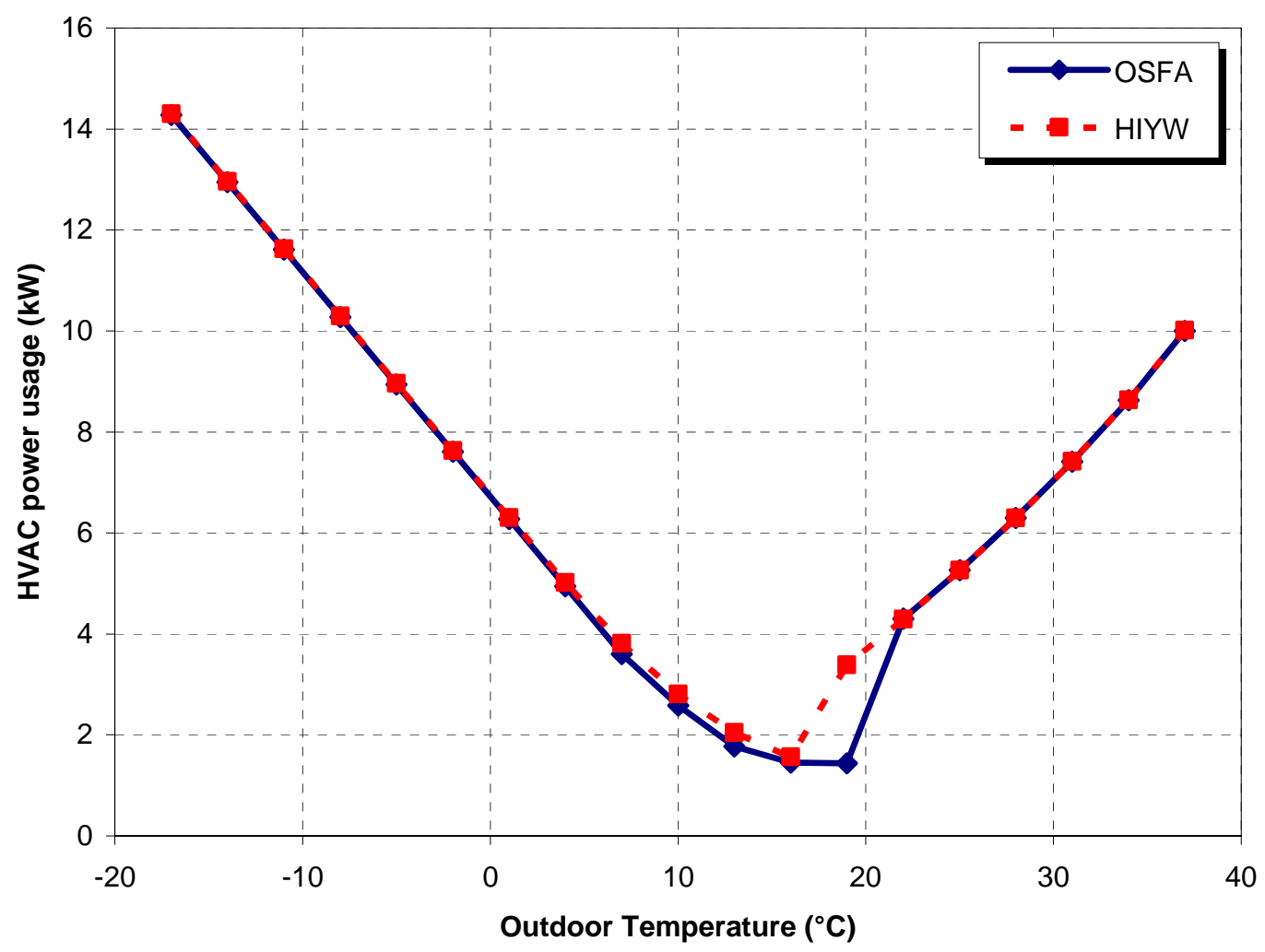

Figure 4.6 - HVAC power usage for OSFA and HIYW baseline building model case in Baltimore, MD (city \#7)

First let us analyze the OSFA curve. The curve can be broken down into three sections much like the building load power demand was. The first is a cooling only phase from $23^{\circ} \mathrm{C}$ and greater. In this phase every room is receiving cooling from the heat pump. In the second section, at $19^{\circ} \mathrm{C}$, there is a noticeable dip in the curve. This is due to the presence of the economizer. At this temperature (and lower) the outdoor air is sufficient to meet the building 


\section{Report No. DE-FG02-03ER63694-F1}

cooling load and only the HVAC fan is in use. Remember that the fan uses $1438 \mathrm{~W}$, the value of the OSFA curve at $19^{\circ} \mathrm{C}$, meaning that the entire building is receiving free cooling via the economizer, with the only power expense being that of the HVAC fan. The third section is to the left of the $19^{\circ} \mathrm{C}$ bin. At this point zones begin to require heating; first the corner offices, then the perimeter offices, and finally the center offices. The curve becomes clearly linear at $4^{\circ} \mathrm{C}$ and beyond, since all zones require heating, and cooling is no longer necessary.

The HIYW curve behaves very similarly to the OSFA, with the same three sections. The key difference however, is the temperature at which the economizer becomes active, i.e. the second section. At the $19^{\circ} \mathrm{C}$ bin, the HIYW curve is still in the cooling phase as the economizer is unable to meet the cooling load until the $16^{\circ} \mathrm{C}$ bin. This is compounded by the fact that for the chosen population (see Appendix 6.3) one corner office requires heat as early as the $19^{\circ} \mathrm{C}$ bin. At temperatures lower than $19^{\circ} \mathrm{C}$ offices slowly begin requiring heating, depending on the occupants' neutral temperature and thermostat set point. Once the economizer is able to meet the building load, the two curves begin to approach each other. As the outdoor temperature decreases, the two curves become nearly coincident. This shows that the energy cost incurred by creating small differences in internal temperatures through HIYW is significantly smaller than the overall cost associated with the extreme outdoor temperatures. The difference between these two curves, and where the differences lie, is critical in determining the yearly energy penalty associated with HIYW compared to OSFA.

Figure 4.7 shows the HVAC power usage for San Francisco. There are two important distinguishing features that make this unique from the previous plot. First, within the average TMY2 year, San Francisco never reaches outdoor temperatures below the $1^{\circ} \mathrm{C}$ bin, nor does it reach temperatures above the $34^{\circ} \mathrm{C}$ bin. Hence the HVAC power usage is plotted only over 
this narrow band of temperatures. Second, the slope of the cooling portion of the curve is unique to San Francisco since it is dependent on the specific sizing of the heat pump. A smaller capacity heat pump will be more efficient at lower building loads and will therefore use less power.

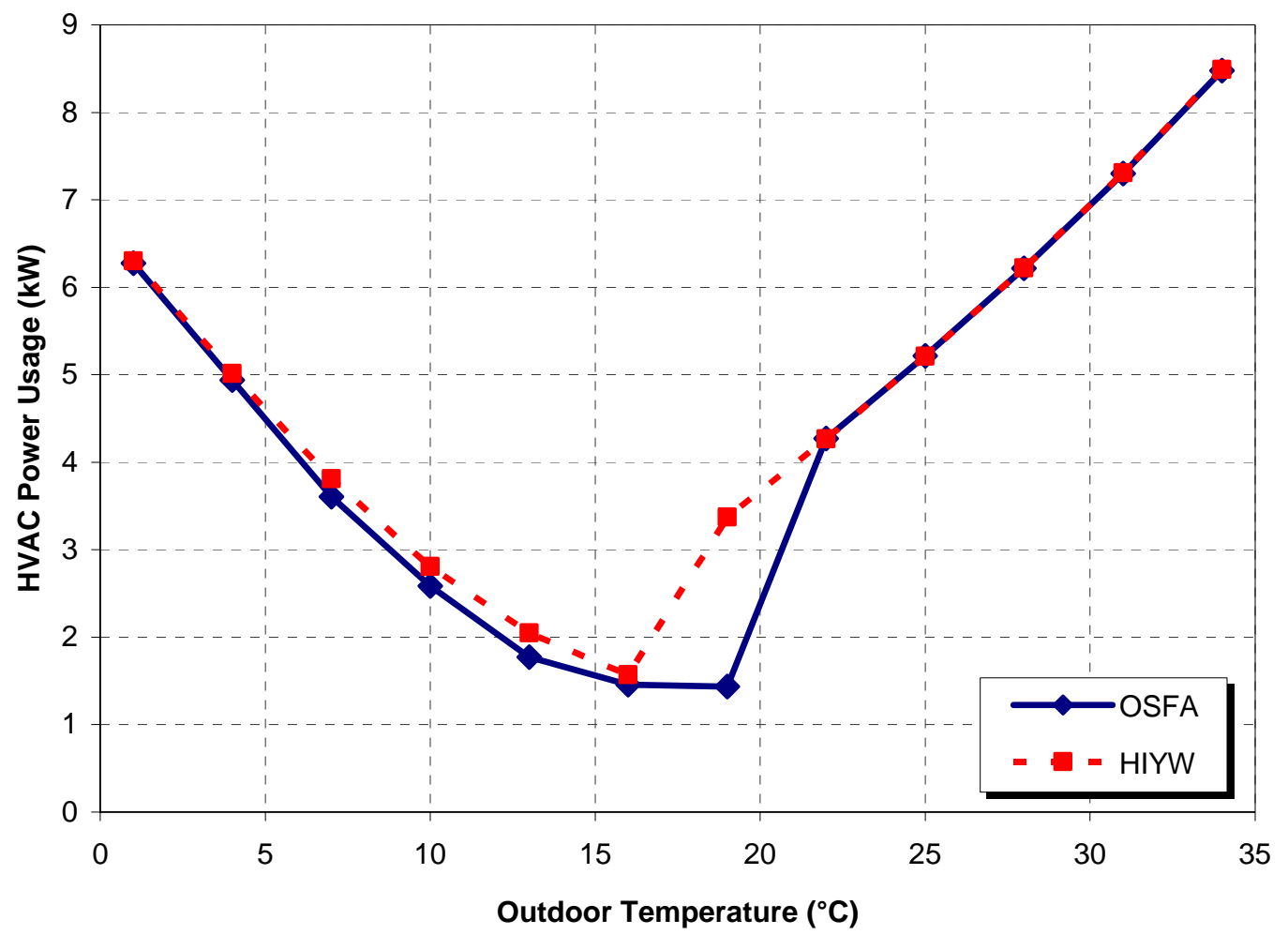

Figure 4.7 - HVAC power usage for OSFA and HIYW baseline building model case in San Francisco, CA (city \#6)

\subsubsection{Energy Cost Associated with HIYW for Various Climates ${ }^{1}$}

Now that we have seen the difference in the HVAC power usage between HIYW and OSFA we need to determine the yearly energy consumption. For each city we will now take the

\footnotetext{
${ }^{1}$ These are non-optimized results. For results of optimization, consult Ari, Cosden, et al. [32].
} 
power usage per bin and multiply by the total number of hours per bin. First we will look at the yearly energy usage (8760 hours) and then the work day only usage (4750 hours).

\subsubsection{Yearly Energy Usage (8760 hours)}

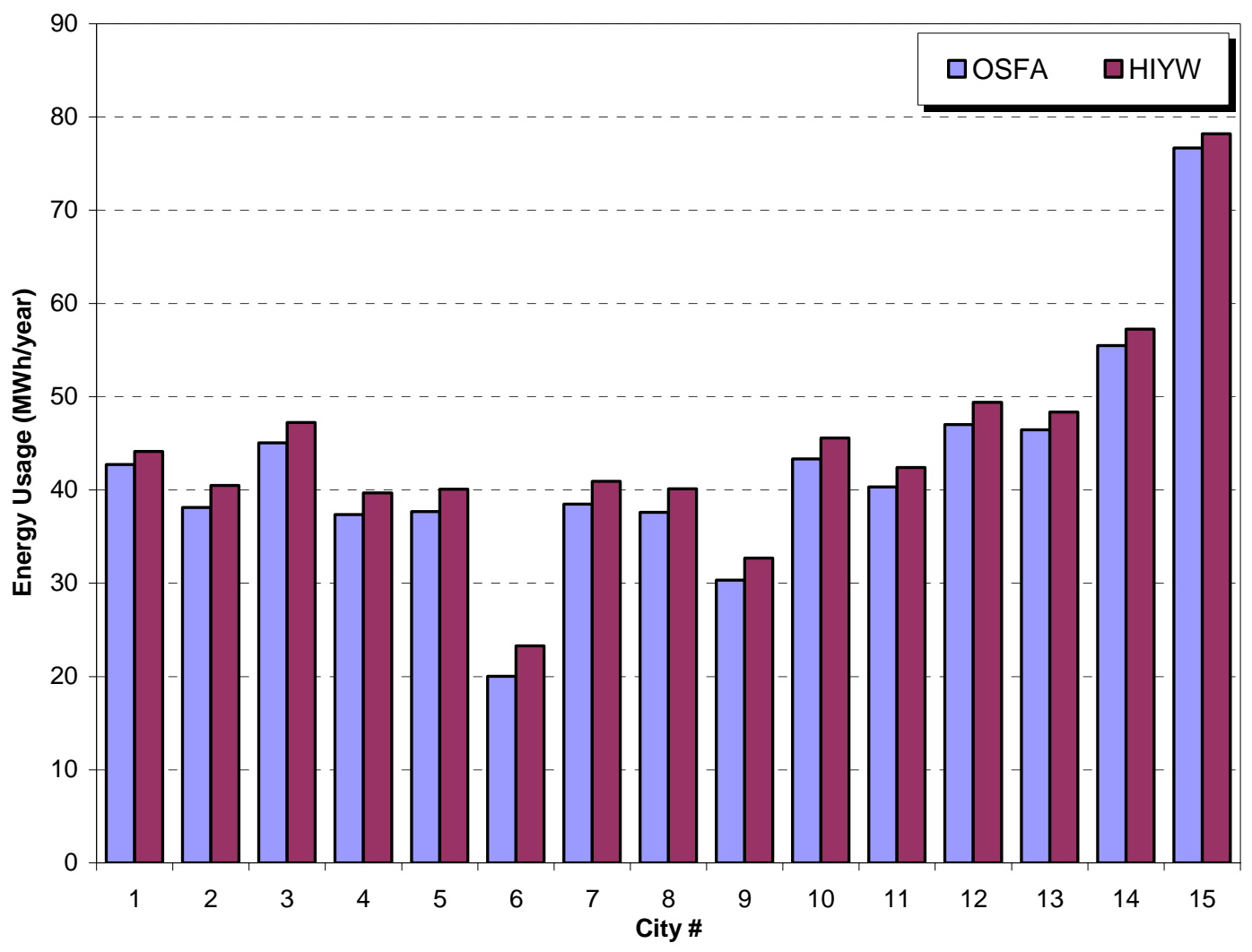

Figure 4.8 - Yearly energy usage for baseline case for OSFA and HIYW

Figure 4.8 shows the integrated yearly energy usage for the two control strategies: OSFA and HIYW. In every case, non-optimized HIYW consumes more energy than OSFA. We can see this more clearly in Figure 4.9, which shows the ratio of HIYW yearly energy usage to OSFA yearly energy usage. Here, a value of 1 indicates that they use exactly the same amount of energy annually, while a value of 1.08 indicates HIYW uses $8 \%$ more energy over the course of 


\section{Report No. DE-FG02-03ER63694-F1}

the year. City \#6, San Francisco, CA, deserves special consideration. San Francisco uses the least amount of energy, for both control strategies, of any city studied. This is due to the frequent use of the economizer. That is, there is a large portion of the year where free cooling can be achieved using the economizer, since the outside air temperature is sufficient for meeting the cooling needs of the building. In cities with a significant number of hours per year in these economizer bins, such as San Francisco, will show the greatest difference between HIYW and OSFA. As we saw before in Figure 4.6 and Figure 4.7, once the economizer is able to meet the cooling needs of the building there is a sharp drop in power consumption. Also, the economizer is unable to meet the cooling load of HIYW at the same outdoor temperature as OSFA. This is due to the economizer activation strategy used. The economizer is activated only if the needs of every zone can be met, which leads to a smaller ambient temperature range when there are 49 different requirements to be met in HIYW, as opposed to three in OSFA. The active economizer bins are the only portion of the graph (Figure 4.7, between $10^{\circ} \mathrm{C}$ and $22^{\circ} \mathrm{C}$ ) where the HIYW and OSFA curves demonstrate a significant difference in power consumption. Integrating this portion of the curve over a large number of hours can create, as it does in San Francisco, a substantial difference between HIYW and OSFA. 
Report No. DE-FG02-03ER63694-F1

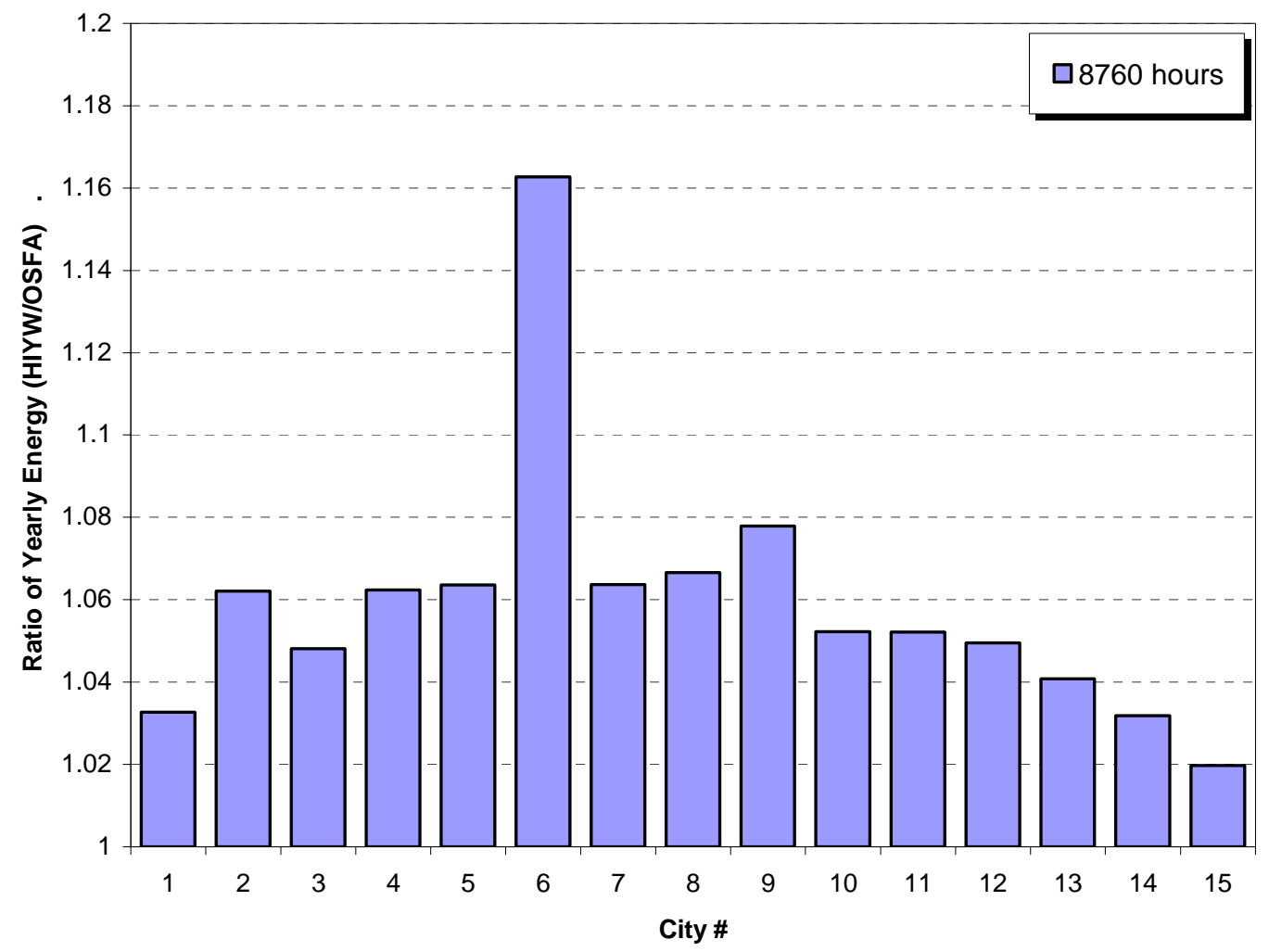

Figure 4.9 - Ratio of HIYW to OSFA energy usage (8760h)

San Francisco, city \#6, is decidedly the worst, needing slightly more than $16 \%$ more energy to provide HIYW over OSFA. Most of the other cities are between 3-8\%, while the extremely cold Fairbanks, AK (city \#15) is less than 2\%. Fairbanks is nearly opposite to San Francisco, as there are so few hours per year in the economizer bins, the energy cost associated with HIYW is nearly the same as OSFA. 


\subsubsection{Work Day Energy Usage (4750 hours)}

As mentioned before in Section 2.7.4 we wish to look at the energy usage of the two control strategies during the work day hours (6am-7pm only). As we did in the previous section we will look at the yearly energy usage and the ratio of HIYW and OSFA.

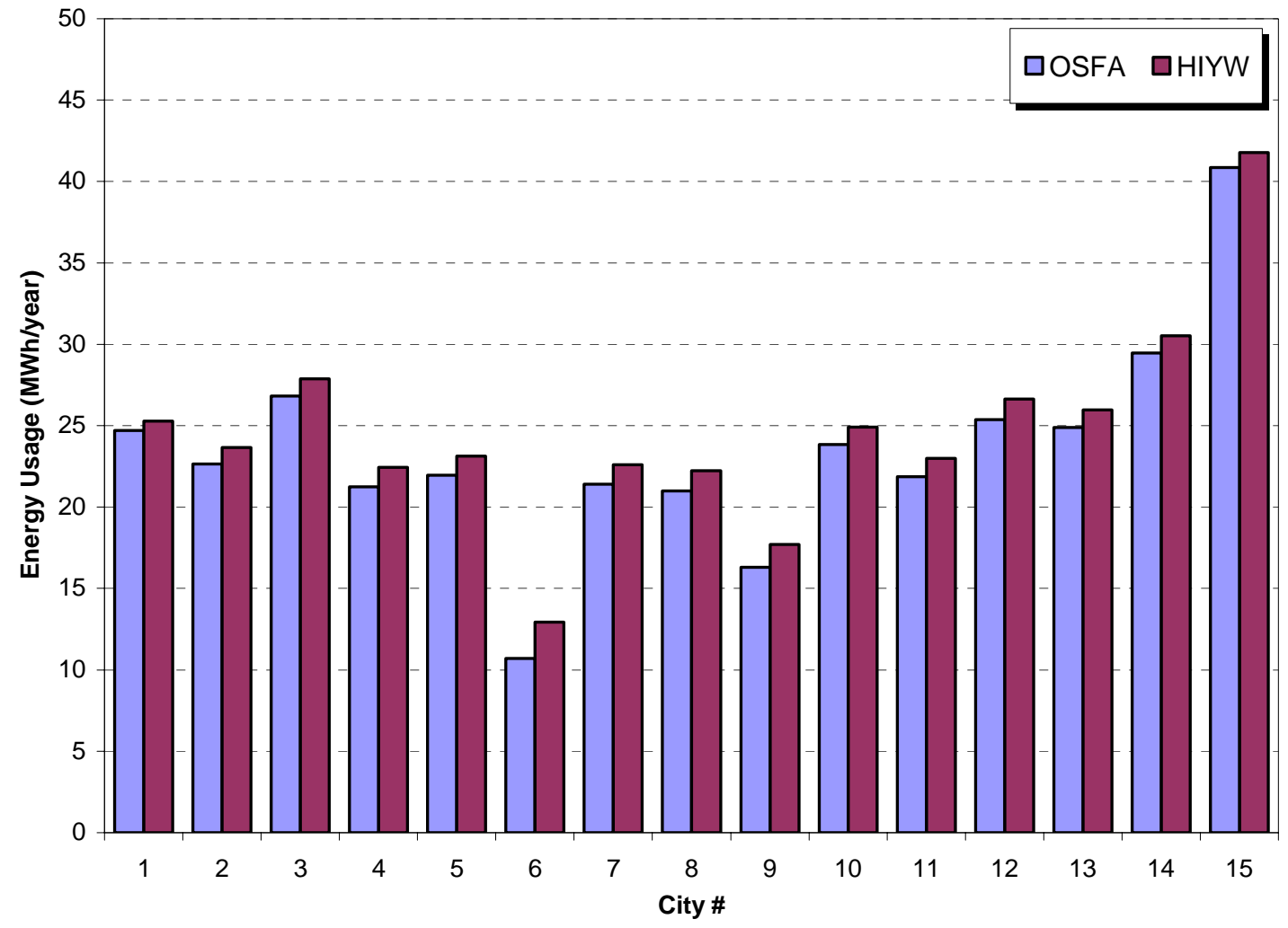

Figure 4.10 - Work day energy usage for baseline case OSFA and HIYW

In Figure 4.10 we can see that qualitatively the energy usage behavior during work hours is very similar to the entire year (Figure 4.8). Obviously the total energy consumption for 4750 hours is less than the 8760 hour case, since we are only considering slightly more than $50 \%$ of the year. However, since we are considering the work day hours only, the hottest temperatures remain since they occur during the daytime, while most of the coldest temperatures are 


\section{Report No. DE-FG02-03ER63694-F1}

truncated since they occur during the nighttime. Again, San Francisco uses significantly less energy than the other cities due to the abundant use of the economizer. Figure 4.11 shows the relative energy usage of HIYW to OSFA for the work day hours, and qualitatively it is also very similar to the $8760 \mathrm{~h}$ (Figure 4.9). In fact, most of the ratios are nearly unchanged from the full year, with only slight changes on the order of $2 \%$. Not surprisingly, the HIYW/OSFA ratio of San Francisco (city \#6) increased more significantly, since a majority of the activated economizer hours, the hours at which HIYW comes at the largest energy penalty, occur during the daytime.

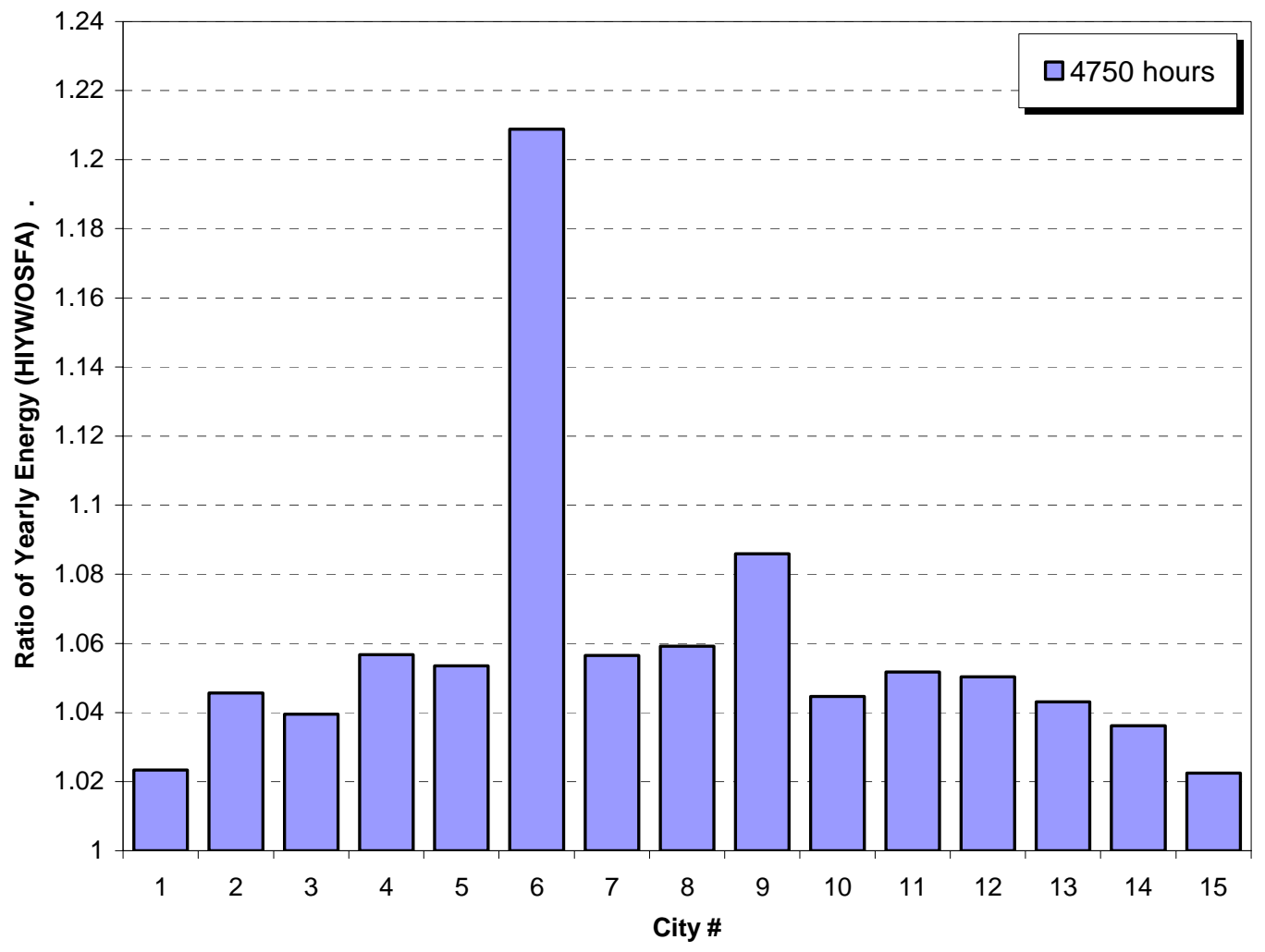

Figure 4.11 - Ratio of HIYW to OSFA energy usage (4750h) 


\section{Report No. DE-FG02-03ER63694-F1}

\subsection{Interior Resistances}

Until now we have been studying only the baseline case, which consists of all private offices. Now we would like to explore other types of offices including cubicles. The interior wall thermal resistance $\left(r_{\text {ivall }}\right)$ in the baseline case corresponded to a common gypsum board inside wall. The value of this thermal resistance is well documented. Unfortunately this is not the case with partial height dividers such as those used in cubicles. The thermal resistance of the solid portion of the divider can be calculated, however the open space above the cubicle must be considered. The mechanism for heat transfer between zones is no longer only conduction as it was with a solid wall from floor to ceiling. Instead there will likely be some advective heat transfer between neighboring zones either above the partitions or through the open doors and corridors. As we have discussed earlier, if we neglect the latent loads, we can still write advective heat flux with a thermal resistance. However, the true thermal resistance, if one such value exists, is not easily calculated. Understanding the airflow behavior between neighboring cubicles and other partial height workstations with different temperature distributions is the subject of current research, but presently there is no accepted method short of computational fluid dynamics (CFD) or detailed measurements to estimate it. Therefore, we will test a variety of resistances that will show us the influence the interior partition resistance has on the building energy consumption.

Table 4.1 lists the six unique trial resistances that we will test in using the TCTB model. Trial one is the baseline building case, exactly as we have used previously. To approximate a partial height thin partition workstation we will use half the thermal resistance of the solid wall. Also, we will explore the effect of the inside convective heat transfer coefficient by using a larger (and therefore less resistive) value, in trials 4-6. In trials $3 \& 6$, an open workspace with no solid partitions is approximated by using the resistance of the film of air between the zones. 


\section{Report No. DE-FG02-03ER63694-F1}

Note that the total interior resistance, $r_{i n}$ is just the sum of the wall resistance and two of the resistance associated with the convective heat transfer coefficient,

$$
r_{\text {int }}=r_{\text {inwall }}+\frac{2}{h_{\text {in }}}
$$

The two convective heat transfer coefficients are due to the presence of two film resistances on each side of the partition. As seen in Table 4.1 the inside convective heat transfer coefficient can have a significant impact on the overall thermal resistance of the partition. This leads to a great deal of uncertainty in the true overall thermal resistance value.

Table 4.1 - Interior resistance trials

\begin{tabular}{|c|c|c|c|c|}
\hline $\begin{array}{l}\text { Trial } \\
\text { No. }\end{array}$ & $\begin{array}{l}\text { Wall Resistance, } \\
\mathrm{r}_{\text {iwall }}\left(\mathrm{m}^{2} \mathrm{~K} / \mathrm{W}\right)\end{array}$ & $\begin{array}{l}\text { Inside Convective Heat } \\
\text { Transfer Coefficient, } \mathrm{h}_{\mathrm{i}} \\
\qquad\left(\mathrm{W} / \mathrm{m}^{2} \mathrm{~K}\right)\end{array}$ & $\begin{array}{l}\text { Resulting } \\
\text { Resistance, } \\
\mathrm{r}_{\text {int }}\left(\mathrm{m}^{2} \mathrm{k} / \mathrm{W}\right)\end{array}$ & Notes \\
\hline 1 & 0.392 & 3.06 & 1.05 & Baseline Case \\
\hline 2 & $0.196^{\mathrm{a}}$ & 3.06 & 0.85 & $\begin{array}{l}\text { Estimated thin partition (i.e. } \\
\text { cubicle) }\end{array}$ \\
\hline 3 & -- & 3.06 & 0.33 & $\begin{array}{l}\text { Approximates open } \\
\text { workspace }^{\text {b }}\end{array}$ \\
\hline 4 & 0.392 & 8.29 & 0.63 & $\begin{array}{l}\text { Baseline with less } \\
\text { conservative } h_{i}[27]\end{array}$ \\
\hline 5 & $0.196^{\mathrm{a}}$ & 8.29 & 0.44 & $\begin{array}{l}\text { Estimated thin partition (i.e. } \\
\text { cubicle) }\end{array}$ \\
\hline 6 & -- & 8.29 & 0.12 & $\begin{array}{l}\text { Approximates open } \\
\text { workspace }^{\mathrm{b}}\end{array}$ \\
\hline
\end{tabular}

Resistances listed for trials $2 \& 5$ are approximated.

${ }^{\mathrm{b}}$ The true resistance value between open space zones is unknown and the subject of current research. Approximated as a single convective heat transfer coefficient 


\section{Report No. DE-FG02-03ER63694-F1}

To observe the effect of the interior resistance we will start with the baseline case and modify only the interior resistances and convective heat transfer coefficients as shown in Table 4.1. It is important to note however, that the inside convective heat transfer coefficient for the outside walls only will be held at the default $3.06 \mathrm{~W} / \mathrm{m}^{2} \mathrm{~K}$ regardless of what the other inside convective heat transfer coefficients are. In other words, the total exterior resistance remains unchanged throughout all trials. One population (Appendix 6.3) will be used for the HIYW control strategy. We will use the HVAC energy usage for work day hours only (4750h). Since the OSFA energy usage is independent of the interior resistance, due to the uniform interior temperature assumption, we can use it as means for comparison and normalization of the HIYW energy usage. For each value of the interior resistance we will take the 15-city average of the energy usage. The average, for both HIYW and OSFA, will be weighted based on the population in each climate zone as described in Section 2.7.3. Figure 4.12 shows the ratio of HIYW to OSFA for the six trials plus an addition trial of $r_{\text {int }}=0.2 \mathrm{~m}^{2} \mathrm{~K} / \mathrm{W}$. This value was needed to clarify the results as seen in Figure 4.12. Since there is a certain level of uncertainty with the true resistance value of partial height partitions, the resulting trend is very important. Through experimental testing or CFD calculations the true thermal resistance value can be found and the proper placement on the curve of partial height cubicles would be known. 
Report No. DE-FG02-03ER63694-F1

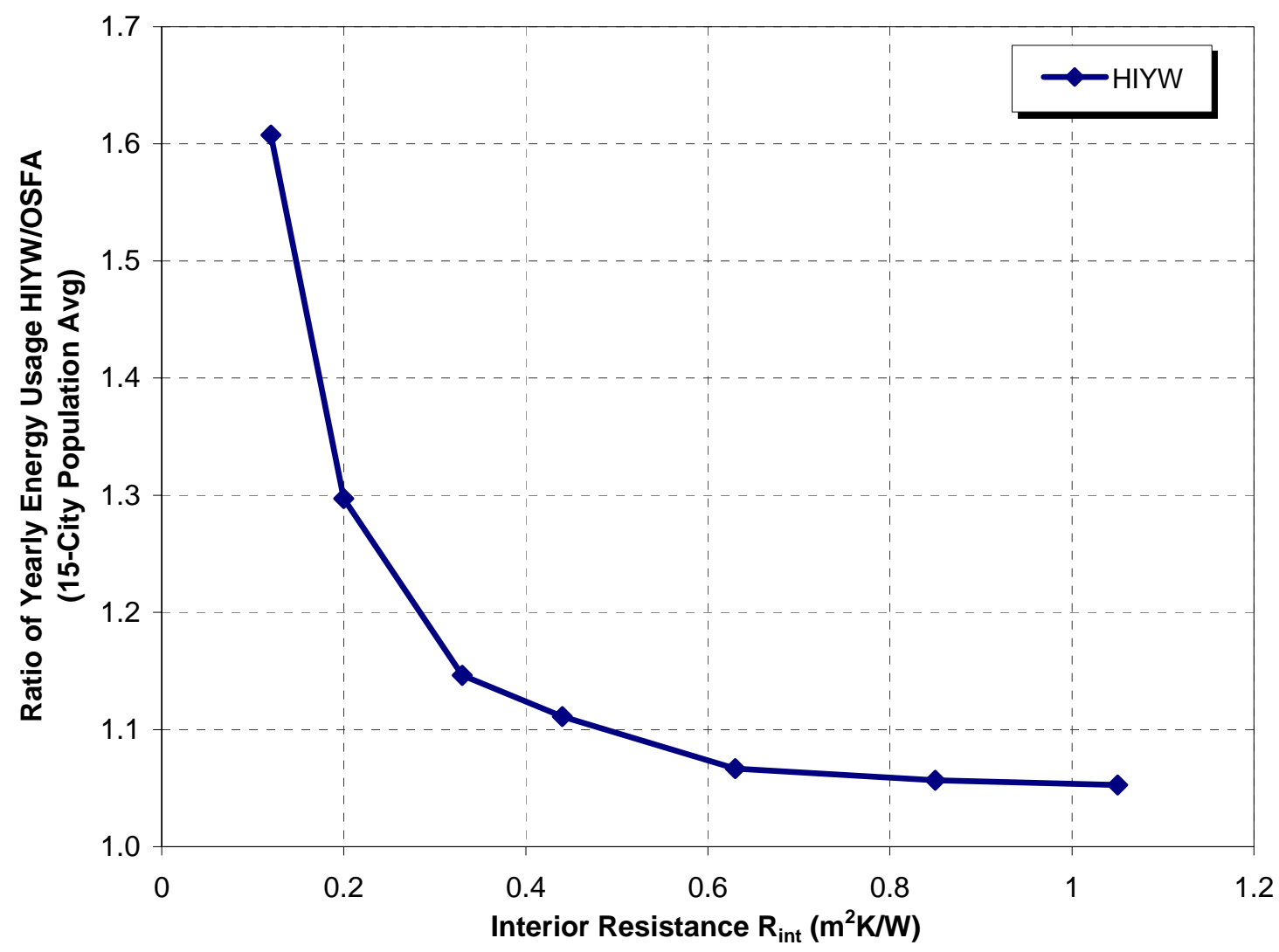

Figure 4.12 - Relative energy cost of HIYW to OSFA for various internal resistances

The rightmost point on the curve in Figure 4.12 corresponds to the baseline case (trial 1). It can be easily seen that slightly reducing the interior resistance from the baseline case has a very small impact on the HIYW energy cost. However, as the resistance between personal zones approaches that of an open workspace the cost of HIYW climbs rapidly ${ }^{1}$ It is also important to note that in the area of the baseline case, small changes in the resistance would have little impact on the overall cost of HIYW. This is especially useful since all interior wall constructions will have slightly different thermal resistances, the results shown for the baseline

\footnotetext{
${ }^{1}$ A well mixed zone assumption, such as the one used here is no longer valid when the individual zones are open to neighboring zones.
} 
Report No. DE-FG02-03ER63694-F1

resistance would be minimally impacted by a slight change in partition thickness or construction.

\subsection{Effect of Population Distribution}

\subsubsection{Random Populations in Baseline Building Model}

Until this point we had considered only one group of people inhabiting the building. To understand what impact the placement and random selection of the building population has on the energy cost we will test 100 different populations. It is expected that there will be certain configurations that will result in similar desired temperatures next to each other and some configurations that will result in vastly different desired temperatures next to each other, however the impact of this on energy consumption is unknown. Each of the 100 populations, of 49 people each, will be chosen at random from the given distribution (Section 2.9.1) and then assigned randomly to a room in the baseline building model. Figure 4.13 shows the range of energy usage of the 100 populations for each city for the baseline case for the work day bin hours (4750h). In the plot, the HIYW energy usage is represented by a box and whiskers; the box indicates the extents of the upper and lower quartiles $(75 \%$ and $25 \%)$ of the energy usage for these trials and the whiskers indicate the range. The line in the middle of the boxes represents the median value, although it may be difficult to see where the data is tightly grouped. The black diamond indicates the OSFA yearly energy usage for each city. 
Report No. DE-FG02-03ER63694-F1

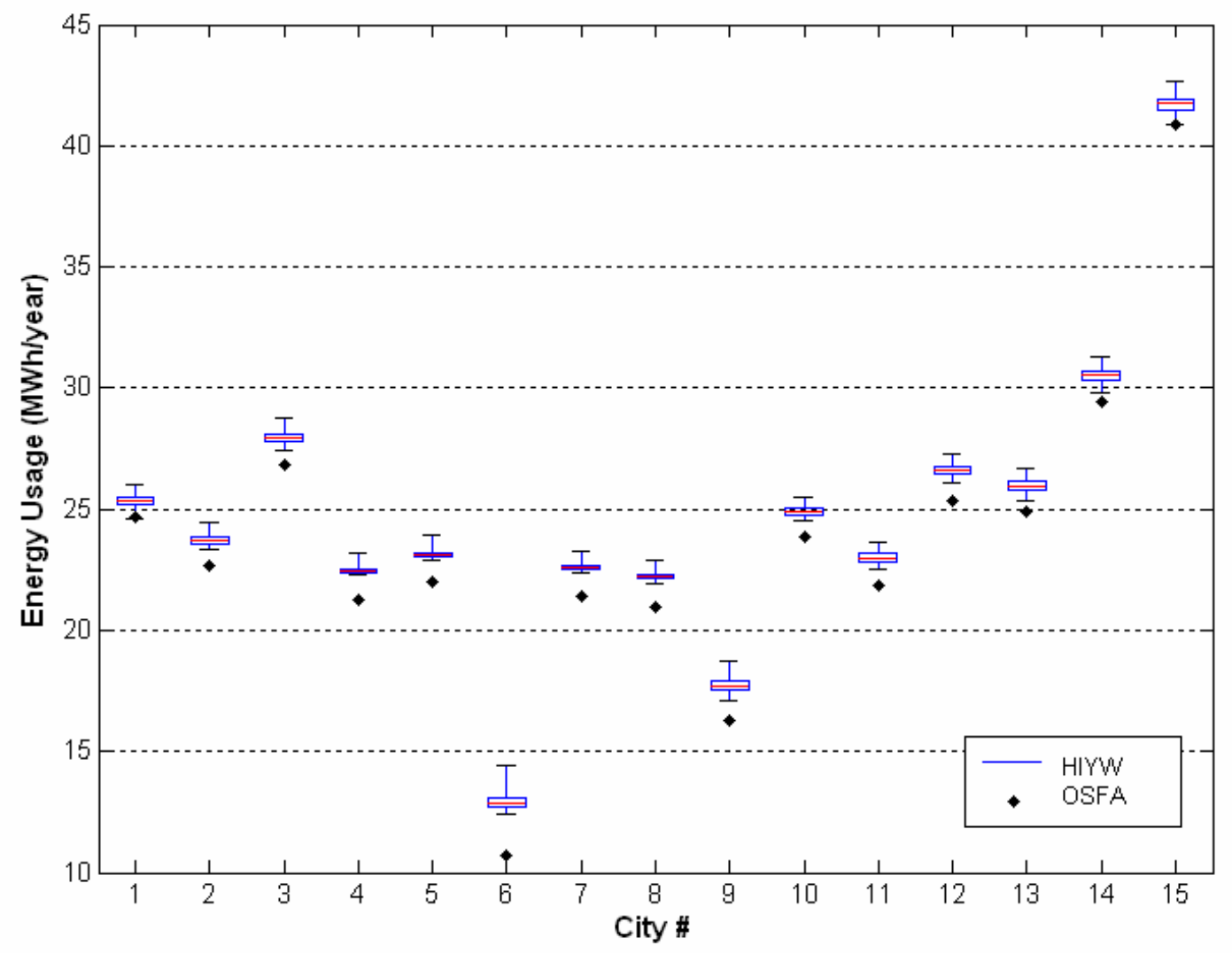

Figure 4.13 - 100 population energy usage comparison for baseline case

In every case except city \#1 (Miami, FL) each building population configuration uses more energy in the HIYW case than the OSFA case. There was one building population configuration that, in Miami used approximately $99.6 \%$ of the OSFA energy; for every other population HIYW used more energy (see Figure 4.14). The energy usage appears to be a weak function of the population within the building. To see the relative effect of the energy cost associated with HIYW, Figure 4.14 shows the ratio of the HIYW energy usage of each random population to the OSFA energy usage. 
Report No. DE-FG02-03ER63694-F1

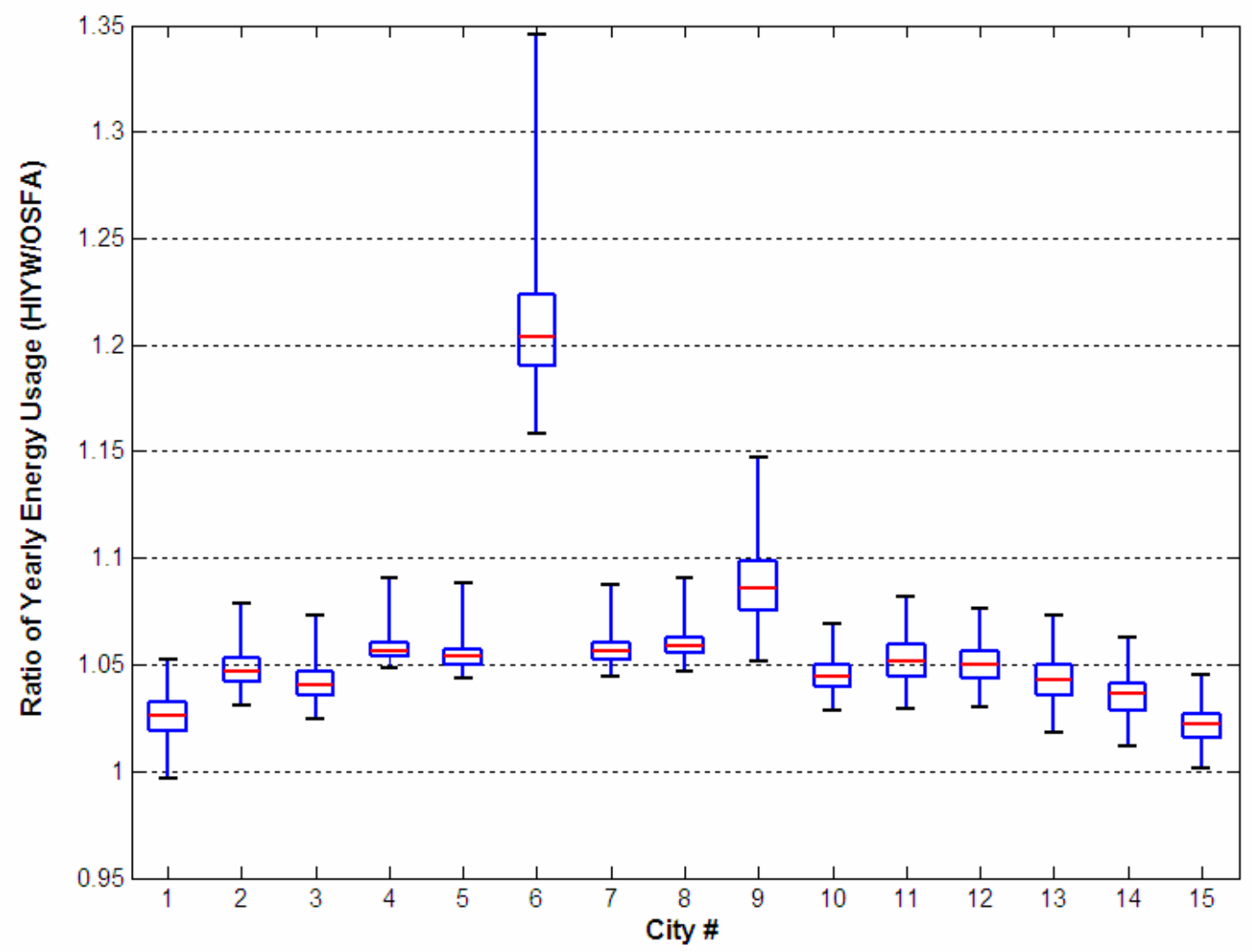

Figure 4.14 - Ratio of HIYW to OSFA energy usage of 100 random populations for baseline case

Here, we can see that the random populations, in most cities can account for approximately $2.5 \%$ increase or decrease from the median value. The obvious exception is city \#6, San Francisco. As mentioned before San Francisco, unlike the other cities has a temperate climate that lends itself well to economizer operation. In the previous plot, Figure 4.13, we saw that San Francisco used considerably less energy over the course of the year than the other cities (both HIYW and OSFA). Therefore a small increase in HIYW energy could actually be significant when compared to OSFA. The effect the random populations have on the energy usage in San Francisco is substantial; ranging from 16\% to 35\% more energy than OSFA. Salem, OR, city \#9, has the next most adverse climate to personal control, and like San 


\section{Report No. DE-FG02-03ER63694-F1}

Francisco, the population inside the building has a significant impact on the energy penalty associated with HIYW.

\subsubsection{Random Populations in Partial Height Partition Building}

Now we will observe the influence of the random populations in a building with partial height partitions (cubicles) as in Section 4.2. We will explore the partial height partition with the lower overall resistance value of $0.44 \mathrm{~W} / \mathrm{m}^{2} \mathrm{~K}$ (trial five in Table 4.1). Figure 4.15 shows the box and whiskers plot for the partial height partition case. As before, the box represents the upper and lower quartile, the whiskers represent the extents of the data, the line in the middle of the box corresponds to the median value, and the black diamond indicates the OSFA energy usage. 
Report No. DE-FG02-03ER63694-F1

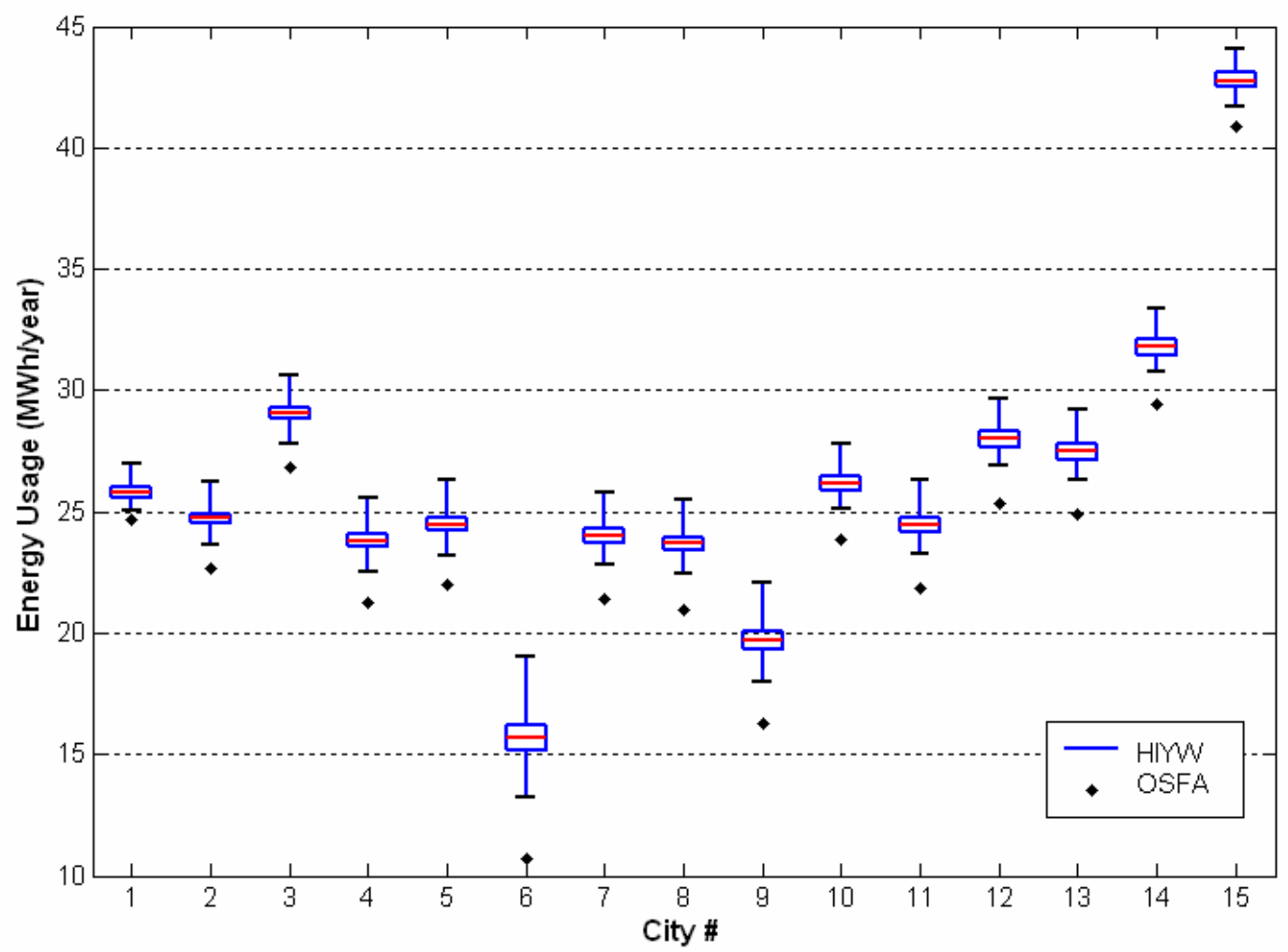

Figure 4.15 - Energy usage by city for 100 random populations for $R_{\text {int }}=0.44 \mathrm{~m}^{2} \mathrm{~K} / \mathrm{W}$ (partial height partition)

For this smaller interior resistance, across the board HIYW uses more energy than OSFA. Also, the populations create a much larger spread in the yearly energy usage compared to the baseline resistances (Figure 4.13). The spread is close to symmetric around the median and now tends to be $\pm 5 \%$ from the median. The energy cost of HIYW as compared to OSFA can be seen in Figure 4.16. 


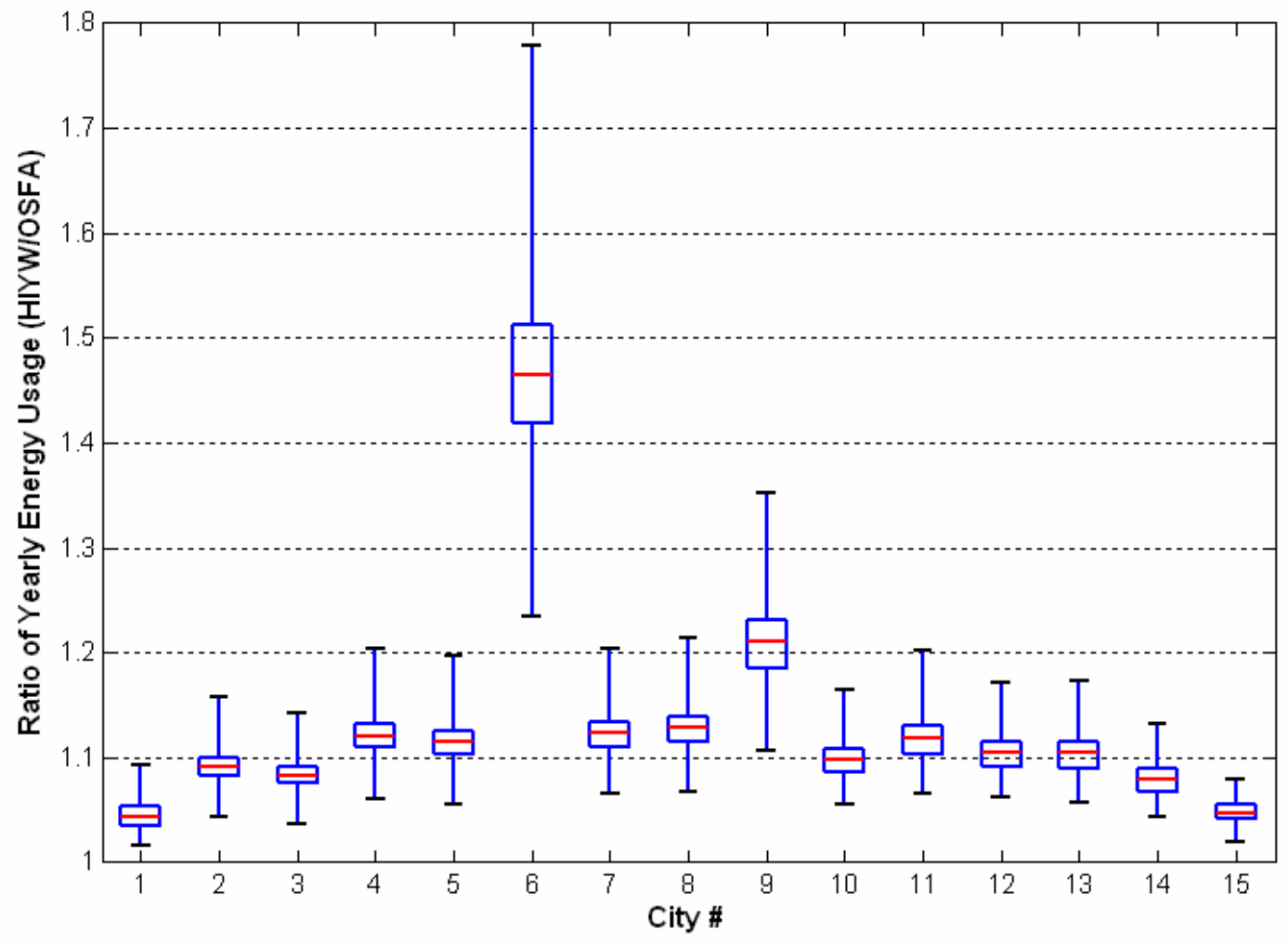

Figure 4.16 - Range of HIYW to OSFA yearly energy usage by city for 100 random populations for $\mathrm{R}_{\text {int }}=0.44 \mathrm{~m}^{2} \mathrm{~K} / \mathrm{W}$ (partial height partition)

Again, city \#6, San Francisco, shows a significant dependence on the population in the building. The energy increase from OSFA can range from $23 \%$ to $78 \%$, depending on the specific distribution of people and their desired temperatures within the building. Salem, OR (city \#9) again shows the second largest energy penalty. As we would expect from our experience with one population, the median value of all the cities increased from the baseline case (Figure 4.14). However, from this plot we can see that not only the does the median increase, but the population has a much larger role in determining the energy cost of HIYW. 


\subsection{Building Size and Shape}

In this section we will explore different building shapes. The baseline case building is a $7 x 7$, 49-zone building. We will try to keep the total number of zones as close as possible to 49, while changing the aspect ratio of the building to other rectangular configurations $(10 \times 5$ and $25 \times 2)$.

\subsubsection{0x5 Building}

The first new building shape configuration we will explore is a rectangular 10x5 zone building. We will keep all other building dimensions the same as the baseline building case. Figure 4.17 shows an example of a possible HIYW temperature distribution within the 10x5 building. Like the baseline case, OSFA will consist of a uniform $24^{\circ} \mathrm{C}$, achieved through three thermostats: one for the corner zones, one for the perimeter zones, and one for the center zones.

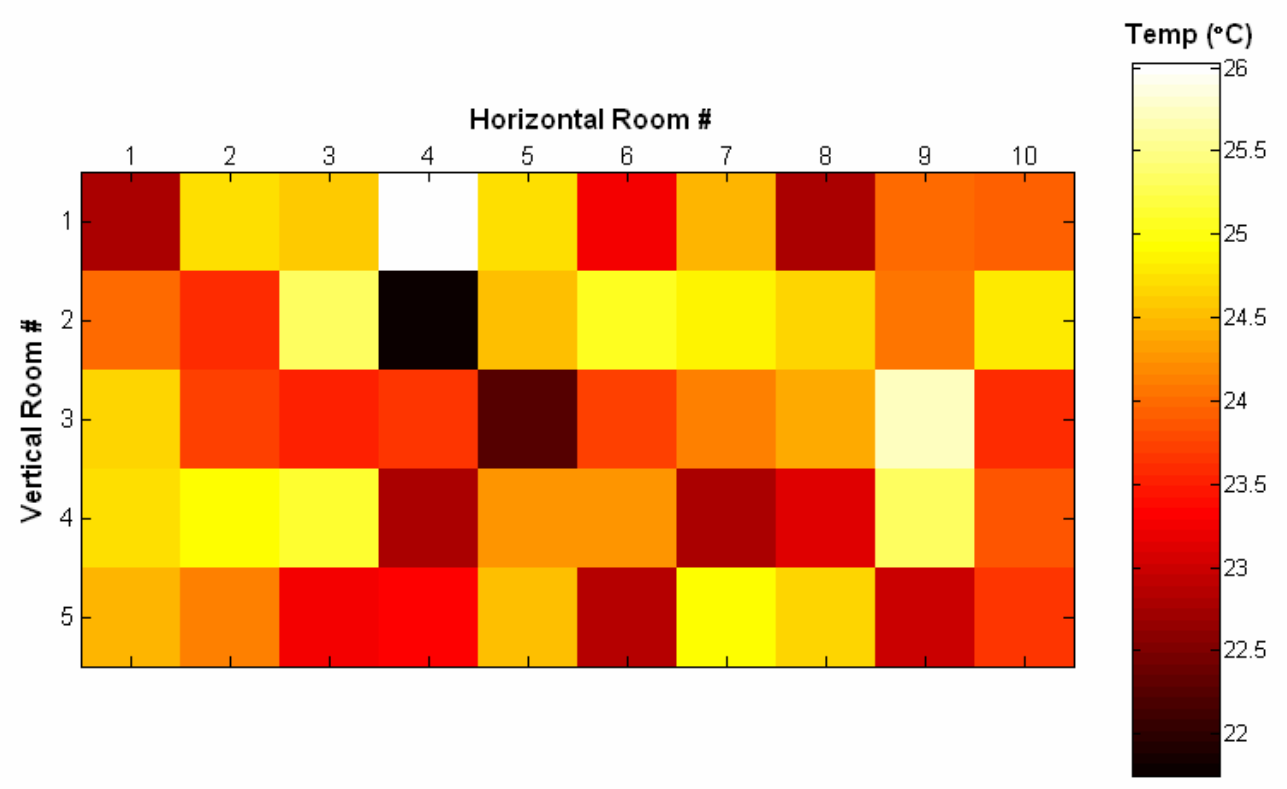

Figure 4.17 - Plan view of an example HIYW temperature distribution for 10x5 building 


\section{Report No. DE-FG02-03ER63694-F1}

All building envelope properties are the same as the baseline case. Interior partitions have a thermal resistance characteristic of wood frame walls (default baseline). We will observe only work day hours. As we did before, to understand the influence of the building inhabitants, we will use 100 random populations assigned randomly to rooms. Figure 4.18 shows both the range of HIYW yearly energy usage and the OSFA yearly energy usage for each of the 15 cities.

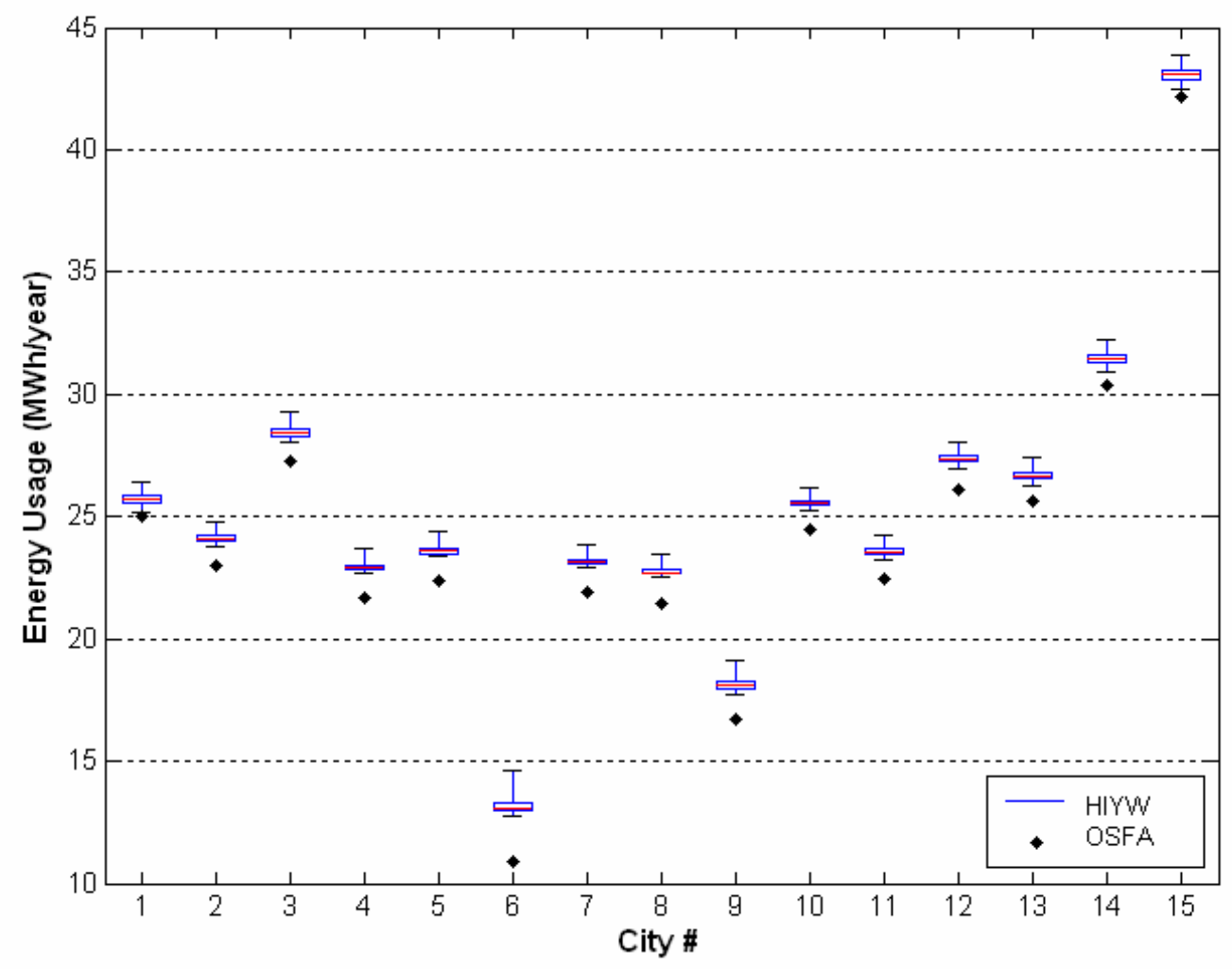

Figure 4.18 - Yearly energy usage for 100 random populations for 10x 5 building

The energy usage shown here is very similar to the baseline $7 \times 7$ building (Figure 4.13). In fact, the OSFA yearly energy usage, which is independent of the building population, is approximately $2 \%$ larger for the $10 x 5$ building than the $7 x 7$. This would appear to be entirely 
due to the unavoidable presence of an extra zone (50 vs. 49$)$. To determine if the HIYW energy usage was similarly affected let us look at the ratio of the HIYW energy usage and the OSFA energy usage (Figure 4.19).

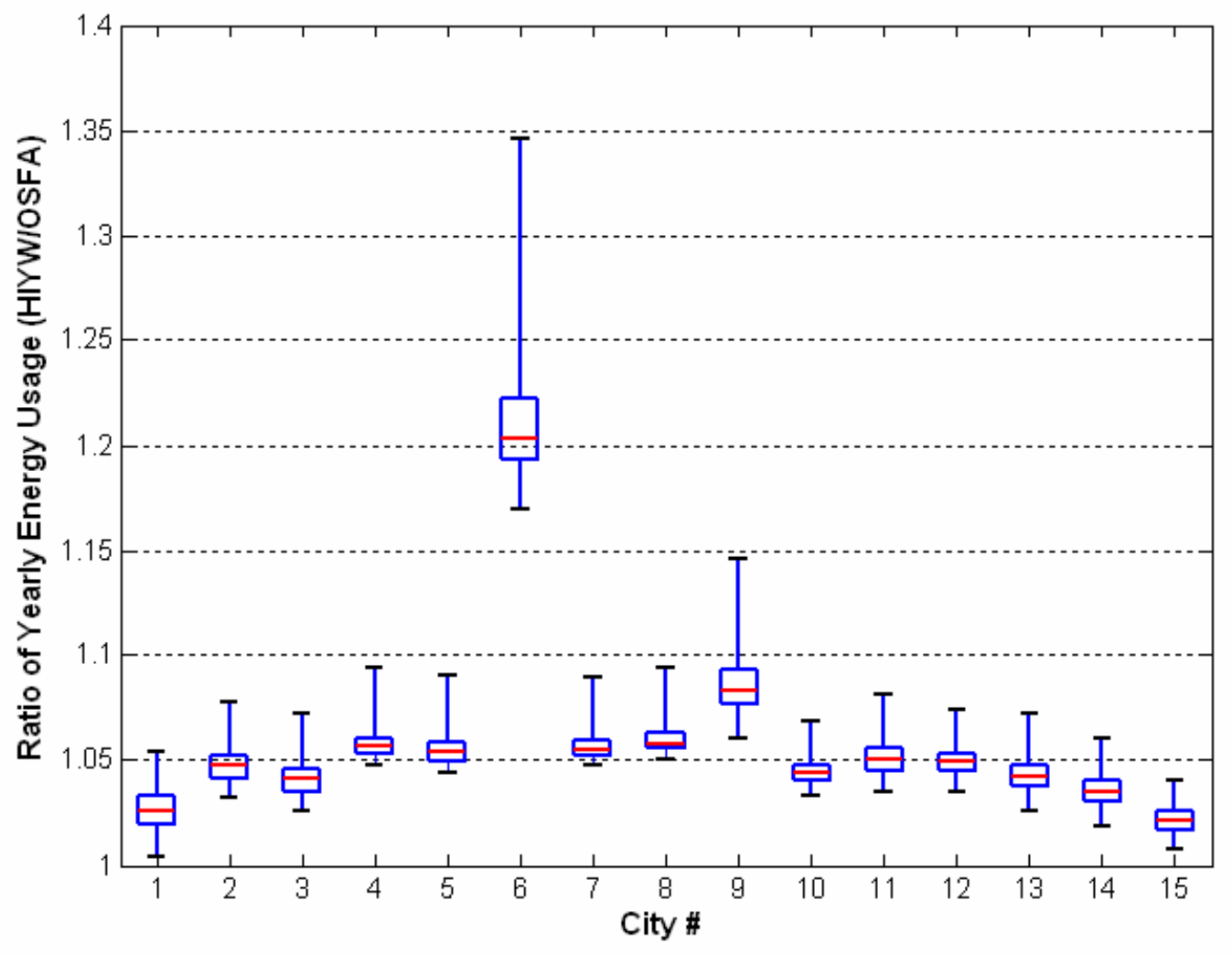

Figure 4.19 - Range of HIYW to OSFA yearly energy usage by city for 100 random populations for $10 \times 5$ building

As with the OSFA energy usage, the ratio between the HIYW and OSFA energy usage for the 10x5 building is nearly identical to the $7 \times 7$ building. The mean value of the 100 energy usage ratios for each city is, in fact, less than $0.1 \%$ different from mean value for the $7 \times 7$ baseline case. Clearly the change in shape from $7 \times 7$ to $10 \times 5$ made no appreciable difference in the energy cost associated with the adoption of HIYW. 


\section{Report No. DE-FG02-03ER63694-F1}

\subsubsection{5x2 Building}

The second new shaped building we will model is a $25 \mathrm{x} 2$ building. This type of configuration could be characteristic of a hallway or corridor lined with offices, or a motel. The 10x5 building produced nearly identical results to that of the baseline $7 \times 7$ building likely because the outside wall surface area increased by only $7.1 \%$ (see Table 4.2). We would expect the $25 \mathrm{x} 2$ building, due to the significant increase in exterior surface area $(92.9 \%)$, to be more likely to show deviations in energy usage from the baseline $7 \times 7$ building.

Table 4.2 - Increase in outside wall surface area from baseline case

\begin{tabular}{|c|c|c|}
\hline Building Shape & $\begin{array}{l}\text { No. of perimeter } \\
\text { walls }\end{array}$ & $\begin{array}{l}\text { Increase in outside } \\
\text { wall surface area }\end{array}$ \\
\hline $7 \times 7$ & 28 & - \\
\hline $10 \times 5$ & 30 & $7.1 \%$ \\
\hline $2 \times 25$ & 54 & $92.9 \%$ \\
\hline
\end{tabular}

Since the orientation of the building is not important in the TCTB model, due to the lack of solar energy accounting, we will arbitrarily choose the 25 to be in the horizontal direction. Figure 4.20 shows one possible temperature distribution within the $25 \times 2$ building. As with the baseline case, we will set the OSFA thermostats to $24^{\circ} \mathrm{C}$. For this building, since there are only two unique types of rooms, corner and perimeter, we only need two thermostats, one in each type, to ensure a uniform $24^{\circ} \mathrm{C}$. 


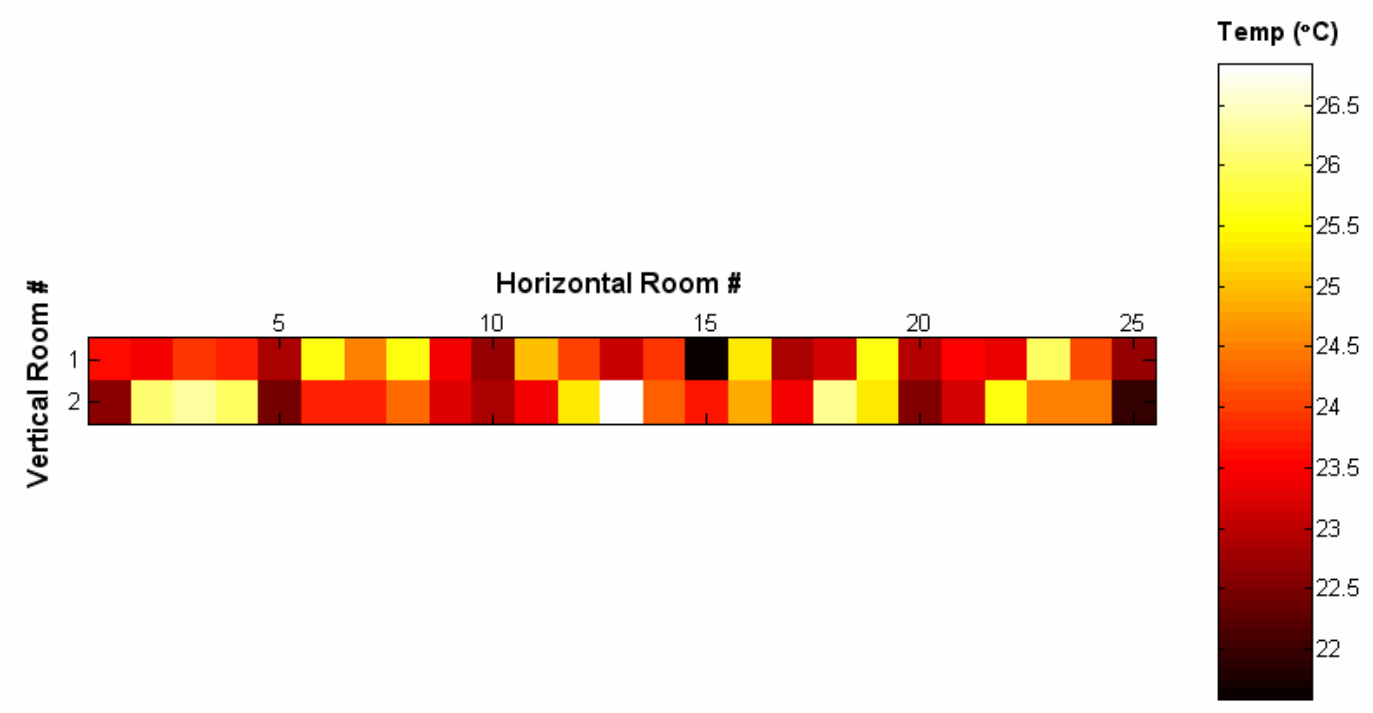

Figure 4.20 - Plan view of an example HIYW temperature distribution for 25x2 building

As we did with the previous building shapes, all the baseline case zonal dimensions and thermal resistances will be used along with the work day bin hours. For the $25 \mathrm{x} 2$ building we will also use 100 random populations assigned randomly to rooms. Figure 4.21 shows a box and whiskers plot of the energy usage of the 100 HIYW populations and the OSFA energy usage for each of the 15 cities. 
Report No. DE-FG02-03ER63694-F1

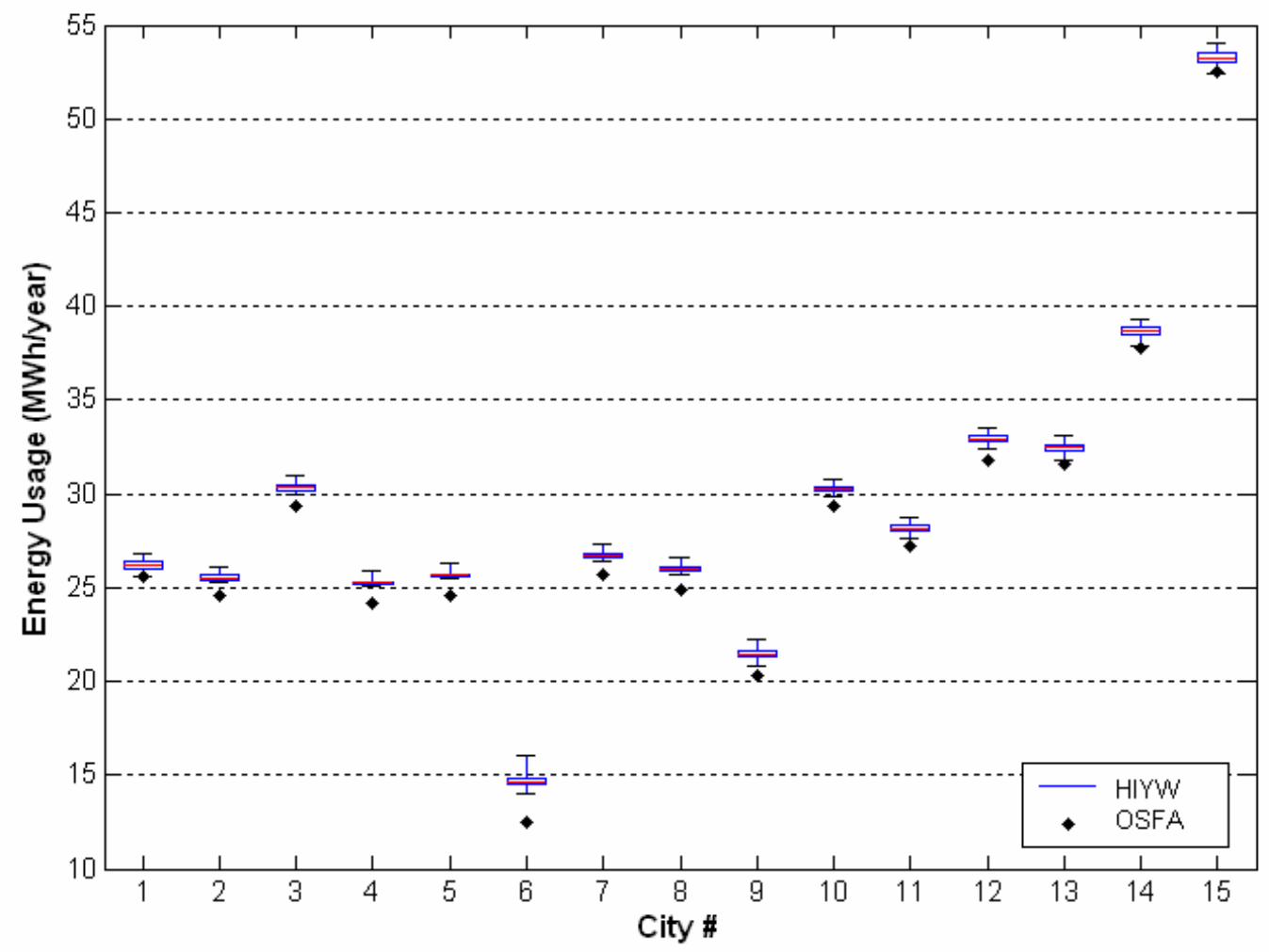

Figure 4.21 - Energy usage for 100 random populations for $25 \times 2$ building

At first glance the plot is very similar to the baseline $7 \times 7$ building (Figure 4.13) and the 10x5 building (Figure 4.18) with a slightly smaller spread amongst the HIYW populations. While the relationship between HIYW and OSFA and the relative energy usage between cities are both very similar to the $7 \times 7$ building. The $25 \times 2$ building uses more energy in every case. This is partially expected since the building contains one more zone (50 vs. 49), however the city average increase in OSFA energy usage from $7 \times 7$ to $25 \times 2$ is $18 \%$, with larger values in the colder climates. This rise in energy is a result of the greater exterior surface area and the resulting increase in heating required. Our main interest is not in the absolute yearly energy but rather the energy cost of the HIYW compared to the OSFA. To explore this case, Figure 4.22 shows a box and whiskers plot of the ratio of HIYW energy usage and OSFA energy usage. 
Report No. DE-FG02-03ER63694-F1

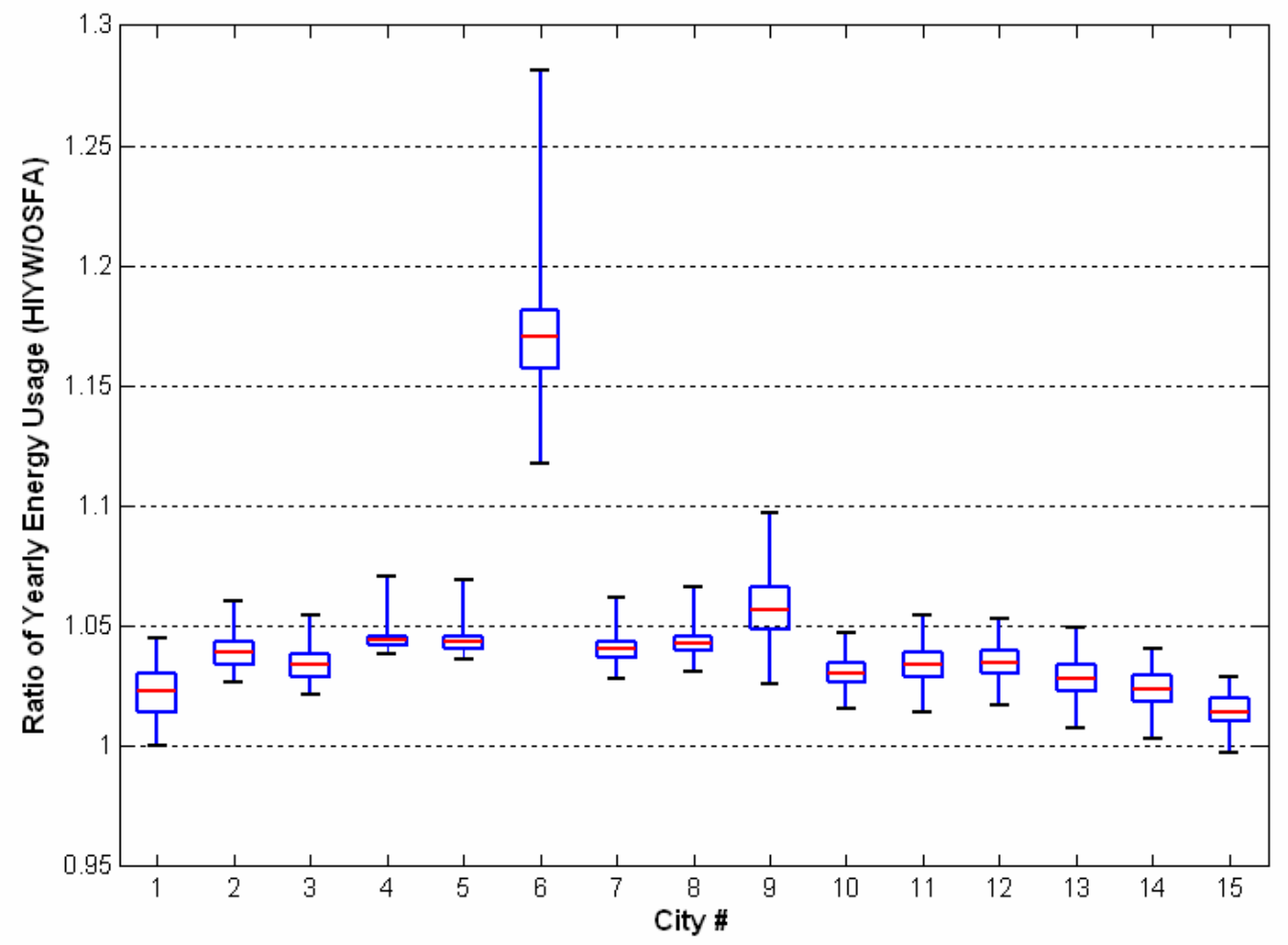

Figure 4.22 - Range of HIYW to OSFA yearly energy usage by city for 100 random populations for $25 \times 2$ building

This again is very similar to the $7 \times 7$ baseline case (Figure 4.14), however there is approximately a $5 \%$ decrease in the ratio for the $25 \times 2$ building, with some cities more than others. Nevertheless, San Francisco (city \#6) still shows the highest energy cost while the other cities are all below 10\% more energy than the corresponding OSFA case. The results suggest that the increase in yearly energy is a function of the building shape and may significantly affect the power consumption for OSFA and HIYW. 
Report No. DE-FG02-03ER63694-F1

\subsection{Deviation from Neutral Temperatures}

Until this point we have considered HIYW to be achieved by providing every building occupant with exactly his/her neutral temperature. This ensures that everyone is perfectly satisfied, thus improving comfort considerably from the OSFA, conventional approach, where only $80 \%$ of occupants are satisfied. It is expected that the majority of people have a certain threshold of sensitivity to temperature. That is, small deviations from the occupant's neutral temperature may not be noticeable, let alone negatively impact comfort. Ari, Cosden, et al. [32] developed a model for this temperature deviation tolerance, and proposed its relation to satisfaction. It may therefore be possible to provide occupants with a temperature very close to that which they desire, but with a slight deviation in an attempt to reduce energy consumption. This is the motivation behind using an optimization routine to try to maximize occupant comfort while decreasing energy usage.

To observe the potential in pursuing such an approach we will observe the average energy cost of HIYW plus some deviation temperature $\left(T_{\text {neutral }}+\Delta \mathrm{T}\right)$. This deviation is the difference between the occupants' neutral temperature and the actual temperature received. We will test $\Delta \mathrm{T}$ from $-3^{\circ} \mathrm{C}$ to $+3^{\circ} \mathrm{C}$. When $\Delta \mathrm{T}=0$, we arrive at the traditional HIYW case, where the neutral temperature is the received temperature. We will run the baseline case, with 10 HIYW populations. For each $\Delta \mathrm{T}$ we will take the average energy consumption for the 10 HIYW populations. The OSFA case, the benchmark for comparison, will remain unchanged at a uniform $24^{\circ} \mathrm{C}$ throughout the building. We will observe three cities, two extreme climates and one mixed. Miami, FL (city \#1) will represent the cooling dominated climates, Fairbanks, AK (city \#15) will represent the heating dominated climates, and Baltimore, MD (city \#7) will 


\section{Report No. DE-FG02-03ER63694-F1}

represent the mixed climates. Figure 4.22 shows the relative energy cost of HIYW compared to OSFA for each of the three cities.

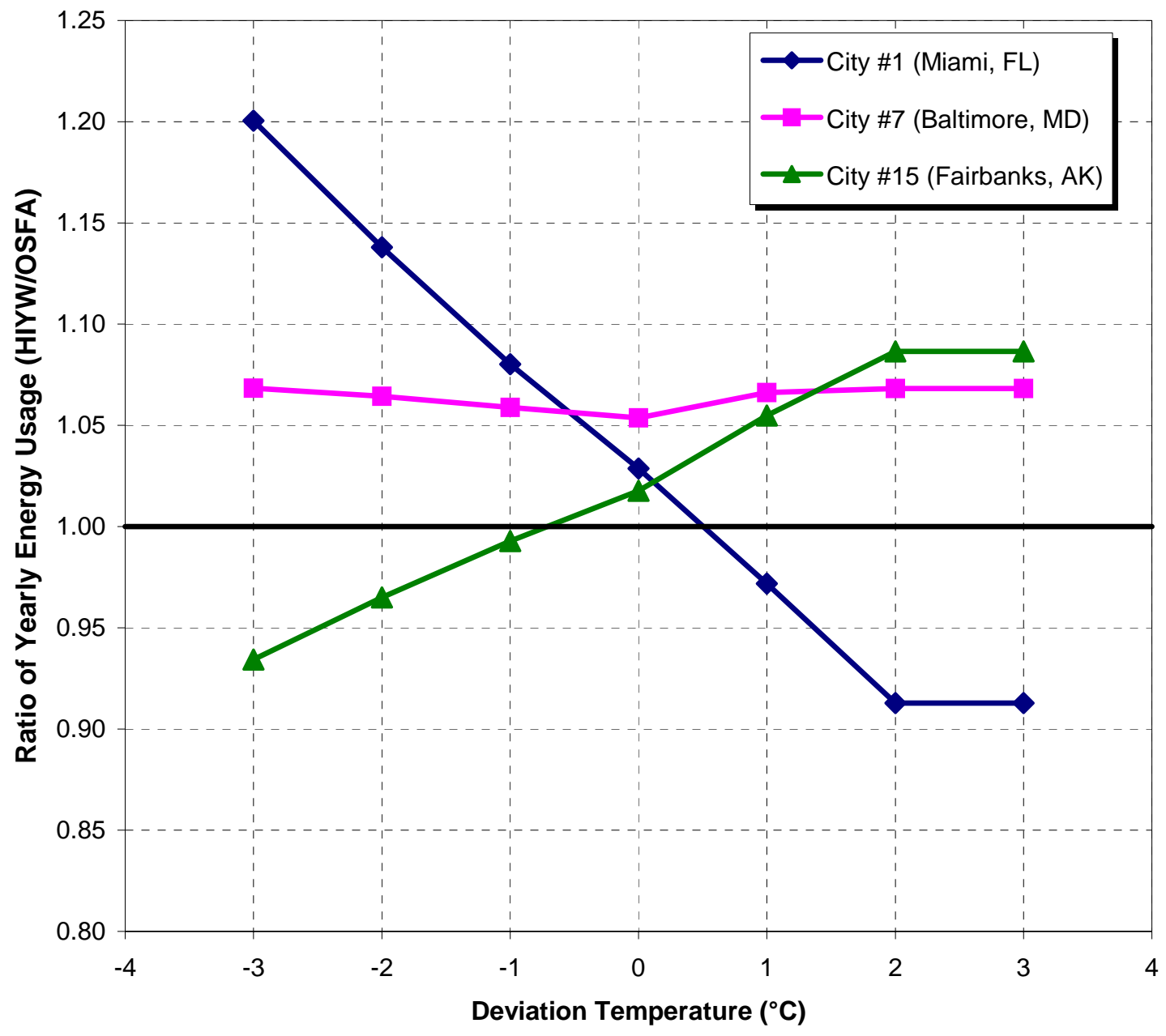

Figure 4.23 - Influence of deviation from neutral temperature on HIYW to OSFA energy usage ratio for Miami, Baltimore, and Fairbanks.

As we can see in Figure 4.22 for a cooling dominated climate (Miami), increasing the occupants' temperatures $(\Delta \mathrm{T}>0)$ results in a net yearly savings over OSFA. Increasing occupants' room temperature $1^{\circ} \mathrm{C}$ above the desired temperature, HIYW energy consumption 
went from a $3 \%$ penalty to a $3 \%$ savings from OSFA. The higher set point temperatures results in a lower building cooling load which resulted in a lower yearly energy usage. In Miami, lowering the occupants' temperatures $(\Delta \mathrm{T}<0)$ however, increased the cooling load and consequently the yearly energy cost. The opposite is true in heating dominated climates. Lowering occupant temperatures resulted in a net yearly savings over OSFA due to the reduced heating load. For the mixed climate (Baltimore) there is no appreciable change regardless of the deviation. Here, when $\Delta \mathrm{T}$ is positive the reduced cooling load in the summer is offset by an increased heating load in the winter, and vise versa when $\Delta \mathrm{T}$ is negative.

With these positive results it may be possible to use a more intelligence approach in deciding how to apply deviations to the occupants' neutral temperature. As we saw before (Figure 4.1) the building power consumption is the lowest at approximately $13^{\circ} \mathrm{C}$. For temperatures greater than $13^{\circ} \mathrm{C}$ the building is mostly cooled, whereas for temperatures below $13^{\circ} \mathrm{C}$ the building is mostly heated. During the cooling phase we will add $\Delta \mathrm{T}$ to the neutral temperature and during the heating phase we will subtract $\Delta \mathrm{T}$. That is,

$$
T_{\text {set }}=\left\{\begin{array}{lll}
T_{\text {neutral }}-\Delta T & \text { if } & T_{\text {out }} \geq 13^{\circ} \mathrm{C} \\
T_{\text {neutral }}+\Delta T & \text { if } & T_{\text {out }}<13^{\circ} \mathrm{C}
\end{array}\right.
$$

We will again test the three ranges of climates, hot, cold, and mixed, for the baseline building model. Again we will use 10 random populations and average the yearly energy usage for each to arrive at a single HIYW yearly energy usage. Figure 4.24 shows the ratio of HIYW to OSFA energy usage for $\Delta \mathrm{T}$ ranging from 0 (traditional HIYW) to $3^{\circ} \mathrm{C}$. 


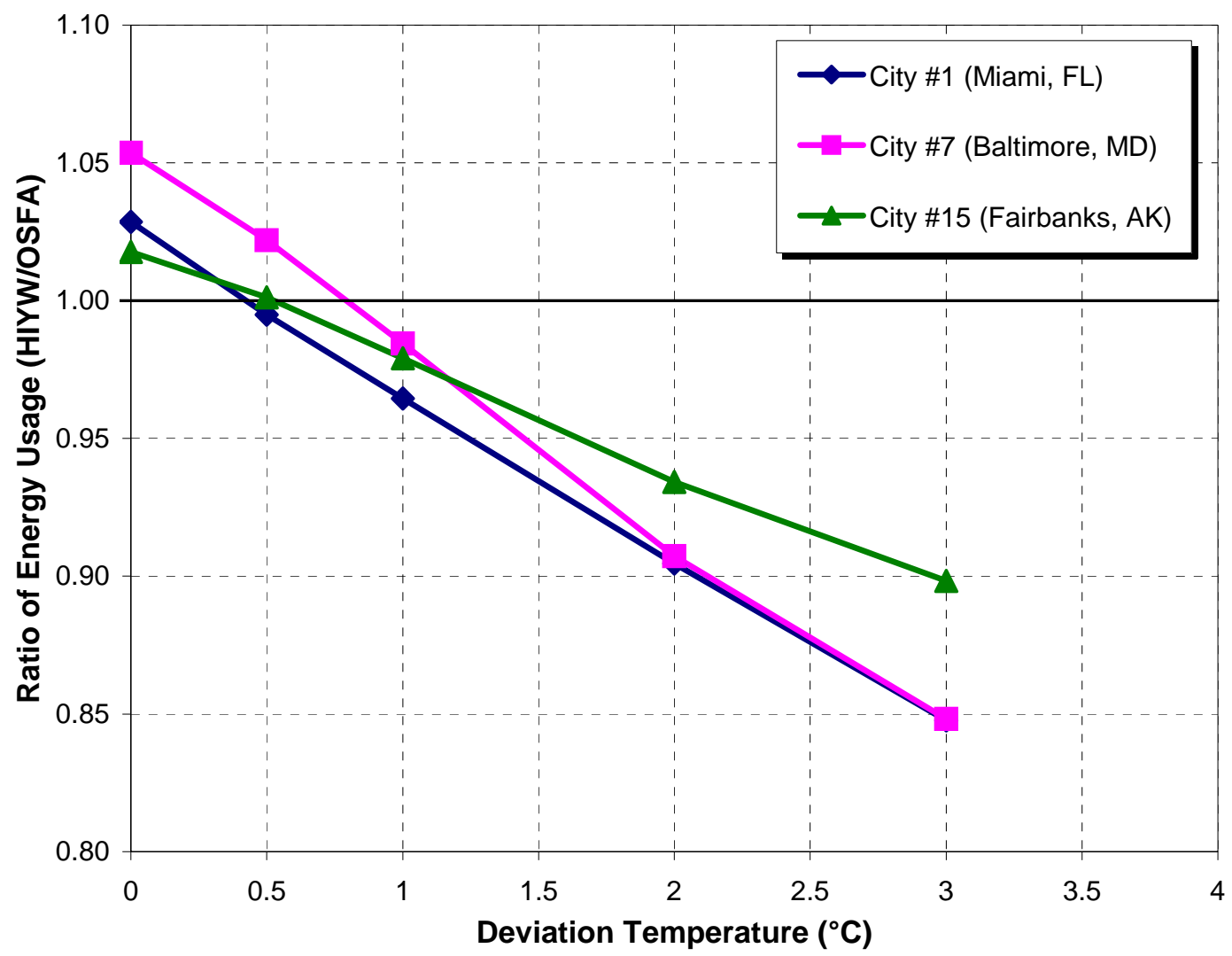

Figure $4.24-$ HIYW to OSFA energy ratio with heating $(-\Delta \mathrm{T})$ and cooling $(+\Delta \mathrm{T})$ temperature deviations

Here we can see the strong effect $\Delta \mathrm{T}$ has on the HIYW energy consumption. Starting at $\Delta \mathrm{T}=0$ (traditional HIYW) HIYW uses more energy than OSFA. However, shifting occupants' set point temperatures slightly depending on the season can quickly lower the energy cost. At a modest $2^{\circ} \mathrm{C}$ shift, HIYW uses approximately $7-9 \%$ less energy than OSFA. Figure 4.25 shows the increase associated with HIYW when a $\Delta \mathrm{T}$ of $1{ }^{\circ} \mathrm{C}$ is used for all the cities studied. We can see that for every city except San Francisco (city\#6) that an energy savings is realized when we slightly lower the occupants' desired temperatures by $1{ }^{\circ} \mathrm{C}$ in the winter and raise the temperatures by $1{ }^{\circ} \mathrm{C}$ in the summer. 
Report No. DE-FG02-03ER63694-F1

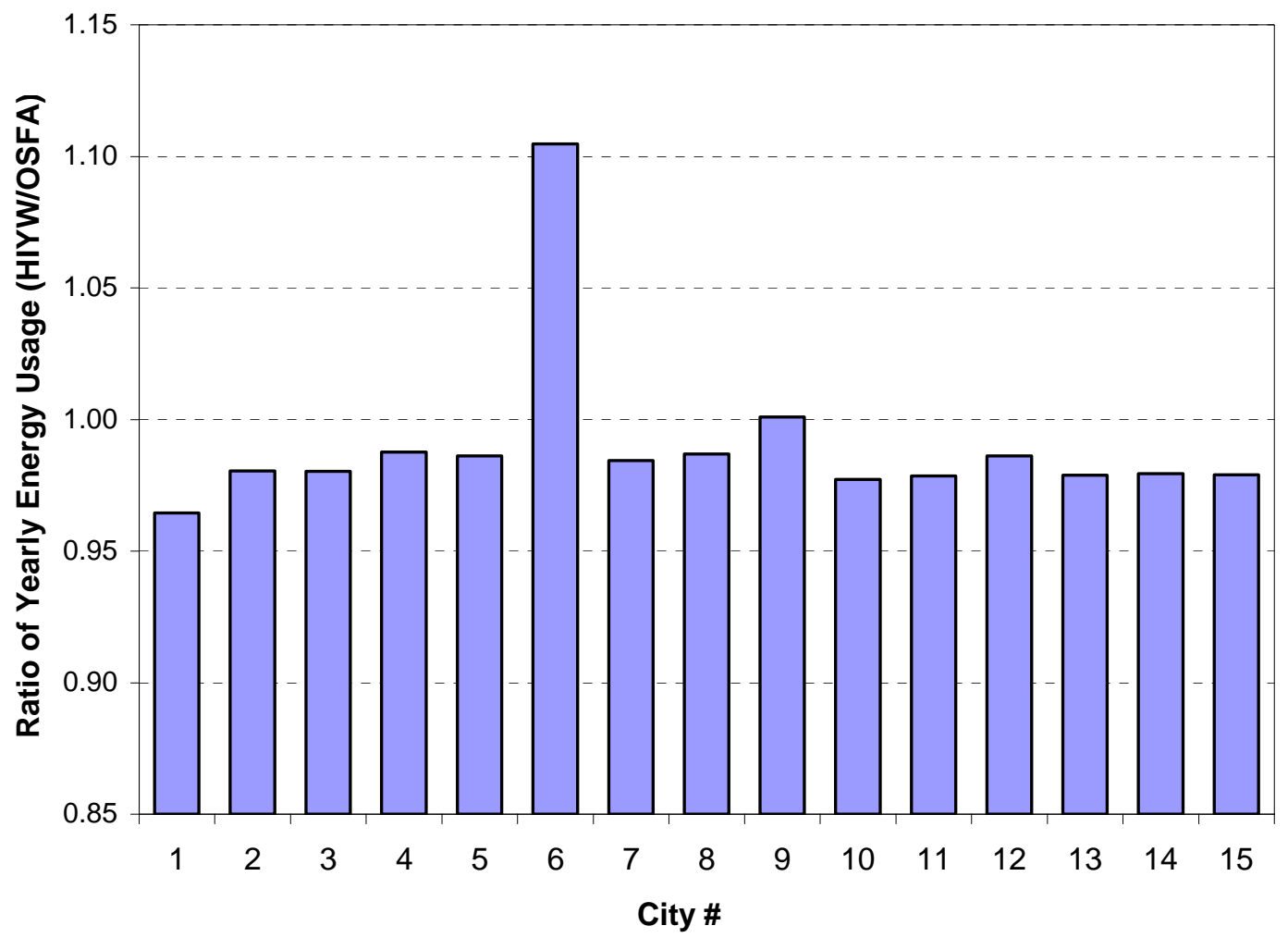

Figure 4.25 - Ratio of yearly energy usage when $\Delta \mathrm{T}=1^{\circ} \mathrm{C}$

These results show the motivation to apply intelligent control to the building system to try to optimize both comfort and energy efficiency. If small deviations are made within each occupants threshold it would be possible to lower the energy consumption without negatively affecting perceived thermal comfort. Ari et al [32], including the author of this study, have used advanced optimization techniques to further reduce energy consumption while maintaining occupant comfort. They have shown that through gradient optimization and fuzzy techniques that it is possible to improve comfort from the traditional OSFA, without increasing energy, or even reducing overall energy usage. Even San Francisco was shown that, by using an optimized HIYW solution, a 5\% energy savings over OSFA could be realized [32]. 


\section{Report No. DE-FG02-03ER63694-F1}

\subsection{Solar Radiation}

One of the major assumptions of the TCTB model is that there are no solar heat gains. To explore the ramifications of neglecting the solar loads we will use TRNSYS and its radiation processors. As an input the radiation processor use the TMY2 data recorded for the total horizontal radiation and direct normal radiation. The TRNSYS radiation processors then calculate the appropriate heat gain based on the azimuth and the slope of each surface. We will orient our square building with each side facing N, S, E, or W. Each exterior wall, and consequently each exterior window, is treated as perfectly vertical and entirely un-shaded. The solar absorptivity of the wall is set to the TRNSYS recommended average value of 0.6 [22]. To try to capture the effect of the solar radiation will look only at the work day hours (6am-7pm) as these are the most likely to be during sunlight hours.

First we will compare the baseline building model with and without solar radiation effects.

Figure 4.26 shows the percentage increase in OSFA yearly energy when we add the effect of solar radiation. The influence of the solar load is clearly large, especially in the warmer, sunnier climates. It is interesting to note that in every climate the addition of solar radiation caused an increase in yearly energy usage. The solar load must increase the cooling load in the summer more than it helps the heating load in the winter. This fact leads us to wonder about the influence of the chosen window (Section 4.6.1). 


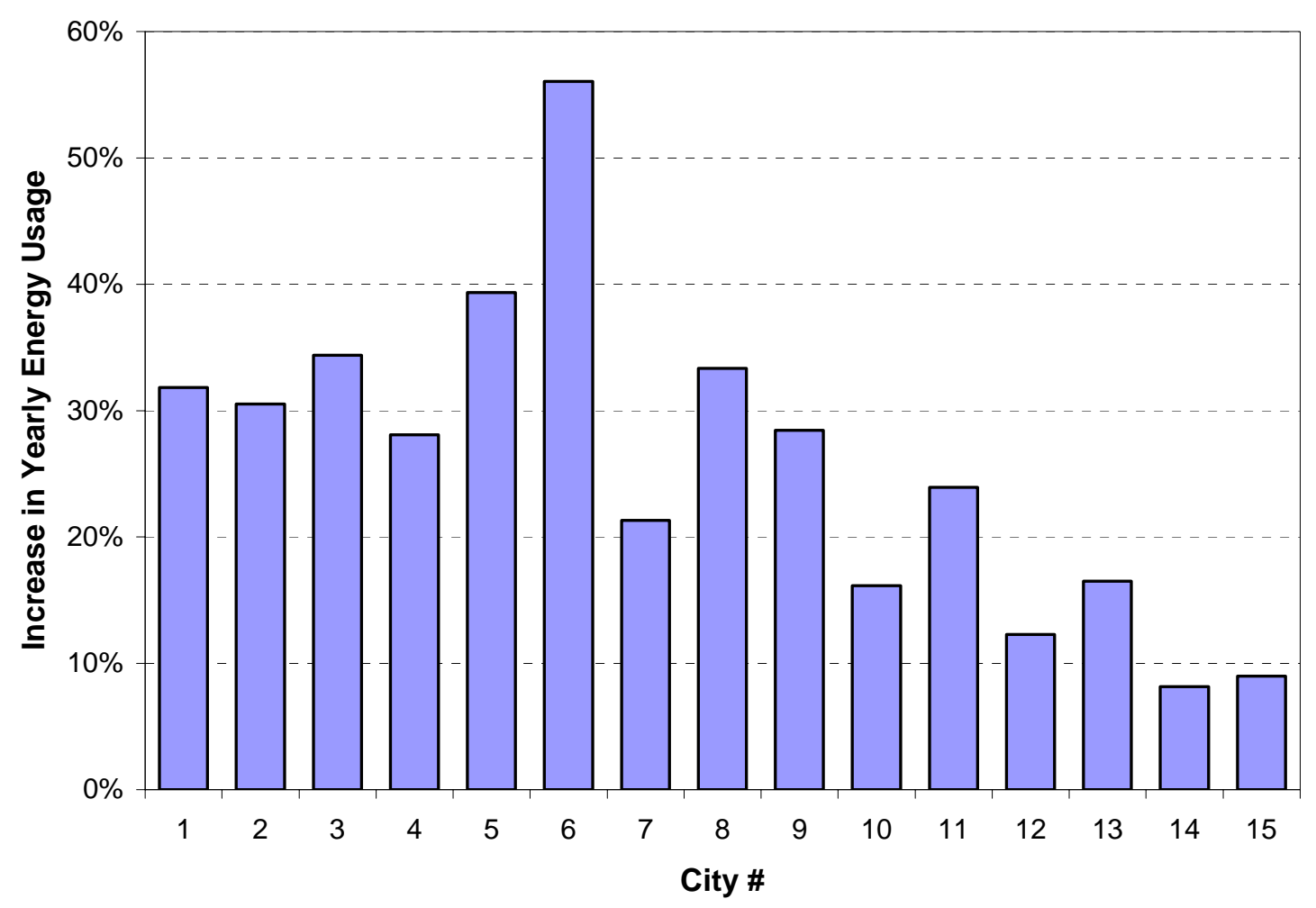

Figure 4.26 - Percent increase in TRNSYS-calculated OSFA yearly energy usage associated with addition of solar radiation

It is interesting to note the effect of the solar radiation on HIYW yearly energy usage. As it did in OSFA, HIYW used considerably more energy over the course of a yearly simulation when solar radiation was added. However, the absolute increase in energy was nearly the same for HIYW. Figure 4.27 shows the comparative increase in yearly energy usage between OSFA and HIYW. It can be seen that the solar radiation gain has a significant impact on the building load but does not vary significantly depending on the interior temperatures. 


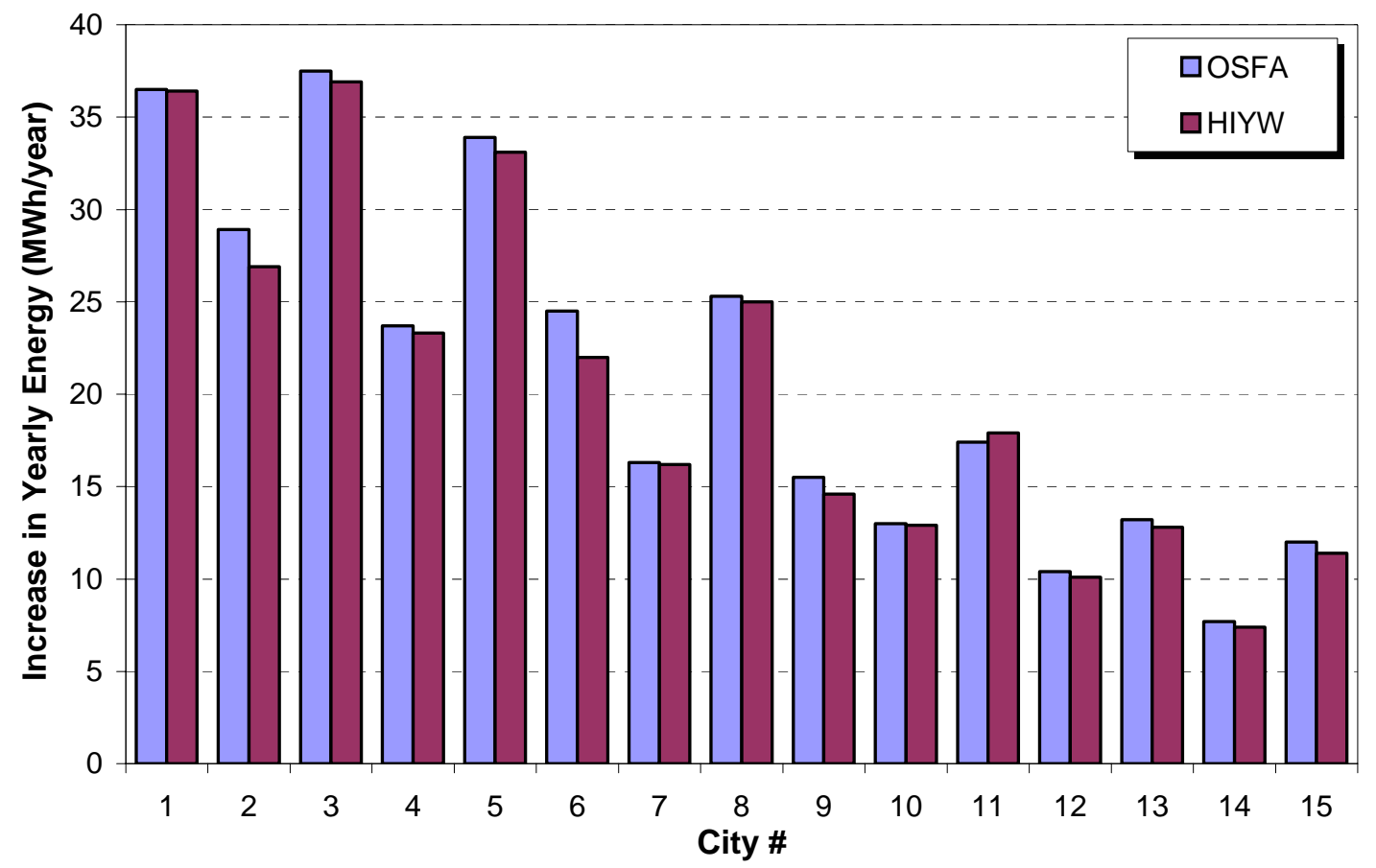

Figure 4.27 - Increase in yearly energy consumption for OSFA and HIYW associated with the addition of solar radiation

This observation is useful. Since the energy increase with the addition of solar gains is on average $3 \%$ different within each city, a single additive factor could be calculated for each city and added to a simulation that neglects solar.

\subsubsection{Solar Radiation without a Window}

As mentioned above we would like to understand the influence of the selected window in the TRNSYS model. We will remove the window and re-run TRNSYS for the baseline building without any exterior windows. The surface area of the outside walls remained the same, just comprised entirely of solid wall materials. All other building and solar characteristics are 


\section{Report No. DE-FG02-03ER63694-F1}

unchanged from the previous simulations. Figure 4.28 shows the percent increase in yearly energy usage when solar radiation is added to a building without windows.

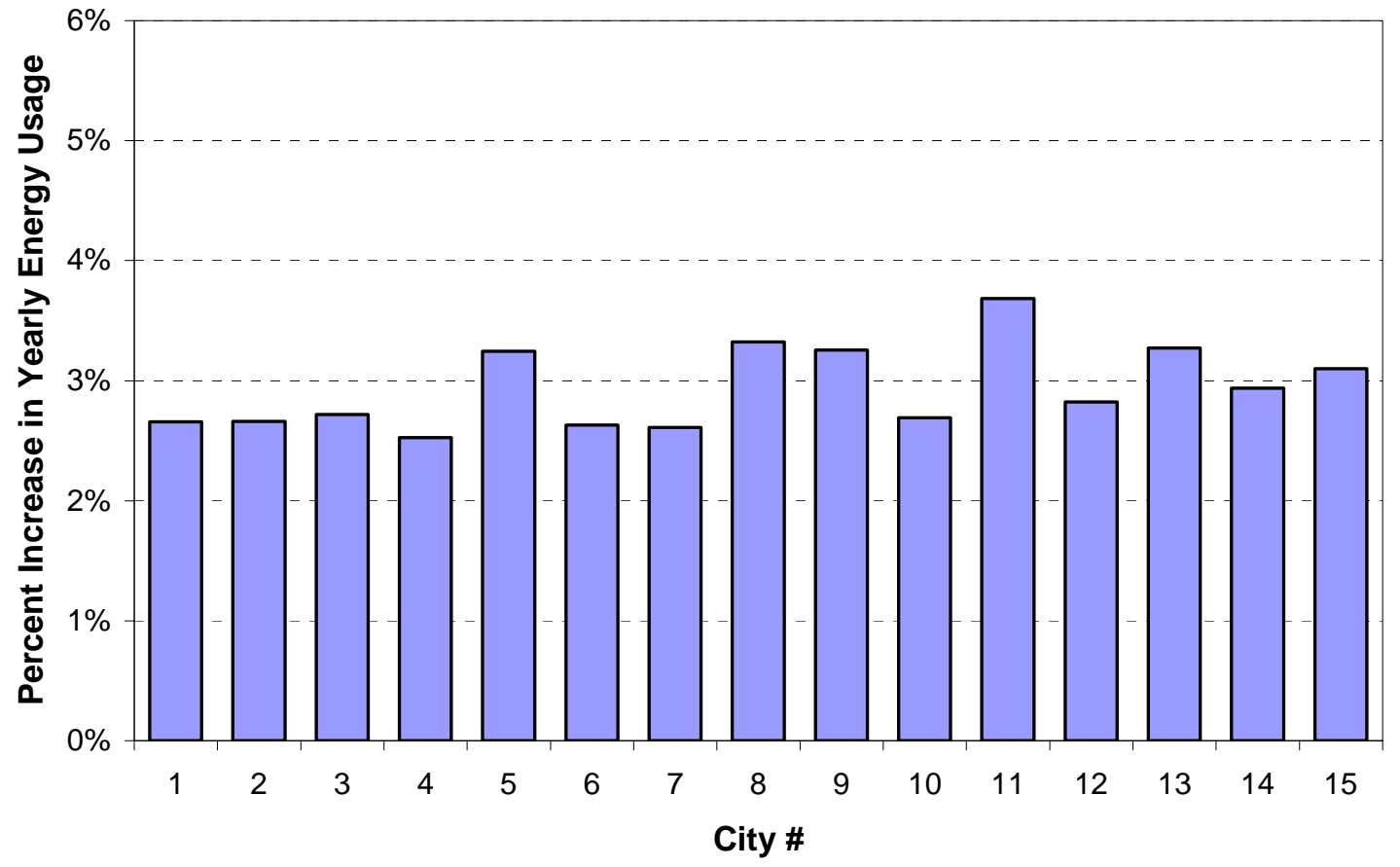

Figure 4.28 - Percent increase in yearly energy usage with the inclusion of solar radiation for baseline building without windows.

Comparing Figure 4.26 and Figure 4.28, clearly the window was the main mechanism for the increased cooling load resulting from the solar radiation. The increase dropped an order of magnitude from an average increase of $29 \%$ to $2.9 \%$ without the windows. The beam radiation that entered directly through the windows appears to be the source of the increased cooling load, as opposed to the radiation absorbed by the building walls. In real buildings windows are often shaded either from the exterior or through internal shading devices in an attempt to reduce large gains from direct beam radiation. It would appear that neglecting solar radiation in these cases is a far less significant assumption than a building with no shading devices. The TCTB model is therefore better suited to calculate the building energy usage for buildings with 


\section{Report No. DE-FG02-03ER63694-F1}

significant shading of the windows, either from blinds or other shading devices, or through exterior shading such as trees or other buildings. 


\section{Report No. DE-FG02-03ER63694-F1}

\section{Conclusions}

The lumped parameter, TCTB model, presented in this study provided us with an accurate and quick method to simulate yearly building energy usage. From the comparison with TRNSYS we were able to determine that, despite neglecting transient effects, for a yearly simulation the TCTB model predicted energy usage within approximately $0.6 \%$ of the transient TRNSYS solution (without solar radiation), while executing in one six-hundredth of the time. The effect of solar radiation was neglected, and proved to be a minor assumption (approximately 3\% energy difference over a yearly simulation) when the direct beam radiation through the windows was removed. The TCTB model's major advantage over the transient TRNSYS simulations is its significantly faster execution time. The TCTB model was able to run a yearly simulation for the two control strategies (HIYW and OSFA) for all 15 cities in approximately thirty seconds. Running the equivalent TRNSYS simulations would require 30 independent simulations each requiring between ten and fifteen minutes of computation time. This would make running large-scale optimization or parametric studies using a TRNSYS nearly impossible in a reasonable time frame. The lumped parameter approach was ideal for the exploration of energy costs in buildings equipped with distributed environmental control systems.

The cost associated with the adoption of a HIYW approach to environmental control is dependent on a number of factors including: climate region, interior partition thermal resistance, building inhabitants preferences, and to a lesser extent building shape (excluding solar heat gain, which depends strong on building shape and orientation).

At extreme temperatures, both hot and cold, the heat transfer associated with the small internal temperature deviations between offices in a building becomes insignificant compared 
to the heat transfer with the outside ambient air. The opposite is true for mild outdoor temperatures. At these temperatures the building energy usage is lowest. Internal heat transfer between offices at different temperatures becomes more significant and has an appreciable effect on building energy use. This is further impacted by the presence of an economizer. In the HIYW case, the economizer is unable to meet the cooling load of the building at outdoor temperatures at which it can in the OSFA case.

The climate in which a building is located is a major factor in the cost associated with HIYW compared to $O S F A$. Cities, and their corresponding climate zones, with a significant portion of the year having extreme temperatures (Miami, Phoenix, Fairbanks, etc.) show the lowest increase in yearly energy usage when switching to HIYW control (between 2-6\%). It is in these cities that adoption of personal environmental control systems, without optimization, appears to be the best choice. Cities with a moderate climate, such as San Francisco, show the largest increase in yearly energy usage, ranging from $10-20 \%$ and would be the least practical to implement personal control. The majority of the US population lives in climates that fall between these two extremes and would likely see a 5-10\% increase with the adoption of personal control.

The thermal resistance of the interior partition of a building also greatly affects the energy cost associated with HIYW. Thermal resistances ranging from partial height cubicles to full office walls, appear to provide sufficient resistance to internal heat transfer to keep building energy usage within reasonable limits. However, open workspaces, where there are no physical boundaries between personal zones, may create significant internal heat transfer that translates to increased energy costs. However, the well-mixed model assumption is a poor representation of the physical problem and more research must be done to determine true energy transfer characteristics of such open office spaces. 
The impact of the randomness of the personal preferences of building occupants varies depending on the climatic location of the building. In most cities the random populations fell within approximately $3-10 \%$ increase for common interior walls and a $4-15 \%$ increase for partial height partitions. For a building in San Francisco, or a comparable climate, the impact of the building occupants' desired temperatures was significantly larger. The HIYW energy usage premium ranged from $10-35 \%$ for common interior walls and $23-78 \%$ for partial height partitions depending on the specific occupants within the building. Regardless of building occupants, in every case, except two populations, HIYW used more energy over the course of a year than OSFA. The only two instances where HIYW saved energy were in extreme climates (Miami and Fairbanks) for an unusually fortunate arrangement of building inhabitants. In both these cases the yearly savings was less than $1 \%$. For all other arrangements and in every other city HIYW resulted in an increase in energy usage, in some cases as high as $78 \%$ more than OSFA.

Building shape appears to have a very small influence on the relative cost between HIYW and OSFA. Most office buildings tend to be rectangular with at least some core offices or cubicles. Small changes in the aspect ratio of the building have a negligible effect on HIYW costs. Even drastic changes, such as a corridor of only exterior offices, actually slightly lowered the energy penalty related to the adoption HIYW.

Allowing building occupants' to receive slightly different temperatures than their desired perfect temperature, energy savings can be realized. No longer will a personalized approach to environmental control result in increased yearly energy costs. A small $1{ }^{\circ} \mathrm{C}$ deviation from the occupants' neutral temperature can help create an energy savings from OSFA in nearly every city, while still catering to the occupants' desires. This promising result has been explored in other studies by the author and others. It was shown that using advanced optimization techniques to 


\section{Report No. DE-FG02-03ER63694-F1}

intelligently deviate occupants' temperature set points, optimized HIYW can result in an energy savings and a better overall thermal satisfaction than OSFA.

The increase in building energy usage is small compared to potential productivity gains. The increase in energy associated with HIYW compared to OSFA in the worst case scenario was approximately $78 \%$. This corresponded to the worst of 100 populations in a partial height partitioned building in San Francisco. If we assume entire building utility cost is comprised of HVAC energy, based on the average commercial building costs, a business would only need to realize a $0.7 \%$ increase in productivity to effectively counteract the increased cost. In most cases, however, the increase in yearly energy usage was closer to $8-10 \%$. To pay for the increase in energy cost, an average business would need only to increase productivity by less than $0.1 \%$. With this in mind, the potential productivity benefits far outweigh the small increase in overall business operating costs that would result from the implementation of distributed environmental control systems.

The TCTB model can be improved in the future to include even more physics, especially solar radiation and humidity effects. The temperature bin approach could be expanded to some form of three dimensional weather bins to include humidity as well as dry bulb temperature. The effect of solar radiation on building energy use is large when windows are involved and would be beneficial to include in the TCTB model, either directly or through some correlation with the outdoor temperature. If it is possible to correlate the solar gain on a vertical surface with different azimuths, a correction factor could be added to the dry bulb temperature. A new effective temperature could then be used for the outer surfaces and windows. Shading factors could then be calculated and a better approximation for windows exposed to solar gains could be made. More work must to be done in an effort to understand and quantify the energy flux 


\section{Report No. DE-FG02-03ER63694-F1}

between partial height partitions. Field testing and experimental office settings may provide some of the answers and help to further validate the computed results. 
Report No. DE-FG02-03ER63694-F1

\section{Appendix}

\subsection{Temperature Bin Data}

Table 6.1 shows the complete temperature bin data extracted from the TMY2 files for the full year (8760 hours). The bins are $3^{\circ} \mathrm{C}$ intervals with centers as shown. 
Report No. DE-FG02-03ER63694-F1

Table 6.1 - Yearly temperature bin data for 15 representative U.S. cities (number of hours per year)

\begin{tabular}{|c|c|c|c|c|c|c|c|c|c|c|c|c|c|c|c|c|c|c|c|c|c|c|c|c|}
\hline \multirow[b]{2}{*}{ City } & \multicolumn{24}{|c|}{ Temperature Bin Center $\left({ }^{\circ} \mathrm{C}\right)$} \\
\hline & 46 & 43 & 40 & 37 & 34 & 31 & 28 & 25 & 22 & 19 & 16 & 13 & 10 & 7 & 4 & 1 & -2 & -5 & -8 & -11 & -14 & -17 & -20 & 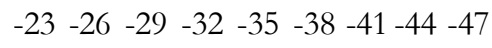 \\
\hline Miami, FL & & & & & 9 & 715 & 2032 & 2926 & 1766 & 665 & 320 & 220 & 81 & 18 & 8 & & & & & & & & & \\
\hline Houston, TX & & & & 3 & 250 & 608 & 1050 & 1622 & 1500 & 989 & 641 & 593 & 473 & 396 & 312 & 213 & 76 & 25 & 8 & 1 & & & & \\
\hline Phoenix, AZ & 14 & 102 & 297 & 494 & 644 & 808 & 959 & 866 & 780 & 815 & 808 & 845 & 657 & 421 & 194 & 48 & 8 & & & & & & & \\
\hline Memphis, TN & & & & 3 & 225 & 492 & 741 & 1115 & 1073 & 870 & 801 & 696 & 717 & 679 & 516 & 384 & 308 & 98 & 33 & 9 & & & & \\
\hline El Paso, TX & & & 3 & 117 & 389 & 552 & 718 & 1014 & 1008 & 884 & 766 & 859 & 758 & 627 & 504 & 325 & 173 & 54 & 9 & & & & & \\
\hline San Francisco, CA & & & & & 6 & 14 & 29 & 72 & 331 & 848 & 1436 & 2892 & 2144 & 769 & 184 & 35 & & & & & & & & \\
\hline Baltimore, MD & & & & 1 & 97 & 202 & 430 & 689 & 993 & 910 & 744 & 705 & 615 & 788 & 857 & 804 & 421 & 267 & 150 & 69 & 17 & 1 & & \\
\hline Albuquerque, NM & & & & 11 & 117 & 308 & 536 & 624 & 812 & 917 & 811 & 763 & 752 & 763 & 723 & 729 & 564 & 252 & 69 & 9 & & & & \\
\hline Salem, OR & & & & & 34 & 76 & 168 & 266 & 423 & 594 & 938 & 1312 & 1449 & 1505 & 1129 & 578 & 205 & 65 & 18 & & & & & \\
\hline Chicago, IL & & & & & 34 & 141 & 302 & 638 & 756 & 829 & 680 & 635 & 649 & 683 & 722 & 881 & 712 & $459 ?$ & 309 & 138 & 81 & 59 & 42 & 10 \\
\hline Boise, ID & & & 2 & 36 & 124 & 221 & 299 & 391 & 513 & 654 & 640 & 813 & 948 & 895 & 969 & 1069 & 608 & 272 & 168 & 71 & 63 & 4 & & \\
\hline Burlington, VT & & & & 4 & 14 & 40 & 185 & 360 & 658 & 832 & 746 & 786 & 725 & 615 & 655 & 733 & 548 & 535 & $468 ?$ & 350 & 195 & 146 & 107 & 526 \\
\hline
\end{tabular}


Report No. DE-FG02-03ER63694-F1

Helena, MT

Duluth, MN

Fairbanks, AK $\begin{array}{lllllllllllllllllllllll}1 & 44 & 100 & 198 & 276 & 404 & 558 & 757 & 793 & 814 & 868 & 949 & 910 & 647 & 458 & 302 & 147 & 122 & 157 & 99 & 89 & 56 & 11\end{array}$

$\begin{array}{llllllllllllllllllllll}13 & 82 & 203 & 330 & 513 & 630 & 777 & 874 & 734 & 646 & 663 & 746 & 544 & 473 & 417 & 332 & 248 & 215 & 144 & 112 & 58 & 6\end{array}$

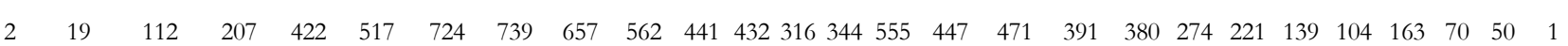




\section{Report No. DE-FG02-03ER63694-F1}

\subsection{Thermodynamic Properties}

The pertinent properties of air are a weak function of temperature so it was decided to assume them to be constant throughout all calculations. The values used in both the TCTB and TRNSYS model are listed below in Table 6.2.

Table 6.2 - Thermodynamic properties of air used in simulations

\begin{tabular}{ll}
\hline Property & Value \\
\hline Density of air & $1.204 \mathrm{~kg} / \mathrm{m}^{3}$ \\
Specific heat of air & $1012 \mathrm{~J} / \mathrm{kgK}$ \\
\hline
\end{tabular}

\subsection{HIYW Temperatures}

Figure 6.1 shows the frequently used HIYW distribution of neutral temperatures. Both the numerical values and the location were chosen at random. The temperature values come from the population distribution described in Section 2.9.1. Table 6.3 lists the numerical value of the set points of the same distribution within the building. 


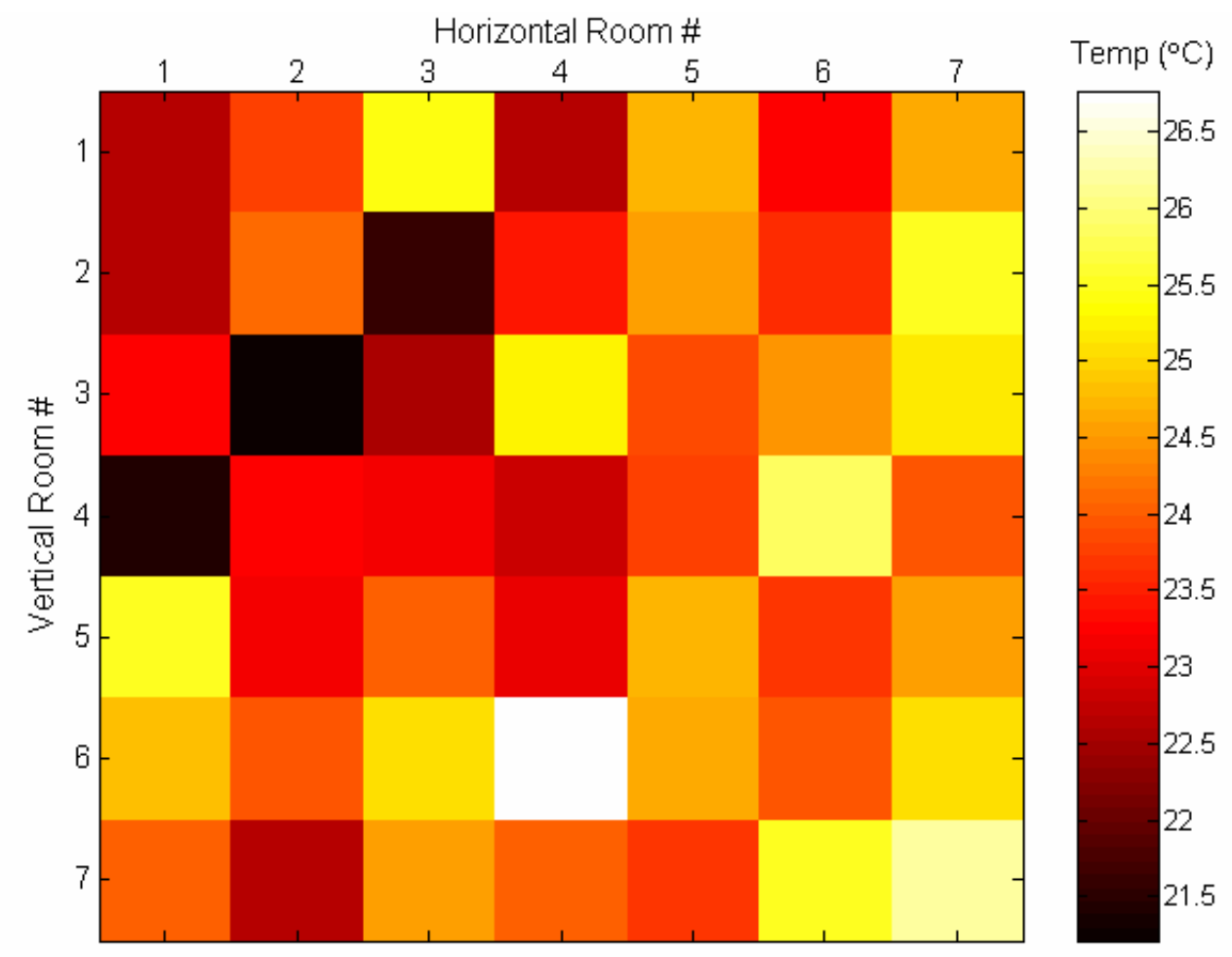

Figure 6.1 - HIYW neutral temperature (thermostat set point) random room assignment distribution within building

Table 6.3 - HIYW neutral temperatures (thermostat set points) by assigned room (in ${ }^{\circ} \mathrm{C}$ )

\begin{tabular}{|l|l|l|l|l|l|l|}
\hline 22.63 & 22.68 & 23.24 & 21.46 & 25.47 & 24.80 & 24.07 \\
\hline 23.75 & 24.10 & 21.21 & 23.23 & 23.17 & 23.91 & 22.67 \\
\hline 25.43 & 21.60 & 22.52 & 23.15 & 24.01 & 25.07 & 24.58 \\
\hline 22.66 & 23.41 & 25.27 & 22.78 & 23.06 & 26.77 & 23.99 \\
\hline 24.76 & 24.55 & 23.86 & 23.78 & 24.70 & 24.63 & 23.67 \\
\hline 23.28 & 23.62 & 24.46 & 25.83 & 23.70 & 23.99 & 25.53 \\
\hline 24.66 & 25.48 & 25.13 & 23.95 & 24.58 & 25.10 & 26.24 \\
\hline
\end{tabular}


Report No. DE-FG02-03ER63694-F1

\subsection{TCTB MATLAB code}

Listed here is the TCTB model, written in MATLAB. The main loop contains four subroutines: Rcalc.m, Lcalc.m, COPcalcCity.m, CYCdegcalc.m. Each subroutine is listed under a separate heading.

\subsubsection{Main loop}

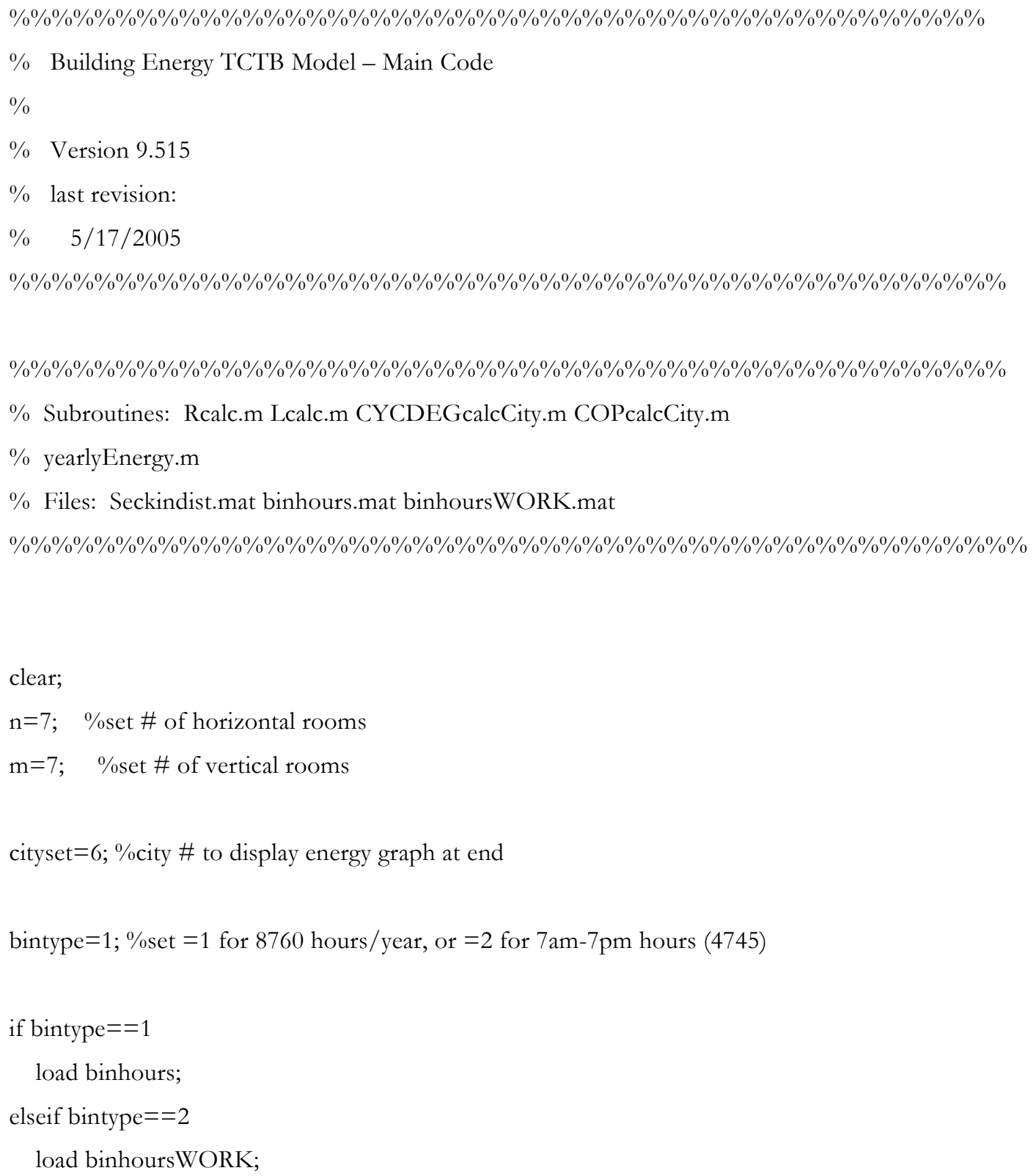




\section{Report No. DE-FG02-03ER63694-F1}

binhours=binhoursWORK;

else

disp('Error with bintype, using 4745 hours')

load binhoursWORK;

end

$\%$ controltype $=$ input('Thermostat control 1: 3TFA, 2: Single Thermo 3: TTFA :')

\%uncomment if other than 3TFA is desired

controltype $=1$;

if controltype $==1 \%$ set thermo control rooms for 3TFA in subroutine Lcalc

elseif controltype $==2$

$\mathrm{X}=25$;

else

$\mathrm{X}=25$;

$\mathrm{X} 2=1$;

end

$\mathrm{ci}=$ clock;

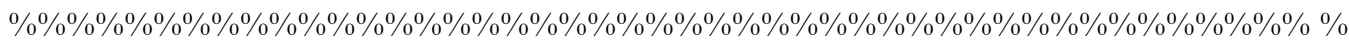

\%load constants, resistances, and ventilation

Rcalc;

$\% \% \% \% \% 0 \% 0 \% 0 \% 0 \% 0 \% 0 \% 0 \% 0 \% 0 \% 0 \% 0 \% 0 \% 0 \% 0 \% \% \% \% \% \% \%$

$\%$ initialize heating and cooling power vectors

PowerCOOL $=$ zeros $(1,67)$;

PowerHEAT $=$ zeros $(1,67)$;

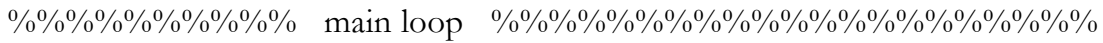

$\%$ counter $=1$ for conventional

$\%$ counter $=2$ for HIYW

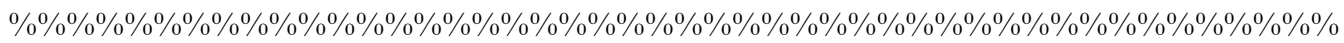

for counter $=1: 2$

if counter $==1$

$\mathrm{T}=24{ }^{*}$ ones $\left(1, \mathrm{n}^{*} \mathrm{~m}\right) ; \%$ uniform $24 \mathrm{C}$ setpoints

elseif counter $==2$ 


\section{Report No. DE-FG02-03ER63694-F1}

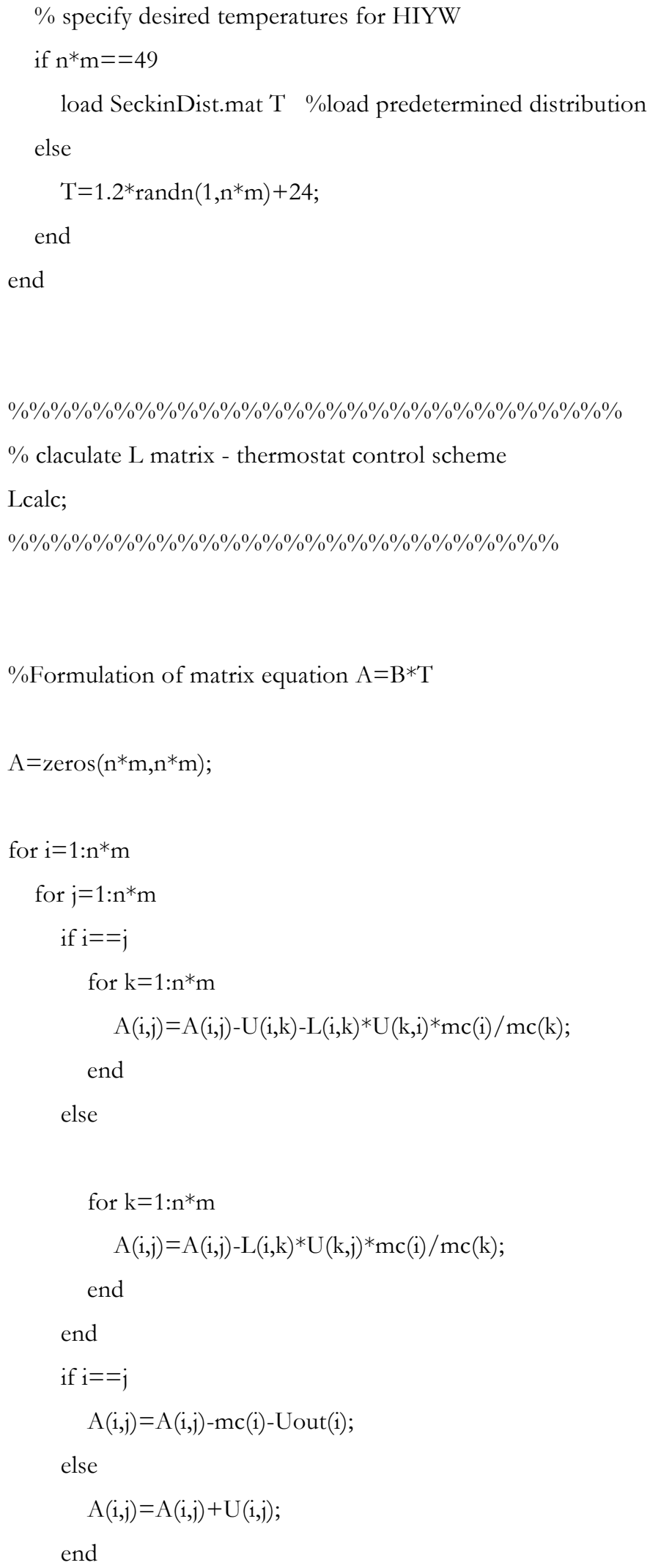




\begin{abstract}
end
end

COPbin=0;

PLCOP $=0$;
\end{abstract}

$\%$ Set thermostat setpoints

Tstar=T;

$\% \% \%$ City loop for 15 DOE cities

$\%$ See list for corresponding numbers/cities

for citynum $=1: 15$

$\% \% \%$ weather bin loop

for bin=1:length $(\mathrm{W})$

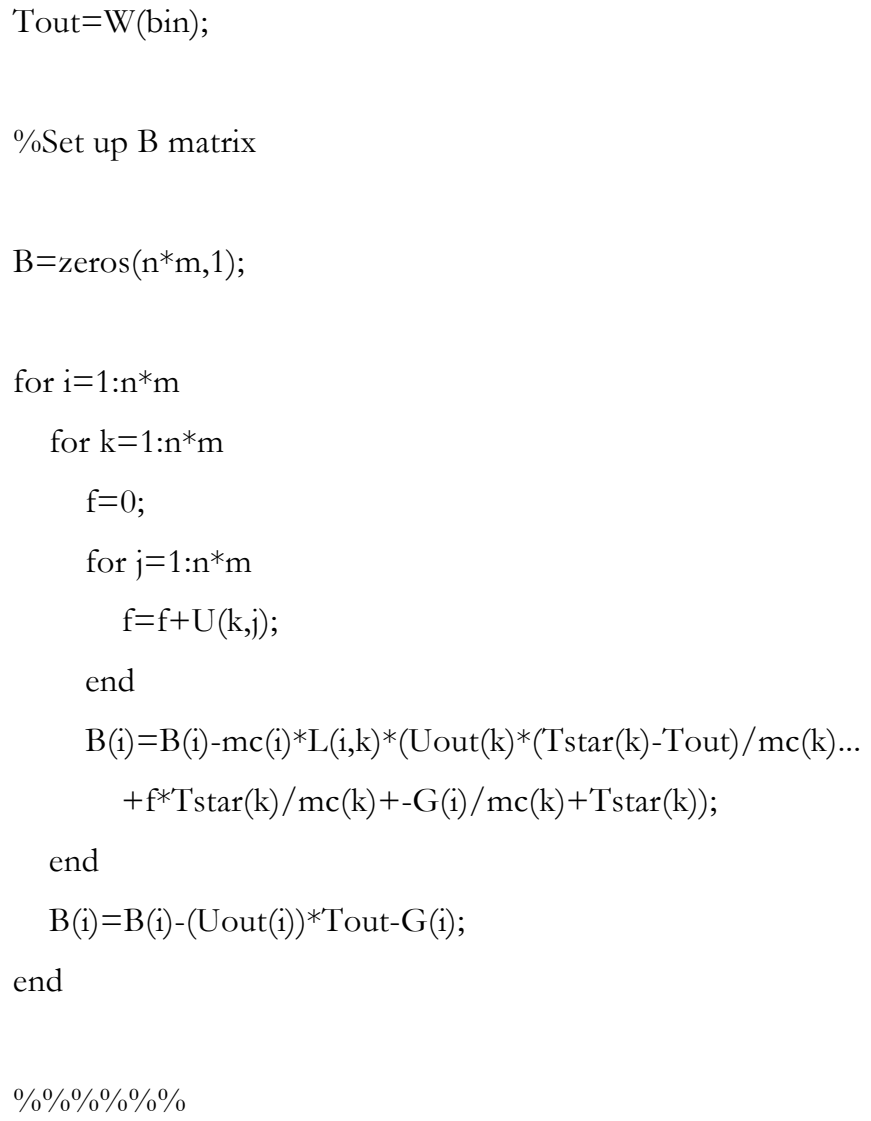




\section{Report No. DE-FG02-03ER63694-F1}

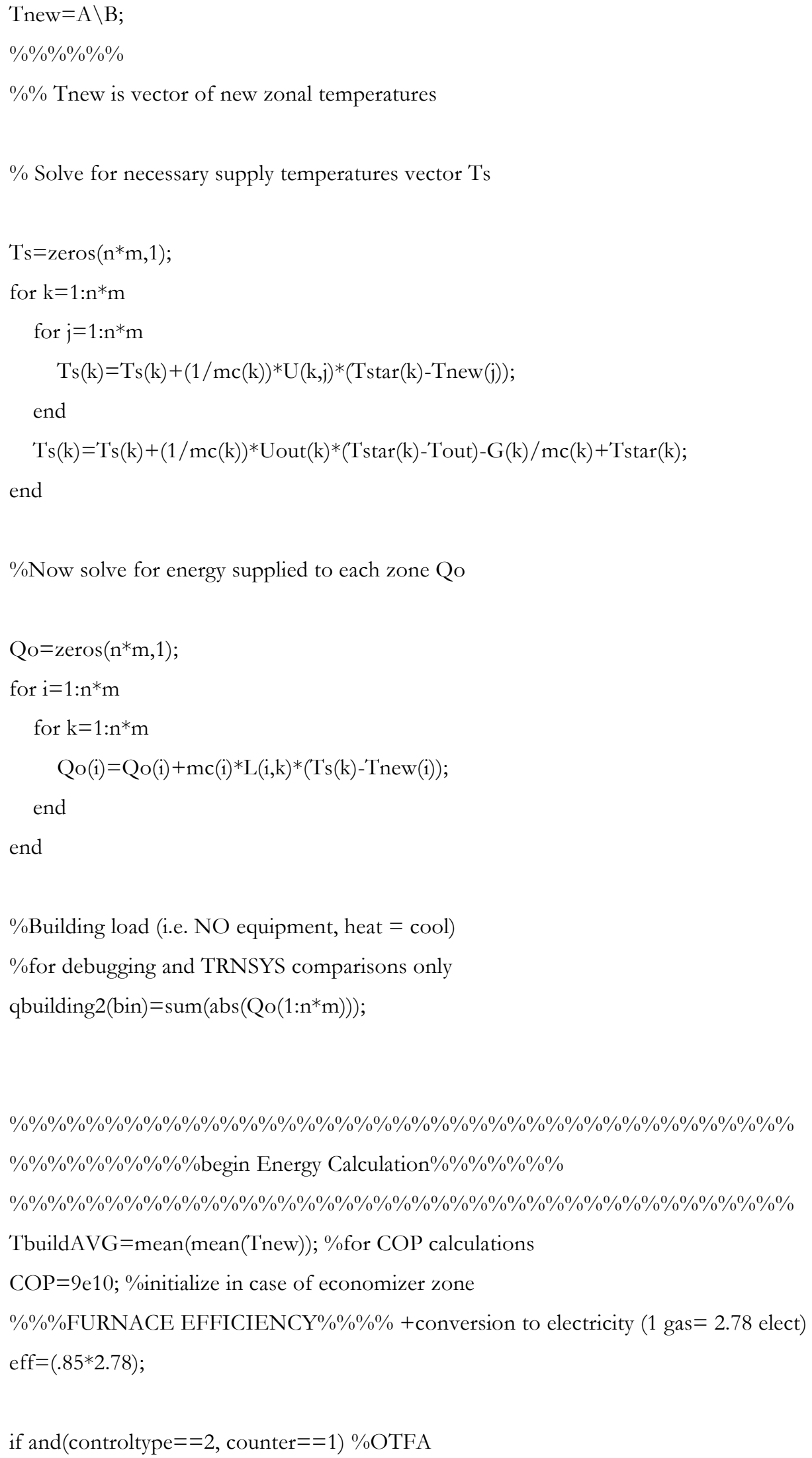




\section{Report No. DE-FG02-03ER63694-F1}

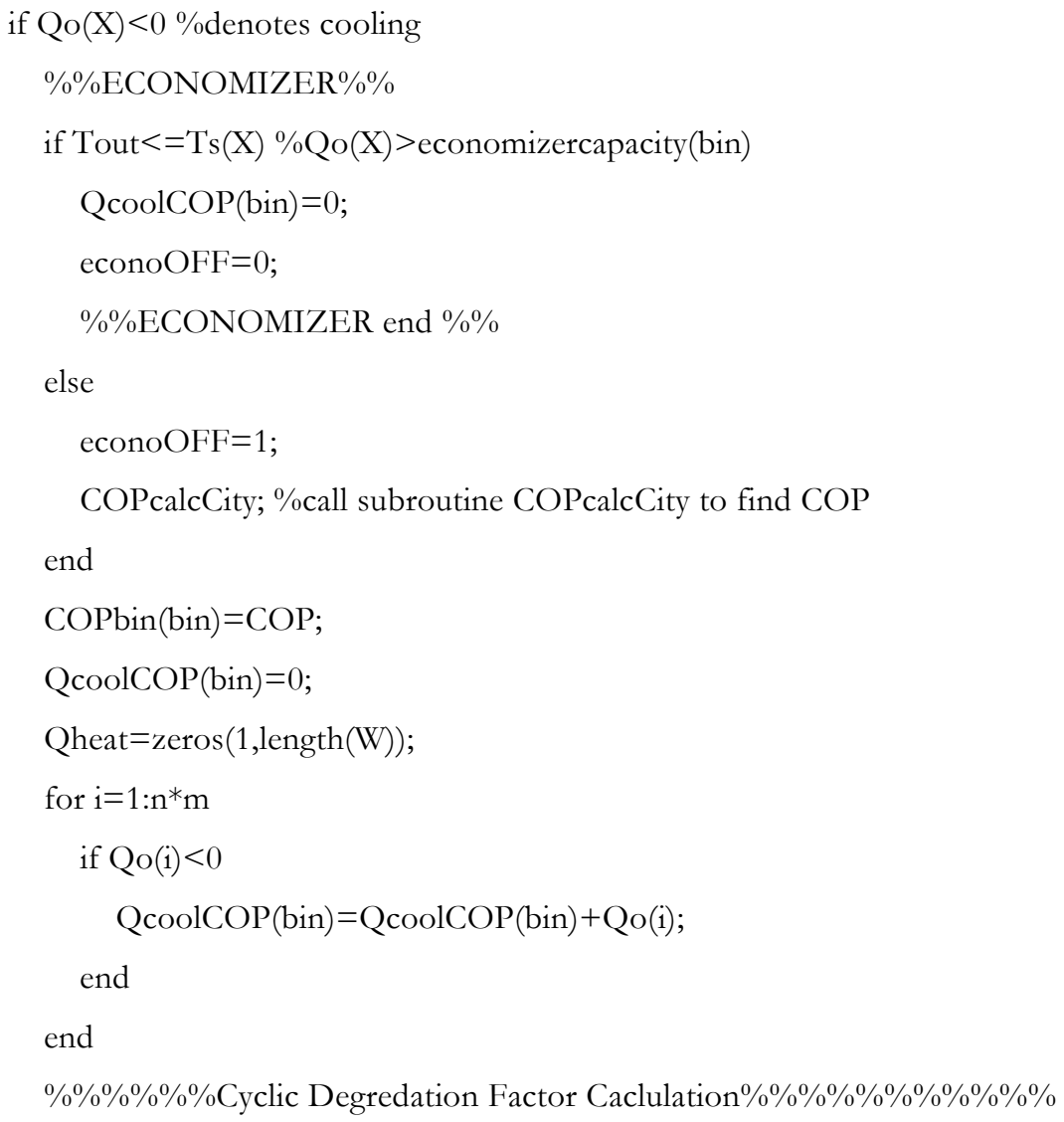




\section{Report No. DE-FG02-03ER63694-F1}

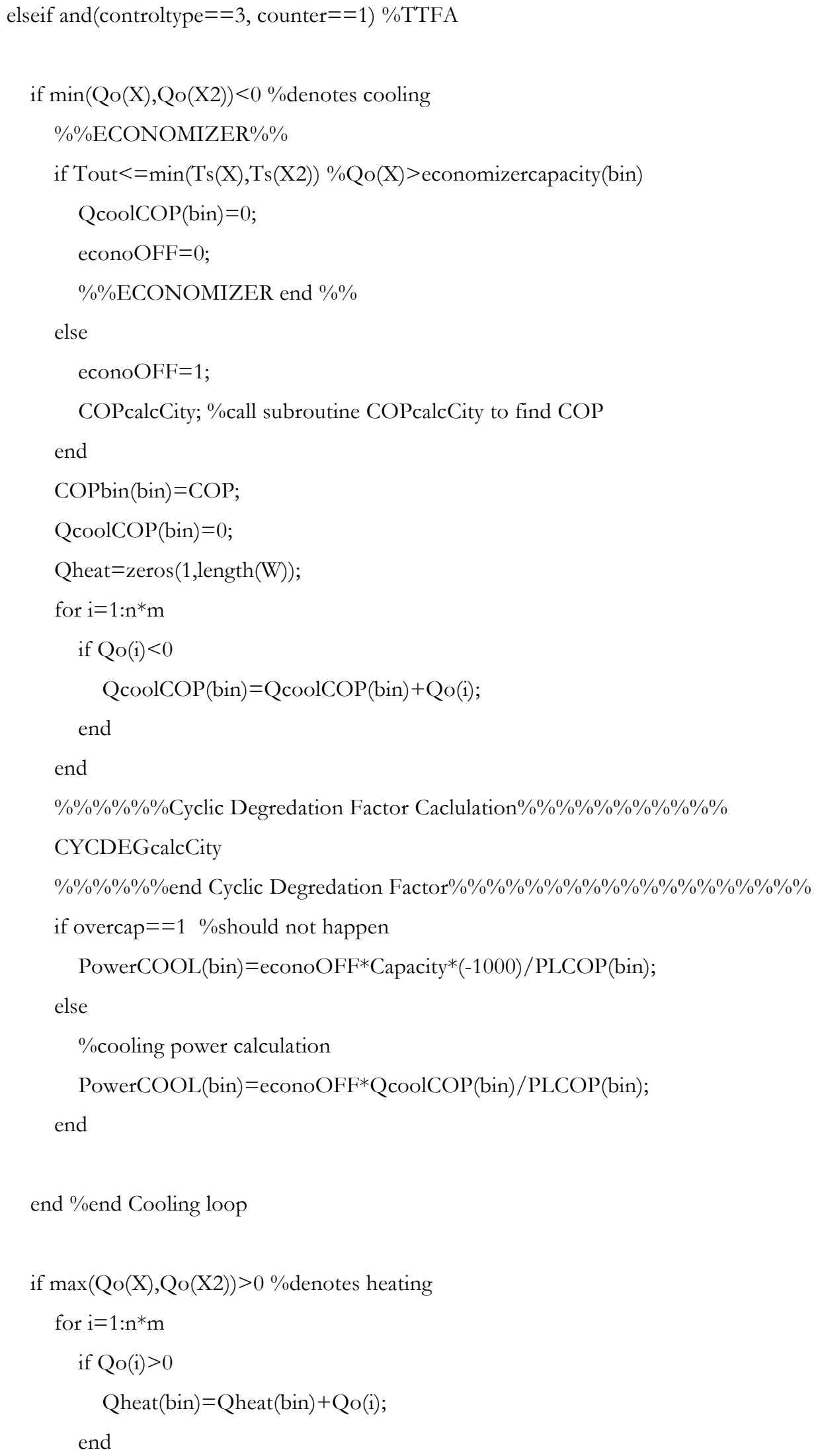




\section{Report No. DE-FG02-03ER63694-F1}

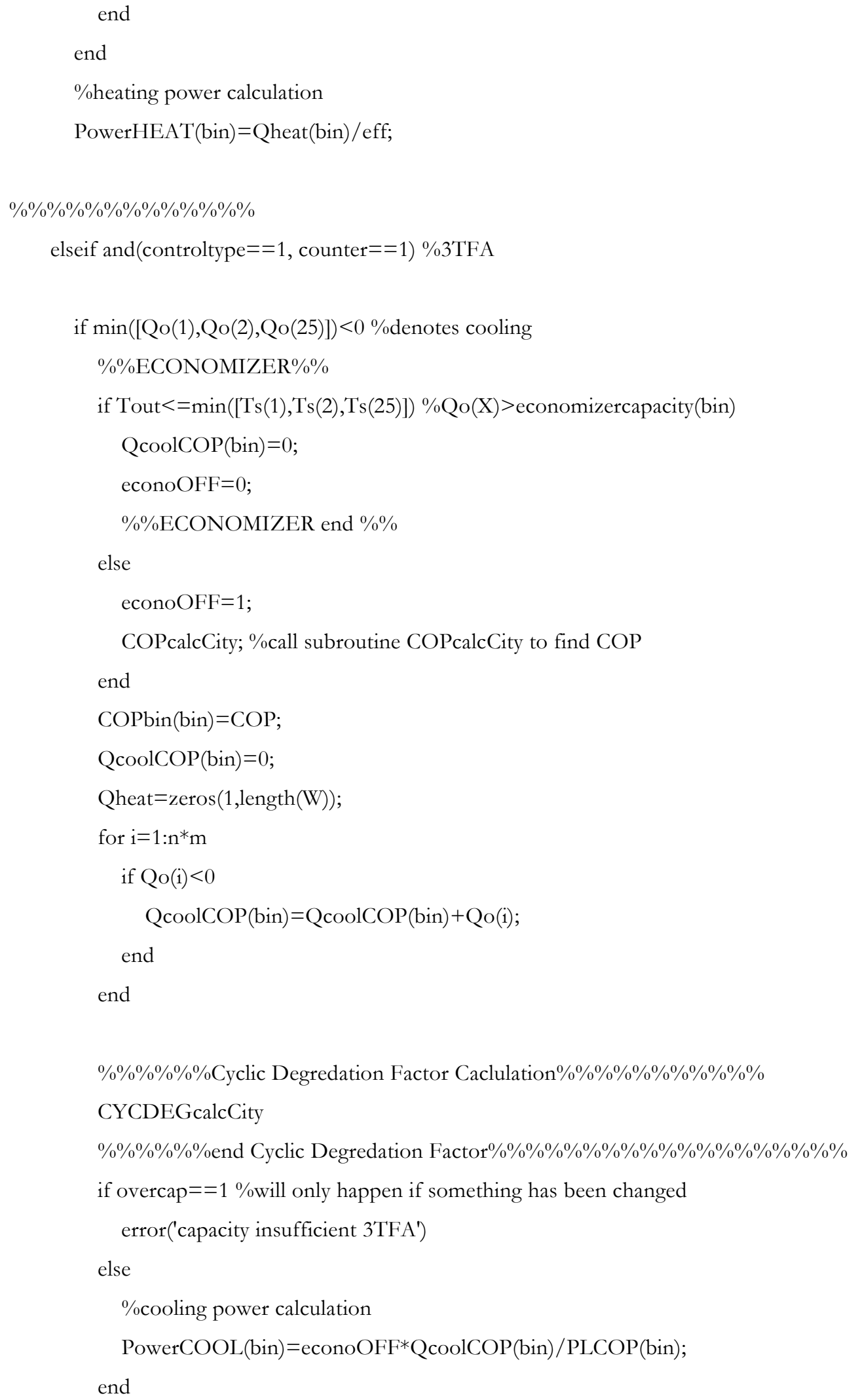


end \%end Cooling loop

if $\max ([\mathrm{Qo}(1), \mathrm{Qo}(2), \mathrm{Qo}(25)])>0 \%$ denotes heating

for $i=1: n^{*} m$

if $\mathrm{Qo}(\mathrm{i})>0$

Qheat $($ bin $)=Q$ heat $($ bin $)+Q o(i)$;

end

end

end

$\%$ heating power calculation

PowerHEAT $($ bin $)=$ Qheat $($ bin $) /$ eff;

$\% \% \% \% \% \% \% \% \% \% \% \% \% \% \% \% \% \% \% \% \% \% \%$

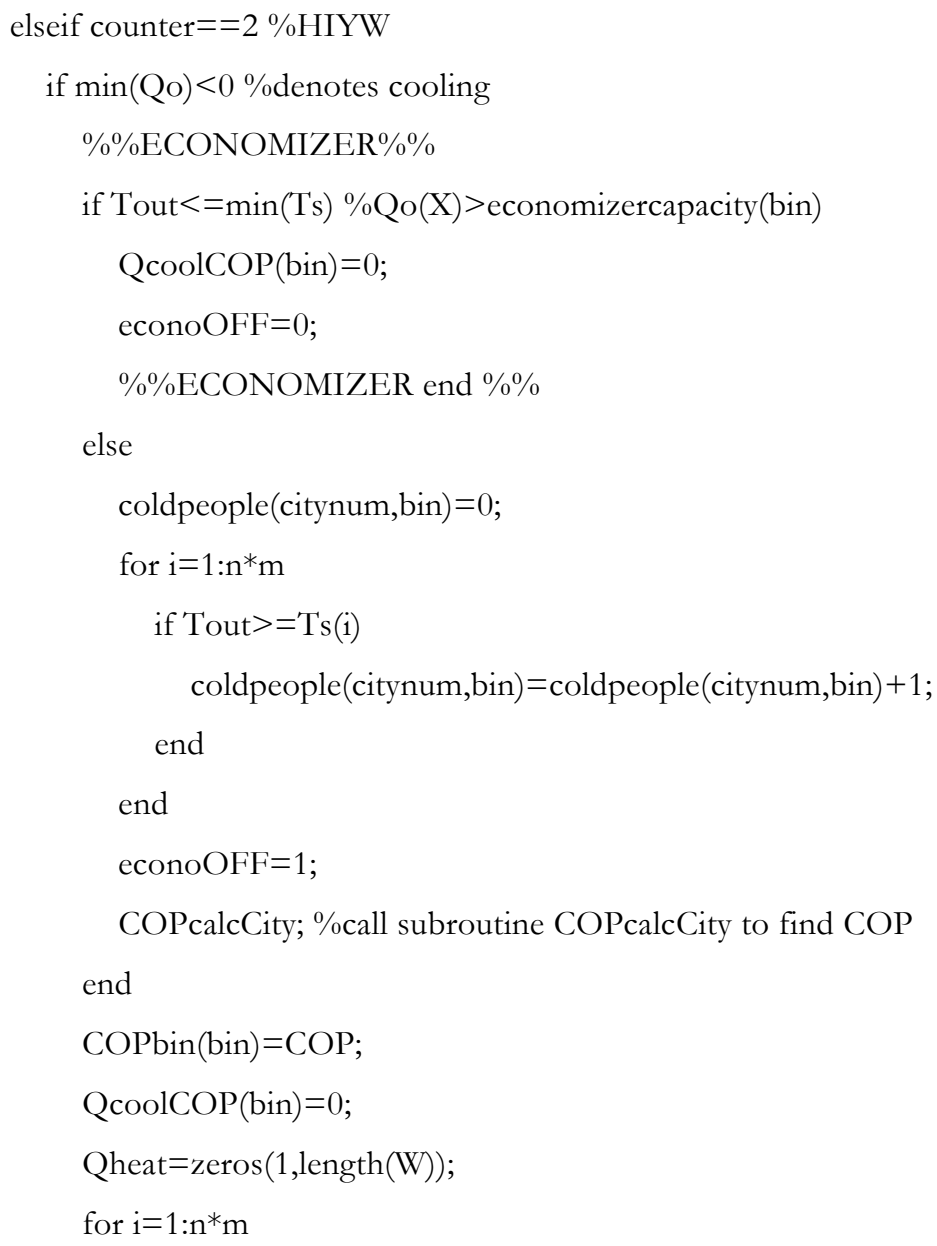




\section{Report No. DE-FG02-03ER63694-F1}

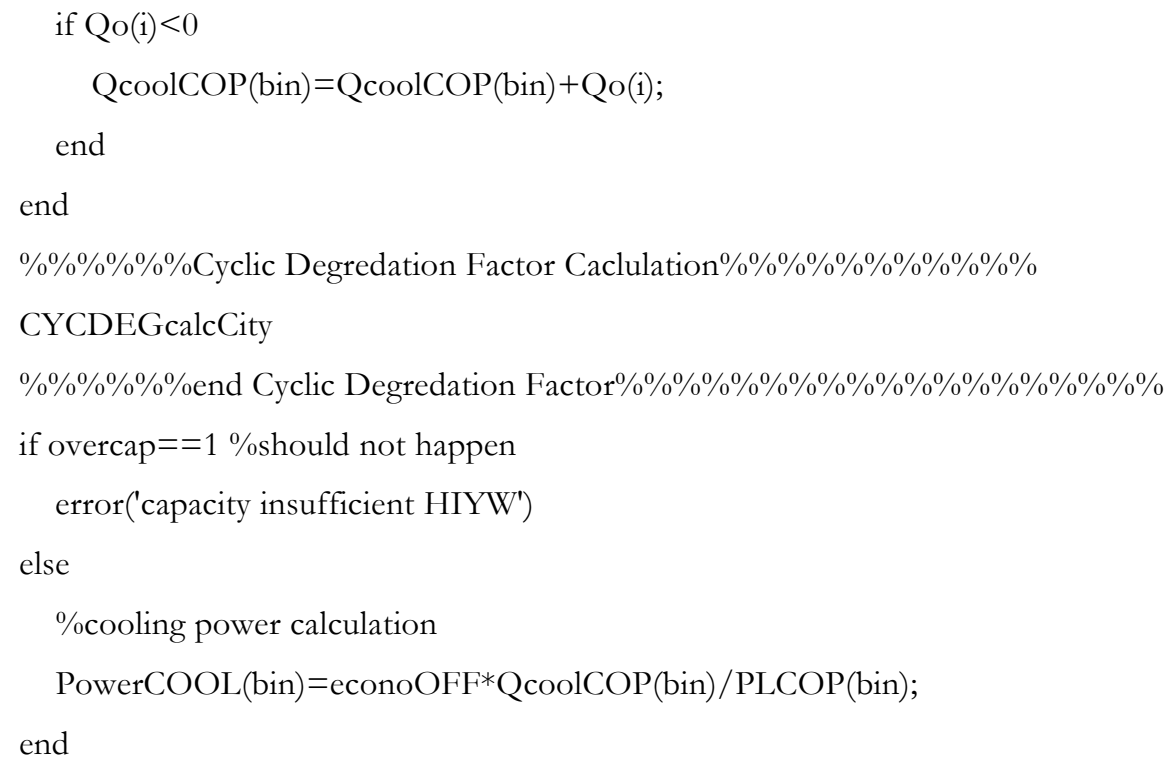




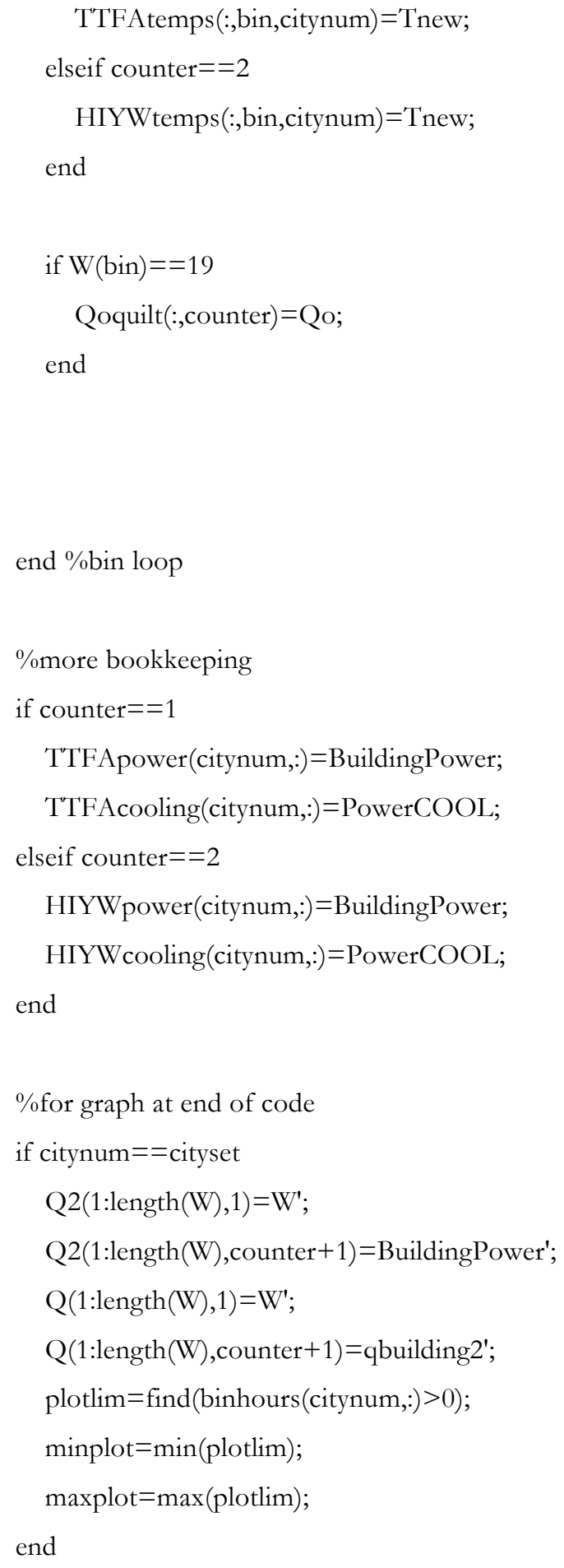




\section{Report No. DE-FG02-03ER63694-F1}

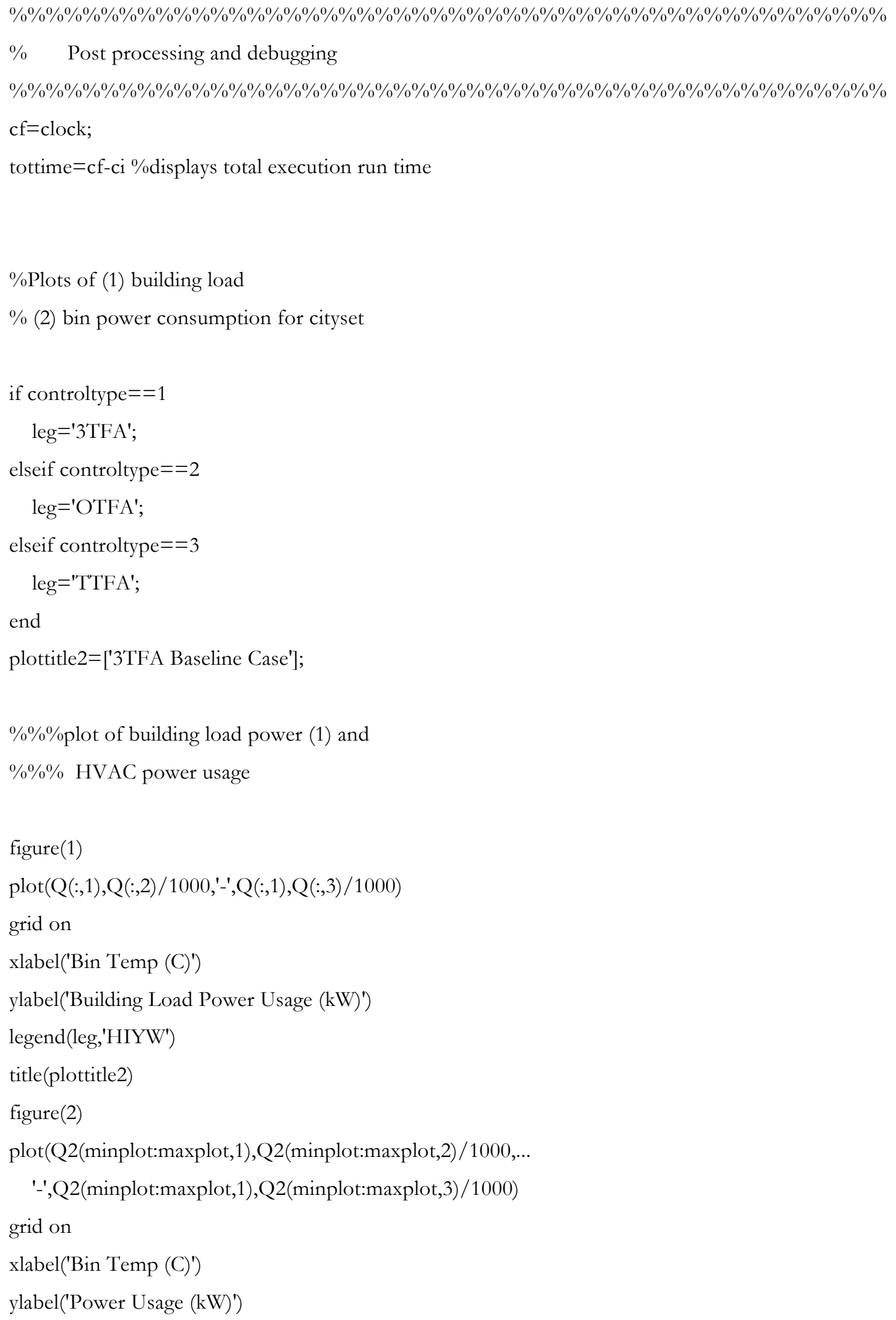




\section{Report No. DE-FG02-03ER63694-F1}

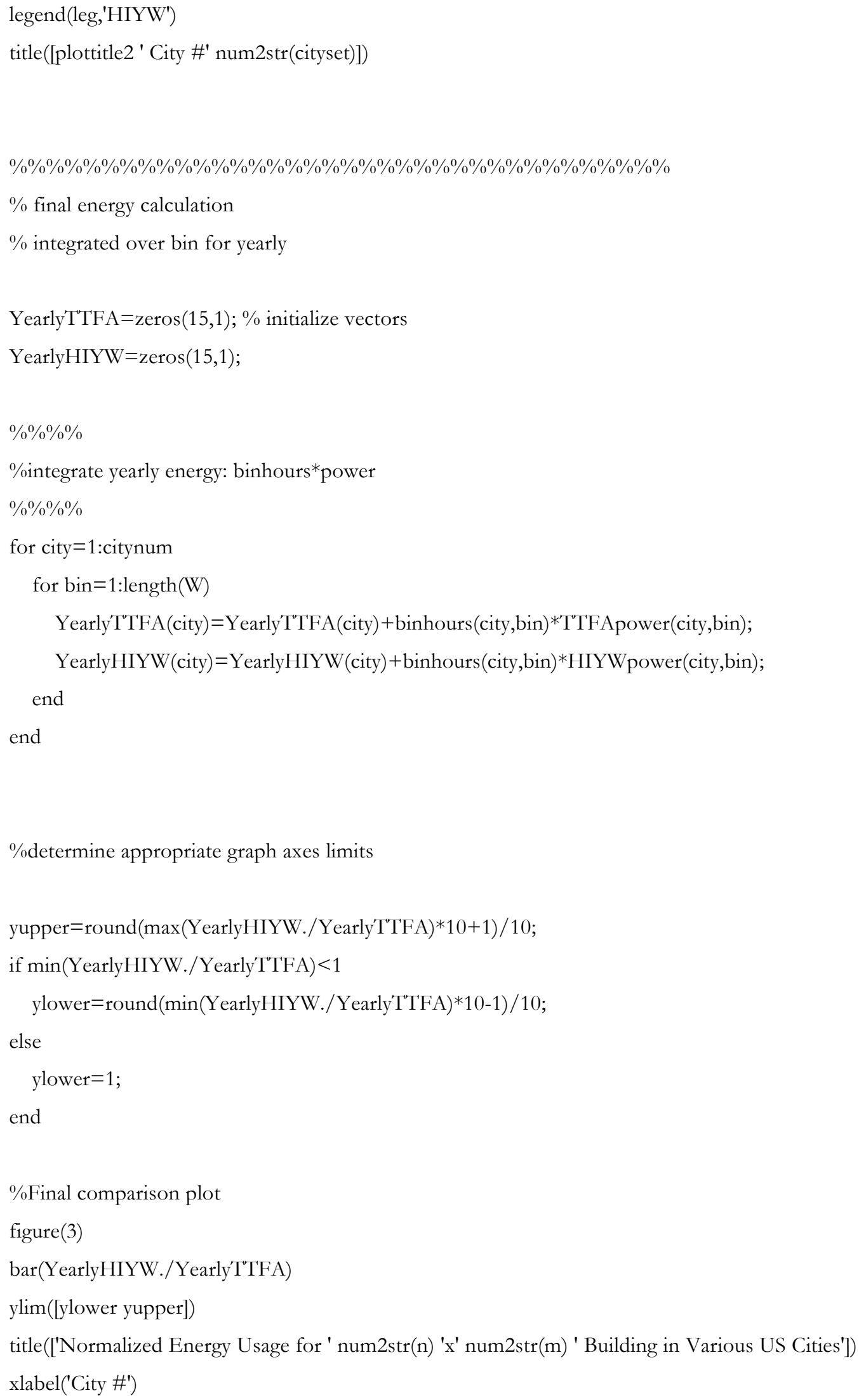


Report No. DE-FG02-03ER63694-F1

ylabel('Normalized Energy Usage (HIYW/3TFA)')

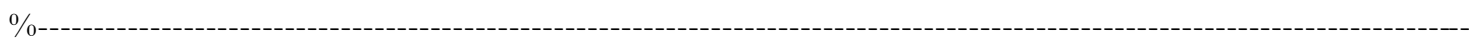




\section{Report No. DE-FG02-03ER63694-F1}

\subsubsection{Resistance Subroutine (Rcalc.m)}

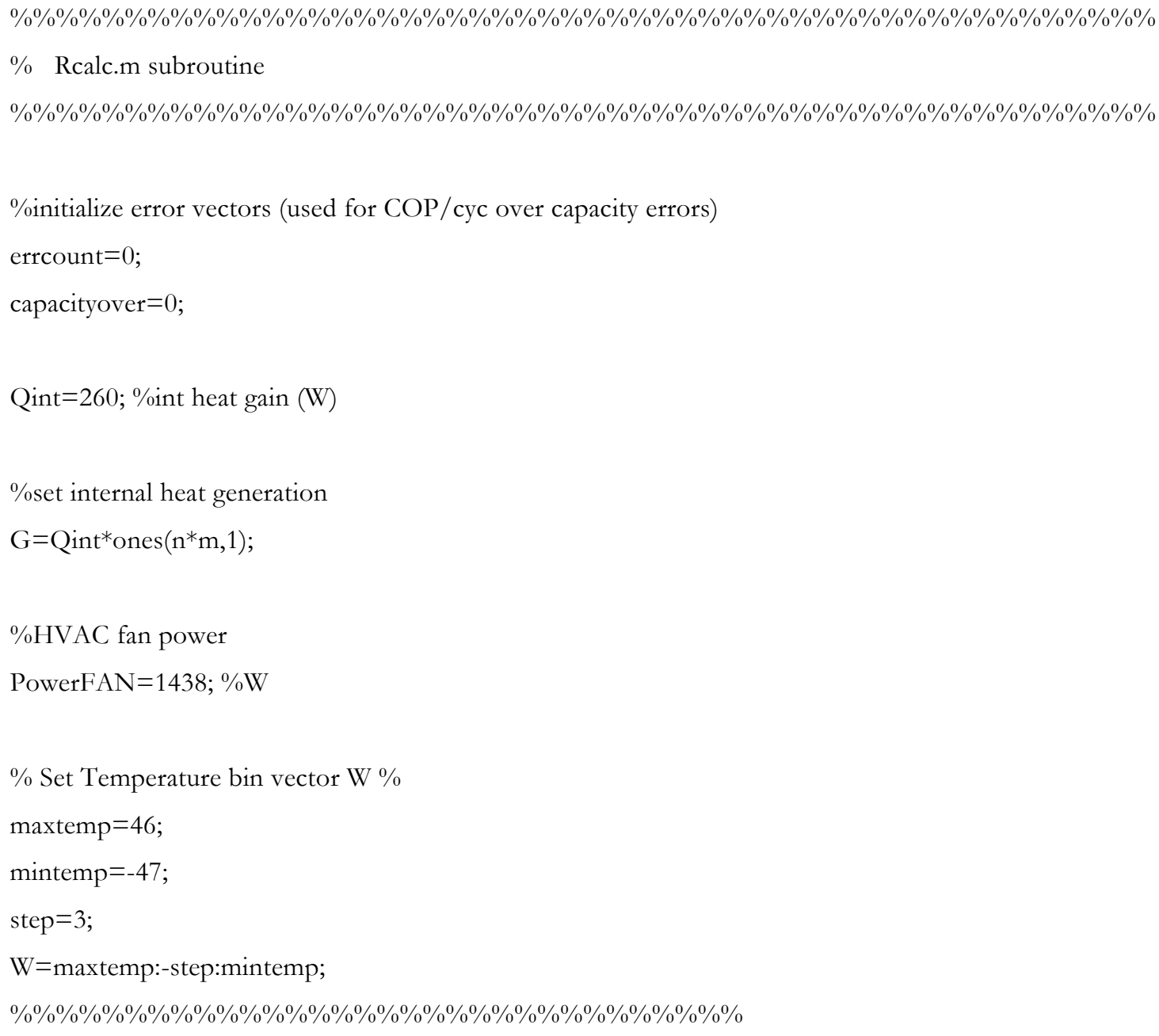




\section{Report No. DE-FG02-03ER63694-F1}

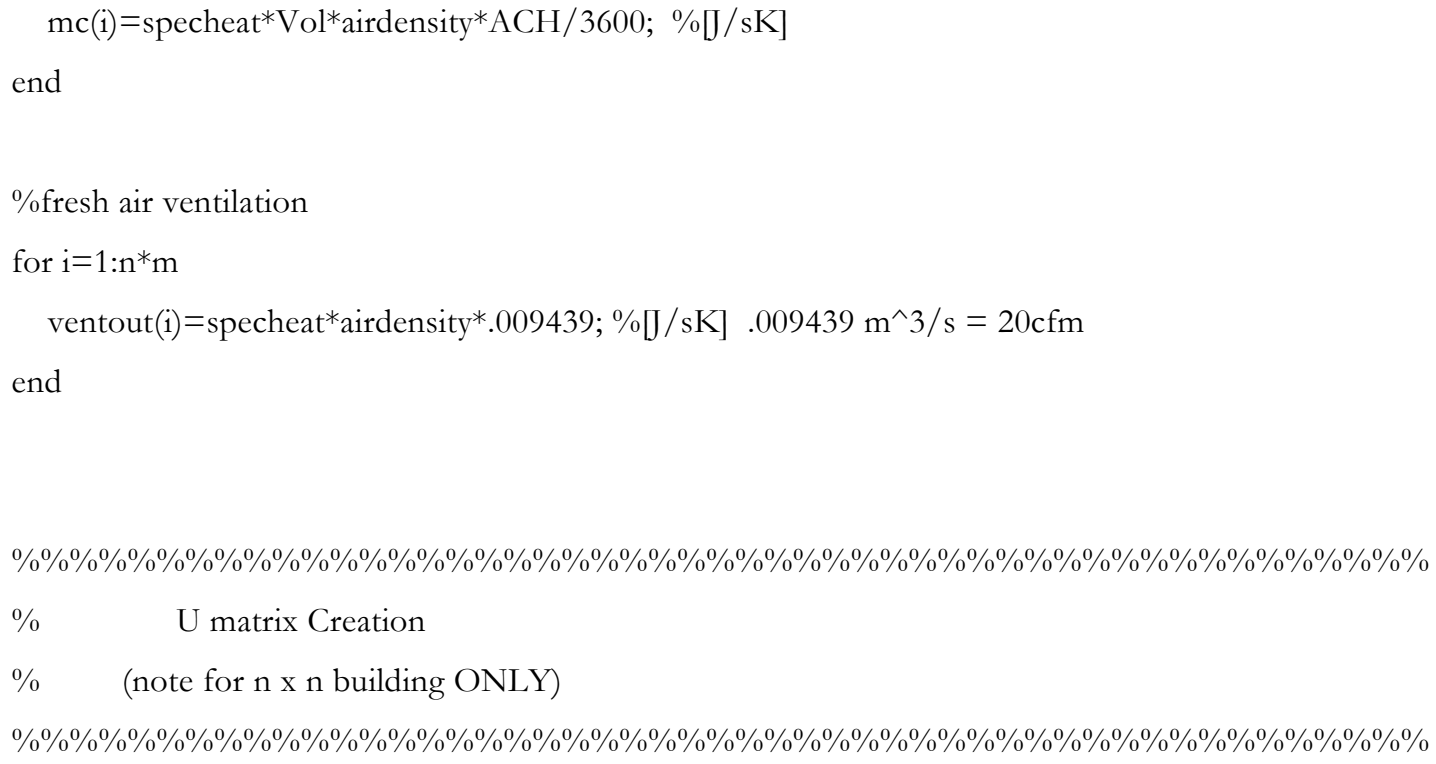




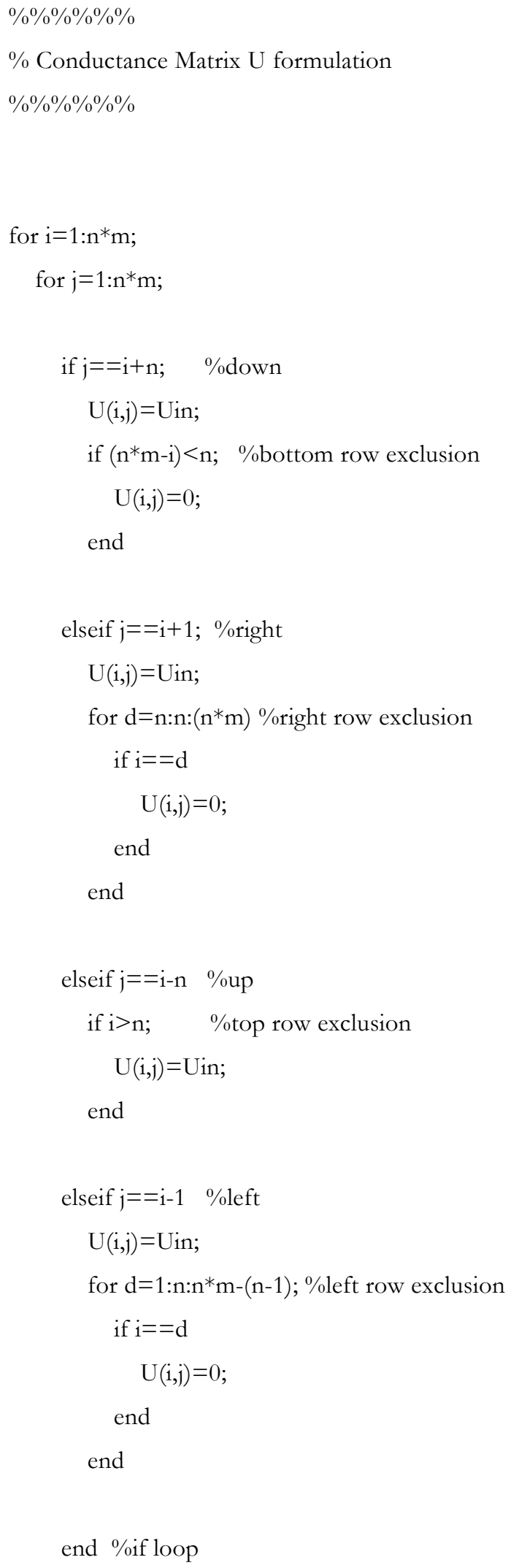




\section{Report No. DE-FG02-03ER63694-F1}

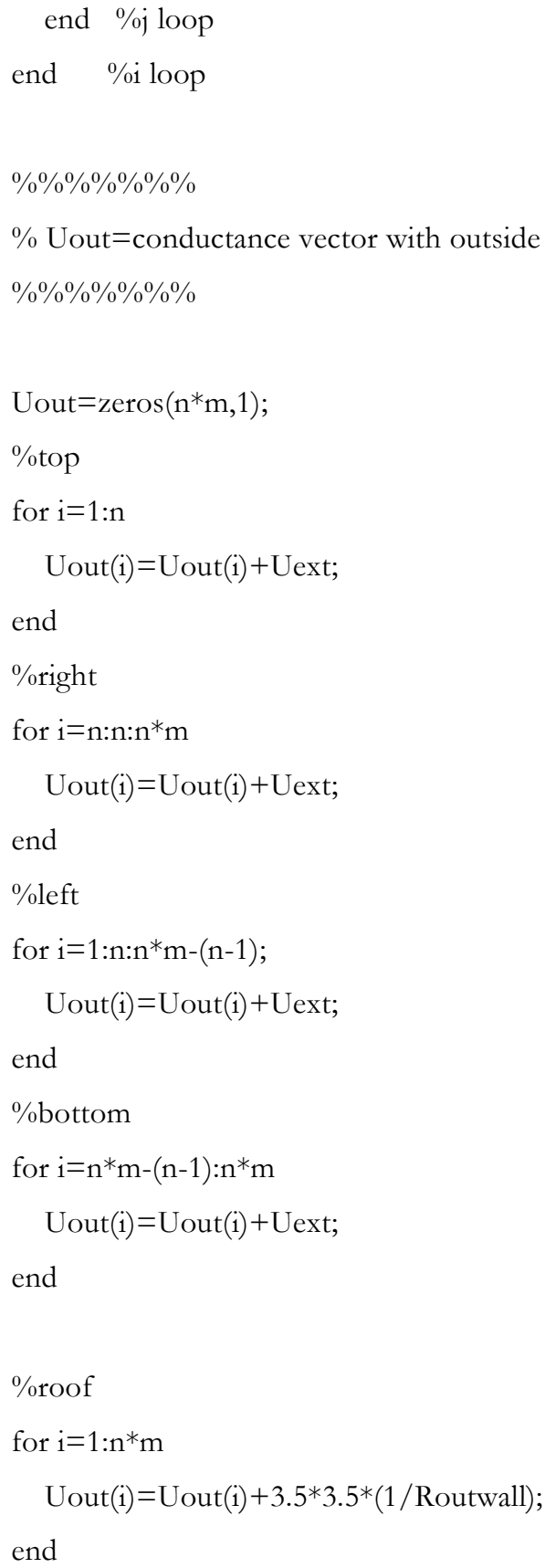


Report No. DE-FG02-03ER63694-F1

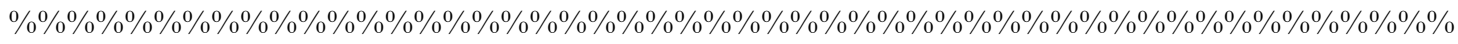

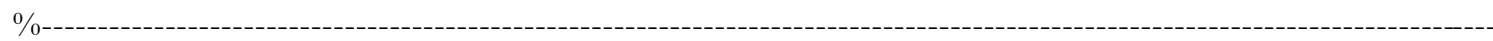




\section{Report No. DE-FG02-03ER63694-F1}

\subsubsection{Thermostat Control Subroutine (Lcalc.m)}

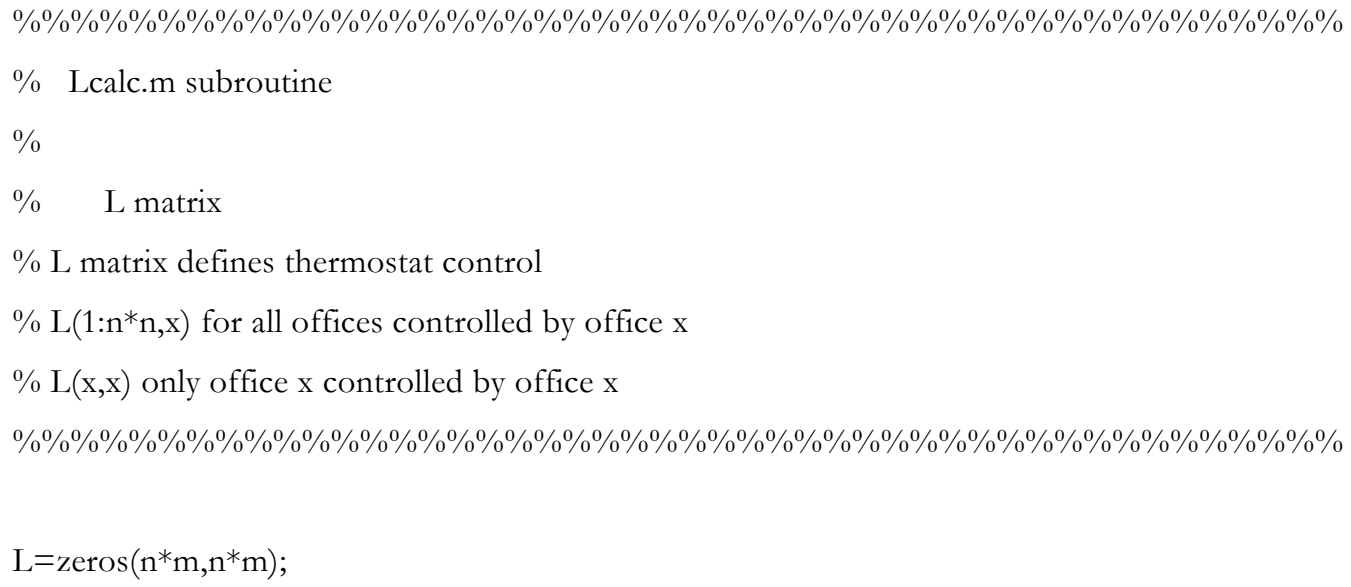


Report No. DE-FG02-03ER63694-F1

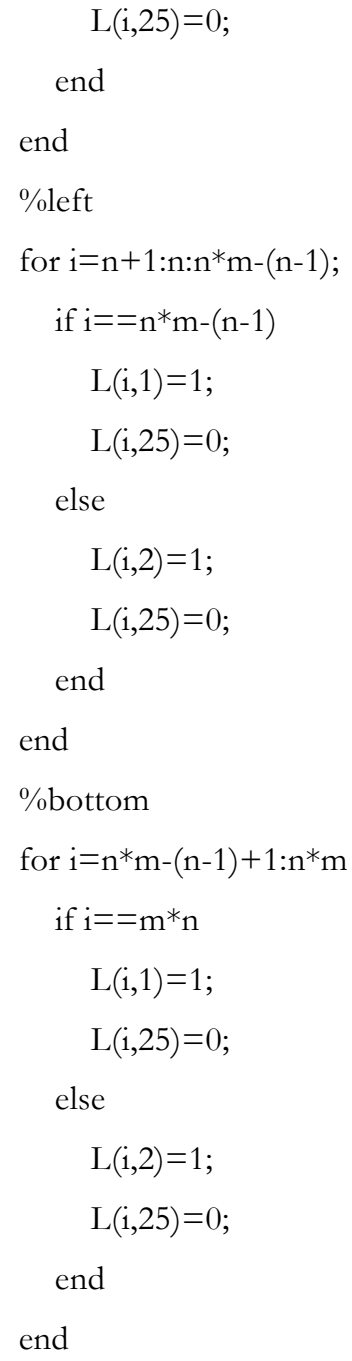


Report No. DE-FG02-03ER63694-F1

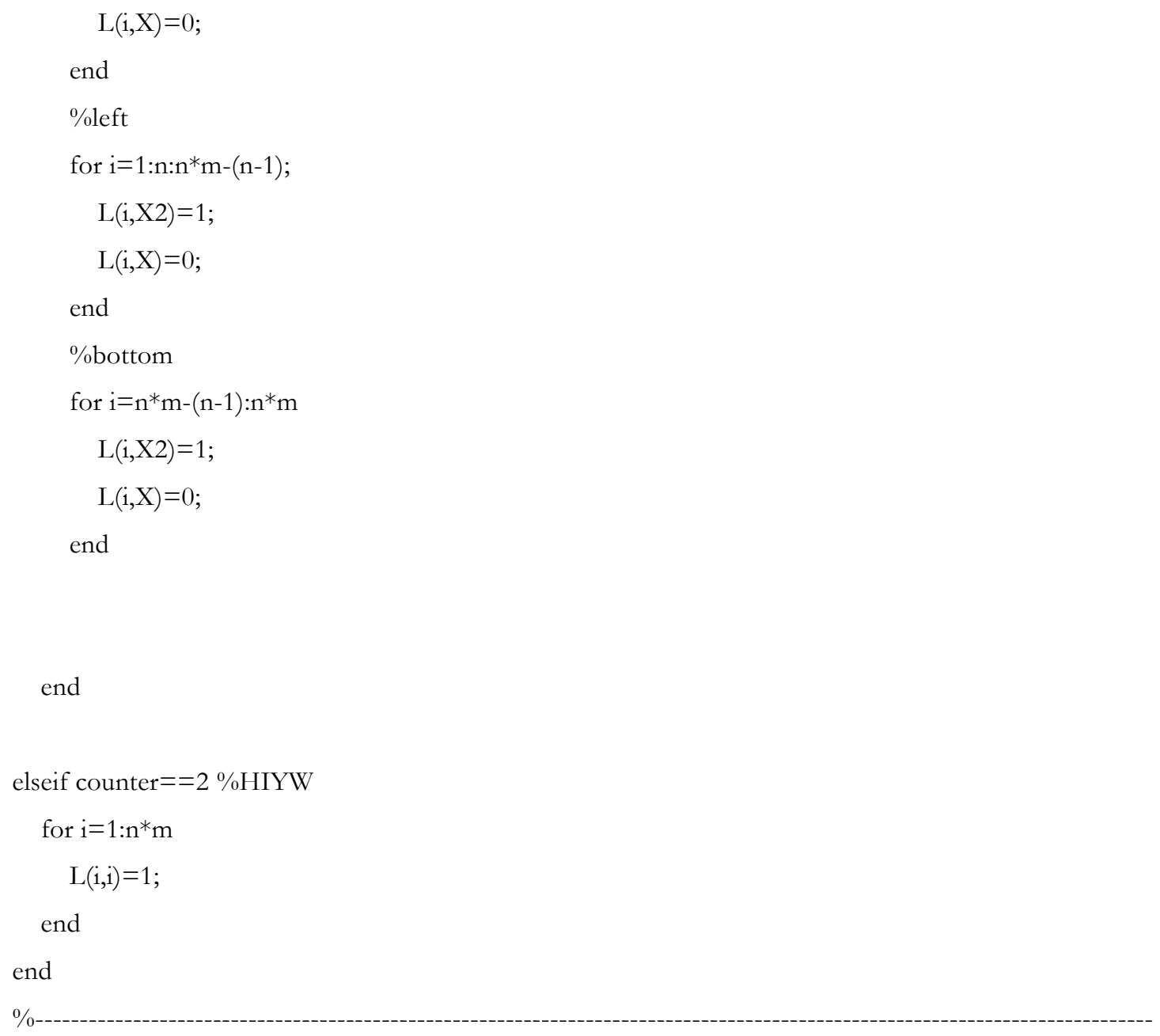




\section{Report No. DE-FG02-03ER63694-F1}

\subsubsection{COP Calculation Subroutine (COPcalcCity.m)}

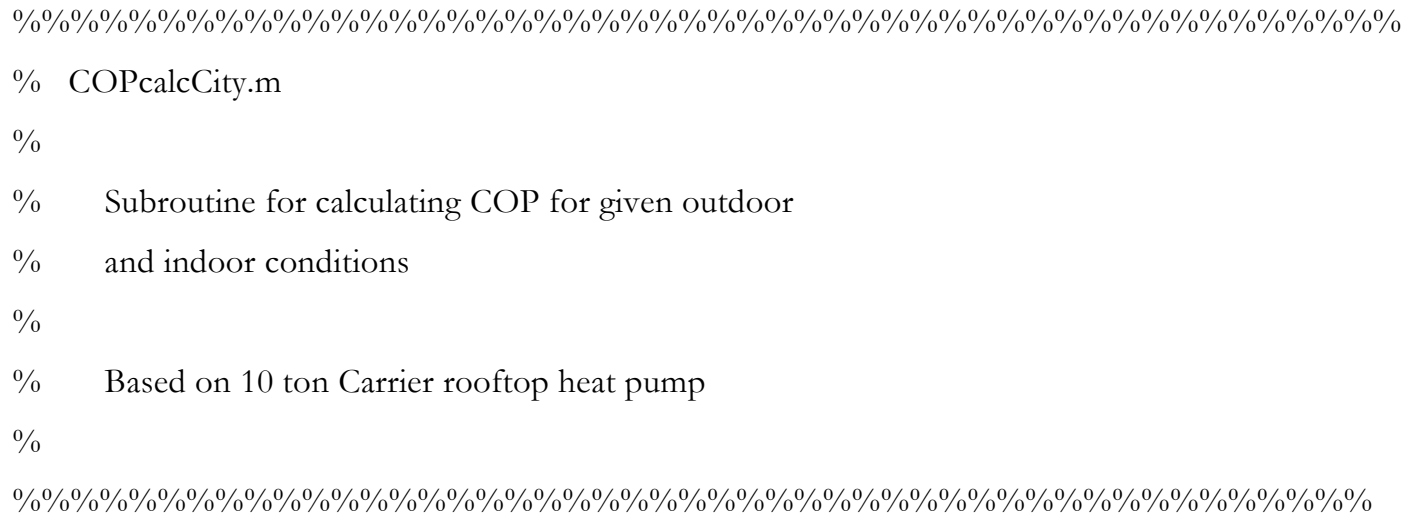

$\begin{array}{cccccc}\mathrm{COPP}=[5.41 & 5.23 & 4.97 & 4.76 & 4.52 & 4.29 \\ 5.17 & 4.98 & 4.74 & 4.53 & 4.30 & 4.08 \\ 4.92 & 4.74 & 4.51 & 4.30 & 4.09 & 3.87 \\ 4.67 & 4.50 & 4.27 & 4.08 & 3.87 & 3.66 \\ 4.43 & 4.25 & 4.04 & 3.85 & 3.65 & 3.45 \\ 4.18 & 4.01 & 3.81 & 3.63 & 3.43 & 3.24 \\ 3.93 & 3.77 & 3.58 & 3.40 & 3.21 & 3.03 \\ 3.69 & 3.52 & 3.35 & 3.17 & 3.00 & 2.82 \\ 3.44 & 3.28 & 3.11 & 2.95 & 2.78 & 2.61 \\ 3.19 & 3.04 & 2.88 & 2.72 & 2.56 & 2.40] ;\end{array}$


Report No. DE-FG02-03ER63694-F1

COP $=0.0667 *$ TbuildAVG +3.3887 ;

['error in COP ' 'Tout=' num2str(Tout)]

end

\%-- 


\section{Report No. DE-FG02-03ER63694-F1}

\subsubsection{Cyclic Degradation COP calculation (CYCDEGcalc.m)}

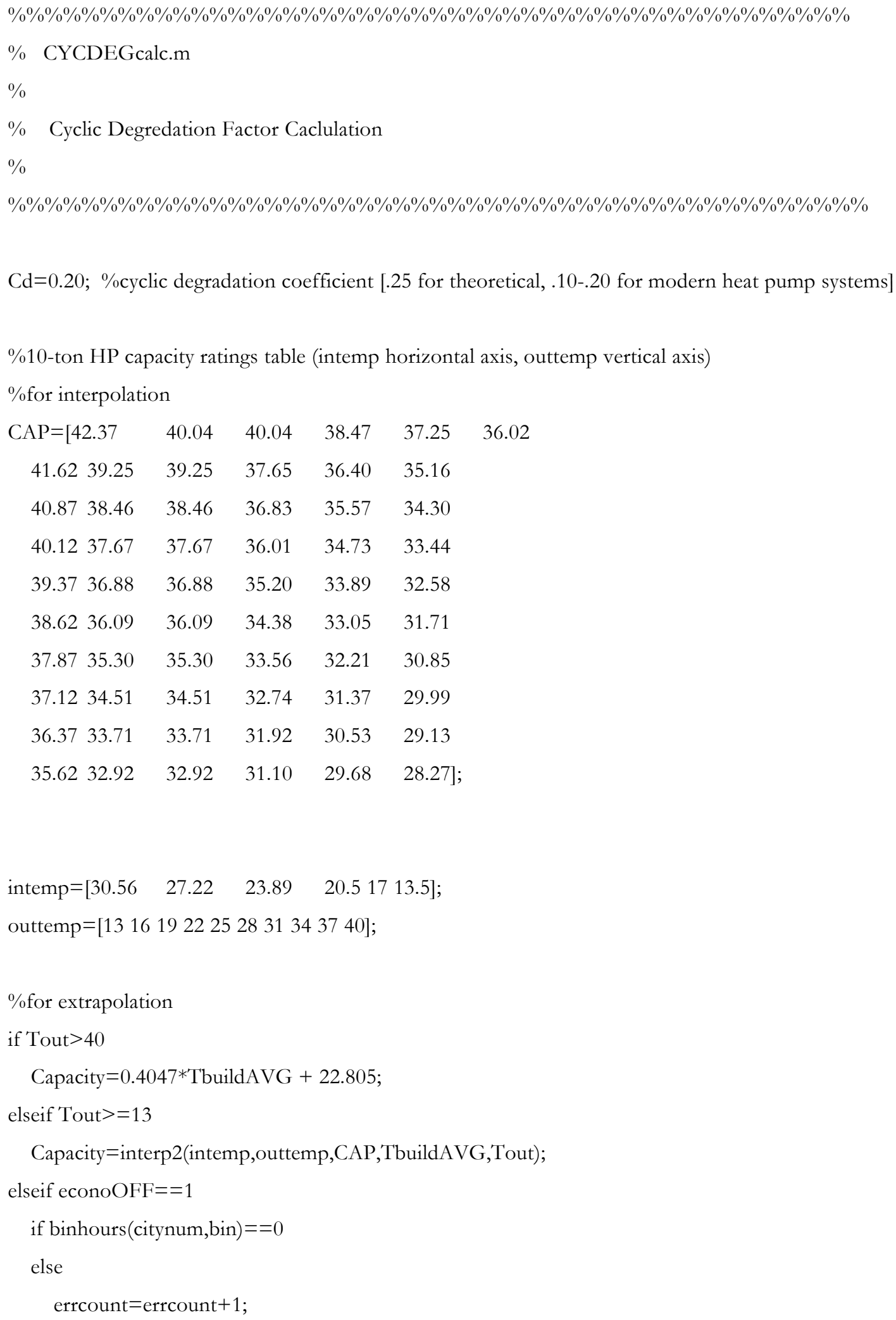

\begin{tabular}{|c|c|c|c|c|}
\hline $\mathrm{CAP}=[42.37$ & 40.04 & 40.04 & 38.47 & 37.25 \\
\hline $41.62 \quad 39.25$ & 39.25 & 37.65 & 36.40 & 35.16 \\
\hline 40.8738 .46 & 38.46 & 36.83 & 35.57 & 34.30 \\
\hline 40.1237 .67 & 37.67 & 36.01 & 34.73 & 33.44 \\
\hline 39.3736 .88 & 36.88 & 35.20 & 33.89 & 32.58 \\
\hline 38.6236 .09 & 36.09 & 34.38 & 33.05 & 31.71 \\
\hline 37.8735 .30 & 35.30 & 33.56 & 32.21 & 30.85 \\
\hline 37.1234 .51 & 34.51 & 32.74 & 31.37 & 29.99 \\
\hline 36.3733 .71 & 33.71 & 31.92 & 30.53 & 29.13 \\
\hline 35.6232 .92 & 32.92 & 31.10 & 29.68 & 28.27]; \\
\hline
\end{tabular}




\section{Report No. DE-FG02-03ER63694-F1}

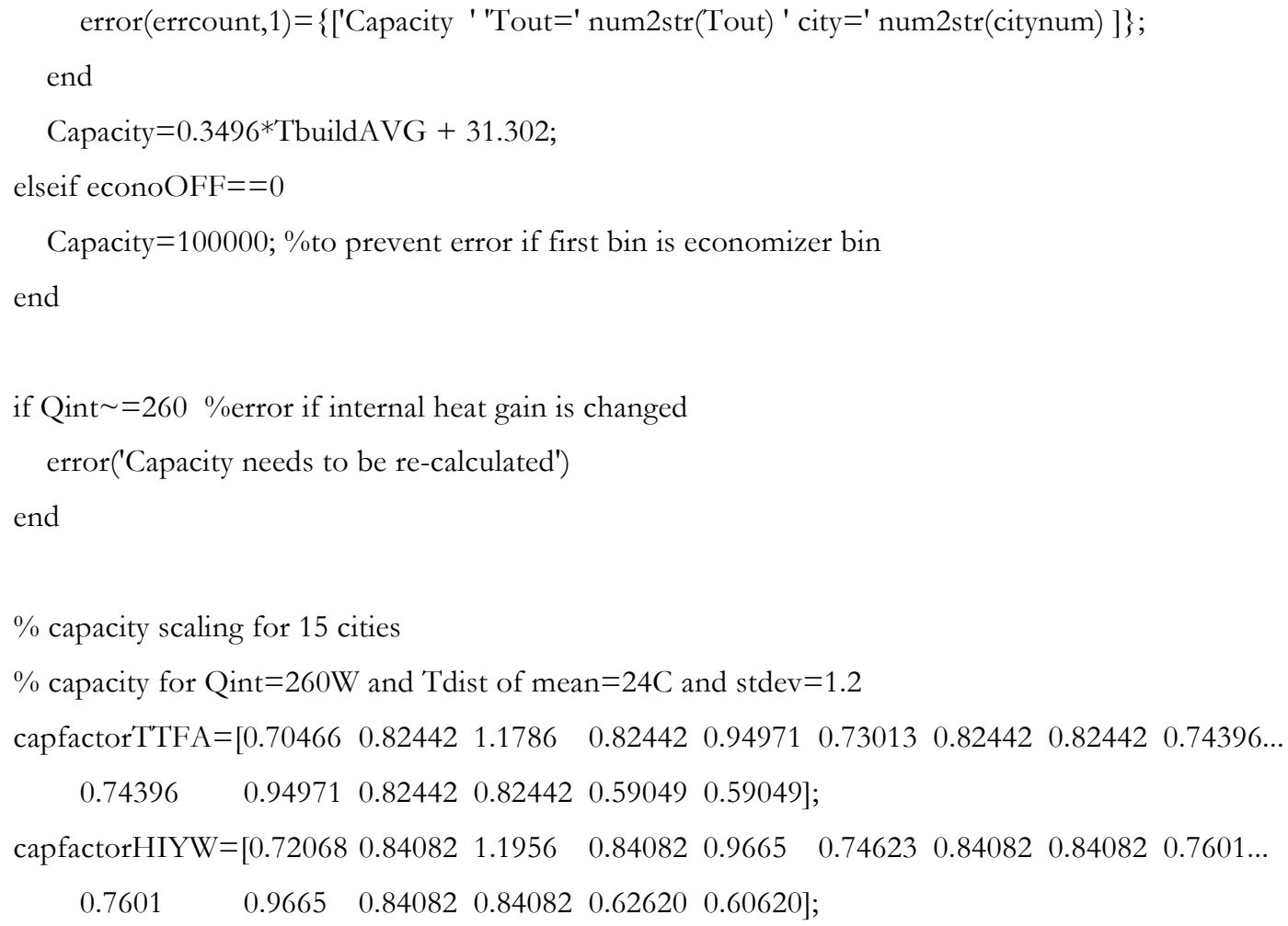




\section{Report No. DE-FG02-03ER63694-F1}

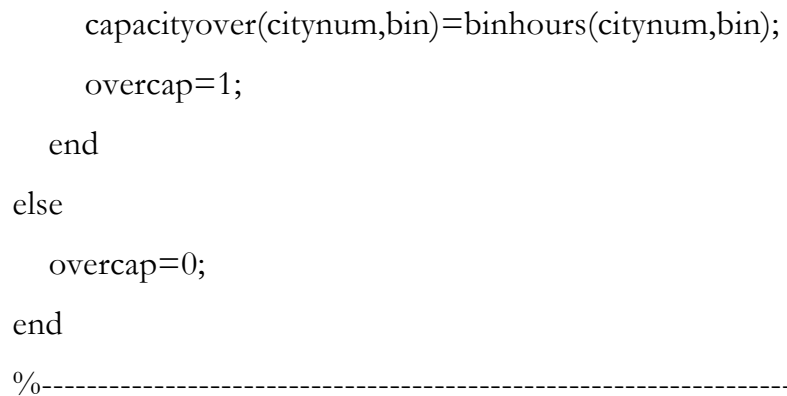


Report No. DE-FG02-03ER63694-F1

\section{References}

[1] United States Department of Energy and United States Environmental Protection Agency, "Sustainable Building Technical Manual," 1996.

[2] Awbi, H., Ventilation of Buildings, Spon Press, London, 2003.

[3] ASHRAE Standard 55-2004, “Thermal Environmental Conditions of Human Occupancy."

[4] IS0/DIS 7730:2003, "Ergonomics of the thermal environment - Analytical determination and interpretation of thermal comfort suing calculation of the PMV and PPD indices and local thermal comfort."

[5] Lomonaco, C. and D. Miller. "Comfort and Control in the Workplace," ASHRAE Journal v39, 1997.

[6] Schiller, G.E., E.A. Arena, F.S. Bauman, C. Benton, M. Fountain, and T Doherty, "A field study of thermal environments and comfort in office buildings," ASHRAE Transactions, 94, 1988.

[7] Bauman, F.S., "Giving Occupants What they Want: Guidelines for Implementing Personal Environmental Control in Your Buildings," World Workplace 99 Proceedings, October 1999.

[8] Bauman, F.S., L.P. Johnston, H. Zhang, E.A. Arens, "Performance testing of a floorbased, occupant-controlled office ventilation system.” ASHRAE Transactions 97, 1991.

[9] Bauman, F.S., "Underfloor Air Distribution (UFAD) Design Guide," Final Report: ASHRAE Research Project RP-1064, Jan. 2003.

[10] Bauman, F.S., H Zhang, and E.A. Arens. "Localized Comfort Control with a Desktop Task Conditioning System: Laboratory and Field Measurements," ASHRAE Transactions, 1993.

[11] Johnson Controls Inc., "White Paper: Environmental Satisfaction, Personal Control and the Positive Correlation to Increased Productivity"

[12] Fisk, W. J. "Health and Productivity Gains from Better Indoor Environments and Their Implications for the US Department of Energy," Indoor Environment Department, Lawrence Berkeley National Laboratory, Berkeley, CA, LBNL-4745, 2000.

[13] Fisk, W.J., “How IAQ Affects Health, Productivity,” ASHRAE Journal, May 2002.

[14] Lorsch, H.G. and O.A. Abdou, "The Impact of the Building Indoor Environment on Occupant Productivity - Part 2: Effects of Temperature," ASHRAE Transactions, Vol. 100, 1994. 
[15] Tham, K.W., "Effects of temperature and outdoor air supply rate on the performance of call center operators in the tropics," Indoor Air 2004, Vol. 14, 119-125.

[16] Wyon, D.P., “Indoor Environmental Effects on Productivity,” IAQ '96: Paths to Better Building Environments, 1996.

[17] Wyon, D.P., "The effects of indoor air quality on performance and productivity," Indoor Air 2004, Vol. 14, 92-101.

[18] Johnson Controls Inc., "Case Study: West Bend Mutual Insurance Company, West Bend, WI" available online: http://www.johnsoncontrols.com/CG-

Cases/cs WestBend.pdf

[19] Indoor Air Quality Update, Oct 1996, v.9 no.10, p.1-5.

[20] Lorsch, H.G. and O.A. Abdou, "The Impact of the Building Indoor Environment on Occupant Productivity - Part 1: Recent Studies, Measures, and Costs," ASHRAE Transactions, Vol. 100, 1994.

[21] Seem, J.E. and J.E. Braun. "The Impact of Personal Environmental Control on Building Energy Use,” ASHRAE Transactions, 48, 1992.

[22] Klein et al, “TRNSYS 16: a TRaNsient SYstem Simulation program,” Solar Energy Laboratory, University of Wisconsin-Madison, 2004.

[23] Glicksman, L.R. and S. Taub. "Thermal and Behavioral Modeling of Occupantcontrolled Heating, Ventilating, and Air Conditioning Systems," Energy and Buildings, 25, 1997.

[24] Crawley, D.B, L. K. Lawrie, C.O. Pedersen, and F.C. Winkelmann, "EnergyPlus: Energy Simulation Program,” ASHRAE Journal, April 2000.

[25] Incropera F.P. and D.P. DeWitt, Introduction to Heat Transfer, John Wiley \& Sons, New York, 2002.

[26] Cengel Y.A and M.A. Boles, Thermodynamics: An Engineering Approach, McGrawHill, New York, 2002.

[27] ASHRAE, Handbook of Fundamentals, 1997.

[28] Marion, W. and K. Urban, “User's Manual for TMY2s: Typical Meteorological Year," National Renewable Energy Laboratory, June 1995.

[29] Briggs R.S., R.G. Lucas, and Z.T. Taylor, "Climate Classification for Building Energy Codes and Standards," Pacific NW National Laboratory, March 2002.

[30] "Results of the 2003 IECC public hearings" available online: http://www.energycodes.info 
[31] United States Census Bureau, "2000 Gazetteer," available online: http://www.census.gov/geo/www/gazetteer/gazette.html

[32] Ari, S., I.A. Cosden, H.E. Khalifa, J.F. Dannenhoffer, P. Wilcoxen, and C. Isik. "Constrained Fuzzy Logic Approximation for Indoor Comfort and Energy Optimization," IEEE NAFIPS, 2005.

[33] Carrier Corp, “WEATHERMASTER ${ }^{\circ}$ 50HJQ004-016 WEATHERMAKER® 50TFQ004-016 Single Package Rooftop Standard and High Efficiency Heat Pump Units Product Data," available online at: http://www.xpedio.carrier.com/idc/groups/public/documents/techlit/50h,t-3pd.pdf

[34] Henderson, H., Y.J. Huang, and D. Parker, "Residential Equipment Part Load Curves for use in DOE-2," LBNL, February 1999.

[35] ENERGY STAR, http://www.energystar.gov.

[36] Energy Information Administration, http://www.eia.doe.gov.

[37] MathWorks products, MATLAB, release 13.

[38] Blair, N. and S. Holst, "BESTEST Results and Experiences using the latest TRNSYS building model (TRNSYS 14.2 version)" 1997 TRNSYS User Day, Stuttgart, Germany, 1997.

[39] National Renewable Energy Laboratory, http://www.nrel.gov. 


\title{
D. INDOOR COMFORT AND ENERGY OPTIMIZATION
}

\author{
By \\ Seckin Ari \\ (Ph.D. Candidate, Syracuse University)
}

June 2006

Approved:

Dr. Can Isik

Date: 


\section{Table of Contents}

\section{List of Figures}

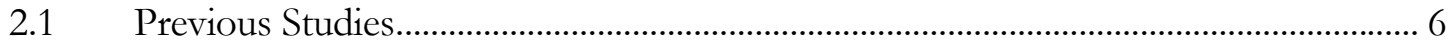

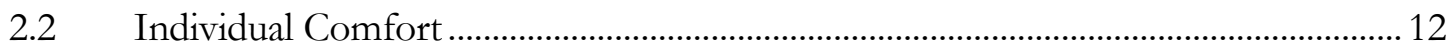

$\underline{3}$ HVAC Control 17

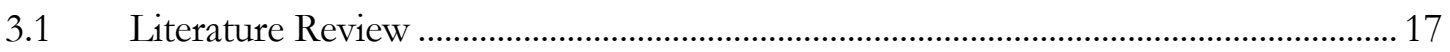

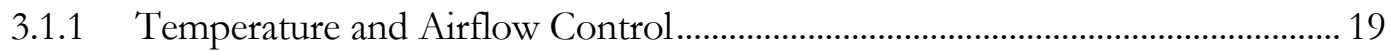

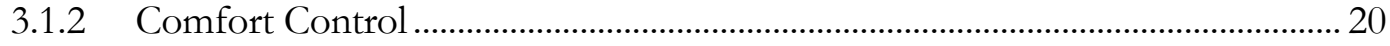

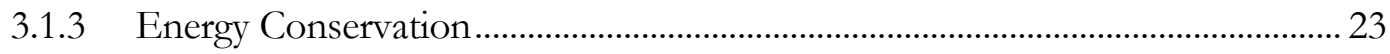

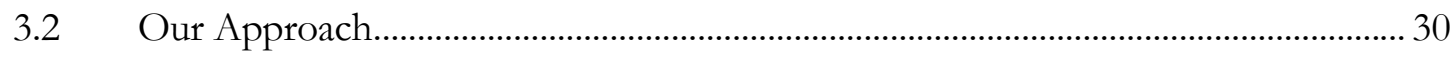

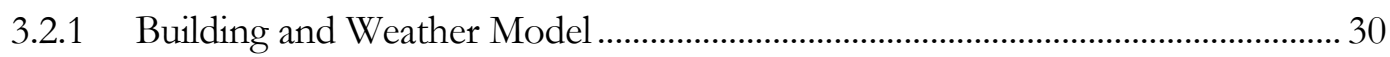

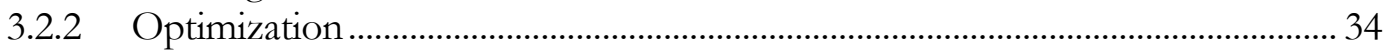

3.2.3 Fuzzy Logic Approximation ............................................................................. 38

4 Results and Conclusion $\quad 42$

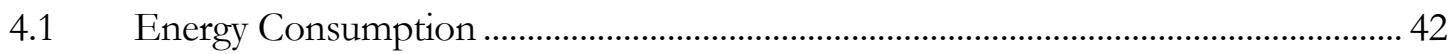

4.2 Effects of Internal Resistance on Optimization............................................................ 43

4.3 Effects of Non-uniform Internal Heat Load on Optimization .................................. 46

$\underline{5}$ Appendix - MATLAB Codes 49

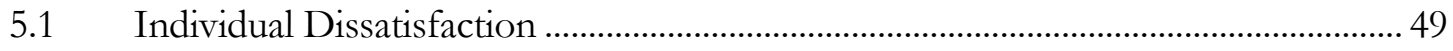

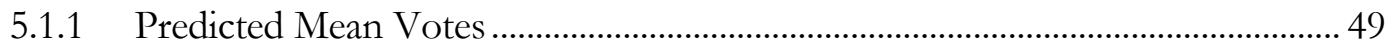

5.1.2 Optimal Settings for Individual Satisfaction Model - Monte Carlo Simulation51

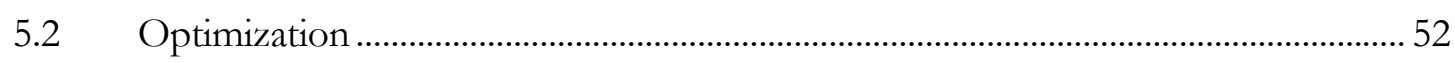

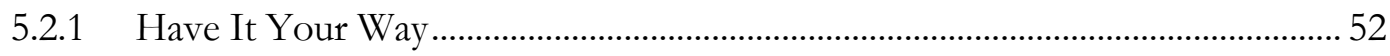

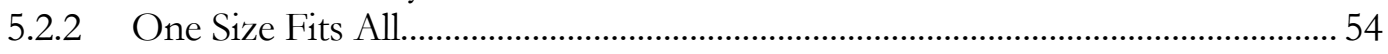

References $\quad 56$ 
Report No. DOE \ER63694-1 


\section{Report No. DOE\ER63694-1}

\section{List of Figures}

Figure 1.1 - Average annual commercial building expenses (in 1995 US dollars) [23]................ 4

Figure 2.1 - Predicted Percentage of Dissatisfied (PPD) as a function of ....................................... 8

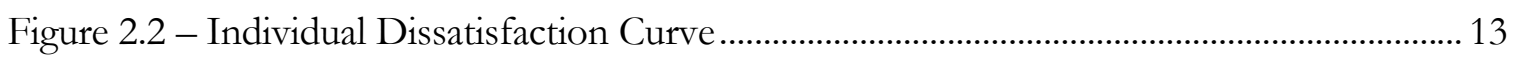

Figure 2.3 - Individual Votes ...................................................................................................... 14

Figure 2.4 - Histogram of 49 occupants' neutral temperature ......................................................... 15

Figure 2.5 - An example of the DID curves for a given population ............................................ 15

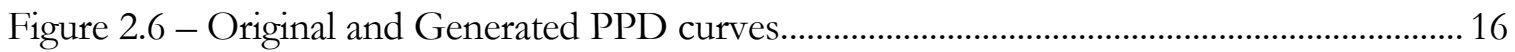

Figure 3.1 - Example of thermal network circuit around zone i ..................................................... 32

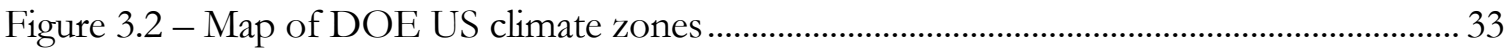

Figure 3.3 - Example of desired temperatures of randomly generated 49 individuals, HIYW. 33

Figure 3.4 - Example of 49 optimal temperature settings of optimized HIYW ........................... 35

Figure 3.5 - Degree of Individual Dissatisfaction Levels of 49 occupants from the Figure 3.335

Figure 3.6 - Example of uniform temperature distribution of OSFA .............................................. 36

Figure 3.7 - Thermostat placements, and three (Corner, Perimeter, and Interior) zones of

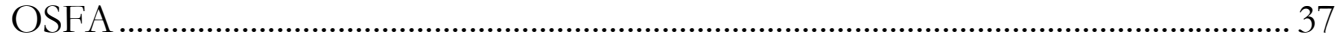

Figure 3.8 - DID level of occupants in OSFA case for the temperature setting in Figure 3.6.37

Figure 3.9 - Sensor communication requirements of fuzzy logic approximation ....................... 39

Figure 3.10 - Sensor connectivity for perimeter FIS........................................................................ 40

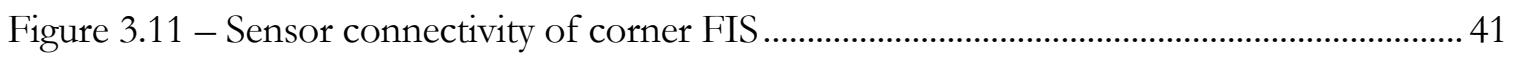

Figure 4.1 - Energy savings per year relative to OSFA. ................................................................... 43

Figure 4.2 - Internal resistance effects on optimization .................................................................... 45

Figure 4.3 - Example of thermostat set points................................................................................... 45

Figure 4.4 - Example of non-uniform heat load.................................................................................. 47

Figure 4.5 - Power results with non-uniform loads, and uniform load .......................................... 47 


\section{Report No. DOE\ER63694-1}

\section{List of Tables}

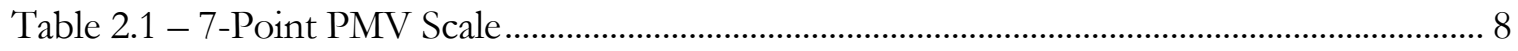

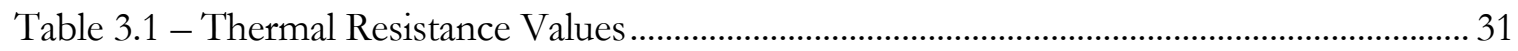

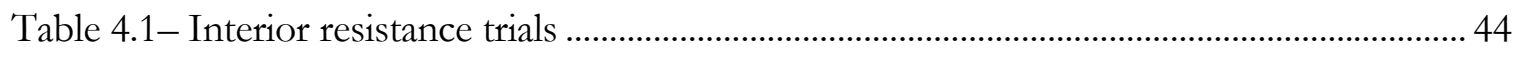


Report No. DE-FG02-03ER63694-F1

\section{Introduction}

Buildings utilize one-third of energy consumed in the U.S., and $40-60 \%$ of the overall energy consumption in buildings can be attributed to typical heating, ventilating, and air-conditioning (HVAC) systems for maintaining thermal comfort [1]. Before the oil crisis of 1973, HVAC systems were designed to provide quality occupant comfort without considering energy saving. Following the energy crisis, building designs targeted to lower energy usage by focusing on building thermal insulation, air-tightness of the building envelopes, and heat recovery while keeping building temperatures within acceptable levels [2]. This design strategy, when taken to extremes without proper monitoring, resulted in reduced comfort and unhealthy indoor air quality (IAQ). The quality of the indoor environment is critically important to human health

and performance. In today's technologically developed societies, a large proportion of the population (more than 95\%) spend more than $90 \%$ of their time in built structures with artificially maintained environments (buildings, vehicles, etc.) [3][4].

American Society of Heating Refrigeration and Air-conditioning Engineers (ASHRAE) and the International Organization for Standardization (ISO) have established and have been maintaining the standards for human thermal comfort. Specifically, the ASHRAE Standard 55-2004 [5] "Thermal Environmental Conditions for Human Occupancy," and ISO Standard 7730 [6], specify the criteria for providing acceptable thermal conditions to a majority of the occupants wherever man-made climates are provided for human occupancy based on a combination of personal factors (thermal resistance of the clothing and activity level) and environmental factors (air temperature, mean radiant temperature, relative air velocity, and relative humidity). These standards assume that a building's occupants are subjected to the same environmental conditions. However, because of the differences in individual occupants' 


\section{Report No. DE-FG02-03ER63694-F1}

preferences, it is impossible to satisfy everyone at the same time. Therefore standards inherently are based on an average population comfort. The current conventional "one-sizefits-all” HVAC systems are usually designed by these thermal comfort standards so as to provide acceptable thermal comfort for a group of people. The thermal conditions are considered acceptable when at least $80 \%$ of a building's occupants are satisfied.

ASHRAE Standard 55-2004 specifies a comfort zone of temperatures to be applied uniformly throughout a building that is regulated by the small number of thermostats. The characteristic differences between zones within a building, due to variations in factors such as internal heat load, local delivery of heating or air conditioning, can make this consistent temperature distribution unachievable. Some studies showed that providing thermal satisfaction to $80 \%$ of a building's occupants may be far from achieved in practice. According to Haghighat et al., even though thermal comfort parameters in buildings were set according to the ASHRAE standards, it has been found that $32 \%$ of the occupants were dissatisfied [7]. In a follow-up study, they found more than $56 \%$ of the occupants dissatisfied with overall office air quality only $63.4 \%$ of their indoor observations fell into the summer comfort zone, and $26.9 \%$ into the winter zone in their feedback survey, conducted in 12 mechanically ventilated buildings with 877 occupants [8], . In many other studies, it has been found that while ASHRAE thermal comfort, air quality, etc. requirements were met in the design and maintenance of facilities, many more than $20 \%$ of the occupants were dissatisfied with thermal comfort and indoor air quality [9], [10], [11], [12].

There is a growing list of researchers and practitioners who have found that allowing occupants control over their personal environment leads to increased comfort and productivity. Considering a traditional approach to thermal comfort, acceptable to $80 \%$, overall workplace productivity is likely more affected by large numbers of slightly dissatisfied 


\section{Report No. DE-FG02-03ER63694-F1}

employees rather than a very few hypersensitive employees [10]. Therefore, if productivity is to be increased effectively the majority of employees, not just those that complain, must be given some level of personal control. Fisk [18] estimates the potential U.S. annual productivity gain from improved worker performance from changes in thermal environment and lighting to be between $\$ 20$ billion and $\$ 160$ billion (1996 U.S. $\$$ ). Wyon [21] predicted that by providing individual control equivalent to $\pm 3^{\circ} \mathrm{C}$, productivity would increase $2.7 \%$ for logical thinking, $7.0 \%$ for general office work, 3.4\% for very skilled manual work, and $8.6 \%$ for very rapid manual work. In a later study with field observations, Wyon [22] showed that poor air quality, resulting from low outdoor air supply rate and uncomfortable temperatures can have a negative effect on office productivity in the range of $6-9 \%$.

Figure 1.1 shows average annual commercial building costs in dollars per square foot. Employee salaries are nearly ten times more costly than the next highest expense, rent. Utilities which we can consider to include all HVAC operation costs, is less than $1 \%$ of employee salaries. This means that if it were possible to increase the productivity of the employees by $1 \%$, the equivalent monetary gain is equal to the entire building utility cost. Surely this is motivation enough to explore the ability to increase worker productivity through increased comfort. 
Report No. DE-FG02-03ER63694-F1

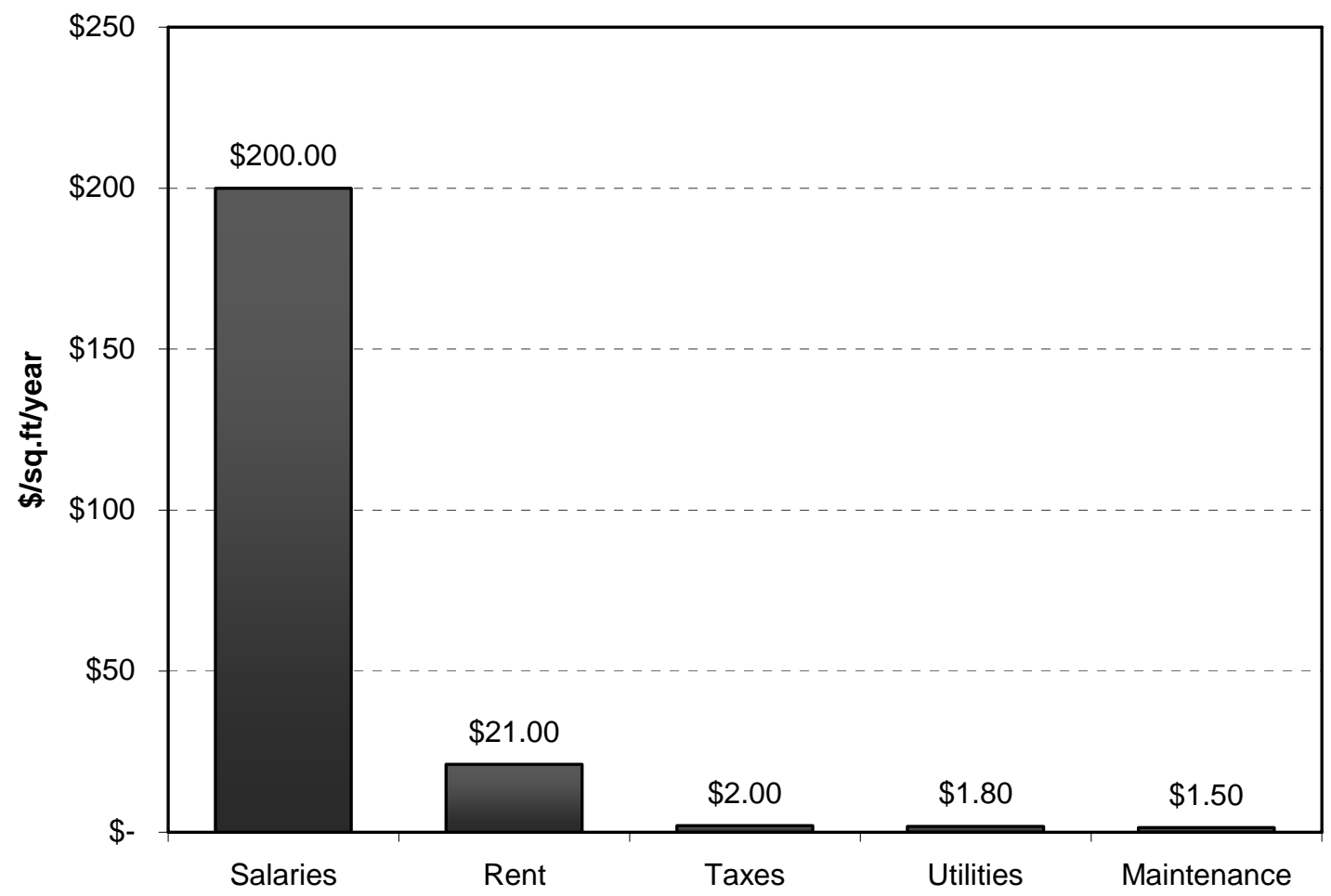

Figure 1.1 - Average annual commercial building expenses (in 1995 US dollars) [23].

With the benefits of personal environmental control (PEC) so overwhelmingly clear, there must be a reason for the industry's reluctance for widespread adoption of individual control. The explanation is two fold: (1) the high uncertainty of measuring an increase in productivity, and (2) increased energy costs. As mentioned before, typical HVAC systems account for between $40-60 \%$ of a commercial building's overall energy consumption. Building owners are reluctant to adopt personal environmental control systems that will likely increase the energy usage of a building for the promise of more productive employees, given the high uncertainty of the latter. Energy costs, while small compared to employee salaries, are still a major commercial expense and are very easily measured, whereas productivity gains due to improved indoor environmental quality are much more difficult to measure. In addition, there is an associated increase in initial construction costs. Lorsch and Abdou [24] note one instance of a 


\section{Report No. DE-FG02-03ER63694-F1}

federal building manager who spent $\$ 1$ million on personal control and never saw concrete evidence of an increase in productivity. With experiences like this it is easy to understand why building owners are hesitant to risk the increased costs associated with personal environmental control. Unfortunately the true energy cost associated with the adoption of personal environmental control is relatively unknown. Often PEC systems are installed in conjunction with other green building, energy saving enhancements that make it difficult to isolate the PEC contribution to building energy costs observed in field studies [25].

This study proposes an intelligent control strategy, which approaches the "have it your way" (HIYW) ideal with no increase, or possibly even a savings in energy consumption by taking advantage of the tolerance that individuals have to small variations in their environmental conditions.

In the next section thermal comfort will be discussed, including a literature survey and a proposed model of individual thermal satisfaction. Then, in section three, different approaches to the selection of thermal profile in a building, and their implication in thermal comfort and energy consumption will be explained. 


\section{Report No. DE-FG02-03ER63694-F1}

\section{Thermal Comfort}

\subsection{Previous Studies}

Comfort of occupants is a vital aspect in the control of indoor environments. There have been a variety of studies about thermal comfort indices in the literature. The realistic model of energy exchange between the skin surface and the ambient environment has been the key factor of thermal comfort indices. The indices of human response to the thermal environment based on this rational model have been successful. The first study about thermal comfort was proposed by Houghton and Yaglou [26]. They presented the first temperature scale in terms of dry bulb and humidity in order to measure the thermal comfort of the environment, and this scale, which is the original American Society of Heating and Ventilating Engineers (ASHVE) Effective Temperature comfort chart, was widely used for almost 50 years over the world. Yaglou reduced the effect of humidity towards lower temperatures in his scale [27], and Winslow et al. defined a skin wettedness index of thermal discomfort in terms of the part of the body surface [28]. Two concentric cylinders, which are a core cylinder and a thin skin cylinder surrounding it, had been used to model the human body. His index predicts thermal discomfort using skin wettedness and temperature. After 1930s, there was a considerable increase in the studies about making a rational predict of comfort and the body and the skin temperatures. In 1971, Gagge et al. showed how important dry bulb temperature and humidity are in considering comfort [29]. The introduced new Effective Temperature (ET ${ }^{*}$ ), and $\mathrm{SET}^{*}$ scale by Gagge et al. are based on the rationalization of the original ASHVE ET comfort chart. $\mathrm{SET}^{*}$ stands for an effective temperature relative to a standard person in a standard environment. They used the same cylindrical model of the human body as the one Winslow used. Fanger presented a new and rational approach to thermal comfort at [30]. 
Based on his experiments among college students, his indicatory comfort equation generated the combination of environmental parameters in order to produce optimal thermal comfort. But this comfort equation was not applicable for the prediction of a certain environmental condition. Starting from this comfort equation, Fanger introduced a new thermal sensation index, which makes it possible to estimate thermal satisfaction of the whole population under any environmental conditions [3]. Unlike Gagge et al.'s ET*, which specifically takes into account temperature and humidity, Fanger's Predicted Mean Vote (PMV) consists of 7-point scale, as shown in Table 2.1, and accounts for the following six principal variables:

1. metabolic rate (met)

2. clothing (clo)

3. air temperature $\left({ }^{\circ} \mathrm{C}\right)$

4. $\quad$ radiant temperature $\left({ }^{\circ} \mathrm{C}\right)$

5. air velocity $(\mathrm{m} / \mathrm{s})$

6. relative humidity $(\%)$.

PMV has been widely accepted as an overall comfort index, and also accepted as a seven-point psycho-physical ASHRAE scale. In addition to this PMV scale, Fanger also explained how acceptable the mean vote is by giving a relationship between the "Predicted Percentage of Dissatisfied" (PPD) and PMV, based on experiments conducted with 1296 subjects. In his experiments, he allowed subjects to vote about their thermal satisfaction based on his sevenpoint scale. Using Gagge et al.'s definition of dissatisfaction [31], Fanger classified the people voting -3 (cold), -2 (cool), +2 (warm), and +3 (hot) as dissatisfied, and the people voting -1 (slightly cool), 0 (neutral), and +1 (slightly warm) as satisfied. This relationship, which is shown by the curve in Figure 2.1, has been adopted by ASHRAE and ISO as a standard of the thermal satisfaction of human occupancy [5][6] and widely accepted by the industry. 
Report No. DE-FG02-03ER63694-F1

Table 2.1 - 7-Point PMV Scale

\begin{tabular}{cl}
\hline Vote & \multicolumn{1}{c}{ Meaning } \\
\hline-3 & Cold \\
-1 & Cool \\
0 & Slightly cool \\
+1 & Slightly warm \\
+2 & Warm \\
+3 & Hot
\end{tabular}

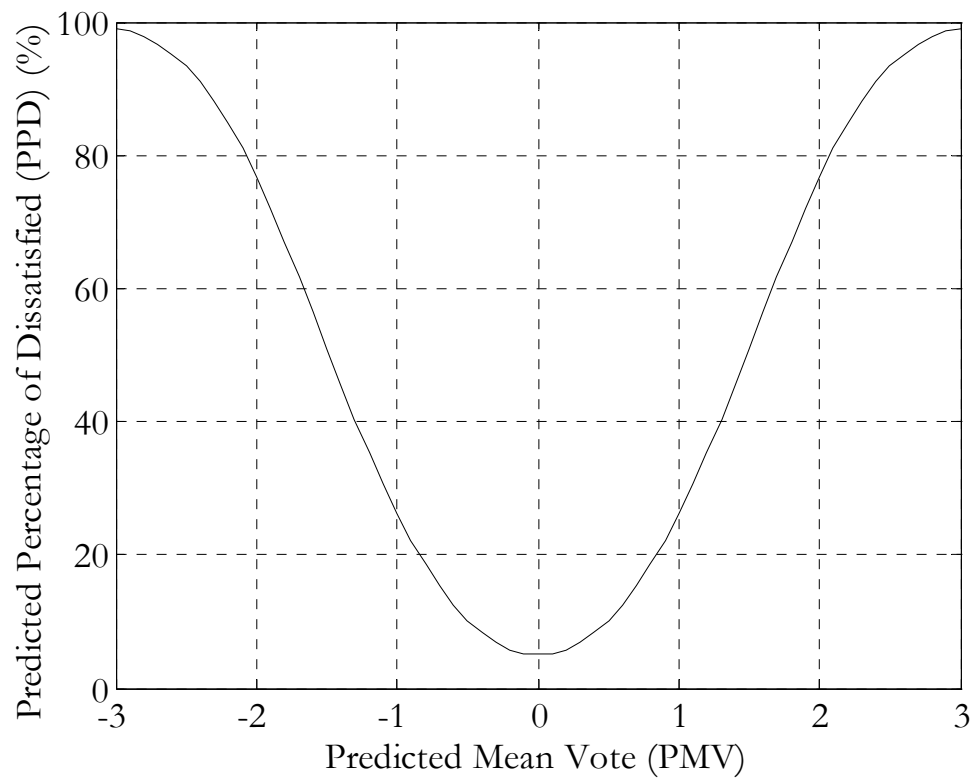

Figure 2.1 - Predicted Percentage of Dissatisfied (PPD) as a function of

Predicted Mean Vote (PMV)

Both PMV and ET ${ }^{*}$ use the steady-state heat transfer between a clothed human body and the environment. In order to take account of humidity effects better, Gagge et al. modified 


\section{Report No. DE-FG02-03ER63694-F1}

Fanger's Predicted Mean Vote equation by introducing a new thermal sensation index, called $\mathrm{PMV}^{*}$ [32]. They proposed the new $\mathrm{PMV}^{*}$ index for any humid or dry environment by substituting operative temperature in Fanger's PMV equation with SET*. PMV is based on the thermal comfort for a large group of people for any environmental conditions. But, in general, the proper thermal comfort variables for one occupant will be different for one another. These variables also depend on national-geographic location, age, sex, health, and weight. McNall et al.'s showed women more sensitive than men to the temperature variations [33]. In order to deal with personal differences, Federspiel and Asada derived a new thermal sensation index based on the same idea as Fanger's Predicted Mean Vote with some basic assumptions [34]. Their user-adaptive PMV-like index is based on estimating the parameters according to the obtained thermal sensation ratings of the occupant. With this adaptation, the predicted thermal sensation rating more closely matches the actual rating acquired of the occupant. Another adaptive model of thermal comfort has been proposed by de Dear et al. for Naturally Ventilated (NV) buildings [35]. They derived an adaptive model from the temperature preferences in terms of outdoor effective temperature in NV buildings by using the field experiments results of ASHRAE RP-884. They built a large database of research results of the 22346 observations compiled in the 160 buildings from all over the world. In this field experiments, occupants of NV buildings have been found comfortable in a wider range of temperatures than the occupants of buildings with centrally controlled HVAC. They also showed an explicit dependency of the adaptive model to the indoor comfort temperatures [2]. In their next study [36], they investigated how personal control of operable windows impacts on occupant comfort and local thermal conditions with another ASHRAE field project RP1161. They collected 1000 survey responses of the occupants having the degree of control over the windows in a NV building in two main seasons. Their results indicated significantly 
Report No. DE-FG02-03ER63694-F1

diverse thermal responses of the occupants with different degrees of personal control, even when they were at the same clothing, activity levels and thermal environmental conditions. This wider comfort zone in the NV buildings, which is provided by personal control, has the potential of significant energy savings by lightening up thermal comfort standards and tolerating more variable indoor temperatures.

There have only been a handful of researchers who have modeled the energy usage of buildings equipped with occupant controlled distributed environmental control systems. Seem and Braun [37] compared HVAC and lighting costs of personal environmental control (PEC) with those of conventional variable air volume (VAV) systems through computer simulations. Each occupant had a desktop PEC equipped with an occupancy sensor. The sensor also controlled local task lighting. The PEC allowed occupants to control the temperature and flow rate of the airflow through a radiant heating panel and local fan. In addition to each PEC, underfloor diffusers were also included to provide overall conditioning to the space. Cooling was provided through a chilled water system while heat was provided through localized heating coils. Seem and Braun modeled one floor (45,000 sq. ft) of a multi-story building in Madison, WI with 360 total occupants. The building was modeled using TRNSYS [38] by dividing the building into three well mixed zones. Each PEC had different occupied and non-occupied heating and cooling set points, so when an occupant leaves his/her workstation the set points are relaxed. Electricity was used to provide cooling power while natural gas was used to provide heating power at an assumed one-third the cost of electricity. Radiant heating panels, however, used electricity. In their analysis Seem and Braun neglected the heat transfer between the individual workstations/offices. In each of the three zones, there were at least 80 occupants, all of whom were modeled as having the same temperature. The energy costs associated with maintaining different personal temperatures within these 


\section{Report No. DE-FG02-03ER63694-F1}

zones was not considered. That is, each workstation/office was assumed to be maintained at the same temperature when occupied. Overall the operating costs of PEC systems costs were found to be between $2-15 \%$ greater than the VAV systems. However, if occupancy rates of workstations fell below $60 \%$ then PEC systems resulted in a net savings of energy because of the occupancy sensor adjusting the lighting and workstation temperature.

Glicksman and Taub [39] modeled occupant controlled HVAC systems using computer simulations. Unlike the previous study by Seem and Braun, Glicksman and Taub accounted for the heat transfer between individual zones. A model was created where individual nodes represented conditioned cubicles equipped with occupant controlled HVAC devices. The area between the cubicles and the ceiling was treated as a single well-mixed zone. Glicksman and Taub modeled an interior region on a floor of a multiple story office building. This interior region was an open-plan office space consisting of only cubicles with no interaction with outside weather conditions. A conventional mixing ventilation system was compared with a new occupant controlled underfloor air distribution system. Random processes were used to model the inhabitance of the occupants at their workstations as well as their desired temperature set points. The local occupant fans as well as the lighting and equipment (computer) is controlled by an occupant sensor. The preferred temperature for each occupant was taken from a Gaussian distribution with a mean of $23^{\circ} \mathrm{C}$ and a standard deviation of $1.5^{\circ} \mathrm{C}$. From this distribution, temperatures were taken and assigned at random within the building model. This study estimated a substantial savings in the adoption of a floor based system. They predicted an annual energy savings of $13 \%$ for a non-uniform temperature distribution and a $21 \%$ savings for a uniform temperature distribution over a conventional mixing ventilation system using the same HVAC equipment. This is largely a result of the stratification within the room, reduced conditioning of the corridor, and occupancy sensors 
Report No. DE-FG02-03ER63694-F1

which reduce the heat load in unoccupied rooms. The energy penalty $(8 \%)$ associated with the non-uniform (i.e. individual control) temperature distribution over a uniform distribution was driven by the energy penalty of the cooler than average rooms.

\subsection{Individual Comfort}

All the work in the literature related to thermal comfort and its control applications have been based on the thermal satisfaction of a population of building occupants. They have not taken into account individual thermal satisfaction. For instance, when $90 \%$ of population is satisfied according to ASHRAE Standard 55-2004, the remaining occupants may be persistently dissatisfied at the same conditions. Research has indicated that personally controlled systems increase productivity, and remain people comfortable in a wide range of thermal conditions. In this study, we approached the thermal comfort from the individual point of view.

The occupant comfort has been modeled by a method inspired by Fanger's PMV and PPD [3]. We developed a model of personal dissatisfaction that generates population results closely match the well-established PPD-PMV curve by introducing a new measure: the Degree of Individual Dissatisfaction (DID):

$$
\operatorname{DID}(\text { vote })=\frac{1+\tanh (2 \mid \text { vote } \mid-3)}{2}
$$

where an individual's vote (Figure 2.3) is in terms of the surrounding indoor temperature (T) and two parameters unfolding individual preferences: $\mathrm{T}_{0}$, which is the desired temperature of occupant, and $\Delta \mathrm{T}$, which is the temperature tolerance of the occupant at the vote value of 1.5 , or -1.5 . 
Report No. DE-FG02-03ER63694-F1

$$
\operatorname{vote}(T)=\left\{\begin{aligned}
+3, & T>T_{0}+2 \Delta T \\
-3, & T<T_{0}-2 \Delta T \\
1.5 \frac{T-T_{0}}{\Delta T}, & \text { otherwise }
\end{aligned}\right.
$$

Figure 2.2 demonstrates a satisfaction curve of a given individual. Unlike the previous studies, which accepted two-state satisfaction criteria, we proposed a fuzzy satisfaction curve (Figure 2.2) as a function of thermal index instead of binary satisfaction. While a person might be comfortable within a certain range of temperature in the binary case, he or she will be dissatisfied even a tenth of a degree of outside the range with this comfort criterion. In addition to our linear comfort index, which is an individual vote (Figure 2.3), we generated these curves for individual thermal preferences.

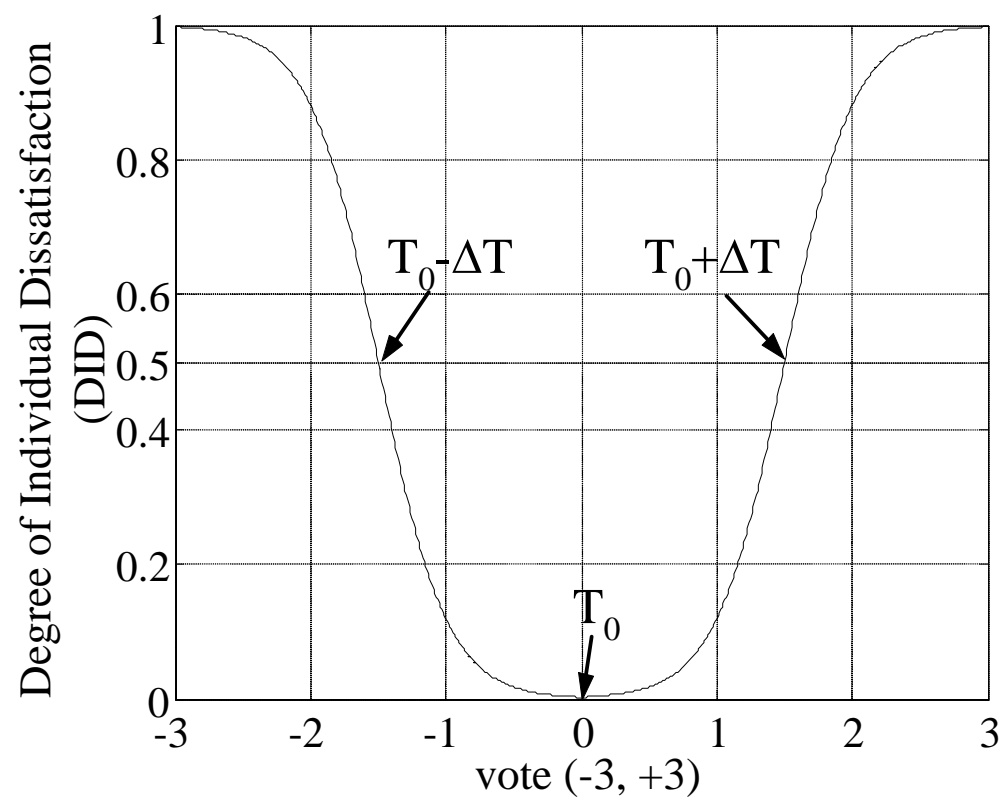

Figure 2.2 - Individual Dissatisfaction Curve 


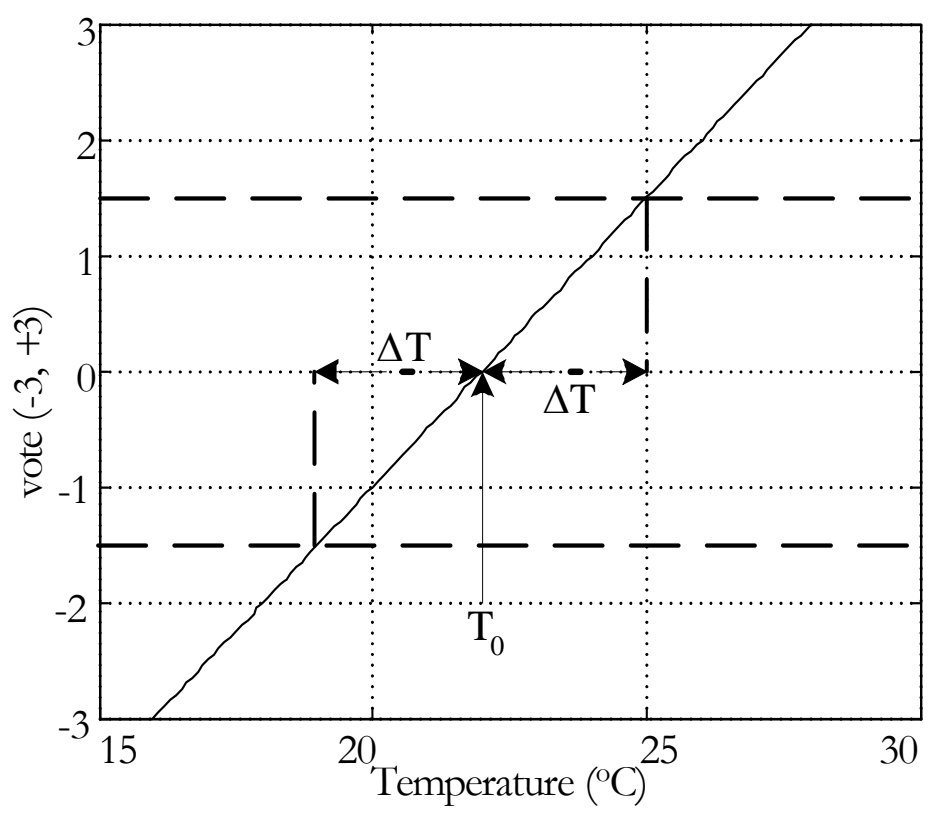

Figure 2.3 - Individual Votes

We assumed $\mathrm{T}_{0}$ and $\Delta \mathrm{T}$ are normally distributed random variables. We run the Monte-Carlo simulation for the mean values and standard deviations of $T_{0}$ and $\Delta T$ by generating 1000 individuals of a population at each simulation step. We validated these individual equations by comparing the cumulative population curve of the percentage of dissatisfied, which is the mean values of 1000 individuals' DID values like an example given in Figure 2.5, with the wellknown original PPD curve of ASHRAE Standard 55 (Figure 2.1). Figure 2.6 has given the closest match to the PPD curve with the root mean squared error of $\sim 1 \%$. We have found the mean value of desired temperatures is $24^{\circ} \mathrm{C}$, and the standard deviation of $1.2^{\circ} \mathrm{C}$. Figure 2.4 is a histogram of 49 occupants' neutral temperatures. 


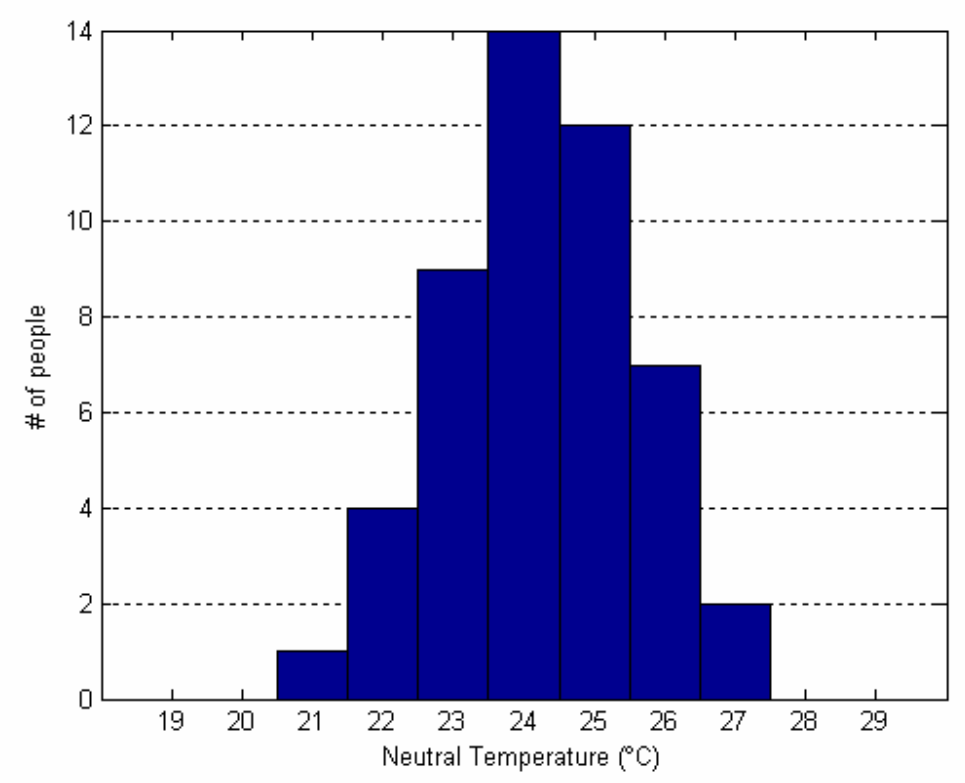

Figure 2.4 - Histogram of 49 occupants' neutral temperature

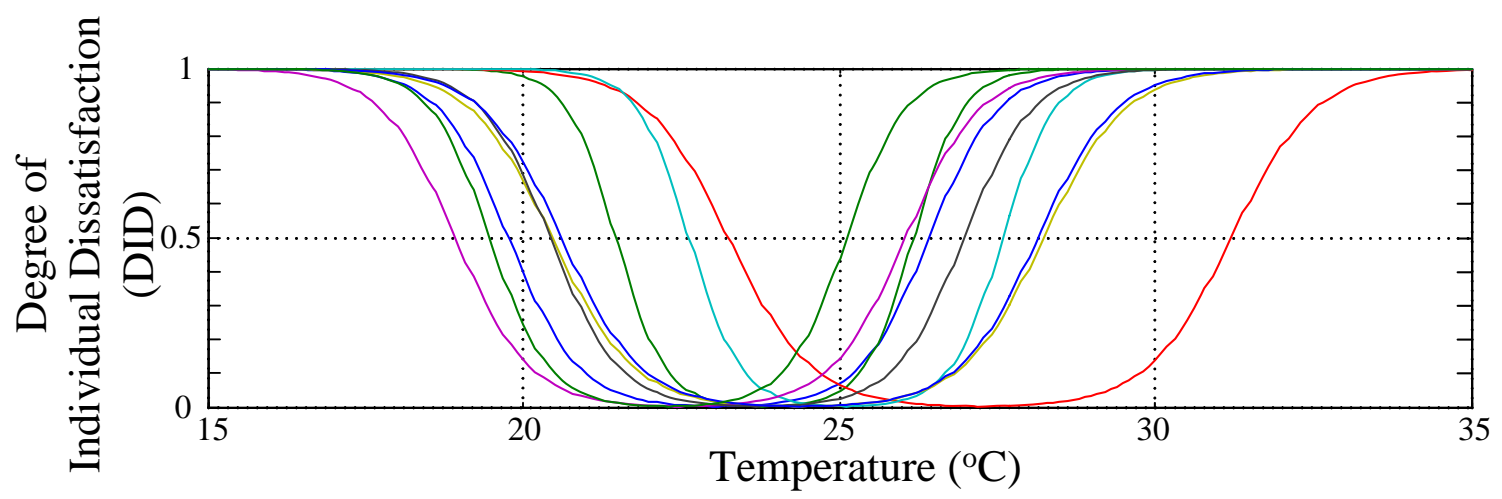

Figure 2.5 - An example of the DID curves for a given population 
Report No. DE-FG02-03ER63694-F1

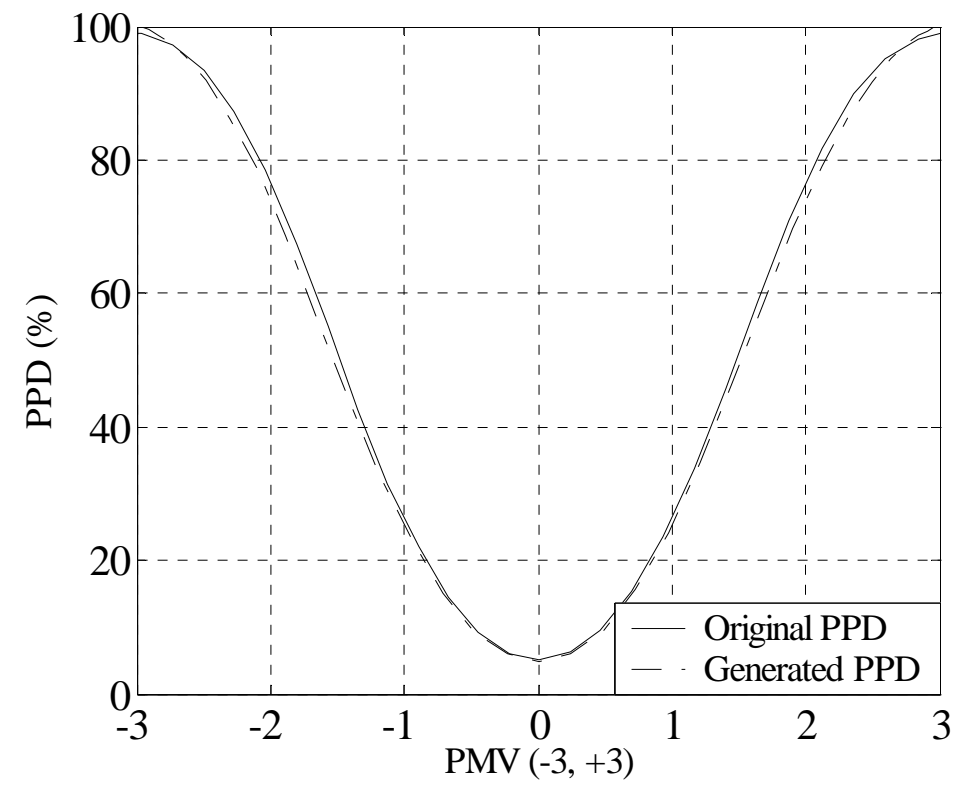

Figure 2.6 - Original and Generated PPD curves 
Report No. DE-FG02-03ER63694-F1

\section{Building Temperature Control}

Here we will discuss the previous building temperature control studies. Then we will introduce our approach to minimize energy consumption of building temperature control systems without sacrificing thermal comfort.

\subsection{Literature Review}

\subsubsection{Historic Perspective}

The studies about automatic control for building applications have been started by Butz with the invention of a thermostat in 1885 [40]. He called his simple device as damper flapper. The need of mixing air components was mentioned at the first ASHVE meeting in 1894 [41]. After this meeting, it has been started to control indoor environment with the inherent pneumatic control systems rather than the manual control like opening a window when the indoor was hot. This control system had become a primary control method of the building applications until 1970s.

The need of air temperature regulation was mentioned by Kinealy in 1903 [42]. He defined the indoor temperature as a function of occupant's activities, and suggested some temperature intervals according to the activity, and healthiness of the occupant. He also defined necessary conditions for temperature control. Jones proposed a method for controlling the airflow rate by using dampers in 1906 [43]. In 1912, Donnelly recommended night setback control methodology and optimum start time in order to conserve energy and improve comfort in his study, which became the first research related to energy conservation in environmental control systems [44]. Winslow and Maglott maintained the proper amount of ventilation air by 
Report No. DE-FG02-03ER63694-F1

measuring the carbon dioxide $\left(\mathrm{CO}_{2}\right)$ level in the space, and they also recommended a satisfactory level of $\mathrm{CO}_{2}$ amount at their study made in a large office building [45].

The researches related to energy conservation had been increased with the first energy crises, which lasted three years in 1914. From the mid-1920s to mid-1930s there had been many control systems introduced [41]. Before the used of electrical control devices and the thermocouples for measuring temperature instead of pneumatic control devices, control was restricted to on/off control. Parmalee and Huebscher ignited the studies about temperature and space control of HVAC systems by proposing the use of thermocouples [46]. Because of the continuous increase in the energy cost after the second energy (oil) crises in 1973, the demand of energy conservation had been increased. With this energy crisis, the night setback control came back with the day setback function. The studies about the energy savings had been accelerated with the introduction of the first computers with advanced technology. Shavit proposed the first energy conservation algorithm by using a microprocessor-based system in 1976 [47]. He proposed the annual savings results of the fan system optimization algorithm such as enthalpy control and reset of cooling coil from the zone of the highest demand with floating space temperature [48].

There have been a variety of studies related to HVAC systems in the literature. Many advanced control strategies have been applied to HVAC systems such as the conventional proportional-integral-derivative (PID) control, auto-tuned PI or PID control, predictive model control, optimal control, decentralized control, fuzzy control, neural networks, genetic algorithms, and evolutionary algorithms. We can classify them into three subcategories: HVAC System Performance related to temperature or airflow control, Comfort Control, and Energy Conservation. We will discuss these categorizes at the following sections. 
Report No. DE-FG02-03ER63694-F1

\subsubsection{Temperature and Airflow Control}

After the introduction of the direct digital control (DDC), PID control became the competitor of the traditional pneumatic control. But the PID control embedded on a microprocessor was not accepted immediately [41]. Today, a microprocessor is the center of every control strategies.

In the literature, there have been many control studies related to HVAC system performance. But mostly, HVAC systems have been treated as temperature control problems. Cherchas et al. proposed an optimal DDC algorithm by using a PID in order to control dry bulb temperature in a single zone space [49]. They simulated their algorithms with the bilinear mathematical model of an HVAC system. Nesler [50] and Pinella et al. [51] introduced a selftuning controller in 1986. While Kasahara et al. [52] and Qu et al. [53] introduced a PI and PID controller, respectively by assuming HVAC system as a first order plus dead time system, Wang et al. used second order plus dead time function for modeling HVAC system to simulate their multivariable PID controller [54]. In order to adjust PID parameters automatically, a variety of methods have been applied for temperature control such as genetic algorithms (GA) [55] and pattern recognition approach [56]. Xu et al. concerned about the airflow control rather than the temperature by applying a different technique for tuning PID parameters [57], while $\mathrm{Bi}$ et al. used their PID to control both airflow and temperature [58].

Since PID has to be tuned for other applications, some model-based predictive control methods have been implemented for temperature and airflow control. In these studies, in order to estimate future state of the HVAC process, researchers used a method for modeling HVAC system such as a system identification technique [59], neural network [60][61] or fuzzy logic [62][63][64][65]. While some used state space equations of HVAC system for simulation, some used real systems. 
Report No. DE-FG02-03ER63694-F1

Fuzzy logic also has been used in some applications to control HVAC systems directly . Lea et

al. introduced a fuzzy logic controller for regulating the airflow to the separate zones[66][67]. Results of the model-predictive control strategies have been judged against those of the conventional PI or PID control to show the improvements of the their methods.

There was no consideration of comfort and energy conservation in all these studies; the only aim has been controlling temperature and airflow consistently.

\subsubsection{Comfort Control}

As mentioned in the previous section, most HVAC systems are considered as air temperature regulator, and they don't account for the thermal comfort of the population. Although it is obvious to make the occupants comfortable rather than the air temperature control, there haven't been a variety of studies about thermal comfort control unlike temperature and airflow control due to its limitations. Researchers mostly developed control systems, which depend on the well-known Fanger's PMV index for the prediction of thermal sensation. Since PMV is based on the average thermal sensation of the occupants, which means the thermal sensation of the whole population, the appropriate parameters of thermal sensation for the population may not be suitable for some occupants among that population. Due to the individual biological differences it will be unattainable to satisfy everyone in the same environment at the same time. Because of this limitation of PMV index, our thermal comfort approach has been based on individual preferences. PMV has been derived as a comfort equation in terms of six variables, which are metabolic rate (activity level), clothing insulation, air temperature, radiant temperature, air velocity, and relative humidity. Since only iterative solutions exist for these parameters, it is hard to apply this index to the real applications. All the environmental 
Report No. DE-FG02-03ER63694-F1

measurements of these six variables have to be done on the occupant, which is not applicable for real time control problems [68].

Even though it has some limitations, On/Off, PID, and fuzzy logic control, which applied to comfort index control, has been found in the literature. Zhou et al. introduced a PID control strategy of IAQ with if-then rules [69]. They developed a time-dependent iterative model of $\mathrm{CO}_{2}$ concentration in the zone.

Thermal comfort has been concerned as a fuzzy concept in some studies rather than the crisp standardized comfort zone. Dounis et al. introduced a fuzzy logic controller (FLC) using a set of fuzzy rules for PMV control [70]. They used calculated PMV and ambient temperature values as controller inputs in order to determine auxiliary heating, cooling and opening angle of the windows. They emulated the indoor temperature with the mathematical models of heat flux through window, auxiliary heating, and heat transfer due to the ventilation. Their simulation results showed FLC could maintain the PMV index between -0.2 and +0.2 range for two seasons, January and June. Dounis et al. also presented their results with additional mathematical models at [71]. They also used the mathematical models of heat transfer due to convection, and indoor air's relative humidity for indoor temperature. In addition to previous study, they also modeled the wall temperatures with radiative heat exchange, and heat flow through walls, and emulated the outdoor climate such as temperature, wind speed, and outdoor relative humidity. Gouda et al. compared the comfort control strategies based on PID and FLC [72]. Their results indicated that the performance of FLC based on PMV was better than that of PID. They simulated the HVAC system and its controllers in MATLAB/Simulink. Chu et al. preferred to use the effective temperature (ET ${ }^{*}$ ) rather than the PMV index [73], since it is a simpler index, which is based on the combination of humidity and temperature, than PMV, which is based on the six different environmental variables. They 


\section{Report No. DE-FG02-03ER63694-F1}

proposed two separate FLC based on the estimation of the least enthalpy. While one of their controllers infers the action from indoor temperature and relative humidity, the other one does it from the outdoor temperature and the current ET* They compared the experimental results of FLC with those of the thermostat controlled another room. While LEE-based FLC provided the average thermal comfort level of $99.84 \%$, the conventional temperature control did those of $87.84 \%$.

Some of the comfort control studies have been considered with the energy conservation together. For instance, Gouda et al. [72] and Chu et al. [73] presented energy conservation results while providing thermal comfort. Gouda et al. achieved $20 \%$ energy savings by using FLC with respect to the conventional PMV based PID controller. Chu et al. reached 35\% savings with FLC relative to the conventional temperature control. These studies will be discussed in detail with those related to the energy consumption in the next section.

All these studies mentioned above used either PMV or ET ${ }^{*}$ indices, which are the populationbased thermal sensation indices. Even when they provide acceptable PMV or ET ${ }^{*}$ ranges, there will always be some occupants dissatisfied due to the individual differences. Federspiel and Asada proposed an approach based on a particular occupant rather than the average person under the assumption of a linear relationship between environmental variables and thermal sensation index [34]. Since it is based on a specific occupant, their comfort criterion has been the closest one to ours from the comfort point of view. Their linearly parameterized user-adaptable thermal sensation index was derived based on the steady-state heat transfer between the human and the nearby environment like PMV. They estimated the parameters of their index with the acquired thermal sensation ratings of the occupant by using constrained recursive least squares algorithm. After the estimation of the parameters, they showed the 
Report No. DE-FG02-03ER63694-F1

simulation results of on/off and PID control of their index by using a lumped parameter model of HVAC system in heating and cooling mode.

Unlike our approach, they used a binary satisfaction criterion like Fanger such that they considered people, voting $-3,-2,+2$, and +3 , dissatisfied. While a person might be comfortable within a certain range of temperature, he or she will be dissatisfied even a tenth of a degree of outside the range with this comfort criterion. In addition to our linear comfort index, which is an individual vote (Figure 2.3), we proposed a fuzzy satisfaction curve (Figure 2.2) as a function of thermal index instead of binary satisfaction. We generated these curves for individual thermal preferences.

\subsubsection{Energy Conservation}

The second energy crisis in 1973 had increased the importance of energy conservation tremendously in HVAC system control applications. While only energy savings has been considered by itself in some studies, the others have concerned both energy conservation and thermal comfort together as we did. One of the studies was traditional night setback control to reduce energy consumption. It returned again after its introduction in the fist energy crisis at the World War I such as Schade's recommendation [74]. By lowering the space temperature at night, he achieved from $5.5 \%$ to $20 \%$ savings depending on the climate in his computer simulation. There have been many different strategies applied to HVAC system energy conservation with advancing technology such as optimal control, PID, fuzzy logic control, and evolutionary algorithms.

Some researchers took in hand optimal control with a few variables by maintaining a fixed temperature set point to reduce energy consumption in HVAC systems. Nizet et al. [75], Boyens et al. [76], Ke et al. [77], and House et al. [78] tried to minimize a cost function 
depending on only energy consumption of HVAC system without considering thermal comfort. House and Smith took into consideration the reducing of energy consumption with thermal comfort in their studies by adding Fanger's PMV index into their cost function. They tried to find the optimal set points of temperature, airflow, and PMV under time varying thermal load conditions [79], and in another study, they determined the night setback strategy by optimal controller [80]. They used a nonlinear programming technique in their studies. Simmonds also minimized a cost function based on energy and PMV in his study [81]. Unlike these studies, Liu and He proposed an optimal control strategy based on Federspiel's useradaptable comfort index instead of PMV and power consumption by using steepest descent optimization [82]. All these optimal control studies tried to find optimal setpoints to minimize energy consumption. However, Wright and Hanby concerned to minimize energy consumption during the design and installation process of HVAC system [83]. They suggested the optimum selection of system components and operating points of the components together.

In addition to optimal control strategies, auto-tuned PID based control techniques also used to reduce energy consumption. PID wasn't accepted immediately after its introduction in 1981. However, it has been widely used in this area today due to its simplicity and low cost like onoff control. PIDs are mostly used for reducing energy consumption without thermal comfort consideration. Kaya et al. proposed an optimal PI control to find out the energy savings in HVAC equipped space [84]. So et al. proposed a self-tuning PID controller for air handling unit (AHU) of HVAC system by using recursive least squares system identification to reduce energy consumption [85]. They achieved $6.62 \%$ energy savings when comparing the simulation results of AHU computer model with those of a well-tuned PID controller. Kulkarni and Hong tried to improve thermal comfort and reduce energy consumption with 
respect to two-position (on/off) control by using PI control [86]. They simulated the statespace building model in MATLAB/Simulink, they also modeled the outdoor temperature on a sinusoidal function. So et al presented a computer vision based control system in another interesting study [87]. They tried the find the number of residents, and estimated the variations in thermal load to assist the conventional PID controller. They proposed five control rules for handling the sudden rush and evacuations. By comparing the experimental results with PID alone, they succeeded in energy conservation with up to $10.7 \%$ savings. Some authors concerned PMV control with both saving energy consumption and improving thermal comfort. MacArthur suggested the use of PMV-based comfort control to maintain uniform comfort level, and to reduce power consumption [88]. He applied the PI control of PMV, and he reached $10.1 \%$ savings in his comparison of the computer simulation results with conventional on-off control. Yang and Su developed an intelligent control to adjust air velocity automatically and to maintain PMV index within comfort zone [89]. They built two separate rooms, which was installed their intelligent control, and the conventional temperature control, respectively in their field experiment. They achieved significant energy savings with respect to conventional control. Kolokotsa et al. and Calvino et al. introduced an adaptive fuzzy PID controller to reduce energy consumption, and maintain thermal comfort. Kolokotsa et al. used PMV, $\mathrm{CO}_{2}$ index, and luminance level as controller inputs [90]. After the performance of their simulations in MATLAB/Simulink toolbox, Sibil, they succeeded in the reduction of energy consumption up to $25-30 \%$ with respect to conventional PID and on-off controller. Calvino et al. proposed a fuzzy logic PID controller, which avoided the modeling of indoor environment and outdoor climate, in their study [91]. Their aim was to reduce fan power and keep the PMV index within the comfort zone. They used PMV error, and its derivative as FLC inputs. 


\section{Report No. DE-FG02-03ER63694-F1}

Since PID settings are not universally applicable, undesirable response and increased energy consumption may occur when it is applied to another system with different parameters. Fuzzy logic has also been used to reduce energy consumption by itself rather than modeling PID controller with fuzzy rules, and membership functions. Yamada et al. modeled the PMV index with neural network by using its six variables to estimate the current PMV value [92]. In order to reach target PMV value, they proposed a FLC, which input is PMV error, and output is temperature set point. They reached $18 \%$ savings in their simulation as compared with the conventional control system. Gouda et al. also simulated a fuzzy logic control of PMV by modeling the internal, and outdoor enthalpy and saturation pressure in MATLAB [72]. They reached 20\% savings with respect to the conventional PID control. Chu et al. proposed a fuzzy controller for thermal comfort and energy savings [73]. Unlike other studies, they preferred to use effective temperature $\left(\mathrm{ET}^{*}\right)$ as a comfort index rather than the PMV index. They proposed two separate FLC based on the estimation of the least enthalpy. While one of their controllers infers the action from indoor temperature and relative humidity, the other one does it from the outdoor temperature and the current ET* They compared the experimental results of FLC with those of the thermostat controlled another room. While LEE-based FLC provided the average thermal comfort level of $99.84 \%$, the conventional temperature control did those of $87.84 \%$. They also saved 35\% energy over the conventional control with FLC based on ET* Alcala et al. presented a solution to improve the performance of fuzzy logic controller by genetic algorithms [93]. They performed it to optimize the membership functions of FLC depending on different criteria such as energy consumption, occupants' thermal comfort, and indoor air quality. They implemented the FLC with PMV, difference between supply and room temperature, $\mathrm{CO}_{2}$ concentration, outdoor temperature, and HVAC system actuators such as valve position, fan speed, etc. Both simulated and experimental 
results showed $11.6 \%$ and $13.34 \%$ savings over the conventional on-off controller. Alcala et al. also proposed different strategy concerning both energy consumption and indoor comfort requirements by FLC with GA [94]. Genetic algorithm process was evaluated to perform rule weight derivation of linguistic fuzzy rules and their selections. They implemented the simulations of on/off, FLC alone, FLC considering rule weights (w), and FLC considering both rule weights and selections (ws) by using an HVAC model based on manufacturer data. Their comparisons of three FLCs with on-off control showed that significant savings on energy consumption while keeping the population within comfort zone. FLC resulted in neutral PMV with 9.5\% energy savings. Improved energy savings was achieved with proposed methodologies, which are FLCw, and FLCws, resulting in $13.21 \%$ and $14.05 \%$ savings over conventional control, respectively. However, while FLC considering rule weights resulted in 0.1 PMV, the other one did 0.6 PMV.

With the proceeding technology, evolutionary algorithms, such as genetic algorithms, etc., have also been applied to reduce energy consumption in HVAC system applications. Jin et al. proposed an approach based on the prediction of energy performance of HVAC system [95]. $\mathrm{CO}_{2}$ concentration of each zone, and outdoor air, supply air flow, temperature, and humidity, outdoor air temperature, and humidity have been used as input data. After the online estimation of the system energy performance, a genetic algorithm was used to find the optimal set point of outdoor air ratio of AHU by minimizing the energy increment function without thermal comfort consideration. Their results achieved the approximately $7.8 \%$ energy savings over the reset control. Lu et al. introduced a modified genetic algorithm to find set points of HVAC system to minimize overall system energy performance [96][97]. Adaptive NeuroFuzzy system has been used to model duct and pipe network. Their results achieved from 2\% to $12 \%$ energy savings over the fixed set point control for different cases such as optimal 
supply temperature vs. fixed one, etc. Fong et al. have applied an evolutionary algorithm by considering reset the suitable operating parameters would have the potential for energy conservation without sacrificing thermal comfort [98]. Evolutionary algorithm was used to setup a reset scheme of chilled water and supply air temperature for HVAC system in a local subway station. By just optimizing chilled water temperature, they reached almost same energy consumption as that of current settings. However, when they applied optimization to both settings yearly, and monthly, they achieved $2.86 \%$ and $6.74 \%$ energy savings, respectively over the existing settings. Nassif et al. preferred to use two objectives at the same time for real-time minimization of energy consumption and thermal discomfort in HVAC system [99]. The twoobjective genetic algorithm was performed with 70 zone temperatures, supply duct static pressure, and supply air temperature as design variables in the simulation of a simplified VAV system. They developed the mathematical models of fan, damper, and cooling coil. While one of the objective functions was to reduce energy usage of HVAC system, they employed predicted percentage of dissatisfied (PPD) instead of PMV alone to improve thermal objective function. The constraints were the limitations on the HVAC system operation such as minimum airflow rates of zone, and fan, and the design capacity of the components. Multiobjective genetic algorithm (MOGA) such as their study gives a set of optimal solutions rather than a one optimal solution, which is called Pareto-optimal set. Energy savings from $18.8 \%$ to $19.5 \%$ has been achieved while satisfying the minimum requirements of zone airflow and thermal comfort over the actual energy usage. They applied MOGA with an additional design variable, which is chilled water supply temperature of a simplified VAV system [100]. Determining optimal set points for an existing HVAV system based on PPD and energy consumption minimization by MOGA resulted in 19.5\% less energy consumption than the actual usage for 2 summer days. In another study [101], they reduced the number of design 
Report No. DE-FG02-03ER63694-F1

variables by using 5 zone temperature set points instead of 70 ones. Their MOGA result showed 16\% energy savings for 2 summer months while assuring the minimum requirements of zone airflow and thermal comfort over the actual energy consumption. MOGA has also been applied to HVAC system during the design process by Wright et al. [102]. They considered the design procedure of buildings as a multi-objective optimization problem. Their method identified the optimum pay-off between the occupants' thermal comfort and the energy cost of a building, which means the components of building thermal design. They simulated their method with 200 variables with set points and the sizes of the system components by using a fan model derived from the manufacturer data. Their results of Pareto-optimal set of optimal solutions based on PPD and energy cost of building showed a large potential for building optimization problems.

\subsubsection{Individual comfort}

A variety of studies about HVAC systems, temperature control systems, and thermal comfort have been reviewed so far. While some authors considered only the HVAC system performance, some just considered thermal comfort of occupants, and energy consumption. We mostly focused on the studies related to both thermal comfort and energy consumption of HVAC system. However, It has been obvious that the common denominator of all these studies is that they are concerned with the comfort of the majority of a population, by using in general Fanger's predicted percentage of dissatisfied which is related to his PMV equation. Thus far, there have been no studies that optimize the comfort level of all individual occupants as well as energy consumption. 
Report No. DE-FG02-03ER63694-F1

\subsection{Our Approach}

Improvement of the thermal satisfaction of a population in a building with control approaches does not take into consideration of individual thermal satisfaction. However, some of the occupants might have persistent dissatisfaction in a space where $90 \%$ of the population is satisfied. Our approach prevents intolerable individual dissatisfaction, while keeping the population PPD at or below 10\%. In order to do this, we developed a model of personal dissatisfaction (Figure 2.2) that generates population results that match the well-established PPD-PMV curve (Figure 2.6) [103]. We will discuss the building model, and optimization procedures, which we performed in this study.

\subsubsection{Building and Weather Model}

Our approach focuses on a typical single story office building, which we modeled with a thermal circuit network coupled with a typical HVAC system consisting of a heat pump and a furnace. The typical $600 \mathrm{~m}^{2}$ office building, which is divided into $7 \times 7$ zones 0 , was modeled using a temperature-bin-based lumped parameter approach in MATLAB [104]. Each zone, which corresponds a node in a thermal circuit network, will be considered as a well-mixed zone of constant temperature. Continuing with the electrical circuit analogy, each node in the network is connected to the other nodes and outside node via a thermal resistance. Typical resistance values of common building materials have been implemented for interior walls, exterior walls, and windows. 
Report No. DE-FG02-03ER63694-F1

Table 3.1 - Thermal Resistance Values

\begin{tabular}{|c|c|c|c|}
\hline Type & Notation & Value & Notes \\
\hline Interior wall & $\mathrm{r}_{\text {iwall }}$ & $0.392 \mathrm{~m}^{2} \mathrm{~K} / \mathrm{W}$ & $\begin{array}{l}\text { Gypsum wall board wood frame } \\
{[105]}\end{array}$ \\
\hline $\begin{array}{l}\text { Exterior wall } \\
\text { (and roof) }\end{array}$ & $\mathrm{r}_{\text {ewall }}$ & $2.47 \mathrm{~m}^{2} \mathrm{~K} / \mathrm{W}$ & Insulated wood frame [105] \\
\hline Exterior window & $r_{\text {window }}$ & $0.353 \mathrm{~m}^{2} \mathrm{~K} / \mathrm{W}$ & Double paned air-filled window [38] \\
\hline $\begin{array}{l}\text { Inside convective heat } \\
\text { transfer coefficient }\end{array}$ & $\mathrm{h}_{\mathrm{in}}$ & $3.06 \mathrm{~W} / \mathrm{m}^{2} \mathrm{~K}$ & $\begin{array}{l}\text { Common building energy simulation } \\
\text { indoor value [38] }\end{array}$ \\
\hline $\begin{array}{l}\text { Outside convective } \\
\text { heat transfer } \\
\text { coefficient }\end{array}$ & $\mathrm{h}_{\text {out }}$ & $17.78 \mathrm{~W} / \mathrm{m}^{2} \mathrm{~K}$ & $\begin{array}{l}\text { Common building energy simulation } \\
\text { outdoor value [38] }\end{array}$ \\
\hline
\end{tabular}

Each occupied office has been considered having three different sources of internal heat generation: a computer, task lighting, and an occupant himself. Figure 3.1 shows a cutout example of a fraction of a thermal circuit network. Zone $i$ (at temperature $T_{i}$ ) is connected to three neighboring zones (at temperatures $T_{i-1}, T_{i+1}$, and $\left.T_{i+n}\right)$ by a proper resistance value $\left(R_{i n}\right)$ for interior wall. It is also connected to the outside (at temperature $T_{0}$ ) by a proper resistance for exterior walls $\left(R_{o u t}\right)$. Each of the various zones will be connected to each other in the building and the outside temperature in this way. 


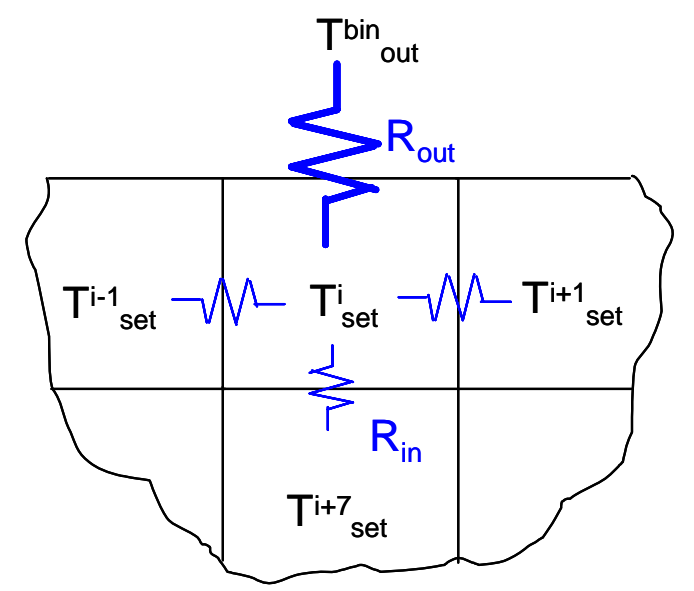

Figure 3.1 - Example of thermal network circuit around zone i

The outside temperature was modeled using temperature bins extracted from the Typical Meteorological Year (TMY2) weather files [106], which stores the 30-year average weather data such as temperature, solar radiation, wind speed, etc. Hourly temperature data, which is 8760 hours per year, was used for 15 different cities in the US representing different climate zones (Figure 3.2) [107]. The building HVAC sizing varied in each city depending on the restriction on design temperature to prevent same building model for each city. 


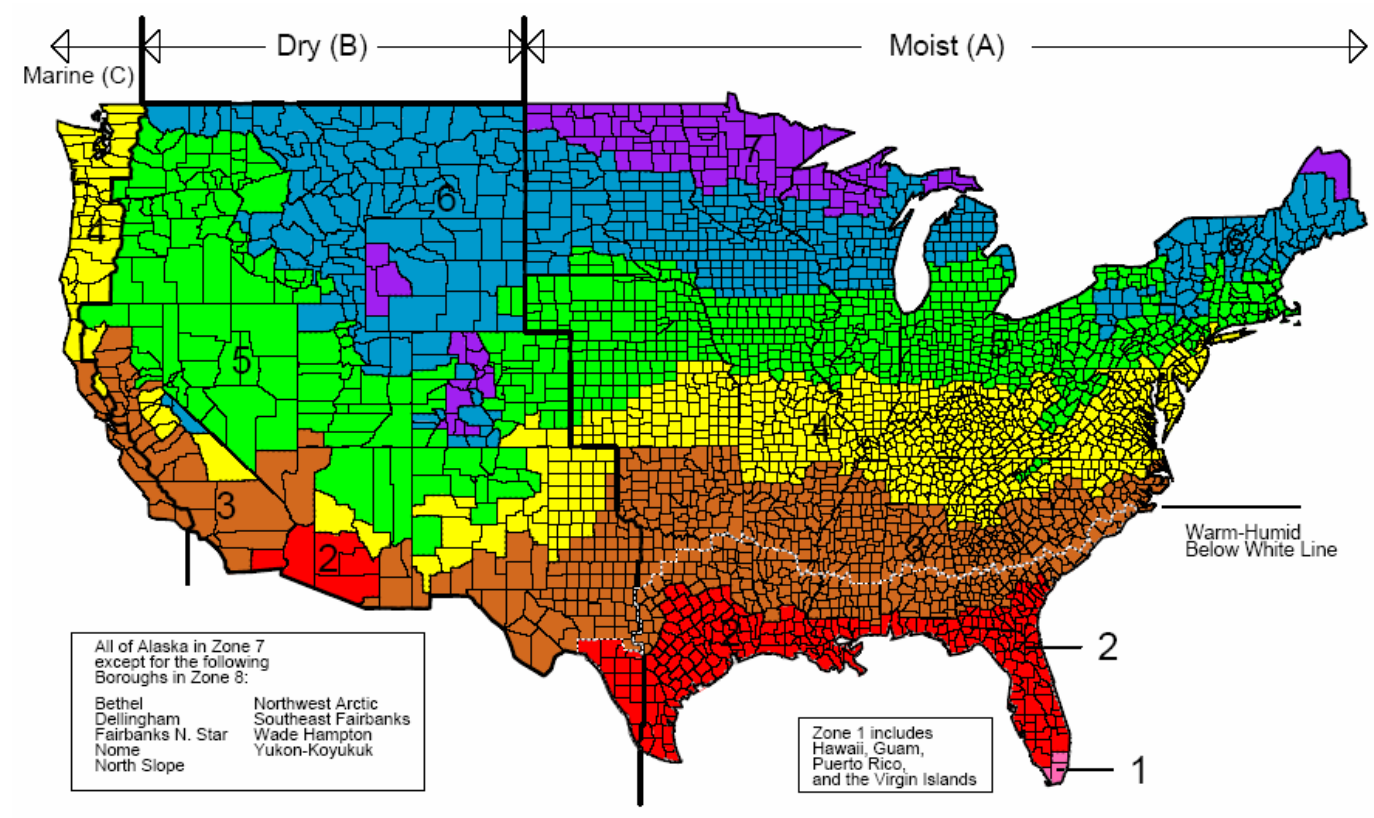

Figure 3.2 - Map of DOE US climate zones

\subsection{2}

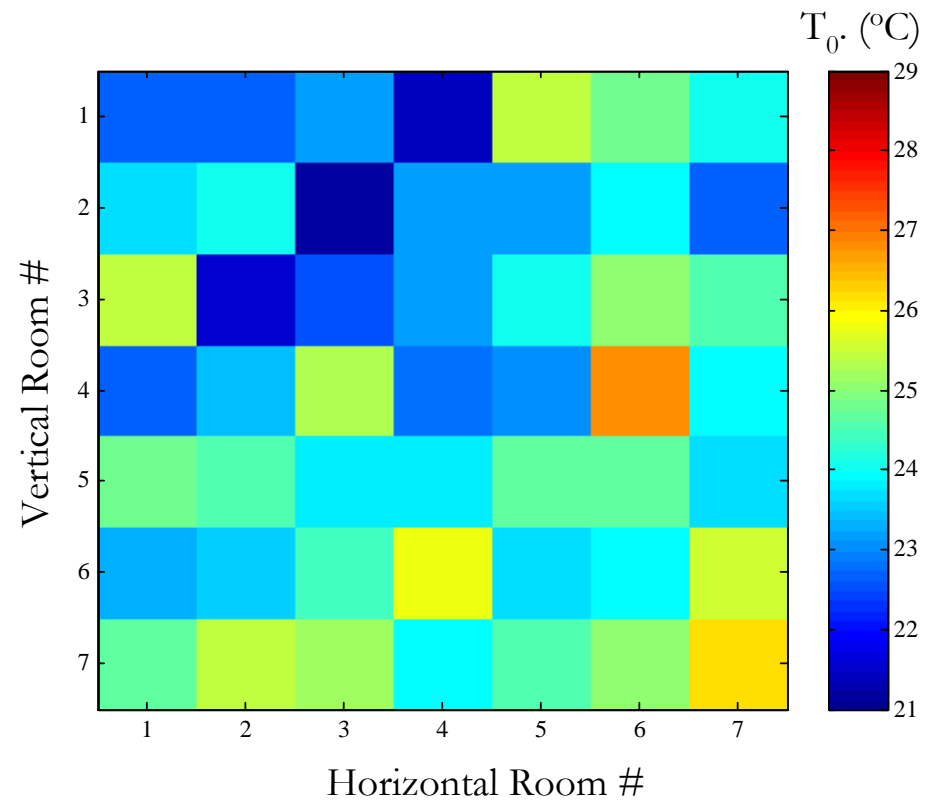

Figure 3.3 - Example of desired temperatures of randomly generated 49 individuals, HIYW 
Report No. DE-FG02-03ER63694-F1

\subsubsection{Optimization}

In this study, we consider satisfaction of each occupant, and we wanted to satisfy individual comfort needs. In order to do that, it is easy to give them a right to adjust their own environments, which is called HIYW here. Figure 3.3 is an example of HIYW system, which is personalized HVAC system. Each office consists of a thermostat in this system for getting exactly what individuals would like by adjusting his/her own thermostats. However, it requires more energy than the current systems do. By taking advantage of individual comfort range in temperature scale, energy consumption has been minimized. Our approach employs a gradient-based scheme to reduce energy consumption by varying office temperatures within an acceptable comfort range while keeping the dissatisfaction of overall population less than $10 \%$ PPD. As we mentioned before, only PPD constraint doesn't guarantee the satisfaction of each individual. While $90 \%$ population is thermally satisfied, some occupants might be miserably dissatisfied at the same environmental conditions. In order to prevent this miserable dissatisfaction for any occupant in a given population, additional constraint has been utilized for occupants' comfort. Each individual dissatisfaction was restricted to the DID level of $20 \%$. Desired temperatures $\left(\mathrm{T}_{0}\right)$ of each individual have been employed as initial conditions. We called it "Optimized HIYW". Figure 3.4 is an example of optimal temperature settings of the example population, given in Figure 3.3, at a given ambient temperature bin. Energy consumption has been minimized by varying occupants' temperature settings within a tolerable range, $20 \%$ DID, while keeping the thermal comfort requirement of the population, ASHRAE Standard 55-2004 [5]. Figure 3.5 shows Degree of Individual Dissatisfaction level of 49 occupants while providing them optimal temperature settings (Figure 3.4). It is also shown that there is no occupant over 20\% DID by Figure 3.5 . 


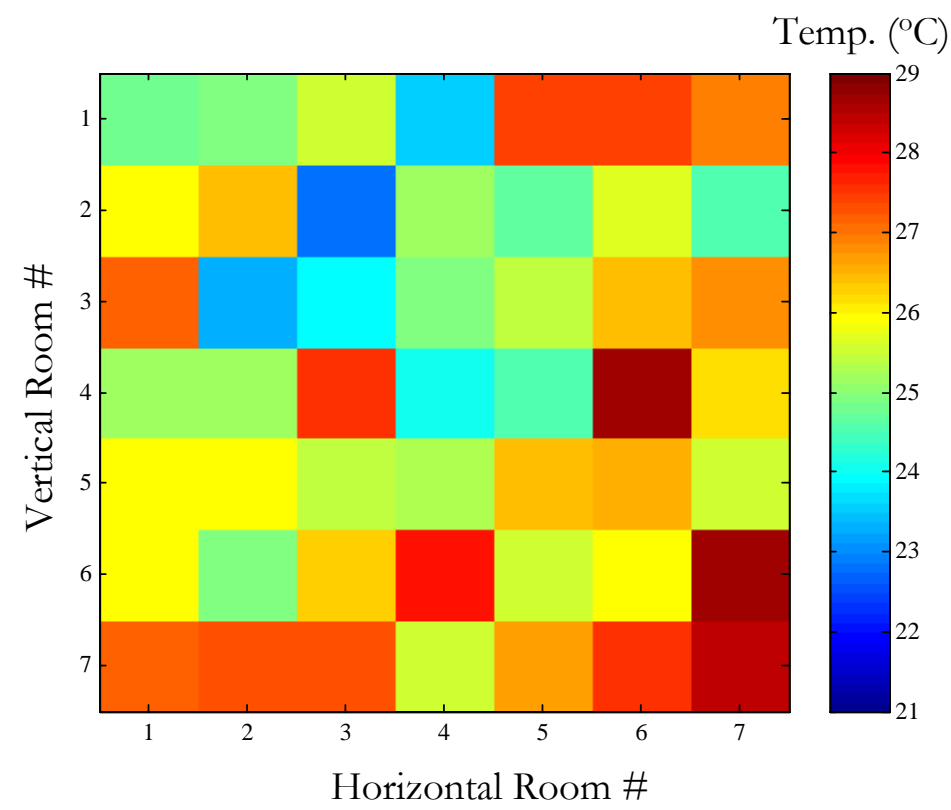

Figure 3.4 - Example of 49 optimal temperature settings of optimized HIYW

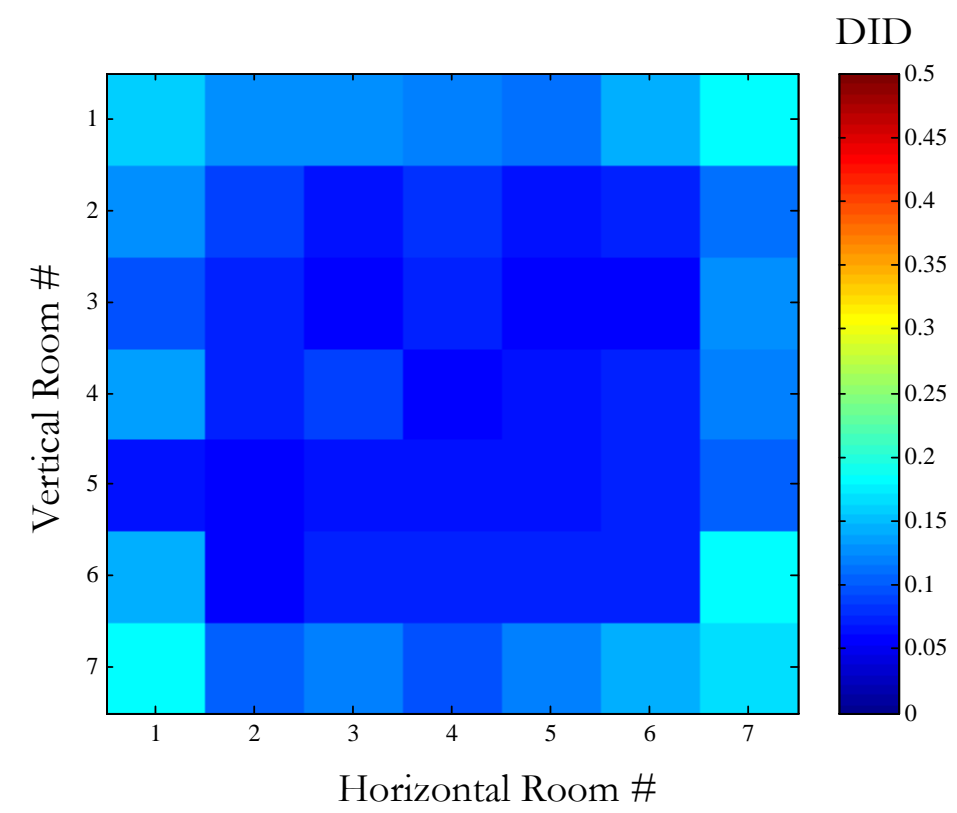

Figure 3.5 - Degree of Individual Dissatisfaction Levels of 49 occupants from the Figure 3.3

In the simulations, 50 different populations have been obtained by our Gaussian distributed functions of desired temperatures $\left(T_{0}\right)$, and temperature ranges $(\Delta \mathrm{T})$. These individuals could 
be assigned randomly to the offices in the building. Figure 3.3 also is an example of desired temperatures of randomly distributed occupants in a single story $7 \times 7$ building. Average of the optimal results of these 50 populations has been compared with the conventional HVAC control as baseline model. Since it provides uniform thermal conditions (Figure 3.6) over the building through small number of thermostats, the conventional control is called OSFA here.

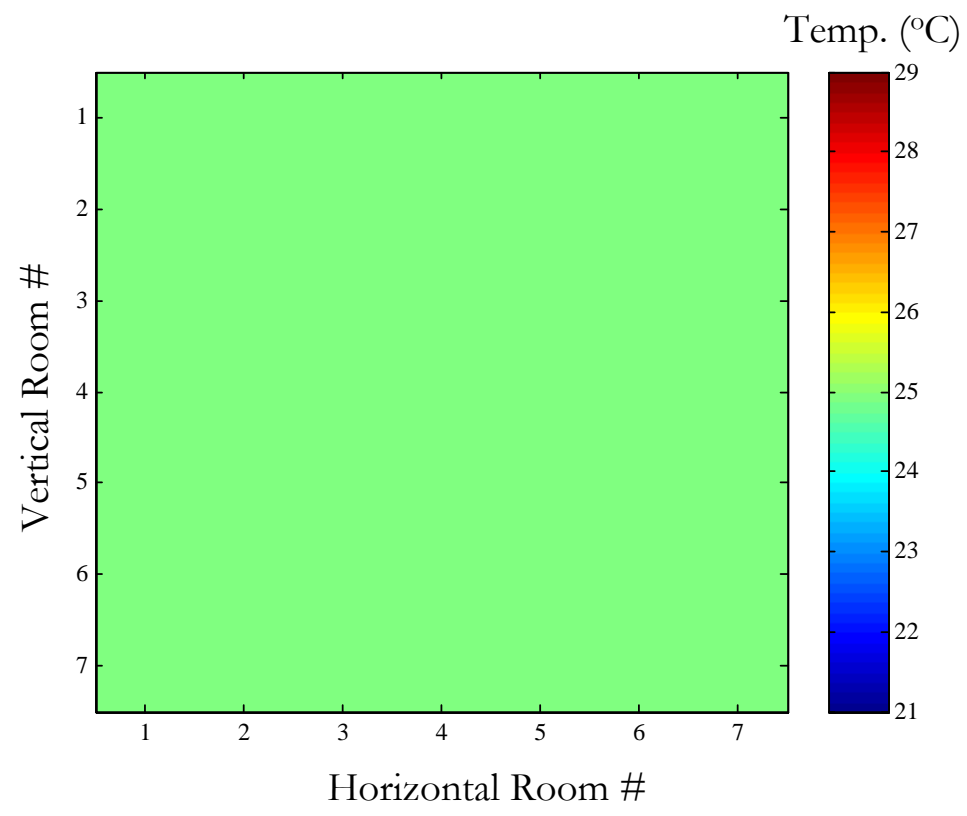

Figure 3.6 - Example of uniform temperature distribution of OSFA

Our base line model has three types of zones: interior (center), perimeter, and corner zones to provide uniform temperature distribution over the building for the case of the conventional OSFA control as shown in Figure 3.7. OSFA has three thermostats in three offices that control the other 46 remaining offices as well. 


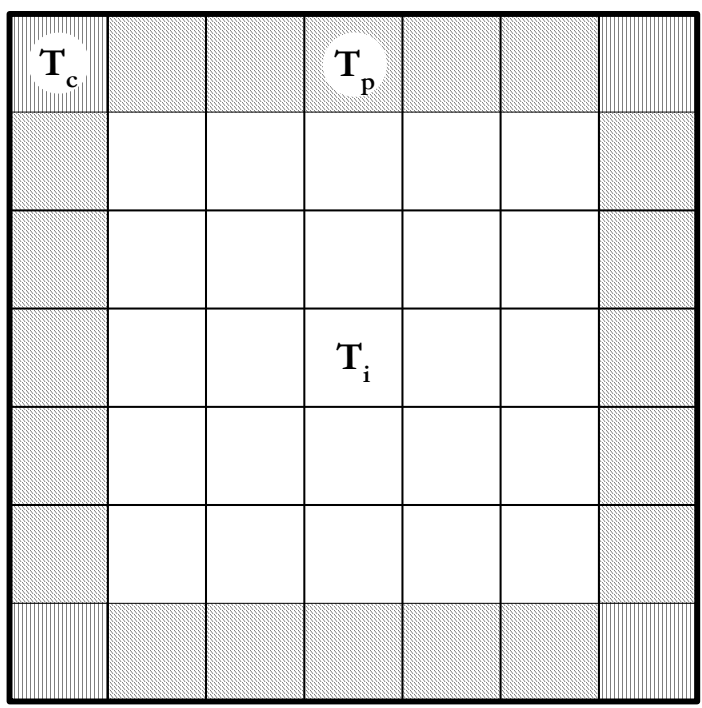

Figure 3.7 - Thermostat placements, and three (Corner, Perimeter, and Interior) zones of OSFA

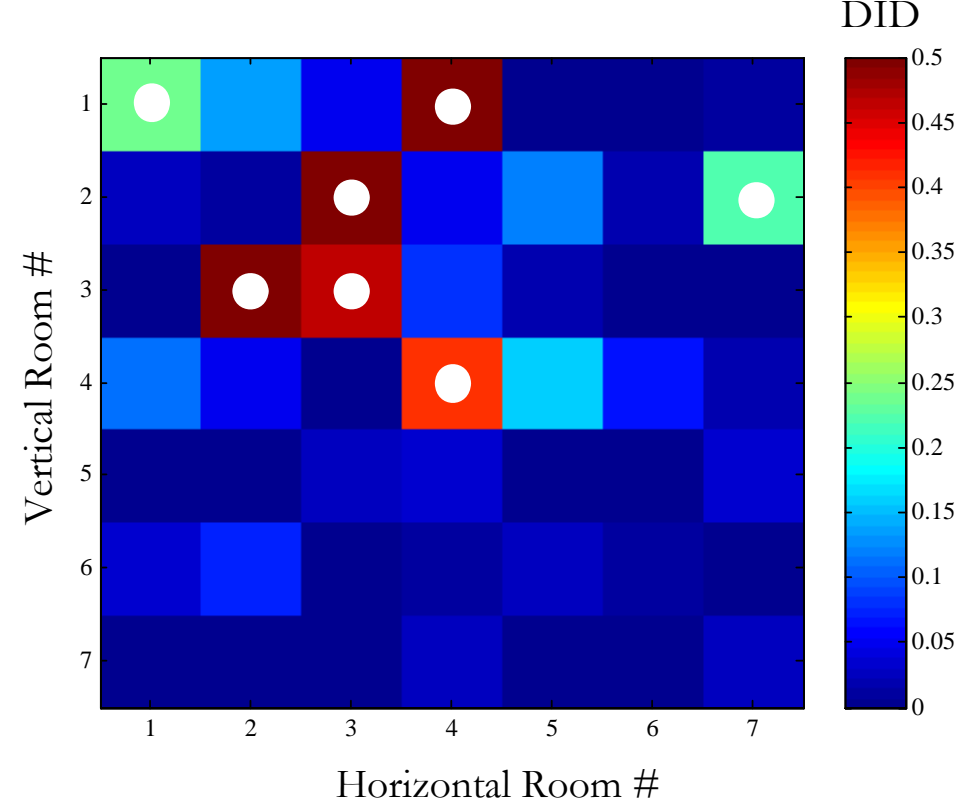

Figure 3.8 - DID level of occupants in OSFA case for the temperature setting in Figure 3.6

In order to make fair comparison, we also optimized conventional OSFA system while keeping the population under 10\% PPD. Unlike optimized HIYW, OSFA might provide dissatisfaction level of over $20 \%$ for some occupants when it provides optimal uniform 
temperature setting for the overall building. Figure 3.8 is an example of DID levels of the occupants in OSFA case. White dots represent the dissatisfied occupants over 20\% DID in this figure. Red colors show the DID level of over $50 \%$.

We compared the both optimization results based on the yearly energy consumption. Total yearly energy consumption has been determined by using both optimal energy consumption results for OSFA and HIYW, and extracted number of hours from TMY2 weather files for each of 32 outside temperature bins from $-47^{\circ} \mathrm{C}$ to $+46^{\circ} \mathrm{C}$. Energy consumption has been optimized to adjust the thermostat set point temperatures by utilizing "fmincon" function in MATLAB optimization toolbox [108].

\subsubsection{Fuzzy Logic Approximation}

Our approach, similar to other global optimization approaches, requires information acquired from all offices and cubicles, while providing a control strategy that brings energy consumption, individual comfort, and population comfort together. The optimization employs the personal satisfaction curves of all occupants in order to seek an optimal solution of temperature settings in each office. In order to do that, gradient-based optimization needs full central sensor connectivity from each office. Any thermostat that does not work properly can cause the whole system to operate unsatisfactorily. Last, but not least, if there is any change in the distribution of the individuals in a building, say due to an illness or other absenteeism, the optimization process needs to be repeated for the system to work as designed.

In order to circumvent these disadvantages and to improve the applicability of this approach in practical situations, we propose using a fuzzy logic approximation approach, with simplified sensor connectivity, and with generalized principles of the gradient optimization 


\section{Report No. DE-FG02-03ER63694-F1}

results rather than a literal application of optimization. Fuzzy logic approximation utilizes the temperature settings from the neighboring offices only, as shown in Figure 3.9.

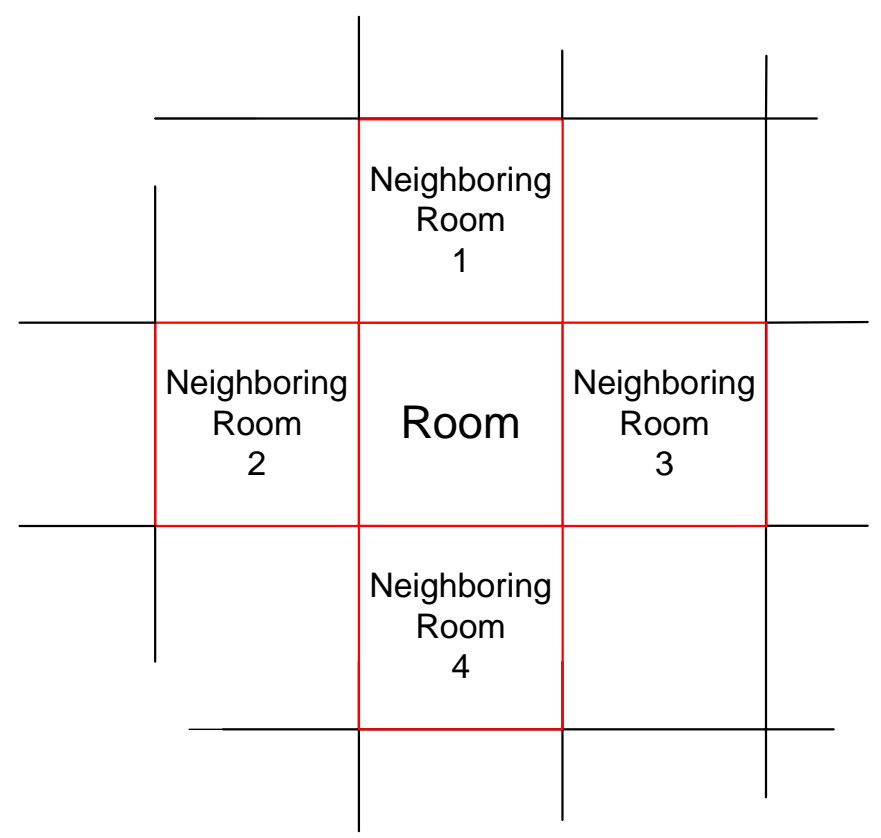

Figure 3.9 - Sensor communication requirements of fuzzy logic approximation

Simplifying sensor connectivity with fuzzy logic also reduces the computational complexity of the system. For example, while gradient-based optimization gives the results for a city within 10 to 15 minutes, it takes less than a second with our fuzzy logic approximation. If any occupant's preference or location changes, the optimization of whole system should be repeated with new settings. Another advantage of using fuzzy logic with our model is that there is no need for the optimization of complete system for varying preferences or thermal conditions with fuzzy logic approximation. It generalizes the gradient-based optimization results by using less sensor connectivity. It is easy to retrofit in an existing building. By using "genfis2" function in MATLAB fuzzy logic toolbox [108], we designed a Takagi-Sugeno type fuzzy inference system (FIS) [109]. However, other statistical modeling methods, including a variety of fuzzy logic and neural network methods can be used instead of this specific tool. 
We considered the buildings having three types of zones: corner, perimeter, and interior zones. Outside temperature mostly affects corner, and perimeter offices, while interior ones are mostly affected by heat exchange with the neighboring offices. Due to this kind of the buildings' characteristic, we generated three different FISs for the corner, perimeter and interior zones. In order to estimate the optimal temperature set points of each office, which is an output of FIS, four neighboring rooms' and its own temperature set points have been used as inputs for our interior FIS (Figure 3.9). In order to generate FIS of perimeter offices, the temperature set points of three neighboring offices, and itself, and the outside temperature have been used as inputs (Figure 3.10).

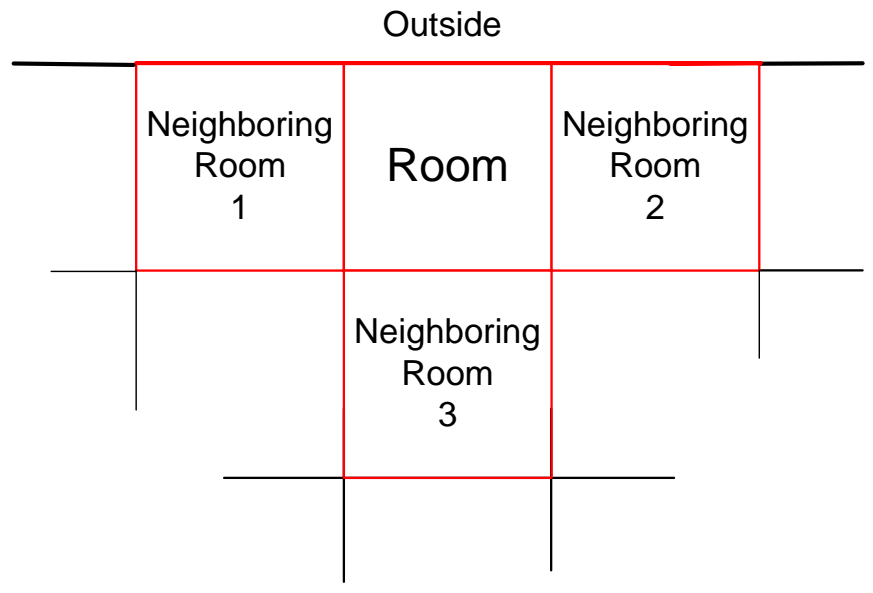

Figure 3.10 - Sensor connectivity for perimeter FIS

Unlike perimeter offices, FIS of corner offices uses two-neighboring temperature set points and outside temperature as shown in 


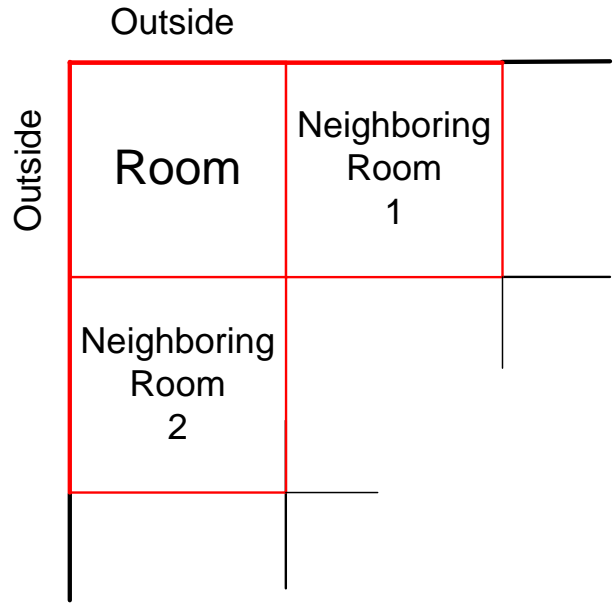

Figure 3.11 - Sensor connectivity of corner FIS

The results of gradient-based optimization have been imitated by using fuzzy logic. The energy consumption of 50 generated populations for each of 32 outside temperatures in three different cities, representing three different climate regions in the US, has been minimized by gradient-based scheme while keeping the population at 10\% PPD, and the individuals at 20\% DID constraints. These cities are Phoenix, AZ (hot-dry), San Francisco, CA (warm-marine), and Chicago, IL (cool-humid). Desired temperatures and these optimal temperature settings, which are results of gradient-based optimization, have been used to train our FIS for three zones of a building. Fuzzy approximation has been implemented to both train (50 populations), and test (10 different populations) data. Thermal satisfaction criteria and energy consumption results, which were found in gradient-based scheme, have been achieved by this suboptimal system approximation. 
Report No. DE-FG02-03ER63694-F1

\section{Results and Conclusion}

\subsection{Improved Individual Thermal Comfort with Reduced Energy Consumption}

The concern about increased energy consumption when providing individual thermal comfort has prevented the adoption of individual thermal control in a large majority of buildings. This study has shown that, it is possible to provide improved thermal comfort to all occupants of a building, not only without any increase in energy consumption, but even with some energy savings.

More specifically, in this study a methodology called Optimized "Have It Your Way" (HIYW) has been developed. This methodology takes advantage of an individual's region of insensitivity to small deviations from his/her preferred temperature setting, and minimizes the annual energy consumption of the temperature control in a building subject to a maximum dissatisfaction level constraint for all individual occupants. A straightforward gradient based optimization, as well as a fuzzy logic generalization of the underlying principles of the optimum solutions have been used as alternative implementations. Both results have been compared with a traditional "One Size Fits All" (OSFA) solution to demonstrate their improvements in both thermal comfort and energy consumption. 


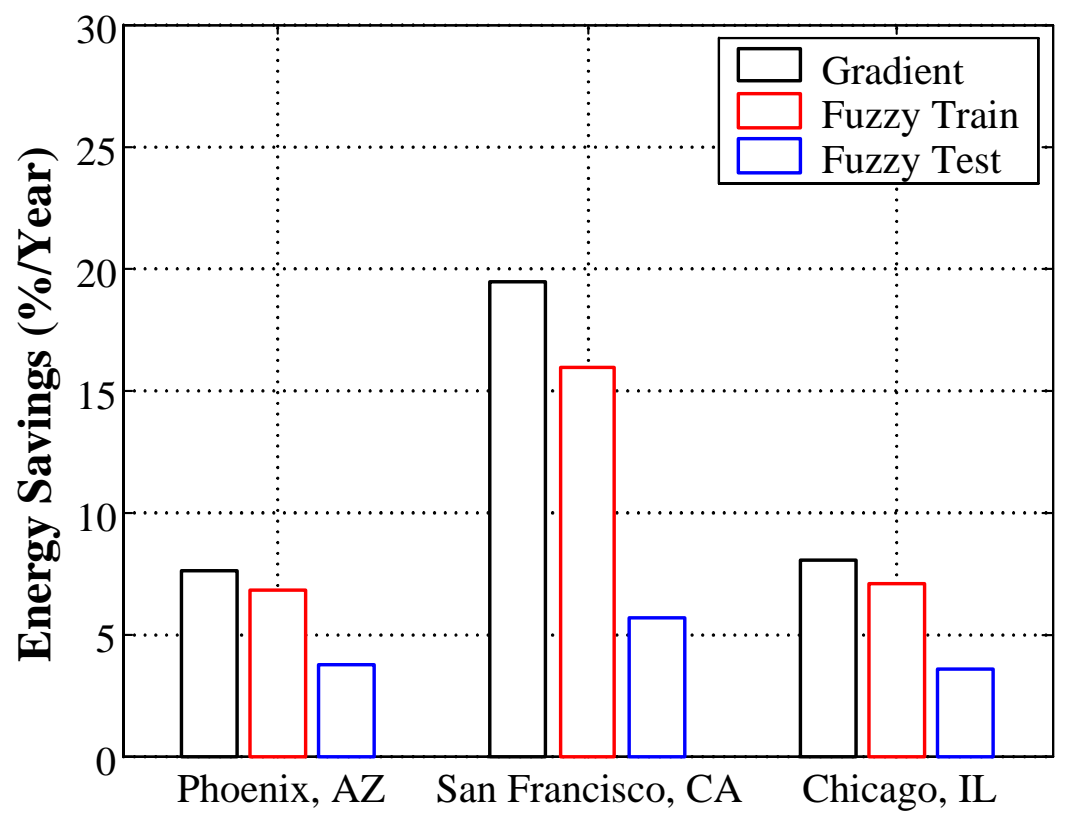

Figure 4.1 - Energy savings per year relative to OSFA.

The numeric results about individual dissatisfactions showed that both HIYW and fuzzy logic approximation provide a Degree of Individual Dissatisfaction (DID) level of no more than $20 \%$ for each occupant. The OSFA method, on the other hand, does not guarantee any level of individual thermal comfort. For instance, for Phoenix, AZ, OSFA is likely to cause the DID level to exceed the $20 \%$ limit for $\sim 15 \%$ of the occupants, and to exceed $50 \%$ for $\sim 5 \%$ of the occupants. Similar violations of individual comfort levels by the OSFA method were observed for other cities as well.

In the rest of this section, results of several parameter variations are reported.

\subsection{Effects of Internal Resistance on Optimization}

The behavior of the lumped parameter thermal energy model mostly depends on the thermal resistance values of the interior and exterior walls. Table 4.1 gives some examples of wall resistance values. During the optimization process in this study, the internal resistance values corresponding to private separate offices were selected. The interior wall thermal resistance in 


\section{Report No. DE-FG02-03ER63694-F1}

the baseline case corresponds to a common gypsum board inside wall. In order to investigate the effect of variations in internal resistance values the optimization of energy consumption was repeated for a variety of internal resistance values in three different cities with 20 different populations.

Table 4.1- Interior resistance trials

\begin{tabular}{|c|c|c|c|c|}
\hline $\begin{array}{l}\text { Trial } \\
\text { No. }\end{array}$ & $\begin{array}{l}\text { Wall Resistance, } \\
\mathrm{r}_{\text {iwall }}\left(\mathrm{m}^{2} \mathrm{~K} / \mathrm{W}\right)\end{array}$ & $\begin{array}{l}\text { Inside Convective Heat } \\
\text { Transfer Coefficient, } \mathrm{h}_{\mathrm{i}} \\
\left(\mathrm{W} / \mathrm{m}^{2} \mathrm{~K}\right)\end{array}$ & $\begin{array}{c}\text { Resulting } \\
\text { Resistance, } \\
\mathrm{r}_{\text {int }}\left(\mathrm{m}^{2} \mathrm{k} / \mathrm{W}\right)\end{array}$ & Notes \\
\hline 1 & 0.392 & 3.06 & 1.05 & Baseline Case \\
\hline 2 & $0.196^{\mathrm{a}}$ & 3.06 & 0.85 & $\begin{array}{l}\text { Estimated thin partition (i.e. } \\
\text { cubicle) }\end{array}$ \\
\hline 3 & -- & 3.06 & 0.33 & $\begin{array}{l}\text { Approximates open } \\
\text { workspace }^{\text {b }}\end{array}$ \\
\hline 4 & 0.392 & 8.29 & 0.63 & $\begin{array}{l}\text { Baseline with less } \\
\text { conservative } h_{i}[105]\end{array}$ \\
\hline 5 & $0.196^{a}$ & 8.29 & 0.44 & $\begin{array}{l}\text { Estimated thin partition (i.e. } \\
\text { cubicle) }\end{array}$ \\
\hline 6 & -- & 8.29 & 0.12 & $\begin{array}{l}\text { Approximates open } \\
\text { workspace }^{\text {b }}\end{array}$ \\
\hline
\end{tabular}

Since OSFA attempts to provide a uniform temperature over the building, the energy consumption in this case is not affected by internal resistances. However, the lower internal resistances result in higher energy usage in the HIYW case, due to increased energy loss between neighboring offices [104]. In order to see the effects of internal resistance on optimal energy consumption, we implemented the gradient-based optimization algorithm while keeping same thermal comfort requirements for individuals (20\% DID), and population (10\% PPD) as constraints. We took the average of 20 different populations from extremely low resistance values to our base line case $\left(\mathrm{R}_{\mathrm{int}}=1.05 \mathrm{~m}^{2} \mathrm{~K} / \mathrm{W}\right)$. Figure 4.2 gives the annual energy savings results with respect to OSFA for Miami, FL, Baltimore, MD, and Fairbanks, AK. It has been interpreted, as internal resistance doesn't give significant effect on annual energy savings even for the low resistance values. 


\section{Report No. DE-FG02-03ER63694-F1}

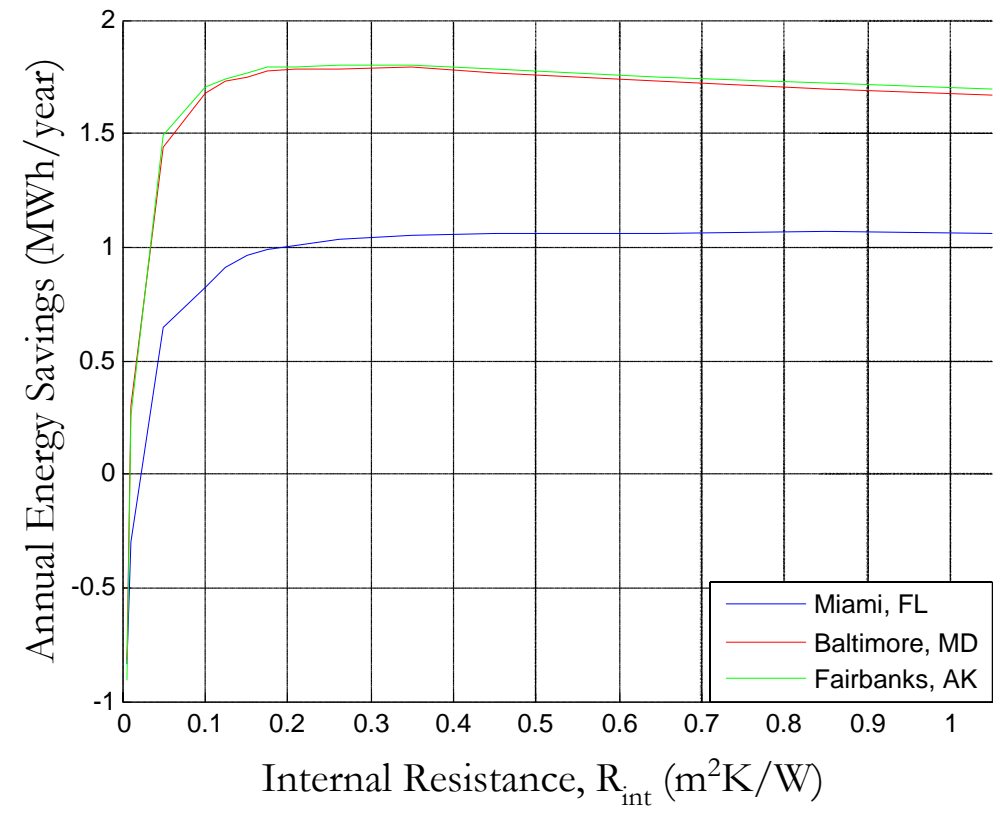

Figure 4.2 - Internal resistance effects on optimization

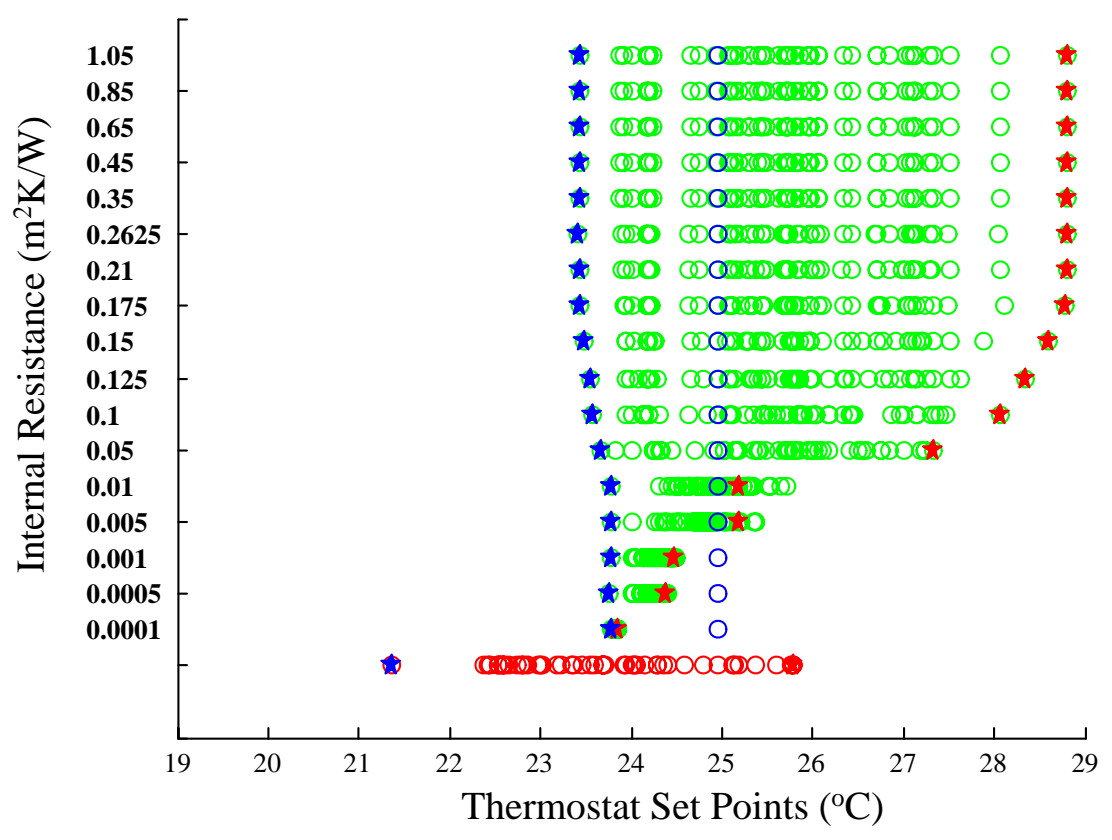

Figure 4.3 - Example of thermostat set points

Figure 4.3 is an example of a population in a given ambient temperature. While red color represents the desired temperature set points of the individuals, green ones are the optimal 
Report No. DE-FG02-03ER63694-F1

ones for the corresponding internal resistance values. Blue color is the optimal set point for OSFA for this illustration population. Blue and red stars represent the people, who would like to get coolest, and warmest temperature in the population, respectively. Optimization reduces the energy loss due to the heat exchange between neighboring offices to minimize energy consumption. In order to reduce energy usage when internal resistance is low, it provides almost identical temperatures to occupants satisfying thermal comfort requirements of both individuals and population.

\subsection{Effects of Non-uniform Internal Heat Load on Optimization}

During the all simulation process mentioned above, we assumed the internal heat loads are identical in each office cubicles. OSFA can provide uniform temperature distribution under this assumption. However, it is not acceptable for the real world applications. In order to investigate the effects of non-uniform heat loads on the optimization results, we implemented the simulations by varying heat loads. A 3-state discrete probability distribution has been used, which consists of $0 \mathrm{~W}, 260 \mathrm{~W}$, and $512 \mathrm{~W}$ with the probability of $0.2,0.8$, and 0.2 , respectively. Figure 4.4 is an example of a non-uniform heat load, which was generated by using this distribution. We applied the optimization to a chosen population, in Baltimore, MD, for 100 different heat load distributions. While optimal results of HIYW did guarantee both individual and population thermal constraints in all cases, there is no significant changes in energy consumption. However, OSFA ensured the population constraint for only 8 cases. It didn't reach feasible solutions for 92 cases under the constraint of 10\% PPD. Figure 4.5 shows the average of optimal energy consumption results of 100 different non-uniform cases, and that of uniform one. 


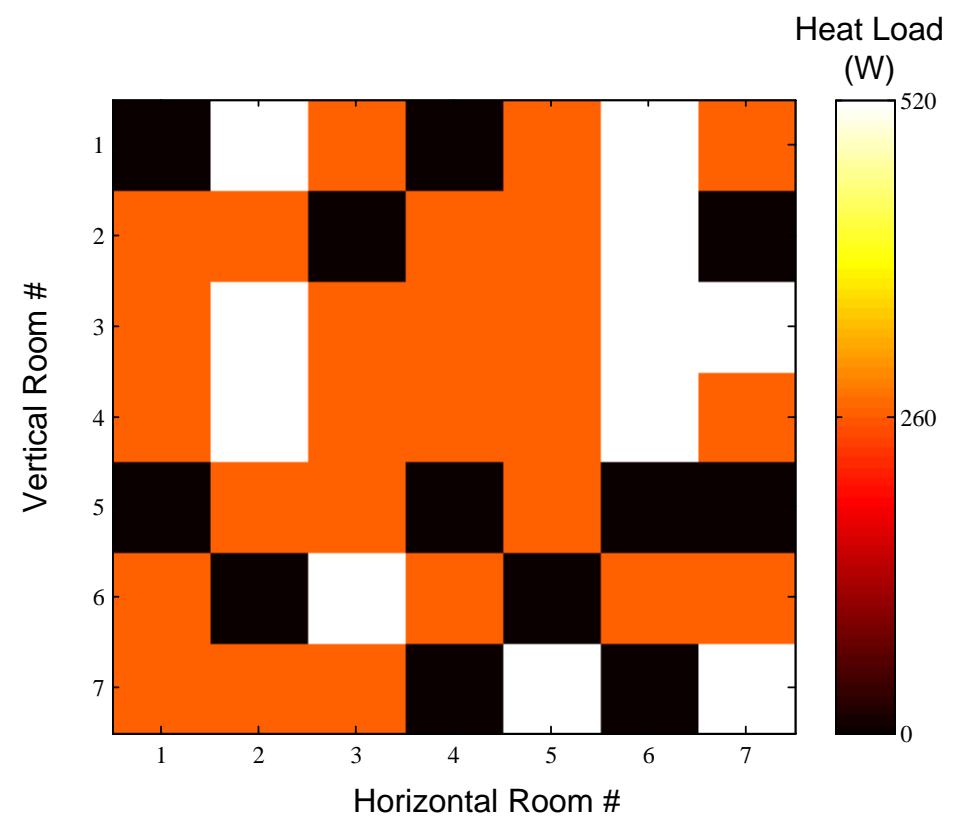

Figure 4.4 - Example of non-uniform heat load

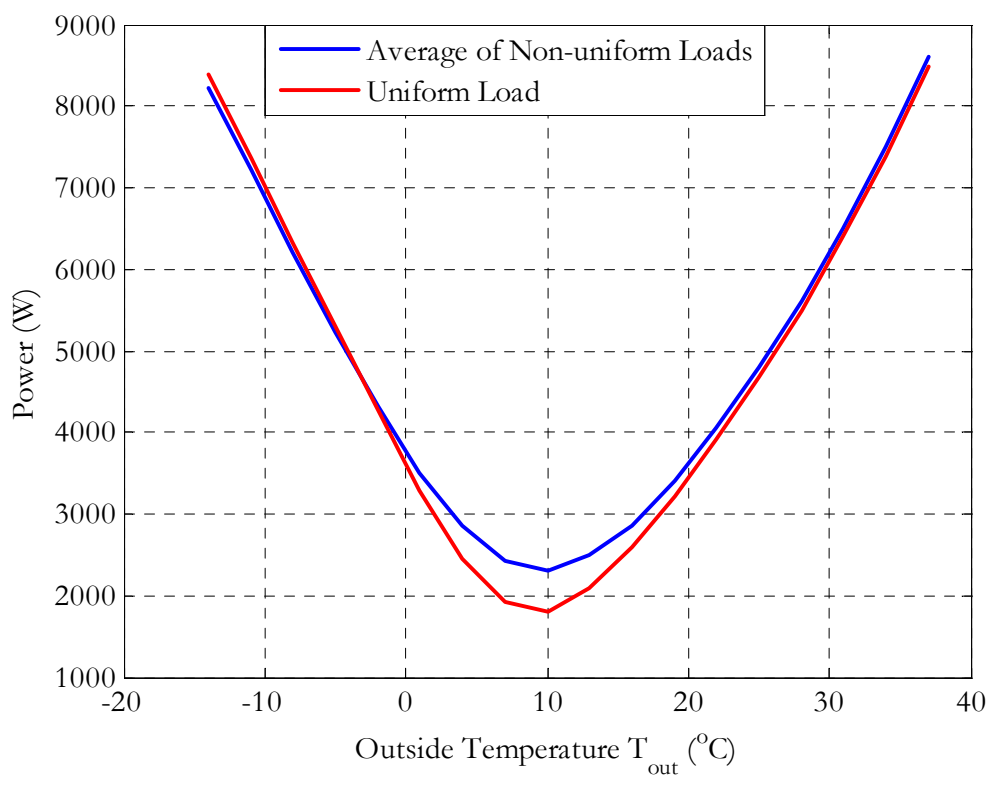

Figure 4.5 - Power results with non-uniform loads, and uniform load

Occupants' comfort has been improved while reducing energy consumption by optimized HIYW system. While HIYW approach requires the use of all sensor network connectivity in 


\section{Report No. DE-FG02-03ER63694-F1}

the building, it is not required in fuzzy logic approximation. Reduction of sensor connectivity would not only reduce system complexity, but also cause modest decrease in energy savings relative to fully connected HIYW system. Optimal results for lower internal resistances, and non-uniform heat loads are also encouraging to apply HIYW system in real world applications. There is always between trade-off between energy consumption and thermal comfort. However, while optimized HIYW make occupant reasonably satisfied within an acceptable temperature range, it also improves the cost of the system under the different thermal conditions such as outside temperature, desired temperatures of occupants, heat loads in offices, and resistance values of internal walls. 


\section{Appendix - MATLAB Codes}

\subsection{Individual Dissatisfaction}

\subsubsection{Predicted Mean Votes}

\section{[5]}

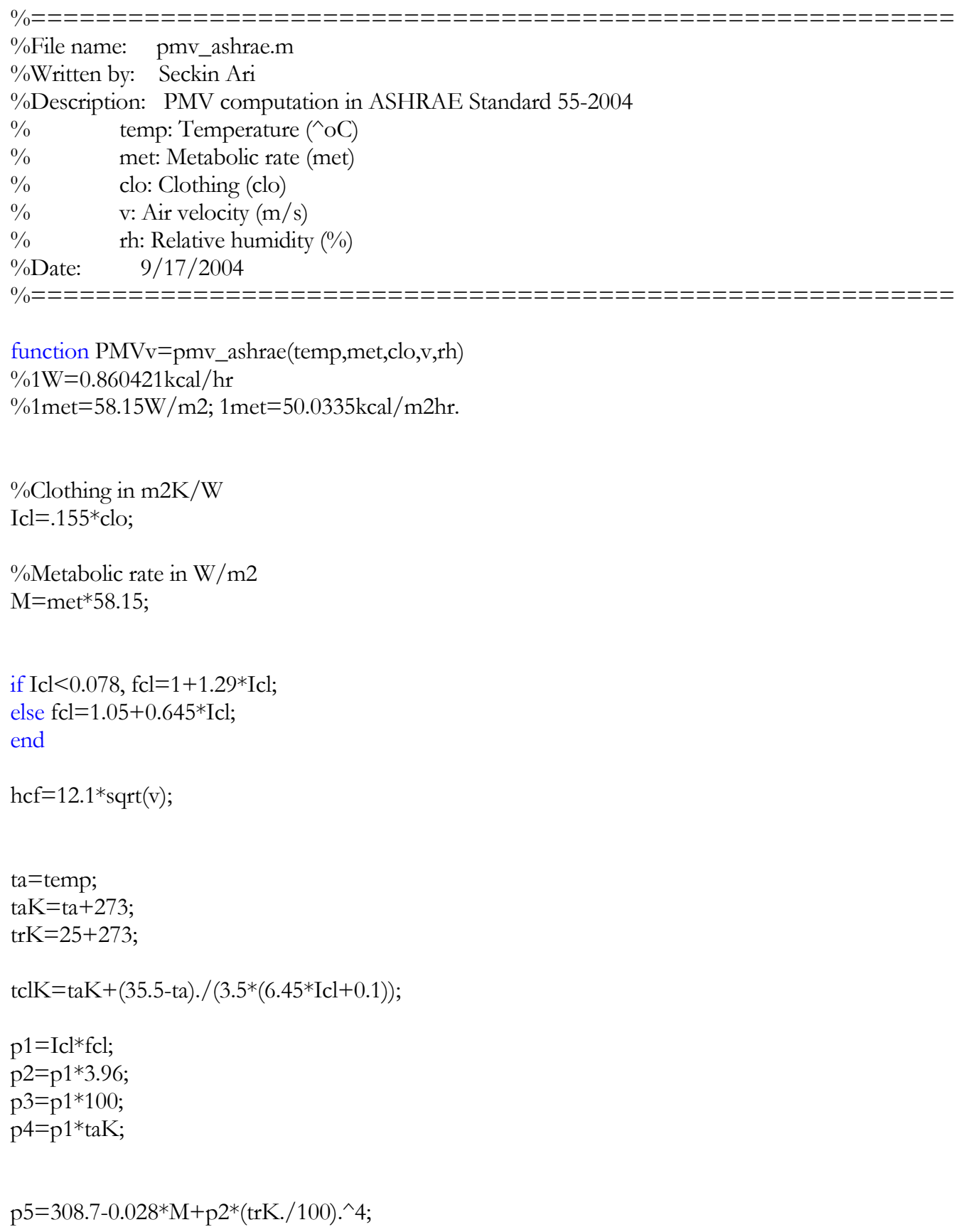


$\mathrm{xn}=\mathrm{tclK} . / 100$

$\mathrm{xf}=\mathrm{xn}$;

eps $=0.00015$;

while(true)

$\mathrm{xf}=(\mathrm{xf}+\mathrm{xn}) \cdot / 2$;

$\mathrm{hc}=2.38^{*} \mathrm{abs}\left(100^{*} \mathrm{xf}-\mathrm{taK}\right) . \wedge 0.25$;

$\mathrm{i}=$ find $(\mathrm{hc}<\mathrm{hcf})$;

hc(i) $=$ hcf;

$\mathrm{xn}=\left(\mathrm{p} 5+\mathrm{p} 4 .{ }^{*} \mathrm{hc}-\mathrm{p} 2 .{ }^{*} \mathrm{xf} .{ }^{\wedge} 4\right) \cdot /\left(100+\mathrm{p} 3{ }^{*} \mathrm{hc}\right) ;$

if $\operatorname{abs}(x n-x f)<=e p s$, break end

end

$\mathrm{tcl}=100 * \mathrm{xn}-273$

tmrt $=$ ta;

$\mathrm{taK}=\mathrm{ta}+273$;

$\operatorname{tmrtK}=\mathrm{taK}$;

$\mathrm{ps}=\exp (16.6536-4030.183 . /(\mathrm{ta}+235))$;

$\mathrm{pa}=\mathrm{ps} . * \mathrm{rh} * 10$;

\%heat loss diff. through skin

HL1=3.05*.001*(5733-6.99*M-pa);

$\%$ heat loss by sweating

HL2 $=.42 *(\mathrm{M}-58.15)$;

$\%$ latent respiration heat loss

HL3=1.7e-5*M*(5867-pa);

$\%$ dry respiration heat loss

HL4=.0014*M*(34-ta);

$\%$ heat loss by radiation

HL5=3.96*fcl*(xn.^4-(tmrtK./100).^4);

$\%$ heat loss by convection

HL6 $=$ fcl*hc.*(tcl-ta);

PMVv $=(0.303 * \exp (-0.036 * \mathrm{M})+0.028) *(\mathrm{M}-\mathrm{HL} 1-\mathrm{HL} 2-\mathrm{HL} 3-\mathrm{HL} 4-\mathrm{HL} 5-\mathrm{HL} 6)$;

$\mathrm{i}=$ find $\left(\mathrm{PMV}_{\mathrm{v}}>3\right)$;

$\operatorname{PMVv}(\mathrm{i})=3$;

$\mathrm{i}=$ find $(\mathrm{PMVv}<-3)$;

$\operatorname{PMVv}(\mathrm{i})=-3$; 
Report No. DE-FG02-03ER63694-F1

\subsubsection{Optimal Settings for Individual Satisfaction Model - Monte Carlo Simulation}

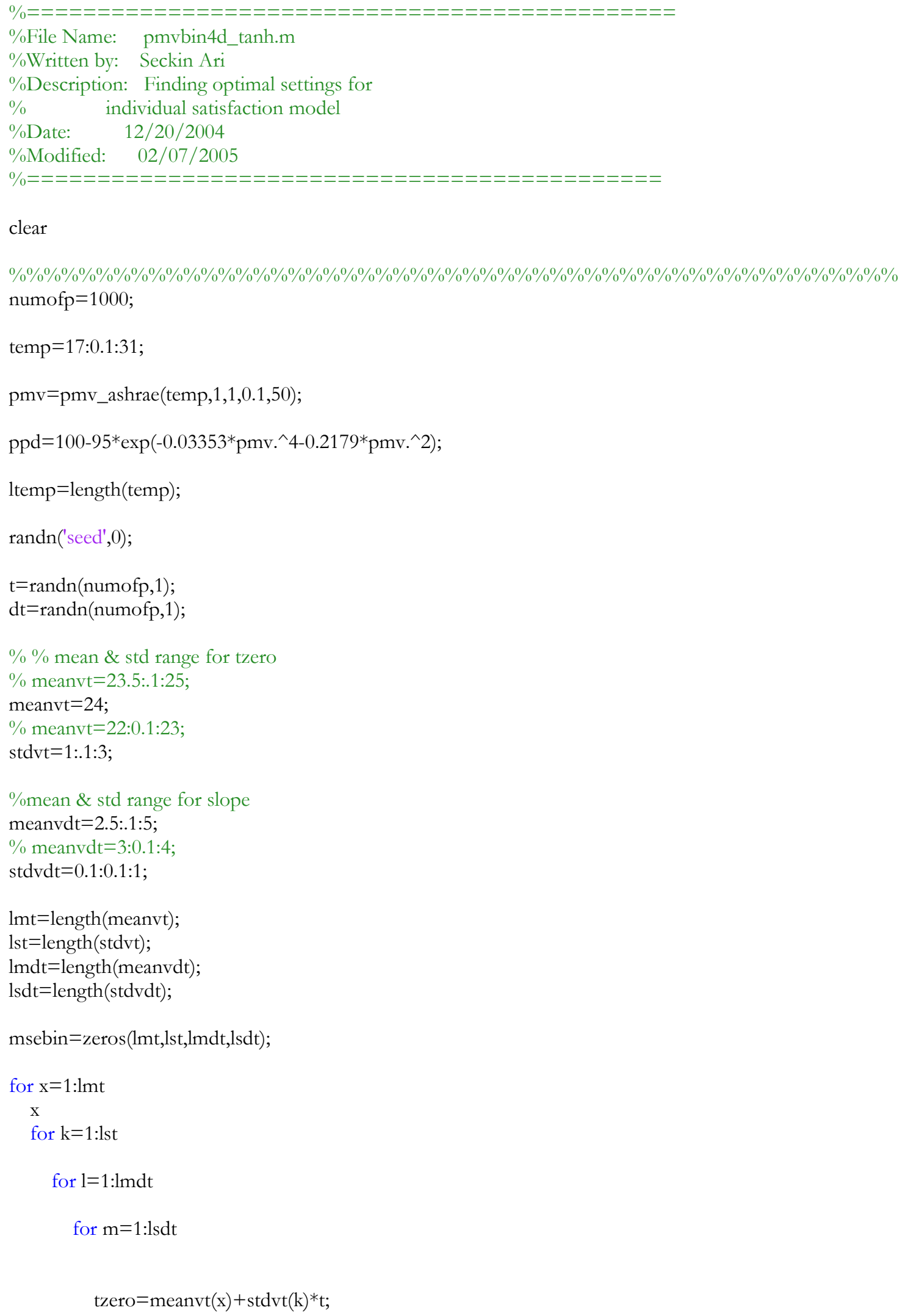




\section{Report No. DE-FG02-03ER63694-F1}

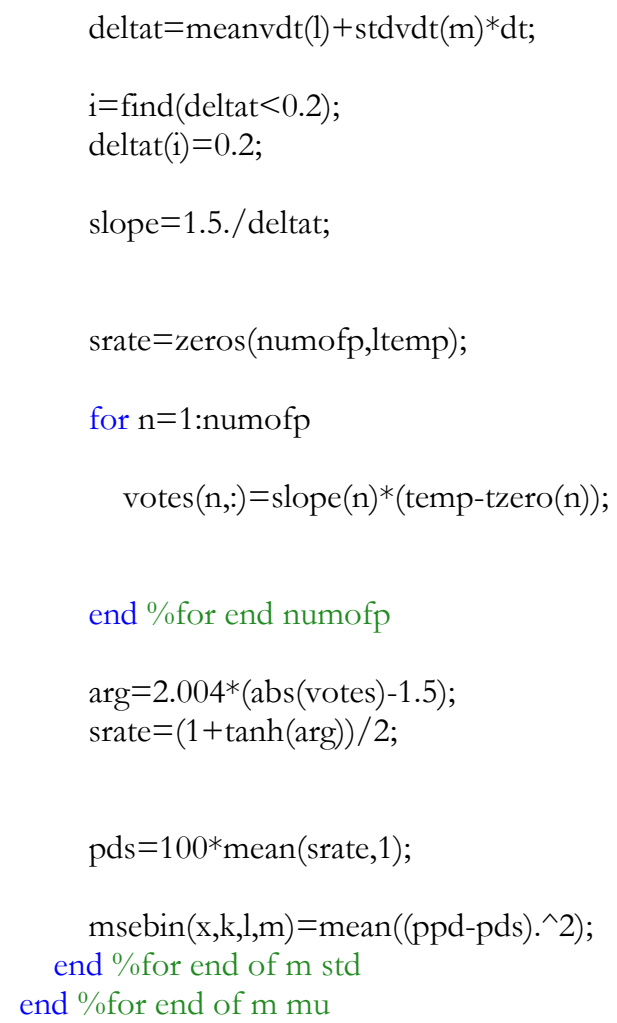

\subsection{Optimization}

\subsubsection{Have It Your Way}

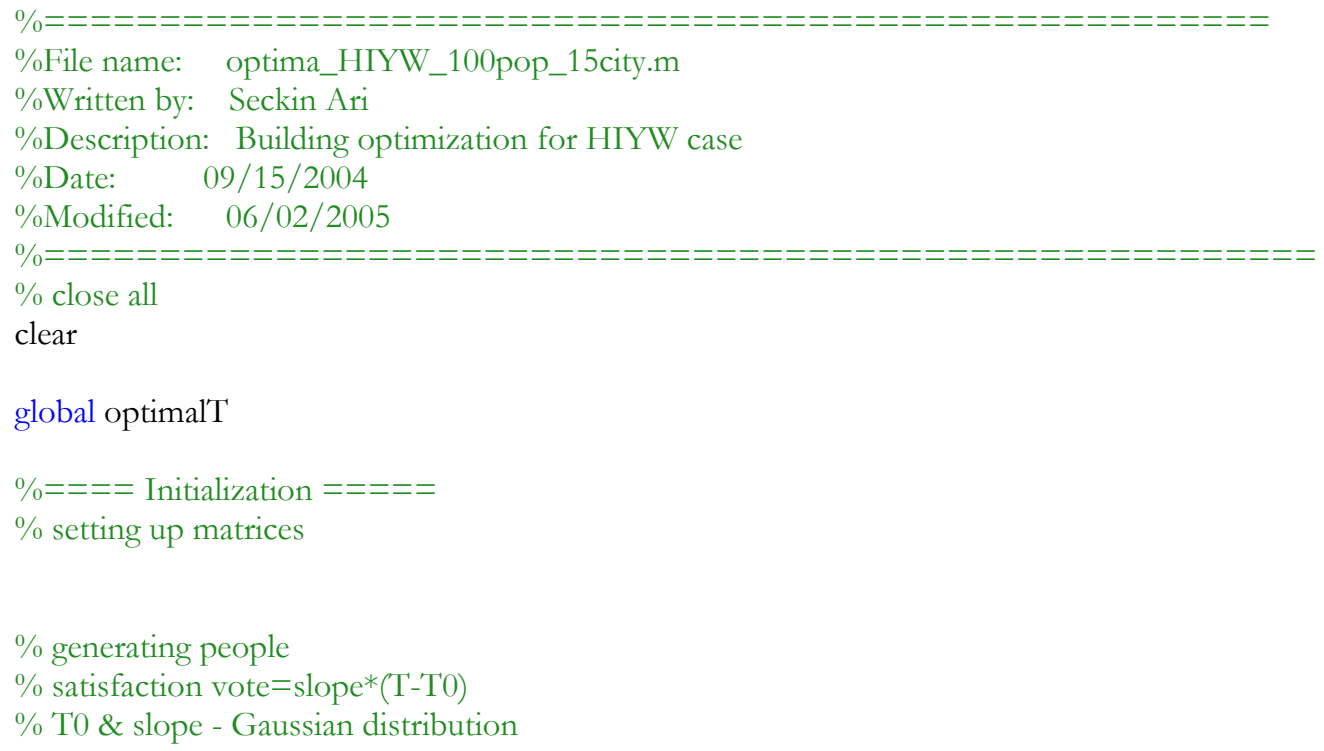




\section{Report No. DE-FG02-03ER63694-F1}

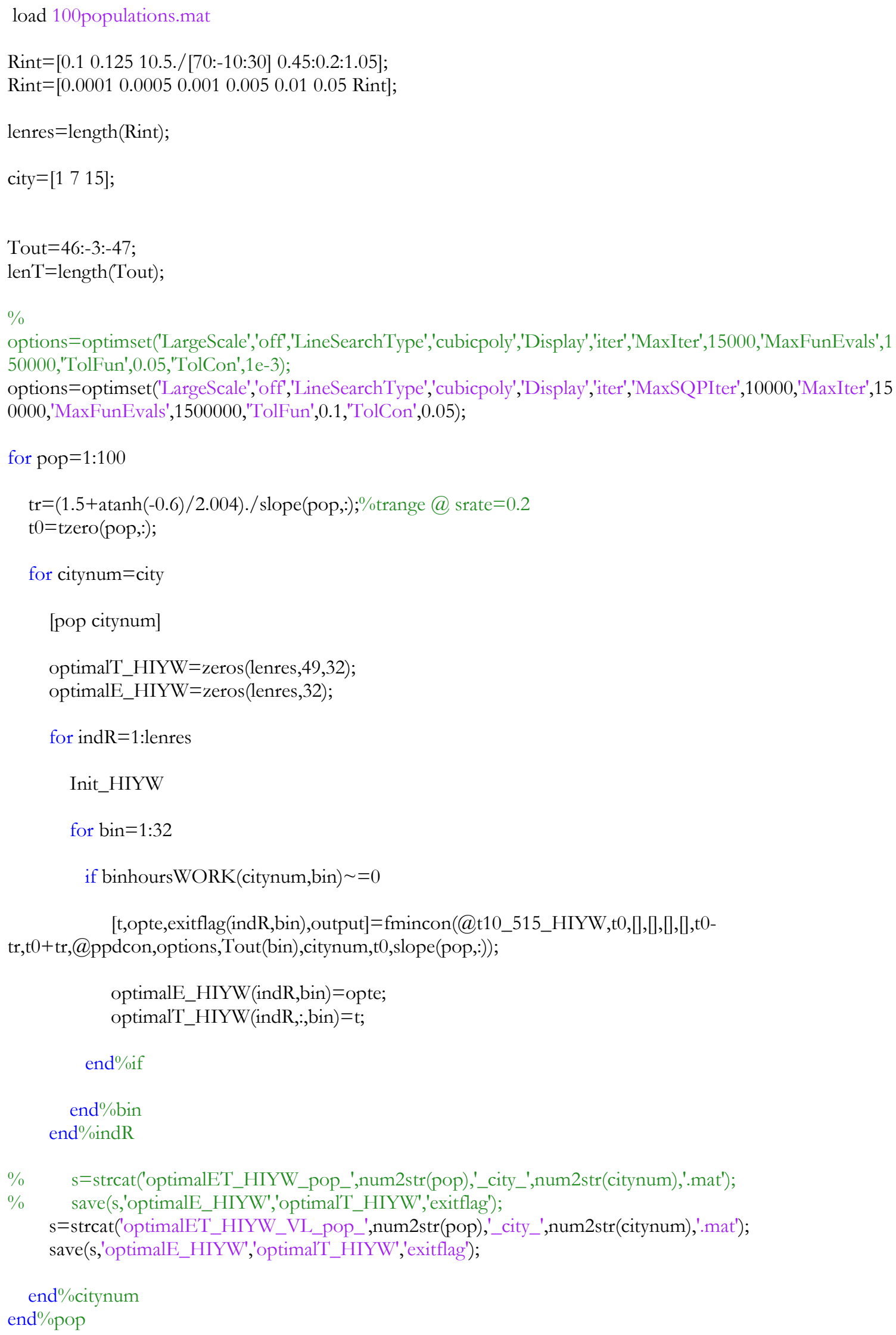




\section{Report No. DE-FG02-03ER63694-F1}

\subsubsection{One Size Fits All}

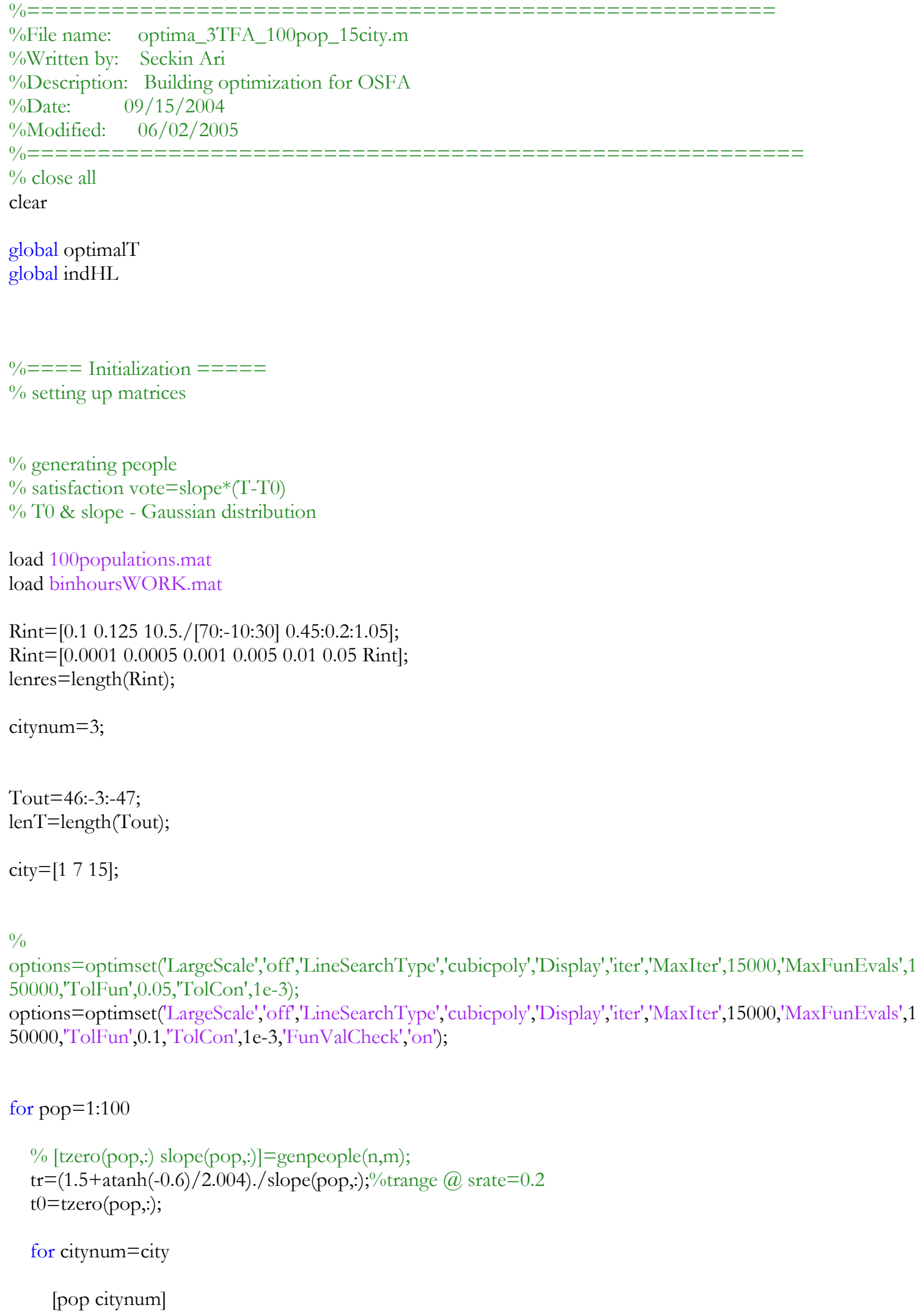




\section{Report No. DE-FG02-03ER63694-F1}

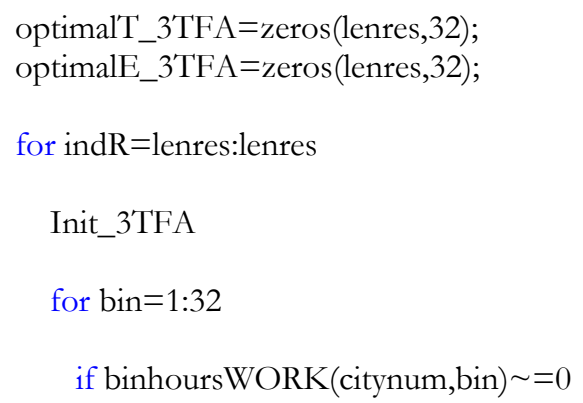

[t,opte,exitflag(indR,bin),output]=fmincon(@t9_515_3TFA,24,[,[,],[,15,30,@ppdcon,options,Tout(bin),cityn um,t0,slope(pop,:));

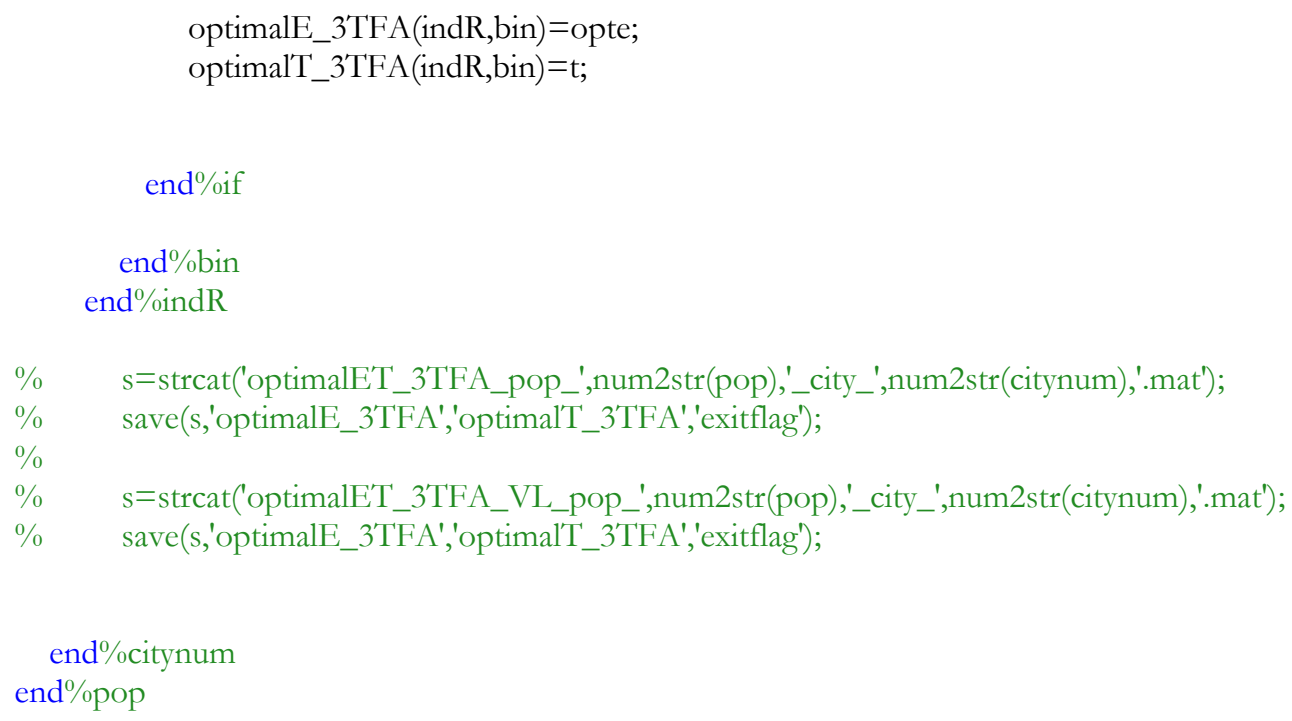


Report No. DE-FG02-03ER63694-F1

\section{References}

[1] United States Department of Energy and United States Environmental Protection Agency, "Sustainable Building Technical Manual," 1996.

[2] de Dear, R., and G.S. Brager, "The adaptive model of thermal comfort and energy conservation in the built environment," Int. J. Biometeorol, vol. 45, pp. 100-108, 2001

[3] Fanger, P.O., Thermal Comfort: Analysis and Applications in Environmental Engineering, McGraw-Hill, NY, 1972

[4] Awbi, H., Ventilation of Buildings, Spon Press, London, 2003.

[5] ASHRAE Standard 55-2004, “Thermal Environmental Conditions of Human Occupancy."

[6] IS0/DIS 7730:2003, "Ergonomics of the thermal environment - Analytical determination and interpretation of thermal comfort suing calculation of the PMV and PPD indices and local thermal comfort."

[7] Haghigtat, F., G. Donnini, and R. D’Addaria, "Relationship Between Occupant Discomfort asPerceivedandasMeasuredObjectively," Indoor Environment, vol. 1, pp. 163-174, 1992

[8] Haghigtat, F., and G. Donnini, "Impact of psycho-social factors on perception of the indoor air environment studies in 12 office buildings," Building and Environment, vol. 34, pp. 479-503, 1999

[9] Davidge, R.O.C., "ASHRAE Standards: A Guarantee of Occupant Satisfaction?," Managing Indoor Air for Health and Energy Conservation; Proceedings of the ASHRAE Conference IAQ, pp. 171-177, 1986.

[10] Lomonaco, C. and D. Miller. "Comfort and Control in the Workplace," ASHRAE Journal v39, 1997.

[11] Schiller, G.E., E.A. Arena, F.S. Bauman, C. Benton, M. Fountain, and T Doherty, "A field study of thermal environments and comfort in office buildings," ASHRAE Transactions, 94, 1988.

[12] Bauman, F.S., "Giving Occupants What they Want: Guidelines for Implementing Personal Environmental Control in Your Buildings," World Workplace 99 Proceedings, October 1999.

[13] Bauman, F.S., L.P. Johnston, H. Zhang, E.A. Arens, "Performance testing of a floorbased, occupant-controlled office ventilation system." ASHRAE Transactions 97, 1991. 
Report No. DE-FG02-03ER63694-F1

[14] Bauman, F.S., "Underfloor Air Distribution (UFAD) Design Guide," Final Report: ASHRAE Research Project RP-1064, Jan. 2003.

[15] Bauman, F.S., H Zhang, and E.A. Arens. "Localized Comfort Control with a Desktop Task Conditioning System: Laboratory and Field Measurements," ASHRAE Transactions, 1993.

[16] Johnson Controls Inc., "White Paper: Environmental Satisfaction, Personal Control and the Positive Correlation to Increased Productivity"

[17] Fisk, W. J. "Health and Productivity Gains from Better Indoor Environments and Their Implications for the US Department of Energy," Indoor Environment Department, Lawrence Berkeley National Laboratory, Berkeley, CA, LBNL-4745, 2000.

[18] Fisk, W.J., “How IAQ Affects Health, Productivity,” ASHRAE Journal, May 2002.

[19] Lorsch, H.G. and O.A. Abdou, "The Impact of the Building Indoor Environment on Occupant Productivity - Part 2: Effects of Temperature," ASHRAE Transactions, Vol. 100, 1994.

[20] Tham, K.W., "Effects of temperature and outdoor air supply rate on the performance of call center operators in the tropics," Indoor Air 2004, Vol. 14, 119-125.

[21] Wyon, D.P., "Indoor Environmental Effects on Productivity," IAQ '96: Paths to Better Building Environments, 1996.

[22] Wyon, D.P., "The effects of indoor air quality on performance and productivity," Indoor Air 2004, Vol. 14, 92-101.

[23] Indoor Air Quality Update, Oct 1996, v.9 no.10, p.1-5.

[24] Lorsch, H.G. and O.A. Abdou, "The Impact of the Building Indoor Environment on Occupant Productivity - Part 1: Recent Studies, Measures, and Costs,” ASHRAE Transactions, Vol. 100, 1994.

[25] Johnson Controls Inc., "Case Study: West Bend Mutual Insurance Company, West Bend, WI" available online: http://www.johnsoncontrols.com/CGCases/cs WestBend.pdf

[26] Houghton, F.C. and C.P. Yaglou, "Determining Lines of Equal Comfort," ASHVE Transactions, vol. 28, pp. 163-176, 1923.

[27] Yaglou, C.P., “A Method for Improving the Effective Temperature Index,” ASHVE Transactions, vol. 53, pp. 307-326, 1947.

[28] Winslow, C.-E.A., L.P. Herrington, L.P. Gagge, and A.P. Gagge, "Relation Between Atmospheric Conditions, Physiological Reactions and Sensations of Pleasantness," American Journal of Hygiene, vol. 26, pp. 103-115, 1937. 
[29] Gagge, A.P., J.A.J. Stolwijk, and Y. Nishi, "An Effective Temperature Scale Based on a Simple Model of Human Physiological Regulatory Response," ASHRAE Transactions, vol. 77, pt. 1, pp. 247-262, 1971.

[30] Fanger, P.O., "Calculation of Thermal Comfort: Introduction of a Basic Comfort Equation,” ASHRAE Transactions, vol. 73, pt. 2, 1967.

[31] Gagge, A.P., A.C. Burton, and H.C. Bazett, "A Practical System of Units for the Description of the Heat Exchange of Man with His Environment," Science, vol. 94, pp. 428-430, 1941.

[32] Gagge, A.P., A.P. Fobelets, and L.G. Berglund, "A Standard Predictive Index of Human Response to the Thermal Environment," ASHRAE Transactions, vol. 92, pt. 2b, pp. 709-731, 1986.

[33] McNall, P.E., J. Jaax, F.H. Rohles, R.G. Nevins, and W. Springer, “Thermal Comfort (Thermally Neutral) Conditions for Three Levels of Activity," ASHRAE Transactions, vol. 73, pt. 1, pp. 1-13, 1967.

[34] Federspiel, C.C. and H. Asada, "Adaptive Control of Thermal Comfort Based on Human Responses and a Model of Human Thermal Sensation," Winter Annual Meeting of ASME, Control of Systems with Inexact Dynamic Model, vol. 33, pp. 161-167, 1991.

[35] de Dear, R.J. and G.S. Brager, "Developing An Adaptive Model of Thermal Comfort and Performance,” ASHRAE Transactions, vol. 104, pt. 1a, pp. 145-167, 1998.

[36] Brager, G.S., G. Paliaga, and R.J. de Dear, "Operable Windows, Personal Control, and Occupant Comfort,” ASHRAE Transactions, vol. 110, pt. 2, pp. 17-35, 2004.

[37] Seem, J.E. and J.E. Braun. "The Impact of Personal Environmental Control on Building Energy Use,” ASHRAE Transactions, 48, 1992.

[38] Klein et al, “TRNSYS 16: a TRaNsient SYstem Simulation program,” Solar Energy Laboratory, University of Wisconsin-Madison, 2004.

[39] Glicksman, L.R. and S. Taub. "Thermal and Behavioral Modeling of Occupantcontrolled Heating, Ventilating, and Air Conditioning Systems," Energy and Buildings, 25, 1997.

[40] Honeywell History, available online at: http://www.honeywell.com/sites/honeywell/ourhistory.htm

[41] Shavit, G., “The Evolution of Control During the Past 100 Years,” ASHRAE Transactions, vol. 101, pt. 1, pp. 538-544, 1995.

[42] Kinealy, J.H., “Temperature Regulation,” ASHVE Transactions, vol. 10, 1903.

[43] Jones, A.O., "An Improved Application for Hot Air Heating," ASHVE Transactions, vol. 13, 1906. 
[44] Donnelly, J.A., “The Time Element in Heating Apparatus,” ASHVE Transactions, vol. 19, 1912.

[45] Winslow, C.-E.A. and G.F. Maglott, "A Study of Heating and Ventilating Conditions in a Large Office Building,” ASHVE Transactions, vol. 22, 1915.

[46] Parmalee, G.V. and R.G. Huebscher, "The Shielding of Thermocouples from the Effect of Radiation," ASHVE Transactions, vol. 53, 1946.

[47] Shavit, G., "A Microprocessor Based System for Energy Management," $13^{\text {th }}$ IEEE Conference, 1976.

[48] Shavit, G., "Energy Savings Through Computer Control of Fan Systems with Floating Space Temperature," ASHRAE Transactions, vol. 83, pt. 1, pp. 374-383, 1977.

[49] Cherchas, D.B., A. Abdelmessih, and M. Townsend, "A Direct Digital Control Algorithm for Control of a Single Environmental Space," ASME Journal of Dynamic Systems, Measurement, and Control, vol. 107, pp. 324-331, 1985.

[50] Nesler, C.G., "Automated Controller Tuning for HVAC Applications,” ASHRAE Transactions, vol. 92, pp. 189-201, 1986.

[51] Pinella, M.J., E. Wechselberger, D.C. Hittle, C.O. Pederson, "Self-Tuning Digital Integral Control,” ASHRAE Transactions, vol. 92, pp. 202-210, 1986.

[52] Kasahara, M, T. Matsubu, Y. Kuzuu, T. Yamazaki, Y. Hashimoto, K. Kamimura, and S. Kurosu, "Design and Tuning of Robust PID Controller for HVAC Systems," ASHRAE Transaction, vol. 105, pt. 2, pp. 154-166, 1999.

[53] Qu, G. and M. Zaheeruddin, "Real-time Tuning of PI Controllers in HVAC Systems," International Journal of Energy Research, vol. 28, pp. 1313-1327, 2004.

[54] Wang, Q.-G., C.C. Hang, Y. Zhang, and Q. Bi, "Multivariable Controller Auto-Tuning with Its Application in HVAC Systems," Proceedings of American Control Conference, pp. 4353-4357, 1999.

[55] Huang, W. H.N. Lam, "Using Genetic Algorithms to Optimize Controller Parameters for HVAC Systems,” Energy and Buildings, vol. 26, pp. 277-282, 1997.

[56] Seem, J.E., "A New Pattern Recognition Adaptive Controller with Application to HVAC Systems,” Automatica, vol. 34, i. 8, pp. 969-982, 1998.

[57] Xu, M., S. Li, and W. Cai, "Practical Receding-Horizon Optimization Control of the Air Handling Unit in HVAC Systems," Industrial Engineering Chemistry Research, vol. 44, pp. 2848-2855, 2005.

[58] Bi, Q., W.-J. Cai, Q.-G. Wang, C.-C. Hang, E.-L. Lee, Y. Sun, K.-D. Liu, Y. Zhang, and B. Zou, "Advanced Controller auto-tuning and its application in HVAC Systems," Control Engineering Practice, vol. 8, pp. 633-644, 2000. 
[59] MacArthur, J.W. and M.A. Woessner, "Receding Horizon Control: A Model Based Policy for HVAC Applications,” ASHRAE Transactions, vol. 99, pt. 1, pp. 139-148, 1993.

[60] Curtiss, P.S., J.F. Kreider, and M.J. Brandemuehl, "Adaptive Control of HVAC Process Using Predictive Neural Networks,” ASHRAE Transactions, vol. 99, pt. 1, pp. 496-504, 1993.

[61] Jeannette, E., K. Assawamartbunlue, P.S. Curtiss, and J.F. Kreider, "Experimental Results of a Predictive Neural Network HVAC Controller," ASHRAE Transactions, vol. 104, pt. 2, pp. 192-197, 1998.

[62] Sousa, J.M., R. Babuska, and H.B. Verbruggen, "Fuzzy Predictive Control Applied to an Air-Conditioning System," Control Engineering Practice, vol. 5, i. 10, pp. 1395-1406, 1997.

[63] da Costa Sousa, J.M. and U. Kaymak, "Model Predictive Control Using Fuzzy Decision Functions," IEEE Transactions on Systems, Man, and Cybernetics, vol. 31, i. 1, pp. $54-$ 65, 2001.

[64] He, M., W.-J. Cai, and S.-Y. Li, "Multiple Fuzzy Model-Based Temperature Predictive Control,” Information Sciences, vol. 169, pp. 155-174, 2005.

[65] Thompson, R. and A. Dexter, "A Fuzzy Decision-Making Approach to Temperature Control in Air-Conditioning Systems," Control Engineering Practice, vol. 13, pp. 689698, 2005.

[66] Lea, R.N., E. Dohmann, W. Prebilsky, and Y. Jani, “An HVAC Fuzzy Logic Zone Control System and Performance Results," IEEE Conference on Fuzzy Systems, vol. 3, pp. 2175-2180, 1996.

[67] Rahmati, A., F. Rashidi, and M. Rashidi, "A Hybrid Fuzzy Logic and PID Controller for Control of Nonlinear HVAC Systems," IEEE Conference on Systems, Man, and Cybernetics, pp. 2249-2254, 2003.

[68] Federspiel, C.C. and H. Asada, "User-Adaptable Comfort Control for HVAC Systems," ASME Journal of Dynamic Systems, Measurement, and Control, vol. 116, i. 3, pp. 474486, 1994.

[69] Zhou, H., M. Rao, and K.T. Chuang, "Intelligent System for Indoor Air Quality Control,” Environment, vol. 20, i. 4, pp. 457-467, 1994.

[70] Dounis, A.I., M.J. Santamouris, C.C. Lefas, and A. Argiriou, "Design of a Fuzzy Set Environment Comfort System,” Energy and Buildings, vol. 22, pp. 81-87, 1995.

[71] Dounis, A.I. and D.E. Monalikis, "Design of a Fuzzy System for Living Space ThermalComfort Regulation,” Applied Energy, vol. 69, pp. 119-144, 2001. 
Report No. DE-FG02-03ER63694-F1

[72] Gouda, M.M., S. Danaher, and C.P. Underwood, "Thermal Comfort Based Fuzzy Logic Controller,” Building Service Engineering Research Technology, vol. 22, i. 4, pp. $237-$ 253, 2001.

[73] Chu, C.-M., T.-L. Jong, and Y.-W. Huang, "Thermal Comfort Control on Multi-Room Fan Coil Unit System Using LEE-Based Fuzzy Logic," Energy Conversion \& Management, vol. 46, pp. 1579-1593, 2005.

[74] Schade, G.R., "Saving Energy by Night Setback of a Residential Heat Pump System," ASHRAE Transactions, vol. 84, pt. 1, pp. 786-797, 1978.

[75] Nizet, J.L., J. Lecomte, and F.X. Litt, "Optimal Control Applied to Air Conditioning in Buildings,” ASHRAE Transactions, vol. 90, pt. 1b, pp. 587-600, 1984.

[76] Boyens, A. and J.W. Mitchell, "Experimental Validation of a Methodology for Determining Heating System Control Strategies," ASHRAE Transactions, vol. 97, pt. 2, pp. 24-30, 1991.

[77] Ke, Y.-P. and S.A. Mumma, "Optimized Supply-Air Temperature (SAT) in VariableAir-Volume (VAV) Systems,” Energy, vol. 22, i. 6, pp. 601-614, 1997.

[78] House, J.M., T.F. Smith, and J.S. Arora, "Optimal Control of a Thermal System," ASHRAE Transactions, vol. 97, pt. 2, pp. 991-1001, 1991.

[79] House, J.M. and T.F. Smith, "A System Approach to Optimal Control for HVAC and Building Systems,” ASHRAE Transactions, vol. 101, pt. 2, pp. 647-660, 1995.

[80] House, J.M. and T.F. Smith, “Optimal Control of Building and HVAC Systems," IEEE American Control Conference, vol. 6, pp. 4326-4330, 1995.

[81] Simmonds, P., "Thermal Comfort and Optimal Energy Use," ASHRAE Transactions, vol. 99, pt. 1, pp. 1037-1048, 1993.

[82] Liu, S. and X. He, “A Distributed-Parameter Model Approach to Optimal Comfort Control in Air Conditioning Systems," IEEE American Control Conference, vol. 3, pp. 3454-3458, 1994.

[83] Wright, J.A. and V.I. Hanby, "The Formulation, Characteristics, and Solution of HVAC System Optimized Design Problems," ASHRAE Transactions, vol. 93, pt. 2, pp. 21332145, 1987.

[84] Kaya, A., C.S. Chen, S. Raina, and S.J. Alexander, "Optimum Control Policies to Minimize Energy Use in HVAC Systems," ASHRAE Transactions, vol. 88, pt. 2, pp. 235-248, 1982.

[85] So, A.T.P., W.L. Chan, T.T. Chow, and W.L. Tse, "New HVAC Control by System Identification,” Building and Environment, vol. 30, i. 3, pp. 349-357, 1995. 
[86] Kulkarni, M.R. and F. Hong, "Energy Optimal Control of a Residential SpaceConditioning System Based on Sensible Heat Transfer Modeling," Building and Environment, vol. 39, pp. 31-38, 2004.

[87] So, A.T.P., W.L. Chan, and T.T. Chow, "A Computer-Vision-Based HVAC Control System,” ASHRAE Transactions, vol. 101, pt. 2, pp. 661-678, 1995.

[88] MacArthur, J.W., "Humidity and Predicted-Mean-Vote-Based (PMV-Based) Comfort Control,” ASHRAE Transactions, vol. 92, pt. 1b, pp. 5-17, 1986.

[89] Yang, K.H. and C.H. Su, "An Approach to Building Energy Savings Using the PMV Index," Building and Environment, vol. 32, i. 1, pp. 25-30, 1997.

[90] Kolokotsa, D., D. Tsiavos, G.S. Stavrakakis, K. Kalaitzakis, and E. Antonidakis, "Advanced Fuzzy Logic Controllers Design and Evaluation for Buildings' Occupants Thermal-Visual Comfort and Indoor Air Quality Satisfaction," Energy and Buildings, vol. 33, pp. 531-543, 2001

[91] Calvino, F., M. la Gennusa, G. Rizzo, G. Scaccianoce, “The Control of Indoor Thermal Comfort Conditions: Introducing a Fuzzy Adaptive Controller," Energy and Buildings, vol. 36, pp. 97-102, 2004.

[92] Yamada, F., K. Yonezawa, S. Sugawara, N. Nishimura, "Development of AirConditioning Control Algorithm for Building Energy-Saving," IEEE Conference on Control Applications, vol. 4, pp. 1579-1584, 1999.

[93] Alcala, R., J.M. Benitez, J. Casillas, O. Cordon, and R. Perez, "Fuzzy Control of HVAC Systems Optimized by Genetic Algorithms,” Applied Intelligence, vol. 18, pp. 155-177, 2003.

[94] Alcala, R., J. Casillas, O. Cordon, A. Gonzalez, F. Herrera, “A Genetic Rule Weighting and Selection Process for Fuzzy Control of Heating, Ventilating and Air Conditioning Systems," Artificial Intelligence, vol. 18, pp. 279-296, 2005.

[95] Jin, X., H. Ren, and X. Xiao, "Prediction-Based Online Optimal Control of Outdoor Air of Multi-Zone VAV Air Conditioning Systems," Energy and Buildings, vol. 37, pp. 939944, 2005.

[96] Lu, L., W. Cai, L. Xie, S. Li, and Y.C. Soh, "HVAC System Optimization - in-Building Section," Energy and Buildings, vol. 37, pp. 11-22, 2005.

[97] Lu, L., W. Cai, Y.C. Soh, and L. Xie, "Global Optimization for Overall HVAC SystemsPart II Problem Solution and Simulations," Energy Conversion and Management, vol. 46, pp. 1015-1028, 2005.

[98] Fong, K.F., V.I. Hanby, and T.T. Chow, "HVAC System Optimization for Energy Management by Evolutionary Programming," Energy and Buildings, vol. 38, pp. 220$231,2006$. 
[99] Nassif, N., S. Kajl, and R. Sabourin, "Two-Objective On-Line Optimization of Supervisory Control Strategy," Building Service Engineering Research, vol. 25, i. 3, pp. 241-251, 2004.

[100] Nassif, N., S. Kajl, and R. Sabourin, "Evolutionary Algorithms for Multi-Objective Optimization in HVAC System Control Strategy," IEEE NAFIPS, vol. 1, pp. 51-56, 2004.

[101] Nassif, N., S. Kajl, and R. Sabourin, "Optimization of HVAC Control System Strategy Using Two-Objective Genetic Algorithm," International Journal of HVAC and R Research, vol. 11, i. 3, pp. 459-486, 2005.

[102] Wright, J.A., H.A. Loosemore, and R. Farmani, "Optimization of Building Thermal Design and Control by Mulri-Criterion Genetic Algorithm," Energy and Buildings, vol. 34, pp. 959-972, 2002.

[103] Ari, S., I.A. Cosden, H.E. Khalifa, J.F. Dannenhoffer, P. Wilcoxen, and C. Isik, "Constrained Fuzzy Logic Approximation for Indoor Comfort and Energy Optimization,” IEEE NAFIPS, pp. 500-504, 2005.

[104] Cosden, I.A., "Modeling the Energy Efficiency of Distributed Environmental Control Systems,” MS Thesis, Syracuse University, 2005.

[105] ASHRAE, Handbook of Fundamentals, 1997.

[106] Marion, W. and K. Urban, "User's Manual for TMY2s: Typical Meteorological Year," National Renewable Energy Laboratory, June 1995.

[107] Briggs R.S., R.G. Lucas, and Z.T. Taylor, "Climate Classification for Building Energy Codes and Standards," Pacific NW National Laboratory, March 2002

[108] Hanselman, D. and B. Littlefield, Mastering Matlab 7: Comprehensive Tutorial and Reference, Prentice Hall, 2005

[109] Takagi, T. and M. Sugeno, "Fuzzy Identification of Systems and Its Applications to Modeling and Control," IEEE Transactions on Systems, Man, and Cybernetics, vol. 15, pp. 116-132, 1985. 


\title{
E. MODIFIED THERMAL CIRCUIT MODEL FOR UNDERFLOOR VENTILATION SYSTEMS IN A TYPICAL OFFICE BUILDING
}

\author{
By
}

Xuanhang (Simon) Zhang

\author{
REPORT \\ Submitted in partial fulfillment of the requirements for the \\ degree of Master of Science in Mechanical Engineering \\ in the Graduate School of Syracuse University
}

May 2006

Approved:

Dr. H. Ezzat Khalifa

Dr. John Dannenhoffer

Date: 
Report No. DE-FG02-03ER63694-F1

Nomenclature

\begin{tabular}{|c|c|c|}
\hline$\varrho$ & Density & $\mathrm{kg} / \mathrm{m}^{3}$ \\
\hline $\mathrm{k}$ & Thermal conductivity & $\mathrm{W} / \mathrm{mK}$ \\
\hline $\mathrm{u}$ & velocity & $\mathrm{m} / \mathrm{s}$ \\
\hline $\mathrm{h}$ & heat transfer coefficient & $\mathrm{W} / \mathrm{m}^{2} \mathrm{~K}$ \\
\hline$\dot{q}$ & Heat flux & $\mathrm{W} / \mathrm{m}^{2}$ \\
\hline$\dot{m}$ & Mass flow rate of conditioning air & $\mathrm{kg} / \mathrm{s}$ \\
\hline $\mathrm{C}$ & Combined mass flow rate and specific heat & $\mathrm{J} / \mathrm{sK}$ \\
\hline $\mathrm{F}$ & View Factor & - \\
\hline $\mathrm{L}$ & Linkage matrix & - \\
\hline $\mathrm{R}$ & Thermal resistance of zonal interface & $\mathrm{K} / \mathrm{W}$ \\
\hline $\mathrm{U}$ & Overall heat transfer coefficient & $\mathrm{W} / \mathrm{K}$ \\
\hline$\dot{Q}$ & Energy & W \\
\hline$T$ & Temperature & ${ }^{\circ} \mathrm{C}$ \\
\hline$T^{*}$ & Desired room temperature & ${ }^{\circ} \mathrm{C}$ \\
\hline$T_{i}^{s}$ & Supply air temperature to room i & ${ }^{\circ} \mathrm{C}$ \\
\hline$T_{i}^{e}$ & Exhaust air temperature from room $\mathrm{i}$ & ${ }^{\circ} \mathrm{C}$ \\
\hline$T_{i}^{o}$ & Operative temperature of person in room i & ${ }^{\circ} \mathrm{C}$ \\
\hline$T_{i}^{a}$ & Mean air temperature around person in room i & ${ }^{\circ} \mathrm{C}$ \\
\hline $\bar{T}_{i}^{r}$ & Mean radiant temperature in room $\mathrm{i}$ & ${ }^{\circ} \mathrm{C}$ \\
\hline$T_{i}^{c}$ & Average air temperature around wall in room $\mathrm{i}$ & ${ }^{\circ} \mathrm{C}$ \\
\hline$T_{j}^{i w}$ & The $j$ Wall temperature of room $i$ & ${ }^{\circ} \mathrm{C}$ \\
\hline \multicolumn{3}{|c|}{ Subscripts and Superscripts } \\
\hline $\mathrm{adv}$ & Advection & \\
\hline cond & Conduction & \\
\hline gen & Generation & \\
\hline HVAC & HVAC system & \\
\hline $\mathrm{cl}$ & cloth & \\
\hline $\mathrm{c}$ & convection & \\
\hline $\mathrm{r}$ & radiation & \\
\hline $\mathrm{w}$ & wall & \\
\hline
\end{tabular}


Report No. DE-FG02-03ER63694-F1

Copyright (C) 2006 Xuanhang Zhang

All Rights Reserved 
Report No. DE-FG02-03ER63694-F1

\section{Table of Contents}

Nomenclature

2

Table of Contents 4

1 Introduction 6

$1.1 \quad$ Background and Research Objective .............................................................................

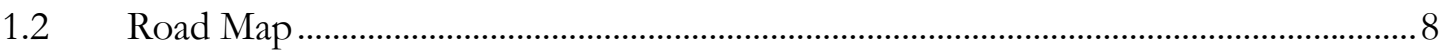

$\underline{2}$ Model Development 9

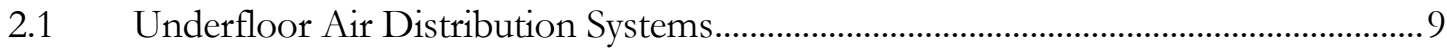

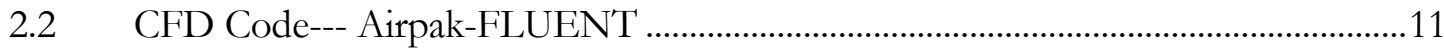

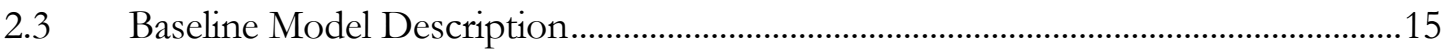

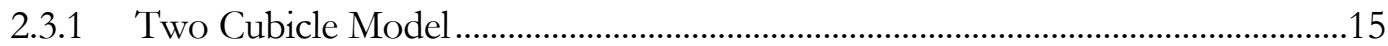

2.3.2 Internal Heat Generation ....................................................................................18

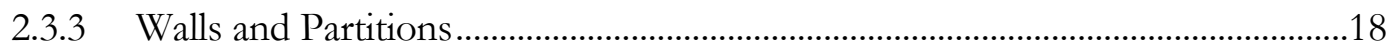

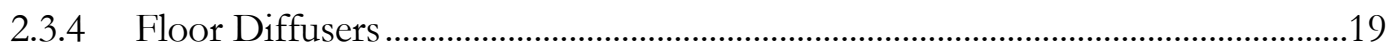

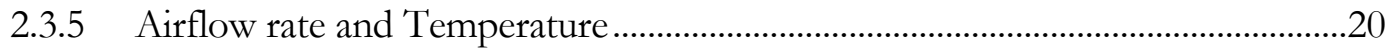

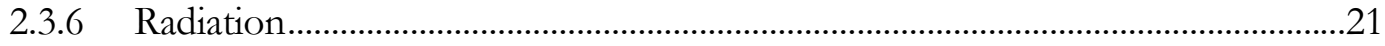

2.3.7 Turbulence modeling .............................................................................................22

$\underline{3}$ Baseline Cases and Computed Results $\quad 27$

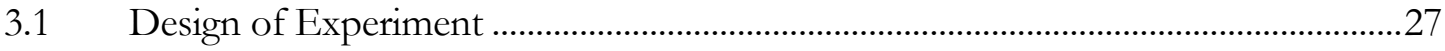

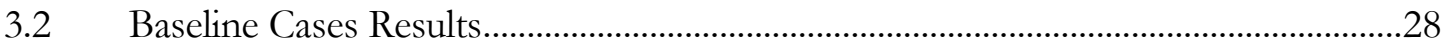

3.2.1 Middle Full Wall Insulated (FWI) .........................................................................28

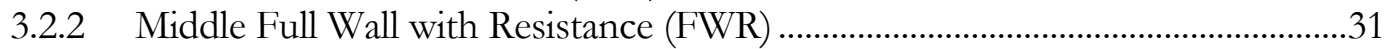

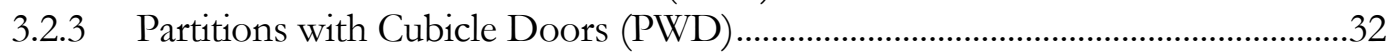

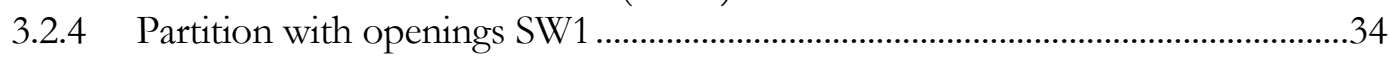

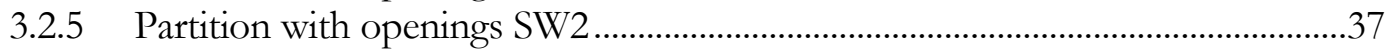

3.3 Other Modes and Comparisons...........................................................................................

4 Methodology of the Modified Thermal Circuit Model 41

4.1 Thermal Environment of Human Occupant ................................................................

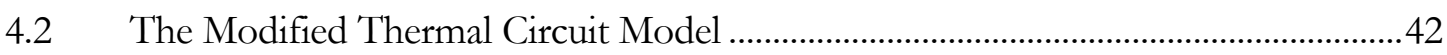

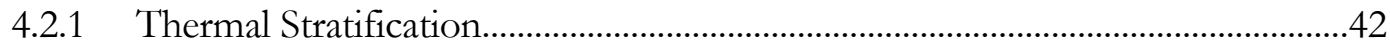

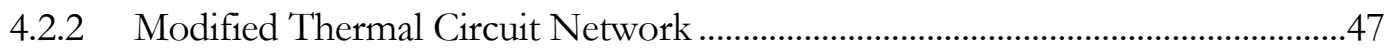

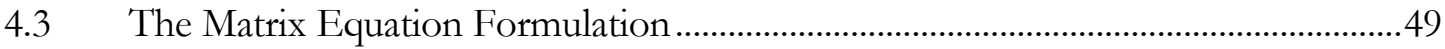




\section{Report No. DE-FG02-03ER63694-F1}

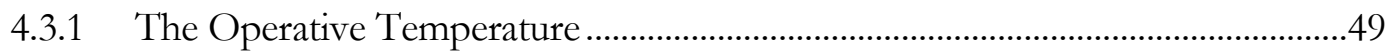

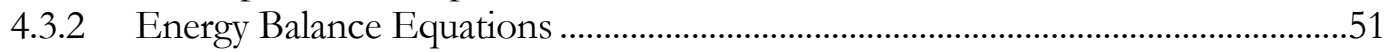

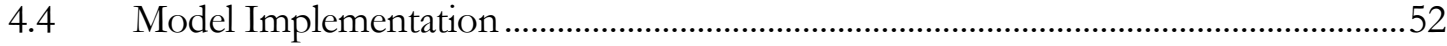

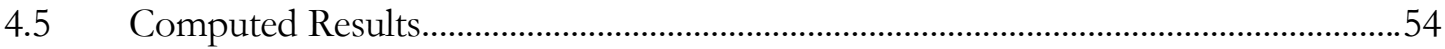

$\underline{5}$ Conclusions $\quad 58$

6 Appendix $\quad 60$

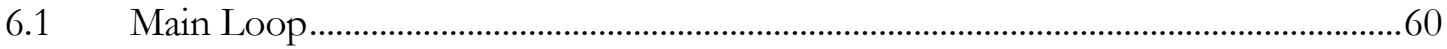

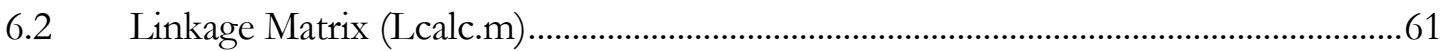

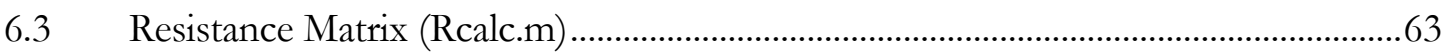

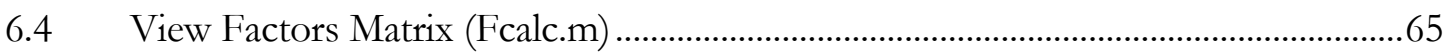

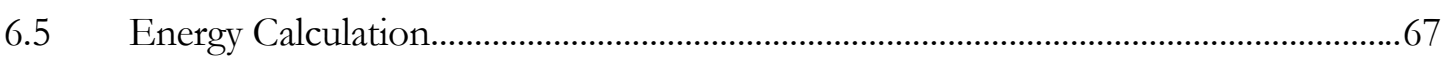

\begin{tabular}{ll} 
References & 76 \\
\hline
\end{tabular} 


\section{Introduction}

\subsection{Background and Research Objective}

In recent years, there has been interest in the modeling of the energy usage of a building with an occupant-controlled distributed environmental control system. For example, Cosden (2005) modeled energy consumption with a new method developed using a temperature-bin-based lumped-parameter approach. In this model, which is based on the analogy to an electrical circuit network, each micro-environment in the building is treated as a node in a circuit, and the heat paths between the zones are modeled as resistors. The air in each zone is assumed to be wellmixed, and hence at a uniform temperature. Energy fluxes between zones are calculated based on the thermal resistance between neighboring zones. Unlike most generally-available building energy models (such as TRNSYS), Cosden's model is not time-dependant and does not account for the transient inertia of building environments. Even though the energy consumption at any particular moment may not be correct in the thermal-circuit model, Cosden showed his model to predict yearly energy consumption within approximately $0.6 \%$ of an equivalent transient simulation, while executing in one six-hundredth of the time.

However, Cosden made several simplifying assumptions in his model that somewhat limits its utility. For example, Cosden's well-mixed approximation makes it only valid for traditional overhead ventilation systems. Modern systems, such as underfloor air distribution (UFAD) systems in which temperature stratification is an essential ingredient, cannot be adequately modeled using the well-mixed approach. Additionally, the well-mixed assumption cannot capture the fact that occupants are located close to the floor, and so only the temperature in the lower part of the room contributes to the occupant's comfort. 


\section{Report No. DE-FG02-03ER63694-F1}

Another assumption of the original thermal circuit model is that there is no air flow between zones in the system, and hence energy flux is only by conduction/convection and not by advection. This is appropriate for buildings composed of offices, but is not appropriate of those composed of workstations divided by partitions and connected by corridors.

Therefore, the objective of this research is to extend the thermal-circuit model in three significant ways: (1) to relax the "office wall" requirement by allowing energy to flow between zones via advection as well as conduction, (2) to improve the comfort model to account both for radiation as well as convection heat transfer, and (3) to support ventilation systems in which the temperature is stratified, such as in underfloor air distribution systems.

The approach to be followed is to perform detailed computational fluid dynamics (CFD) calculations for portions of a building with partitions and adjoining corridors. Through parametric studied, factors such as supply temperature differences and configuration changes will be studied to determine which of these has the strongest influence on the occupants' temperatures, and hence comfort. Also, the simulation results will provide data for analysis of the air stratification behavior due to thermal buoyancy. Subsequently, the temperature profile of each zone will be extracted from CFD results and a simple model will be constructed that can be incorporated into a new thermal-circuit model. This new physics will be incorporated in such a way that the new model will remain linear and will be quickly solvable for use in a control-system optimization process. 


\section{Report No. DE-FG02-03ER63694-F1}

\subsection{Road Map}

In this report, first we will give a short introduction on the underfloor air distribution (UFAD) systems in Section 2.1. A brief summary of the CFD method and code that we used in this research are illustrated in the following section. In Section 2.3, we will introduce the baseline office model, consisting of two cubicles and an adjoining corridor, and all the features of the model will be described in detail. The turbulence modeling of the CFD cases is presented in Section 2.4, because turbulence modeling and near wall treatment is a relatively important part in CFD modeling. In Chapter 3, the baseline case experiment will be explained in 3.1 and the computed results will be shown in Section 3.2 and 3.3. We will then discuss the method that we used to modify the original thermal circuit model in Chapter 4. The idea of personal comfort and thermal stratification will be introduced in Section 4.1 and Section 4.2 respectively. The matrix formulation and the model implementation will be shown in later sections. Results can be found in Section 4.5, and the comparisons were made between the modified and the original thermal circuit model. Finally, the report will end with some conclusions and a listing of the newlymodified thermal-circuit model. 
Report No. DE-FG02-03ER63694-F1

\section{Model Development}

This chapter describes the various components that are assembled into the computational model. It begins by describing the physical problem in general, then discusses the computational tools, and finally combines them into the actual computational model that is used.

\subsection{Underfloor Air Distribution Systems}

An Underfloor air distribution (UFAD) system is an innovative ventilation method that uses the underfloor plenum below a raised floor to deliver heating and air conditioning to offices and commercial buildings. The market for UFAD technology is expanding in North America because of the benefits that it offers over conventional overhead air distribution.

Originally introduced in the 1950s in spaces having high heat loads (e.g., computer rooms, control centers, and laboratories), underfloor air distribution has proven to be the most effective method to deliver conditioned air to occupied zones. Thermal comfort of occupants can be improved by allowing individual control of their local thermal environment. (Bauman 2003) Improvements in ventilation and indoor air quality at the breathing level can also be expected by delivering the fresh supply air at floor level or near the occupant. In addition, Bauman (Bauman 2003) reports UFAD also reduces energy usage by reducing total air volume and static pressure requirements, reduces life-cycle building costs, reduces floor-to-floor height in new construction, and improves productivity and health. 


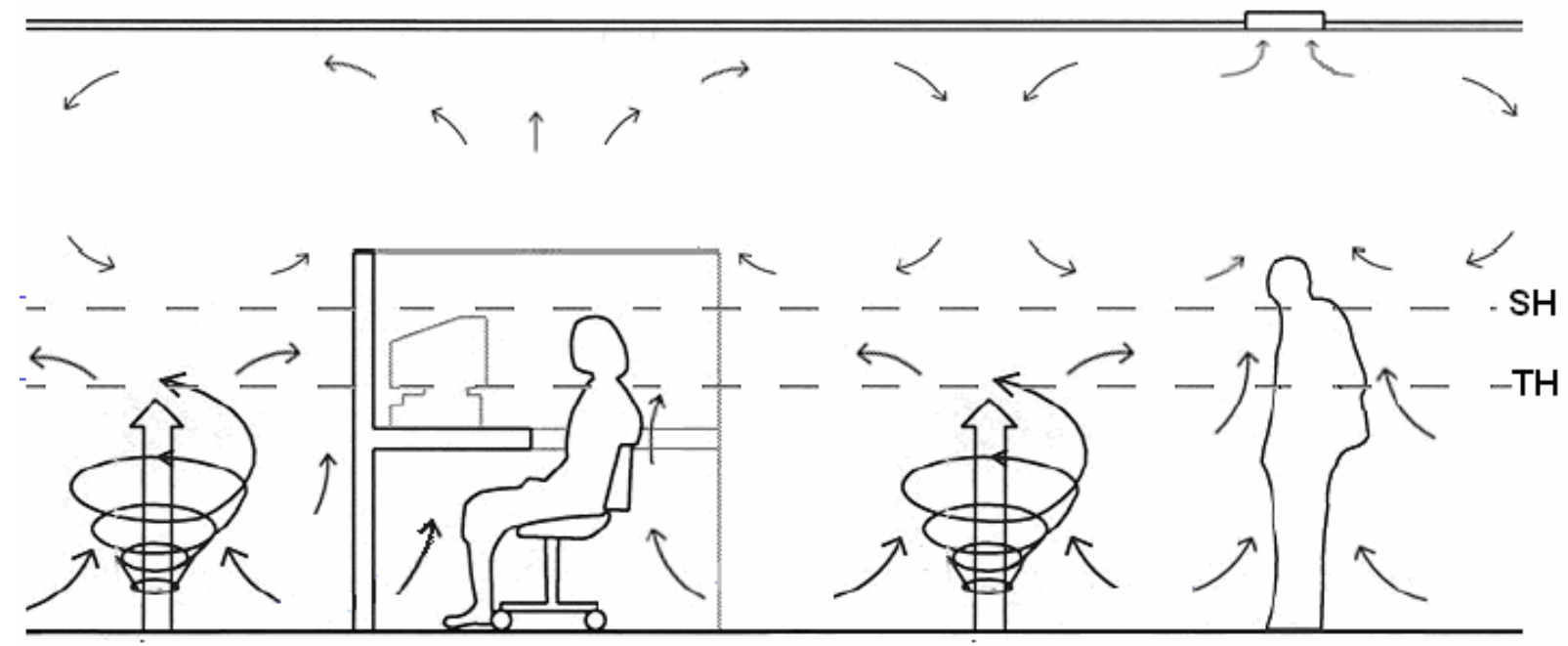

Figure 1.1 Underfloor air distribution systems with diffusers throw below the stratification height (Bauman)

Figure 1.1 shows a typical underfloor room air distribution model. The primary difference between underfloor air distribution systems and displacement ventilation (DV) systems is that UFAD systems have higher velocity inlet air through smaller outlets and allow personal control for local air supply conditions.

Bauman (2003) has shown that there are two characteristic heights, the throw height (TH) and the stratification height $(\mathrm{SH})$, which divide the room into three zones. The Lower (mixed) Zone is between the floor and the TH. Air in this zone is relatively well mixed. The supply air reaches a terminal velocity of around $0.25 \mathrm{~m} / \mathrm{s}$ at the upper boundary of this layer. The vertical temperature gradient is reduced in this zone due to greater mixing compared to DV systems. The throw height (TH) is largely dependent on the throw of the supply outlets and the ratio of the space heat load to the supply airflow. The Middle (Stratified) Zone is a transition layer between $\mathrm{TH}$ and $\mathrm{SH}$. The air in this region is entirely driven by the rising thermal plume around heat sources in the room. The vertical temperature gradient tends to be greatest in this stratified 
zone. This middle zone disappears if $\mathrm{TH}$ is higher than SH. The Upper (mixed) Zone is comprised of warm air accumulated by the rising heat plumes. Air in this region is also relatively well mixed because of the high velocity thermal plumes entering through the lower boundary. The upper mixed zone disappears if the supply air flow rate is equal to or greater than the volume of the heat plumes generated. In that case, it can be modeled as a two-zone model, composed of lower zone and the stratified zone.

\subsection{CFD Code--- Airpak-FLUENT}

The computational fluid dynamics (CFD) software used in this research, Airpak, is a popular design tool for assessing indoor air quality, building airflow and thermal comfort, which is distributed as a companion to the FLUENT system. As in all CFD simulations, Airpak consists of four main phases: model definition, mesh generation, simulation and post-processing. The basic structure of the program is shown in Figure 1.2. 


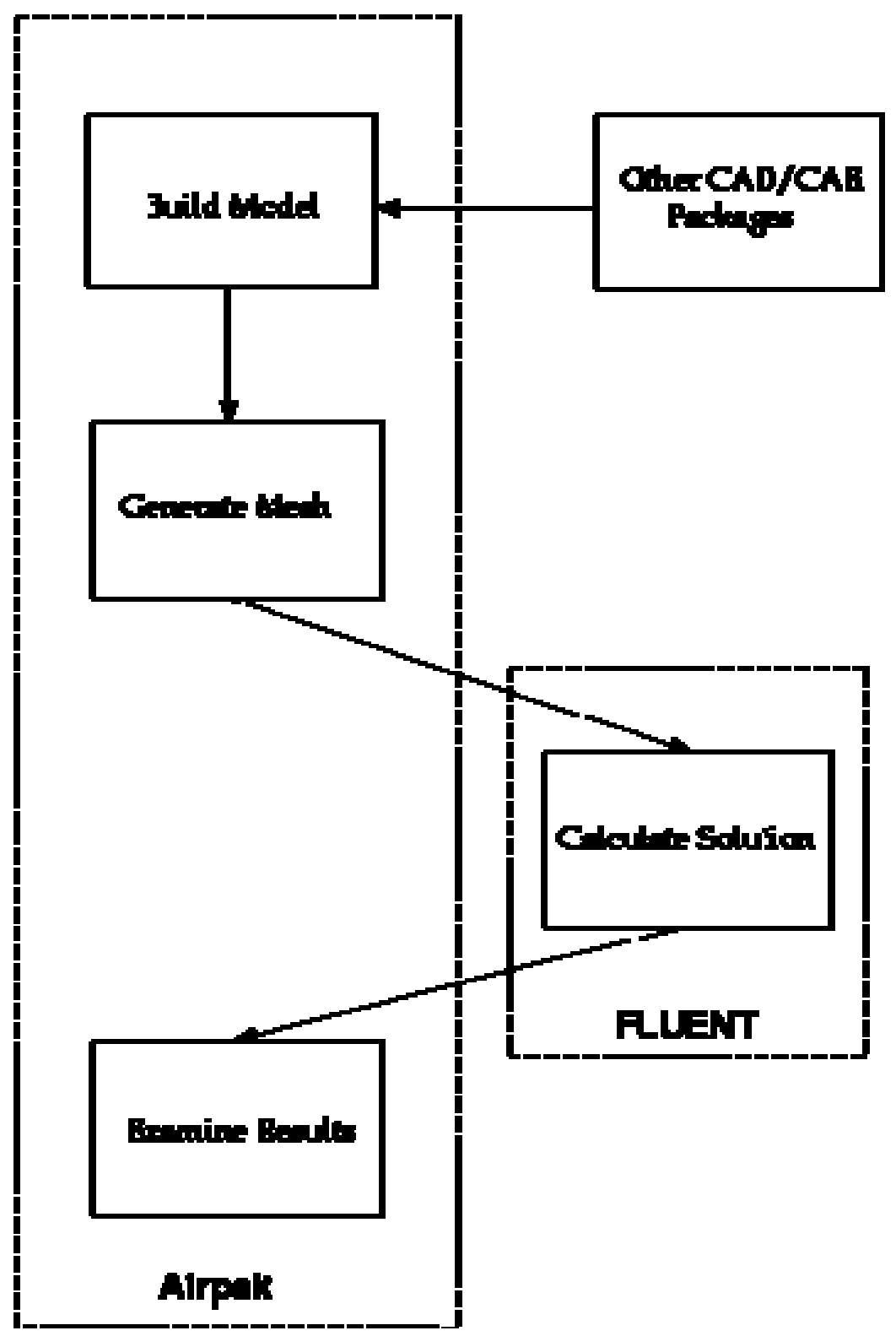

Figure 1.2 Basic Program Structure of Airpak (Airpak Manual 2002)

At first, the user can build a model selecting from the predefined objects, including rooms, people, blocks, fans, partitions, vents, openings, sources, walls, sources, resistances, ducts etc. The object-based procedure can save considerable time in constructing the model. The model can also be imported from other design tools such as CAD/CAE packages. 
After defining all the boundary conditions for the computational model, Airpak will generate a mesh automatically based on the user-specified mesh parameters. It provides full user control of meshing parameters and mesh deployment.

Before running simulations, users also need to define the physical model by choosing a turbulence model, a radiation model, a species transport model, and steady-state or transient analyses etc. In the solver setup, a discretization scheme, under-relaxation factors and convergence criteria can be specified. Some advanced setups can be done through editing the case file directly in text file before running the simulation.

In the simulation phase, five governing partial differential equations will be solved together with equations that model turbulence. The governing equations are the continuity equation:

$$
\frac{\partial \rho}{\partial t}+\frac{\partial}{\partial x_{i}}\left(\rho u_{i}\right)=0
$$

The momentum (in each coordinate direction):

$$
\frac{\partial}{\partial t}\left(\rho u_{i}\right)+\frac{\partial}{\partial x_{j}}\left(\rho u_{i} u_{j}\right)=-\frac{\partial p}{\partial x_{i}}+\frac{\partial \tau_{i j}}{\partial x_{j}}+\rho g_{i}+F_{i}
$$

where $\rho g_{i}$ is the gravitational body force and $F_{i}$ contains other source terms. $\tau_{i j}$ is the stress tensor given by

$$
\tau_{i j}=\left[\mu\left(\frac{\partial u_{i}}{\partial x_{j}}+\frac{\partial u_{j}}{\partial x_{i}}\right)\right]-\frac{2}{3} \mu \frac{\partial u_{l}}{\partial x_{l}} \delta_{i j}
$$

The energy equation for a fluid in terms of sensible enthalpy, $\mathrm{h}\left(\mathrm{h}=\int_{T_{\text {ref }}}^{T} c_{p} d T, T_{\text {ref }}\right.$ is 298.15), is

$$
\frac{\partial}{\partial t}(\rho h)+\frac{\partial}{\partial x_{i}}\left(\rho u_{i} h\right)=\frac{\partial}{\partial x_{i}}\left(k+k_{t}\right) \frac{\partial T}{\partial x_{i}}+S_{h}
$$


where $k$ is the molecular conductivity, $k_{t}$ is the conductivity due to turbulence transport $\left(k_{t}=c_{p} \mu_{t} / \mathrm{Pr}_{t}\right)$, and $S_{h}$ is the source term that includes all the volumetric heat sources.

For solid regions, the energy equation is written as

$$
\frac{\partial}{\partial t} \rho h=\frac{\partial}{\partial x_{i}}\left(k \frac{\partial T}{\partial x_{i}}\right)+q^{\prime \prime}
$$

where $q^{\prime \prime}$ is the volumetric heat source.

As a default, Airpak uses a first-order algorithm while solving the momentum equations, in space (upwind method) and time. The first-order scheme gives a relatively quick and reasonably accurate solution. The second-order scheme is also available for more accurate solutions, but is recommended to be used based on converged first order solutions. The SIMPLE pressurevelocity coupling algorithm is applied and the discretization scheme for the pressure was body force weighted. In this research, first-order scheme and SIMPLE algorithm were used in all the simulations. Stable convergent solutions were achieved by setting the under-relaxation factor to 0.7 for pressure and 0.2 for momentum for the cases studied in this report.

In the post-processing phase, Airpak includes a full-function 3D object-based post-processor, which allows the user to generate visualizations of the flow field and reports. Users can employ a variety of methods in the post-processing, including vector plots and contour plots, object face plots, animation etc. to present air, temperature, contaminant distribution and other parameters. Finally it can generate a report customized to the user's specifications. Post-processing can also be done in FLUENT, which provides more flexible capabilities of reporting. 


\section{Report No. DE-FG02-03ER63694-F1}

\subsection{Baseline Model Description}

\subsubsection{Two Cubicle Model}

Many commercial buildings consist of different types of cubicles and corridors. The office space is divided to separate workstations by partition walls, and then connected by corridors. A typical office suite layout is shown in Figure 2.1. There are different spatial combinations of two cubicles in a typical office building. Six typical combinations of the two adjacent cubicles can be found in a simplified building layout, shown as in Figure 2.2. In each mode (combination), the air flow and energy flux between the two cubicles will have different kind of behavior according to the configuration of the cubicles.

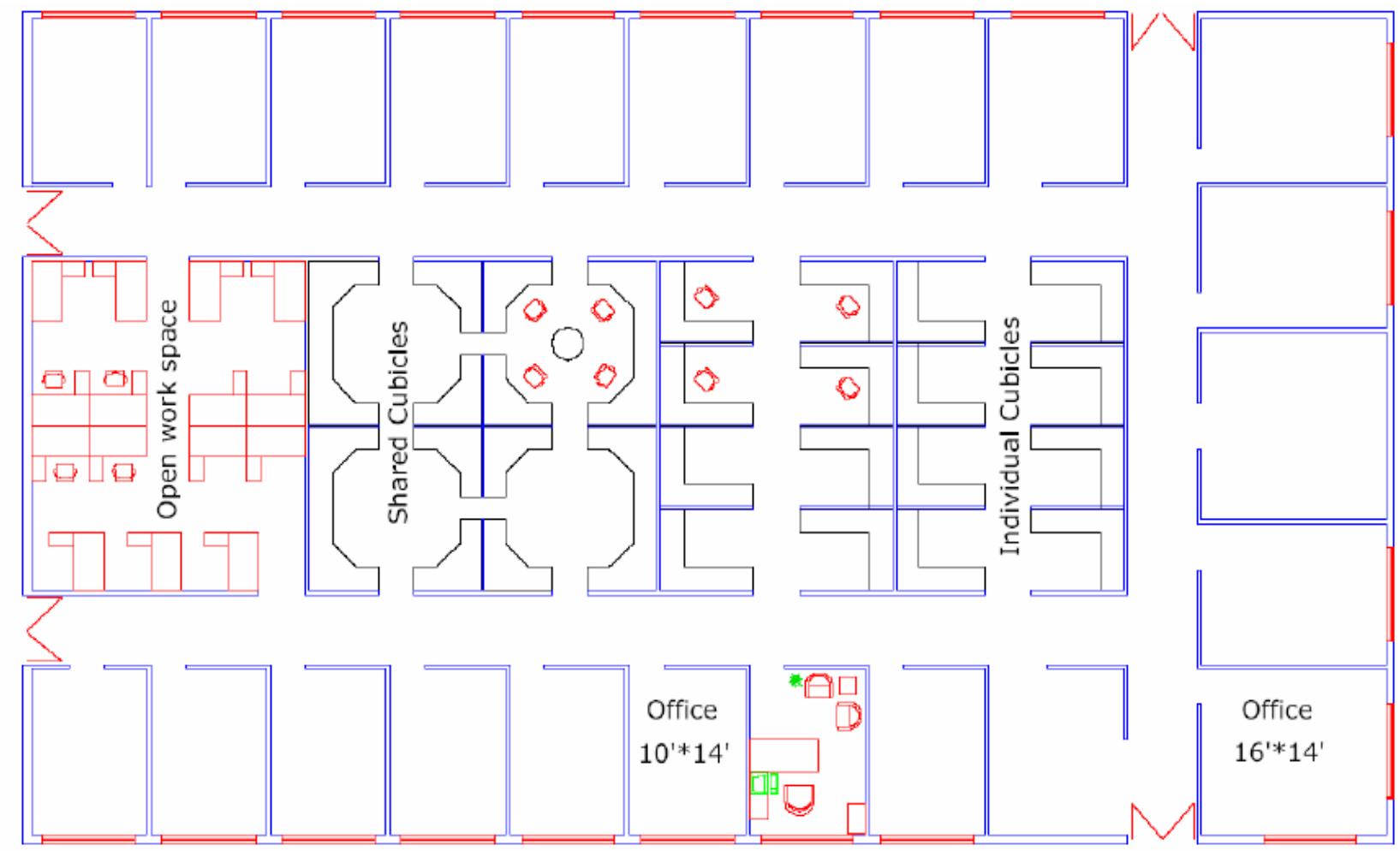

Figure 2.1 A Typical Office Suite Layout 


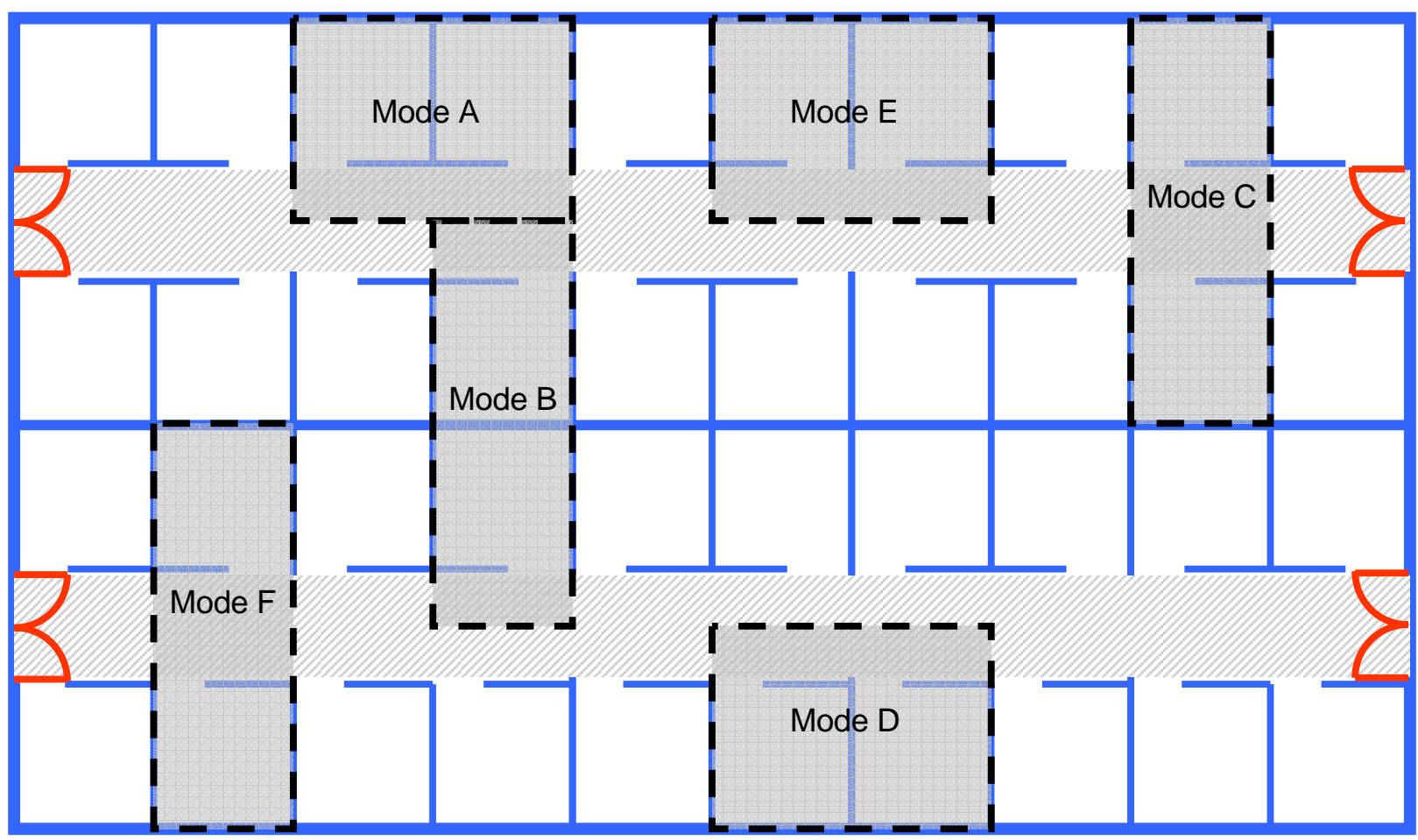

Figure 2.2 A Simplified Typical Office Suite Layout

For this project, the baseline case is Mode A. We will assume that the cubicles repeat themselves symmetrically at each boundary, i.e. the cubicles next to the pair being represented are exactly identical to it in geometry and boundary conditions.

The specific geometry of the two-cubicle-model used throughout this study is shown in Figures 2.3 to 2.5 


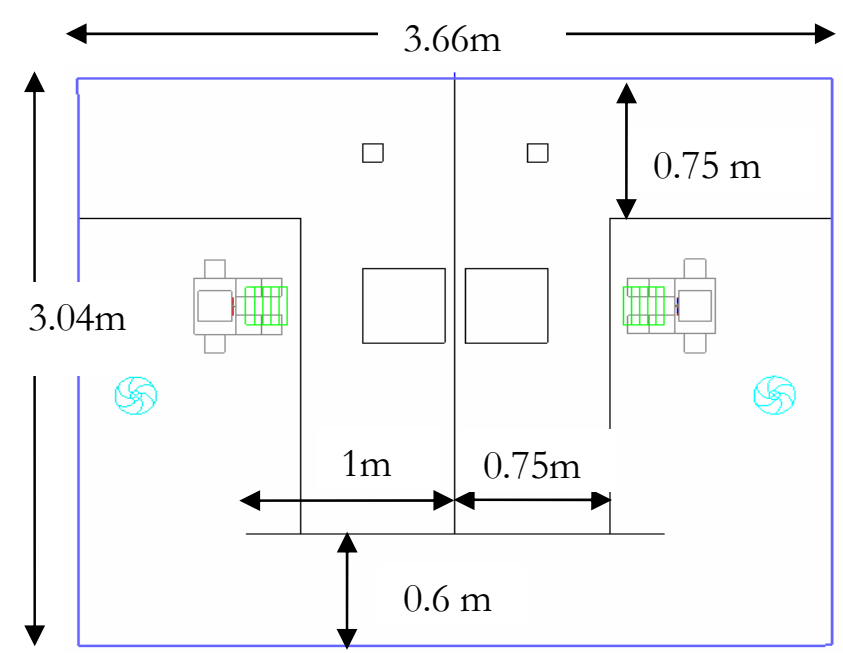

Figure 2.3 Mod A (Top View)

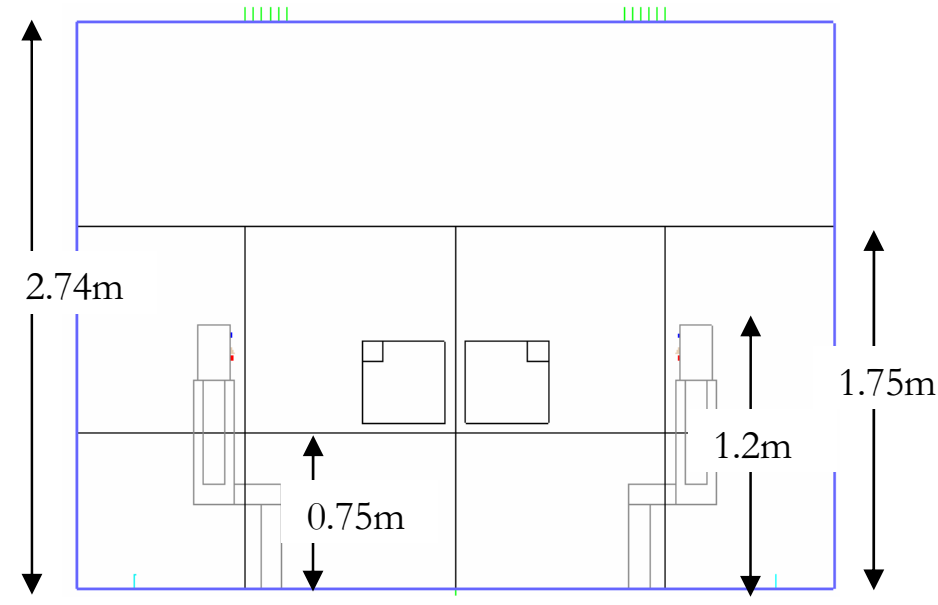

Figure 2.4 Mod A (Side View)

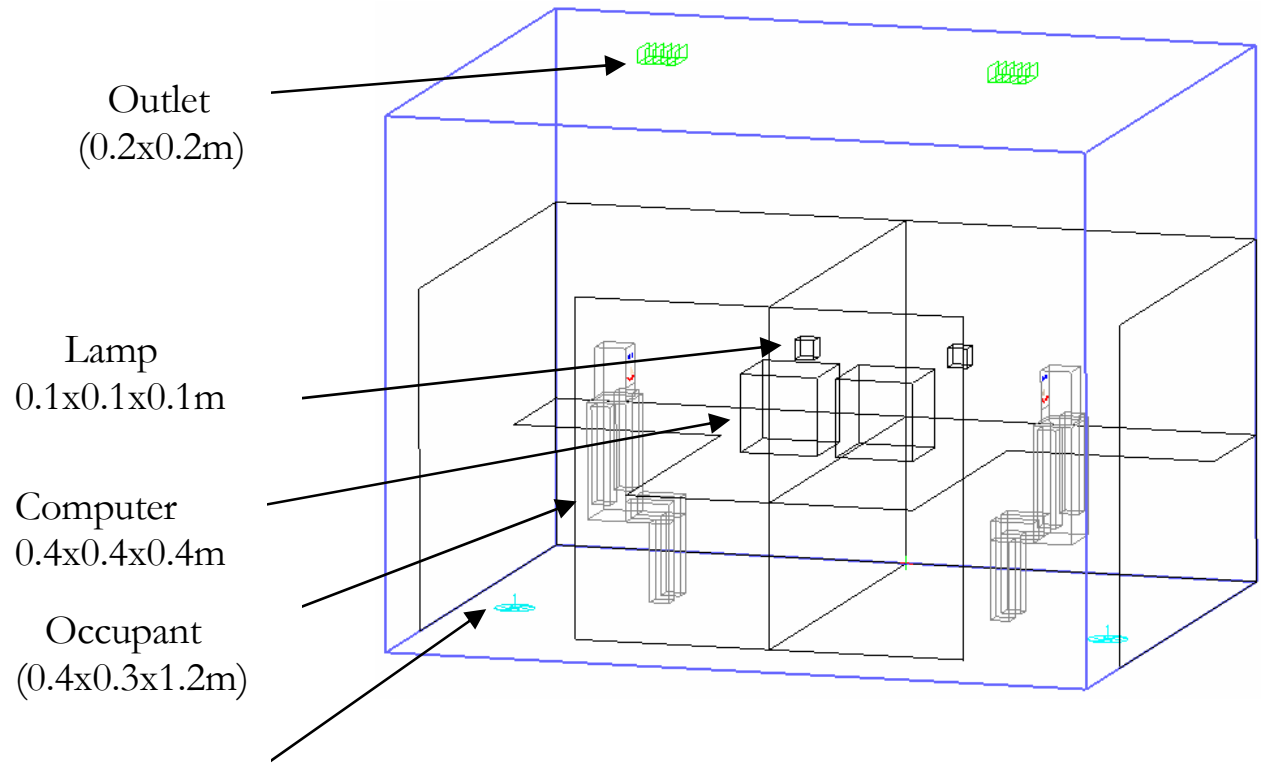

Swirl Inlet $(\mathrm{d}=0.2 \mathrm{~m})$

Figure 2.5 Two-cubicle-model of mod A (Plain View)

The model for each cubicle incorporates an L-shaped desk, an occupant, a computer, a desk lamp and panel partitions, which represents a typical office setup in North America. To simplify 
the CFD model, the seated occupants were replaced by heated blocks, with fixed internal heat generation. At the meantime, simple blocks were also used to represent the computers (CPU box and monitor) and the desk lamps.

\subsubsection{Internal Heat Generation}

In a typical office room or a cubicle, there is a human occupant doing moderate office work with a computer and a lamp. To define these internal heat sources inside each zone, the specific heat gain values can be obtained from the ASHRAE handbook of Fundamentals (2001). For a sedentary office occupant with a skin surface area of $1.8 \mathrm{~m}^{2}$, the sensible heat is $75 \mathrm{~W}$, in which $38 \sim 58 \%$ is radiant heat. (Ch29, ASHRAE handbook 2001) The latent heat is not accounted for because humidity is not included in our model. As for the computer, it generates an average of $55 \mathrm{~W}$ from CPU box and 55W from the monitor. Our computer model combines these two components and is simplified by a single block, $5 \mathrm{~cm}$ above the desk, with total power $110 \mathrm{~W}$. In our model, the back and top of the computer cube is a "hot" surface, whereas the others are "cold".. The heat generated from the hot surface is about 2.5 times greater than the heat generated from the cold surface (Abanto et al. 2004). A 75W desk lamp is also included in the model. Therefore, the total internal heat generation in each office (cubicle) is $260 \mathrm{~W}$.

\subsubsection{Walls and Partitions}

For this project, it is not of interest to model heat transfer between offices on different floors. Therefore, adiabatic conditions were chosen for the floor and the ceiling. Partitions, which are $1.75 \mathrm{~m}$ high, divide the working space into cubicles. The thermal resistance of the interior wall partition is $0.392 \mathrm{~m}^{2} \mathrm{~K} / \mathrm{W}$, which agrees with Cosden's thermal circuit model (Cosden 2005). 
Further, it is assumed that the two cubicles are adjacent to other cubicles and connected by a corridor. The space above the partitions is open. Symmetric boundary condition are imposed on the boundary surfaces that connecting the open spaces. For this boundary condition there is no flow across the boundary and no scalar flux across the boundary.

\subsubsection{Floor Diffusers}

UFAD systems have a larger number of supply diffusers compared to standard overhead systems (http://www.cbe.berkeley.edu/underfloorair/diffusers.htm). Many of these diffusers are in close proximity to the occupants, delivering a smaller amount of air than traditional ceiling diffusers. The swirl diffuser is the most commonly installed type of diffuser in UFAD systems. There is a wider variety of swirl diffusers available than any other type of diffusers. Figure 2.6 gives some examples of currently available underfloor swirl diffusers manufactured by Carrier.
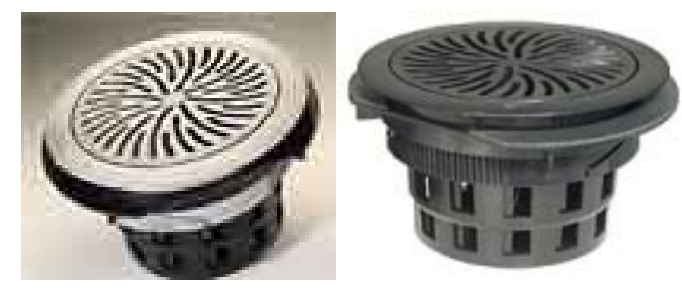

Figure 2.6 Swirl Diffusers (Carrier)

Rapid mixing of supply air with room air up to the throw height will be caused by the swirling pattern of the discharged flow. Generally, the swirl diffusers will be placed in the interior zones, as shown in Figure 2.7, allowing occupants to have some control of their local environment. The volume, direction, and sometimes temperature of the incoming air supply can be adjusted by occupants. The swirl diffusers can be installed as passive diffusers (with pressurized underfloor plenums) or active diffusers (fan-driven). In this simulation, active diffusers are used. 


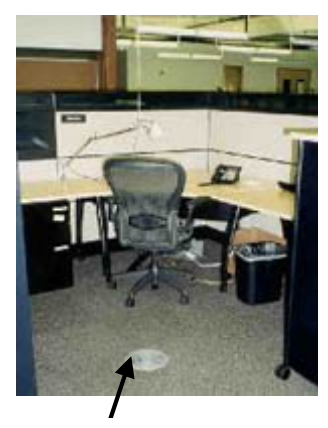

Floor diffuser

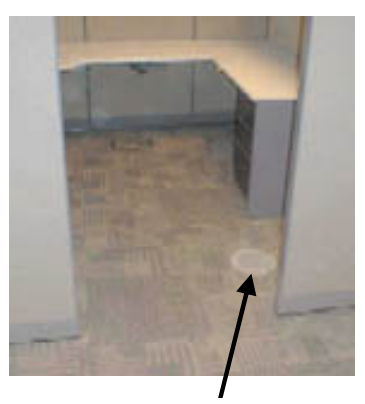

Floor diffuser

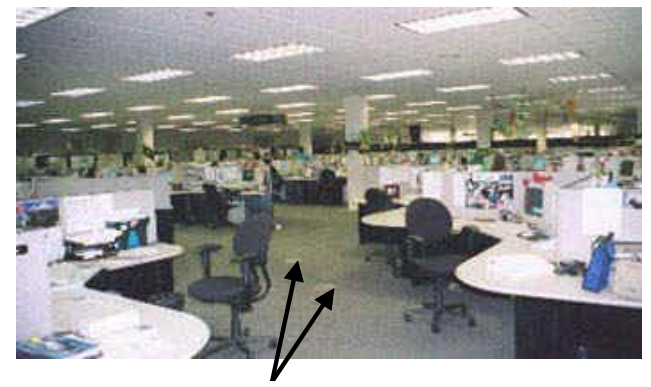

Floor diffusers

Figure 2.7 Floor Diffusers and a typical layout (Bauman's Case Study)

\subsubsection{Airflow rate and Temperature}

The total volume of one "zone", including the cubicle and the corresponding corridor, is $1.83 \mathrm{~m}$ x $3.04 \mathrm{~m} \times 2.74 \mathrm{~m}(6 \mathrm{ft} \times 10 \mathrm{ft} \times 9 \mathrm{ft})$, or about $15.24 \mathrm{~m}^{3}\left(540 \mathrm{ft}^{3}\right)$. The air change rate for each zone was chosen to be 6 air changes per hour $(6 \mathrm{ACH})$, which is typical of office ventilation systems. (Awbi, 2003) Therefore for the baseline case, we have the total airflow rate of $0.0254 \mathrm{~m}^{3} / \mathrm{s}$ (54cfm).

Because air is supplied directly into the occupied zone, the minimum supply air temperature should not be less than $16^{\circ} \mathrm{C} \sim 18^{\circ} \mathrm{C}$ (Bauman 2003). Currently, other supplies of air to offices for personal control allow temperatures to vary over a wider range ---- up to $13^{\circ} \mathrm{F}\left(7^{\circ} \mathrm{C}\right)$ for desktop outlets and $9^{\circ} \mathrm{F}\left(5^{\circ} \mathrm{C}\right)$ for floor diffusers (Tsuzuki et al. 1999). For the purpose of this study, we extended the range for the floor diffuser to $8^{\circ} \mathrm{C}\left(16 \sim 24^{\circ} \mathrm{C}\right)$.

The exhaust vent is located in the center of the ceiling for each cubicle. This location represents a typical exhaust of a real individual office cubicle. The pressure at the vent is same as ambient. 


\section{Report No. DE-FG02-03ER63694-F1}

The outside temperature of the vent is equal to the ambient temperature $22^{\circ} \mathrm{C}$ (needed in cases where the is backflow out of the vent).

\subsubsection{Radiation}

In addition to conduction and convection, radiation heat transfer also needs to be taken into account. It is said that about $38 \sim 58 \%$ of sensible heat from the occupant is radiant heat (ASHRAE handbook 2001). Unlike conduction and convection, radiation does not require a medium for transmission. Also, for most situations, conductive and convective heat transfer rates are linearly proportional to temperature difference, while radiant heat transfer is proportional to differences in temperature raised to the fourth power. The heat transfer rate is defined as (Fluent user's guide 2003):

$$
q=\sigma e F\left(T_{\text {surface }}^{4}-T_{\text {remote }}^{4}\right)
$$

where $T_{\text {surface }}$ is the temperature of the object surface, $T_{\text {remote }}$ is the temperature of the surface to which the object radiates heat, $\mathrm{F}$ is the view factor indicating the fraction of energy that is intercepted by the surface of the object, e is the emissivity of the surface of the object and $\sigma$ is the Stefan-Boltzmann constant $\left(\sigma=5.67 \times 10^{-8} \mathrm{JK}^{-4} \mathrm{~m}^{-2} \mathrm{~s}^{-1}\right)$.

Two radiation models are available in Airpak: the discrete ordinates (DO) radiation model and the surface-to-surface (S2S) radiation model. The latter provides an economical way to account for radiation in most applications, but its accuracy greatly depends on the view factor calculation. Airpak calculates the view factors automatically based on a very coarse mesh after the model is 
set up and carry those through the whole calculation. The DO model, on the other hand, solves the radiative transfer equation (RTE) for a finite number of discrete solid angles, each associated with a vector direction fixed in the global Cartesian system. It utilizes the current grid to do iterative calculation, in a similar manner to the continuity, momentum and energy equations. In order to get more accurate results, we choose the DO radiation model in our radiation calculation. A comparison of the results from S2S model and DO model will be discussed in Chapter 3.2.

\subsubsection{Turbulence modeling}

Since the majority of indoor air flows are turbulent, it is necessary to include some representation of turbulence in computational models of flow processes. There are no exact solutions for the Navier-Stokes equations for turbulent flows. Numerical solutions can be obtained by direct numerical simulations (DNS) (Moin et al. 1998), in which all temporal and spatial scales are solved accurately, but at an extraordinarily high computational cost. Since it is unrealistic to simulate the detailed fluctuations, the transient governing equations can be time-averaged to remove the small scale and high frequency fluctuations. The classical turbulence models are based on time-averaged Reynolds Navier-Stokes equations. They include mixing length model (zero equation model), $\mathrm{k}-\varepsilon$ model (two-equation model), Reynolds stress equation model as well as others. Besides DNS and the Reynolds-Averaged approach, large eddy simulation (LES) approach can serve as a compromise. It directly solves the large energy-containing spatial scales (like DNS), while modeling the influence of small scale turbulence as well (like ReynoldsAverage) (Moin 1997). 


\section{Report No. DE-FG02-03ER63694-F1}

\subsubsection{The standard k-E model}

Of the conventional turbulence models, the $\mathrm{k}-\varepsilon$ model, proposed by Launder and Spalding (1974), is the most widely used and validated semi-empirical model based on model transport equations for the turbulent kinetic energy $(\mathrm{k})$ and its dissipation rate $(\varepsilon)$. It solves the two separate transport equations simultaneously which allow the turbulent velocity and length scales to be independently determined. The turbulent viscosity, $\mu_{t}$, is computed by combining $\mathrm{k}$ and $\varepsilon$ as follows:

$$
\mu_{t}=\rho C_{\mu} \frac{k^{2}}{\varepsilon}
$$

where $C_{\mu}$ is an empirical constant specified in the turbulence model, 0.09 (standard k- $\varepsilon$ model) and 0.0845 (RNG k- $\varepsilon$ model) as set by FLUENT.

The transport equations for the standard $k-\varepsilon$ model are:

$$
\frac{\partial}{\partial t}(\rho k)+\frac{\partial}{\partial x_{i}}\left(\rho k u_{i}\right)=\frac{\partial}{\partial x_{j}}\left[\left(\mu+\frac{\mu_{t}}{\sigma_{k}}\right) \frac{\partial k}{\partial x_{j}}\right]+G_{k}+G_{b}-\rho \varepsilon-Y_{M}+S_{k}
$$

and

$$
\frac{\partial}{\partial t}(\rho \varepsilon)+\frac{\partial}{\partial x_{i}}\left(\rho \varepsilon u_{i}\right)=\frac{\partial}{\partial x_{j}}\left[\left(\mu+\frac{\mu_{t}}{\sigma_{\varepsilon}}\right) \frac{\partial \varepsilon}{\partial x_{j}}\right]+C_{1 \varepsilon} \frac{\varepsilon}{k}\left(G_{k}+C_{3 \varepsilon} G_{b}\right)-C_{2 \varepsilon} \rho \frac{\varepsilon^{2}}{k}+S_{\varepsilon}
$$

In these Equations, $\mathrm{Gk}$ is the generation of turbulence kinetic energy due to the mean velocity gradient. Gb represents the generation of turbulence kinetic energy due to buoyancy. YM accounts for the contribution of the fluctuating dilatation in compressible turbulence to the overall dissipation rate. $\mathrm{C} 1 \varepsilon, \mathrm{C}_{2} \varepsilon$ and $\mathrm{C} 3 \varepsilon$ are constants. $\sigma \mathrm{k}$ and $\sigma \varepsilon$ are the turbulence Prandtl numbers for $\mathrm{k}$ and $\varepsilon$, respectively. Sk and $\mathrm{S} \varepsilon$ are source terms. (Fluent user's guide 2003) 


\section{Report No. DE-FG02-03ER63694-F1}

\subsubsection{Initialize k-e model}

The turbulence intensity of the inlet boundary condition is defined as $10 \%$, a typical value used for indoor office environment. The initial values for $\mathrm{k}$ and $\varepsilon$ are then calculated using equations:

$$
k=\frac{3}{2}\left(I u_{a v g}\right)^{2}
$$

and

$$
\varepsilon=C_{\mu}^{3 / 4} \frac{k^{3 / 2}}{l}
$$

where $\mathrm{I}$ is the turbulence intensity. The turbulence length scale is defined as $1=0.07 \mathrm{~L}(\mathrm{~L}$ is the hydraulic diameter of the duct) (Nielsen 2003).

\subsubsection{Near-wall treatment}

Some turbulence models, such as the $k-\varepsilon$ model, is primarily valid only for fully turbulent flows, which means they assumed the molecular viscosity is negligible. Therefore, attention should be paid to the wall-bounded flows and how to modify these models to make them accurate in that region. Numerous experiments have shown that the near-wall region can be subdivided into three main layers, as illustrated in Figure 2.8. The layer closest to the wall is called the 'viscous sublayer' in which the molecular viscosity dominates momentum, heat and mass transfer. The buffer layer (blending region) connects the viscous sublayer and the outer layer. In the fullyturbulent layer, turbulence dominates momentum, heat and mass transfer and it is known as the 'log-law region’.

Two models are included in Airpak to simulate flows in the near-wall region: the Wall Functions model and the Near-Wall model. The wall functions are used to bridge the molecular viscosityaffected region between the fully-turbulent region and the wall, typically used for most highReynolds-number flows. The near-wall model can resolve viscosity-affected region by solving a 
modified turbulence model all the way down to the wall with a very fine mesh near the wall. For the flow we are dealing with, the Reynolds number is relatively low. Therefore it is recommended that the near-wall model approach will provide more accurate solutions (Fluent user's guide 2003).

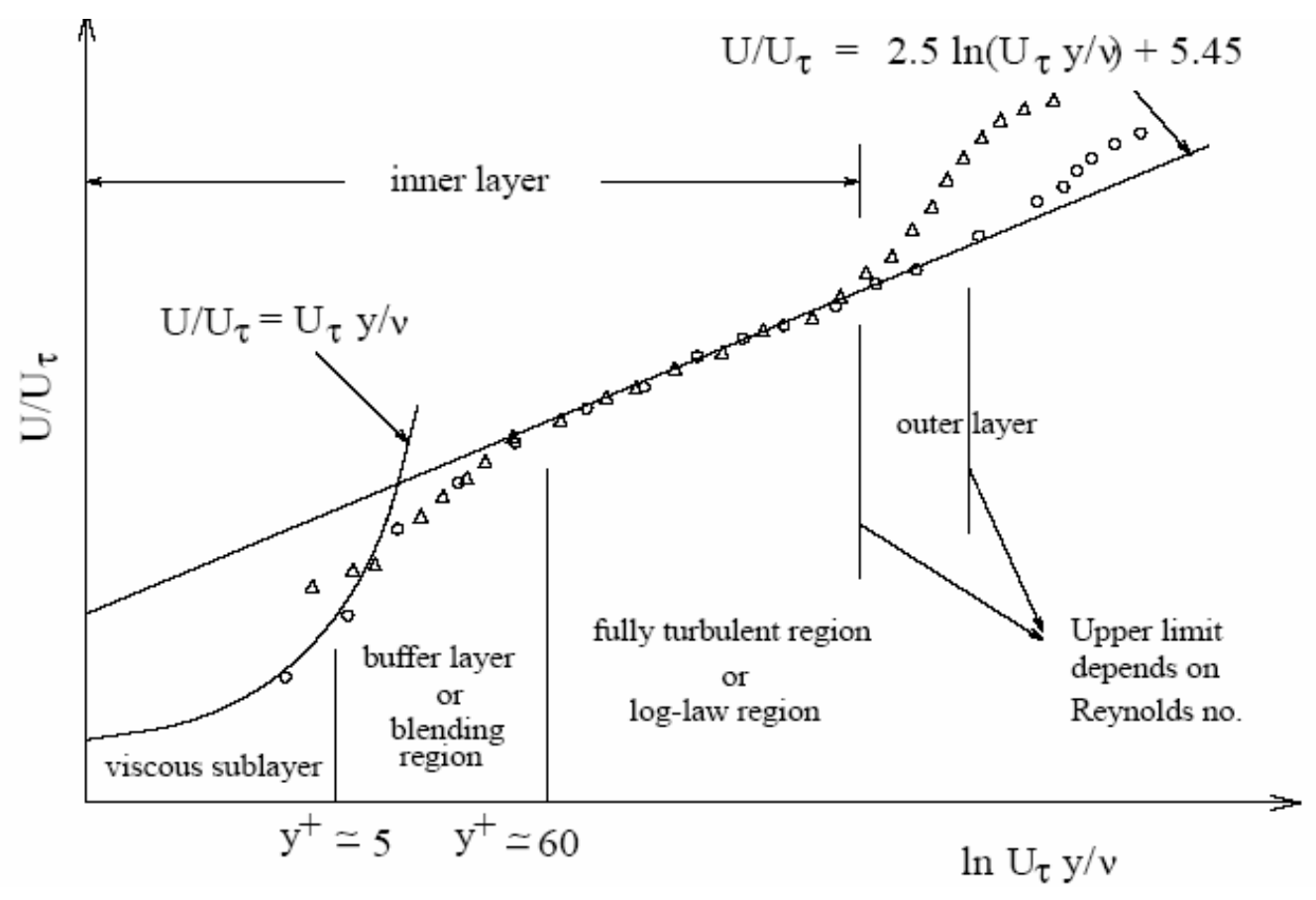

Figure 2.8 subdivisions of the Near-wall Region (FLUENT manual)

Low-Reynolds number models have been applied for plenty of indoor airflow modeling (Chen 1995, Awbi 1998). In low-Reynolds number modeling (Launder and Spalding 1974) grid points are placed within the boundary layer, including the laminar region. As a result, some of the empirical constants will vary with the local turbulence Reynolds number. In the early 1900's, Prandtl first proposed a two-layer model to model the near wall region. But in the computational schemes, sufficiently fine mesh should be placed near walls, which will dramatically increase 


\section{Report No. DE-FG02-03ER63694-F1}

computational burden. Fluent (Fluent user's guide 2003) offered a method to combine the traditional two-layer model with enhanced wall functions (Kader 1993).

When the enhanced wall treatment is employed, $y^{+}$, defined as $y^{+}=\rho u_{\tau} y / \mu$, at the walladjacent cell should be less than $4 \sim 5$ to enable the cell to be within the viscous sublayer. Also, at least 10 cells should be placed within the viscosity-affected near-wall layer to resolve turbulent quantities in this region (Fluent user's guide 2003). In this research, $y^{+}$are less than 3 around the person surfaces. 
Report No. DE-FG02-03ER63694-F1

\section{Baseline Cases and Computed Results}

This chapter describes the three-dimensional computational simulations that were performed for two-cubicle-with-corridor configurations.

\subsection{Design of Experiment}

The purpose of the simulations is to test the degree of coupling in the relationship between the personal temperatures in these two cubicles. In other words, if personal air conditioning is implemented in each cubicle, how much does the air temperature in one cubicle affect that of the other cubicle? The design of the experiment is shown in Table 3.1 below:

\begin{tabular}{|c||c|c|c|}
\hline Ts1 $\left({ }^{\circ} \mathrm{C}\right)$ & Ts2 $\left({ }^{\circ} \mathrm{C}\right)$ & Vs1 $(\mathrm{ACH})$ & $\mathrm{Vs} 2(\mathrm{ACH})$ \\
\hline 16 & 16 & 6 & 6 \\
\hline 20 & 16 & 6 & 6 \\
\hline 24 & 16 & 6 & 6 \\
\hline
\end{tabular}

Table 3.1 Design of the Experiment

For a given mode (combination of two cubicles), the supply temperature (Ts) and volume flow $(\mathrm{Vs})$ rate of the two cubicles are adjustable parameters. For the temperature control cases, the ventilation rate of each zone will be fixed at $6 \mathrm{ACH}$. The supply temperature of zone 1 varies from $16^{\circ} \mathrm{C}$ to $24^{\circ} \mathrm{C}$ with constant supply temperature of $16^{\circ} \mathrm{C}$ in zone 2 . It can be expected that if the two zones are perfectly decoupled, the person temperature in zone $1(\mathrm{Tp} 1)$ will increase as 
Ts1 increases, while the person temperature in zone $2(\mathrm{Tp} 2)$ will remain constant, since there is no change for Ts2. On the contrary, if the two zones are coupled, we would expect that Tp2 will increase due to the increase of Ts1, and Tp1 will not increase as much as the ideally decoupled case because of the effect of a lower Ts2. Figure 3.1 and Figure 3.2 show the plots of the correlation of $\mathrm{Tp}$ and $\mathrm{Ts}$ with respect to degree of coupling of the two zones. The more the two zones coupled, the closer $\mathrm{Tp} 1$ and $\mathrm{Tp} 2$ are on the plots.

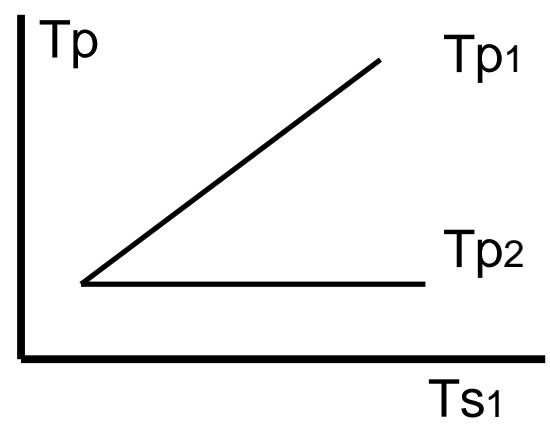

Figure 3.1 2-zones perfectly decoupled case

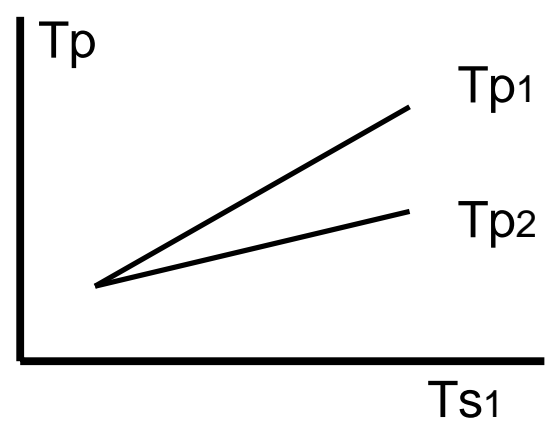

Figure 3.2 2-zones coupled case

\subsection{Baseline Cases Results}

\subsubsection{Middle Full Wall Insulated (FWI)}

Firstly, a full wall, from front to back and from floor to ceiling, was put in to separate the two zones, as shown in Figure 3.3. Although it is not realistic in real life, it is the good beginning to test the model at the extreme perfectly decoupled situation. When the middle full wall is insulated, the perfectly decoupled behavior of the two zones will be evident and a plot similar to Figure 3.1 would be expected. 


\section{Report No. DE-FG02-03ER63694-F1}
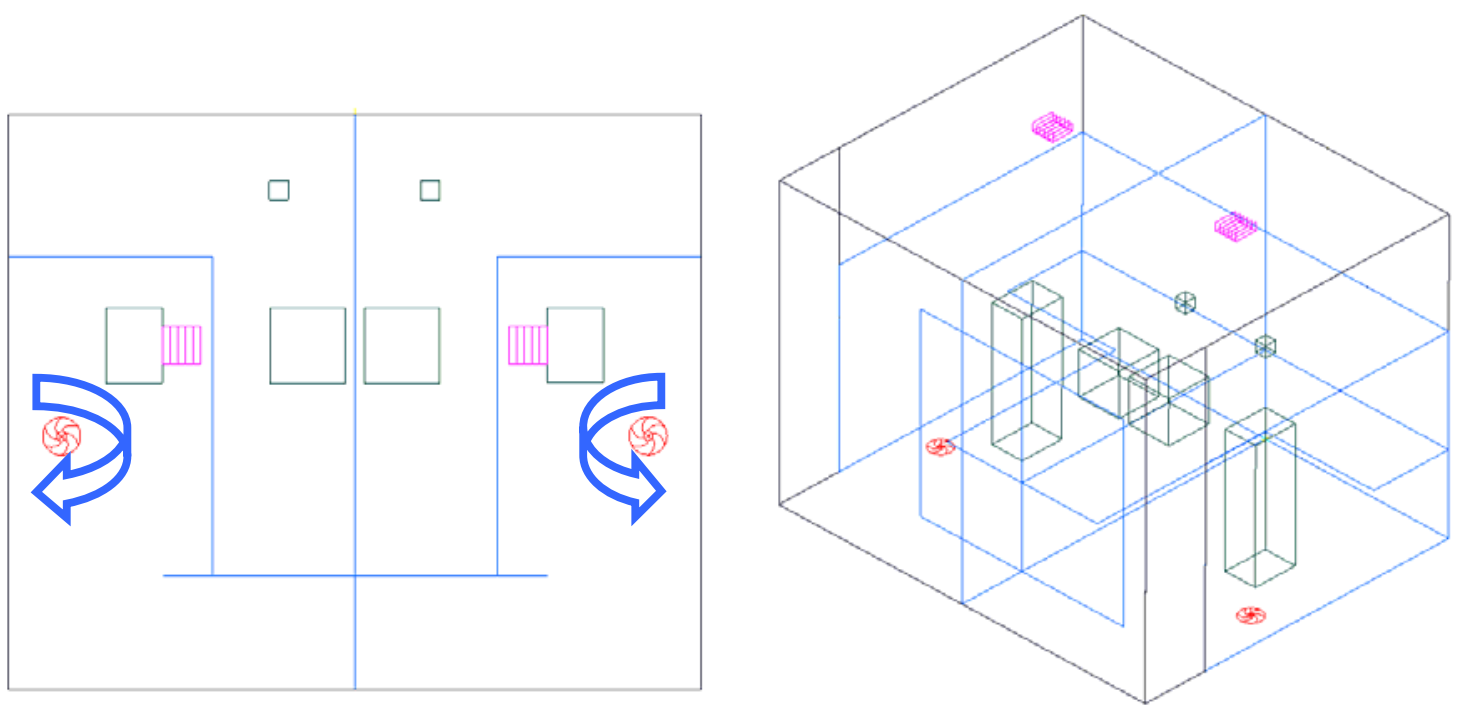

Figure 3.3 Mode A with full wall in middle SW1

The swirl direction for the two floor supplies was chosen to be symmetric and the direction shown in Figure 3.3 above is defined as Swirl Direction 1 (SW1). 


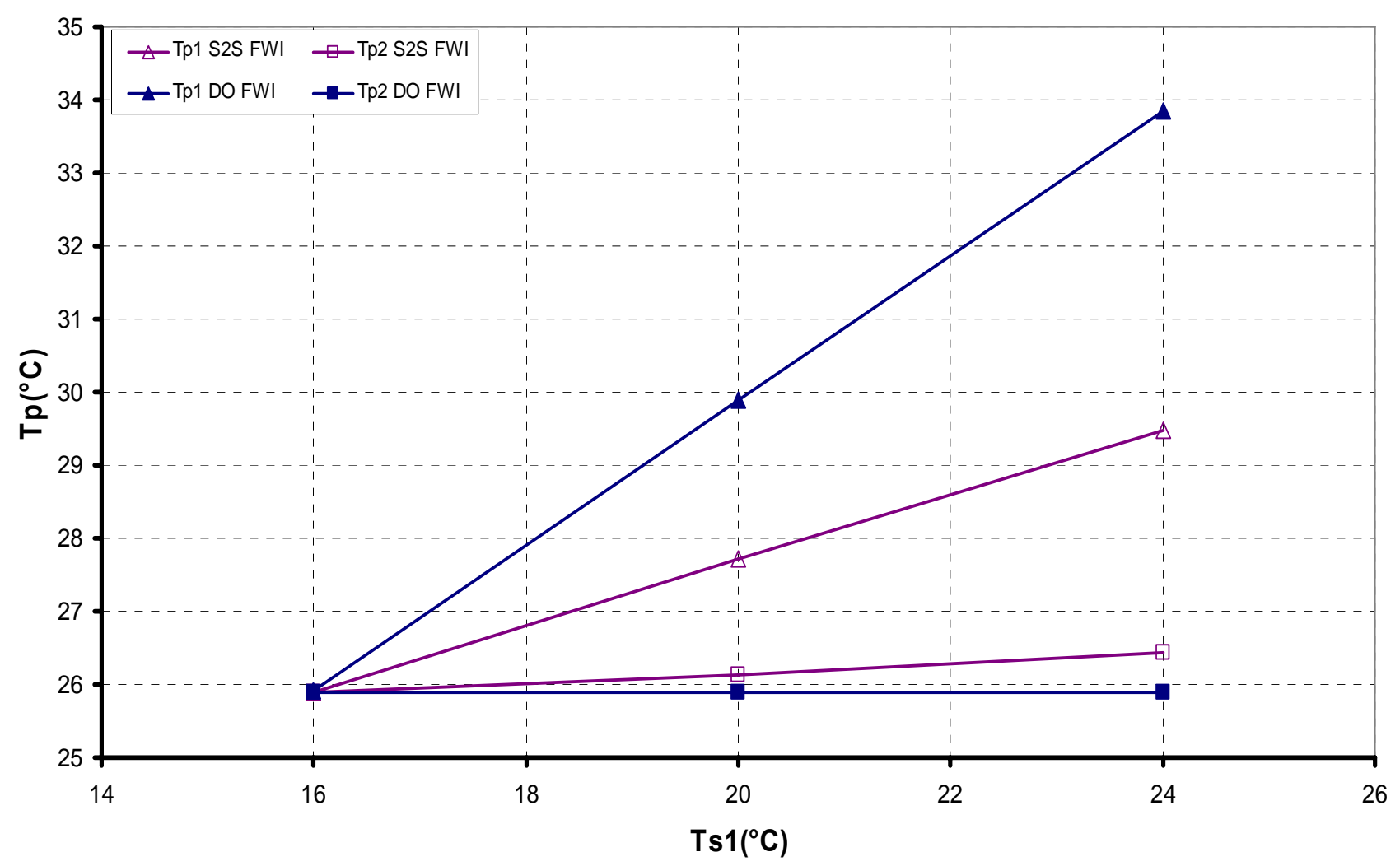

Figure 3.4 The effect on Tp in two cubicles by adjusting Ts1 (FWI)

Figure 3.4 shows the change of the personal surface temperature in the two zones by adjusting the supply temperature in zone 1 while maintaining the supply temperature in zone 2 as a constant. The personal surface temperature was calculated based on an area-weighted average temperature on the surface of each person, post-processed in FLUENT. Two radiation models were used: the Surface-to-Surface (S2S) model and the Discrete Ordinates (DO) model. The results from the DO model are as expected: $\mathrm{Tp} 1$ increases as Ts1 rises and $\mathrm{Tp} 2$ remains constant due to the unchanging Ts2. Also, it indicates that Tp1 will increase the same amount as Ts1 does, for the DO model. On the other hand, the results from the S2S model illustrate an increase of Tp2 with Ts1, which is physically wrong. Furthermore Tp1 doesn't increase as much as Ts1. Perfectly decoupled behavior is not present when using S2S radiation model. The possible cause 


\section{Report No. DE-FG02-03ER63694-F1}

of the problem for the S2S model is the way Airpak calculates the view factors, as discussed previously. Based on these results, DO model is chosen to calculate radiation for all further simulations.

\subsubsection{Middle Full Wall with Resistance (FWR)}

The next item of interest is how the conduction through the middle wall will affect the degree of coupling of the two zones. A thermal resistance of $0.392 \mathrm{~m}^{2} \mathrm{~K} / \mathrm{W}$, a typical thermal resistance for the interior wall, was chosen for the middle full wall (Cosden 2005).

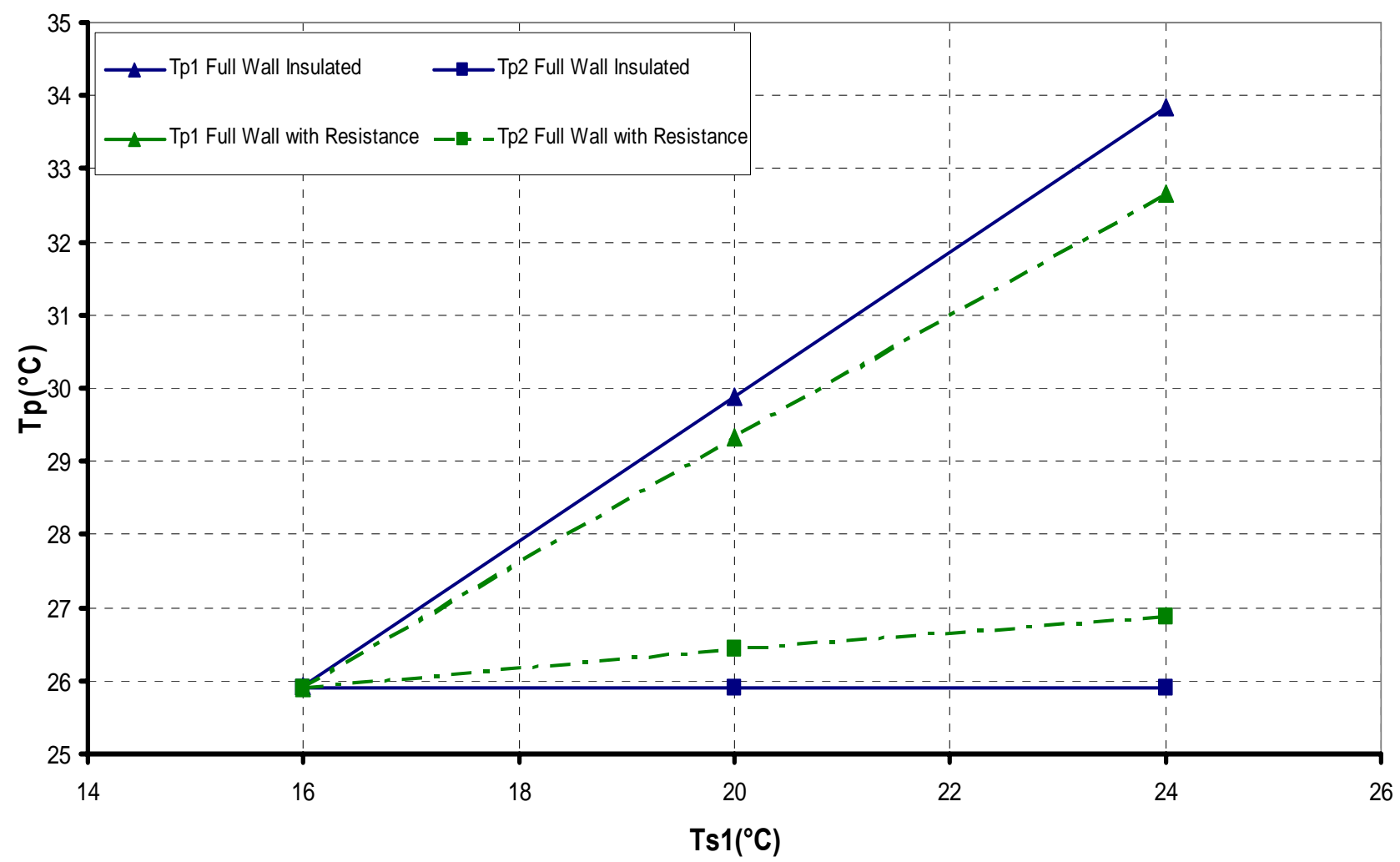

Figure 3.5 The effect on Tp in two cubicles by adjusting Ts1 (FWR vs. FWI) 


\section{Report No. DE-FG02-03ER63694-F1}

Figure 3.5 shows the comparison between the FWI and the FWR case. It can be found that for the case FWR, Tp1 drops a little bit while Tp2 increases almost the same amount compared to the FWI case as Ts1 increases. The increase of Tp2 for the FWR case indicates that Ts1 has influenced it through by conduction heat transfer through the middle wall.

\subsubsection{Partitions with Cubicle Doors (PWD)}

The results above indicated that by putting allowing heat transfer through the middle wall causes the personal temperature of the two cubicles to be slightly coupled. Here, instead of using a full wall from front to back and from floor to ceiling, a typical partition with the height of $1.75 \mathrm{~m}$ was inserted to separate the two working spaces. If a partial height partition is chosen as a divider between the cubicles, airflow interaction will occur over the partition and the energy flux between the two cubicles will consist of not only conduction but also advection. It is of interest to test the effects of the energy flow in the open space above the partial height partition. The set up of the model is shown in Figure 3.6. Doors were put on the cubicle openings to prevent advection through the corridor.

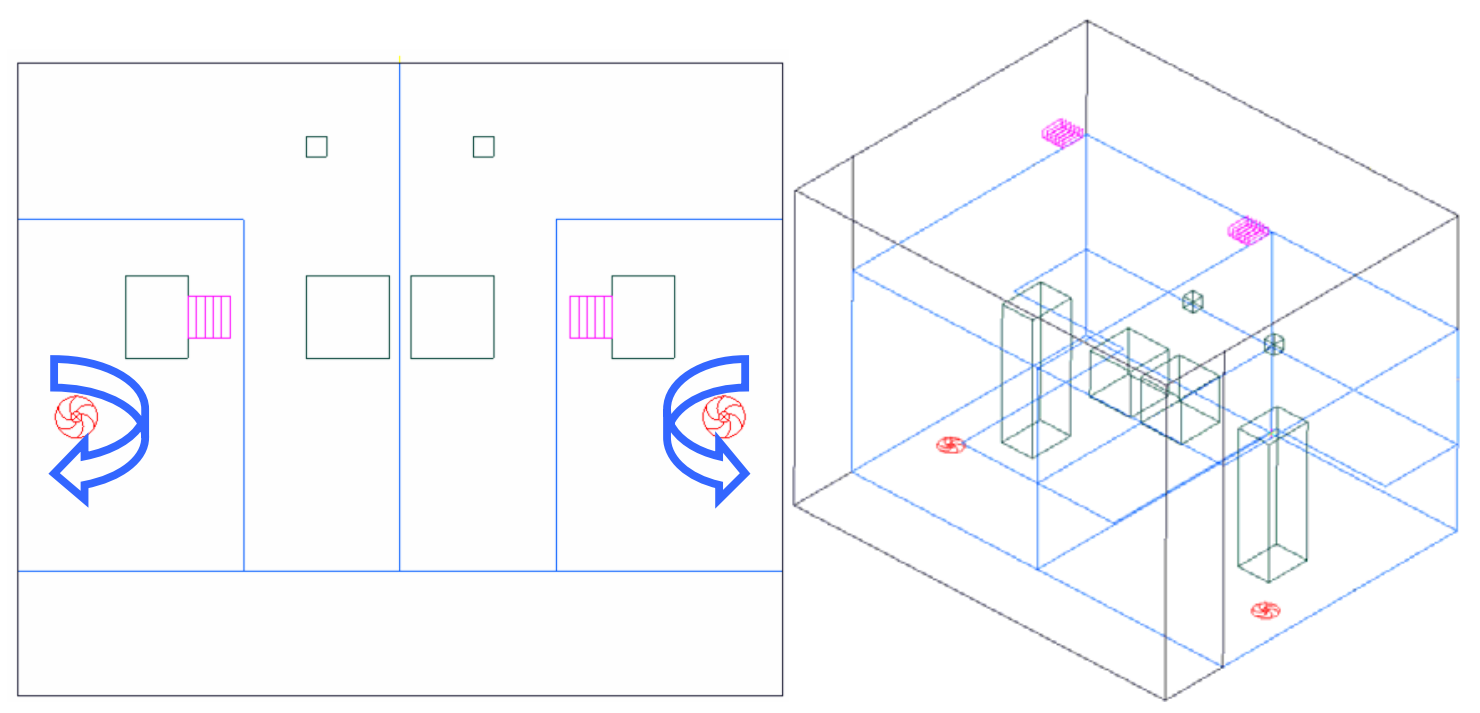

Figure 3.6 Mode A partition with doors SW1 


\section{Report No. DE-FG02-03ER63694-F1}

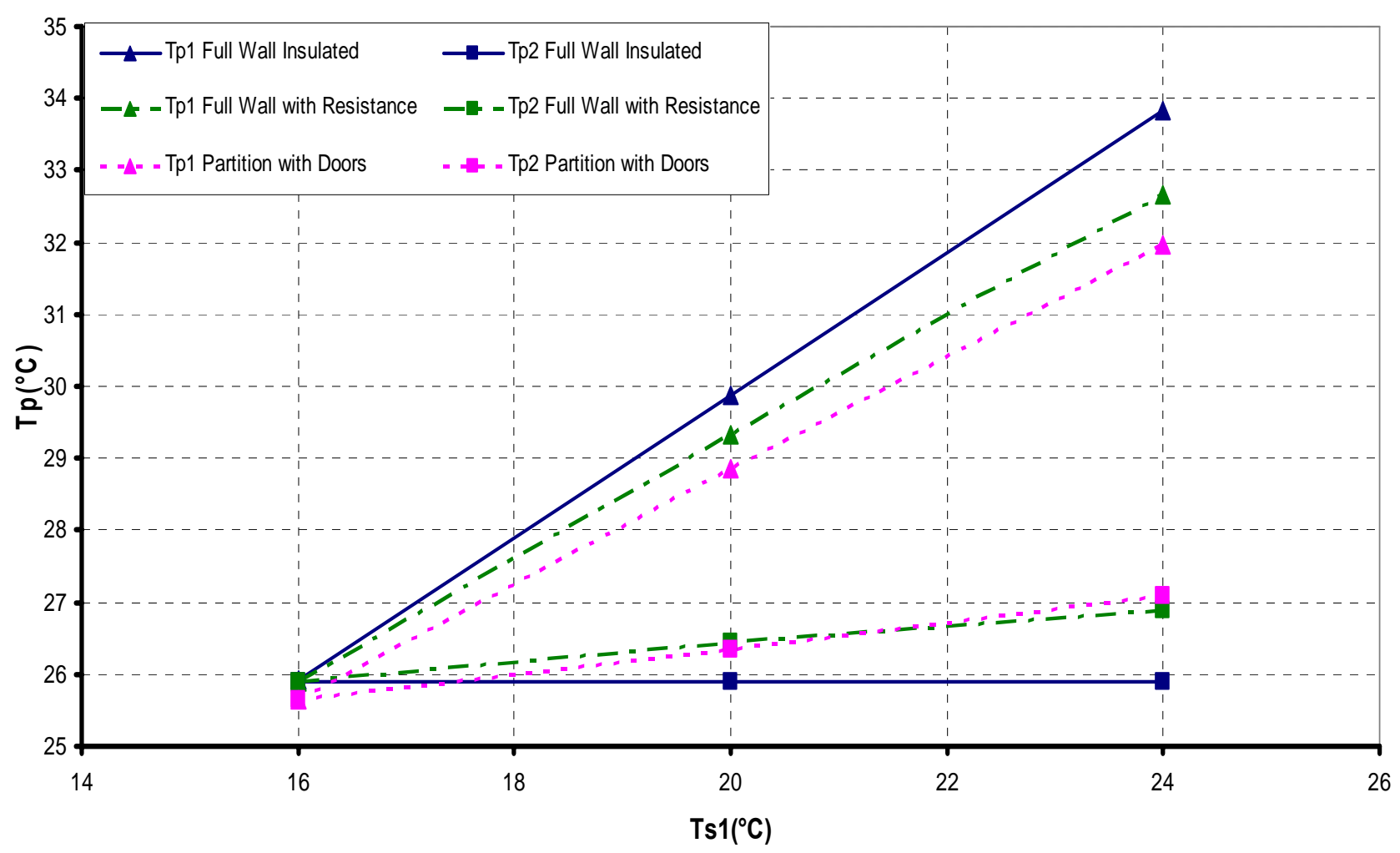

Figure 3.7 The effect on Tp in two cubicles by adjusting Ts1 (PWD)

The results of the PWD cases are plotted on Figure 3.7, compared with the results from the previous full wall configurations. The Tp1 and Tp2 profiles of the PWD cases have very similar behavior as that of the FWR case, but the difference of Tp is slightly smaller, indicating an advection energy flux over the top of the partition that has an effect that is comparable to the conduction through the full middle wall. The other thing need to be noticed is that for the case with same supply temperatures at $16^{\circ} \mathrm{C}$, the PWD case causes Tp to be lower than all other cases. That is expected because by closing cubicle doors, each cubicle will receive more cooling since no air leaves from it to the corridor. 
Report No. DE-FG02-03ER63694-F1

\subsubsection{Partition with openings SW1}

After testing the model with the middle wall extending from floor to ceiling and the case with doors on cubicle openings, the next scenario of interest is that with a partial height divider between the two cubicles and with the allowance of flow in the corridor. Based on the model described in Chapter 2.4, the doors to the corridor were eliminated, allowing flow in the corridor. Figure 3.8 shows the configuration of the model with a partition divider.
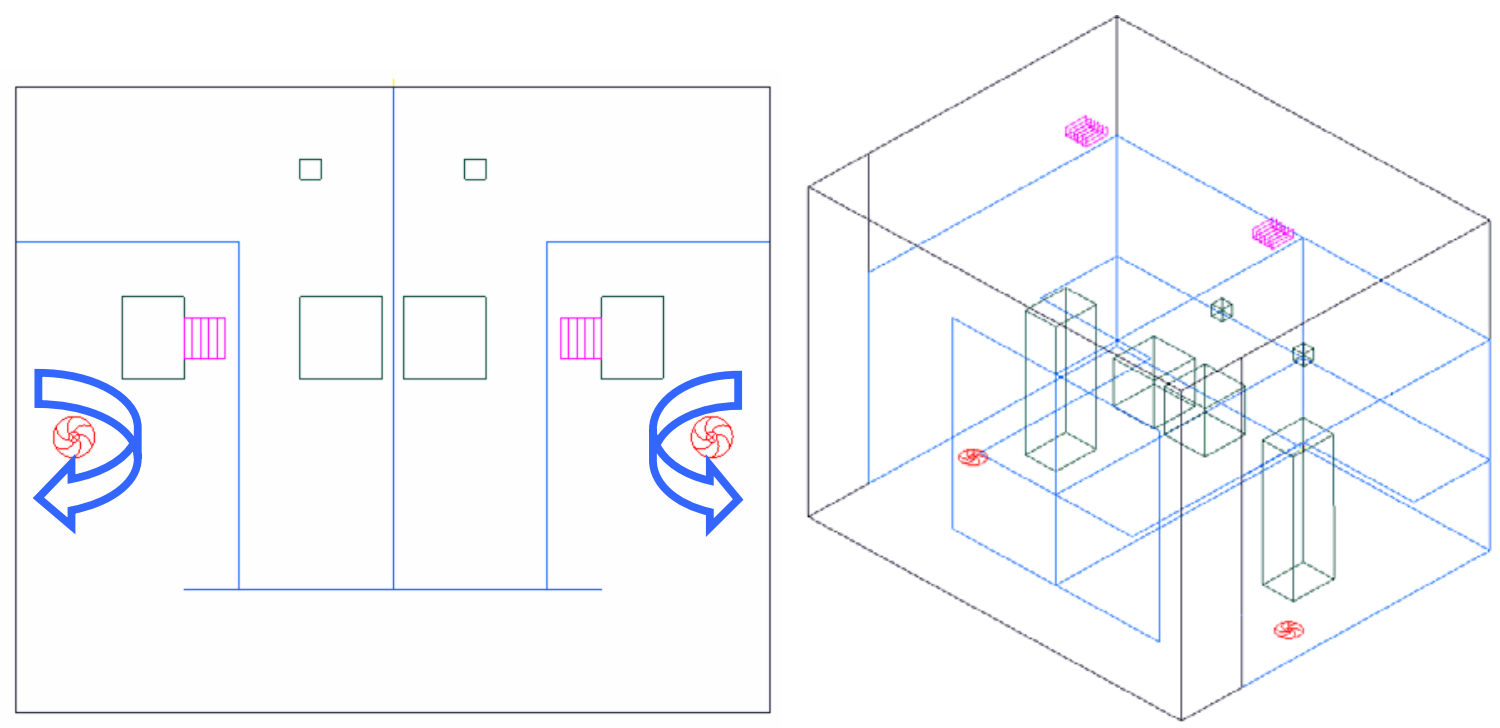

Figure 3.8 Mode A with partitions SW1

It can be expected that the two zones would become more coupled due to the open space above the middle partition, as well as the flow through the corridor. The results from these simulations are shown in Figure 3.9. Strong coupling behavior of the personal temperature in the two zones is illustrated by the decreased slope of the Tp1 profile and the increased slope of the Tp2 profile. In the extreme case, which is an $8^{\circ} \mathrm{C}$ difference in the supply temperature, the personal temperature in the two zones show only a $1.8^{\circ} \mathrm{C}$ difference. This result clearly shows there is advective heat transfer through the corridor and through the open space above the middle 


\section{Report No. DE-FG02-03ER63694-F1}

partition and the corridor flow has the dominant effect on the coupling behavior of the personal temperature in the two cubicles.

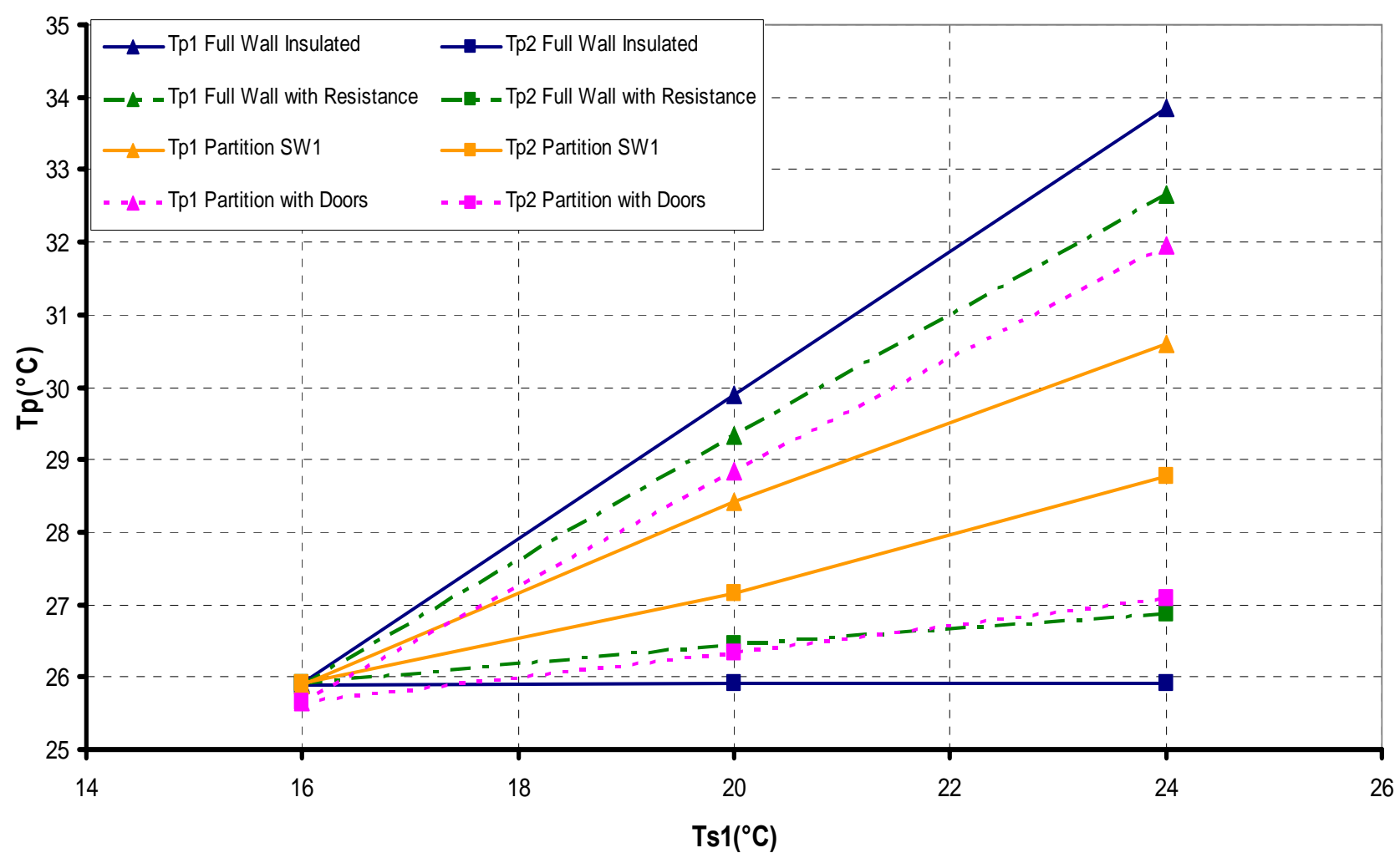

Figure 3.9 The effect on Tp in two cubicles by adjusting Ts1 (Partition SW1)

Figure 3.10 shows the particle trace from one of the supply diffuser, swirl diffuser 1 (SD1) with Ts1 at $24^{\circ} \mathrm{C}$. Figure 3.11 shows a particle trace from the other swirl diffuser, SD2, with Ts2 at $16^{\circ} \mathrm{C}$. In Figure 3.10, a significant portion of the air flow can be observed leaving cubicle 1 entering cubicle 2 through the upper part of the corridor. It is clearer in Figure 3.11 that after falls down from the 'fountain', the cold air seeps to the other cubicle through the lower part of the corridor. These results are unexpected but explainable: the difference in supply temperature creates a pressure difference in the two zones due to buoyancy and hence drives the flows in the corridor. 
Report No. DE-FG02-03ER63694-F1

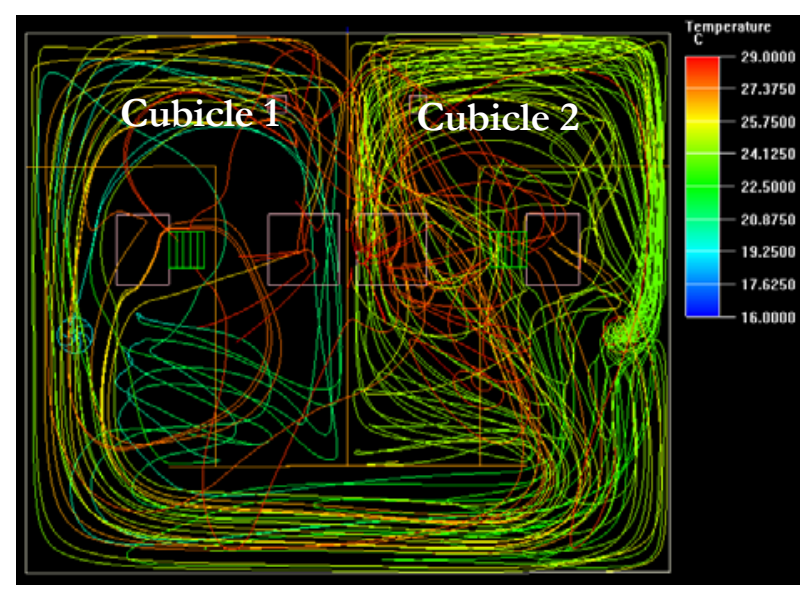

Top View

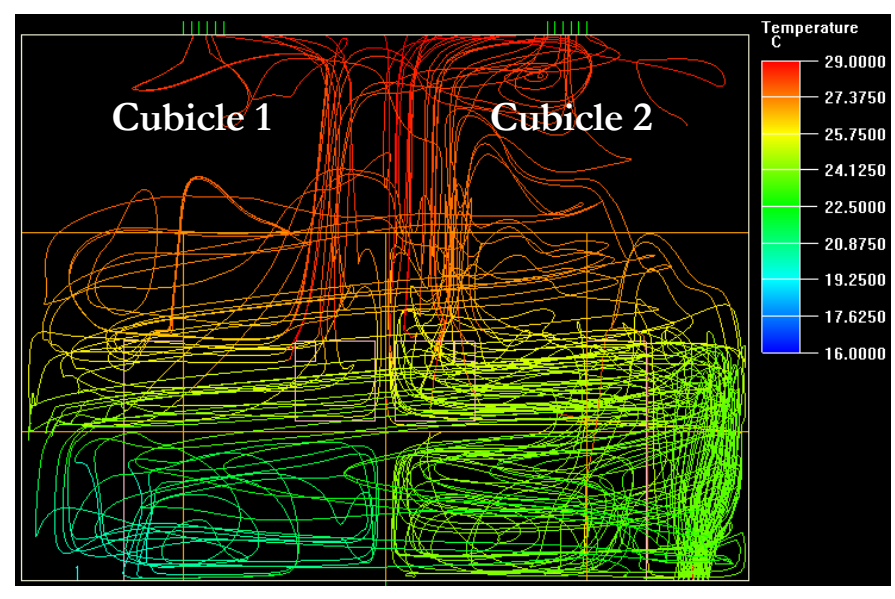

Side View

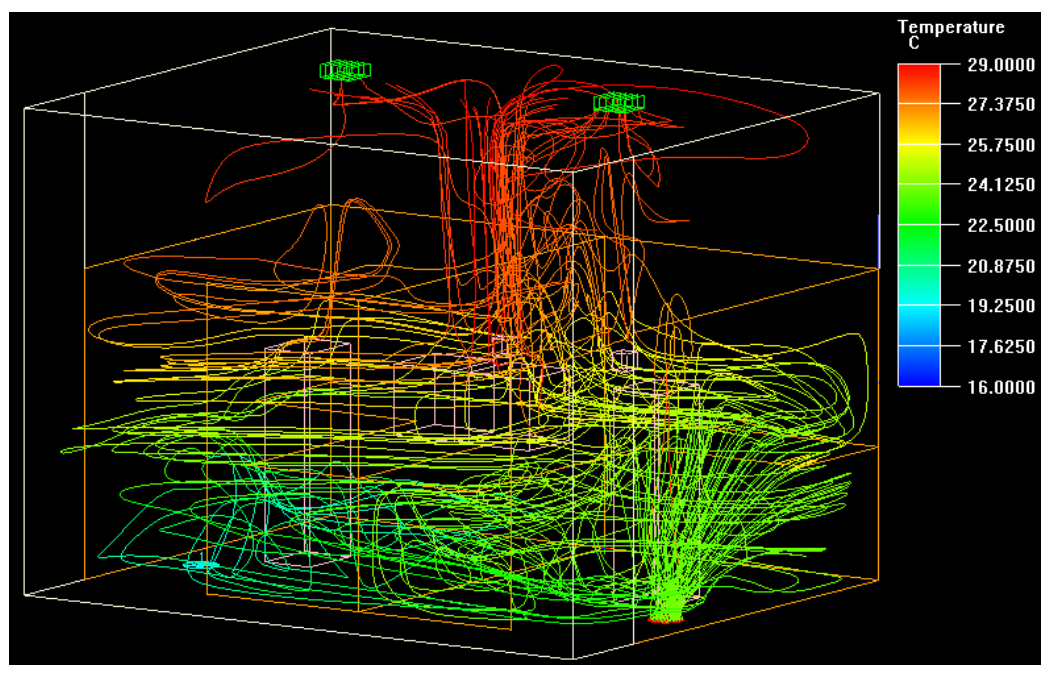

Plain View

Figure 3.10 Particle trace from Swirl Diffuser $1\left(\mathrm{Ts} 1=24^{\circ} \mathrm{C}\right)$ 


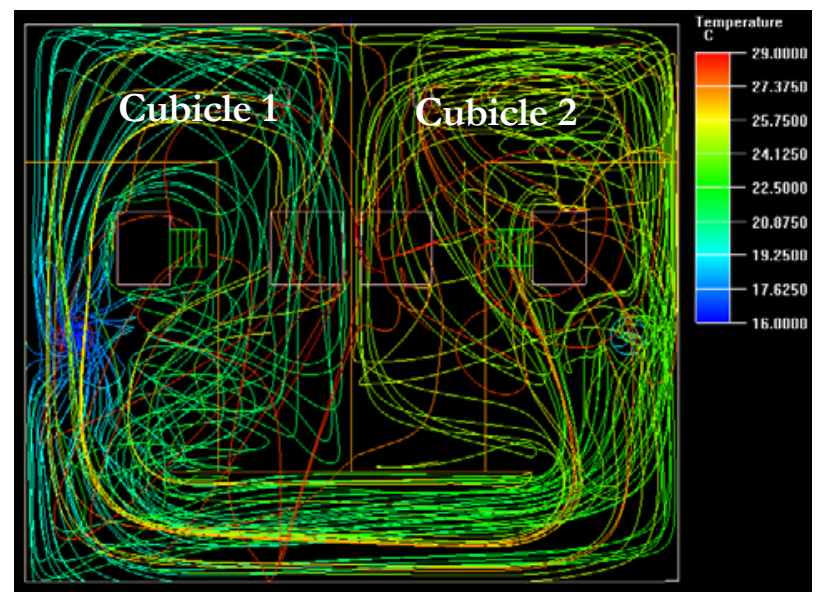

Top View

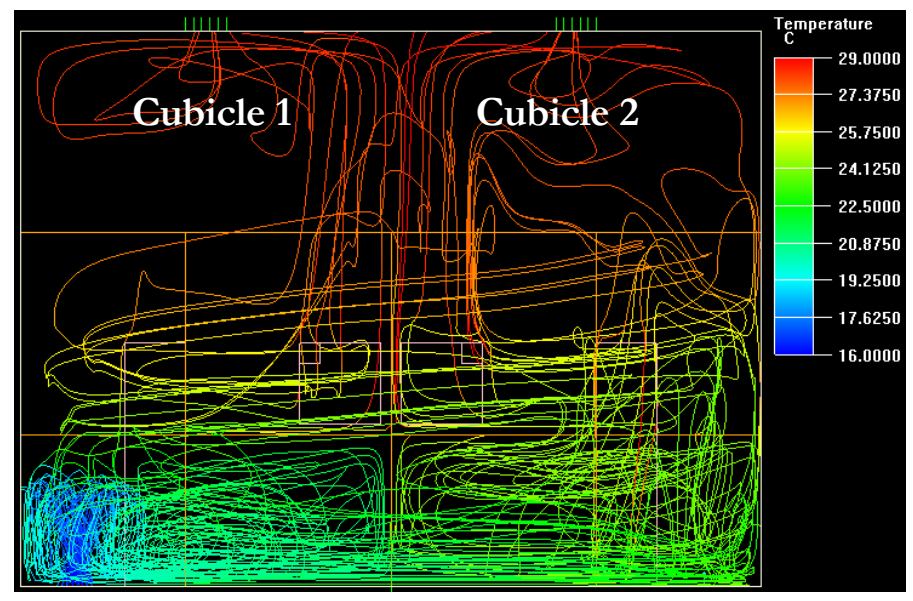

Side View

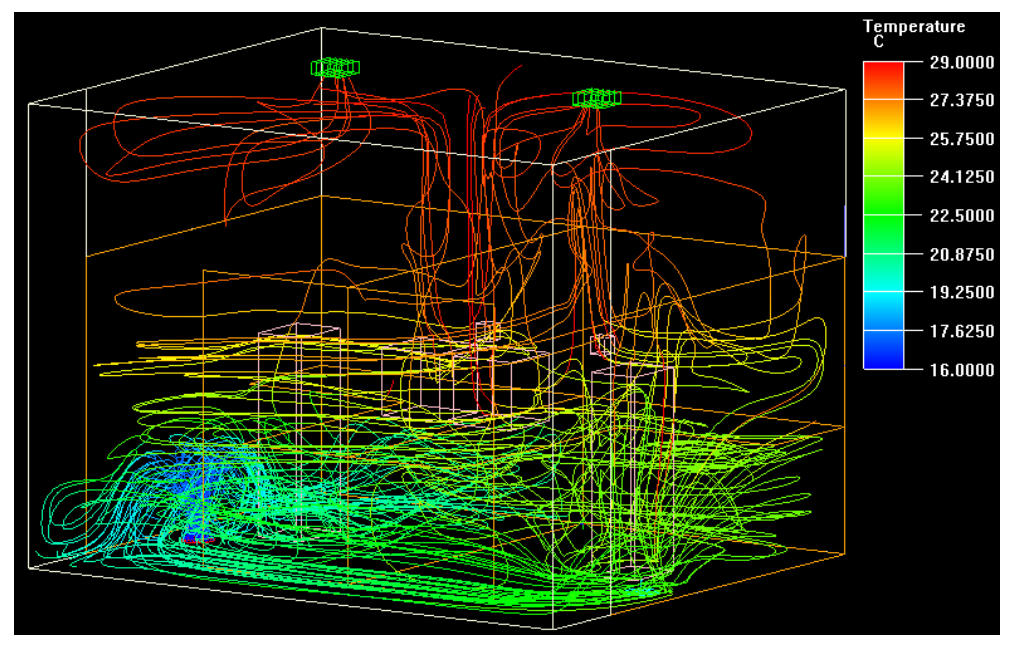

Plain View

Figure 3.11 Particle trace from Swirl Diffuser $2\left(\mathrm{Ts} 2=16^{\circ} \mathrm{C}\right)$

\subsubsection{Partition with openings SW2}

It is possible that the swirl directions of the floor diffusers can affect the coupling behavior of the two cubicles. Therefore, another swirl direction was applied, shown as in Figure 3.12. 


\section{Report No. DE-FG02-03ER63694-F1}

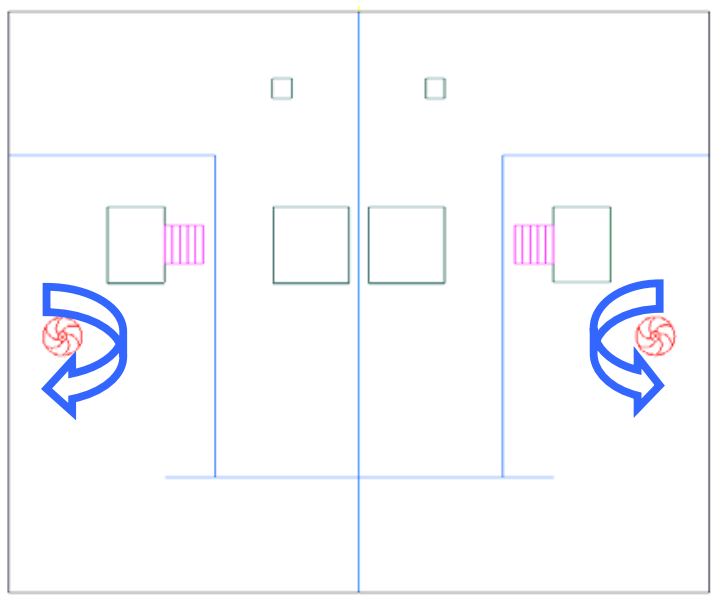

SW 1

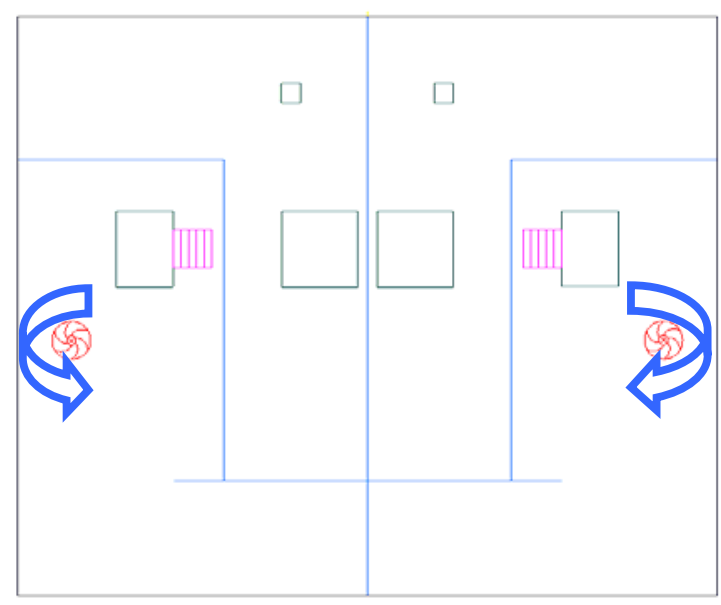

SW 2

Figure 3.12 Swirl Directions of Mode A

The symmetric features of the diffusers were still preserved. The new swirl direction is called

SW2. The results of the SW2 case is shown in Figure 3.13, compared with previous results.

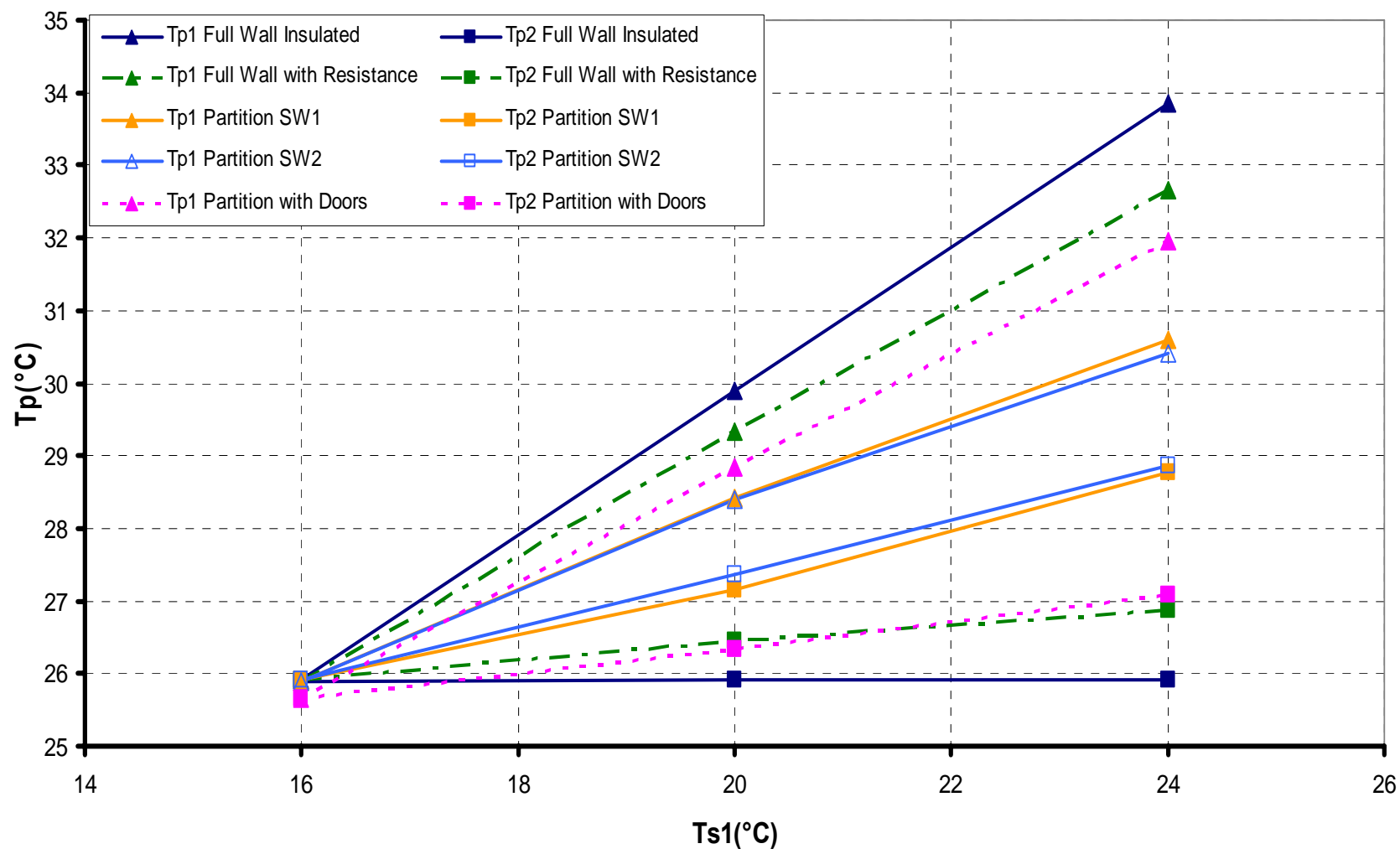

Figure 3.13 The effect on Tp in two cubicles by adjusting Ts1 (Partition SW2) 
A very slight difference between the two swirl directions can be observed on the plot. Therefore for this configuration, swirl direction only changes the flow pattern without changing the degree of coupling of the two cubicles.

\subsection{Other Modes and Comparisons}

The results presented to this point are for a cubicle configuration referred to as mode A (see Figure 3.14). Since there are many other combination of the two cubicles (other modes), it is also important to look into the flow pattern and the coupling behavior of those modes. Figure 3.14 shows all the configuration of the modes. The small arrows illustrate the swirl directions. Symmetric swirl directions are presented in all modes except for Mode D and the asymmetrical Mode F.

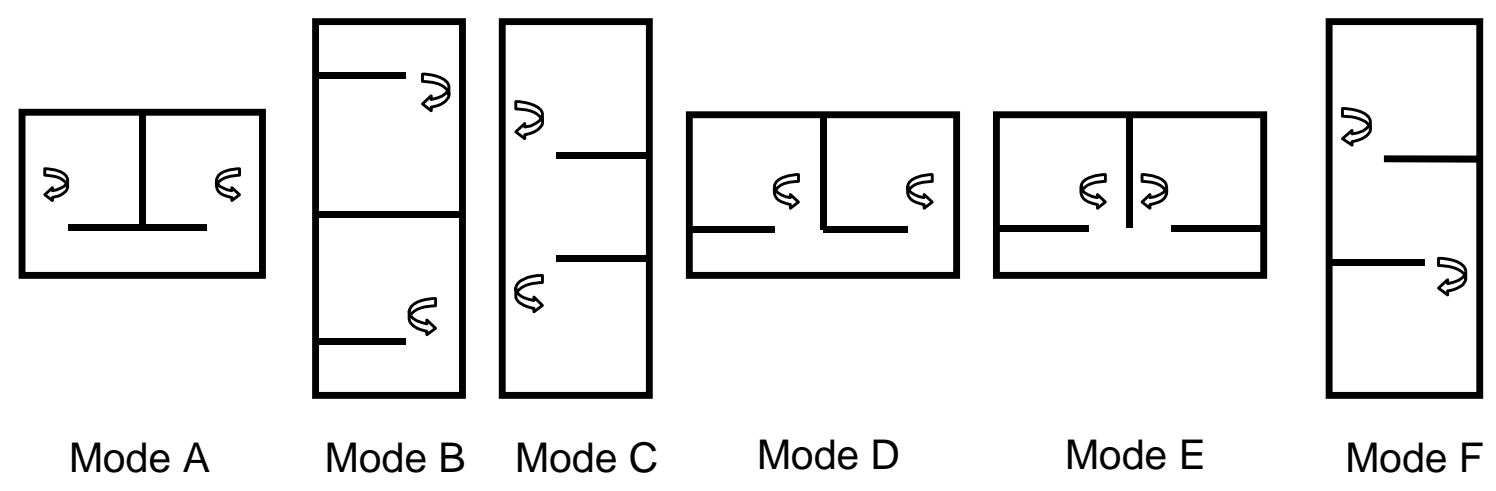

Figure 3.14 Configuration of All the modes

Only the extreme cases $\left(\mathrm{Ts} 1=24^{\circ} \mathrm{C}\right.$ and $\left.\mathrm{Ts} 2=16^{\circ} \mathrm{C}\right)$ are of interest since all the previous results show essentially linear behavior of $\mathrm{Tp} 1$ and $\mathrm{Tp} 2$ with respect to Ts1. Simulations were performed based on these other modes (Mode B, C, D, E, F) for the cases with partition openings facing the corridor and the case with partitions having doors. The personal 
temperature difference of the two occupants is of interest and hence the results are compared based on $\Delta \mathrm{Tp}$ for all the different modes.

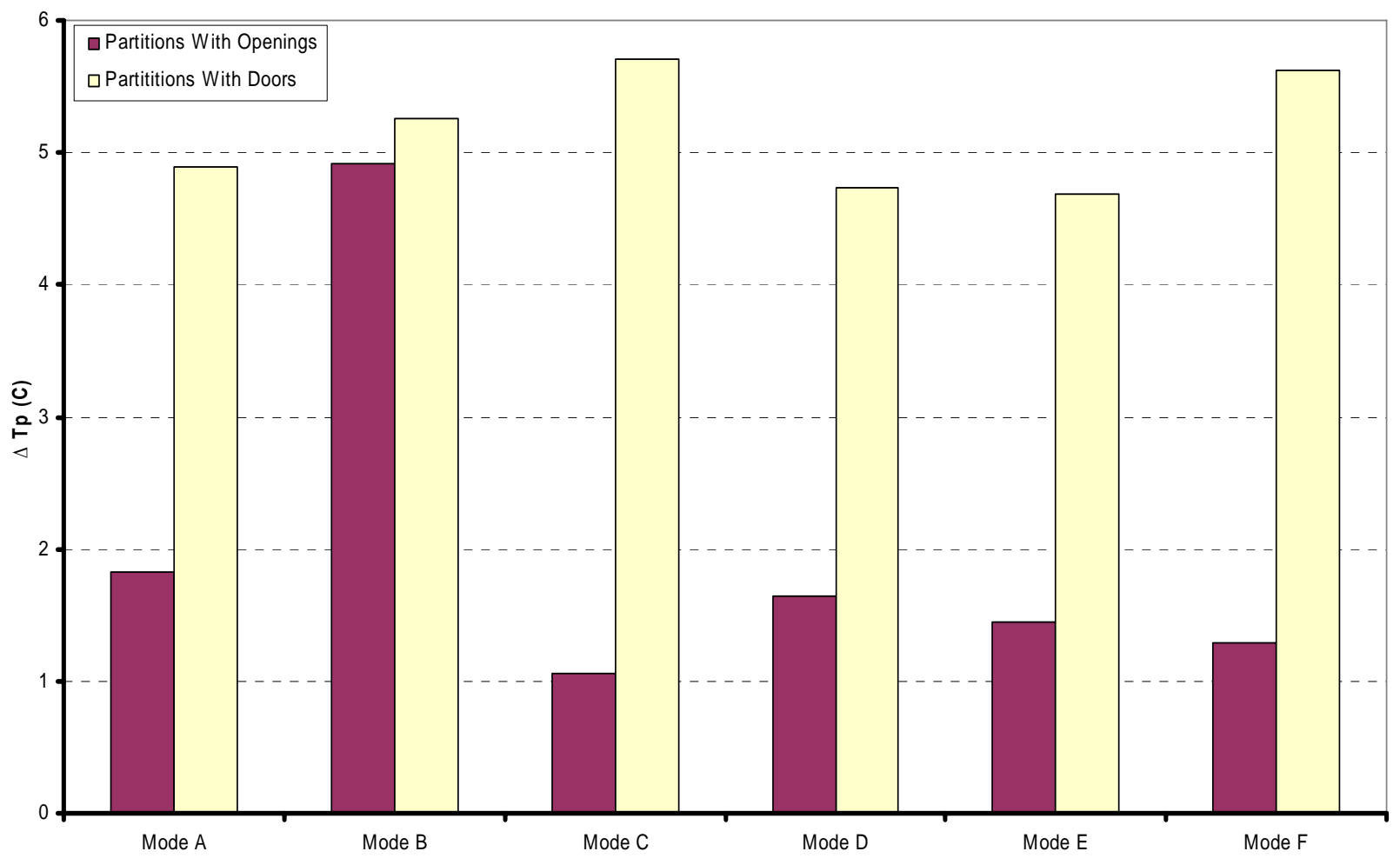

Figure 3.15 Personal Temp Differences in Two Zones for Different Modes $\left(24^{\circ} \mathrm{C} 16^{\circ} \mathrm{C}\right)$

Figure 3.15 shows the comparison of all the modes for the 'partitions with opening' and 'partitions with doors' cases. For the partitions with openings to the corridor cases, $\Delta$ Tp of all modes range from $1^{\circ} \mathrm{C}$ to $1.8^{\circ} \mathrm{C}$, except Mode $\mathrm{B}$, which has no corridor connecting the two cubicles. These values are relatively small compared to the supply temperature difference $\left(8^{\circ} \mathrm{C}\right)$. However if the openings to the corridor are blocked by putting doors on them, $\Delta \mathrm{Tps}$ dramatically increase to around $5^{\circ} \mathrm{C}$ and hence improved decoupled behavior of the two cubicles is evident. 
Report No. DE-FG02-03ER63694-F1

\section{Methodology of the Modified Thermal Circuit Model}

The previous chapter described the CFD models that were performed for two-cubicle-withcorridor configurations. Those computational results will now be applied to a simplified thermal-circuit model that is suitable for building optimization studies.

\subsection{Thermal Environment of Human Occupant}

Research has been done to calculate the heat exchange between people and the environment quantitatively (Fanger 1967, 1970; Rapp and Gagge 1967). The thermal exchange of the human body with the environment represents a combination of the sensible heat loss and the evaporative heat loss from the skin. Since the models studied here do not account for humidity, the latent heat loss of the occupant will not be considered. Therefore, in this report, the heat exchange between the human body and the environment will only be the sensible heat loss which consists of both convective and radiative heat loss (ASHRAE handbook 2001):

The convective heat transfer is modeled as

$$
C=f_{c l} h_{c}\left(T^{c l}-T^{a}\right)
$$

whereas the radiative part is modeled as

$$
R=f_{c l} h_{r}\left(T^{c l}-\bar{T}^{r}\right)
$$

where $h_{c}$ is the convective heat transfer coefficient, $\mathrm{W} /\left(\mathrm{m}^{2} \mathrm{~K}\right), h_{r}$ is the linear radiative heat transfer coefficient, $\mathrm{W} /\left(\mathrm{m}^{2} \mathrm{~K}\right), f_{c l}$ is the clothing area factor to account for the actual surface area of the clothed body ( $f_{c l}=1$ for nude body), $T^{c l}$ is the mean temperature of the outer surface of the clothed body $(\mathrm{K}), T^{a}$ is the mean ambient air temperature $(\mathrm{K})$ and $\bar{T}^{r}$ is the mean radiant temperature $(\mathrm{K})$. 
The total sensible heat exchange can be described in terms of a combined heat transfer coefficient $h$ and an operative temperature $T^{o}$ (ASHRAE handbook 2001):

$$
Q_{p}=C+R=f_{c l} h\left(T^{c l}-T^{o}\right)
$$

where

$$
\begin{gathered}
h=h_{c}+h_{r} \\
T^{o}=\frac{h_{c} T^{a}+h_{r} \bar{T}^{r}}{h_{c}+h_{r}}=\frac{h_{c}}{h_{c}+h_{r}} T^{a}+\frac{h_{r}}{h_{c}+h_{r}} \bar{T}^{r}
\end{gathered}
$$

The operative temperature is defined as the combination of a convection part and a radiation part of temperature. It is equal to the temperature at which a specified hypothetical environment would support the same heat loss from a human body as the actual environment. In a certain environment, the actual temperature that people feel will be the operative temperature.

\subsection{The Modified Thermal Circuit Model}

\subsubsection{Thermal Stratification}

As discussed in Section 2.1, air distribution in a room with UFAD system will have stratification behavior due to the configuration and thermal buoyancy. In such a non-uniform environment, the calculation of air temperature around the person and the wall should account for the stratified temperature distribution. Therefore, it is helpful to observe the actual temperature distribution in each zone, i.e. the temperature profiles with respect to different height of the room. Figure 4.1 shows the normalized air temperature profiles for different configurations, calculated from CFD simulations described in the previous chapter. 


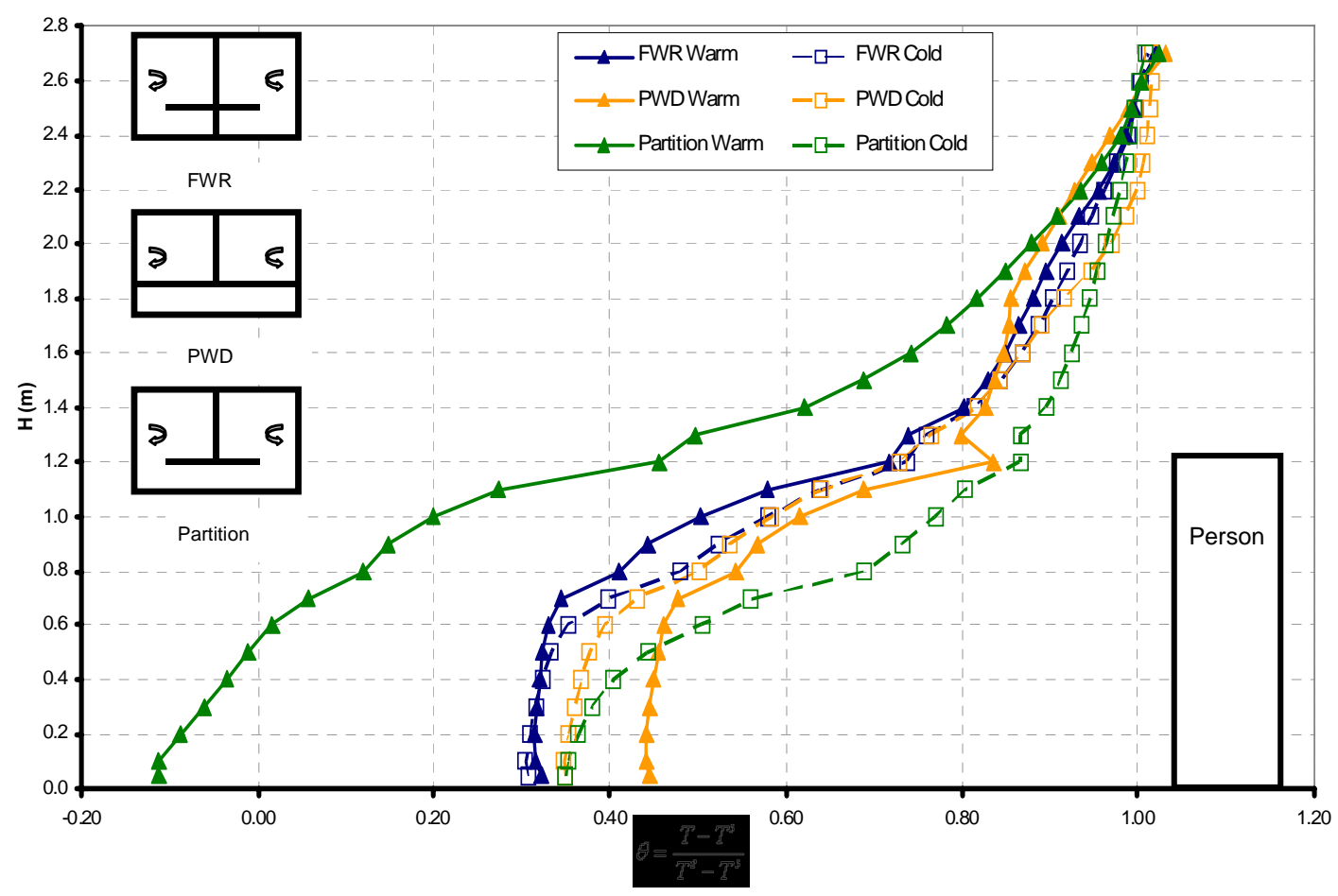

Figure 4.1 Normalized Air Temp. Profiles Comparison for Different Configurations

$$
\text { (Mode A, } 24^{\circ} \mathrm{C} / 16^{\circ} \mathrm{C} \text { ) }
$$

The profiles depict the non-dimensional normalized temperature difference at different height. The difference between the air temperature $T$ and the supply temperature $T^{s}$ is weighted by the temperature difference between the exhaust temperature $T^{e}$ and the supply temperature of the zone, as shown in equation (3-6):

$$
\theta=\frac{T-T^{s}}{T^{e}-T^{s}}
$$

The air temperature of each zone is obtained by performing area-weighted average calculation of temperature for every plane at different height in the two zones. The dark blue profiles show the temperature profiles of the scenario of FWR, full wall with resistance. The normalized temperature profiles of the two zones are very similar. The orange profiles illustrate the scenario of PWD, partition with doors. Compared to the FWR profiles, the PWD's move a little bit to 
the right, meaning they had higher $\theta$ s, while keeping the same shapes. But when it comes to the Partition case, the profiles are greatly skewed, especially the 'Warm' zone profile. The reason is the same as that discussed in Section 3.2.4; the interactive advection flow between the cold zone and the hot zone through the corridor. Figure 4.2 shows the 'Partition' case with different supply temperature difference in the two neighboring zones. When the supply temperatures of the two cubicles are the same $\left(\right.$ at $\left.16^{\circ} \mathrm{C}\right)$, the temperature profiles of the two zones will fall very close to each other. With the increase of the supply temperature difference between the two zones, the profiles will depart more and more from the original overlapped profiles (the green two), especially the profiles of the warm zones. It indicates that for the cases with openings to the corridor, the shapes of the temperature profiles will greatly depend on the supply temperature difference between the two zones.

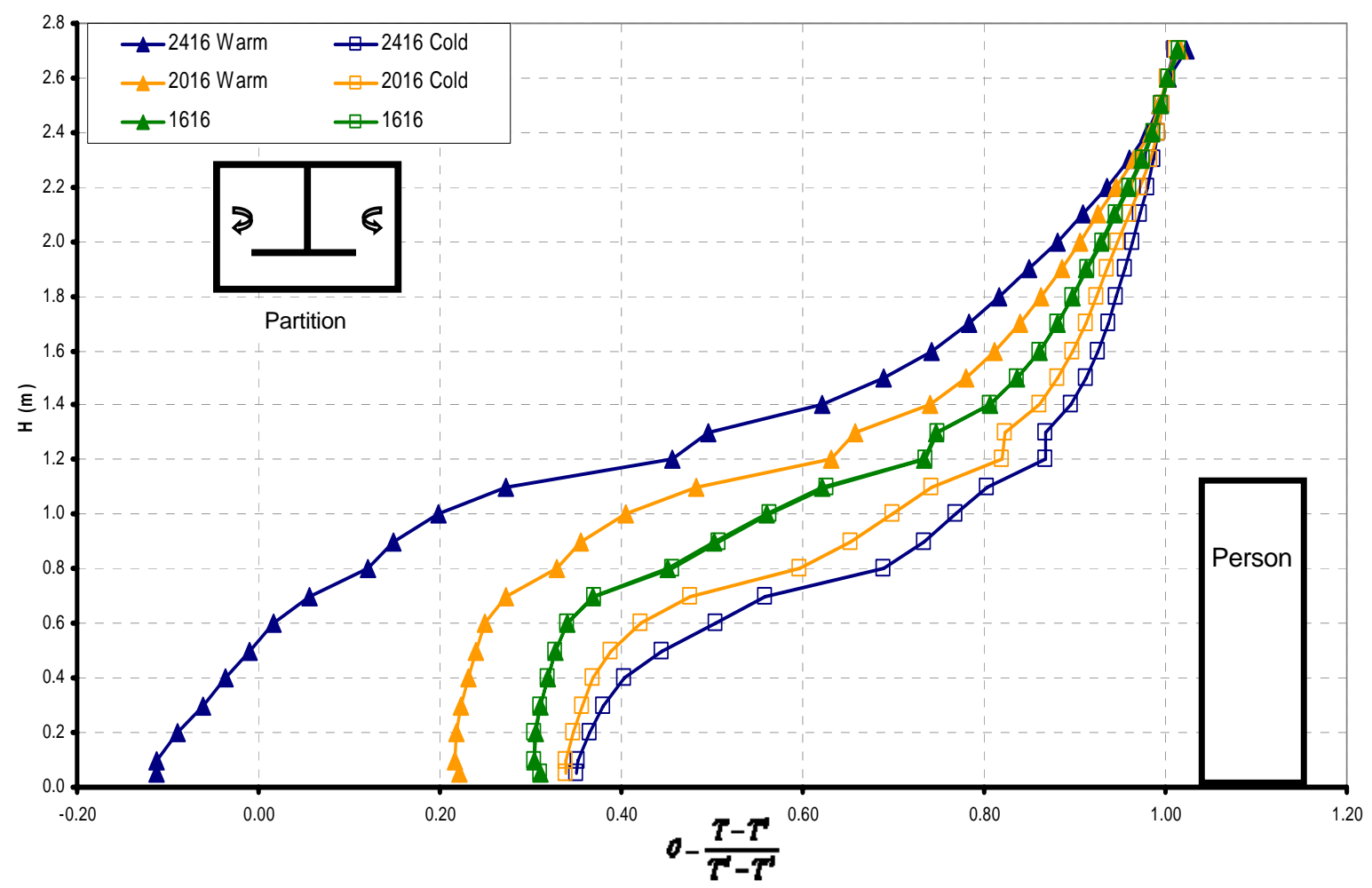

Figure 4.2 Normalized air temp. profiles comparison with different $\Delta$ Ts (Mode A) 
Since the shapes of the temperature profiles of FWR and PWD cases are fairly similar, an analysis was performed as shown in Figure 4.3. The 'example' temperature profile can be broken down into three layers: from floor to the height of the desk $(0 \sim 0.8 \mathrm{~m})$, from the desk top to the top of the computer and person $(0.8 \sim 1.2 \mathrm{~m})$, and the space above the computer and occupant to the ceiling $(1.2 \sim 2.74 \mathrm{~m})$. When looking at the corresponding actual physical model, it is also consistent with the calculated temperature profiles, in terms of heat generation in different layers.

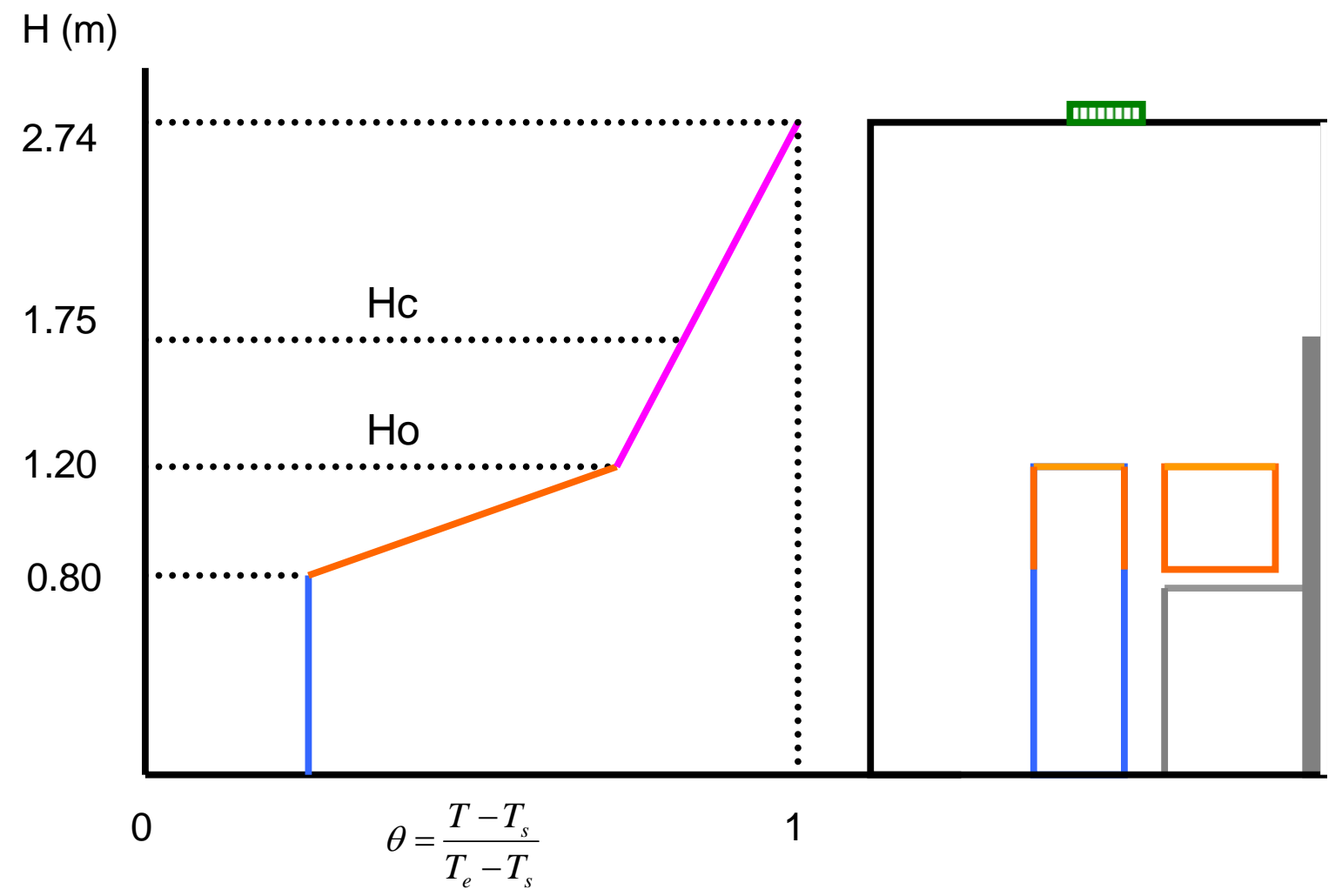

Figure 4.3 Example Temperature Profile and corresponding physical model

The actual air temperature in the UFAD model should be related with the air distribution profiles. The air temperature around the person and the wall are no longer the same, as assumed in Cosden's model, but should be calculated based on a weighted average temperature. The 


\section{Report No. DE-FG02-03ER63694-F1}

mean air temperature $T^{a}$ is considered to be the ambient air around the occupant. For the underfloor ventilation systems, the calculation of $T_{i}^{a}$ for certain zone $i$ should account for the stratification of the air in the room due to thermal buoyancy. An assumption is made that the mean air temperature around the person is a linear combination of the supply temperature $T_{i}^{s}$ and the exhaust temperature $T_{i}^{e}$ :

$$
T_{i}^{a}=\alpha T_{i}^{e}+(1-\alpha) T_{i}^{s}
$$

Here $\alpha$ is a certain constant number between 0 and 1 . The method to achieve $\alpha$ will be discussed later.

Applying the same concept as used in $T^{a}$, the air temperature around walls, $T^{c}$, can also be treated as a linear combination of the exhaust temperature $T^{e}$ and the supply temperature $T^{s}$, while using another similar constant $\beta$ :

$$
T_{i}^{c}=\beta T_{i}^{e}+(1-\beta) T_{i}^{s}
$$

For a given a temperature profile, the constants $\alpha$ and $\beta$ can be found by an appropriate integral of the temperature profile model. Namely,

$$
\begin{gathered}
\alpha=\int_{0}^{H_{o}} \theta d h / H_{o} \\
\beta=\int_{0}^{H_{c}} \theta d h / H_{c}
\end{gathered}
$$

where $H_{o}$ is the height of the seated occupant, equals to $1.2 \mathrm{~m}, H_{c}$ is the height of the wall, which could be a full wall $(2.74 \mathrm{~m})$ or a partition $(1.75 \mathrm{~m})$. For the FWR cases, $\alpha$ is 0.4 and $\beta$ is 0.7 (full wall, $\mathrm{Hc}=2.74 \mathrm{~m}$ ). For the PWD cases, $\alpha$ is 0.5 and $\beta$ is 0.6 (partition, $\mathrm{Hc}=1.75 \mathrm{~m}$ ). 


\subsubsection{Modified Thermal Circuit Network}

A building model using a thermal circuit network has been established by Cosden (2005). Figure 4.4 shows a cut-out example of the thermal circuit network around zone i. Each zone (including the outside zone) is treated as a node in the analogous electrical network and connected to all its neighbors by a resistance appropriate to the dividing interface $\left(R^{\text {in }}\right.$ for interior wall and $R^{\text {out }}$ for exterior wall).

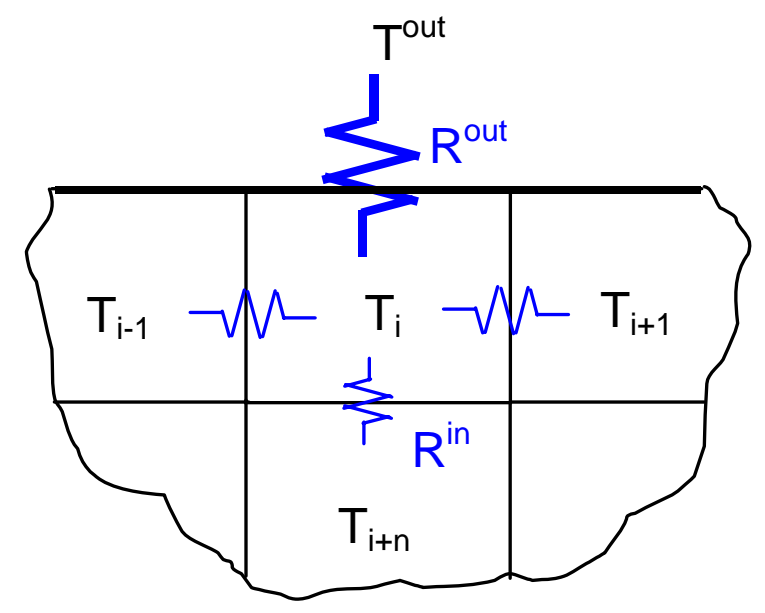

Figure 4.4 Cut-out Example of a thermal circuit network around zone i (Cosden, 2005)

Cosden's thermal circuit model assumes each zone is fully mixed, in other words, the air temperature around the person, near the walls and at the exhaust are the same. Furthermore, the model doesn't account for the thermal comfort of the occupants. Therefore, Cosden's model is only valid for the mixing ventilation system (e.g. the overhead HVAC system). For a building with UFAD system, air temperature stratification should be considered. Hence the originally thermal circuit model should be modified accordingly. Figure 4.5 shows a side view of the modified thermal circuit model. 


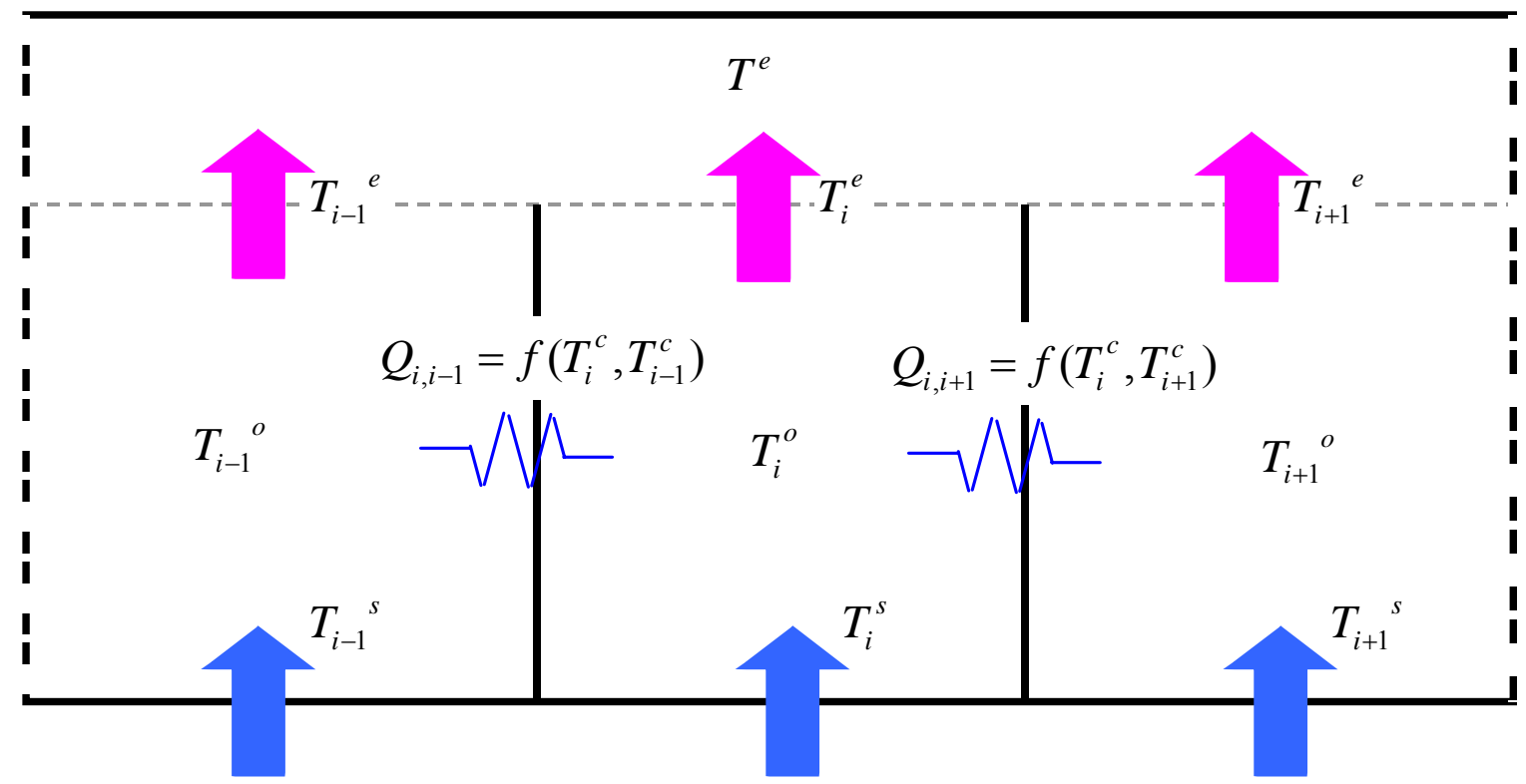

Figure 4.5 Cut-out Example of the modified thermal circuit network around zone i (Side View)

The supply air flow comes into each zone at $T^{s}$ and leaves it at $T^{e}$. The desired set point temperature will equal to the operative temperature $T^{o}$ in each zone, which can be expressed by equation (4-5). The energy transfer between zones will be based on the actual air temperature $T^{c}$ near the walls. Temperature stratification will be account for by using the parameter $\alpha$ and $\beta$, discussed in Section 3.2.1. Thus, personal thermal comfort and nonuniform temperature distribution will be included in the modified model. Unlike the original thermal circuit model, the exhaust temperatures are also involved in the calculation as well. Therefore, for a building with $\mathrm{N}$ zones, $2 \mathrm{~N}$ variables $\left(\mathrm{N} T^{s}\right.$ and $\mathrm{N} T^{e}$ ) need to be solved. They can be solved based on the $2 \mathrm{~N}$ equations ( $\mathrm{N}$ operative temperature equations and $\mathrm{N}$ energy balance equations). 
Report No. DE-FG02-03ER63694-F1

\subsection{The Matrix Equation Formulation}

\subsubsection{The Operative Temperature}

The desired set point temperature, $T^{*}$, will be considered to be perfectly achieved through the HVAC system. Once the desired set point temperature is determined by the occupant or the system, the actual temperature the occupant will experience, the operative temperature, will be equal to the desired temperature. A linkage matrix $L_{i j}$ can be applied (Cosden 2005) to account for different thermostat control strategies, e.g. Three Thermostat for all (TTFA), or Have It Your Way (HIYW). To obtain the desired temperature, the mean air temperature $T^{a}$ and the mean radiant temperature $\bar{T}^{r}$ need to satisfy equation (3-5).

The calculation of the mean air temperature around the person $T^{a}$ is already discussed in Section 4.2.1.

$$
T_{i}^{a}=\alpha T_{i}^{e}+(1-\alpha) T_{i}^{s}
$$

The mean radiant temperature $\bar{T}^{r}$ is also an important variable in making thermal calculations for the human body. It is defined as a uniform temperature of an imaginary enclosure in which radiant heat transfer from the human body equals the radiant heat transfer in the actual nonuniform enclosure (ASHRAE handbook 2001). The mean radiant temperature can be calculated from the temperature of the surrounding walls and surfaces and their positions with respect to the occupant. For an enclosure with relatively small temperature difference between surfaces, $\bar{T}^{r}$ of a certain zone i can be calculated by equation:

$$
\bar{T}_{i}^{r}=T_{1}^{i w} F_{p-1}+T_{2}^{i w} F_{p-2}+\cdots+T_{N}^{i w} F_{p-N}
$$

where $T_{N}^{i w}$ is the surface temperature of surface $\mathrm{N}$ of zone $\mathrm{i}(\mathrm{K}), F_{p-N}$ is the angle factor between a person and surface N. It can be assumed that a wall temperature is the average of the 
air temperatures at both sides of the wall. The angle factors can be expressed by a matrix $F_{i j}$, showing the angle factor between the person in zone $\mathrm{i}$ and the surface between zone $\mathrm{i}$ and the neighboring zone $\mathrm{j}$. Thus the mean radiant temperature $\bar{T}_{i}^{r}$ can be expressed by equation (4-12):

$$
\bar{T}_{i}^{r}=\sum_{j=0}^{N} F_{i j}\left(\frac{T_{j}^{c}+T_{i}^{c}}{2}\right)
$$

Notice here $\mathrm{j}$ is from 0 to $\mathrm{N}$, the total number of zones. $\mathrm{j}$ equals 0 presents an outside wall. In this research, since the occupant is relatively in the middle of the workstation and the four walls around him have very similar areas, it is reasonable to assume the angle factor between the person and each surface around it to be the same and equals 0.25 (the angle factor between the person and the floor or the ceiling is relatively small and is ignored). The view factors can be easily changed in the code for different type of building configurations and walls.

As for the convective heat transfer coefficient, a recommended $h_{c}$ can be obtained from ASHRAE handbook (2001): for seated person with moving air at the velocity $0<V<0.2 \mathrm{~m} / \mathrm{s}$, $h_{c}=3.1 \mathrm{~W} /\left(\mathrm{m}^{2} \mathrm{~K}\right)$. Also $h_{r}$ is stated nearly constant at a value of $4.7 \mathrm{~W} /\left(\mathrm{m}^{2} \mathrm{~K}\right.$ ) (ASHRAE handbook 2001).

Substituting equation (4-9), (4-10), and (4-12) into equation (4-5), and after rearranging the equation, it can be written as:

$$
A T^{e}+B T^{s}=J
$$

where,

$$
A_{i j}=\beta \cdot \frac{h_{r}}{h_{c}+h_{r}} \cdot \frac{F_{i j}}{2}, \quad i \neq j,
$$




$$
\begin{aligned}
& A_{i i}=\alpha \cdot \frac{h_{c}}{h_{c}+h_{r}}+\beta \cdot \frac{h_{r}}{h_{c}+h_{r}} \cdot \sum_{j=0}^{N} \frac{F_{i j}}{2}, \\
& B_{i j}=(1-\beta) \cdot \frac{h_{r}}{h_{c}+h_{r}} \cdot \frac{F_{i j}}{2}, \quad i \neq j, \\
& B_{i i}=(1-\alpha) \cdot \frac{h_{c}}{h_{c}+h_{r}}+(1-\beta) \cdot \frac{h_{r}}{h_{c}+h_{r}} \cdot \sum_{j=0}^{N} \frac{F_{i j}}{2}, \\
& J_{i}=T_{i}^{o}-\frac{h_{r}}{h_{c}+h_{r}} \cdot \frac{F_{i o}}{2} \cdot T_{o u t} .
\end{aligned}
$$

$T^{e}$ and $T^{s}$ are temperature vectors. The operative temperatures $T_{i}^{o}$ are given for all the zones as the desired set point temperature. $T_{\text {out }}$ is the outside temperature.

\subsubsection{Energy Balance Equations}

For each zone (office) in a system (building), first law of thermodynamics must be satisfied, i.e. the heat flux into and out of each zone must equal zero. The heat flux consists of three terms: the conduction and advection (cond + adv), internal heat generation of the zone (gen) and the HVAC supplied energy flux (HVAC):

$$
\sum \dot{Q}_{i}^{\text {cond }+a d v}+\sum \dot{Q}_{i}^{g e n}+\dot{Q}_{i}^{H V A C}=0
$$

The three terms can be broken down to (Cosden 2005):

$$
\sum_{j=0}^{N} U_{i j}\left(T_{j}^{c}-T_{i}^{c}\right)+G_{i}+C_{i i}\left(T_{i}^{s}-T_{i}^{e}\right)=0
$$

$U_{i j}$ in the first term is a heat transfer coefficient defined as:

$$
U_{i j}=C_{i j}+\frac{1}{R_{i j}}
$$

$C_{i j}$ is the effective flow rate between zone $i$ and zone $\mathrm{j}$ with the units $\mathrm{J} / \mathrm{sK} . R_{i j}$ is the conductive and convective resistance of the interface between the two zones. $j=0$ indicates an outside wall. 
$T_{j}^{c}$ and $T_{i}^{c}$ are the effective air temperature near the wall in zone $j$ and zone $i$. The method to obtain these temperatures has been already discussed in Section 3.2.1. $G_{i}$ is the sum of internal heat generation in each zone i. $C_{i i}$ is treated as the effective flow rate coming into zone i from the HVAC system.

Substitute equation (4-9) into equation (4-15), the resulting equation can be written in the following form:

$$
D T^{e}+E T^{s}=K
$$

where,

$$
\begin{aligned}
& D_{i j}=\beta \cdot U_{i j}, i \neq j, \\
& D_{i i}=-\beta \cdot \sum_{j=0}^{N} U_{i j}-C_{i i}, \\
& E_{i j}=(1-\beta) \cdot U_{i j}, i \neq j, \\
& E_{i i}=-(1-\beta) \cdot \sum_{j=0}^{N} U_{i j}+C_{i i}, \\
& K_{i}=-G_{i}-U_{\text {io }} \cdot T_{\text {out }} .
\end{aligned}
$$

Again, $T^{e}$ and $T^{s}$ are temperature vectors. $T_{\text {out }}$ is the outside temperature.

\subsection{Model Implementation}

MATLAB, a commercially available software, is well known for its excellent capability in calculations involving matrices and vectors (Matlab release 13). The MATLAB code was written based on the methods described earlier. The complete code is included in the Appendix. The outline of the format and structure of the code is shown in Figure 4.6. 
The combine linear equation sets (4-13) and (4-17), equations can be written in an overall matrix notation, with unknown supply temperature vector $T^{s}$ and exhaust temperature vector $T^{e}$ :

$$
\left[\begin{array}{ll}
A & B \\
D & E
\end{array}\right]\left[\begin{array}{l}
T^{e} \\
T^{s}
\end{array}\right]=\left[\begin{array}{l}
J \\
K
\end{array}\right]
$$

Coefficient matrix A, B, D, and E have already been defined above. In the code, the overall coefficient matrix consists of $\mathrm{A}, \mathrm{B}, \mathrm{D}$, and $\mathrm{E}$ is called $\mathrm{H}$. The right hand side vector $\mathrm{J}$ and $\mathrm{K}$ are also known and the overall right-hand side vector is defined as $\mathrm{S}$ in the code. The only unknowns are vectors $T^{e}$ and $T^{s}$. From equation (3-18), it can be easily solved for the unknown temperature vectors, given the stratification parameters from CFD ( $\alpha$ and $\beta$ ), the view factors matrix $\left(F_{i j}\right)$, the thermal resistance matrix $\left(U_{i j}\right)$, the thermostat control strategy $\left(L_{i j}\right)$, the HVAC air flow rate $\left(C_{i i}\right)$, the internal heat generation from each zone $\left(G_{i}\right)$, and the heat transfer coefficient for the occupants $\left(h_{c}\right.$ and $\left.h_{r}\right)$. Although the supply temperature vector $T^{s}$ is the only temperature of interest, $T^{e}$ has to be solved at the same time for closure of the linear equations. 
Report No. DE-FG02-03ER63694-F1

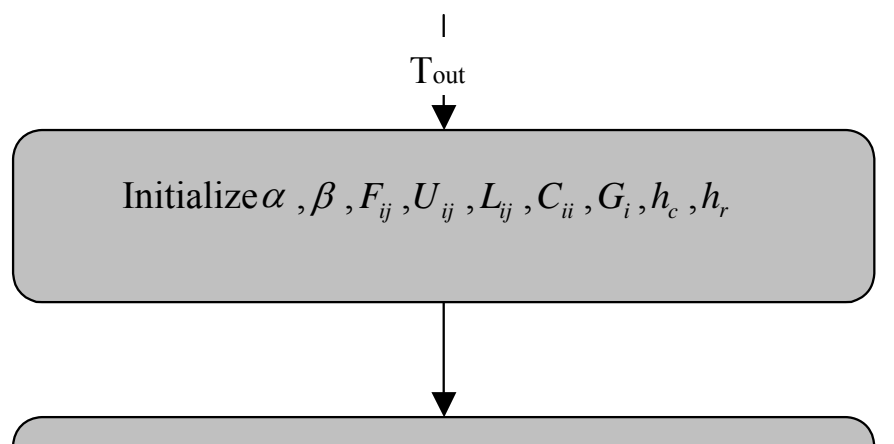

Solve for desired zonal temperature $T^{o}$

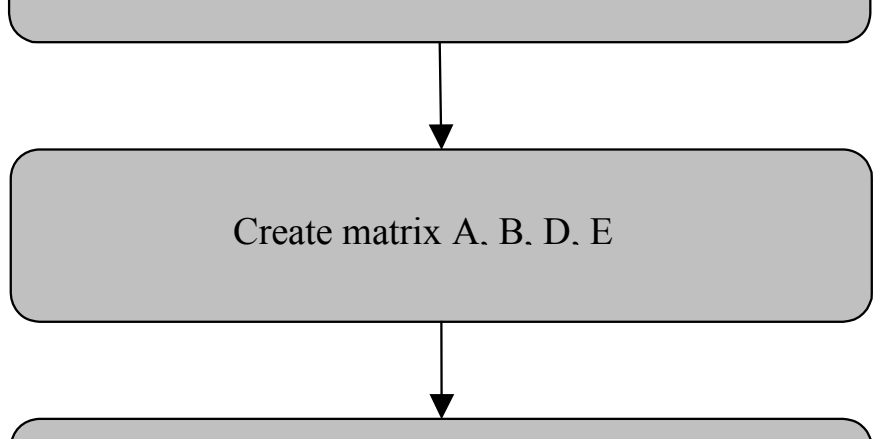

Create the overall matrix $\mathrm{H}$

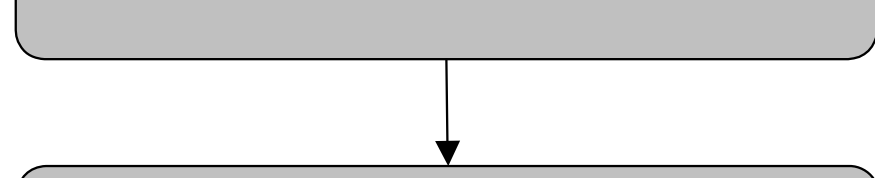

Create overall right-hand side vector S

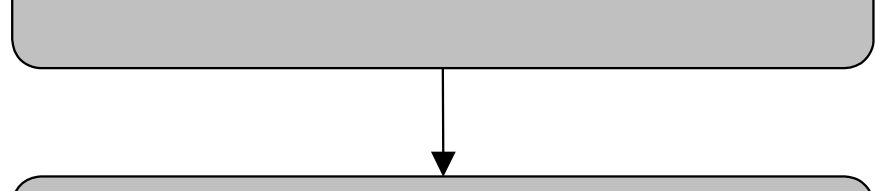

Solve for required supply temps. ( $T^{s}$ vector)

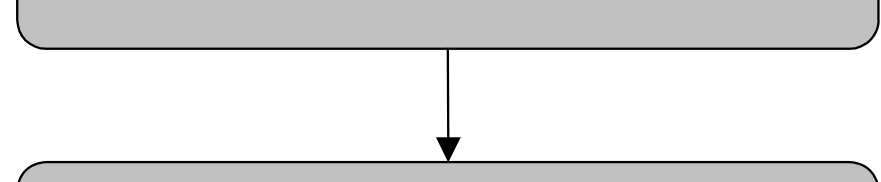

Building HVAC power usage calculation

Next Tout

Figure 4.6 Flow Chart of the modified thermal circuit model calculation 


\section{Report No. DE-FG02-03ER63694-F1}

\subsection{Computed Results}

Cosden has developed a model for building energy calculation using the thermal circuit model combined with a temperature bin method (Cosden 2005). The idea is grouping the yearly temperature profile of a city by number of hours per year in various temperature intervals (bins). The building energy consumption calculation will be based on a steady state calculation for each constant outdoor temperature bin and then multiplied by the number of hours in each bin. Common practice in the U.S. is to use a $5^{\circ} \mathrm{F}\left(3^{\circ} \mathrm{C}\right)$ temperature bin.

Cosden's thermal circuit model can be replaced by the modified thermal circuit model in order to calculate the appropriate supply temperatures for the UFAD systems, while the temperature bin method can still be applied for building energy calculation.

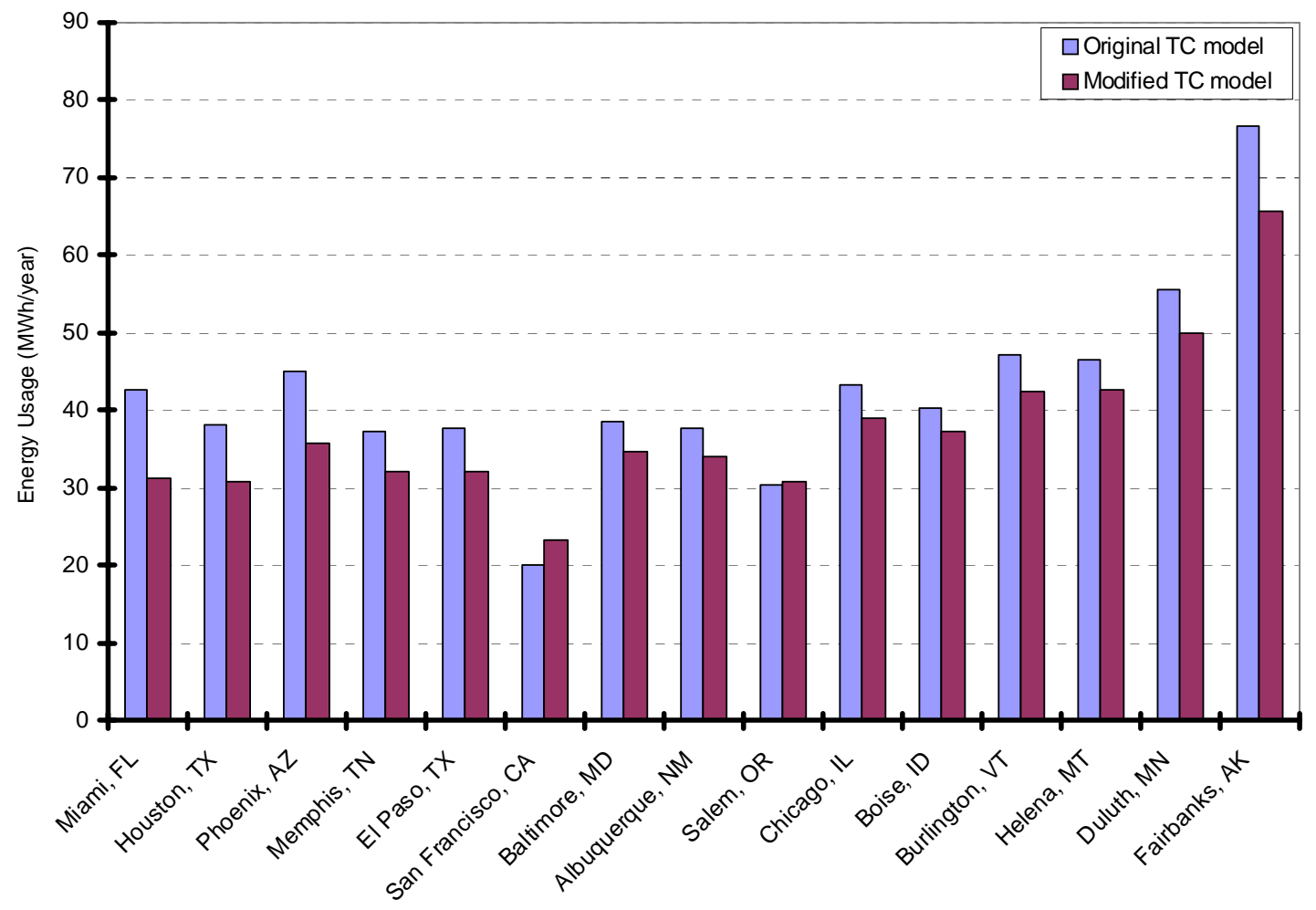

Figure 4.7 Yearly Energy Usage Comparisons for the Original and Modified TC Models (TTFA) 


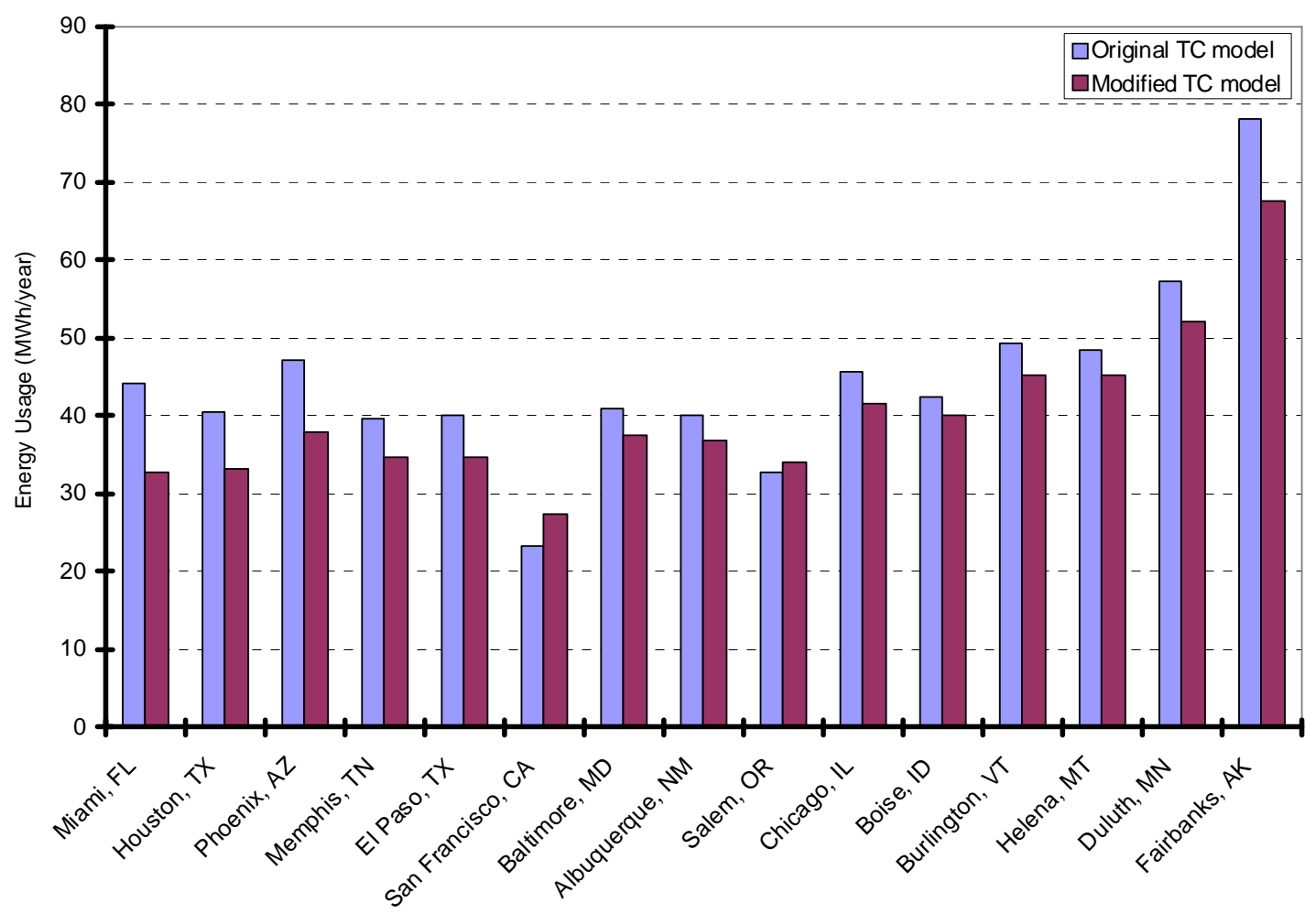

Figure 4.8 Yearly Energy Usage Comparisons for the Original and Modified TC Models (HIYW)

Figure 4.7 and Figure 4.8 show the integrated yearly energy usage for the two control strategy: TTFA and HIYW. Comparisons were made between the original and the modified thermal circuit models. Here, the parameters $\alpha$ and $\beta$ are chosen to be 0.4 and 0.7 from the FWR case. It can be seen that for most of the cities, applying the modified thermal circuit model will result in a $10 \sim 26 \%$ energy savings for both thermostat control strategies. This is because for cooling and heating in UFAD systems, it only needs to cool/heat up to certain height to meet the occupant's satisfaction, while leave the space above the occupied zone alone. Therefore it works relatively well for cities in hot climate zones (Miami) or very cold zones (Fairbanks). Special attention should be paid to San Francisco, CA and Salem, OR. More energy consumption is required for the modified TC model. This can be explained by the frequent use of economizer. For these two cities, there is a large portion of the year where free air conditioning can be 
achieved by the economizer. Because the outside air temperature is sufficient for ventilating the entire building. Figure 4.9 presents the ratio of HIYW yearly energy usage to TTFA yearly energy usage and again comparisons were made between the original and the modified TC model.

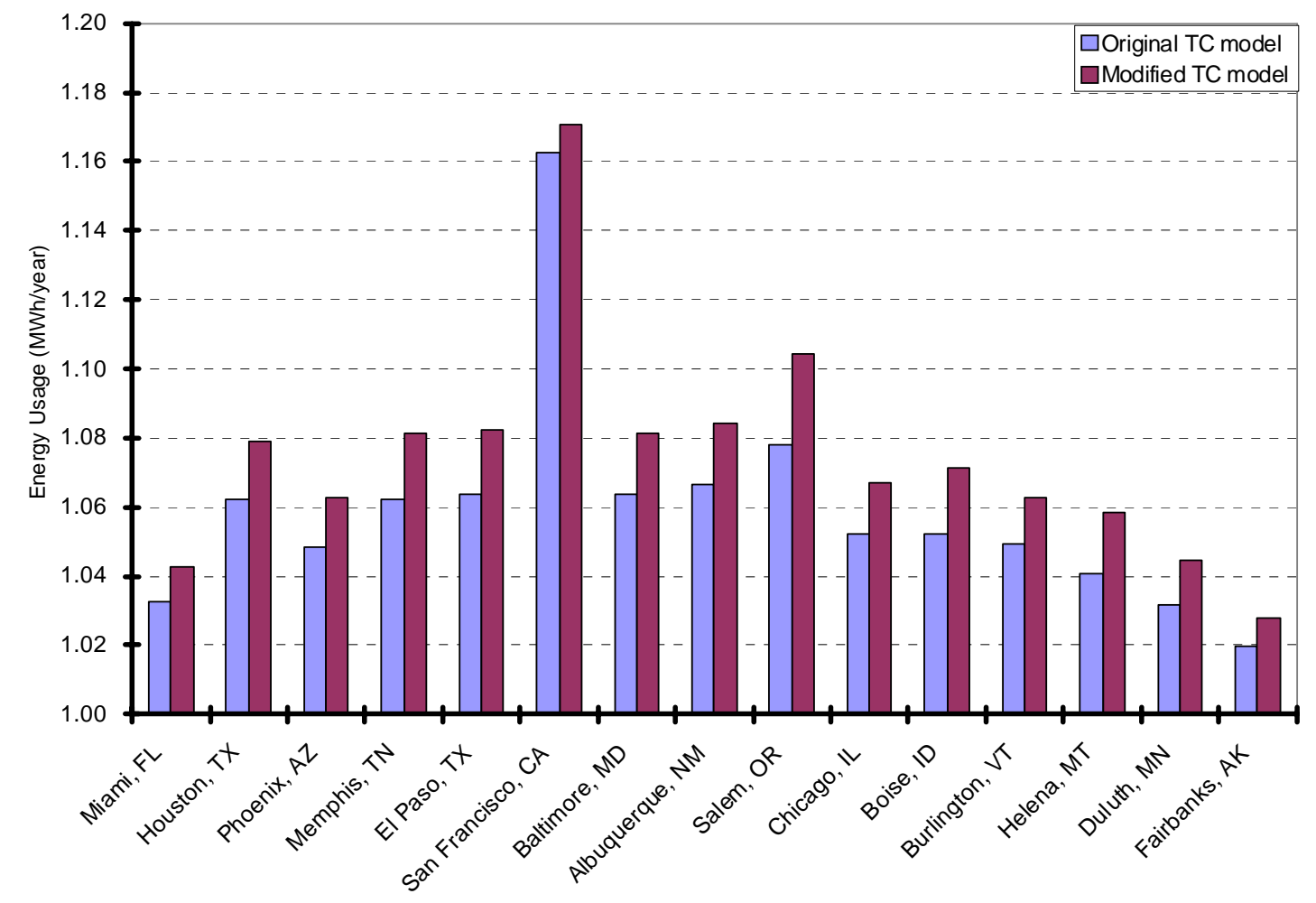

Figure 4.9 Ratio of HIYW to TTFA Comparisons for the Original and Modified TC Models

Here, a value of 1 indicates that HIYW and TTFA have exactly the same yearly energy consumption. Qualitatively, the ratios for all the cities are very similar using the two thermal circuit models. The modified TC model will only resulting in $1 \sim 2 \%$ increase on the ratio of HIYW to TTFA. Again (Cosden 2005), San Francisco is decidedly the worst since it needs 17\% more energy to achieve HIYW for the modified TC model. All the other cities will still remain in the range of $3 \sim 8 \%$. 


\section{Report No. DE-FG02-03ER63694-F1}

\section{Conclusions}

For cases composed of two cubicles and an adjoining corridor, the advective flow between zones is very significant and severely limits the occupants' ability to control the personal micro-environments via inlet temperature control. CFD calculations were performed for pairs of cubicles with an adjoining corridor in which the supply temperatures in the two cubicles differed by up to 8 degrees $C$. In cases where the air was allowed to freely traverse through the corridor, the temperatures "felt" by the occupants in the two cubicles differed by less than $25 \%$ of the supply temperature difference. Flow visualization of the CFD results indicated that this loss of personal temperature control was caused by the cold supply flow sinking to the floor and traversing through the corridor into the other cubicle; at the same time, the warm supply in the other cubicle ascended into the space above the cubicles and had little effect on the temperatures "felt" by the occupant. Through a series of parametric studies it was found that the effectiveness of the personal environment control could best be improved by installing doors at the openings between the cubicles and the corridor; other strategies, such as changing the height and insulation of the cubicle walls, changing the configuration of the cubicles, and altering the swirl direction and location of the inlet diffusers had a negligible effect on the control of temperature in the two cubicles.

A thermal comfort model that accounts for stratified temperature profiles and radiation is required for configurations that employ an underfloor air distribution (UFAD) system. While Cosden's original thermal circuit (TC) model is suitable for predicting the annual energy consumption of buildings with mixing ventilation, its well-mixed assumption is not valid for the stratified flows associated with UFAD. In the current work, detailed CFD simulations were performed to predict the vertical air temperature profiles associated with UFAD, and a simple model was developed to approximate 


\section{Report No. DE-FG02-03ER63694-F1}

these profiles. The thermal circuit model was subsequently modified to account for the thermal stratification, both for the temperature experienced by the occupant and also for the energy transfer between zones via convection and conduction. Additionally, Cosden's model was modified to include a thermal comfort model that accounts both for convection/conduction and radiation to/from the occupant. Care was taken to include these additional physical phenomena into Cosden's model via linear relationships, and thus had a very small impact on the overall execution time of the model.

The modifications to the thermal circuit model reduce the predicted energy usage for UFAD in non-temperate cities by between 10 and 26\% annually, as compared with Cosden's model. These results apply both to Cosden's three-thermostats-for-all (T'TFA) and have-it-your-way (HIYW) control strategies. This reduction is attributed to the temperature stratification associated with UFAD, which Cosden's model ignores. For cities with moderate climates such as San Francisco, the modified model predicts about $17 \%$ more energy is required, due largely to increased frequency of use of the economizer. Only slight changes were observed on the ratio of HIYW yearly energy usage to TTFA yearly energy usage using the modified thermal circuit model. 


\section{Report No. DE-FG02-03ER63694-F1}

\section{Appendix}

Listed here is the code for modified thermal circuit model, written in MATLAB. The main loop contains three subroutines: Lcalc.m, Rcalc.m, Fcalc.m.

\subsection{Main Loop}

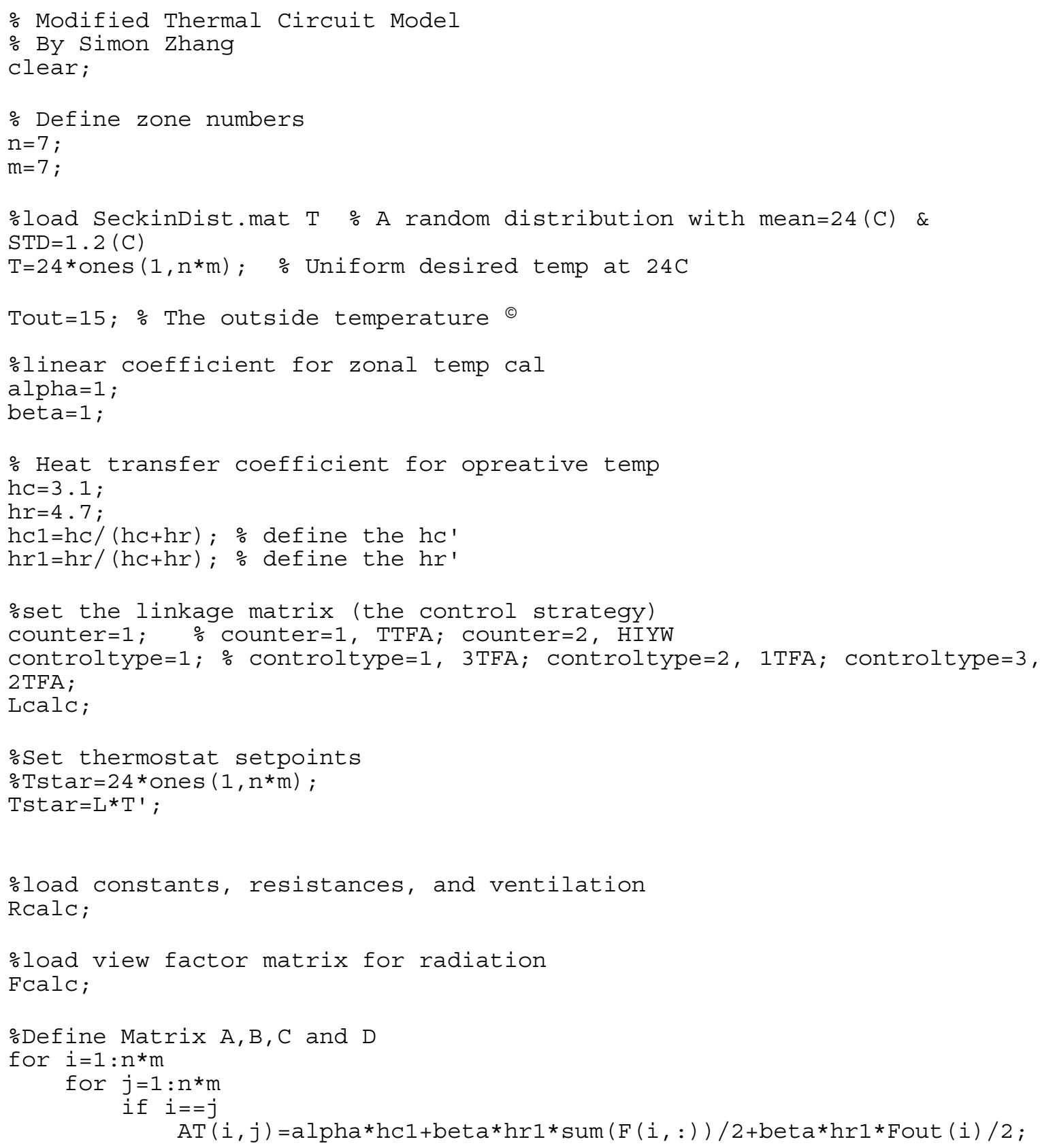




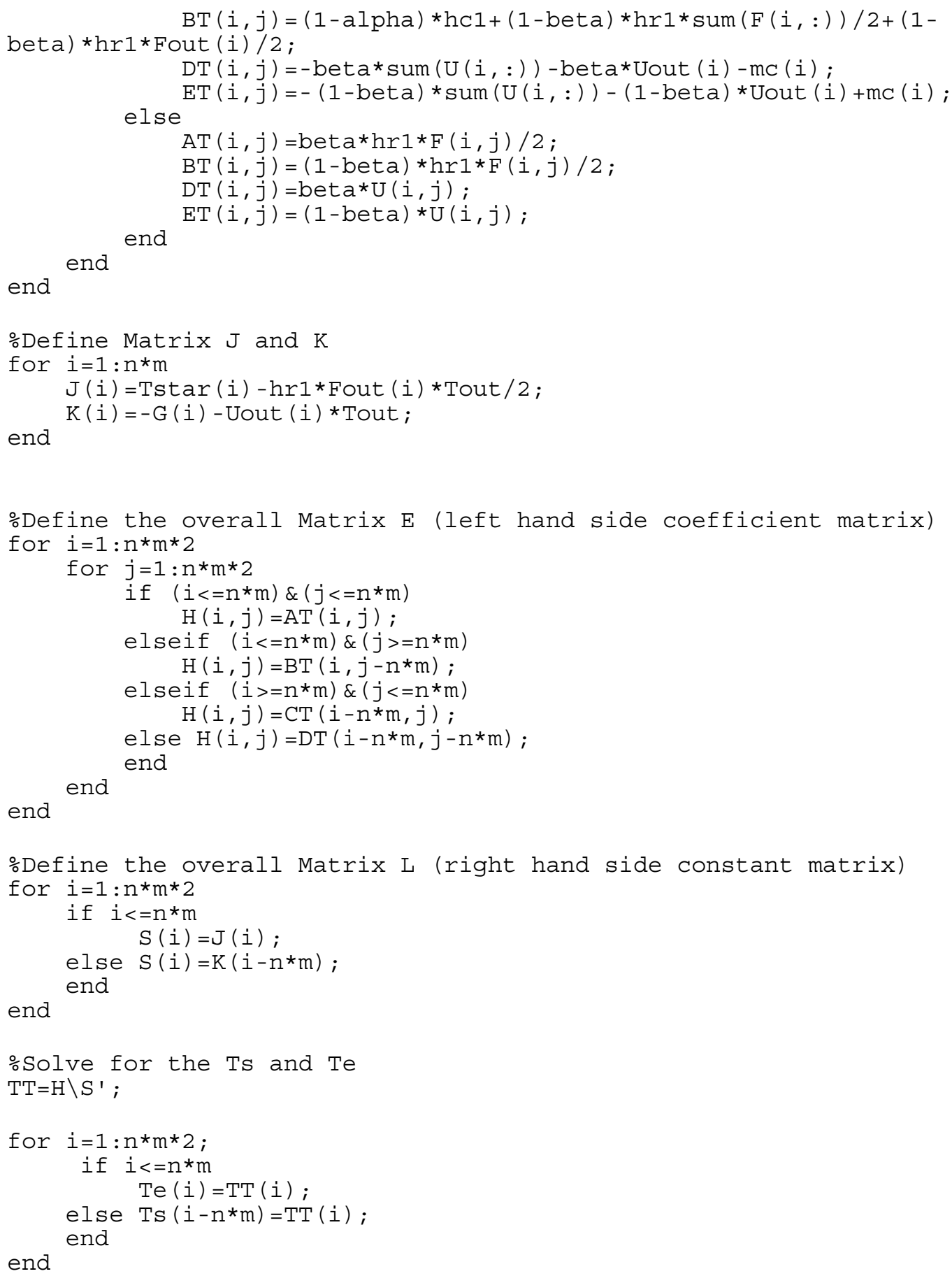

\subsection{Linkage Matrix (Lcalc.m)}

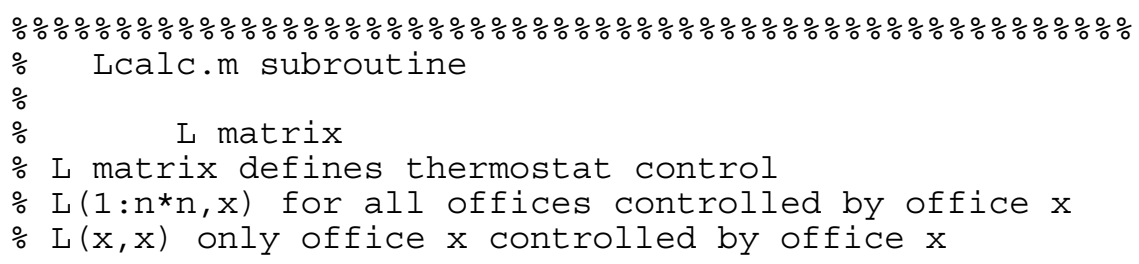




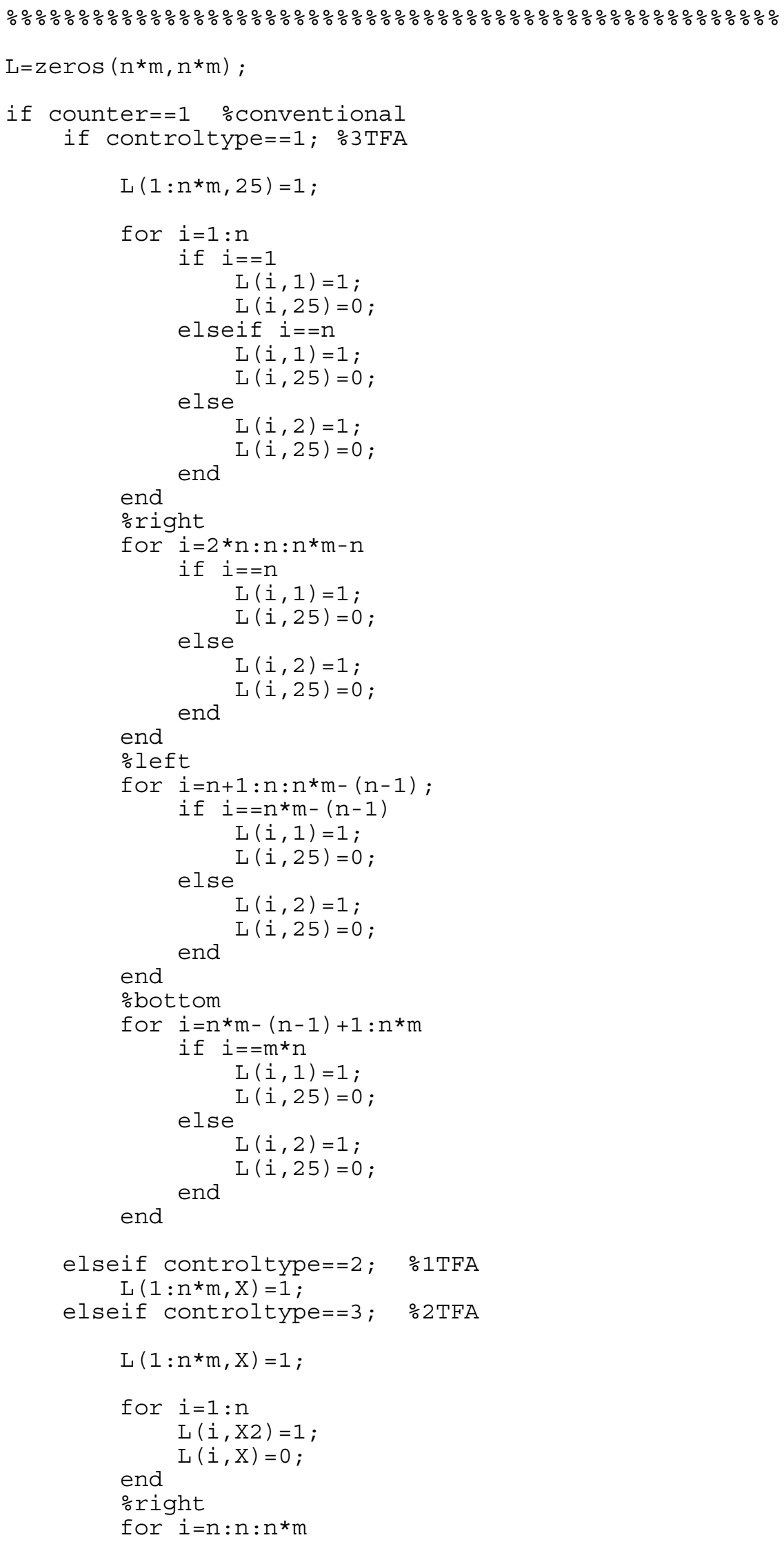


end

$$
\begin{aligned}
& \mathrm{L}(\mathrm{i}, \mathrm{X} 2)=1 ; \\
& \mathrm{L}(\mathrm{i}, \mathrm{X})=0 ;
\end{aligned}
$$

\%left

for $i=1: n: n * m-(n-1)$;

$$
\mathrm{L}(\mathrm{i}, \mathrm{X} 2)=1 \text {; }
$$

end

$$
\mathrm{L}(\mathrm{i}, \mathrm{X})=0 \text {; }
$$

\%bottom

for $i=n * m-(n-1): n * m$

$$
\mathrm{L}(\mathrm{i}, \mathrm{X} 2)=1 \text {; }
$$

end

$$
\mathrm{L}(\mathrm{i}, \mathrm{X})=0 \text {; }
$$

end

elseif counter $==2 \%$ HIYW

for $i=1: n * m$

end

$$
L(i, i)=1 \text {; }
$$

end

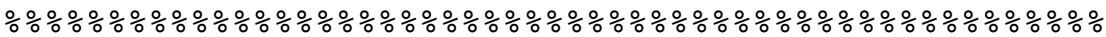

\subsection{Resistance Matrix (Rcalc.m)}

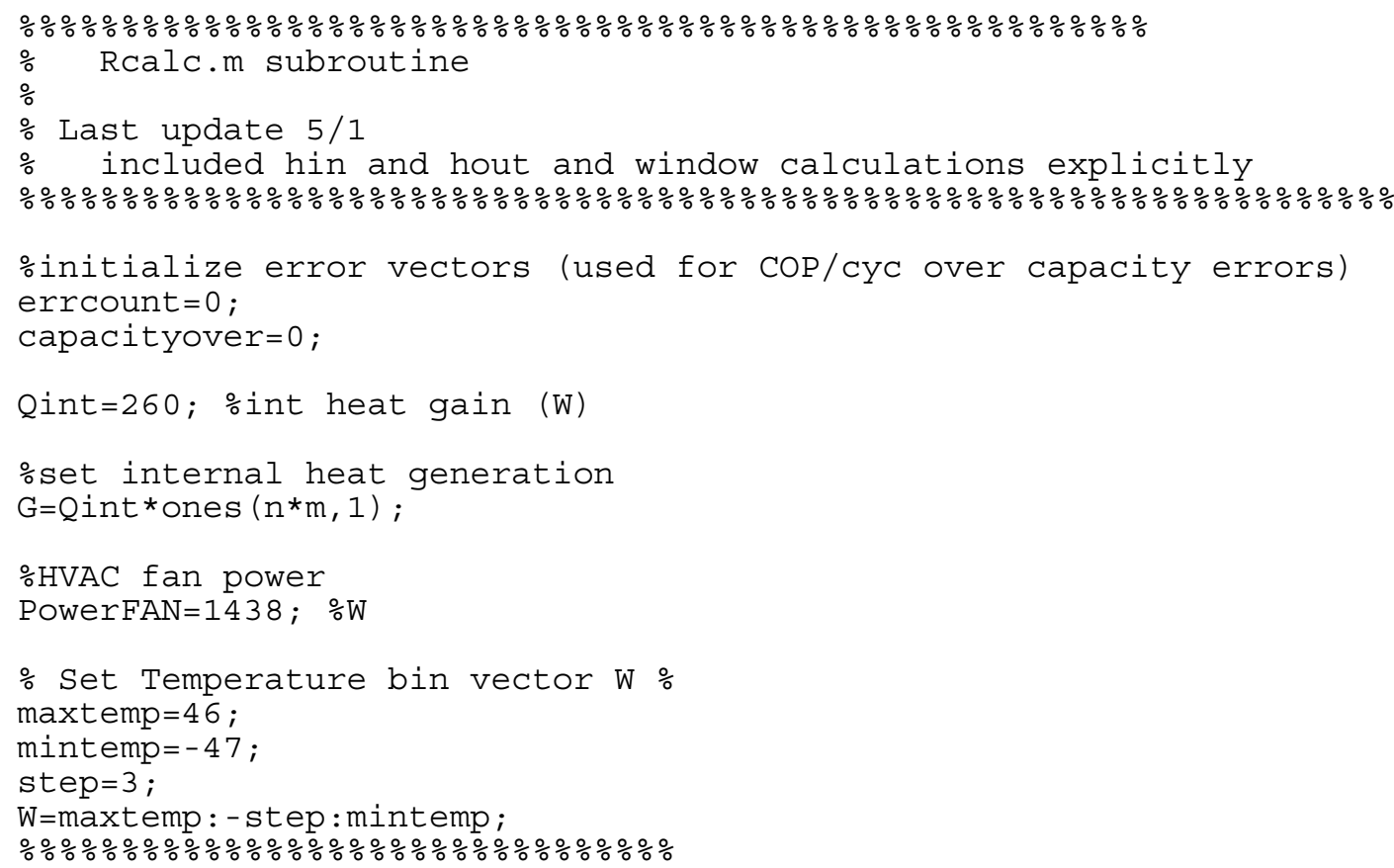




\section{Report No. DE-FG02-03ER63694-F1}

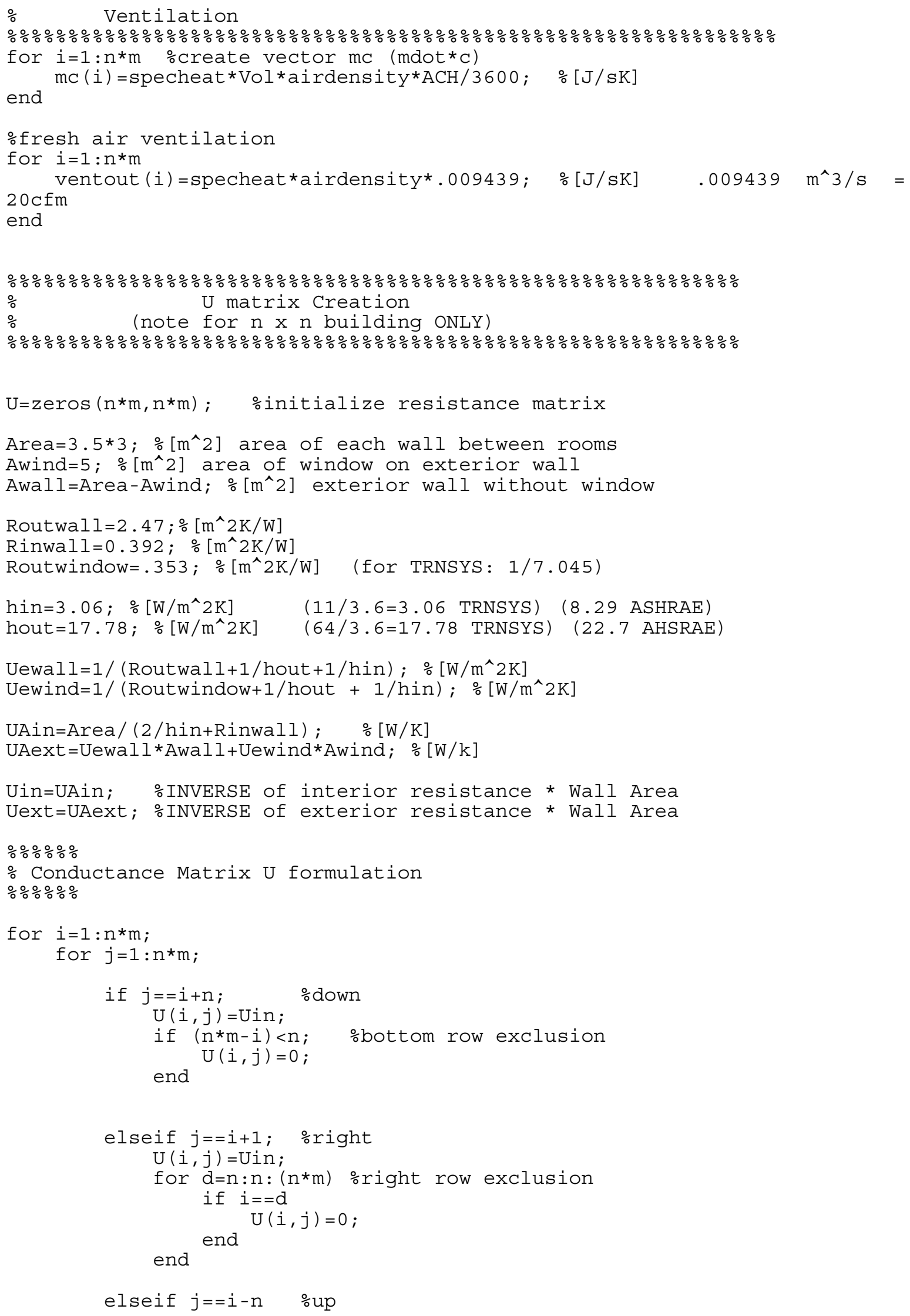


Report No. DE-FG02-03ER63694-F1

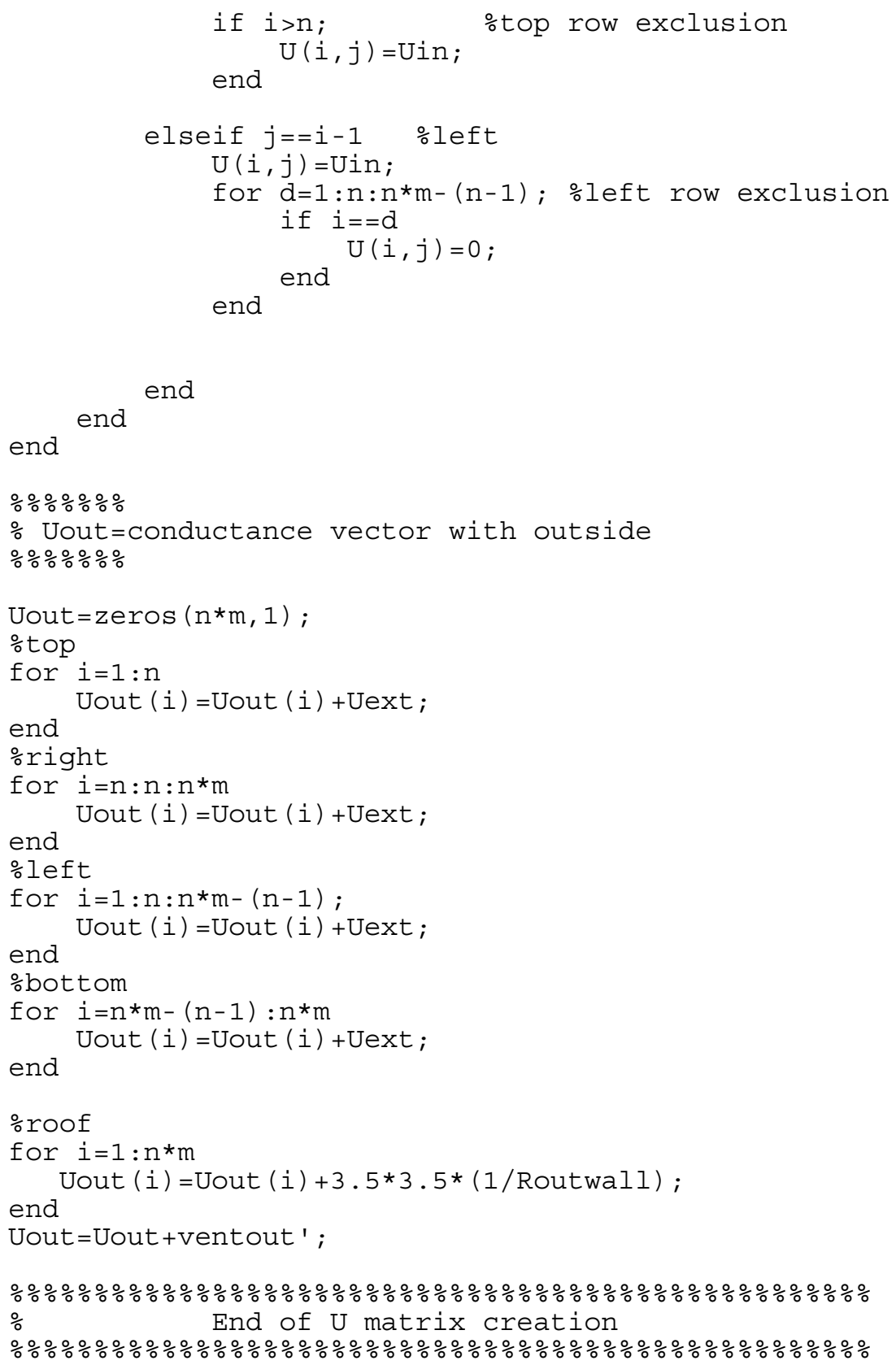

\subsection{View Factors Matrix (Fcalc.m)}

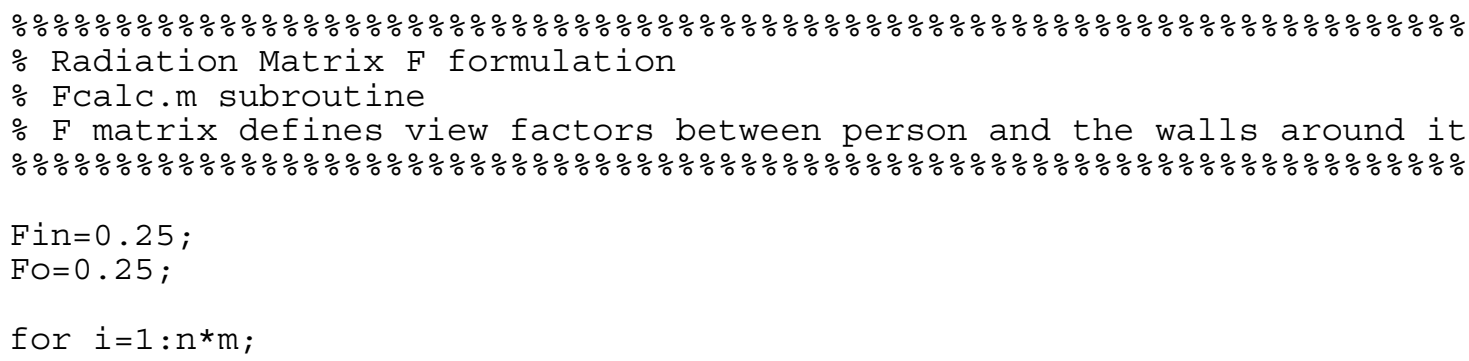




\section{Report No. DE-FG02-03ER63694-F1}

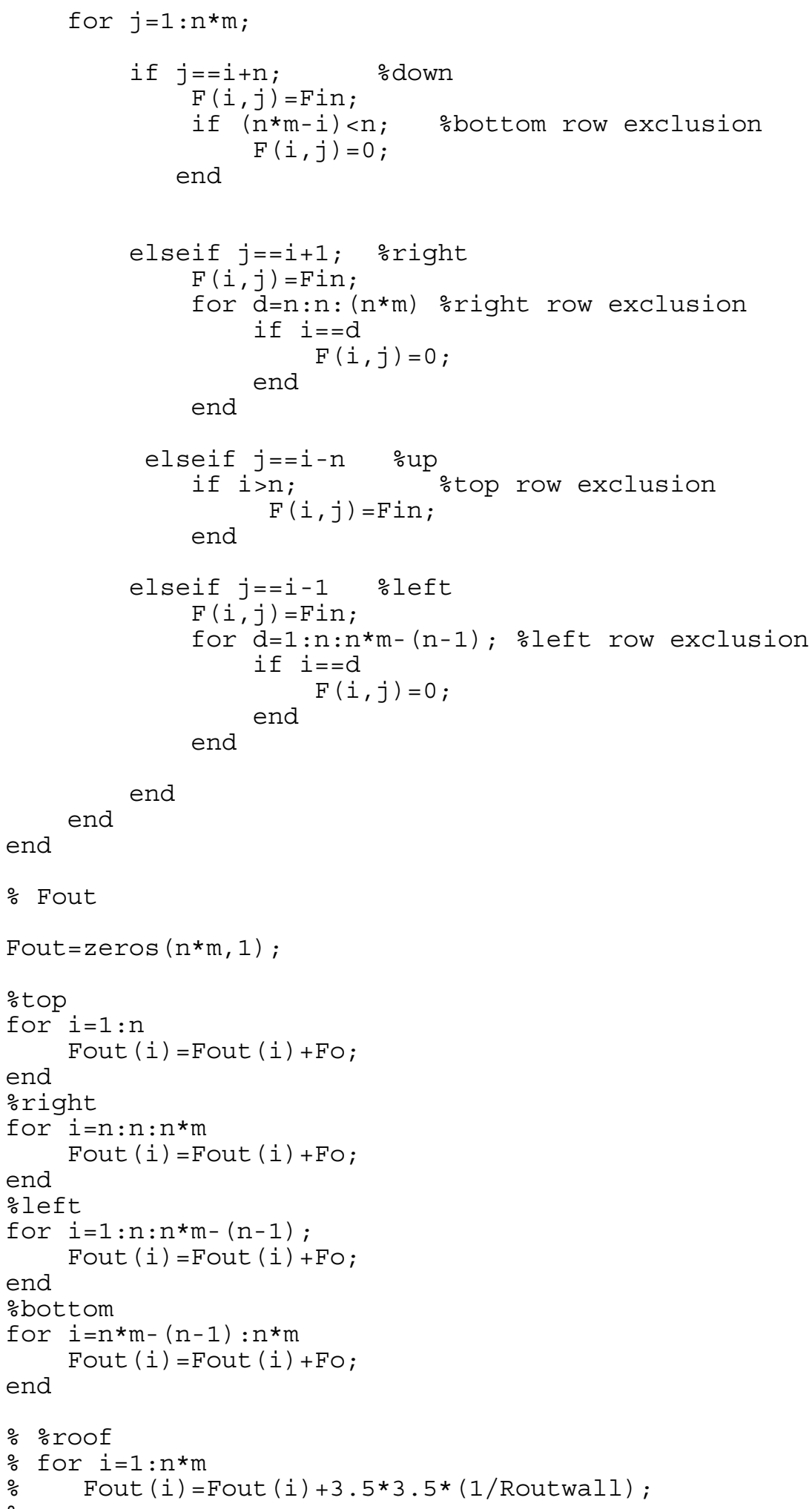




\section{Report No. DE-FG02-03ER63694-F1}

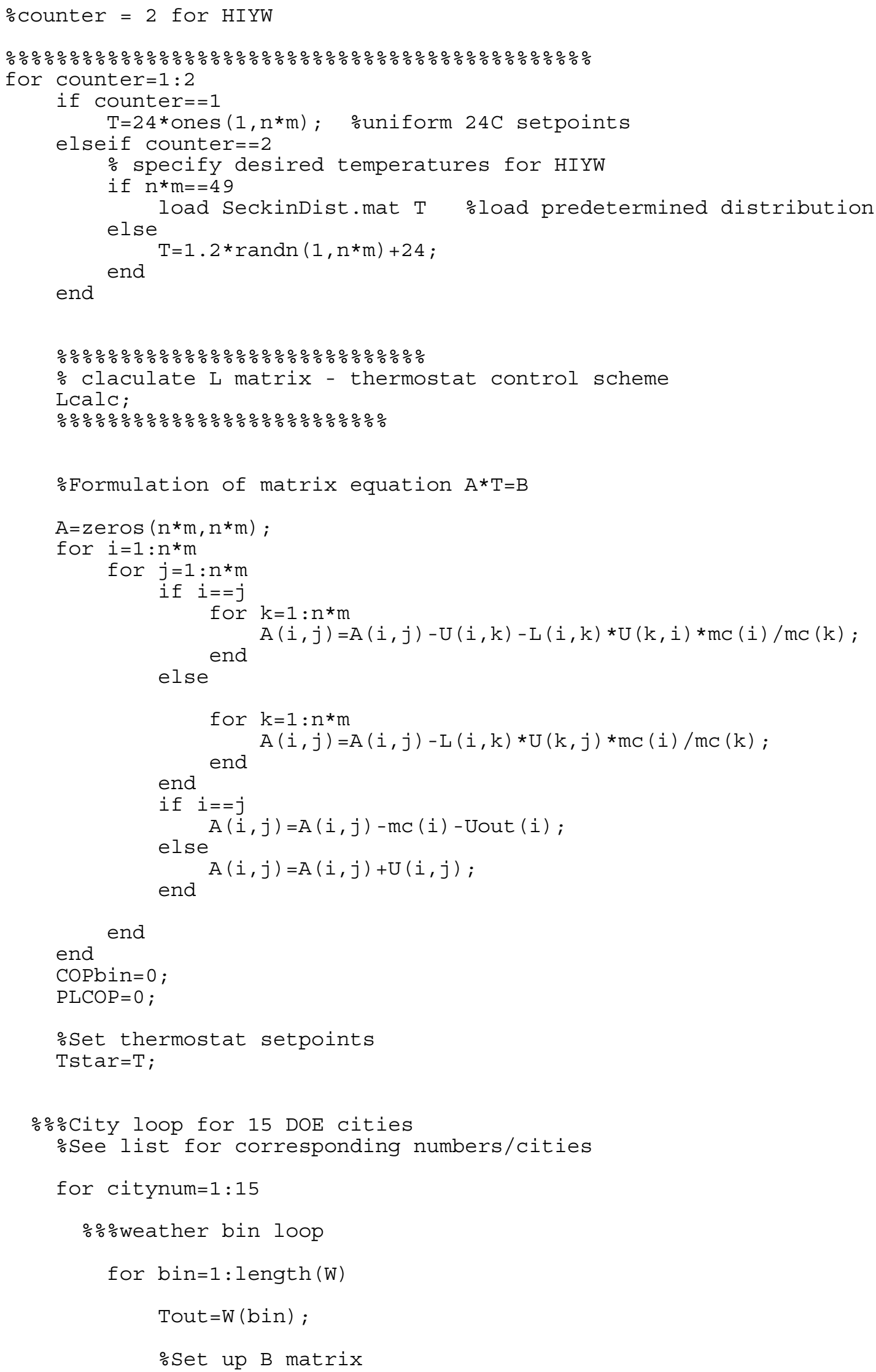




\section{Report No. DE-FG02-03ER63694-F1}

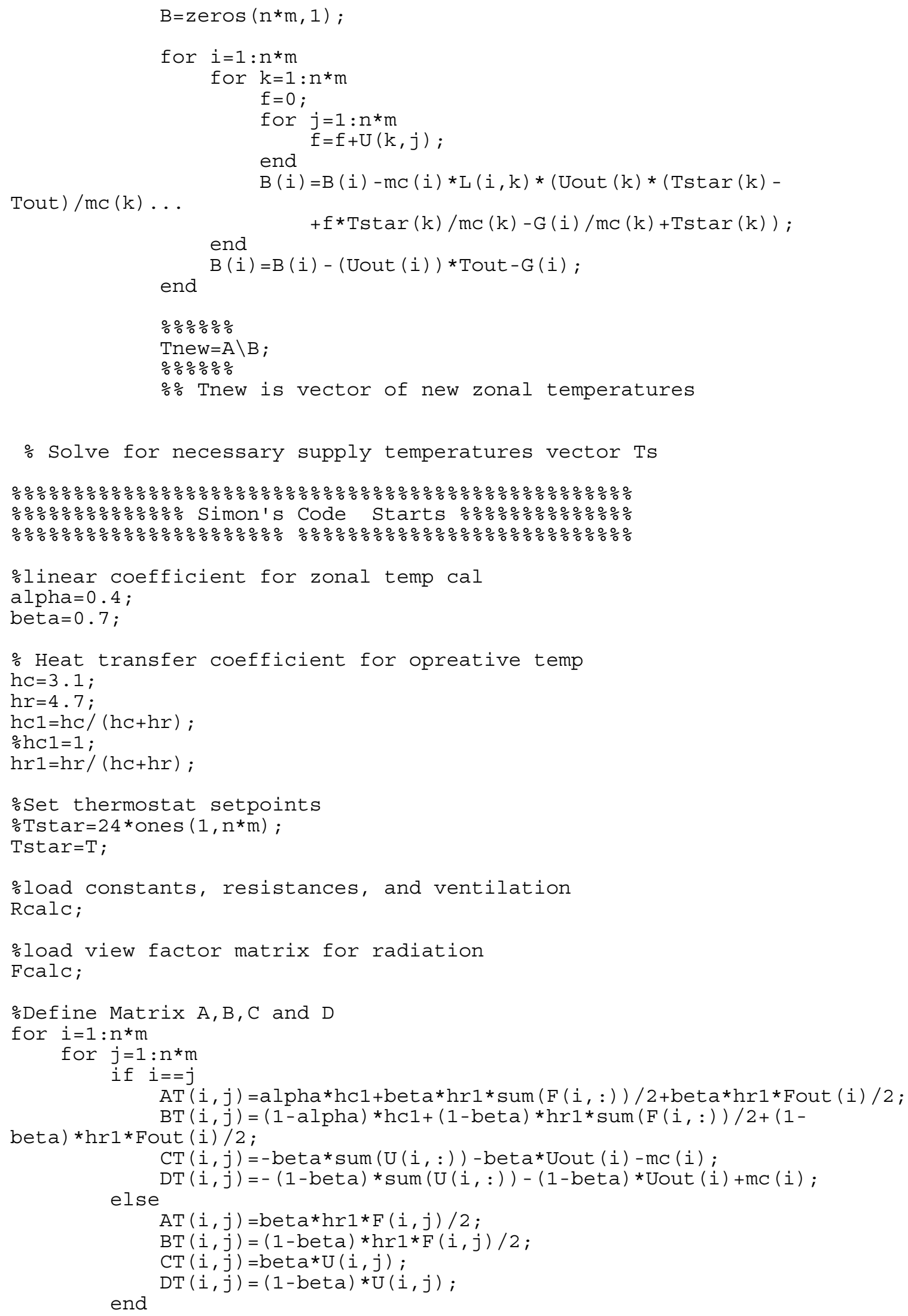




\section{Report No. DE-FG02-03ER63694-F1}

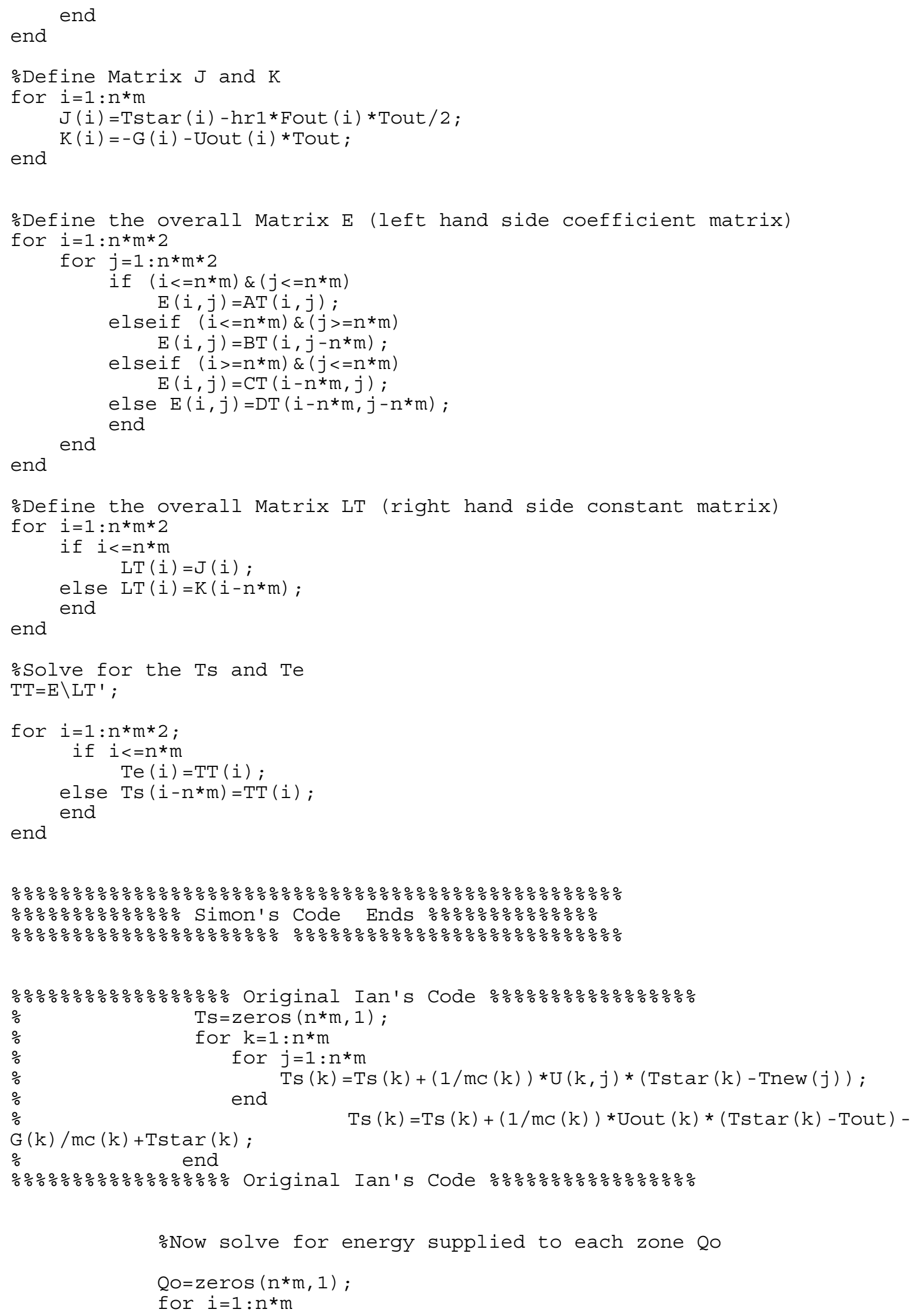




\section{Report No. DE-FG02-03ER63694-F1}

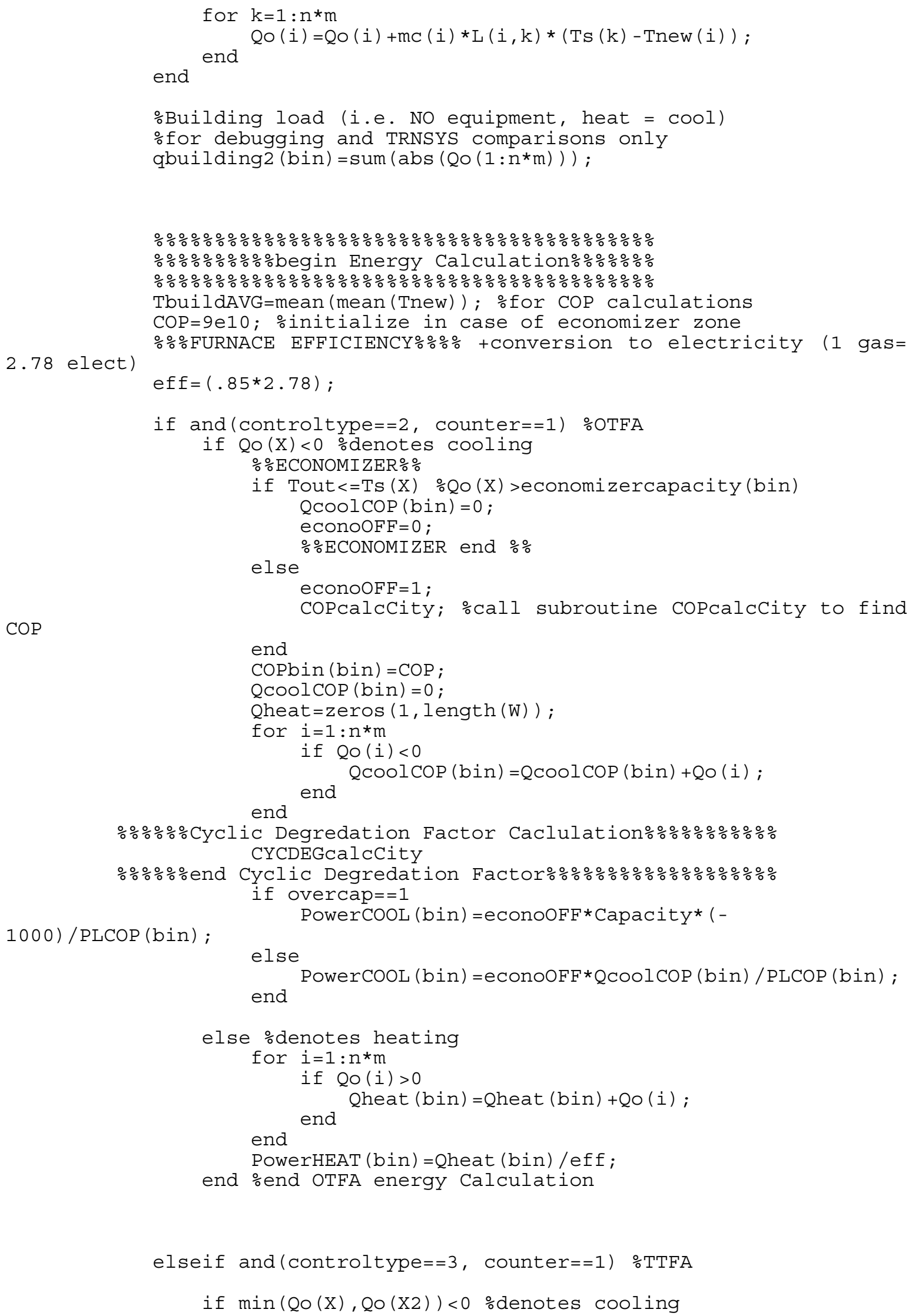


Report No. DE-FG02-03ER63694-F1

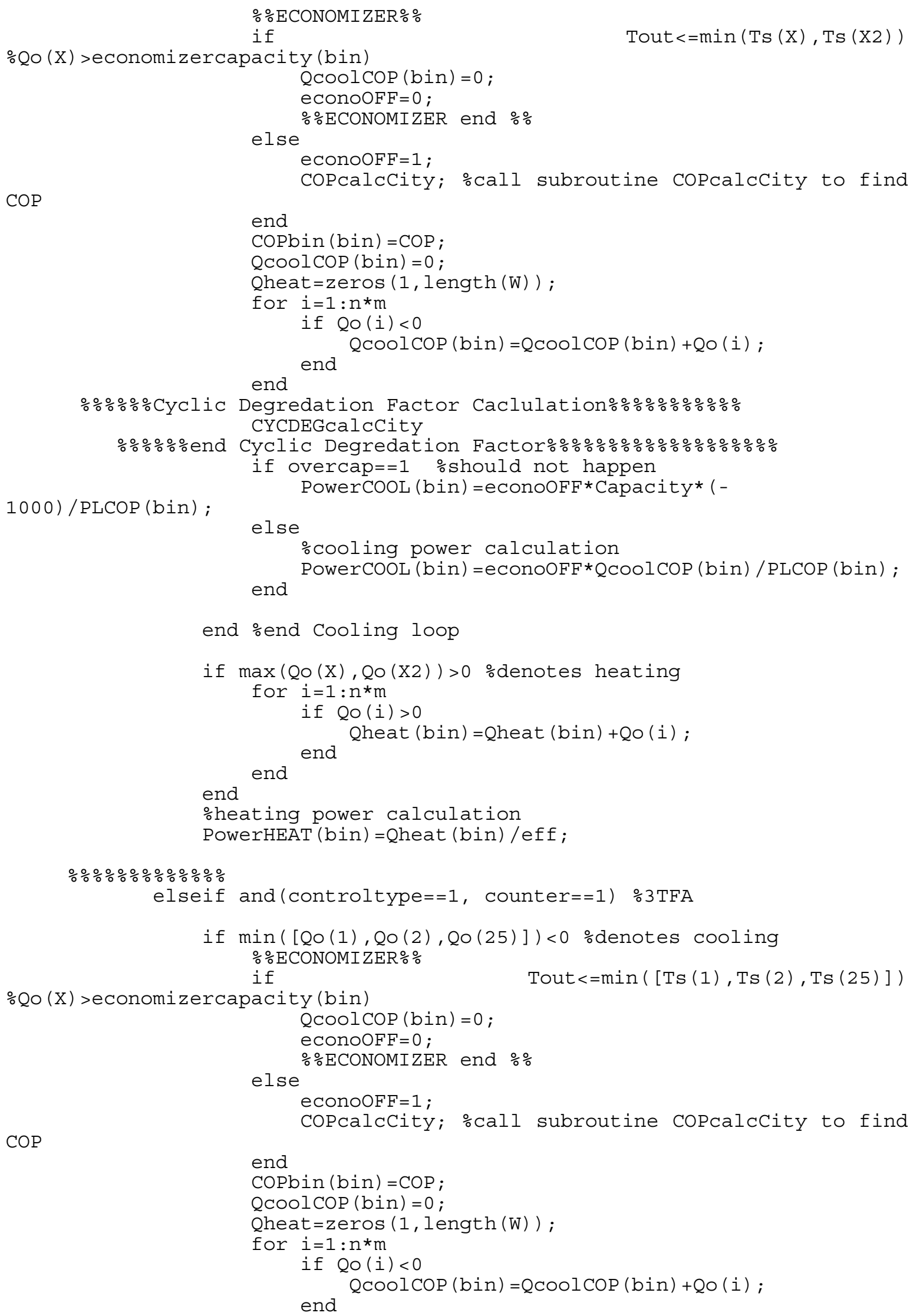




\section{Report No. DE-FG02-03ER63694-F1}

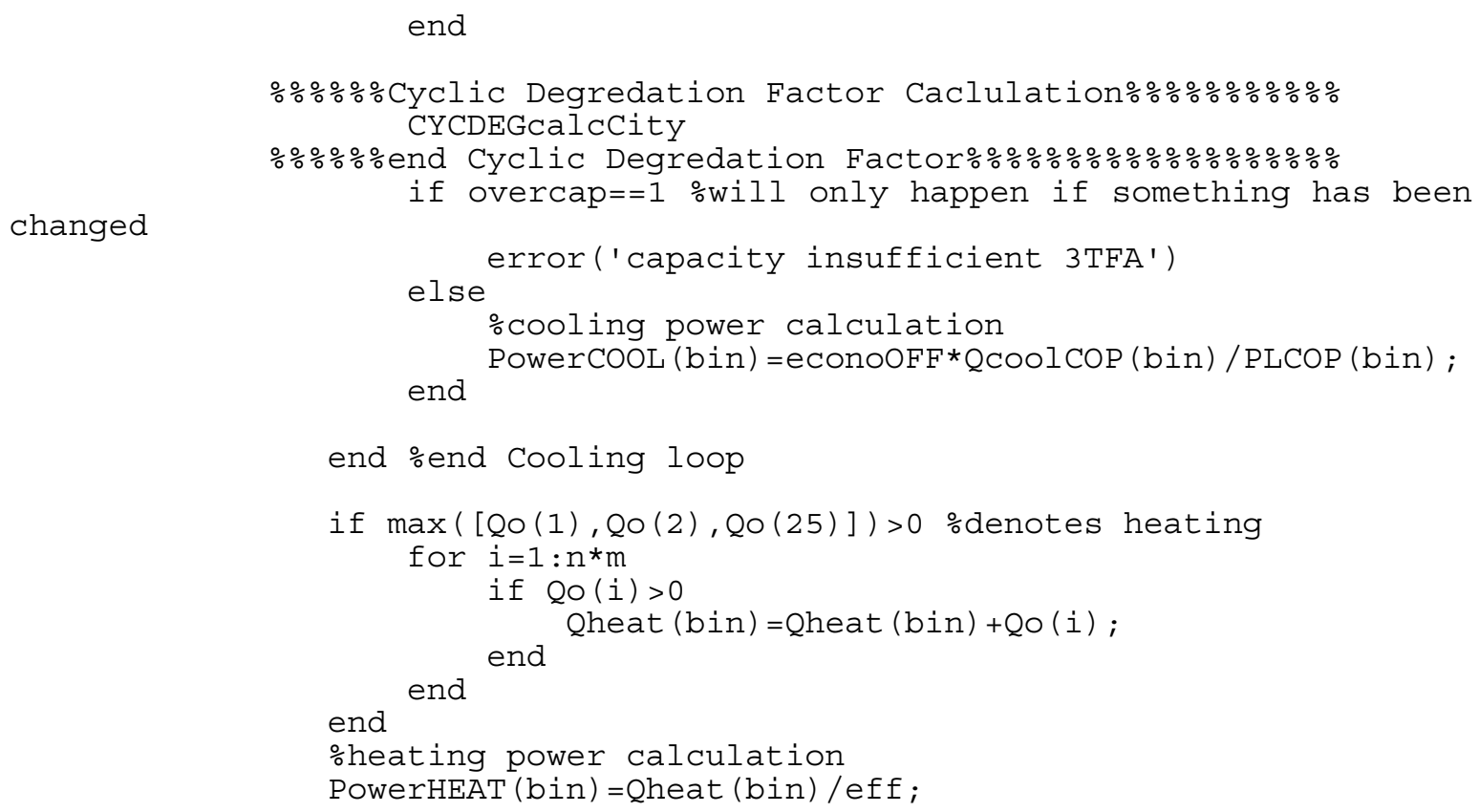

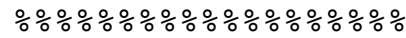

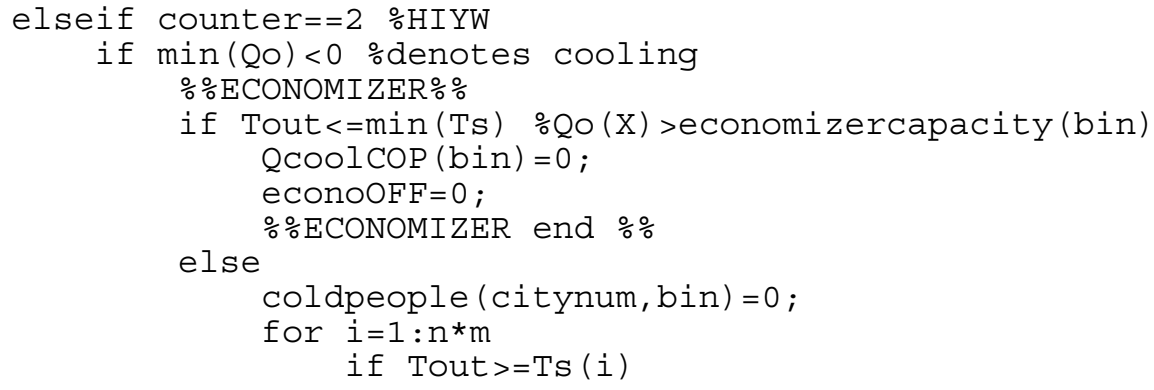




\section{Report No. DE-FG02-03ER63694-F1}

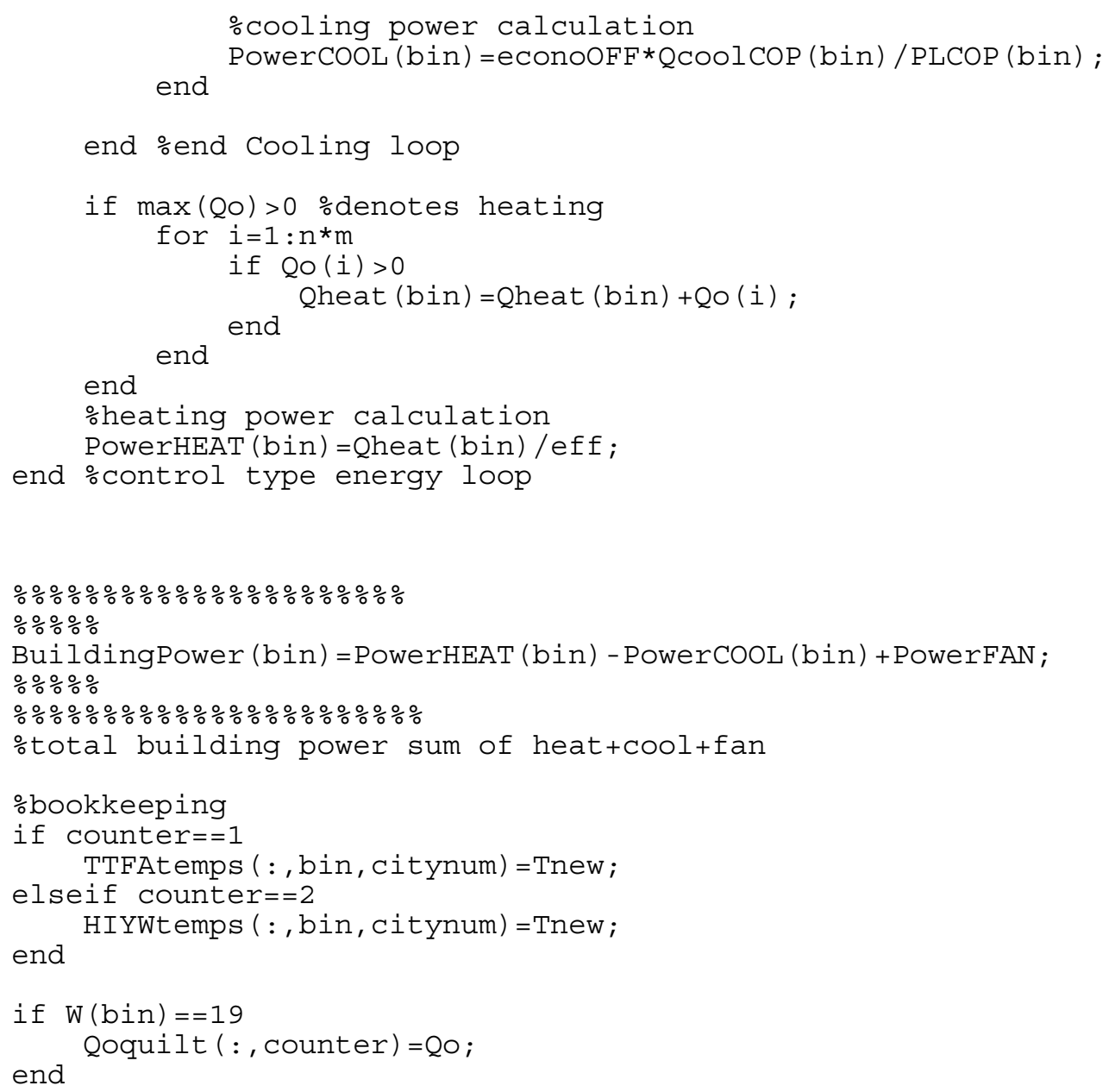

end \%end city loop 


\section{Report No. DE-FG02-03ER63694-F1}

end \%counter loop

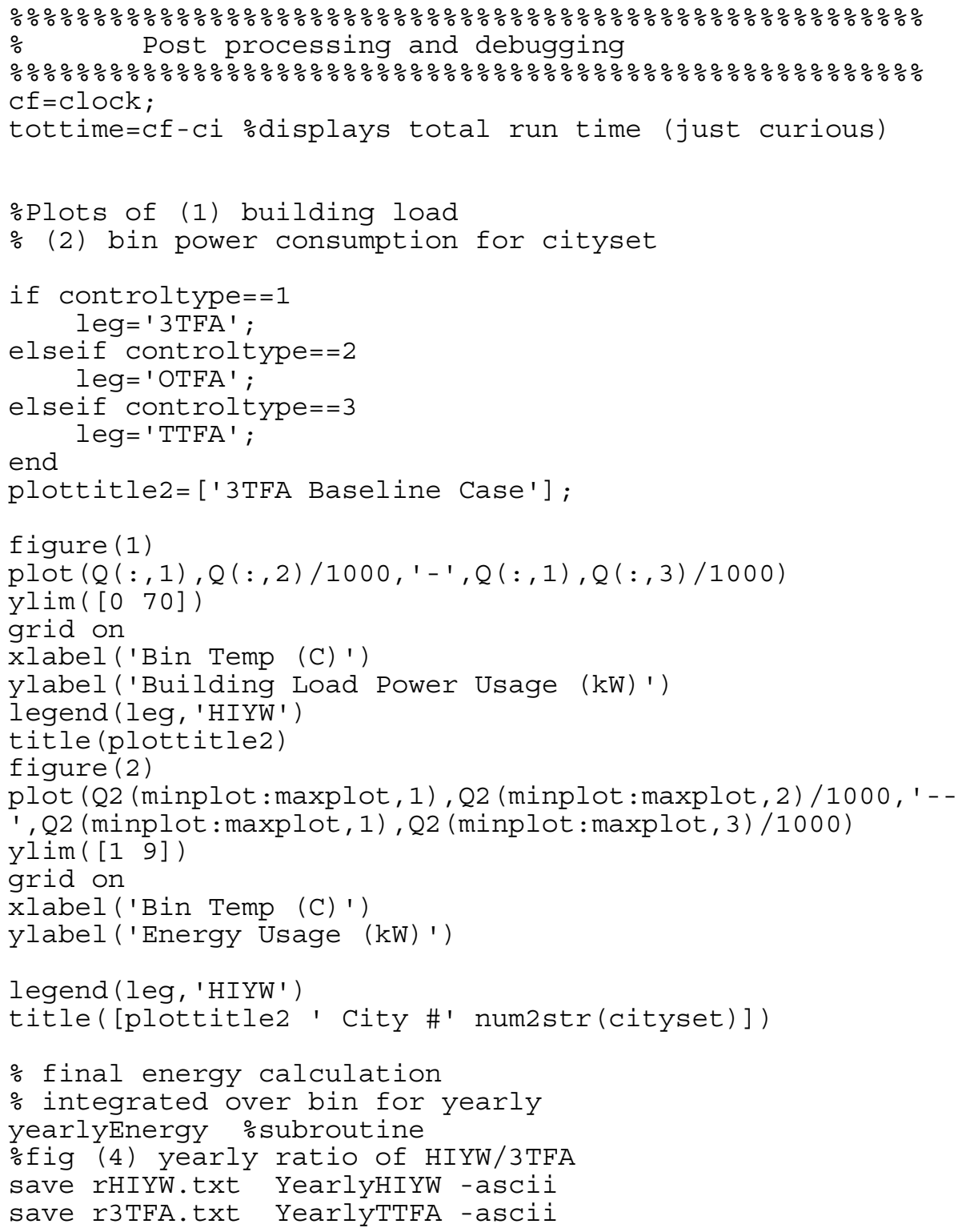




\section{References}

[1] Abanto, J., D. Barrero, M. Reggio, B. Ozell. (2004) Airflow modeling in a computer room, Building and Environment, V 39, n 12, December, 2004, p 1393-1402.

[2] ASHRAE. (2004) Thermal Environmental Conditions of Human Occupancy, ASHRAE standard 55-2004.

[3] ASHRAE. (2001) Thermal comfort, ASHRAE handbook of fundamentals, Atlanta, chapter 8 .

[4] Awbi, H. (2003) Ventilation of Buildings, Spon Press, London.

[5] Bauman, F.S. (2003) Underfloor air distribution (UFAD) design guide, ASHRAE, Atlanta.

[6] Chen, Q. (1995) Comparison of different k- $\varepsilon$ models for indoor air flow computations, Numerical Heat Transfer, (part B), 353-369.

[7] Chen, Q., W. Xu. (1998) Zero-equation turbulence model for indoor airflow simulation, Energy and Buildings, v 28, n 2, Oct, p 137-144.

[8] Cosden, I.A. (2005) Modeling the Energy Efficiency of Distributed Environmental Control Systems, Master Thesis, Syracuse University.

[9] Gao, N. and J. Nui. (2004) CFD study on micro-environment around human body and personalized ventilation, Building and Environment, 39: 795-805.

[10] Fanger, P.O. (1967) Calculation of thermal comfort: Introduction of a basic comfort equation. ASHRAE Transactions 73 (2):III.4.1.

[11] Fanger, P.O. (1970) Thermal comfort analysis and applications in environmental engineering. McGraw-Hill, New York.

[12] Fluent Inc. (2002) AIRPAK 2.1 Manual C Fluent Inc. 2002-04-19.

[13] Fluent Inc. (2003) Fluent 6.1. user's guide. Fluent Inc.

[14] Gosman, A. D., P. V. Nielsen, A. Restivo and J. H. Whitelaw. (1980) The Flow Properties of Rooms With Small Ventilation Openings, Journal of Fluids Engineering, Vol. 102, pp. 316-323.

[15] Incropera, F.P. and D.P. DeWitt. (2002) Introduction to Heat Transfer, John Wiley \& Sons, New York.

[16] Kader, B. (1993) Temperature and Concentration Profiles in Fully Turbulent Boundary

[17] Layers, Int. J. Heat Mass Transfer, 24(9):1541 1544. 
[18] Launder, B.E. and D.B. Spalding. (1972) Lectures in Mathematical Models of Turbulence, Academic Press, London and New York.

[19] Launder, B.E. and D.B. Spalding. (1974) The numerical computation of turbulent flows,

[20] Comp.Meth. Appl. Mech. Energy, 269-289.

[21] Math Works products, MATLAB, release 13

[22] Moin, P. (1997) Progress in large eddy simulation of turbulent flows, AIAA pap. 97-0749.

[23] Moin, P. and K. Mahesh. (1998) Direct Numerical Simulation: A Tool in Turbulence Research, Annu. Rev. Fluid Mech. 30:539-78.

[24] Murakami, S., S. Kato, J. Zeng. (1997) Flow and temperature fields around human body with various room air distribution, CFD study on computational thermal manikin---Part I, ASHRAE Transactions, 103: 3-15.

[25] Murakami, S., S. Kato, J. Zeng. (2000) Combined simulation of airflow, radiation and moisture transport for heat release from a human body, Building and Environment, 35: 489-500.

[26] Nielsen, P. V. (1990) Specification of a Two-Dimensional Test Case, IEA Annex 20 Report, Aalborg University, ISSN 0902-7513 R9040.

[27] Nielsen, P.V., S. Murakami, S. Kato, C. Topp and J.H. Yang. (2003) Benchmark Tests for a Computer Simulated Person, Version of 7, November 2003.

[28] Rapp, G. and A.P. Gagge. (1967) Configuration factors and comfort design in radiant beam heating of man by high temperature infrared sources. ASHRAE Transactions 73 (2):III.1.1.

[29] Sørensen, D.N., L.K. Voigt. (2003) Modeling flow and heat transfer around a seated human body by computational fluid dynamics, Building and Environment, 38: 753-62.

[30] Tsuzuki, K., E.A. Arens, F.S. Bauman, and D.P. Wyon. (1999) Individual thermal comfort control with desk-mounted and floor-mounted task/ambient conditioning (TAC) systems, Proceedings of Indoor Air '99, Edinburgh, Scotland, 8-13 August.

[31] Versteeg, H.K. and W. Malalasekera. (1995) An Introduction to Computational Fluid Dynamics, Longman Group Ltd.

[32] http://www.cbe.berkeley.edu/underfloorair/casestudies.htm

[33] http://www.cbe.berkeley.edu/underfloorair/diffusers.htm 